Walter Kahlenborn, Jens Clausen, Siegfried Behrendt, Edgar Göll (Hg.)

AUF DEM WEG ZU EINER

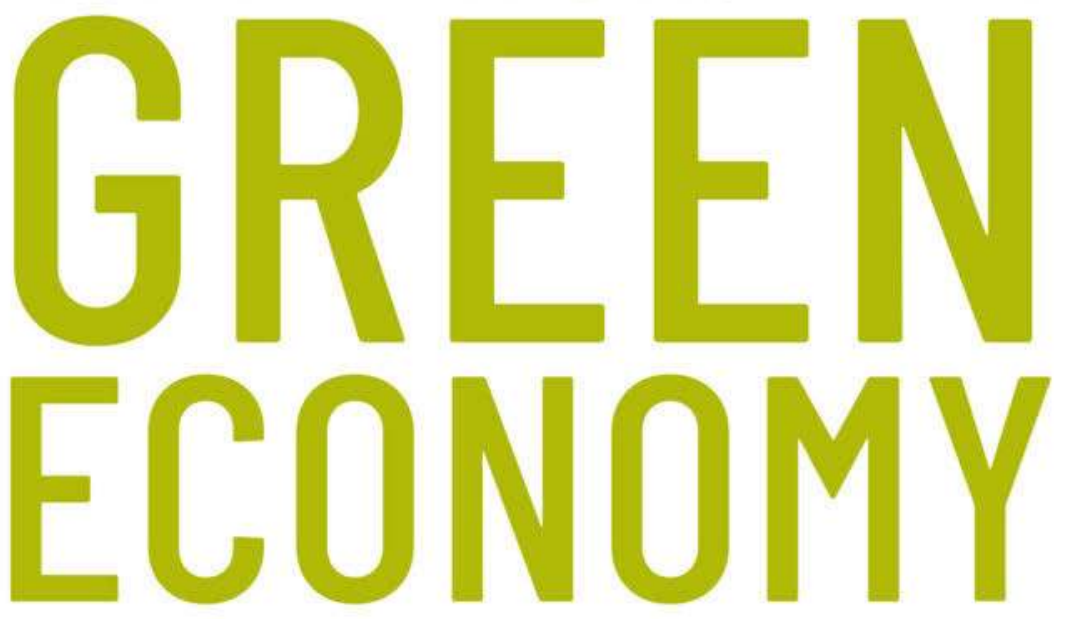

Wie die sozialökologische Transformation gelingen kann

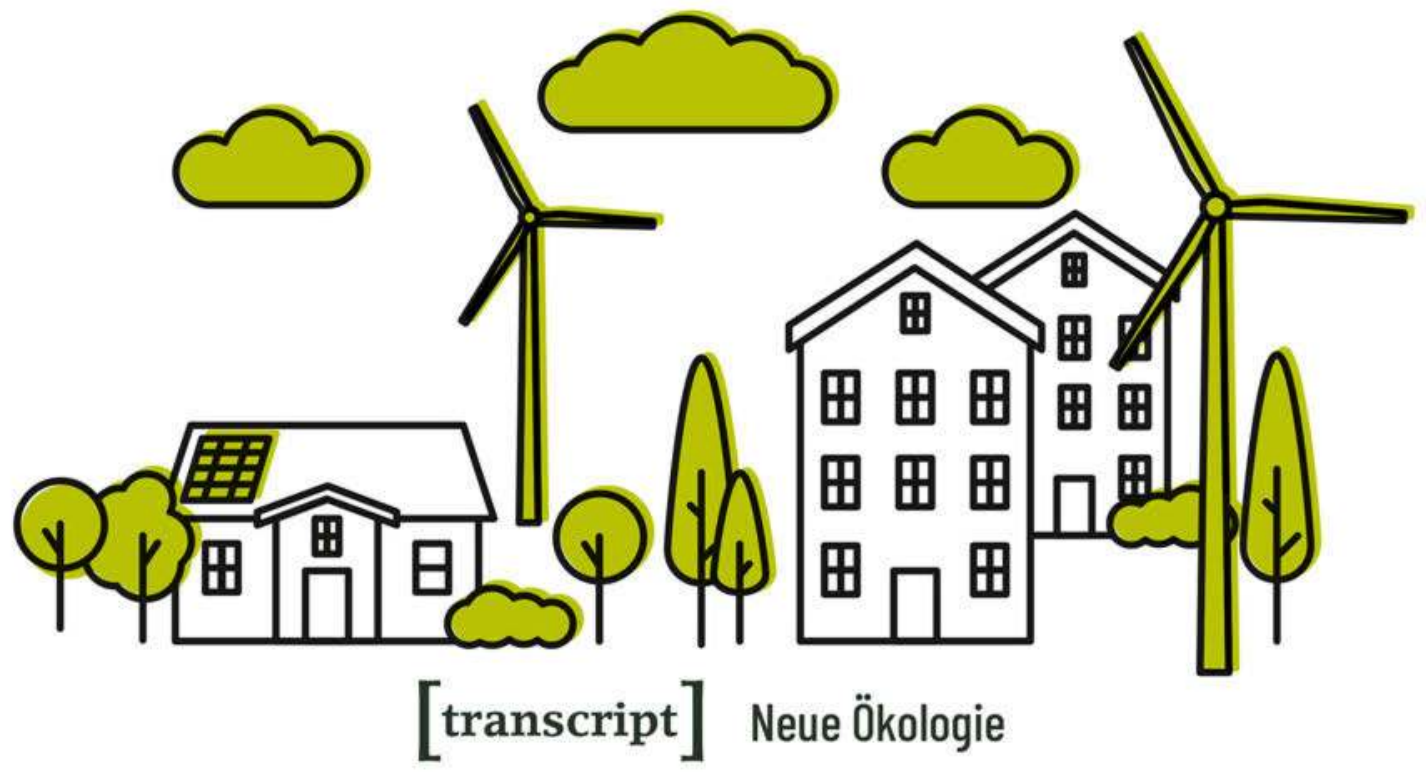


Walter Kahlenborn, Jens Clausen, Siegfried Behrendt, Edgar Göll (Hg.) Auf dem Weg zu einer Green Economy

Neue Ökologie | Band 3 
Walter Kahlenborn ist Mitbegründer und Geschäftsführer der Berliner Denkfabrik adelphi. Seit mehr als 20 Jahren leitet der Wirtschaftswissenschaftler Forschungs- und Beratungsprojekte in den Themenfeldern Green Economy, Nachhaltiges Wirtschaften sowie Klima und Energie. Er berät Regierungen und Nichtregierungsorganisationen, internationale Organisationen, die Europäische Kommission sowie Verbände und Unternehmen. Er ist Autor von mehr als 200 Büchern, Aufsätzen und Fachartikeln und gilt als einer der profiliertesten umwelt- und klimapolitischen Berater Deutschlands.

Jens Clausen(Dr.) ist Mitgründer des Borderstep Instituts. Der Diplomingenieur für Maschinenbau leitet als Senior Researcher das Borderstep Büro Hannover. In seinen Arbeiten beschäftigt er sich mit Gründungs-, Innovations- und Transformationsforschung. Sein besonderes wissenschaftliches Interesse gilt den Themen Wärme, Elektromobilität und Digitalisierung. Nach seinem Studium arbeitete er als Entwicklungsingenieur und Anwendungstechniker für die Continental AG. Von 1991 bis zur Gründung des Borderstep Instituts im Jahr 2005 war er als Senior Researcher am Institut für ökologische Wirtschaftsforschung im Forschungsfeld »Ökologische Unternehmenspolitik« in Berlin und Hannover tätig.

Siegfried Behrendt (Dr.) ist seit 1990 im Institut für Zukunftsstudien und Technologiebewertung (IZT) Berlin tätig. Der Politologe und Biologe leitet dort das Cluster »Technologie und Innovation«. Er lehrt außerdem als Dozent an der Beuth-Hochschule für Technik und an der Hochschule für Wirtschaft und Recht in Berlin die Fächer Ökologisches Wirtschaften, Industrielle Ökologie, Grüne Zukunftsmärkte und Öko-Controlling. Im Mittelpunkt seiner Forschungs- und Beratungstätigkeit steht die Früherkennung und Bewertung von Nachhaltigkeitschancen, Risiken und Nebenfolgen von Innovationen für sozial-ökologische Transformationsprozesse in Wirtschaft und Gesellschaft.

Edgar Göll (Dr.) ist seit 1995 im Institut für Zukunftsstudien und Technologiebewertung (IZT) in Berlin tätig und als Sozialwissenschaftler Forschungsleiter des Clusters »Zukunftsforschung und Partizipation«. Seine Arbeitsschwerpunkte sind Konzepte und Methoden der Zukunftsforschung, Nachhaltige Entwicklung, Governance und Partizipation. Er ist seit 2010 Dozent im Masterstudiengang »Zukunftsforschung« am Institut Futur der FU Berlin. 
Walter Kahlenborn, Jens Clausen,

Siegfried Behrendt, Edgar Göll (Hg.)

Auf dem Weg zu einer Green Economy

Wie die sozialökologische Transformation gelingen kann

[transcript] 


\section{(a) \\ adelphi}

\section{Bibliografische Information der Deutschen Nationalbibliothek}

Die Deutsche Nationalbibliothek verzeichnet diese Publikation in der Deutschen Nationalbibliografie; detaillierte bibliografische Daten sind im Internet über http://dnb.d-nb.de abrufbar.

\section{()) $(1) \Theta \Theta$}

Dieses Werk ist lizenziert unter der Creative Commons Attribution-NonCommercial-NoDerivs 4.0 Lizenz (BY-NC-ND). Diese Lizenz erlaubt die private Nutzung, gestattet aber keine Bearbeitung und keine kommerzielle Nutzung. Weitere Informationen finden Sie unter https://creativecommons.org/licenses/by-nc-nd/4.o/deed.de Um Genehmigungen für Adaptionen, Übersetzungen, Derivate oder Wiederverwendung $\mathrm{zu}$ kommerziellen Zwecken einzuholen, wenden Sie sich bitte an rights@transcript-verlag.de

Die Bedingungen der Creative-Commons-Lizenz gelten nur für Originalmaterial. Die Wiederverwendung von Material aus anderen Quellen (gekennzeichnet mit Quellenangabe) wie z.B. Schaubilder, Abbildungen, Fotos und Textauszüge erfordert ggf. weitere Nutzungsgenehmigungen durch den jeweiligen Rechteinhaber.

\section{(C) 2019 transcript Verlag, Bielefeld}

Umschlaggestaltung: Maria Arndt, Bielefeld

Satz: Michael Rauscher, Bielefeld

Druck: Majuskel Medienproduktion GmbH, Wetzlar

Print-ISBN 978-3-8376-4493-7

PDF-ISBN 978-3-8394-4493-1

https://doi.org/10.14361/9783839444931

Gedruckt auf alterungsbeständigem Papier mit chlorfrei gebleichtem Zellstoff. Besuchen Sie uns im Internet: https://www.transcript-verlag.de Bitte fordern Sie unser Gesamtverzeichnis und andere Broschüren an unter: info@transcript-verlag.de 


\section{Inhalt}

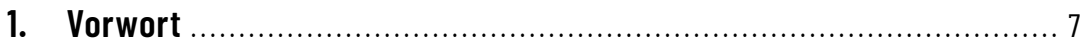

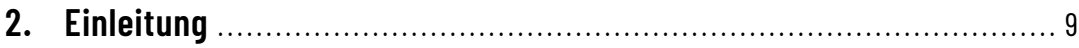

3. Theoretische Ansätze zum Verständnis von Transformationsprozessen in Richtung einer Green Economy .... 21

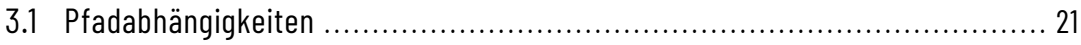

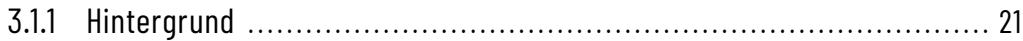

3.1.2 Pfadabhängigkeiten als Analyserahmen für »evolution2green« ............26

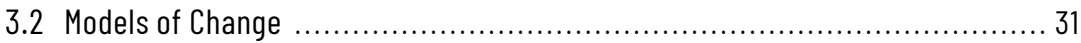

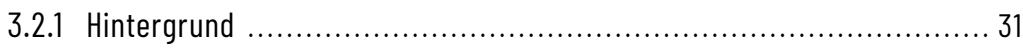

3.2.2 Der MoC-Ansatz als Analyserahmen für »evolution2green « ................ 36

3.3 Effizienz, Konsistenz und Suffizienz

als Basisstrategien einer Green Economy ........................................... 51

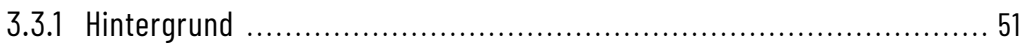

3.3.2 Effizienz, Suffizienz und Konsistenz als Analyserahmen

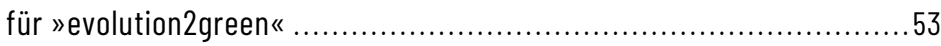

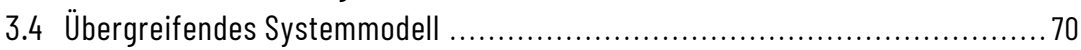

3.4.1 Die Analyseansätze im Überblick ............................................. 71

3.4.2 Konturen eines übergreifenden Systemmodells ..............................73

3.4.3 Verwendung im Rahmen des Buches ............................................. 75

4. Pfadabhängigkeiten, Hindernisse und Erfolgsfaktoren für Transformationsprozesse in Richtung einer Green Economy ....... 77

4.1 Pfadabhängigkeiten und Hindernisse aus 15 Transformationsfeldern in den Bereichen Wärme, Mobilität, Ressourcen und Landwirtschaft .............. 77

4.1.1 Die Auswahl der 15 Transformationsfelder ..................................... 77

4.1.2 Pfadabhängigkeiten in den vier Wendethemen .............................. 81

4.1.3 Pfadabhängigkeiten: Typen und Zusammenhänge ........................... 111

4.2 Erfolgsfaktoren aus 20 Transformationsbeispielen in den Bereichen

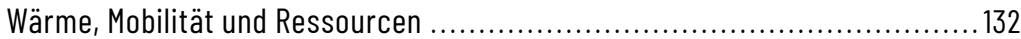

4.2.1 Transformationsbeispiele und Analysemethodik ............................133 


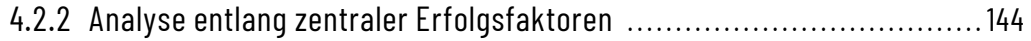

4.2.3 Analyse entlang unterschiedlicher Typen

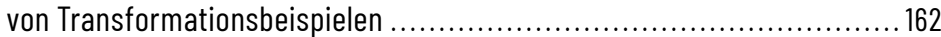

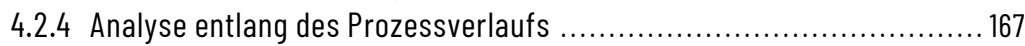

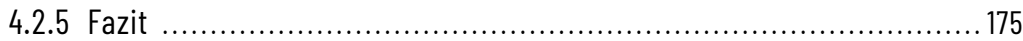

5. Den Pfadwechsel gestalten Handlungsansätze für die Wende in drei Transformationsfeldern ....183

5.1 Pfadwechselkonzeption für eine Green Economy .................................. 183

5.2 Roadmap für eine Wärmewende im Gebäudesektor ................................. 186

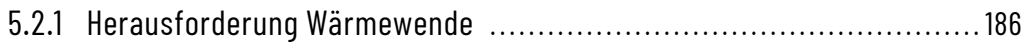

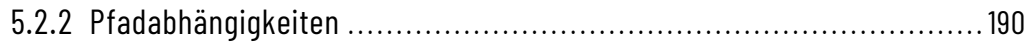

5.2.3 Den Pfadwechsel gestalten: Politische Maßnahmen und Initiativen ...... 197

5.2.4 Roadmap Wärmewende im Überblick ....................................... 206

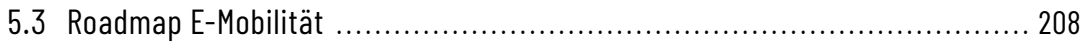

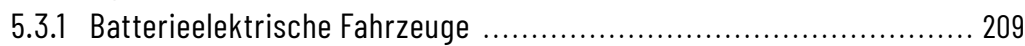

5.3.2 Beurteilung der alternativen Antriebe mit Blick auf das Jahr 2050 ....... 211

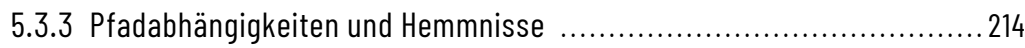

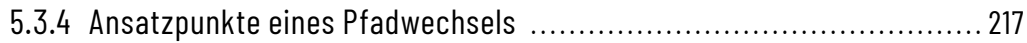

5.3.5 Maßnahmen und politische Initiativen ....................................... 219

5.3.6 Roadmap Elektromobilität im Überblick ....................................... 226

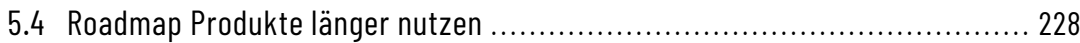

5.4.1 Das Problem der Obsoleszenz ................................................. 228

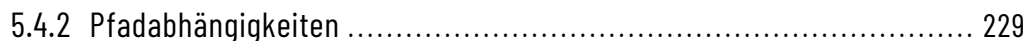

5.4.3 Herausforderungen und Maßnahmen für einen Pfadwechsel ............. 233

5.4.4 Akteure und Zeithorizont .................................................... 245

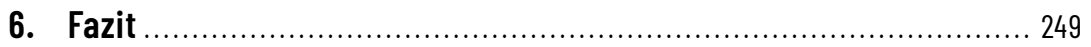

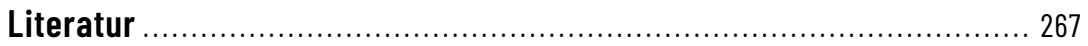

Autorinnen und Autoren der Kapitel .............................................. 299 


\section{Vorwort}

Die Relevanz eines Buches zu nachhaltigem Wirtschaften in Zeiten des Klimawandels ist offensichtlich: Die »Klimakrise« bestimmt zunehmend politische und gesellschaftliche Debatten. Doch so sehr sich die (meisten) Beteiligten über die Problemlage grundsätzlich einig sind, so schwierig gestaltet sich angesichts gesellschaftlicher Trägheiten und kurzsichtiger Einzelinteressen die Mobilisierung politischen Willens und die Ausformulierung eines kohärenten und ambitionierten Politikrahmens. Dabei zeigt insbesondere die »Klimakrise«, dass klassische Umweltpolitik an ihre Grenzen stößt. Denn Ausmaß und Charakter von Umweltproblemen haben sich in den letzten Jahrzehnten deutlich geändert: Sie sind globaler und systemischer Natur und ihre Lösung bedarf der Transformation ganzer gesellschaftlicher und wirtschaftlicher Systeme - hin zu einer Green Economy.

Sowohl Politik als auch Gesellschaft spielen in einer Green Economy wichtige Rollen, um den Wandel zu initiieren und voranzutreiben. Gleichwohl ist die Politik in der Verantwortung, durch entsprechende Reformmaßnahmen und Änderungen rechtlicher Rahmenbedingungen die notwendigen Gelegenheitsstrukturen und Anreize für nachhaltiges Handeln zu schaffen.

adelphi, das Boderstep Institut für Innovation und Nachhaltigkeit und das Institut für Zukunftsstudien und Technologiebewertung (IZT) haben sich intensiv mit dem Themenbereich »Green Economy« und den komplizierten Wegen dorthin auseinandergesetzt. Die gemeinsame Arbeit im Forschungsprojekt »evolutionzgreen « und das daraus entstandene vorliegende Buch haben gezeigt, dass viele der Erfolgs- wie auch der Hemmnisfaktoren von nachhaltigen Transformationen politischen Maßnahmen zugänglich sind.

Wie ein solcher Wandel aussehen kann, stellt dieses Buch vor. Im Rahmen des zugrunde liegenden Forschungsprojektes »evolutionzgreen« sind vielfältige Faktoren identifiziert worden, die für die Frage von Erfolg oder Misserfolg eines Transformationsprozesses von Bedeutung sind. Aus den verschiedenen Fallbeispielen sind zahlreiche Erkenntnisse erwachsen. Auf der Basis dieser Erkenntnisse ist es möglich, diverse Anregungen für die Gestaltung einer transformativen Umweltpolitik zu formulieren.

Wir möchten uns bei den Mitwirkenden von adelphi, Borderstep und dem IZT bedanken. Das gesamte Projekt hat enorm von der fachlichen Expertise, wis- 
senschaftlichen Neugier und dem unermüdlichen Einsatz der Kolleginnen und Kollegen profitiert. Insbesondere erwähnt werden sollen an dieser Stelle Valentin Tappeser, Daniel Weiss, Moritz Bursian und Jan Hefter, die die Erstellung des Buches betreut haben.

Beim transcript Verlag möchten wir uns für die gute Zusammenarbeit bedanken.

Dem Bundesministerium für Bildung und Forschung danken wir für das Vertrauen und die Förderung des Forschungsvorhabens »evolution2green«.

Ein ganz besonderer Dank gilt darüber hinaus dem Beirat des Forschungsvorhabens, bestehend aus: Dagmar Dehmer (Bundesgesellschaft für Endlagerung, vormals bei Der Tagesspiegel), Ansgar Gessner (German Marshall Fund), Professor Dr. Joseph Huber (emeritiert, Universität Halle), Dr. Kora Kristof (Umweltbundesamt), Professor Dr. Christa Liedtke (Wuppertal Institut für Klima, Umwelt, Energie), Hans Mönninghoff (Umwelt- und Wirtschaftsdezernent der Landeshauptstadt Hannover a. D.), Florian Nehm (Axel Springer), Dr. Steffi Ober (Naturschutzbund Deutschland), Dr. Joachim Spangenberg (UFZ Helmholtz Zentrum für Umweltforschung/Sustainable Europe Research Institute SERI Germany), Dr. Alfred Strigl (plenum).

Ein herzlicher Dank geht zudem an alle Interviewpartnerinnen und -partner sowie Teilnehmerinnen und Teilnehmer der Workshops, die sich Zeit für Gespräche im Rahmen des Forschungsvorhabens genommen haben.

Dem Öko-Institut danken wir für die gute Zusammenarbeit bei der gemeinsamen Abschlusskonferenz »transformation2green«. 


\section{Einleitung}

Schon seit mehreren Jahrzehnten werden die weitreichenden negativen Auswirkungen des menschlichen Handelns auf die globale Umwelt immer deutlicher. Der bereits spürbare Klimawandel, globale Umweltverschmutzung, Biodiversitätsverlust und steigender Ressourcenverbrauch sind nur einige Beispiele komplexer Problemlagen, denen die weltweite Umwelt- und Nachhaltigkeitspolitik zu begegnen versucht. Dabei werden die Rufe nach einer veränderten und besser integrierten Umweltpolitik immer lauter (BMUB 2016; Hendricks 2016). Als Hauptursache hierfür kann gesehen werden, dass die Folgen der menschlichen Präsenz auf das Erdsystem verstärkt zu spüren sind. Der menschliche Einfluss auf die globale Umwelt ist so ausgeprägt, dass von einer neuen geologischen Epoche gesprochen werden kann, dem »Anthropozän«, in der die Menschheit selbst zu einer globalen geophysikalischen Kraft wurde (Steffen et al. 2011).

Eine der Konsequenzen menschlichen Handelns ist der Klimawandel. Veränderte Niederschlagsmuster und Temperaturen sind weltweit zu beobachten und führen zu signifikanten Schäden durch Überflutungen oder Dürren, zu Ernteausfällen und dem Verlust von Ökosystemleistungen (IPCC 2014). Die zehn weltweit wärmsten Jahre seit Beginn der Temperaturaufzeichnungen wurden alle in der Periode seit 1998 gemessen (Blunden et al. 2018). Die globale Durchschnittstemperatur liegt nun etwa $1^{\circ} \mathrm{C}$ höher als noch in der vorindustriellen Zeit (IPCC 2018). Neben dem Klimawandel werden weitere durch den Menschen verursachte Umweltbelastungen, die ein gefährdendes Niveau erreicht haben, durch das Konzept der »Planetaren Grenzen« in den Fokus gerückt. Mit planetaren Grenzen werden ökologische Grenzen der Erde bezeichnet, bei deren Überschreitung gravierende ökologische Folgeveränderungen auf kontinentaler oder globaler Ebene drohen, mit potenziell katastrophalen Konsequenzen für die Menschheit (Rockström et al. 2009). Viele der Belastungsgrenzen sind bereits überschritten (Steffen et al. 2015).

Zwar konnten in der Vergangenheit verschiedene Umweltprobleme in Deutschland durch umweltpolitische Eingriffe signifikant reduziert werden. Als Beispiele lassen sich hier die Verbesserung verschiedener Aspekte von Luft- und Wasserqualität nennen. Der entscheidende Unterschied zu den aktuellen Herausforderungen besteht aber darin, dass diese Umweltprobleme relativ klar abzugrenzen 
waren, eine tendenziell leicht nachvollziehbare Auslöser-Wirkung-Beziehung aufwiesen und durch vergleichsweise einfache Regulierung einzudämmen waren.

Die heutige Umweltpolitik sieht sich zunehmend mit hochkomplexen beziehungsweise globalen Umweltproblemen konfrontiert, denen mit bestehenden umweltpolitischen Instrumenten nicht $\mathrm{zu}$ begegnen ist. Verschiedene aktuelle Problemlagen entziehen sich dem verfügbaren Regulierungsrahmen, sodass die Instrumente klassischer Umweltpolitik, wie etwa die einfache ordnungsrechtliche Festlegung von Grenzwerten, nicht die benötigten ökologischen Verbesserungen erzielen. Zum einen handelt es sich um Umweltprobleme, die wir aufgrund ihrer Komplexität bisher kaum in den Griff bekommen haben und bei denen das bisherige Instrumentarium auch schon im Ansatz nur wenige Lösungen bietet. Hierzu zählen insbesondere die zahlreichen und unkontrollierten Einträge von künstlichen Substanzen (Plastik, Pestizide, Pharmazeutika etc.) in die Umwelt. Zum anderen geht es um Umweltprobleme, die im Laufe der Jahre zwar immer weiter reduziert werden konnten, die jedoch deutlicher zusätzlicher Anstrengungen bedürfen - Anstrengungen, die kaum noch finanzierbar sind oder die für bestimmte Bevölkerungskreise zu gesellschaftlich nicht mehr tolerierten Nachteilen führen würden. Der Fall der Stickoxidbelastung der Atemluft ist hier ein Beispiel. Die Probleme bei der notwendigen Beschränkung von weiteren Stickstoffeinträgen aus der Landwirtschaft sind ein anderes Beispiel für diese Art von Umweltbelastungen. Eine dritte grundsätzliche Problemlage, die die Grenzen bisheriger Umweltpolitik aufzeigt, ist die umfassende Verlagerung von Umweltbelastungen aus Industrieländern in weniger entwickelte Länder, deren Regulierungssysteme und -fähigkeiten diesen Herausforderungen gar nicht gewachsen sind. Viele Umweltverbesserungen der letzten Jahre in Deutschland und Europa beruhen lediglich auf einer Verlagerung von Produktion oder Entsorgung und der damit verbundenen schädlichen Emissionen in andere Länder. Die Erfolge der nationalen bzw. europäischen Umweltpolitik sind hier teils (wenngleich nur in geringem Maße) sogar noch ursächlich für diese Verlagerungen. Die bisherigen Ansätze, wie die Förderung der Verbreitung von Umwelttechnologien, die Förderung von umweltrechtlichen Standards oder die Unterstützung beim Aufbau einer Umweltadministration in den betroffenen Ländern, haben die Situation bisher kaum verbessert.

Als vierte Problemklasse sind die Bereiche einzusortieren, bei denen Umweltbelastungen und Umweltfolgen einen globalen Maßstab haben. Hier wurden zwar im internationalen Rahmen vereinzelt erhebliche Erfolge erzielt, etwa beim Schutz der Ozonschicht. Die vielfache Überschreitung der schon erwähnten planetaren Grenzen verdeutlicht aber, dass noch erheblicher Handlungsbedarf besteht, der sich wiederum den bestehenden umweltpolitischen Ansätzen und Möglichkeiten oftmals entzieht. Die Erfolge und Misserfolge der internationalen Klimapolitik stehen hier stellvertretend für die verschiedenen globalen Umwelt- 
probleme, die in den nächsten Jahren und Jahrzehnten zunehmend greifbar werden.

Die Adressierung der genannten umweltpolitischen Herausforderungen, sei es der Klimawandel, seien es künstliche Stoffeinträge oder der zu hohe Ressourcenverbrauch, ist auch deshalb so komplex, weil dies ein Zusammenspiel und ein Verständnis unterschiedlicher Akteure, politischer Rahmenbedingungen, Marktund Infrastrukturen, Technologien und soziokultureller Praktiken erfordert. All diese Aspekte müssen im Zusammenhang gesehen und als System betrachtet werden, in dem Veränderungen in einem Bereich Auswirkungen auf andere Faktoren haben. Zielführend ist somit nur ein integrierter Ansatz, der Probleme nicht isoliert betrachtet, sondern in ständiger Interaktion mit unterschiedlichen Sektoren, Regierungsebenen sowie räumlichen und zeitlichen Dimensionen (DeFries et al. 2012). Die Kombination aus zunehmenden globalen und von hoher Komplexität geprägten Umweltproblemen mit nicht länger wirksamen umweltpolitischen Instrumenten zwingt dazu, neue Handlungsoptionen zu erarbeiten. Es reicht nicht mehr, dass die Umweltpolitik versucht, Umweltauswirkungen wirtschaftlicher oder gesellschaftlicher Aktivitäten mehr oder minder punktuell zu reduzieren. Die klassische Umweltpolitik ist hier an ihre Grenze gestoßen. Eine Umweltpolitik, die auf den Konzepten des letzten Jahrhunderts basiert, ist an ihrem Ende.

Notwendig ist eine Umweltpolitik, die eine Transformation von Wirtschaft und Gesellschaft initiiert, fördert und gestaltet; eine Umweltpolitik, die eine Transformation hin zu einer Green Economy leistet.

Sowohl der Begriff der "Transformation« als auch der der "Green Economy» ist schillernd. Beide sollen daher kurz angesprochen werden, weil sie die Basis für die weiteren Überlegungen darstellen.

In der Literatur werden die Wörter transformation und transition vor allem im englischsprachigen Raum häufig gleichbedeutend verwendet, wobei von einzelnen Wissenschaftlerinnen und Wissenschaftlern hier eine Unterscheidung vorgenommen wird (Geels und Kemp 2006). Im Folgenden wird keine Differenzierung der beiden Begriffe vorgenommen und Transformation als eine Veränderung der fundamentalen Eigenschaften eines Systems verstanden (IPCC 2012). Transformationsprozesse sind als langfristige Wandlungsprozesse zu sehen, denn sie umfassen weitreichende Veränderungen in verschiedenen gesellschaftlichen Teilbereichen: von Produktions- und Konsummustern über rechtliche Konzepte und Organisationsformen bis hin zu kulturellen Wertvorstellungen (Brand und Brunnengräber 2013; IPCC 2012). Insofern ist es präziser, von Transformationsprozessen zu sprechen, da es sich nicht um eine einzige Transformation handelt, sondern um parallel und auf unterschiedlichen Ebenen stattfindende Prozesse, die das gesamte Leben durchziehen.

Die wissenschaftliche Debatte um eine Transformation von Gesellschaft und Wirtschaft ist in ihren Grundlagen nicht neu. Sie findet ihren Ursprung in den 
Gedanken des Wirtschaftshistorikers Karl Polanyi. In seinem Hauptwerk »The Great Transformation - The Political and Economic Origins of Our Time« aus dem Jahr 1944 beschreibt Polanyi den gesamtgesellschaftlichen Wandel im 19. und 20. Jahrhundert und seine Ursachen, die er einerseits in der Industrialisierung beziehungsweise der Entwicklung der Dampfmaschine, andererseits in der parallelen Etablierung des Nationalstaatensystems sieht. Den politischen, ökonomischen und gesellschaftlichen Prozess, der zu tief greifenden Veränderungen führte, bezeichnet er als große Transformation (Polanyi 2001). Die Feudalgesellschaft wurde durch die Industriegesellschaft abgelöst. Dies veranschaulicht auch, dass Transformation per se nicht nachhaltig sein muss. Als zentrales Problem sieht Polanyi die Entfesselung der Wirtschaft aus der Gesellschaft und die daraus resultierenden Auswirkungen auf den Menschen und seine Arbeitskraft, die Umwelt und wirtschaftliche Stabilität. So schreibt er:

Unsere These ist die, dass die Idee des selbstregulierenden Marktes schlicht und einfach eine Utopie implizierte. Eine solche Institution könnte niemals bestehen bleiben, ohne die menschliche und natürliche Substanz der Gesellschaft gänzlich zu vernichten. (Übersetzung nach Polanyi [2001]: xxiv)

Laut Polanyi braucht es einen neuen Gesellschaftsvertrag, um moderne Industriegesellschaften zu stabilisieren. Ein solcher Gesellschaftsvertrag umfasst die Steuerung von Marktdynamiken, rechtsstaatliche und demokratische Prinzipien sowie »wohlfahrtsstaatliche Arrangements« (WBGU 2011).

In einem anderen Verständnis von Transformation (auch als societal transition bezeichnet; Feola 2015) sind bestehende Systeme Gegenstand der Betrachtung. Im Gegensatz zur Idee eines neuen Gesellschaftsvertrags wie bei Karl Polanyi besteht der Transformationsgedanke hier aus einem evolutionären Rekonfigurationsprozess: einem neuen Gleichgewicht aus Technologien, kulturellen Praktiken und Institutionen, welches durch unterschiedliche Innovationen in den verschiedenen Bereichen kreiert werden kann (Geels 2002; Jacob et al. 2015b). Diese Entwicklungen in Teilsystemen sind interdependent und koevolutionär. Der Endpunkt einer Transformation ist hier nicht ein anderer Gesellschaftsvertrag, sondern ein neues Gleichgewicht, das immer wieder von neuen, konkurrierenden Innovationen herausgefordert wird. Nischen, die Antrieb und einen schützenden Raum für die Entwicklung von Innovationen bieten, sind dabei von erheblicher Bedeutung (Kemp et al. 1998; Smith und Raven 2012). So wurde die Dampfmaschine beispielsweise ursprünglich dazu entwickelt, Wasser aus Minen zu pumpen. Aus dieser Nische heraus entwickelte sich die Dampfmaschine anschließend (mit anderen Faktoren) zu einem der Haupttreiber der Industrialisierung und zog eine Reihe von sozioökologischen Veränderungen nach sich. 
Im Kontext der Umweltpolitik findet der Transformationsbegriff in den letzten Jahren vermehrte Verwendung vor allem durch das Konzept der transformativen Umweltpolitik, das ja auch diesem Buch zugrunde liegt. Die Erkenntnis, dass einzelne Maßnahmen der Umweltpolitik in Zeiten globaler Umweltprobleme nicht mehr ausreichend sind, ist nicht neu. Young et al. (2006) prägten den Begriff der "gekoppelten sozialökologischen Systeme«, welcher die starken wechselseitigen Beziehungen und Abhängigkeiten der unterschiedlichen Bereiche voneinander unterstreicht. Kemp et al. (1998) erkannten, dass einzelne technologische Innovationen, z. B. die Entwicklung von Elektromotoren, für einen Wandel des Regimes hin zur Nachhaltigkeit nicht ausreichend sind. Das Konzept des strukturellen Wandels oder Systemwandels im Kontext der Nachhaltigkeit und die Frage, wie dieser zu erreichen sei, erhielten seitdem akademischen Aufschwung (Kemp et al. 1998; Geels 2002; Elzen und Wieczorek 2005; Geels und Kemp 2006). Obwohl die Bezeichnung der transformativen Umweltpolitik hier nicht explizit verwendet wurde, tritt immer wieder der Gedanke einer nachhaltigen Transformation in den Vordergrund. So wird eine nachhaltige Transformation (sustainability transition) als dauerhafte, radikale und sich gegenseitig verstärkende Veränderungen in den wirtschaftlichen, technologischen, institutionellen und sozio-kulturellen Domänen eines Systems mit gesellschaftlichen Funktionen definiert (Elzen und Wieczorek 2005). Dabei wird auch der Unterschied zwischen einzelnen inkrementellen Prozessen (z. B. einzelne technologische Innovationen, die individuell betrachtet keinen Wandel der Gesellschaft hervorrufen) und reeller Transformation betont.

Auch das Intergovernmental Panel on Climate Change (2014) hebt die Unterscheidung zwischen inkrementeller Anpassung und transformativer Anpassung hervor. Inkrementelle Anpassung wird hier als Set von Maßnahmen definiert, bei denen das zentrale Ziel der Erhalt der Integrität eines Systems oder Prozesses ist; transformative Anpassung hingegen verändert die fundamentalen Eigenschaften eines Systems. Wo einzelne Maßnahmen des Umweltschutzes an Grenzen stoßen, können transformative Maßnahmen zielführend sein (IPCC 2014).

Das Konzept einer sozialökologischen Transformation sieht es als langfristiges Ziel aktueller Transformationsvorhaben, einen neuen Weltgesellschaftsvertrag für eine klimaverträgliche und nachhaltige Wirtschaftsordnung zu schaffen (WBGU 2011; DeFries et al. 2012; Pelling 2011). Das Konzept stellt damit einen Lösungsansatz für heutige Umweltproblematiken dar, der über einzelne isolierte Anpassungsmaßnahmen hinausgeht.

Bei einer transformativen Umweltpolitik, die eine solche sozialökologische Transformation verfolgt, geht es um »den grundlegenden Umbau unserer Produktions-, Handels-, Dienstleistungs- und Konsummuster« (BMUB 2016, S. 27). Umweltpolitik soll nicht nur ihrem eigenen Zweck dienen, sondern stärker in unterschiedlichen Lebensbereichen verankert werden. Somit ist transformative Umweltpolitik 
als "Motor des Wandels« hin zu einer nachhaltigen Entwicklung zu verstehen (BMUB 2016, S. 15). Transformative Umweltpolitik ist der Versuch, transformatives Potenzial in einer Gesellschaft zu identifizieren und dieses im Sinne einer sozialökologischen Transformation auszuschöpfen, d.h. das Verhältnis der Menschen zueinander und zur Natur vermehrt in den Fokus zu rücken.

Das Transformationsverständnis im hier beschriebenen Kontext beinhaltet eine Anzahl von Facetten. Eine wichtige Variable des Transformationsverständnisses ist dabei die Rolle des Staates innerhalb des Transformationsprozesses und das Ausmaß, in dem der Staat über Eingriffs- und Gestaltungsmöglichkeiten verfügt. Neben den staatlichen Eingriffsmöglichkeiten beinhaltet eine transformative Umweltpolitik das Finden neuer gesellschaftlicher Organisationsformen und die Bildung neuer Allianzen, die eine sozialökologische Transformation gemeinsam gestalten und gesellschaftlich vorantreiben.

Eine wichtige Fragestellung ist somit die der möglichen Governance einer solchen Transformation. Nach Mayntz beschreibt der Begriff»Governance« auf der gesellschaftlichen Makro-Ebene »den Tatbestand der Kombination, des Nebenund Miteinanders verschiedener Arten kollektiver Regelung gesellschaftlicher Sachverhalte « (Mayntz 2008, S. 45). In den Kontext der sozialökologischen Transformation übersetzt bedeutet diese Perspektive, dass der Staat den Wandel nicht im Alleingang "von oben « verordnet, sondern dass Transformationsprozesse auch aus der Gesellschaft und aus der Wirtschaft heraus initiiert und gefördert werden (Benz und Dose 2010, S. 18). Burns beschreibt eine solche Form der Transformation als organic transformation. Der Wandel manifestiert sich hier durch kollektives Handeln einer Vielzahl von Akteuren, die autonome und dennoch ähnliche Entscheidungen auf lokaler, Meso- und Makroebene treffen. In Summe können diese Maßnahmen einen Paradigmenwechsel und eine neue Ordnung hervorbringen (Burns 2011, 99 ff.). Nach Benz und Dose können Transformationsprozesse somit als ein »Management von Interdependenzen« zwischen verschiedenen politischen, gesellschaftlichen und wirtschaftlichen Gruppen verstanden werden (Benz und Dose 2010, S. 18). Das Resultat eines solchen Prozesses ist die Veränderung bestehender Governance-Strukturen mit anderen, neuen Akteurinnen und Akteuren, Zielen und Methoden (Burns 2011, 99 ff.). Ein Beispiel hierfür ist Burns zufolge aktuell in der weltweiten sustainability revolution (Burns 2011, S. 102) zu beobachten, die er in Ausmaß und Bedeutung mit den Umbrüchen der industriellen Revolution vergleicht. Eine Charakteristik dieser Revolution ist die sogenannte "green governance«: Diese umfasst neue regulatorische Mechanismen, die zwischen guten - also grünen - und schlechten - also nicht-grünen - Innovationen und Entwicklungen unterscheiden. Auch werden zunehmend verschiedene Stakeholder in politische und wirtschaftliche Entscheidungsprozesse einbezogen. Das Erreichen von green governance erfordert hier eine Neuordnung bestehender Prioritäten und Governance-Strukturen (Burns 2011, $104 \mathrm{ff}$.). Die Aufgabe des 
Staates kann darin gesehen werden, die Interessen der verschiedenen Akteurinnen und Akteure, Netzwerke und Politikprozesse wahrzunehmen, zu koordinieren und zu moderieren (Benz und Dose 2010, S. 18; Loorbach 2010, S. 178).

Dabei bilden sich unterschiedliche Ansichten heraus, inwieweit der Staat in dieser Transformation eine Steuerungsfähigkeit ausüben kann. Eine Transformation wird vielfach als polyzentrischer Veränderungsprozess beschrieben und die Fähigkeit des Staates, diese Veränderungen gezielt zu koordinieren, in Frage gestellt. Colander und Kupers (2014) vertreten die Ansicht, dass der Ansatz einer Regierung, eine Transformation "von oben « einzuleiten, nicht zielführend ist. Der Staat müsse eine »moralische Stärke« (moral strength) besitzen, die die Menschen dazu ermutigt ihre Maßnahmen freiwillig und zur Steigerung des Allgemeinwohls zu koordinieren. Dies steht im Gegensatz zu »von oben« verordneten Zwangsmaßnahmen (erzwungen durch staatliche coercive strength), welche die Entstehung einer sozialen Kohäsion der Zivilgesellschaft und damit eine nachhaltige Transformation untergräbt. Der Staat kann auch als ein Verbund komplexer sozialer Beziehungen gesehen werden, in dem die mächtigen Interessensgruppen Verbindungen eingehen. Somit ist der Staat per se ein Resultat eines Machtungleichgewichtes und wird eine nachhaltige und sozial gerechte Transformation alleine nicht zielführend einleiten können und wollen (Brand 2013). Allerdings birgt auch der Aufruf zur gemeinschaftsbasierten (community-based) Transformation Gefahren, da hier der »Rückzug« des Staates aus der Verantwortung der Governance nicht zwingend in einem Erstarken der Gesellschaft resultiert. Es besteht das Risiko, dass nun die freie Marktwirtschaft zum Haupttreiber der Transformation wird und damit die Ziele der Nachhaltigkeit nicht erfüllt werden können (Avelino und Wittmayer 2016). Zum Transformationsverständnis einer transformativen Umweltpolitik gehören somit verschiedene Akteurinnen und Akteure, Einflüsse und Regelungsebenen, die in der Gesamtheit die Governance einer Transformation bilden. Der Staat kann hier aber durchaus eine gestaltende Rolle einnehmen. Für die politischen und staatlichen Eingriffsmöglichkeiten ist gleichzeitig relevant, dass Transformationsprozesse von hoher Komplexität und einem nicht-linearen Ablauf geprägt sind. Somit kann kein chronologisch ablaufender Maßnahmenkatalog definiert werden. Nach Kristof sind Transformationen aus diesem Grund nicht unmittelbar steuerbar (Kristof 2010b).

Neben der Frage der Steuerungsmöglichkeiten und der Steuerbarkeit von Transformationsprozessen durch staatliche Akteure ist eine weitere zentrale Frage für eine transformative Umweltpolitik die des Ziels der Transformation.

Das Umweltbundesamt definiert dies als »Vorbeugung weiterer Umweltzerstörung, indem systemische Ursachen adressiert werden oder ohnehin stattfindende Wandlungsprozesse genutzt werden" (Wolff et al. 2018, S. 5). Unter welchen Umständen diese Zielsetzung erreicht wird, hängt davon ab, welcher Ansatz und welches Verständnis einer transformativen Umweltpolitik angewendet wer- 
den. So ist das konkrete Ziel des deutschen Klimaschutzplans 2050 eine Transformation hin zu einer weitgehend treibhausgasneutralen Wirtschaft und Gesellschaft bis Mitte des Jahrhunderts unter Nutzung von Chancen für Wohlstand, Beschäftigung, Umweltschutz und Innovation (BMUB 2016).

Wie bereits angedeutet, bestehen verschiedene Zielverständnisse des Konzepts einer transformativen Umweltpolitik. Auf einer grundlegenderen Ebene kann man drei Zielvorstellungen voneinander unterscheiden. Einen Ansatz für einen gesamtgesellschaftlichen Wandel zu einer nachhaltigen Entwicklung stellt das Postwachstumskonzept dar, im englischen Sprachraum auch als degrowth bezeichnet. Der Ansatz strebt grundlegende Veränderungen des derzeitigen wirtschaftlichen und sozialen Systems an und basiert dabei auf einer Abkehr vom Paradigma des kontinuierlichen wirtschaftlichen Wachstums und teils mehr explizit, teils mehr implizit auf einer Verringerung der Wirtschaftsleistung. Dabei steht der Widerspruch zwischen Nachhaltigkeit und wirtschaftlichem Wachstum im Zentrum (Jackson 2009). Die Postwachstumsdebatte widmet sich der Frage, wie Wohlstand (well-being) erreicht werden kann, ohne dabei das monetär oder materiell gemessene Wohlergehen (well-having) zu priorisieren (Latouche 2010, S. 52; Schulz und Affolderbach 2015, S. 7). So soll der aktuelle Wachstumszwang, von dem unsere Wirtschaft geprägt ist, überwunden werden und zugunsten einer Gesellschaft weichen, die u. a. auf einer gerechteren Verteilung von Einkommen und Ressourcen sowie einer größeren Suffizienz beruht (Asara et al. 2015). Dies setzt eine tiefgreifende sozioökonomische Transformation der Gesellschaft voraus, in der Wohlstand nicht mehr auf materiellem Reichtum und Konsum basiert (Kallis et al. 2012).

In klarer Abgrenzung dazu hat sich der Begriff des "green growth « etabliert: Ursprünglich als Antwort auf die wirtschaftlichen Krisen der 2000er-Jahre konzipiert, wird green growth als das Streben nach Wirtschaftswachstum bei gleichzeitiger Erhaltung ökologischer Nachhaltigkeit definiert (Allen und Clouth 2012, S. 33). Hier steht also nach wie vor quantitativ messbares Wachstum im Vordergrund, im Gegensatz zum Fokus auf eine sinkende wirtschaftliche Leistung und damit geringeren materiellen Wohlstand des Degrowth-Ansatzes. Der GreenGrowth-Ansatz ist somit durch einen wesentlich geringeren Transformationscharakter geprägt, als dies beim Postwachstumskonzept der Fall ist. So legt die Green-Growth-Strategy der OECD (2011) ihren klaren Schwerpunkt auf wirtschaftliches Wachstum (innerhalb des bestehenden Systems) durch ökologische Modernisierung. Eine wichtige Rolle spielen hierbei Investitionen und Innovationen, die nachhaltiges Wachstum untermauern und zu neuen wirtschaftlichen Chancen führen (OECD 2011). Das Ziel ist ein kontinuierliches Wirtschaftswachstum bei gleichzeitiger Schonung natürlicher Ressourcen und Gewährleistung von Nachhaltigkeit. 
Einen Mittelweg zwischen diesen beiden Konzepten stellt das Leitbild einer »Green Economy« dar, mit dem ein Konzept einer Transformation der Gesellschaft und Wirtschaft Verbreitung fand, das einen weniger drastischen Einschnitt in das wachstumsbasierte Wirtschaftsmodell vorsieht als der Postwachstumsansatz. Gleichzeitig beschreibt es klar die Notwendigkeit einer Transformation des bestehenden sozio-ökonomischen Systems, in Abgrenzung zum Konzept des "green growth«. Jedoch wird wirtschaftliches Wachstum in einer Green Economy nicht als fundamental unvereinbar mit der Beachtung der ökologischen Belastungsgrenzen der Erde angesehen (Diyar et al. 2014; Hamdouch und Depret 2010). Das Konzept der »Green Economy« hat sich mittlerweile als Leitbegriff in internationalen Nachhaltigkeitsdebatten etabliert und ist zum festen Bestandteil internationaler und nationaler Nachhaltigkeitsstrategien geworden (UNEP 2011; ICC 2012; Europäische Kommission 2019). So definiert das Umweltprogramm der Vereinten Nationen (UNEP) eine Green Economy als eine Wirtschaft, die menschliches Wohlergehen und soziale Gerechtigkeit steigert und gleichzeitig Umweltrisiken und Ressourcenknappheit entgegenwirkt. Im Speziellen bedeutet dies einen Wandel zu einer kohlenstoffarmen, ressourceneffizienten und sozial integrativen Wirtschaft (UNEP 2011). Für den Wandel zu einer Green Economy besteht die Rolle der Politik darin, durch entsprechende Reformmaßnahmen und Änderungen rechtlicher Rahmenbedingungen die notwendigen Anreize zu schaffen. Damit einher geht ein kultureller Wandel hin zu einer Gesellschaft, die einerseits den Wert von biologischer Vielfalt und Ökosystemleistungen als wichtiges Wirtschaftsgut erkennt, andererseits eine Erhaltung dieser Faktoren in ihrem eigenen Interesse sieht (Renault und Schwietring 2016; UNEP 2011). Als eine von drei zentralen Erkenntnissen argumentiert UNEP, dass eine Green Economy mittelfristig neben Zugewinnen in Gemeingütern und Naturkapital auch ein höheres BIP-Wachstum hervorbringt (UNEP 2011, $23 \mathrm{ff}$.). Somit ist Wirtschaftswachstum weiterhin ein Bestandteil einer grünen Wirtschaft. Jedoch wird von einem neuen ökonomischen Paradigma gesprochen, in dem materieller Wohlstand nicht mit erhöhten Umweltrisiken und ökologischer Knappheit einhergeht (UNEP 2011). Während die Green-Growth-Strategie der OECD weniger konkrete Zielsetzungen aufweist und Wirtschaftswachstum mit Klimaschutz $\mathrm{zu}$ vereinbaren versucht, um kostenintensiver Umweltzerstörung und ineffizientem Ressourcenverbrauch vorzubeugen, verfolgt der Ansatz der Green Economy das Ziel, nicht zwingend Wirtschaftswachstum, sondern Wohlbefinden (well-being) und soziale Gerechtigkeit zu verbessern, wobei Umweltrisiken und ökologischem beziehungsweise Ressourcenmangel entgegengewirkt wird (UNEP 2011).

Die vorliegende Buchpublikation folgt den hier dargelegten Überlegungen zum Transformationsverständnis und zum Verständnis einer Green Economy. Die Publikation geht, wie erwähnt, von einem breit gefassten Transformationsbegriff aus und stellt die Frage nach der Rolle des Staates im Transformationspro- 
zess in den Fokus, ohne die anderen Akteure dabei zu vernachlässigen. Das Buch möchte einen Beitrag leisten zu der Frage, wie Deutschland am ehesten das Ziel einer Green Economy erreichen kann. Es fußt dabei auf einem Forschungsvorhaben, dass vom Bundesministerium für Bildung und Forschung (BMBF) unterstützt wurde: »evolution2green « (e2g, https://www.evolution2green.de/).

Das BMBF hat bereits vor vielen Jahren in seiner Forschungsplanung die Transformation zu einer Green Economy aufgegriffen. Das Forschungsministerium möchte den Wandel hin zu einer nachhaltigen Wirtschaft und Gesellschaft unterstützen und hat diese Absicht in verschiedenen Strategien verdeutlicht. Im Rahmen seiner Forschungsförderung zur Sozial-ökologischen Forschung wurde speziell ein Schwerpunkt ergänzt, der den Übergang Deutschlands hin zu einer Green Economy unterstützen soll. Dabei wird Green Economy ganz im Sinne des zuvor Gesagten als die Verbindung von wirtschaftlicher Wettbewerbsfähigkeit mit Umwelt- und Naturschutz sowie mit Inklusion und Gerechtigkeit verstanden.

Teil der entsprechenden Forschungsagenda ist die Fördermaßnahme "Nachhaltiges Wirtschaften«, mit der das BMBF 30 Forschungsprojekte unterstützt hat (vgl. https://nachhaltigeswirtschaften-soef.de), die »Entwicklungsperspektiven für eine Wirtschaft aufzeigen, die Nachhaltigkeit in all ihren Dimensionen berücksichtigt « (BMBF 2018). Durch diese Förderung wurden unter anderem Forschungsvorhaben unterstützt, die eine systemische Perspektive einnehmen und „Vorschläge für die Rahmenbedingungen einer Green Economy« erarbeiten (BMBF 2018b). Spezifisch war es auch das Ziel, Antworten auf die Frage zu erhalten, wie gesellschafts- und wirtschaftspolitische sowie sonstige Politikinstrumente ausgestaltet sein müssen, um eine Green Economy schrittweise zu erreichen.

An dieser Stelle setzt »evolutionzgreen« an. Das Forschungsvorhaben wurde von den drei Forschungsinstituten adelphi, Borderstep und Institut für Zukunftsstudien und Technologiebewertung konzipiert und durchgeführt. Das Ziel des Projektes bestand in einem wissenschaftlich fundierten Erkenntnisgewinn über Transformationspfade hin zu einer Green Economy und der Gestaltung von Pfadwechseln. Diese Erkenntnisse tragen dazu bei, eine umweltpolitische Gesamtstrategie für einen Pfadwechsel hin zu einer Green Economy und einer Transformation des derzeitigen Gesellschafts- und Wirtschaftssystems zu entwickeln.

Aufbauend auf konzeptionellen Vorarbeiten setzte sich das Projekt zusammen aus 15 Transformationsfeldanalysen, 20 Fallbeispielen erfolgreicher Transformationen, drei Zukunftswerkstätten, mehreren Roadmaps für den Wandel ausgewählter Handlungsfelder und einer abschließenden Auswertung der verschiedenen Teilergebnisse.

Die vorliegende Publikation bündelt die Kernergebnisse aus dem Projekt »evolutionzgreen« und stellt die vielfältigen Anknüpfungspunkte für eine aktive Transformationsgestaltung dar. Sie setzt sich zusammen aus drei Teilen: $\mathrm{Zu}$ nächst werden unterschiedliche konzeptionelle Ansätze vorgestellt, die einen 
Analyserahmen für ein umfassenderes Verständnis von Transformationsprozessen bilden. Ein erster Schwerpunkt liegt dabei auf Pfadabhängigkeiten, die häufig ein entscheidendes Hindernis für erfolgreiche Transformationen sind. Ein zweites Konzept besteht im Models-of-Change-Ansatz (MoC-Ansatz), der hier weiterentwickelt wird. Die transformatorischen Leitstrategien »Effizienz«, »Konsistenz « und »Suffizienz « werden als dritter Ansatzpunkt vertieft betrachtet. Aus den verschiedenen konzeptionellen Ansätzen wird ein übergreifendes Systemmodell entwickelt, das in den weiteren Kapiteln als Analyserahmen sukzessive zur Anwendung kommt.

Der Analyserahmen dient im zweiten Teil der Publikation einer Analyse und Diskussion von Hindernissen und Erfolgsfaktoren für Transformationsprozesse in Richtung einer Green Economy. Dabei werden zunächst die Ergebnisse von 15 Transformationsfeldanalysen auf Basis des Pfadabhängigkeiten-Ansatzes dargestellt. Die zugehörigen Fallbeispiele wurden in vier Themenfeldern ausgewählt, in denen ein besonderer Transformationsbedarf besteht: Pkw-Mobilität, Landwirtschaft und Ernährung, Wärmeversorgung und Rohstoffversorgung. Für diese vier Themenbereiche werden die relevanten Pfadabhängigkeiten und mögliche Transformationsansätze erläutert. In weiteren 20 Fallstudien wurden im Rahmen des Vorhabens die Prozesse und Mechanismen erfolgreicher Transformationen unter Anwendung des Models-of-Change-Ansatzes untersucht. Die Resultate dieser Analyse werden ebenfalls im zweiten Teil der Publikation im Rahmen einer Querschnittsanalyse dargestellt, aus der zentrale Erfolgsfaktoren für Transformationsprozesse abgeleitet werden.

Der dritte Teil der Publikation beschäftigt sich mit der praktischen Anwendung der gewonnenen Erkenntnisse und einer hierauf basierenden Pfadwechselkonzeption. In Form von Roadmaps werden umfassende Strategien für einen Pfadwechsel in den drei Transformationsfeldern E-Mobilität, Wärmewende und der längeren Nutzung von Produkten entwickelt. Sie geben konkrete Handlungsempfehlungen, wie innerhalb dieser Bereiche die Transformation zu einer Green Economy gelingen kann. Im abschließenden Fazit werden die Implikationen der im Rahmen des Projektes erlangten Erkenntnisse für die Politikgestaltung diskutiert. Anhand der identifizierten Erfolgsfaktoren, Hemmnisse und Handlungsansätze für die Gestaltung einer Transformation werden Steuerungsmöglichkeiten für die Politik abgeleitet. 



\section{Theoretische Ansätze zum Verständnis von Transformationsprozessen in Richtung einer Green Economy}

\subsection{Pfadabhängigkeiten}

\subsubsection{Hintergrund}

Viele Arbeiten in der Innovationsforschung nutzen das Konzept der Pfadabhängigkeiten, um die Tatsache zu erklären, dass Veränderungen oft schwer zu erreichen sind, vor allem in Bezug auf die Transformation zu einer Green Economy. Die überwiegende Mehrheit der Beiträge erklärt Pfadabhängigkeiten als vorwiegend technisches Phänomen. Die QWERTY-Tastatur (David 1985; Liebowitz und Margolis 1995) und die Videokassettensysteme Betamax und VHS (Vergne und Durand 2010) werden häufig als Beispiele herangezogen. Pfadabhängigkeiten gehen jedoch weit über technische Lock-ins hinaus. Das Projekt »evolution2green« hat u. a. zum Ziel, wesentliche Pfadabhängigkeiten als Hindernisse für die Transformation zu analysieren und dabei einen besonderen Schwerpunkt auf sozioökonomische Pfadabhängigkeiten in 15 verschiedenen Transformationsbereichen in Deutschland zu setzen.

In einem routinemäßigen Pfad bestehen in der Regel beträchtliche Bindungen, die ihn über lange Zeiträume stabilisieren und ihn resistent gegen Veränderungen jeglicher Art machen können. Eine Abweichung vom bestehenden Pfad ist schwierig und konkurrierende Pfade können ausgeriegelt werden. Die Evolutorische Ökonomik greift das Konzept der Pfadabhängigkeiten auf, um sowohl die Bindungen als auch die Kräfte, die Alternativen ausschließen, zu analysieren und zu erklären (Nelson und Winter 1982; Nelson 1987). In diesem Zusammenhang differenzieren Gavetti und Levinthal (2000) einerseits rückwärtsgerichtete Dynamiken, die auf Erfahrungen aufbauen, und andererseits Dynamiken, die nach vorn gerichtet sind und von kognitiven Annahmen unterstützt werden (Gavetti und Levinthal 2000; Gardini et al. 2009).

Nill (2009; Nill et al. 2005) unterscheidet zwischen Lock-ins im Wettbewerb einer etablierten Technologie und einer neuen Lösung (»Alt-neu-Wettbewerb«) einerseits und im Wettbewerb zwischen zwei neuen Lösungen (»Neu-neu-Wett- 
bewerb«) auf der anderen Seite. Bei Pfadabhängigkeiten im Falle konkurrierender neuer Technologien wurden von Arthur (1989) increasing returns gefunden, also Skalen- und Lerneffekte sowie Vorteile durch bessere Koordination und Netzwerke, sogar im Falle nicht unbedingt überlegener Alternativen. Durch kontingente Ereignisse kann dabei eine der Alternativen einen ersten Vorteil erlangen und später den Markt dominieren. Andere Autoren untersuchen Pfadabhängigkeiten im Hinblick auf konkurrierende neue Technologien in der Phase der Nischenbildung (Nill 2009) und im Nischenmanagement (Kemp 1994).

Vergne und Durand führen eine formale Definition der Pfadabhängigkeit ein, die zwischen Pfadabhängigkeit und anderen Mechanismen, die die Bedeutung von Geschichte einbeziehen, unterscheidet. Fälle von Pfadabhängigkeit werden hier mit stochastischen Prozessen, Kontingenzereignissen, selbstverstärkenden Mechanismen und Lock-ins verknüpft (Vergne und Durand 2010, S. 741). Garud et al. (2010) diskutieren und verwerfen Vergne und Durand systematisch und eröffnen damit den Raum für eine Diskussion über Mechanismen der Pfadkreation. Es muss aber angemerkt werden, dass der Prozess, den sie als Pfadkreation beschreiben (Garud et al. 2010; Karnøe und Garud 2012), deutliche Überschneidungen mit der Entrepreneurship-Forschung (Casson 1982; Schumpeter 1983) sowie mit Arbeiten zu sozialen Netzwerken von Entrepreneuren aufweist (Aldrich und Zimmer 1986; Jarillo 1988). Weiter sind Parallelen zur Clusterforschung (KärcherVital 2002; Porter 1998) festzustellen.

Mit Blick auf die Praktikabilität und die politische Relevanz des Konzepts der Pfadabhängigkeit ist diese hier wie folgt sehr breit definiert:

Der Begriff Pfadabhängigkeit beschreibt, dass ein Ereignis von vorangegangenen Ereignissen oder bislang üblichen Denk- und Verhaltensweisen abhängig ist. Das Konzept der Pfadabhängigkeit besagt allgemein, dass eine kausale Wirkung von früheren Ereignissen der Ereigniskette A, B, C, D, E ... auf spätere vorliegt. (Clausen et al. 2011)

Bei dieser Definition wurde berücksichtigt, dass die hierauf basierende Analyse Pfadabhängigkeiten mit Schwerpunkt auf dem »Alt-neu-Wettbewerb« identifiziert, wobei im Allgemeinen eine alte, nicht nachhaltige Technologie oder Lösung mit einer neuen, nachhaltigeren Technologie oder Lösung konkurriert. Die Definition ist viel breiter als die, die z. B. von Vergne und Durand genutzt wurde und bringt damit die Gefahr mit sich, auch Effekte zu umfassen, die andere allgemein als Hemmnisse beschreiben könnten.

Damit stellt sich die Frage, wie ein Hemmnis gegenüber einer Pfadabhängigkeit abgegrenzt werden kann. Hierzu wäre zunächst das Hemmnis zu definieren: 
Ein Hemmnis oder Hindernis ist etwas, was sich hemmend oder erschwerend auf die (erwünschte) Entwicklungsrichtung eines Prozesses auswirkt, unabhängig davon, ob es historisch bedingt (Pfadabhängigkeit) oder ein Faktor der aktuellen Kontextbedingungen ist.

Sowohl aktuelle Kontextbedingungen als auch eine Pfadabhängigkeit können sich also erschwerend auf einen Prozess auswirken. Das Spezifikum der Pfadabhängigkeit liegt darin, dass die Hemmung historischer Natur, also von vorangegangenen Ereignissen oder bisher üblichen Denk- und Verhaltensweisen abhängig ist. Es mag zur Abgrenzung der Begriffe weiterführen, den in den Transformationsfeldstudien gefundenen Pfadabhängigkeiten eine Reihe von Hemmnissen gegenüberzustellen, die z. B. als wesentliche Faktoren für die (Nicht-)Durchführung von Umweltmaßnahmen in Unternehmen identifiziert wurden (Hitchens et al. 2003, $65,107,151,224)$, die also letztlich die Diffusion von Umweltinnovationen hemmen:

- Schwierigkeiten in der Beschaffung von Kapital

- Zu lange Amortisationszeit von möglichen Investitionen

- Mangel an qualifiziertem Personal

- Unsicherheiten in der Anwendung neuer Technologien

- Andere Prioritäten des Managements

- Mangel an Managementzeit

- Dominanz des Profitmotivs über das Umweltschutzmotiv

- Mangel an qualifizierten Zulieferern oder Beraterinnen und Beratern

Die meisten dieser Hemmnisse sind nicht unmittelbar durch Pfadabhängigkeiten begründet. Nur der Mangel an qualifiziertem Personal, Zulieferern und Beraterinnen und Beratern ist ohne einen historischen Vorlauf nicht erklärbar. Die anderen Hemmnisse begründen sich primär durch Sichtweisen auf die Gegenwart oder Zukunft, Prioritäten oder Meinungen und lassen sich durchaus klar von Pfadabhängigkeiten abgrenzen.

D'Este et al. (2012) kategorisieren ein ähnliches Spektrum an Hemmnissen, wieder mit dem Fokus auf Unternehmen. Sie unterscheiden Kostenfaktoren (hohe ökonomische Risiken, zu hohe Innovationskosten, Kosten und Verfügbarkeit der Finanzierung), Wissensfaktoren (Mangel an qualifiziertem Personal sowie an Wissen über Technologie und Markt), Marktfaktoren (dominierende etablierte Unternehmen, Unklarheiten bezüglich der Nachfrage) und regulative Faktoren (Notwendigkeit der Erfüllung von nationalen und EU-Regeln). Mit Bezug auf Pfadabhängigkeiten ist in den von D'Este aufgeführten Hemmnissen auf den Mangel an qualifiziertem Personal und Wissen wie auch auf die Dominanz etablierter Firmen im Zielmarkt hinzuweisen. Bei den anderen untersuchten Hemmnissen kann der Charakter einer Pfadabhängigkeit auch hier nicht erkannt werden. 
In der Literatur, die sich mit Pfadabhängigkeiten beschäftigt, werden verschiedene Arten von Rückkopplungsschleifen und selbstverstärkenden Effekten diskutiert (Lehmann-Waffenschmidt und Reichel 2000, S. 349). In vielen Fällen wird dabei keine systematische Unterscheidung zwischen verschiedenen Arten von Pfadabhängigkeiten gemacht. Dies wäre aber wichtig, um Chancen für bewussten Wandel und Innovation besser zu erkennen und zu verstehen. Ein interessantes Beispiel eines differenzierteren systematischen Ansatzes findet sich bei Nill (2009, S. 138), der sechs Arten von Lock-in-Verstärkern unterscheidet:

»1. Irreversible Investitionen in die etablierte Technologie,

2. Wechselkosten aufgrund von technischen Komplementaritäten,

3. Unsicherheit über die Güte der neuen Technologien,

4. set-up<-Kosten der neuen Technologie,

5. Koordinationsprobleme seitens der Akteure, die die neuen Technologien vorantreiben sowie

6. Institutionelle Wechselhemmnisse.«

Unruh (2000) setzt Pfadabhängigkeit in den Kontext der Entwicklung technologischer Systeme. Lock-in entsteht bei ihm, wenn zusammenhängende Komponenten eines Systems einen hohen Wert für die Anwenderinnen und Anwender sowie für Produzenten entwickeln und sich in spezifischen und oft sehr stabilen Produktions- und Konsumverhältnissen ausdrücken. Als Beispiel für ein technisches System erwähnt Unruh (2000, S. 822) Autos und den Individualverkehr einschließlich der Zulieferindustrie, der Erdölproduktion und -verteilung, der Kautschukherstellung und des Straßenbaus. Er beschreibt so ein umfängliches System, das größer ist, mehr Leistung erbringt und schwieriger zu ändern ist als eine einzelne Technologie. Die Langzeitstabilität eines solchen Systems wird dadurch gestärkt, dass Unternehmen an die Entwicklung des dominanten Designs gebunden sind und sich so auf inkrementelle Innovationen konzentrieren, um ihre Produkte zu optimieren. Diese Unternehmen sind selten die Quelle radikaler Innovationen. Da etablierte Unternehmen in der Regel höhere Gewinne und Cashflows generieren als mögliche neue Wettbewerber mit neuen (nachhaltigen) Produkten, haben sie einen besseren Zugang zu Kapital, um ihre Investitionen zu finanzieren und so die Lock-in-Bedingungen weiter zu verschärfen. Zusätzlich zu den so entstehenden Unterschieden in der Verfügbarkeit von endogenem Kapital folgt die Verfügbarkeit externer Finanzierungen der gleichen Dynamik. Finanzinstitute sind generell in ihrer Kreditvergabe risikoavers und vergeben Kredite häufiger an etablierte Unternehmen als an Wettbewerber mit neuen und weniger bewährten Produkten (Unruh 2000, S. 823). Wenn das System an Größe wächst, entstehen Industrieverbände und Gewerkschaften, die ihre Interessen bündeln. Die Anwenderinnen und Anwender sind ebenso in das System eingebunden, da 
sich der hohe praktische Nutzen der Systemdienste in Verhaltensinstitutionen und sozialen Normen manifestiert. Die wichtigsten Arten von Pfadabhängigkeiten, die Unruh sieht, sind (Unruh 2000, S. 822-823):

- Ein erfolgreiches technisches System, das sich im Laufe der Zeit entwickelt hat und physische, soziale und informative Elemente umfasst

- Produzenten des dominanten Designs, die ihre Produktionsanlagen besitzen und Profit machen, mit der langfristigen Wirkung, nur im Rahmen des dominanten Designs zu denken

- Industrieverbände und Gewerkschaften, die von dem etablierten Weg profitieren und Lobbyismus für ihre Interessen betreiben

- Benutzerinnen und Benutzer des dominanten Designs, die die jeweiligen Produkte besitzen und in den täglichen Routinen und Kulturen des Gebrauchs gebunden sind

Unruh nutzt den Fall der Stromnetze, um den folgenden Überblick über die zugrunde liegende Systemdynamik zu geben:

Abbildung 1: Der techno-industrielle Komplex aus Stromproduktion, Transport und Verbrauch

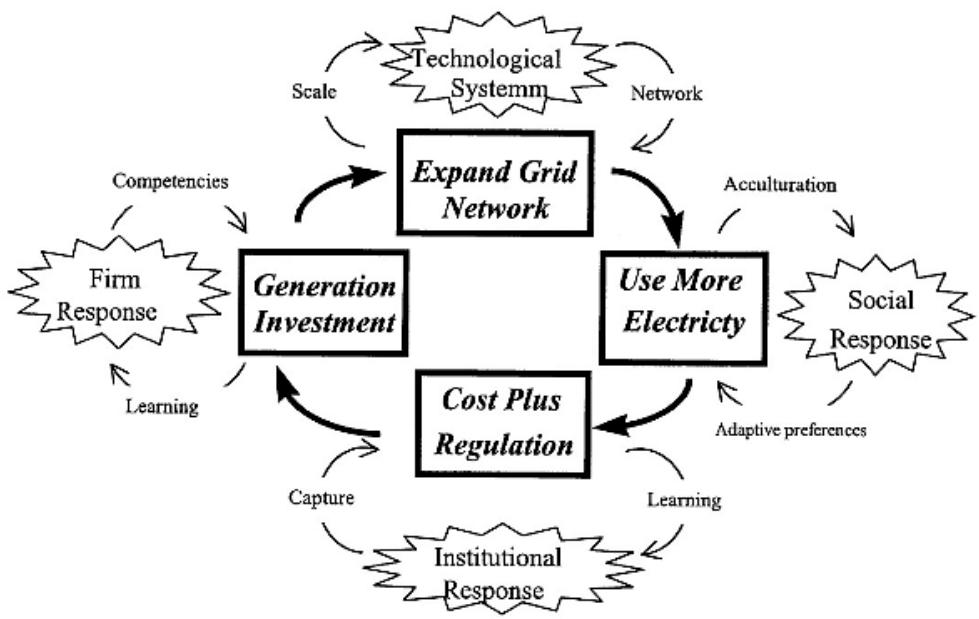

Quelle: Unruh (2000, S. 325).

Öffentliche Institutionen spielen nach Ansicht von Unruh eine besondere Rolle. Während die oben erwähnten Pfadabhängigkeiten alle Elemente einer Marktlogik sind (ähnlich auch bei Kirchner 2008, S. 331), in denen ein stabiles Wechselspiel der Akteurinnen und Akteure von spezifischen Produktions- und Konsummus- 
tern profitiert, kann die öffentliche institutionelle Politik die Marktlogik außer Kraft setzen (Unruh 2000, S. 324). Wenn politische und regulative Institutionen erst einmal geschaffen sind, neigen sie dazu, über lange Zeiträume zu bestehen. Akteurinnen und Akteure des etablierten Regimes nutzen bei ihren Lobby-Bemühungen diese Stabilität oft, um ihre Interessen zu wahren und dominante Pfade trotz veränderter Marktbedingungen zu verlängern. Aus bestimmten Gründen kann jedoch eine Regierung ihre regulatorische Macht auch nutzen, um einen Pfad zu ändern. Solche Gründe könnten die nationale Sicherheit oder die öffentliche Sicherheit, die Bereitstellung eines Universaldienstes für alle Bürgerinnen und Bürger oder Fälle von so genannten natürlichen Monopolen sein (Unruh 2000, S. 325). Auch in den Fällen eines sehr erfolgreichen Technologiesystems können schädliche Auswirkungen auf die Umwelt ein Grund für die Regierung sein, durch politische Maßnahmen einen Pfadwechsel herbeizuführen. Es kann vermutet werden, dass die Akteurinnen und Akteure des etablierten Pfades versuchen werden, die Regierung davon abzubringen und - wenn es ihnen nicht gelingt - sie zumindest daran zu hindern, Regulierungen umzusetzen, die zur Änderung des Pfades wirklich effektiv sind. Folglich nutzt Nill (2009, S. 471) den Begriff der »Pfadwirksamkeit« bei der Bewertung von Pfadänderungskonzepten.

\subsubsection{Pfadabhängigkeiten als Analyserahmen für »evolution2green«}

In diesem Buch wird eine Abgrenzung verschiedener Arten von Pfadabhängigkeiten vorgestellt, die hauptsächlich auf der Arbeit von Unruh (2000) und Beiträgen von Fichter und Clausen (2013, S. 90, 2016) basiert. Folgende Typen von Pfadabhängigkeiten werden unterschieden:

\section{Rechtliche Pfadabhängigkeiten}

Unter rechtlichen Pfadabhängigkeiten sollen hier solche verstanden werden, bei denen sich Bindungskräfte aufgrund gesetzlicher Regeln oder Standards entwickeln. Rechtliche Regeln, also Gesetze und andere formale Regelsysteme, können in erheblichem Maße technische und ökonomische Systeme stabilisieren. Es können eine Reihe von verschiedenen Typen rechtlicher Pfadabhängigkeiten identifiziert werden:

- Rechtliche Unmöglichkeiten, z. B. das Verbot der Nachzucht von Pflanzen durch Einzellandwirtinnen und -landwirte durch das Sortenrecht

- Das Steuer- und Abgaberecht, aufgrund dessen vielfach die »Preise nicht die ökologische Wahrheit« sagen, z. B. durch niedrige Steuern auf fossile Energieträger, die regenerative Lösungen unwirtschaftlich machen, durch niedrige Rohstoffsteuern oder den Förderrahmen der Gemeinsamen Agrarpolitik, der 
in völlig unzureichender Weise ökologische Ziele verfolgt und stattdessen industriellen und umweltbelastenden Landbau fördert

- Auch das Umgehen von Regelungen, z. B. durch die Nichterfüllung von Selbstverpflichtungen oder die Vorspiegelung gesunkener Benzinverbräuche durch phantasievolle Interpretation der Messvorschriften, ist von Bedeutung, wobei hierdurch meist ein durch die Regelung unterstützter bzw. gewollter Pfadwechsel verhindert werden soll.

- Normen wirken sich als konkrete Pfadabhängigkeiten eher im Kontext von kleinschrittigen Innovationen aus, in denen ein bereits genormtes Artefakt so verändert werden soll, dass es der Norm nicht mehr entspricht.

\section{Ökonomische Pfadabhängigkeiten}

Wirtschaftliche Positionen und Interessen können erhebliche Kräfte zur Pfadstabilisierung freisetzen. Unter ökonomischen Pfadabhängigkeiten sollen hier solche verstanden werden, bei denen sich höhere Bindungskräfte aufgrund ökonomischer Dynamiken entwickeln. Eine Reihe solcher Dynamiken wird diskutiert:

- Die aus Investitionen resultierende Kapitalbindung führt generell dazu, dass die Eigentümerin oder der Eigentümer einer Investition diese so lange wie möglich nutzen will. Dieses Nutzungsinteresse ist unabhängig von Abschreibungszeiten und bemisst sich eher an noch erzielbaren Gewinnen aus den vorhandenen Anlagen. Nill (2009, S. 138) bezeichnet diese Pfadabhängigkeit als »irreversible Investitionen in die etablierte Technologie«. Ein Beispiel ist das Interesse, Kohlekraftwerke unabhängig von sämtlichen klimapolitischen Bedenken möglichst lange nutzen und mit ihnen Gewinn erzielen zu wollen.

- Das Ausmaß der ökonomischen Aktivitäten geht mit einer zunehmenden Anzahl von Arbeitsplätzen einher. Das Interesse der Arbeitnehmerinnen und Arbeitnehmer an dem Erhalt ihres Arbeitsplatzes stellt eine weitere ökonomische Pfadabhängigkeit dar. Gewerkschaften wirken gegenwärtig sowohl in der Energie- wie auch in der Autobranche als pfadstabilisierende Akteure.

- Die mittels großer Produktions- und Absatzmengen (economies of scale) verringerten Stückkosten etablierter Produkte erschweren neuen und Nischenprodukten den Marktzugang. Der Effekt der economy of scale kann aber sowohl zu Beginn eines Pfadwechsels pfadstabilisierend wie auch später pfadverändernd wirken (Clausen et al. 2011; Göll und Henseling 2017).

Der Wert von Unternehmen, gemessen an der Marktkapitalisierung, stellt keine historische Pfadabhängigkeit dar, sondern ist eher von Erwartungen an die Zukunft abhängig. Man könnte diesen Wert auch als Ergebnis des Prozesses der Pfadbildung sehen. Der Energiesektor zeigt beispielhaft, dass eine pfaderhaltende Strategie, unterstützt durch starke Lobbyarbeit von Unternehmen und 
Gewerkschaften, in Bezug auf die Marktkapitalisierung nicht erfolgreich war. Etwa 50 Prozent der Marktkapitalisierung der 16 größten europäischen Energieunternehmen gingen in den Jahren 2008 bis 2016 verloren (Clausen und Fichter 2016, S. 14). Dabei wäre es die Aufgabe des Top-Managements gewesen, vorausschauend zu sein und in die Lösungen der Zukunft zu investieren - nicht in die Vergangenheit. Nur so können die Werte der Aktionärinnen und Aktionäre der etablierten Unternehmen langfristig gesichert werden.

\section{Technische Pfadabhängigkeiten}

Technische Pfadabhängigkeiten können auf vorhandenen Artefakten, z. B. Infrastrukturen oder in Nutzung befindlichen Produkten, basieren. In technischen Pfadabhängigkeiten kann sich so die "normative Kraft des Faktischen« manifestieren. Aber auch nicht-vorhandenes Wissen über Alternativen oder mangelnde Fähigkeiten zu deren Realisierung begründen technische Pfadabhängigkeiten:

- Die Frage des schlichten Vorhandenseins oder Nichtvorhandenseins von Ressourcen, z. B. Flächen für die innerstädtische Gewinnung von solarthermischer Wärme oder neue Güterbahnhöfe, aber auch das Aussterben bestimmter Nutztierrassen oder Saatgutsorten determiniert die Möglichkeit ihrer zukünftigen Nutzung.

- Das Vorhandensein von gut ausgebauten Infrastrukturen bietet den auf ihnen basierenden Services Vorteile im Markt und erzeugt bei den Betreibern gleichzeitig das Interesse an der Fortführung ihres Geschäftsmodells, also an der möglichst lang andauernden weiteren Nutzung. Ein Beispiel sind gut ausgebaute Autobahnen für Lkw-Transporte bei gleichzeitig knappen Kapazitäten auf Schiene und Wasserwegen.

- Nicht-vorhandenes technisches Wissen, z. B. bezüglich der Produktion von Batterien für die Elektromobilität, stabilisiert den Pfad des Verbrennungsmotors.

- Einzelne technologische Lösungen, z. B. in der IT, aber auch in der Pflanzenzucht, sind in das Zentrum komplexer Systeme gewandert, sodass ihre Veränderung schwierig wird.

\section{Organisationale Pfadabhängigkeiten}

Organisationale Pfadabhängigkeiten begründen die Trägheit von Organisationen im Falle von Änderungen. Verschiedene Typen organisationaler Pfadabhängigkeiten können identifiziert werden:

- Unhinterfragte Grundannahmen, nach denen Unternehmen geführt oder Politik gestaltet wird, bilden das Zentrum wesentlicher Pfadabhängigkeiten. So geht z. B. die Verkehrspolitik seit Jahrzehnten davon aus, dass mehr Stra- 
ßen zu mehr Wohlstand führen. Dass diese Grundannahme angesichts aktueller Studien aus den OECD-Ländern (Clausen 2017e) eher ein Mythos ist, beeinträchtigt nicht ihre Wirksamkeit.

- Weiter sind Organisationsroutinen von Bedeutung, die aus Unsicherheit beibehalten werden, weil z.B. das Vertrauen in eine neue Technik, ein neues Produkt oder einen neuen Markt fehlt. Dies zeigt sich z. B. daran, dass trotz erklärter Energiewende lange an Geschäftsmodellen, die auf fossile Energien ausgerichtet waren, festgehalten wurde.

- Von Bedeutung sind auch Lobbyaktivitäten der Wirtschaft wie auch der Gewerkschaften. Die Unternehmerseite vertritt z. B. ihre Lobbyinteressen in der Heizungstechnik aktiv und wirksam und plädiert immer wieder für die »Technologieneutralität« von Politiken, sodass erprobte Produkte im Absatz nicht behindert werden.

\section{Nutzungsbezogene Pfadabhängigkeiten}

Die Nutzung bestimmter Produkte oder Services kann bei Anwenderinnen und Anwendern zur Bildung von Routinen und Gewöhnungseffekten bis hin zur Sucht führen. Eine Reihe von Typen nutzungsbezogener Pfadabhängigkeiten können identifiziert werden:

- Die Zufriedenheit der Nutzerinnen und Nutzer mit bestimmten Produkten und Services stabilisiert deren zukünftige Weiterverwendung. Die Ausbildung von Routinen und lieben Gewohnheiten auch über das Individuum hinaus (»Was? Sie waren dieses Jahr nicht mit dem Flieger im Süden?«) können eine starke Pfadabhängigkeit bilden.

- Die Zufriedenheit mit den bekannten Produkten und Services wirkt zusammen mit der Unsicherheit von Konsumentinnen und Konsumenten in Bezug auf das Neue. Besonders die "späte Mehrheit« und die »laggards" (Rogers 2003) unter den Konsumentinnen und Konsumenten warten lange auf Belege dafür, dass neue Produkte oder Services risikofrei genutzt werden können. So werden z. B. auch neun Jahre nach dem Verkaufsstopp der Glühbirnen mit einer Leistung von 100 Watt und mehr diese Produkte immer noch als sogenannte »Restposten« im Internet angeboten.

- Durch Lobbyaktivitäten werden Unsicherheiten in Bezug auf neue Produkte oft gezielt geschürt.

- Ein weiterer stabilisierender Faktor ist mangelndes Wissen über Alternativen oder falsche Vorstellungen über Kosten und Nutzen von Alternativen. 


\subsubsection{Netzwerkbeziehungen von Pfadabhängigkeiten}

Die systematische Analyse von Pfadabhängigkeiten generiert einen guten Überblick über unterschiedliche Gruppeninteressen, mögliche finanzielle Werte, die bei der Transformation zerstört werden würden, die Rechtspositionen von Akteurinnen und Akteuren sowie den rechtlichen Rahmen, der die Transformation leicht oder schwer macht. Die Analyse von Pfadabhängigkeiten darf aber nicht bei einer Aufzählung von Pfadabhängigkeiten stehen bleiben. Es gilt vielmehr, ihre Strukturen zu analysieren sowie Zusammenhänge und Dynamiken zu verstehen. Denn Pfadabhängigkeiten sind innerhalb der soziotechnischen Systeme verknüpft. Die folgende Abbildung zeigt mögliche Netzwerkbeziehungen auf:

Abbildung 2: Netzwerkbeziehungen von Pfadabhängigkeiten

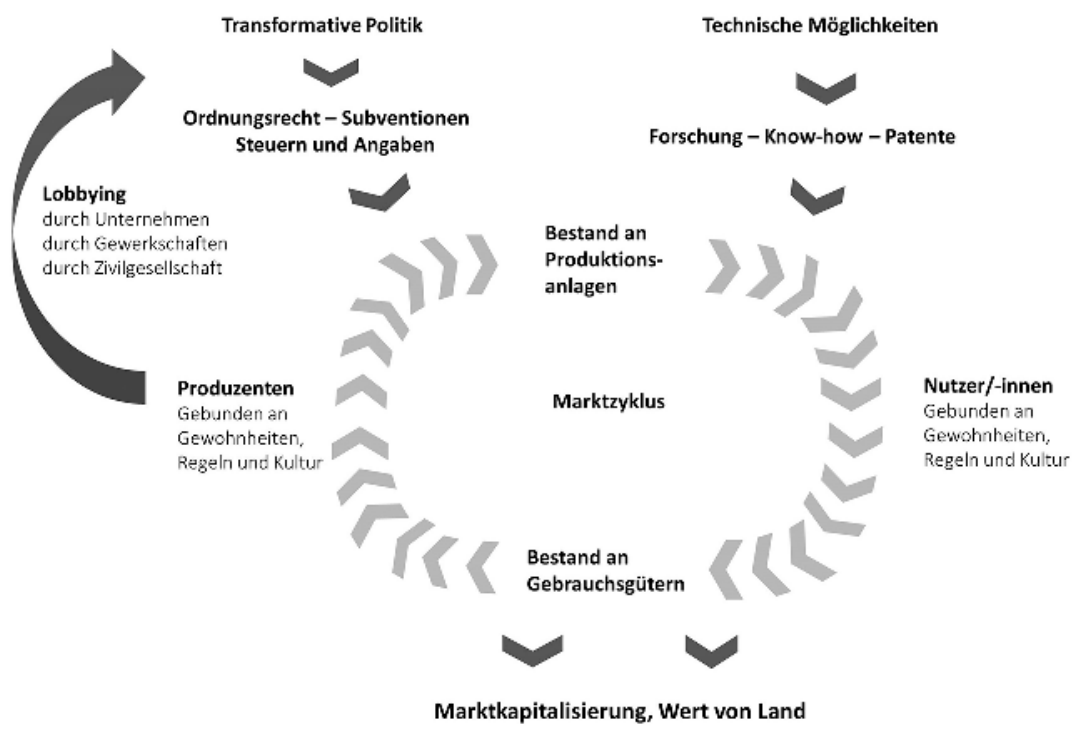

Quelle: Eigene Darstellung, Borderstep Institut für Innovation und Nachhaltigkeit.

Im Zentrum der Transformationsdynamik gibt es eine große Anzahl von Märkten, in denen Waren verkauft und gekauft werden. Viele dieser Güter oder Technologien sind Teil größerer technologischer Systeme und Regimes (Kemp 1994) und die Transformation solcher Märkte ist stark miteinander verknüpft. Diese Güter oder technologischen Systeme können, müssen aber nicht nachhaltig sein. Lieferanten wie auch Nutzerinnen und Nutzer sind in Gewohnheiten oder Organisationsstrukturen verstrickt oder einfach an Investitionen und Infrastrukturen gebunden. All dies macht es einfacher, dem alten Weg zu folgen.

Eine zentrale Voraussetzung für Veränderungen ist es, über die notwendigen technischen Fähigkeiten (Know-how, Technologien) zu verfügen. Zurzeit verfü- 
gen z. B. Asien und die USA über genügend Know-how (gemessen an Patenten sowie an der Zahl der tatsächlich verkauften Autos), um hochwertige Elektroautos $\mathrm{zu}$ bauen, während Europa eher abgeschlagen ist. Forschung und Entwicklung ( F \&E), wenn nötig mit öffentlichen Mitteln, wäre ein zentrales Mittel, um hier ein Lock-out herbeizuführen. Technische Innovationen sind daher von hoher Bedeutung für ein Lock-out.

Eine zentrale Voraussetzung für die Fortführung von erfolgreichen und einträglichen Pfaden besteht darin, diese rechtlich abzusichern. Letztlich entscheiden Parlament und Regierung über die ordnungsrechtliche Zulässigkeit und den ökonomischen Rahmen von Steuern und Abgaben, aus denen sich die Möglichkeit der Pfadfortführung letztlich ergibt. Hier begründet sich die Bedeutung von Lobbying und der Kreation von Mythen und alternativen Fakten, mit denen die Absicht der Politik, Dinge zu verändern, häufig erfolgreich gebremst oder gar ganz in Frage gestellt wird. Dennoch ist gerade der rechtliche Rahmen gleichzeitig ein zentrales Instrument, mit dem ein Lock-out herbeigeführt werden kann.

\subsection{Models of Change}

\subsubsection{Hintergrund}

Der erste Entwurf des Models-of-Change-Ansatzes basiert auf einer Reihe von interdisziplinären Projekten, die in den 1990er-Jahren am Wuppertal Institut durchgeführt wurden und die auf die Verbesserung von Energieeffizienz- und Klimaschutzaktivitäten abzielten. In einer Anzahl von Kommunen und Unternehmen wurden empirische Erkenntnisse zu Veränderungsprozessen gesammelt. Darauf basierend wurde ein Analyseraster entwickelt, mit dessen Hilfe diese Veränderungsprozesse aus drei unterschiedlichen Perspektiven in den Blick genommen werden können:

- Prozess- und Phasenmodell des Veränderungsprozesses

- Klassifizierung zentraler Erfolgsfaktoren

- Differenzierung als Ansatz für erfolgreiche Veränderungsprozesse

Im Prozess- und Phasenmodell werden Veränderungen als sechsphasiger Lernprozess verstanden (Impuls-, Aktivierungs-, Planungs-, Entscheidungs-, Realisierungsphase und Fazit). Zentral sind in diesem ersten Modell zudem drei Ebenen, auf denen sich Erfolgsfaktoren herauskristallisieren. Diese umfassen erstens die Personenkonstellationen und die Interaktion der beteiligten Akteurinnen und Akteure, zweitens explizite und implizite Prozessregelungen und drittens Rahmenbedingungen und Ressourcen. 
Zusätzlich werden sechs Spannungsfelder identifiziert, die wesentlich über den Erfolg und Misserfolg von Veränderungsprozessen entscheiden: 1) Atmosphäre vs. Effizienz, 2) bestimmende Persönlichkeit vs. Teamgeist, 3) »Gerede«vs. Aktionismus, 4) Offenheit vs. Vorstrukturierung, 5) freischwebend vs. verbindlich und 6) do it yourself vs. Hilfe von außen (Irrek und Kristof 2000, S. 68). Diese Spannungsfelder verdeutlichen gegenüberstehende Extreme der Prozessgestaltung, welche jeweils Vor- und Nachteile aufweisen.

Dieses ursprüngliche Modell wurde von Kora Kristof in ihrer Habilitationsschrift »Models of Change. Einführung und Verbreitung sozialer Innovationen und gesellschaftlicher Veränderungen in transdisziplinärer Perspektive« (2010a) sowie ihrem Buch »Wege zum Wandel« (2010b) analysiert und systematisch weiterentwickelt. Dazu hat sie sowohl auf Erfahrungen aus der Praxis als auch auf diverse Forschungsansätze zurückgegriffen.

Ziel der Forschungsarbeit war es, »die Hypothesen zum Modell von Veränderungsprozessen und deren Beeinflussbarkeit, aber auch die identifizierten Erfolgsfaktoren zu testen und [...] deutlich zu verbessern« (Kristof 2010a, S. 2). Dem Ansatz liegt die Annahme zugrunde, dass Veränderungsprozesse zwar nicht im engeren Sinne steuerbar sind, jedoch Ansatzpunkte zur Beeinflussung von Transformationsprozessen existieren, aus denen Erfolgsfaktoren abgeleitet werden können (Kristof 2010a, S. 227).

Kristof führte zu diesem Zwecke 40 Interviews mit Persönlichkeiten aus Verwaltung, Politik und Zivilgesellschaft, die in sozial-ökologische Veränderungsprozesse in Deutschland involviert sind oder waren. Im Fokus standen implizite Annahmen über Veränderungsprozesse, die dem Handeln der befragten Akteurinnen und Akteure zugrunde liegen. Erfragt wurden Einschätzungen zur Gestaltbarkeit von Veränderungsprozessen, die Rolle wesentlicher change agents, Fragen zur Bedeutung des Faktors Zeit, relevante Rahmenbedingungen sowie Problembereiche und Lösungsansätze. In einem zweiten Schritt wurden explizite Veränderungsmodelle aus Betriebs- und Volkswirtschaftslehre, Innovations- und Diffusionsforschung, Organisations- und Gruppenpsychologie sowie Soziologie und Politologie analysiert.

Daraus resultiert ein neues Veränderungsmodell, welches komplex, dynamisch, systemisch und interaktiv ausgerichtet ist (siehe Abbildung 3 für eine bildliche Zusammenfassung). Der Veränderungsprozess wird bei Kristof nicht wie im ursprünglichen Wuppertaler MoC-Ansatz als eine Abfolge konsekutiver Phasen dargestellt. Vielmehr wird er als interdependenter Fließprozess beschrieben, der aus einer Reihe von zentralen Komponenten besteht (Kristof 2010a, S. 526). 
Abbildung 3: Ursprung und Weiterentwicklung des MoC-Ansatzes

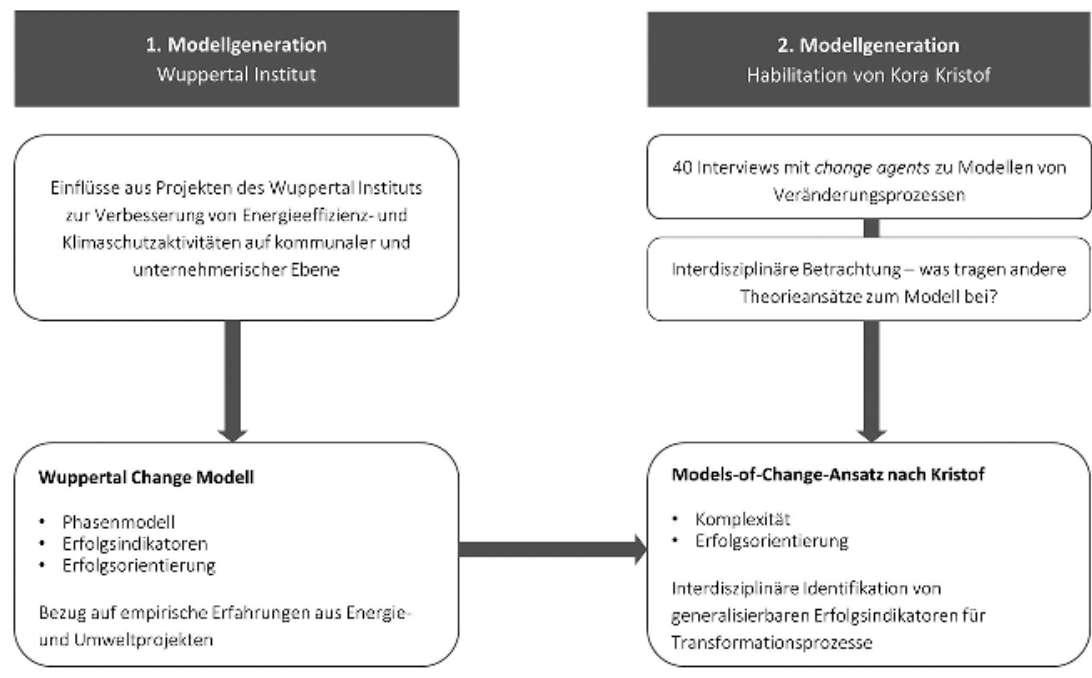

Quelle: Eigene Darstellung, adelphi.

Das so entstandene Veränderungsmodell ist prinzipiell für alle Veränderungsprozesse in demokratischen Kontexten anwendbar. Sowohl die untersuchten Wissenschaftsansätze als auch die Interviewpartnerinnen und Interviewpartner gehen davon aus, dass die grundlegenden Prinzipien ihrer Veränderungsmodelle bereichsübergreifend gelten. Unterschiede in den formalen Entscheidungsstrukturen, konkreten Herausforderungen und damit verbundenen Machtstrukturen können jedoch eine Modifikation des Gesamtmodells erfordern (Kristof 2010a, S. 516).

\section{Ansatzpunkte für Erfolgsfaktoren}

Als konstitutiv für den Erfolg von Veränderungsprozessen werden in der Literatur diverse Faktoren wie Rahmenbedingungen, Regelungen und Akteurskonstellationen (z. B. sogenannte change agents) angesehen. Weitere erwähnte Erfolgsbedingungen sind die konsensuale Tragfähigkeit der Idee von Veränderungsprozessen und der Faktor Zeit (Kingdon 2002). Kristof argumentiert, dass keine »Kochrezepte« (Kristof 2010b, S. 106) für Transformationen identifiziert werden können, da Veränderungsprozesse komplex und dynamisch verlaufen. Dennoch sollten folgende zentrale Ansatzpunkte für eine erfolgreiche Transformation im Blick behalten werden:

- Erfolgreiche Einbindung zentraler Akteurinnen und Akteure (change agents, Zielgruppen, weitere Akteurinnen und Akteure)

- Entwicklung tragfähiger Veränderungsideen und Lösungsvorschläge 
- Adäquate Berücksichtigung von spezifischen Zeitaspekten

- Professionelle Gestaltung des Veränderungsprozesses

- Planung im Kontext politischer Rahmenbedingungen, vorherrschender Veränderungskultur und dynamischer Lernprozesse

Die meisten dieser Ansatzpunkte wurden im MoC-Ansatz auf der Prozessebene identifiziert und sind als interdependente, sich verstärkende oder hemmende Faktoren zu verstehen. Von besonderer Relevanz sind dabei der Instrumentenmix und die Institutionalisierung, aber auch zielorientierte Kommunikations- und Beteiligungsprozesse oder die Ausgestaltung individueller und gruppenorientierter Lernprozesse. Alle Erfolgsbedingungen für Transformationen müssen immer auch kontrafaktisch zu bestehenden Pfadabhängigkeiten analysiert werden. Dies bedeutet, dass die Wahrscheinlichkeit der erfolgreichen Gestaltung eines Veränderungsprozesses immer auch von den vorherrschenden Rahmenbedingungen abhängt, etwa von der vorhandenen Infrastruktur im Energie- oder Verkehrssektor.

Das Modell nach Kristof ist interdisziplinär ausgerichtet und vereint verschiedene Ansatzpunkte. Vier Ebenen (Akteurinnen und Akteure, Zeitaspekte, Veränderungsidee/Lösungsvorschlag, Veränderungsprozess) beschreiben die Bereiche, in denen durch aktives Handeln die Erfolgswahrscheinlichkeit für einen Veränderungsprozess gesteigert werden kann. Die Rahmenbedingungen stellen die Leinwand dar, vor deren Hintergrund die Veränderungen ablaufen. Rahmenbedingungen können aber auch genutzt werden, um Veränderungsprozesse zu institutionalisieren. Kristof verweist auf die Notwendigkeit der Entwicklung von veränderungsfördernden Rahmenbedingungen, die den Wettbewerb um die besten Ideen, Lösungen und Umsetzungswege erleichtern (Kristof 2010a, S. 535).

Der Ansatz von Kristof zeichnet sich durch eine explorative und transdisziplinäre Herangehensweise aus, die dem neuen Veränderungsmodell eine breite theoretische und empirische Fundierung gibt. Durch die Einbeziehung von impliziten persönlichen Ideen von Veränderungsprozessen, die der subjektiven Wahrnehmung von Personen aus Praxis und Wissenschaft entsprechen, wurde zudem eine neue Wissensquelle nutzbar gemacht. Kristof betont, dass der Vergleich von impliziten und expliziten Modellen zur Verbesserung der Anwendbarkeit von Forschungsergebnissen beiträgt. Das Modell erlaubt einen »frischen Blick« auf Veränderungsprozesse und regt an, eigene Annahmen, Perspektiven und theoretische Herangehensweisen zu überprüfen und gegebenenfalls zu erweitern.

\section{Bisherige Anwendungen des MoC-Ansatzes nach Kristof}

Der MoC-Ansatz nach Kristof wurde bereits in verschiedenen Kontexten für die Analyse von Transformationsprozessen eingesetzt. Die Erkenntnisse aus diesen Projekten gaben weitere Impulse für die Anpassung des Analysemodells. Ein 
Beispiel ist das Projekt »Transformationsstrategien und Models of Change für nachhaltigen gesellschaftlichen Wandel« des Umweltbundesamtes (UFOPLANVorhaben - FKZ 371211 103), das wichtige Ansätze für eine weiterführende Operationalisierung im Rahmen von »evolution2green « liefert. Im Rahmen des Projekts wurden u.a. mögliche Erfolgsbedingungen für eine Nachhaltigkeitstransformation untersucht. Insgesamt konnten sieben Faktoren identifiziert werden, die auch in den Analyserahmen von »evolution2green« einfließen (nach Grießhammer und Brohmann 2015):

- Überwindung der infrastrukturell-technischen Pfadabhängigkeiten

- Kultur- und Mentalitätswandel

- Überwindung von Ängsten und Widerständen

- Langfristiges Denken

- Breite Beteiligung und Kooperationx

- Kohärente, nachhaltigkeitsfördernde Politik und Rahmenbedingungen

- Pionier-Handeln im Kontext technischer und sozialer Innovationen

Ein weiteres Vorhaben des Umweltbundesamtes, in dem der Models-of-ChangeAnsatz genutzt und weiterentwickelt wurde, ist »Erfolgsfaktoren im Politikprozess Klimaanpassung. Akteurszentrierte Untersuchung der Erfolgsbedingungen und Hemmnisse für Anpassungsstrategien« (UFOPLAN-Vorhaben - FKZ 371348 101). Hier wurde konkret der Versuch unternommen, den MoC-Ansatz in einen Analyserahmen für Transformationsprozesse $\mathrm{zu}$ transferieren. $\mathrm{Zu}$ diesem Zwecke wurden auch weitere Aspekte aus der politikwissenschaftlichen Forschung in den Analyserahmen integriert. Die daraus resultierenden Erkenntnisse dienen ebenfalls als Grundlage für das in »evolution2green« weiterentwickelte und angepasste Modell von MoC.

Die genannten Forschungsvorhaben weisen darauf hin, dass der MoC-Ansatz nach Kristof durchaus als Basis für die Analyse von Veränderungsprozessen verwendet und projektspezifisch angepasst werden kann. Auch im Rahmen des e2gProjekts ist eine Anpassung des Ansatzes notwendig, um diejenigen Aspekte zu beleuchten, die mit Bezug auf den betrachteten Untersuchungsgegenstand und die verfügbaren Daten sinnvoll operationalisierbar sind. Dementsprechend kann eine Erweiterung und Ergänzung des Analyserasters um relevante Aspekte des spezifischen Veränderungsphänomens für die Identifikation von Erfolgsfaktoren notwendig und hilfreich sein. Die projektspezifische Anpassung und Ergänzung des MoC-Ansatzes für das e2g-Projekt wird in den folgenden Kapiteln dargelegt. 


\subsubsection{Der MoC-Ansatz als Analyserahmen für »evolution2green«}

Auf Basis des oben umrissenen MoC-Ansatzes können die Kernfaktoren, die der weiteren Untersuchung zugrunde liegen und als Basis für das zu erstellende Analyseraster dienen, wie folgt benannt werden:

- Akteurinnen und Akteure und ihre Qualifikationen

- Lösungsidee/Lösungsvorschlag

- Zeitaspekte

- Veränderungsprozesse

- Pfadabhängigkeiten und Rahmenbedingungen

Im Folgenden werden diese zentralen Analysekriterien nach Kristof (2010a, 2010b) erläutert und eine Anpassung und Erweiterung dieser Kernfaktoren des MoC-Ansatzes vorgenommen. In einem weiteren Schritt wird ein Analyseraster für die Problemfeldbetrachtung und die Identifikation von Erfolgsfaktoren im e2g-Projekt entwickelt. Erkenntnisse aus relevanten Praxiserfahrungen der Projektbeteiligten sowie aus einer erweiterten Literaturbetrachtung dienen als Basis, um weitere Theorieansätze, insbesondere aus der Politik- und Wirtschaftswissenschaft zu integrieren.

Zudem gilt es, die spezifischen Grundlagen der weiteren Analyseansätze von »evolutionzgreen«, nämlich einerseits der evolutorischen Ökonomik und der Pfadabhängigkeiten und andererseits der Leitstrategien Konsistenz, Effizienz und Suffizienz, geeignet aufzugreifen (siehe Abbildung 4) und in das Analyseraster zu integrieren.

Abbildung 4: Weiterentwicklung von MoC für »evolution2green«

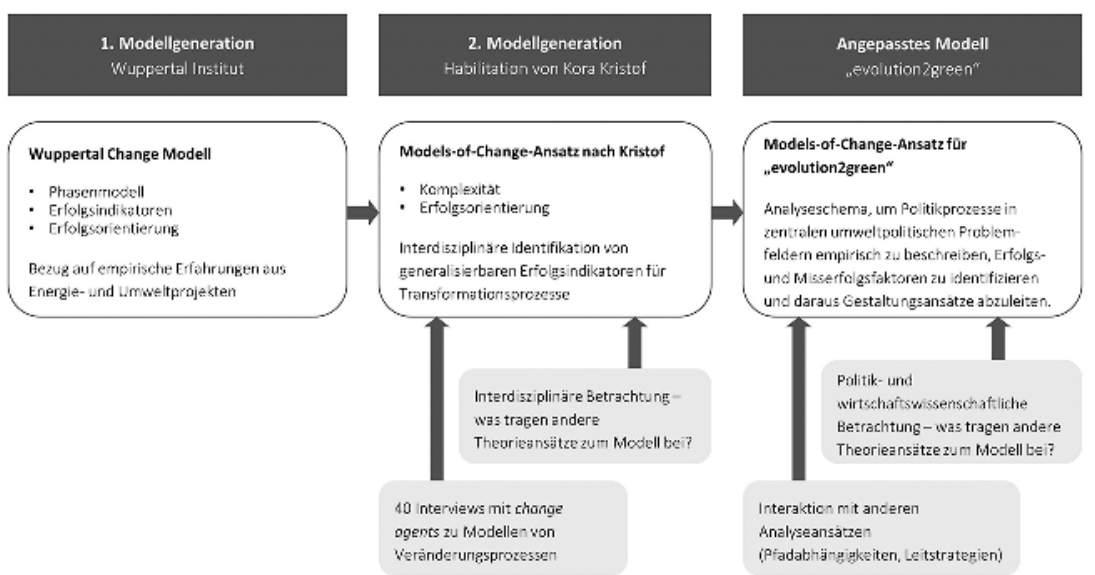

Quelle: Eigene Darstellung, adelphi. 


\subsubsection{Akteurinnen und Akteure und ihre Qualifikationen}

Gesellschaftliche Transformationen werden von Menschen angestoßen und vorangetrieben. Da die Folgen von Veränderungen sehr unterschiedlich wahrgenommen werden, nehmen Akteurinnen und Akteure vielfältige Rollen ein. Sie können als aktive Promotorinnen und Promotoren, Visionärinnen und Visionäre, positive Leistungsträger/-innen, abwartende Skeptiker/-innen sowie Bremser/ -innen und Blockierer/-innen fungieren (Kristof 2010a, S. 140).

Aus dem MoC-Ansatz nach Kristof geht hervor, dass es für einen erfolgreichen Veränderungsprozess aktiver Promotorinnen und Promotoren bedarf, die den Prozess initiieren und gestalten. Die Ausgestaltung bzw. Erfüllung der Rolle von change agents ist ein zentraler Aspekt für den Erfolg von Veränderungsprozessen. Oftmals handelt sich um einzelne Individuen oder eine kleine Personengruppe. Die notwendige Anzahl an change agents, um die Transformation effektiv und effizient voranzutreiben, hängt von der Dimension der angestrebten Veränderung ab. Change agents müssen über eine Reihe von Qualifikationen und Ressourcen verfügen. Anhand des Promotorenmodells lässt sich eine Kategorisierung in Fach-, Prozess-, Macht- und Beziehungspromotorinnen und -promotoren vornehmen (Kristof 2010b, S. 110). Die erste Gruppe zeichnet sich durch objektspezifisches Fachwissen aus und hat zur Aufgabe, Projekte zu initiieren, Alternativen zu entwerfen sowie Probleme zu lösen und Vorhaben zu implementieren. Prozesspromotorinnen und -promotoren besitzen zusätzlich zu Fachwissen einen gewissen Grad an Führungskompetenz und können Probleme im Projekt definieren, gestalten und kommunizieren. Machtpromotorinnen und -promotoren haben Zugriff zu materiellen und immateriellen Ressourcen und können damit Veränderungsprozesse initiieren und deren Erfolg fördern. Außerdem sind Beziehungspromotorinnen und -promotoren vonnöten, die Beziehungen und Netzwerke aufbauen und aufrechterhalten sowie Konflikte und Interaktionen erfolgsorientiert gestalten können. Damit sollen sie Prozesspromotorinnen und -promotoren in Interaktionsprozessen unterstützen.

Komplexe, gesamtgesellschaftliche Veränderungen erfordern eine Vielzahl von Akteurinnen und Akteuren mit unterschiedlichen Qualifikationen und Ressourcen. Vielversprechend ist daher eine Kombination verschiedener Promotorentypen, die untereinander gut vernetzt sind. Nicht nur hartes Faktenwissen, sondern auch "weiche Kompetenzen« wie Kommunikationsstärke, Kreativität, Sozialkompetenz und Vertrauenswürdigkeit zählen zu den Qualifikationsanforderungen, um Widerständen gegen eine Transformation adäquat zu begegnen (Kristof 2010a, S. 523). Diese Widerstände sind »normal und wichtiger Bestandteil (Kristof 2010b, S. 101) eines Veränderungsprozesses, da in den allermeisten Konstellationen einige Akteurinnen und Akteure kritisch gegenüber einer Veränderung eingestellt sind oder diese sogar aktiv behindern wollen. Change agents sollten diese Widerstände als wichtig für einen erfolgreichen Wandel und als Weg 
$\mathrm{zu}$ besseren Lösungen begreifen. Aus der Perspektive von »evolution2green« ist hinzuzufügen, dass nicht nur politische und zivilgesellschaftliche Akteurinnen und Akteure wichtig sind, sondern auch privatwirtschaftliche die Funktion eines change agents einnehmen können - insbesondere hinsichtlich der Transformation der Wirtschaft hin zu einer Green Economy (Weiß 2015). Zudem werden in der Literatur weitere Klassifizierungen von change agents vorgeschlagen. Beispielhaft identifiziert Caldwell (2003) Leadership-, Consulting-, Management- und TeamModelle von change agency, die auf die komplexen Rollenzuschreibungen und besonderen Erfordernisse der Koordination in Veränderungsprozessen hinweisen. Darüber hinaus wird in der Literatur zwischen externen und internen change agents unterschieden (Lacey 1995; Cameron und Green 2012). Während die Letzteren Expertise in der internen Organisationsentwicklung haben, müssen sich externe change agents zunächst in die Spezifika einer anderen Organisation einfinden.

Akteurinnen und Akteure sind zudem auch Betroffene von Veränderungsprozessen, wobei die Breite der Zielgruppe (von nur wenigen Personen bis hin zur gesamten Bevölkerung) vom jeweiligen Veränderungsprozess abhängt. Die wichtigsten Klassifizierungen resultieren aus 1) der Betroffenheit durch die Veränderung, 2) der Haltung gegenüber der Veränderungsidee und dem Veränderungsprozess, 3) der Breite der Zielgruppe, 4) der Stakeholderkonstellation und 5) der gesellschaftlichen Rolle.

$\mathrm{Zu}$ der Zielgruppe und der Dimension der weiteren Akteurinnen und Akteure zählen sowohl politische und öffentliche als auch private Akteurinnen und Akteure, die sich jeweils für oder gegen eine Idee einsetzen. Da der MoC-Ansatz nach Kristof nicht zwischen politischen, öffentlichen und privaten Akteurinnen und Akteuren differenziert, lässt sich im Rahmen dieses Ansatzes keine konkrete akteurszentrierte Analyse vornehmen. Diese Differenzierung ist allerdings ein wesentlicher Punkt, sodass es sinnvoll erscheint, den Fokus zu erweitern und auf Akteurskoalitionen (advocacy coalitions) zu legen (vgl. Sabatier 1998; Weible et al. 2009; Baumgartner et al. 2009; Bulkeley 2000; Biermann et al. 2009). Der Advocacy-Coalition-Ansatz hilft, Akteurskonstellationen innerhalb eines weit gefassten Akteurskreises zu erfassen. Zentral ist die Idee, dass sich unterschiedliche Akteurinnen und Akteure zu Koalitionen zusammenschließen, um größeren Einfluss zu erlangen. Anhand der Betrachtung von Policy-Subsystemen, also durch die Analyse von »Interaktionen von Akteuren verschiedener Institutionen, die an einem Policy-Bereich interessiert sind« (Sabatier 1993, S. 120), lassen sich diese Koalitionen identifizieren. Ziele und Wahrnehmungen dominanter Koalitionen sind im Subsystem des Politikfeldes zentral. Diese zu verändern ist eine komplexe Herausforderung (Weible et al. 2009, S. 124; Sabatier 1993, S. 131). Da der Erfolg politischer Zielsetzungen stark von den Interessen dominanter Koalitionen abhängt, die den Veränderungsprozess behindern können, sind gerade 
diese potenziellen Bremser/-innen von besonderem Analyseinteresse. Der Vetospieler-Ansatz ist ein weiteres politikwissenschaftliches Konzept, welches die Bedeutung von Gegnerinnen und Gegnern eines Vorhabens betont (Tsebelis 2002). Je größer die Anzahl an Vetospielerinnen und -spielern und je kohärenter deren Zusammenarbeit, desto unwahrscheinlicher ist der Erfolg einer Veränderungsinitiative. Ein ähnliches Konzept deklariert diese Akteurinnen und Akteure als Beteiligte mit Status-quo-Interessen, die starken Einfluss auf das Handlungsfeld haben und oftmals Verluste durch Veränderungsprozesse befürchten (Schneider und Veugelers 2010). In das Analysemodell werden deshalb zentrale Akteurinnen und Akteure sowie Koalitionen aufgenommen, die für Transformation eintreten, sowie solche, die einer Transformation skeptisch gegenüberstehen.

Über den MoC-Ansatz von Kristof hinaus sind Mehrebenenansätze, die die Stellung wesentlicher Akteurinnen und Akteure für den Transformationsprozess berücksichtigen, von Bedeutung. Die politikwissenschaftliche Multi-LevelGovernance-Perspektive bezieht sich einerseits auf unterschiedliche politische Ebenen, auf denen Akteurinnen und Akteure mit Entscheidungsgewalt angesiedelt sind, sowie andererseits auf die Frage nach der Rolle und Interaktion von Nationalstaaten und nicht-staatlichen Akteurinnen und Akteuren (Bauknecht et al. 2015a). Die Multi-Level-Perspektive in der Transitionsforschung (Geels 2011) unterscheidet zudem die Ebenen Nische, Regime und Landschaft. Hierbei wird davon ausgegangen, dass Neuerungen in Nischen entstehen, die sich im dominanten Regime behaupten müssen, um zu einer Veränderung auf gesamtgesellschaftlicher Ebene (Landschaft) führen zu können. Für die Entwicklung von Innovationen in den Nischen benötigen Promotorinnen und Promotoren strategische Vorstellungen über das Wachstum dieser Nische sowie deren Entfaltungsmöglichkeiten im Regime. Dabei ist zum einen wichtig, dass transformative Innovationen wahrscheinlicher in diesen Nischen außerhalb des Systems entstehen, zum anderen liegt ein Augenmerk auf der Förderung diverser Nischen mit Entwicklungspotenzial. Auch Regimeakteurinnen und -akteuren wird eine beachtliche Bedeutung zugemessen, da sie das bestehende System stützen, aber sich auch an Nischenaktivitäten beteiligen können (Späth und Rohracher 2010; Geels 2011). Zusammenfassend lassen sich relevante Akteurinnen und Akteuren nach Politikebene sowie nach Regime- oder Nischenzugehörigkeit aufschlüsseln. Für die Operationalisierung sind sowohl Qualifikationen, Interessen und Effizienz der change agents als auch die Konstellation von Akteurinnen und Akteuren sowie von Akteurskoalitionen, die sich für oder gegen eine Veränderungsidee einsetzen, von zentraler Bedeutung. 


\subsubsection{Lösungsidee/Lösungsvorschlag}

Im MoC nach Kristof werden verschiedene Charakteristika einer Lösungsidee benannt, welche die Erfolgswahrscheinlichkeit von Veränderungsprozessen bedingen (Kristof 2010a, S. 525):

- Lösungsideen müssen zielgruppen- und situationsspezifisch sein, d. h. auf die beteiligten Akteurinnen und Akteure und das Problem passgenau abgestimmt sein. Bei der Weiterentwicklung sind proaktive Beteiligte, aber auch potenzielle Kritikerinnen und Kritiker zu berücksichtigen. Im Laufe des Transformationsprozesses sollte sich die Umsetzungslösung zudem dynamisch weiterentwickeln können, indem sie an Rahmenbedingungen und Zeitfaktoren angepasst wird.

- Lösungsideen müssen mitreißend sein, um vor allem zu Beginn des Veränderungsprozesses Menschen zur Mitwirkung zu motivieren. Lösungsvorschläge sollten den Zeitgeist erfassen und anschlussfähig sein. Bei der Öffnung eines window of opportunity sollten bereits konkrete Lösungsvorschläge existieren. Idealerweise gelingt es im Anschluss, einen Wettbewerb um die beste Lösung zu initiieren. Die ausgewählte Lösung muss dann auf Basis ihrer Tragfähigkeit im Diffusionsprozess bestehen.

- Zudem ist der effektive Umgang mit Zielkonflikten und Widerständen wichtig. Diese treten insbesondere bei der Planung und Durchführung eines Transformationsvorhabens auf und sind als Indikatoren für Verbesserungspotenziale zu verstehen.

Der MoC-Ansatz nach Kristof kann auch an dieser Stelle durch Ansätze aus Politik- und Wirtschaftswissenschaft ergänzt werden. Aus politikwissenschaftlicher Perspektive lassen sich beispielsweise politische Strategien als Veränderungsideen betrachten, $z u$ deren Umsetzung der Einsatz von bestimmten Instrumenten als konkreter Lösungsvorschlag gilt. Zielvorgaben in Strategien müssen präzise genug formuliert sein, um deren Interpretation nicht alleinig dem Ermessen der beteiligen Akteurinnen und Akteure zu überlassen. Gleichzeitig müssen die Vorgaben die in komplexen und dynamischen Politikfeldern notwendige Anpassungsfähigkeit zulassen. Diese Flexibilität ermöglicht die Anpassung der Strategie an neueste Erkenntnisse der wissenschaftlichen Forschung, sodass Kosten für ineffiziente Maßnahmen vermieden werden können. Hinsichtlich des Einsatzes von Instrumenten ist wichtig, dass seitens der Beteiligten alle zur Verfügung stehenden Mittel für die Erreichung von Zielen in Betracht gezogen werden (Flanagan et al. 2011; Persson 2004) - selbst wenn diese unbeliebt sind oder hohe Kosten verursachen. Forschungsergebnisse verdeutlichen, dass rechtliche und ökonomische Instrumente eine große Wirkung auf die Dynamik von Veränderungsprozessen entwickeln können (Pollack und Hafner-Burton 2010). 
Auch in der betriebswirtschaftswissenschaftlichen Forschung existieren Konzepte, die Alternativen zu etablierten Mustern aufzeigen. Lean-Ansätze aus der Entrepreneurship-Forschung (lean production, lean management, lean business models etc.) konzentrieren sich auf die wesentlichen Komponenten eines Geschäftsmodells, -prozesses oder -systems. Die optimale Abstimmung von Wertschöpfungsphasen sowie die Vermeidung von Verschwendung sind zentrale Erfolgsfaktoren dieser Ansätze (Groth und Kammel 1994; Dickmann 2008). Agility-Ansätze betonen die Fähigkeit von Unternehmen, sich schnellstmöglich an Veränderungsprozesse anzupassen. Dies umfasst die Aufrechterhaltung und Anpassung einer ursprünglichen Unternehmenskonfiguration etwa in Hinblick auf veränderte Kundenbedürfnisse, Rahmenbedingungen oder Ressourcenvorkommen (Pal und Pantaleo 2005). "Design Thinking « gilt als Innovationsmethode, die anhand von Kreativität und der Zusammenarbeit von Personen aus unterschiedlichen Disziplinen zu möglichst ganzheitlichen, vielfältigen und zielgruppenorientierten Lösungsideen führt (Brown 2008). Mithilfe von »Rapid Prototyping" lassen sich erste Lösungsideen möglichst schnell umsetzen und in der Praxis auf Schwachstellen testen, um iterativ Weiterentwicklungen vorzunehmen (Tripp und Bichelmeyer 1990). Auch Feedbackmethoden wie "360-Degree-Feedback" oder »Instant Feedback« zielen auf eine ganzheitliche, direkte Bewertung von Managementstilen, Innovationen und Veränderungen ab, um eine Lösungsidee möglichst effektiv weiterzuentwickeln (Chappelow 2004). Alle Ansätze unterstützen eine passgenaue Entwicklung von Lösungsstrategien und sollten bei der Analyse von Veränderungsideen im Hinblick auf relevante Erfolgsfaktoren berücksichtigt werden. Etwas anders verhält es sich, wenn eine disruptive Innovation eine Marktsituation unerwartet schnell verändert (Bower und Christensen 1995). Diese Technologien, etwa die subventionierte Diffusion von erneuerbaren Energieträgern, führen zu beschleunigten Veränderungsprozessen und verstärkten Effekten im Bereich der Lösungsstrategien. Wie im Fall der Energiewende ersichtlich, bedarf es jedoch erheblicher, vor allem finanzieller Ressourcen und passionierter change agents, um den Innovationsprozess weiter voranzutreiben.

\section{Beispiel: Elektromobilität}

Ein zentrales Beispiel für eine potenziell transformative Veränderungsidee ist die Elektromobilität. Ziel der Förderung und Weiterentwicklung der Elektromobilität ist es, Treibhausgasemissionen im Bereich Verkehr zu reduzieren und den Straßenverkehr unabhängiger von der Verfügbarkeit von Erdöl zu gestalten. Um Deutschland als Geburtsland des Automobils auch im Bereich Elektromobilität an die Spitze zu bringen, versucht die deutsche Bundesregierung gemeinsam mit der Nationalen Plattform Elektromobilität 
förderliche Rahmenbedingungen für die Entwicklung von Elektroautos zu schaffen.

Um die Elektrofahrzeuge für die Verbraucherinnen und Verbraucher in ihrem Alltag nutzbar zu machen, müssen zwei große Herausforderungen gemeistert werden: Zum einen muss die Speicherkapazität der Batterien erhöht werden, zum anderen fehlt eine flächendeckende Ladeinfrastruktur. Maßnahmen der Bundesregierung, welche die Umsetzung der Veränderungsidee »Elektromobilität « beschleunigen sollen, umfassen Investitionen in F \& E in der Phase der Marktvorbereitung, erste Infrastrukturmaßnahmen sowie Kaufanreize (z. B. Kfz-Steuerbefreiungen, Sonderparkflächen sowie die Erlaubnis zur Mitbenutzung von Busspuren). Um Wettbewerbsverzerrungen und Fehlentwicklungen vorzubeugen, werden Subventionen von der deutschen Bundesregierung vermieden.

Durch Pilotvorhaben und Kampagnen in einzelnen Regionen Deutschlands sollen die Zielgruppen von »marktfähigen, alltagstauglichen Produkten, die sich in der Praxis bewähren und die Bürger überzeugen«, gewonnen werden (BMWi 2011). Trotz umfangreicher Maßnahmen bleiben Absatz und Zulassungszahlen von Elektrofahrzeugen bisher hinter den Erwartungen zurück. Als Grund hierfür gilt vor allem der hohe Anschaffungspreis (Nationale Plattform Elektromobilität 2014). Um neben den early adopters auch breitere Zielgruppen zu erreichen und die Motivation zu stärken, den politisch angestoßenen Transformationsprozess weiter voranzutreiben, ist es notwendig, die Veränderungsidee dynamisch weiterzuentwickeln. Hierzu ist es erforderlich, sich intensiv mit den Schwachstellen der Elektromobilität auseinanderzusetzen, Gegner/-innen und Wettbewerbskonkurrenz zu identifizieren und Rahmenbedingungen anzupassen, sodass die Elektromobilität zu einer attraktiven Lösung für weitere Kundengruppen wird.

\subsubsection{Zeitaspekte}

Die Zeitdimension von Prozessen kann als eine Sequenz von »individual and collective events, actions, and activities unfolding over time in context « betrachtet werden (Pettigrew 1997, S. 338). Als analytische Kategorie ist sie in der Literatur über Veränderungsprozesse unterrepräsentiert. Der MoC-Ansatz nach Kristof untersucht diese teilweise schwer zu operationalisierenden Zeitaspekte von Veränderungsprozessen. Als zentral für erfolgreiche Veränderungen werden deren Auslöser, wichtige Ereignisse, Prozessgeschwindigkeit und Zeitressourcen betrachtet.

Anhand der Untersuchung von Auslösern und Ereignissen lassen sich der Anlass bzw. die Motivation für die Entstehung eines Veränderungsprozesses erkennen (z. B. Transfer aus anderen Ländern, Vorgabe einer anderen Ebene, Problemdruck). Veränderungsprozesse können durch eine Vielzahl von Faktoren initiiert 
werden. $\mathrm{Zu}$ beachten ist, dass Katastrophen keine ausreichende Bedingung für die Einleitung tief greifender Veränderungsprozesse darstellen (siehe z. B. Berkhout et al. 2006, S. 152). Vielmehr wirken solche Ereignisse in Interaktion mit anderen Einflussfaktoren wie ökonomischem Druck oder internationalen Übereinkünften. Auch Lernprozesse und bestehende Best-Practice-Modelle können eine Transformation veranlassen. Aus methodologischer Sicht ist es bisher umstritten, inwiefern die vielfach debattierten gesellschaftlichen tipping points und TippingProzesse rechtzeitig identifiziert und bezüglich ihrer Relevanz für Veränderungen analytisch eingestuft werden können (Brohmann und David 2015, S. 27). Insbesondere ist umstritten, ob ein gesellschaftlicher tipping point, ähnlich wie die planetaren Kipppunkte, einen umfassenden Wandel zur Nachhaltigkeit auslösen kann. Vielmehr wird dem Konzept ein »Gestaltungspotenzial in Teilsystemen« zugesprochen (Brohmann und David 2015, S. 33). Tipping points bieten insbesondere Gelegenheitsfenster für konkrete Veränderung und können den Zeitgeist beeinflussen (Kristof 2010b, S. 485-486). Auch in diesem Kapitel muss auf die Bedeutung von disruptiven Innovationen hingewiesen werden (Bower und Christensen 1995). Diese können den Veränderungsprozess deutlich beschleunigen und potenziell soziale tipping points auslösen. Jedoch kann auch argumentiert werden, dass disruptive Elemente nur Erfolg haben, wenn der Zeitgeist dies zulässt oder windows of opportunity vorhanden sind.

Die adäquate Prozessgeschwindigkeit ist abhängig vom Veränderungsziel und den Zeitvorstellungen beteiligter Akteurinnen und Akteure. Erfolgversprechend ist eine zügige, aber nicht überstürzte Prozessgestaltung mit klaren Abschlusszielen, sodass die Richtung der Veränderung nicht grundsätzlich hinterfragt wird. Eine zu starke Beschleunigung des Prozesses überfordert möglicherweise die Beteiligten, indem nicht genügend Zeit für die Anpassung von Gewohnheiten eingeräumt wird. Eine zu starke Entschleunigung birgt die Gefahr, dass der Prozess ins Stocken gerät und damit auch das Interesse an der Veränderung nachlässt. Diese Überlegungen des MoC-Ansatzes nach Kristof lassen sich anhand von Ansätzen zur Ökologie der Zeit ergänzen, die im Rahmen von Debatten über die gesellschaftlichen Gestaltungsmöglichkeiten von zeitlichen Rahmenbedingungen entstanden sind. Es lassen sich beispielsweise die Taktgeberinnen und Taktgeber von Veränderungsprozessen bestimmen, also jene Rahmenbedingungen, Institutionen oder Individuen, die in der Lage sind, den Rhythmus von Prozessen wesentlich zu bestimmen oder zu beeinflussen (Heitkötter und Schneider 2004). Rhythmus beschreibt das wiederholte Durchlaufen von Zuständen mit etwa gleicher Ausprägung (Heitkötter und Schneider 2004, S. 21). Den Taktgeberinnen und -gebern stehen Taktnehmerinnen und -nehmer gegenüber, die sich den Vorgaben weitgehend anpassen. Dadurch entstehen oftmals Zeitkonflikte zwischen den beiden Gruppen, sodass für wachsende zeitliche Konfliktpotenziale neue Lösungen erforderlich sind. 
Ausreichende Zeitressourcen spielen eine wichtige Rolle im Veränderungsprozess. Change agents müssen über genügend Zeitressourcen verfügen, da Veränderungsprozesse mühsam und langwierig sein können (Kristof 2010a, S. 527). Doch nicht nur für change agents, sondern auch bei der Ausgestaltung des Prozessverlaufes ist die verfügbare Zeit eine zentrale Variable. Die Forschung zur Ökologie der Zeit greift in diesem Sinne verschiedene Aspekte auf (Reisch 2002; Reisch und Bietz 2014). Beispielsweise erfordern Beteiligungsprozesse Zeit; »wieviel hängt neben der Art bzw. dem Grad der erwünschten Partizipation und der Komplexität der zugrundeliegenden Prozesse und anstehenden Entscheidungen von weiteren Faktoren ab, z. B. der Anzahl der Beteiligten, der Homogenität/Heterogenität bzw. Vereinbarkeit ihrer Interessen, der Art und Weise, wie die Durchsetzungsfähigkeit der Interessen unter den Beteiligten verteilt ist, dem Informationsstand sowie der Beurteilungs- und Kommunikationskompetenz der Beteiligten« (Heitkötter und Schneider 2004). Die Forschung zur Ökologie der Zeit thematisiert auch das Konzept des Zeitwohlstands, welches in engem Zusammenhang mit nachhaltiger Entwicklung, insbesondere mit nachhaltigem Konsum, steht. Reisch (2002, S. 48) hat hierfür acht relevante Dimensionen identifiziert: Zeitdauer, Zeitabfolge, Zeitsouveränität und Zeitautonomie, Timing, Synchronizität, angemessene Geschwindigkeit, Rhythmik und Berücksichtigung von Eigen- und Systemzeiten. Aufgrund der gestiegenen Bedeutung fordern Forscher eine explizite Zeitpolitik, die individuelle und gesellschaftliche Zeitarrangements berücksichtigt und in politische Entscheidungen integriert (Grießhammer und Brohmann 2015; Reisch und Bietz 2014).

\subsubsection{Veränderungsprozesse}

Der MoC-Ansatz nach Kristof betont die besondere Bedeutung der Charakteristika des Veränderungsprozesses für den Erfolg von Transformationen. Unabhängig von der Art des konkreten Veränderungsprozesses (etwa der Veränderungsaufgabe, der Haupttriebkraft, der Geschwindigkeit oder dem Leitbild der Veränderungsidee) müssen Elemente des Veränderungsprozesses so kombiniert sein, dass sie dessen Erfolg begünstigen. Folgende Tabelle 1 fasst die zentralen Elemente einer Prozessgestaltung des MoC-Ansatzes nach Kristof zusammen.

Einige dieser Elemente lassen sich im Rahmen eines Analyseprozesses leichter anderen Kernfaktoren zuordnen. Zudem können nicht alle Aspekte zufriedenstellend operationalisiert werden. Von besonderer Relevanz für den Analyseprozess sind folgende Elemente: 1) Interaktion der Akteurinnen und Akteure und Institutionalisierung, 2) Beteiligungs- und Teilhabeprozesse, 3) Veränderungskultur, Wissensbasis und Lernprozesse, 4) Reflexivität und Erfolgskontrolle sowie 5) Ressourcenausstattung (siehe Tabelle 1). Im Folgenden werden diese zu operationalisierenden Aspekte des MoC-Ansatzes erläutert und mit weiterführenden politikwissenschaftlichen Perspektiven angereichert. 
Tabelle 1: Zentrale Elemente einer erfolgreichen Prozessgestaltung

\begin{tabular}{|c|c|}
\hline Ebene der Erfolgsfaktoren & Beschreibung der Erfolgsfaktoren \\
\hline $\begin{array}{l}\text { Gestaltung des Verände- } \\
\text { rungsprozesses }\end{array}$ & 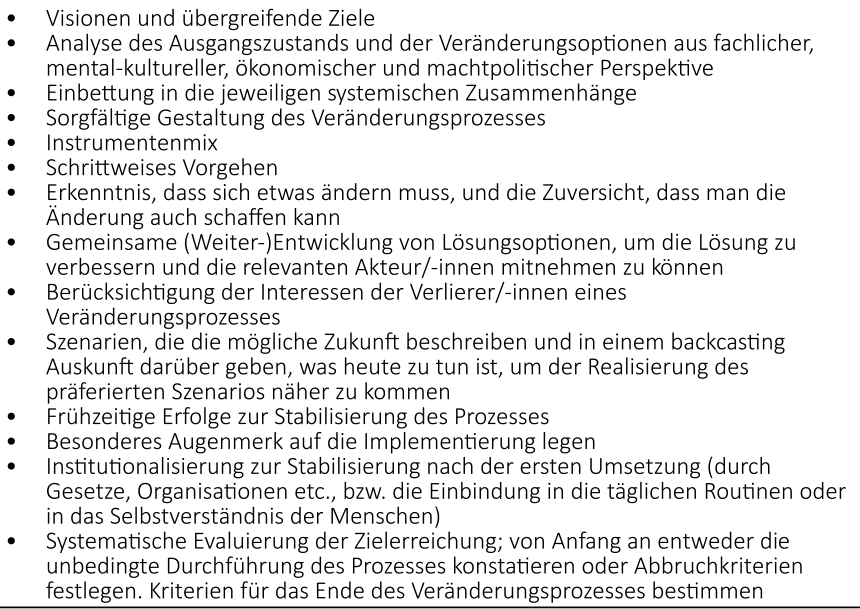 \\
\hline $\begin{array}{l}\text { Akteurinnen und Akteure } \\
\text { und ihre Interaktion }\end{array}$ & $\begin{array}{l}\text { Adäquate Interaktion und Einbindung der Beteiligten (change agents, Zielgruppe, } \\
\text { weitere relevante Akteurinnen und Akteure): } \\
\text { - Beteiligungs- und Teilhabeprozesse } \\
\text { - Souveränes Bewegen im Akteursnetzwerk mit den sehr verschiedenen } \\
\text { Standorten und Prozesssichten } \\
\text { - Am Ziel orientierte Kommunikations- und Beteiligungsprozesse }\end{array}$ \\
\hline Lernprozesse & $\begin{array}{l}\text { Individuelle und gruppenorientierte Lernprozesse zur Veränderung von Denkstrukturen } \\
\text { und Verhaltensweisen, ergänzt durch Lernprozesse, die Kompetenzen zur Gestaltung } \\
\text { von Veränderungsprozessen aufbauen (Lernen zweiter Ordnung) }\end{array}$ \\
\hline \multirow[t]{2}{*}{ Veränderungskultur } & Veränderungsfreundliche Kultur (inkl. Informations- und Kommunikationskultur) \\
\hline & Erhöhte Wahrscheinlichkeit für disruptive Innovationen \\
\hline Ressourcenausstattung & Ausreichende Ausstattung mit Personal, finanziellen Ressourcen und Zeit \\
\hline \multirow[t]{2}{*}{ Systemsicht } & $\begin{array}{l}\text { Umkehrbarkeit, Fehler- und Korrekturfreundlichkeit, flexible Reaktion auf } \\
\text { unbeabsichtigte Wirkungen als Leitlinien der Prozessgestaltung und als Antwort auf } \\
\text { die Komplexität der Systeme }\end{array}$ \\
\hline & $\begin{array}{l}\text { Unsicherheit, Unvorhersehbarkeit und Unplanbarkeit als Grundkonstante jedes } \\
\text { Veränderungsprozesses anerkennen }\end{array}$ \\
\hline
\end{tabular}

Quelle: Eigene Darstellung auf Basis von Kristof (2010a, S. 534).

\section{Die Interaktion der Akteurinnen und Akteure und Institutionalisierung ist}

eine zentrale Variable für erfolgreiche Transformationsprozesse, die sich auf verschiedenen politischen und gesellschaftlichen Ebenen manifestiert. Eine horizontale Integration, also die Koordination politischer Beteiligter unterschiedlicher Ressorts auf derselben Politikebene, gilt insbesondere aus Governance-Perspektive als zentraler Erfolgsfaktor in der Politik (Peters 1998; Kemp et al. 2005). In der Praxis treffen die dafür eingerichteten Koordinationsmechanismen häufig auf Probleme, wenn es an Institutionalisierung, Einflussmöglichkeiten und wichtigen Ressourcen fehlt. Eine vertikale Integration, also die Koordination der Akteurinnen und Akteure verschiedener Politikebenen, ist eine grundlegende Herausforderung für politische Veränderungsprozesse, insbesondere in föderal organisierten Staaten (Casado-Asensio und Steurer 2014; Kemp et al. 2005). Durch 
vertikale Integration lässt sich ein Informationsaustausch zwischen Beteiligten unterschiedlicher Ebenen gewährleisten, sodass nicht beabsichtigte Wirkungen von Maßnahmen einer Ebene für andere politische Ebenen vermieden werden können. Das Prinzip der Subsidiarität ist zu beachten, um Veränderungen und die damit verknüpften Entscheidungen auf der passenden politischen Ebene zu adressieren. Sowohl bei der horizontalen als auch bei der vertikalen Integration sollten die Interessen von Gewinnerinnen und Gewinnern sowie Verliererinnen und Verlierern des Veränderungsprozesses berücksichtigt und in die strategische Planung miteinbezogen werden.

Die Beteiligung und Teilhabe der Zivilgesellschaft an Veränderungsprozessen steigert die Erfolgswahrscheinlichkeiten von Transformationen, da sie zu einer Stärkung des Vertrauens in die Politik und zur Bereitstellung von lokalem Wissen führen kann. $\mathrm{Zu}$ beachten ist, dass relevante Akteurinnen und Akteure über die notwendigen Mittel zu Teilnahme und Teilhabe verfügen müssen. Dadurch wird sichergestellt, dass nicht nur stark organisierte Interessengruppen und Verbände den Beteiligungsprozess dominieren, sondern auch Individuen oder schwach organisierte Interessengruppen vertreten sind. Zudem ist die tatsächliche Berücksichtigung von Standpunkten und nicht die bloße Beteiligung als Legitimierungsmaßnahme von elementarer Bedeutung, damit echte Teilhabe entstehen und lokales Wissen genutzt werden kann. Ziel muss es sein, dass sich möglichst viele Betroffene mit dem Vorhaben identifizieren und somit ein Gefühl von ownership entsteht. Bottom-up-Beteiligungsprozesse beschäftigen sich oftmals verstärkt mit normativen Forderungen, verfügen dabei jedoch vielfach über mangelndes Prozess- und Hintergrundwissen, was eine sinnvolle Einbindung erschwert (Spash 2001; Rydin und Pennington 2000; Cooke und Kothari 2001).

Die Punkte Veränderungskultur, Wissensbasis und Lernprozesse umfassen eine Reihe von Aspekten, welche die Umfeldbedingungen für Veränderungsprozesse beschreiben. Wissen - beispielsweise über den Klimawandel - ist insbesondere bei umfassenden Transformationen wichtig, aber auch von spezifischen Charakteristika wie Komplexität, Langfristigkeit und Unsicherheiten geprägt (Sharman und Holmes 2010). Die Einbindung von Wissenschaft und Forschung zur Bereitstellung der Wissensbasis und zur Förderung von disruptiven Innovationen ist daher ein wichtiger Erfolgsfaktor (Pregernig und Böcher 2012). Dabei sollte schon möglichst früh innerhalb eines Veränderungsprozesses analysiert werden, welche Wissensbasis vorliegt, um Lücken zu identifizieren. Außerdem gilt es, das vorliegende Wissen, z.B. hinsichtlich der Organisation und Koordinierung von Prozessen für die Prozessgestaltung, zu nutzen (Dovers und Hezri 2010, S. 224).

Reflexivität und Erfolgskontrolle sind im gesamten Prozessverlauf von Bedeutung. Dazu zählen beispielsweise Assessments in der Strategieformulierung und -umsetzung, die Einbeziehung von Expertinnen und Experten sowie die Evaluation und das Indikatoren-Monitoring. Die Monitoring-Ergebnisse müssen 
anschließend angemessen verwertet werden, damit sie zu einer Verbesserung des Prozesses führen können. Zudem wird empfohlen, die Erfolgskontrolle möglichst von einer unabhängigen Institution durchführen zu lassen (Casado-Asensio und Steurer 2014). Dabei sind auch die im Abschnitt zu Lösungsideen angeführten betriebswirtschaftlichen Konzepte wie lean management, agility und 360-DegreeFeedback wichtige Tools zur Analyse von Erreichtem und für potenzielle Korrekturmaßnahmen (Schuh 2013; Leybourn 2013; Lepsinger und Lucia 2009). Dies verdeutlicht die außerordentliche Bedeutung von flexiblen Innovationen im Transformationsprozess.

Schließlich hat selbstverständlich auch die Ressourcenausstattung einen großen Einfluss auf den Verlauf von Veränderungsprozessen. Dies umfasst beispielsweise zeitliche und finanzielle Ressourcen, aber auch das Wissen der beteiligten Akteurinnen und Akteure (Reisch und Bietz 2014).

\section{Beispiel: Lokale Agenda 21}

Die Agenda 21 zur Förderung der Nachhaltigkeit wurde 1992 in Rio de Janeiro von über 170 Staaten als Aktionsprogramm mit konkreten Handlungsaufträgen unterzeichnet. Die Lokale Agenda 21 (LA21) stellt ein spezielles Handlungsprogramm zur Umsetzung der nachhaltigen Entwicklung auf der Ebene der Städte und Kommunen dar. Die lokalen Verwaltungen übernehmen als kleinste staatspolitische Organisationseinheiten also eine zentrale Rolle bei der Realisierung der Agenda 21. Laut Kapitel 28 sollen etwa die Kommunalverwaltungen »in einen Dialog mit Bürgerinnen und Bürgern, örtlichen Organisationen und der Privatwirtschaft eintreten und eine kommunale Agenda 21 beschließen« (UN 1992, S. 291).

Durch die vielfältigen Akteurskonstellationen und Problemfelder vor Ort ist bei der Implementierung der LA21 eine flexible Planung und Umsetzung notwendig. Verschiedene Organisationen wie ICLEI haben Leitfäden zur Erstellung einer LA21 präsentiert, die sich am klassischen Projektmanagement orientieren (Walk 2008, S. 225-226; Göll und Nolting 2012). Studien zeigen, dass der Aufbau von Netzwerken sowie eine aktive Informations- und Kommunikationspolitik als Erfolgsfaktoren für Agenda-Prozesse angesehen werden können. »Besonders positiv zu bewerten ist die Einbindung lokaler Bildungsträger in die Agenda-Prozesse. Sie sind in der Lage, Ideen und Projekte der Agenda 21, oftmals in Kooperation mit Umwelt- und Entwicklungsinitiativen, in Seminaren, Mitmachaktionen und Exkursionen lebhaft zu vermitteln" (Born und Kreuzer 2002, S. 10).

Der LA21-Prozess stellt einen partizipativen Multi-Stakeholder-Prozess dar, der die Beteiligung der Kommunalverwaltung, der Bevölkerung, der Nichtregierungsorganisationen sowie der Privatwirtschaft vorsieht. Es soll ein Hand- 
lungsplan erarbeitet werden, der an den Prinzipien nachhaltiger Entwicklung ausgerichtet ist und konsensual von allen Beteiligten verabschiedet wird (Ruschkowski 2002). Eine erste Evaluation der LA21-Implementierung zeigt, dass viele Kommunen erfolgreich Partizipationsmöglichkeiten für örtliche Akteurinnen und Akteure verbessert haben, beispielsweise durch den Aufbau von Agenda-Prozessen mit Hilfe von Vereinen und Bürgerforen sowie AgendaStabsstellen und Kontaktbüros in der Verwaltung. Dennoch mangelte es oft an ausreichenden Finanzmitteln, um ein professionelles und langfristiges Agenda-Management sicherzustellen (Born und Kreuzer 2002). Es zeigt sich, dass die Beteiligungsergebnisse von Politik und Verwaltung meist ernst genommen werden. Strategien zur langfristigen Umsetzung bleiben jedoch oftmals im Unklaren.

Der LA21-Prozess ist als Lernprozess zu betrachten, der zu einem kooperativen und auf Konsensfindung basierenden Verständnis von Politik beiträgt. Zudem werden Monitoring und Anpassungsprozesse des Handlungsprogramms kontinuierlich mittels Indikatoren durchgeführt (Ruschkowski 2002). Das Bundesministerium für Umwelt, Naturschutz, Bau und Reaktorschutz (BMUB) und die kommunalen Spitzenverbände sicherten ihre Unterstützung bei der Bearbeitung einer Lokalen Agenda $21 \mathrm{zu}$. Im Hinblick auf die Ressourcenausstattung ist des Weiteren eine gemeinsam herausgegebene Erklärung der kommunalen Spitzenverbände und der Umweltministerkonferenz hervorzuheben, in der beispielsweise Unterstützungsmöglichkeiten mittels Leitfäden und Modellvorhaben zugesagt wurden (Born und Kreuzer 2002).

\subsubsection{Pfadabhängigkeiten und Rahmenbedingungen}

Wie eingangs erläutert bilden Rahmenbedingungen im MoC-Ansatz nach Kristof den Kontext, vor welchem Veränderungen ablaufen. Sie können den Prozess behindern oder fördern. Zu diesen Rahmenbedingungen zählen Pfadabhängigkeiten, das framing des Problems und windows of opportunity.

Generell werden Transformationen durch ihren historischen Kontext geprägt, da dieser Einfluss auf die Erfahrungen der Akteurinnen und Akteure, den Aufbau von Akteursnetzwerken und deren Positionierung hat. Pfadabhängigkeiten verdeutlichen diese historische Einbettung, indem sie auf die Rolle bereits geschehener Ereignisse für Veränderungen in der Zukunft hinweisen (Leach et al. 2007). Bei einem Transformationsprozess sind deshalb vergangene Entwicklungen zu berücksichtigen, um mögliche zukünftige Hindernisse identifizieren zu können. Pfadabhängigkeiten werden nicht nur im MoC-Ansatz nach Kristof diskutiert, sondern stellen den zentralen Untersuchungsgegenstand der Evolutorischen Ökonomik dar. Unterschieden werden können rechtliche, ökonomische, infrastrukturelle, organisationale und nutzerbezogene Pfadabhängigkeiten (vgl. Kapitel 3.1). 
Während eines Veränderungsprozesses muss beachtet werden, dass vergangene und gegenwärtige Entscheidungen Auswirkungen auf zukünftige Entscheidungsoptionen haben können. Deshalb sollten insbesondere in frühen Stadien der Diffusion reversible Maßnahmen umgesetzt werden, um eine flexible Anpassung zu ermöglichen (Kristof 2010a; Leach et al. 2007). Dies ist insbesondere relevant, um sogenannte Lock-in-Effekte, etwa durch den Aus- und Neubau von Kohlekraftwerken, zu vermeiden. Vertreterinnen und Vertreter von Status-quoInteressen versuchen oftmals das »alte System« $\mathrm{zu}$ erhalten und ökonomische Vorteile aus Pfadabhängigkeiten und Lock-in-Effekten zu ziehen (Fligstein und McAdam 2011).

Weiterhin relevant für den Erfolg einer Transformation ist das framing des zugrundliegenden Problems. Dazu gehören einerseits die Spezifika eines Problems (Komplexität, Unsicherheiten etc.) und andererseits die Wahrnehmung eines Problems, etwa der Rückbezug auf persönliches Erleben. Auf diesem Wege kann durch eine bestimmte Darstellung des Veränderungsprozesses die Wahrscheinlichkeit einer erfolgreichen Transformation erhöht werden (Rein und Schön 1996; Chong und Druckman 2007; Head 2008).

Schließlich verweisen windows of opportunity auf bestimmte Zeitfenster, während welcher der Erfolg der Veränderung wahrscheinlicher ist. Die Bedeutung von Zeitfenstern für Veränderungen wurde von Kingdon und Thurber bereits in den 1980er-Jahren betont und seither zahlreich aufgegriffen. Ereignisse wie beispielsweise Naturkatastrophen, die ein Problem klar sichtbar machen und Dringlichkeit signalisieren, helfen policy windows zu öffnen und die Problemlösung zu beschleunigen. So verweist insbesondere Kingdons »Strom-Analogie» auf die Rolle von Zeitfenstern (Kingdon und Thurber 1984). Demnach ist ein Politikfeld bestimmt durch drei wesentlich voneinander unabhängige Ströme - den politischen Strom (Zustand der Politik einschließlich der öffentlichen Meinung), den Policy-Strom (policy: Lösung für ein Problem) und den Strom an Problemen. Veränderung wird möglich, wenn sich Ströme während eines Zeitfensters treffen - beispielsweise durch einen Wandel in der Problemwahrnehmung oder durch disruptiven technologischen Fortschritt (Birkland 2014). Einmalige tipping points oder Tipping-Prozesse werden dabei als Chancen verstanden, die ein Gleichgewicht in Teilsektoren hin zu nachhaltigeren Lösungen verändern können (Brohmann und David 2015). Wenn diese durch disruptive Innovationen ausgelöst werden, spielen das richtige Timing sowie die Unterstützung von relevanten Akteurinnen und Akteuren eine große Rolle.

Die Rahmenbedingungen für politischen Wandel werden darüber hinaus von der Punctuated-Equilibrium-Theorie thematisiert (Baumgartner und Jones 1993). Demnach wird themenbezogene Stabilität durch politische Monopole geschaffen, die durch die Unterstützung des Status quo durch entscheidende Beteiligte (bzw. Vetospielerinnen und Vetospieler) entstehen. Solche politischen Monopole haben 
kein Interesse an Transformationen und versuchen daher den Veränderungsdruck gering zu halten. Gründe für diese Stabilität sind etwa die Pfadabhängigkeit von Institutionen, starke Interessengruppen und begrenzte Rationalität (Baumgartner und Jones 1993). Dennoch gibt es Anlässe, die zum Zusammenbrechen von Monopolen und ihren Rahmenbedingungen sowie zu einem raschen Politikwandel in einem politischen Teilsystem führen können. Dies ist der Fall, wenn es Gegenspielerinnen und Gegenspielern gelingt, eine Alternative aufzuzeigen und dafür ausreichende Unterstützung und Akzeptanz zu generieren, sodass sich eine neue Phase der Stabilität durch eine grundlegende Veränderung in der Gesellschaft einstellt. Dies kann beispielhaft durch die Diffusion einer revolutionären technologischen Innovation erreicht werden.

\section{Beispiel: Reaktorkatastrophe in Fukushima}

Ein Beispiel für die besondere Rolle von Rahmenbedingungen innerhalb von Veränderungsprozessen ist die Reaktorkatastrophe von Fukushima im März 2011 und das sich daraus ergebende Zeitfenster (Grießhammer und Brohmann 2015). Diese nukleare Katastrophe löste in Deutschland eine Wende in der Atompolitik aus und war somit von großer Bedeutung für Fortschritte in der Energiewende. Obwohl die deutsche Bundesregierung noch wenige Monate zuvor eine Verlängerung der Laufzeiten der deutschen Atomkraftwerke gegen die atomkritischen Oppositionsparteien durchgesetzt hatte, erfolgte im Zuge der Reaktorkatastrophe ein deutlicher Wandel der Atom- und Energiepolitik. Der Atomausstieg wurde wieder eingeleitet und im Juni 2011 vom deutschen Bundestag mit großer, parteiübergreifender Mehrheit beschlossen (Wolling und Arlt 2014). Das »13. Gesetz zur Änderung des Atomgesetzes« regelt die Beendigung der Kernenergienutzung und die Beschleunigung der Energiewende (Deutscher Bundestag 2011).

Weitere Beschlüsse im Rahmen der deutschen Atompolitik nach der Katastrophe in Fukushima umfassten die Anordnung von Sicherheitschecks für alle deutschen Atomkraftwerke, die Verkündung eines Atommoratoriums und die Einsetzung einer Ethikkommission. Der beschleunigte Atomausstieg ist im Rahmen der Energiewende mit dem Ziel, den Ausbau der erneuerbaren Energien als Alternative zur Kernkraft bis zum Jahr 2025 auf 40 bis 45 Prozent und bis zum Jahr 2035 auf 55 bis 60 Prozent auszubauen, ein wichtiger Schritt. Im Zuge der Katastrophe von Fukushima stieß der Ausstieg aus der Kernenergie mit einem beschleunigten, verbindlichen Datum auf breite gesellschaftliche Unterstützung.

Das Beispiel der Reaktorkatastrophe von Fukushima und der dadurch intensivierte Transformationsprozess in Richtung Atomausstieg und Energiewende zeigen, dass das richtige Timing eine zentrale Bedeutung für die Implementie- 
rung oder die Verstärkung von Transformationsprozessen hat. Ein Ländervergleich zeigt aber auch, dass windows of opportunity in umweltpolitischen Transformationsprozessen unterschiedlich stark genutzt werden: Deutschland nimmt hier eine Vorreiterrolle ein. Dies lässt sich auf unterschiedliche Faktoren und Ereignisse zurückführen. Zum einen ist in Deutschland seit vielen Jahren eine vergleichsweise starke Anti-Atomkraft-Bewegung aktiv und der Ausbau der erneuerbaren Energien wurde lange Zeit inhaltlich, technisch und strukturell vorbereitet. Zum anderen gaben auch die Ölkrise (1974), die AntiAtomkraftwerk-Demonstrationen, Tschernobyl (1986), das 100.000-Dächer-Programm (1999) sowie das Erneuerbare-Energien-Gesetz (EEG 2000) wichtige Impulse für den Atomausstieg, der am tipping point der Reaktorkatastrophe von Fukushima beschlossen wurde (Grießhammer und Brohmann 2015).

\subsection{Effizienz, Konsistenz und Suffizienz als Basisstrategien einer Green Economy}

\subsubsection{Hintergrund}

In der Debatte über die Umsteuerung zu einer nachhaltigen Entwicklung und speziell zu nachhaltigem Wirtschaften werden drei mögliche Basis- bzw. Leitstrategien diskutiert: »Effizienz«, »Konsistenz« und »Suffizienz«. Kurzgefasst steht »Effizienz« für weniger Ressourceneinheit pro Serviceeinheit, »Konsistenz« für naturverträgliche Technologien und Strukturen und »Suffizienz« für die Reduktion des belastenden Konsums. Über die Definitionen und Abgrenzungen sowie den Stellenwert dieser Strategien für die Transformation (vgl. WBGU 2011; Grießhammer und Brohmann 2015) von Wirtschaft und Gesellschaft und ihr Verhältnis $\mathrm{zu}$ einer anzustrebenden Green Economy existieren unterschiedliche Ansichten und wird teilweise heftig gestritten (vgl. Huber 2013; Linz 2013; Spangenberg 2013).

Die Effizienz-Strategie gilt dabei in der Wirtschaft als besonders anschlussfähig und wird in zahlreichen Formen praktiziert. Eine Steigerung der Ressourcenproduktivität, der Rohstoff- und Energieeffizienz ist in wirtschaftlicher und ökologischer Hinsicht vorteilhaft, sprich: wirtschaftlich kosteneinsparend und ökologisch belastungsminimierend. Inzwischen spiegelt sich dieser Zusammenhang in einer dynamischen Entwicklung der globalen GreenTech-Märkte wider, deren »Volumen eine völlig neue Dimension« erreicht (BMUB 2014). Der Effizienz-Strategie wird sogar eine »Effizienzrevolution« zugetraut. Andere Akteure sind hingegen deutlich weniger euphorisch, ja skeptisch, und messen der Effizienz-Strategie einen nachrangigen Stellenwert bei. Sie verweisen dabei auf Rebound-Effekte, die Effizienzgewinne geringer ausfallen lassen oder sogar überkompensieren (Santarius 2012; Paech 2012). 
"Konsistenz« wird im Unterschied dazu als grundlegenderer, systemischer Ansatz diskutiert. Ihm werden eine größere Reichweite und ein größerer Impact zugetraut. Während die Effizienz-Strategie mengenorientiert ist - weniger Ressourcenverbrauch bei mehr Ertrag -, zielt die Konsistenz-Strategie auf die Veränderung der Qualität der Stoff- und Energieströme ab. Gemeint sind dabei Stoffe, Produkte und Technologien, die verträglich, eben konsistent, mit natürlichen Stoffkreisläufen sind (Schmidt 2008). Im Kern geht es um die "Schaffung einer metabolisch naturintegrierten Industriellen Ökologie durch basisinnovative Konzeption und Konstitution von technischen `Systemwechseln', also dem Set-up und späteren Take-off neuer Technologien auf neuen Pfaden« (Huber 2000, S. 13).

Besonders leidenschaftlich debattiert wird über den Stellenwert von Suffizienz. Während eine Fraktion behauptet, dass Suffizienz "nicht nur ein relativ geringes Einsparpotenzial« besitzt, sondern »auch ein viel zu geringes sozio-kulturelles Anschluss- und Resonanzpotenzial (Huber 2000, S. 12; siehe auch Fücks 2013), kommt die andere Fraktion zu der Einschätzung: "Suffizienz ist ein notwendiger Baustein in einem ebenso notwendigen Ensemble unterschiedlicher Nachhaltigkeitsstrategien" (Heyen et al. 2013, ähnlich Linz 2012). Auf Gesellschaftsebene wird in dieser Sicht eine »konsequente sozial-ökologische Transformation der Produktions- und Lebensweise und eine demokratisch organisierte Reduktion von Produktion und Konsum" (Schmelzer und Passadakis 2011, S. 67) als notwendig angesehen. Das Konzept »der Postwachstumsökonomie orientiert sich an einer Suffizienz-Strategie und dem partiellen Rückbau industrieller, insbesondere global arbeitsteiliger Wertschöpfungsprozesse zugunsten einer Stärkung lokaler und regionaler Selbstversorgungsmuster" (Paech 2012). Gefordert wird ein »Transformationsdesign", das auf ein »komplett anderes Leben« abzielt, um die sich zuspitzenden Bedrohungen wie Klimakatastrophe zu vermeiden bzw. deren Negativefekte zu mindern - nach dem Motto »Transformation by Design« statt "Transformation by Desaster«. »Wiederverwenden, umnutzen, mitnutzen« ist das Credo einer neuen, "reduktiven Moderne«, die mithin explizit kulturelle Veränderungen erfordert und mit sich bringt (Sommer und Welzer 2014). Einen aktuellen Schub erhält die Diskussion über die drei Basisstrategien der Nachhaltigkeit durch den Diskurs über Postwachstum, da in diesem Kontext über Bereiche von künftigem Wachstum und dessen Art und Qualität sowie die erforderlichen Konzepte und Vorgehensweisen diskutiert wird (z. B. Adler 2014; Hunecke 2013; Paech 2012; Welzer 2011). Darüber hinaus erhält die Thematik »Nachhaltigkeit und Pfadwechsel« in internationaler Perspektive eine besonders große Bedeutung, da eine Verallgemeinerung des westlichen Produktions- und Lebensstils nicht möglich ist und die von der UN und der Weltgemeinschaft definierten Nachhaltigkeitsziele (SDG) auch für die EU-Staaten eine große Herausforderung darstellen und deren Erreichung dringend ermöglicht werden muss (Messner und Scholz 2015; Wuppertal Institut 2005; Steffen et al. 2015; Hege 2019). 
Während die bisherige wissenschaftliche, gesellschaftliche und politische Debatte im Wesentlichen die Vorzüge und Nachteile der jeweiligen Strategien hervorgehoben hat und dabei Positionen und Scheidelinien (z. B. Effizienz und Konsistenz versus Suffizienz oder umgekehrt) markiert, fehlt es an einer vergleichenden Analyse und Einordnung der Transformationspotenziale für eine Green Economy, die auch deren Wechselwirkungen beachtet und konzeptionell miteinander verknüpft, also die komplementären Funktionen und Potenziale herausarbeitet. Mit Blick darauf soll das vorliegende Kapitel dafür einen heuristischen Rahmen aufspannen.

\subsubsection{Effizienz, Suffizienz und Konsistenz als Analyserahmen für »evolution2green"}

Das Strategiemodell »Effizienz, Konsistenz und Suffizienz« ist ein hilfreiches Analyseraster, um Transformationen strukturiert zu diskutieren. Es kombiniert strategische Ebenen auf Makro-, Meso- und Mikroebene und verbindet diese mit einer Akteursperspektive, die es ermöglicht Handlungsspielräume auszuloten. Im Folgenden werden die Strategien bezüglich ihrer Transformationspotenziale für eine Green Economy beleuchtet und eingeschätzt.

\section{Abbildung 5: Strategien für eine Green Economy}

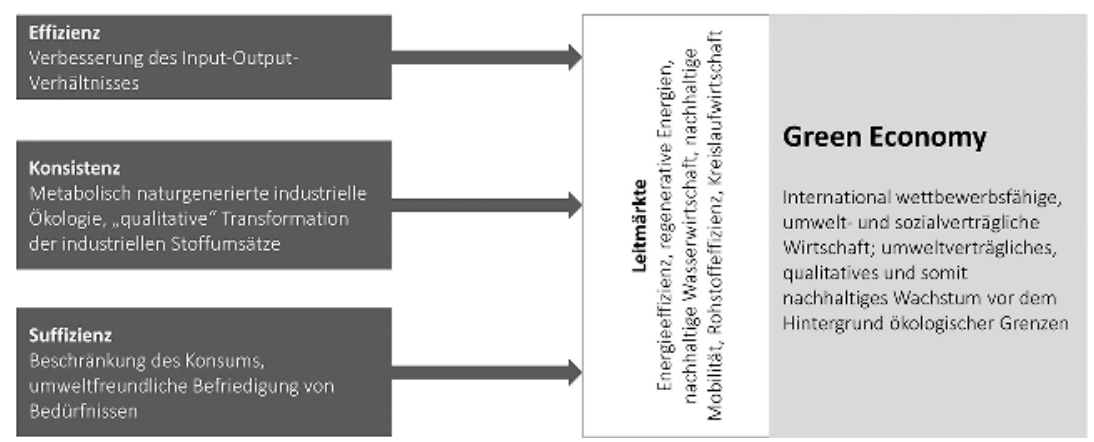

Quelle: Eigene Darstellung, Institut für Zukunftsstudien und Technologiebewertung nach BTEnquete-Kommission (1996); EU, BMUB (2014).

\subsubsection{Effizienz}

Die Effizienz-Strategie zielt darauf ab, eine ökonomische Leistung mit geringstmöglichem Einsatz an Material und Energie zu erstellen, indem das Input-Output-Verhältnis verbessert wird. Konkret bedeutet dies eine Steigerung der Material-, Rohstoff- und Energieeffizienz. Der Effekt besteht in einer relativen Senkung des Ressourcenverbrauchs. Ansätze liegen in der Verbesserung der Technik, der Prozesse und der Produkte. Ein Beispiel sind effizientere Motoren, 
ein anderes Beispiel ist die Wärmedämmung von Gebäuden, die zur Energieeinsparung beiträgt. Man kann mit Blick darauf von der Energieeffizienz (Einsatz an Energie pro Einheit Nutzenleistung) sprechen. Gängige Indikatoren sind beispielsweise im Wohnungsbereich Wärmeenergie pro Fläche (Kilowattstunde pro Quadratmeter) oder Wärmeenergie pro Kopf. Unter Materialeffizienz ist das Verhältnis der Materialmenge in den erzeugten Produkten zu der für ihre Herstellung eingesetzten Materialmenge zu verstehen. Auf volkswirtschaftlicher Ebene dient der Quotient aus Wertschöpfung (BIP) und seinem Rohstoffverbrauch (BIP/ Rohstoffmasse) zur Beschreibung der Rohstoffproduktivität. Energieproduktivität wird ausgedrückt als Einheit BIP (Bruttoinlandsprodukt) im Verhältnis zum Primärenergieverbrauch oder zum Endenergieverbrauch. Die Effizienz ist umso höher, je niedriger der Ressourceneinsatz ist. Die Umkehrung dieser Quotienten wird ebenfalls verwendet und beschreibt die Ressourcenintensität als Verhältnis von genutzter Umweltressource zur erwirtschafteten Leistung einer Wirtschaft. Dabei ist eine sinkende Ressourcenintensität gleichbedeutend mit einer zunehmenden Ressourceneffizienz - oder anders ausgedrückt: Je sparsamer eine natürliche Ressource in Relation zur ökonomischen Wertschöpfung verwendet wird, desto weniger wird die Umwelt belastet.

\section{Transformationspotenziale}

Die Effizienz-Strategie ist jene, »die im vorherrschenden Wirtschaftsgeschehen am ehesten anschlussfähig ist (Huber 1995). Dabei ist der Fokus meist betriebswirtschaftlich gesetzt. In der Effizienz-Strategie »findet sich das Wirtschaftlichkeitsdenken am ehesten wieder: das Maximierungsprinzip, wonach der Ertrag bei konstantem Aufwand zu maximieren ist, und das Minimierungsprinzip, wonach der Aufwand bei konstantem Ertrag zu minimieren ist « (Schmidt 2008).

Es verwundert daher nicht, dass die Effizienz-Strategie von Akteurinnen und Akteuren der Wirtschaft gerne aufgegriffen wird. "Sie steht in keinem Widerspruch zu ökonomischen Zielen, sondern unterstützt diese sogar«. (Schmidt 2008) Im Rahmen der Debatte um eine Green Economy spielt die Effizienz-Strategie eine große Rolle. Im Umwelttechnologie-Atlas des BMUB heißt es: Für die Fortschritte der »Grünen Transformation« spielt die Ressourceneffizienz eine Schlüsselrolle, »denn die Unternehmen dieses Wirtschaftszweiges tragen mit ihren Produkten, Verfahren und Dienstleistungen zur Lösung der ökologischen Herausforderungen bei. Hinzu kommt, dass die Umwelttechnik und Ressourceneffizienz das Greening, das heißt die Grüne Transformation in anderen Wirtschaftszweigen maßgeblich unterstützt« (BMUB 2014). Energieeffizienz, Material- und Rohstoffeffizienz, Wassereffizienztechnologien, Verkehrseffizienz und Kreislaufwirtschaft sind wichtige Leitmärkte. Sogar eine Effizienzrevolution wird für möglich gehalten und auch gefordert (Müller 2007). Denn »würde man die Logik der Effizienzsteigerung und Kostenminimierung noch konsequenter 
als bisher auch auf ökologische Aspekte anwenden, dann, so die Hoffnung, wäre eventuell der erforderliche Material- und Energie-Input für Endleistungen auf einem gleichbleibend hohen Konsumniveau in möglichst kurzer Zeit um einen Faktor vier bis zehn zu verringern « (Huber 2000; siehe Schmidt-Bleek 2014; Weizsäcker et al. 1995; Weizsäcker et al. 2010).

Grundsätzlich bestehen in der Wirtschaft noch erhebliche Effizienzpotenziale. Ihre Erschließung fällt hingegen sehr unterschiedlich aus. Zwar lässt sich ein relatives decoupling bzw. delinking - eine Ent- bzw. Abkopplung von der Entwicklung des Bruttoinlandsproduktes (BIP) - feststellen, absolut sind die Reduktionen aber gering. Vergleichende Länderstudien kommen zu Unterscheidungen nach »Vorreitern«, »Nachzüglern« und »Sitzenbleibern« (Simonis 2004). Dementsprechend muss eine weit höhere Ressourcenproduktivität erzielt werden, wenn Ressourceneffizienz als Transformationsstrategie für eine Green Economy eine starke bzw. absolute Entkopplung erreichen soll - wenn die globale Ökologie also wirklich entlastet werden soll (vgl. Simonis 2004; UNEP 2014). Ressourceneffizienz als Transformationsstrategie für eine Green Economy ist als absolute und nicht nur relative Umweltentlastung nur dann wahrscheinlich, wenn die Ressourceneffizienzzunahme beschleunigt wird, also deutlich schneller als bis dato erfolgt. Aus Transformationssicht in Richtung einer Green Economy geht es dabei nicht nur um inkrementelle Verbesserungen, sondern um »Quantensprünge « für einen schonenderen Umgang mit Ressourcen, die deutlich über dem Wirtschaftswachstum liegen und/oder geringe Rebound-Effekte erwarten lassen. Dies gilt insbesondere dort, wo sich neue Paradigmen im Umgang mit Ressourcen abzeichnen. Beispiele sind: urban mining, non-destructive recycling, Null-Ausschuss-Produktion oder Green IT. Von ihnen kann eine starke gestalterische Kraft ausgehen, die über verschiedene Anwendungsfelder hinweg wirkt und Selbstorganisationsprozesse auslöst. Dadurch eröffnen sich neue Gestaltungsmöglichkeiten (windows of opportunity) für eine effizientere Ressourcennutzung. $\mathrm{Zu}$ fragen ist also, welche weitergehenden Material-, Produkt- und Systeminnovationen möglich sind und wie diese erschlossen werden können (durch welche Akteure, Rahmenbedingungen, Governance etc.).

\section{Grenzen: Wachstumszwänge}

Grenzen der Effizienz-Strategie liegen im vorherrschenden bzw. immer wieder öffentlich proklamierten Wirtschaftswachstum und in Rebound-Effekten. Wirtschaftswachstum treibt den Energie- und Rohstoffverbrauch an und erhöht die Ressourceninanspruchnahme. Liegt die Erhöhung der Ressourcenproduktivität unter der Wirtschaftswachstumsrate, ist nur eine relative Entkopplung möglich, der Ressourcenverbrauch dürfte absolut steigen. Effizienzsteigerungen können außerdem produktions- und konsumsteigernde Auswirkungen haben. Solche Rebound-Effekte schmälern erzielte Effizienzgewinne, können sie auch gänz- 
lich aufheben. Das papierlose Büro ist ausgeblieben, der Verkehr wächst trotz Telekommunikation, die Hardwaremassenströme schwellen trotz Leistungssteigerung und Miniaturisierung der Informations- und Kommunikationstechnik an. Zu solchen Rebound-Effekten tragen verschiedene Mechanismen bei. Sie kommen insbesondere dadurch zustande, dass Effizienzverbesserungen preissenkende und einkommensstärkende und daher konsumsteigernde Wirkungen haben können. Außerdem können Effizienzfortschritte zu Zeitgewinnen führen, die sich wiederum in einem veränderten Konsum niederschlagen. Volkswirtschaften reagieren im Allgemeinen auf Effizienzerhöhungen mit einer Nachfrageausweitung (Binswanger 2001). Die Erschließung neuer Anwendungs- und Absatzmöglichkeiten trägt damit zu Kompensation von Effizienzgewinnen bei. Häufig führen Effizienzsteigerungen auch zu höheren Leistungsanforderungen, sodass sich nicht der Ressourcen-Input pro Produkt reduziert, sondern der Leistungs-Output erhöht (Hilty 2003; Hilty et al. 2015).

\subsubsection{Konsistenz}

Während es bei Effizienz in erster Linie um Mengenaspekte geht, also um weniger Rohstoff- und Energienutzung, hebt die Konsistenz-Strategie auf eine »qualitative Transformation der industriellen Stoffumsätze« (Huber 2000) ab, ist also stärker systemisch und strukturell ausgerichtet.

Konsistenz bedeutet die umweltverträgliche Beschaffenheit von Stoffströmen. Konsistente Stoffströme sind solche, »die entweder weitgehend störsicher im abgeschlossenen technischen Eigenkreislauf geführt werden, oder aber mit den Stoffwechselprozessen der umgebenden Natur so übereinstimmen, dass sie sich, auch in großen Volumina, relativ problemlos darin einfügen« (Huber 2000, S. 81). Konsistenz steht also für eine »metabolisch naturintegrierte industrielle Ökologie«. Damit zielt diese Strategie auf eine Veränderung der »Qualität« der Energie- und Stoffströme ab. Alternativ wird auch der Begriff»Öko-Effektivität« genutzt.

Handlungsfelder für eine Konsistenz-Strategie liegen besonders dort, wo ökologische Grenzen erreicht sind; dies gilt insbesondere mit Blick auf globale, "planetare Grenzen" (Rockström et al. 2009). Vier von neun planetaren Grenzen sind durch den Einfluss des Menschen bereits überschritten: Klimawandel, Biodiversität, Landnutzung und biogeochemische Kreisläufe. Einige der Grenzen, die global noch nicht überschritten sind, übersteigen bereits regionale Toleranzlimits, etwa der Wasserverbrauch in Teilen Südeuropas, Asiens und des Mittleren Ostens. In Deutschland werden Wirkungsschwellen von Ökosystemen aufgrund versauernder und eutrophierender Stoffeinträge des Niederschlags überschritten. Die derzeitigen Säure- und Stickstoffeinträge liegen für viele Ökosysteme über den critical loads. Bei bestimmten Grenzwertüberschreitungen werden tipping points vermutet - mit oft plötzlichen und irreversiblen Reaktionen. 
Vor diesem Hintergrund geht es darum, in ökologisch betroffenen Bereichen konsistente Stoffströme herzustellen. Im Zuge der Nachhaltigkeitsdebatte sind vielfältige Ansätze zu verzeichnen, zum Beispiel

- Bionik (Rechenberg 1973; Gleich 1998)

- Ökologische Modernisierung (Jacob und Jänicke 2006; Huber 1995)

- Management des industriellen Metabolismus (Ayres und Simonis 1994)

- Cradle-to-Cradle (Braungart und McDonough 2008)

- Industrielle Ökologie (Graedel 1994; Gleich und Gößling-Reisemann 2008)

- Bioökonomie (Lahl und Zeschmar-Lahl 2012; BMBF 2014)

- Die übereinstimmende Stoßrichtung der verschiedenen Konzepte liegt darin, grundlegende Technik- und Produktinnovationen herbeizuführen, die auf ein ökologisches re-embedding abzielen.

$\mathrm{Zu}$ den wichtigsten Transformationsfeldern einer metabolisch besser naturintegrierten Wirtschaft gehören die Energieerzeugung, der Verkehrsbereich, die Landwirtschaft, die Grundstoffindustrie und die Chemie. Auf besondere Handlungsbereiche machen verschiedene Studien und Aufsätze (Huber 2000; Simonis 2004; Schmidt 2008) aufmerksam, u.a.

- Erneuerbare Energien wie Solarenergie, Wind- und Wasserkraft, Geothermalenergie

- Brennstoffzellen in mannigfaltigen Anwendungen, von Kraftwerken bis Fahrzeugantriebe

- Wasserstoff-Technologien

- Dezentralisierte Mikroenergieerzeugung

- Industrielle Biotechnologie

- Ersatz gefährlicher Chemikalien

- Biofeedstocks

- Nanotechnologie

- Ökologische Landwirtschaft

- Verzicht auf persistente Stoffe

Die Beispiele machen deutlich, dass Konsistenz gegenüber bestehenden Pfaden vielfach radikale Innovationen verlangt; oftmals wird eine Substitution bestehender Technologien, Produkte oder Stoffe angestrebt, weshalb andere Technologien notwendig sind. Mit einer Konsistenz-Strategie sind nicht nur technische Veränderungen verbunden, sondern auch große organisatorische Änderungen mit hoher Eingriffstiefe bei Design, Produktion, Distribution und Redistribution von Produkten. Konsistenz ist deshalb eine Strategie für einen technischen und wirtschaftlichen Strukturwandel mit weiteren Folgen für rechtliche, politische und 
kulturelle Veränderungen (Huber 2000). Ein Übergang zu einer naturintegrierten Wirtschaft muss offensichtlich mit Basisinnovationen einhergehen, die einen Paradigmenwechsel herbeiführen und breite Teile von Wirtschaft, Politik und Gesellschaft erfassen. »Energiewende«, "Verkehrswende«, "Ernährungswende«, »Bioökonomie« und »Low Carbon Economy« sind aktuelle Beispiele für solche Transformationsfelder.

In einzelnen Bereichen macht sich die Konsistenz-Strategie signifikant bemerkbar. So beispielsweise beim Abbau der Ozonkonzentration in der Stratosphäre. Durch das Verbot ozonzerstörender Substanzen ging deren Emission deutlich zurück, langsam auch die Konzentration in der Ozonschicht. Ein weiteres Beispiel ist das Verbot der als »Dreckiges Dutzend« (dirty dozen) bekannten zwölf Giftstoffe (u.a. Pflanzenschutzmittel, Industriechemikalien und Nebenprodukte von Verbrennungsprozessen). Sie wurden durch die POP-Konvention (Stockholmer Übereinkommen über persistente organische Schadstoffe) bzw. das Stockholmer Übereinkommen (vom 22. Mai 2001) weltweit verboten. Im Energiesektor wurden die energiebezogenen $\mathrm{CO}_{2}$-Emissionen vom Wirtschaftswachstum entkoppelt. In 2014 wuchs die Weltwirtschaft um ca. drei Prozent, während die $\mathrm{CO}_{2}$-Emissionen des Energiesektors konstant blieben. Neben dem Rückgang der Energieintensität ist diese Entwicklung auf die Expansion kohlenstoffarmer Technologien zurückzuführen. Erneuerbare Energieträger, z. B. Wind, Solar oder Wasserkraft, machten fast die Hälfte der in 2014 neu geschaffenen Stromerzeugungskapazität aus (IEA 2015).

\section{Grenzen: Technologieumsetzung}

Die Konsistenz-Strategie besitzt ein hohes Transformationspotenzial für eine Green Economy. Allerdings ist die Eingriffstiefe in bestehende Strukturen und Systeme vielfach hoch, insbesondere dort, wo es um die Substitution bestehender Technologien und/oder Systeme geht, sodass die Konsistenz-Strategie mit der Überwindung von Pfadabhängigkeiten und Blockaden konfrontiert ist (z. B. feedstock change, regenerativ basierte E-Mobilität). Aus diesem Grund wird argumentiert, dass der »vollständige und gleichwertige Ersatz aller derzeitigen Güter und Dienstleistungen in konsistenter Form derzeit noch Zukunftsmusik ist. Und darauf zu setzen, dass die notwendigen Technologiesprünge rechtzeitig stattfinden, bevor der Klimakollaps droht, die fossilen Ressourcen erschöpft sind oder die Artenvielfalt zerstört ist, ist hochriskant" (Fischer und Grießhammer 2013). Tatsache ist, dass viele industrielle Stoffe bis dato nicht in die Natur als »Nährstoffe« integriert werden können (Huber 1995, S. 146). Produkte, die nach dem Cradle-to-Cradle-Konzept (Braungart und McDonough 2008) entweder als biologische Nährstoffe in biologische Kreisläufe zurückgeführt werden können oder als »technische Nährstoffe« kontinuierlich in technischen Kreisläufen gehalten werden, sind bis dato die Ausnahme. Im Gegenteil: Durch den Trend zur 
Miniaturisierung und Leistungsintegration können viele Produkte immer schwerer stofflich verwertet werden. Durch die Komplexität der Wertschöpfungskette wird auch das Recycling schwieriger (Behrendt et al. 2015a). Dies ist beispielsweise bei vielen Technologiemetallen, die für Zukunfts- und Umwelttechnologien von besonderer Bedeutung sind, festzustellen. Die Erfassung und Sammlung von technologiemetallhaltigen Abfällen sowie die Einsteuerung dieser Abfälle in die dafür am besten geeigneten Recyclingketten ist heute völlig unzureichend.

Außerdem können ökologische Risiken und Nebenfolgen auftreten. Sie äußern sich beispielsweise in Form von Nutzungskonflikten für die zur Verfügungen stehenden Räume und Flächen. So lässt sich insbesondere die Bodenfläche für Bioenergie und Biomasse insgesamt kaum ausweiten, ohne Nahrungsproduktion und Naturschutz zu gefährden (Unmüßig et al. 2012). Der Bau von Windkraftanlagen ruft Lärmprobleme und Nutzungskonkurrenzen hervor. Es ist demnach davon auszugehen, »dass eine knappe Ressource nicht ohne weiteres durch eine andere zu ersetzen ist.« (Unmüßig et al. 2012) »Weltweit ist zum Beispiel das verfügbare Ackerland pro Person in den letzten Jahrzehnten von 0,45 ha auf weniger als 0,25 ha gesunken. Mehr noch: Je mehr Technik sich in Naturzyklen integriert, desto mehr wirken deren Rhythmen und Kapazitäten als eine Bremse für übersteigerte Leistungserwartungen.« (Unmüßig et al. 2012).

Die Strategie der Konsistenz hat voraussichtlich das weitreichendste Transformationspotenzial. Indem mit ihr versucht wird, die Stoff- und Energieströme den natürlichen biogeochemischen Stoffkreisläufen und deren Funktionsprinzipien anzupassen, impliziert sie einen tiefgreifenden gesellschaftlichen Wandel (in ökologischen Problemfeldern wie Energie, Verkehr, Landwirtschaft, Chemie usw.). Die damit verbundenen Prozesse sind langfristig, »wegen des erforderlichen wissenschaftlich-technologischen Vorlaufs, wegen der nur langfristig erfolgenden Erneuerung oder Substitution von Kapitalstöcken, der Trägheit von Paradigmenwechseln ebenso wie Personalstrukturen, den Interessenkonflikten zwischen Platzhaltern und Neuerern, wegen der Notwendigkeiten gesellschaftlicher Bewertung und alltagspraktischer Assimilation, auch der Notwendigkeiten rechtlicher Regelungen« (Huber 2000) und vielem mehr. Das Problemlösungspotenzial der Konsistenz-Strategie hängt auch wesentlich davon ab, inwieweit es gelingt, die Prozesse so zu gestalten, dass ökologische Risiken und Nebenfolgen, Nutzungskonkurrenzen und Zielkonflikte früh erkannt und möglichst vermieden werden.

\subsubsection{Suffizienz}

Unter Suffizienz, hier: »Öko-Suffizienz«, verstehen wir »Änderungen in Konsummustern, die helfen, innerhalb der ökologischen Tragfähigkeit der Erde zu bleiben, wobei sich Nutzenaspekte des Konsums ändern« (Heyen et al. 2013, S. 7). Darunter werden diejenigen Schritte, Maßnahmen, Instrumente und Strategien 
von Individuen und Organisationen gefasst, mit denen Ressourcen durch Verhaltensveränderungen eingespart werden können, die mit der Absicht erfolgen, Energie und Rohstoffe anders zu nutzen und von ihnen weniger zu verbrauchen als bisher. »Eine Ressourcen schonende Lebensweise ist also das Ziel der Öko-Suffizienz.« (Linz 2012, S. 75) Mit dieser Basisstrategie bestehen unmittelbare Anknüpfungspunkte bzw. Überschneidungen vor allem $\mathrm{zu}$ »anwenderbezogenen Pfadabhängigkeiten« bzw. entsprechenden Möglichkeiten für einen Pfadwechsel in diesen Kontexten.

Der Suffizienzdiskurs zeichnet sich allerdings noch durch viele weitere Facetten aus: So wird Suffizienz oft mit dem Diskurs um ein »gutes Leben« (Schneidewind und Zahrnt 2013) in Verbindung gebracht oder als ein Konsummuster mit dem »rechtem Maß« (Linz 2013; Oekom 2013). Es findet demnach eine positive Bewertung sowohl eines qualitativ anderen Konsums als auch einer Reduktion von Konsum statt. Besonders greifbar wird dies durch die von Sachs entwickelten »4 E's«(Entschleunigung, Entrümpelung, Entflechtung, Entkommerzialisierung), die von Schneidewind und Zahrnt aufgenommen werden. Brischke ergänzt die 4 E's um ein weiteres E (Emanzipation). Die Befreiung von Überfluss, Beschleunigung und Entfremdung sowie die Emanzipation hin zu einer "Wertschätzung für sich selbst und die Umwelt" (Brischke 2014) versteht Suffizienz als einen notwendigen und erstrebenswerten kulturellen Wandel.

\section{Transformationspotenziale}

Grundsätzliches Ziel des Wirtschaftens ist die Befriedigung von Bedürfnissen der Menschen, die einem Wandel unterliegen und durch äußere, gesellschaftliche Umstände mitgeformt werden. Sowohl im historischen als auch im internationalen Vergleich wird deutlich, wie vielfältig sich die Bedürfnisse sowie die Nachfrage nach Gütern und Dienstleistungen entwickelt haben. In unserer sogenannten »Konsumgesellschaft" (Stengel 2011) haben sich verschiedene Steigerungsdynamiken entwickelt, vor allem die »Beschleunigung « vieler Aktivitäten und Prozeduren in der Wirtschaft, die auch den Stoffumsatz betreffen (vgl. Rosa 2012; Welzer 2019).

Indem alltägliche Verhaltensweisen diese Fehlentwicklung mit bewirkt haben, ist ein Umsteuern unmittelbar von den Gewohnheiten, Verhaltensroutinen und Motiven der Individuen und Organisationen sowie deren Veränderbarkeit und Veränderung abhängig. Dies zeigt sich u. a. bei der Einführung neuer technischer Systeme: So ist das Wunschbild von einem "papierlosen Büro« nicht in Erfüllung gegangen.

Bei intendierten Verhaltensänderungen kommt es darauf an, den jeweiligen Aufwand (»Transaktionskosten«) möglichst gering zu halten. Dazu ist es sinnvoll, den Aufwand im Sinne eines Spektrums bzw. einer Skala zu differenzieren. Fischer und Grießhammer (2013) haben dies am Beispiel von Nutzungsweisen eines 
Kühlschranks exemplarisch dargestellt. Deutlich wird dabei, dass mit höherem Aufwand auch höhere Einspareffekte erzielt werden können. Daraus kann der Schluss gezogen werden - wie die Autoren dies für die schrittweise Einführung von Rauchverboten erwähnen -, dass sukzessives Vorgehen, beginnend möglichst mit erfolgversprechenden, spürbaren Entlastungen, sehr erfolgversprechend sei.

Der folgende Klassifizierungsvorschlag am Beispiel der Kühlgerätenutzung soll der Akzeptanzabschätzung dienen; er baut daher auf den Eingriffstiefen hinsichtlich des Nutzens auf:

Tabelle 2: Suffizienzstufen auf Basis der Eingriffstiefe

\begin{tabular}{|llll|}
\hline Suffizienzstufe & $\begin{array}{l}\text { Empfundene Einschrän- } \\
\text { kung bzw. Aufwand }\end{array}$ & $\begin{array}{l}\text { Art der Änderung des } \\
\text { Konsummusters }\end{array}$ & Beispiel \\
\hline 1 & keine bis wenig & $\begin{array}{l}\text { Nutzung eines } \\
\text { kleineren Gerätes }\end{array}$ & $\begin{array}{l}\text { Kühlschrank mit Drei-Sterne-Fach (101//17l) } \\
\text { statt Kühl-Gefriergerät (171l/41l) }\end{array}$ \\
\hline 2 & mittel & $\begin{array}{l}\text { Nutzung eines Gerätes } \\
\text { mit weniger Komfort }\end{array}$ & Küh/gerät ohne Gefrierfunktion \\
\hline 3 & stark & $\begin{array}{l}\text { Umstellung von } \\
\text { Praktiken }\end{array}$ & $\begin{array}{l}\text { Kühlschrank im Winter nicht nutzen, Lebens- } \\
\text { mittel auf Balkon/vor dem Fenster kühlen }\end{array}$ \\
\hline 4 & sehr stark & $\begin{array}{l}\text { Verzicht auf Gerät, Um- } \\
\text { stellung von Praktiken }\end{array}$ & $\begin{array}{l}\text { Verzicht auf Kühlschrank, dafür häufiger/ } \\
\text { andere Lebensmittel einkaufen, einmachen/ } \\
\text { konservieren }\end{array}$ \\
\hline
\end{tabular}

Quelle: Heyen et al. (2013, S. 11)

An diesem Beispiel wird das Transformationspotenzial von Suffizienz im kleinen Maßstab beschrieben. Nach diesem Schema können Suffizienzpotenziale je Gerätetypus bzw. Bedarfsfeld oder auch je nach Individuum bzw. Organisation (kollektiv/gemeinschaftlich) kalkuliert werden. Vor allem bei Strategien und Maßnahmen für Suffizienz ist zu prüfen, inwieweit und mit welchen Aktivitäten eine breitere Diffusion, ein mainstreaming suffizienter Verhaltensweisen in möglichst viele soziale Milieus und Bereiche erreicht werden kann. Im Zusammenhang mit industrieller Ökologie konstatiert (Huber 2000): »Das Hauptproblem im Innovationsprozess liegt weniger in der Erfindung und Frühentwicklung neuer Technologien als vor allem in der Einführung und allgemeinen Verbreitung.«

Die Umwelt besonders entlastende Verhaltensweisen sind bislang nur für die Bereiche Energie und Klimaschutz relativ gut untersucht worden. »Interessante Handlungsfelder für Suffizienz wären dort aus unserer Sicht der Pkw-Ersatz, das Stromsparen, die Ernährungsumstellung auf mediterrane Kost und die Wohnflächenreduktion. Sie gehören zu den `Top Ten $<$ der Energiesparmöglichkeiten im privaten Haushalt (Grießhammer et al. 2010). Zudem sind Pkw-Ersatz, Stromsparen und Ernährungsumstellung gesellschaftlich vergleichsweise anschlussfähig, Ernährungsumstellung hat zudem ein Potenzial für gesellschaftlichen Strukturwandel (Umstellung der Landwirtschaft). Wohnflächenreduktion dürfte auf mehr Widerstand stoßen und geringe Ausstrahlungswirkung haben, hat aber ein 
hohes Potenzial sowohl für individuellen als auch gesellschaftlichen Strukturwandel.« (Fischer und Grießhammer 2013)

Positive Effekte im Zusammenhang mit Aktivitäten und Maßnahmen der Suffizienz sind bislang für Einzelfälle und auf individueller Ebene (Haushalt) vorgenommen worden. Eine allgemeine Einschätzung der Potenziale ist derzeit noch schwer möglich. Ansätze für eine bessere Gestaltung von Suffizienzmaßnahmen liegen bereits vor.

Während die Basisstrategie »Suffizienz« früher in Bezug auf persönliche Lebensweisen thematisiert wurde, erhält sie inzwischen mehr strukturelle, strategische Bedeutung (z. B. ressourcenschonende Lebensstile, Entschleunigung). Dabei wird Suffizienz als Bereich politischer Gestaltung erachtet und es werden konkrete Suffizienzpolitiken diskutiert. »So führt (aller Voraussicht nach) nichts an einer verordneten Nachhaltigkeit und damit einer verbindlich gemachten Suffizienz vorbei [...].« (Linz 2013, S. 47).

Von einer Suffizienzpolitik prinzipiell förderbare Handlungen sind im Folgenden beispielhaft aufgeführt, aufgeteilt in drei Bereiche (nach Heyen et al. 2013, S. 8):

1. Suffiziente Handlungen aufVerbraucher-Ebene:

- Ein Weniger an (ressourcenintensiven) Gütern und Güternutzung:

- Vollständiger Verzicht auf bestimmte Güter (z. B. Autobesitz, Fernreisen)

- Reduzierung der Menge bestimmter Güter (z. B. Fleisch, Fernsehgeräte)

- Seltenere Nutzung von Gütern (z. B. des Autos, des Fernsehers)

- Evtl. Ersatz durch qualitativ andere, umweltfreundlichere Güter (z. B. Rad statt Auto)

- Wahl einer Variante des (gleichen) Gutes mit einem Weniger an Größe, Funktionen oder Komfort (z. B. kleinerer Fernseher, kleinere Wohnung, Auto ohne Klimaanlage)

- Eigenproduktion, wenn ökologisch vorteilhaft (z. B. ökologischer Gemüseanbau im Garten)

- Gemeinsame Nutzung, wenn ökologisch vorteilhaft (z. B. Nachbarschaftsauto)

- Verändertes, umweltfreundlicheres Nutzungsverhalten (z. B. $\max .120 \mathrm{~km} / \mathrm{h}$ auf Autobahn)

- Verlängerung der Nutzungsdauer, wenn ökologisch vorteilhaft (z. B. Handy, Kleidung)

2. Suffizienzerleichternde Handlungen aufUnternehmensebene:

- Angebot und Bewerbung umweltfreundlicherer Güter mit veränderten Nutzenaspekten (z. B. Produktion eines kleinen Geräts und günstige Platzierung im Geschäft) 
- Nutzen-statt-Besitzen-Angebote für eine intensivere Nutzung (z.B. Werkzeugverleih)

- Herstellung von Gütern mit längerer Haltbarkeit bzw. Angebote, die eine längere Nutzung ermöglichen (z. B. günstige Reparatur und auswechselbarer Akku beim Laptop)

- Reduzierung der neu angebotenen Güter pro Jahr (z. B. Mode-Kollektionen)

- Bereitstellung von Umweltinformationen (z. B. absoluter Energieverbrauch einer Kühltruhe)

- Setzen entsprechender Anreize bei Mitarbeiterinnen und Mitarbeitern (z. B. BahnCard statt Dienstwagen)

3. Suffizienzerleichternde Handlungen Dritter (z. B. Verbände, Initiativen, „Prosumenten «):

- Bereitstellung von Angeboten, die suffizientes Handeln erleichtern (z. B. Verleihladen)

- Durchführung von Kampagnen, Bereitstellung von Informationen, Vergabe von Labels

Im Rahmen eines aktuellen Projektes über den Bereich »Energiesuffizienz« analysiert ein Team des ifeu verschiedene Energiesuffizienz-Strategien und -Instrumente als komplementäre Ergänzung zu Energieeffizienz und Konsistenz auf den Ebenen Geräte, Haushalte sowie urbane Dienstleistungen und Infrastrukturen. Letzteres erfolgt vor allem, um zu prüfen, wie diese »zu suffizienten Praktiken und Alltagsroutinen in Haushalten und zu suffizienten urbanen Lebensstilen beitragen können« (Schmitt et al. 2015, S. 7).

Dabei werden drei prinzipielle Suffizienzansätze unterschieden: Reduktion, Substitution und Anpassung:

- Reduktion: Quantitative Verringerung des Techniknutzens, nach Bewusstmachung der tatsächlich benötigten Bedarfe und Entlastungen, durch verringerten Technikgebrauch (weniger fernsehen) oder bewusste Entscheidungen bei Geräteausstattung (kleinerer Fernseher) oder Haushaltsproduktion (Kleidung seltener waschen).

- Substitution: Qualitative Veränderung von sozialen Praktiken und Alltagsroutinen im Haushalt, die Nutzen mit hohem Aufwand an technischer Energie liefern hin zu Arten des Nutzens mit geringerem Aufwand an technischer Energie (Wäscheleine statt Wäschetrockner, frische Nahrung statt Tiefkühlgerichte, Innenstadt-Wohnung statt Eigenheim am Stadtrand).

- Anpassung: Bewusste Anpassung der gelieferten technischen Dienstleistung an den angeforderten Techniknutzen (bspw. Anpassung der beheizten Herdplattenfläche an die Topfgröße) oder Anpassung des Technikgebrauchs an 
den tatsächlichen Bedarf (modulare Abschaltung von nicht genutztem Kühlvolumen). Der Ansatz kann mittels technischer Sensoren und Automatismen oder auch durch nudging unterstützt werden - und damit ohne umfassende Bewusstmachung erfolgen. Damit ist die Anpassung vor allem eine Verringerung von Überfluss bzw. unnötigem Energieverbrauch.« (Schmitt et al. 2015, S. 8)

Wichtig in Bezug auf die Umsetzbarkeit von Suffizienzmaßnahmen ist gemäß der Erkenntnisse des Projektes zur Energiesuffizienz die kommunale Ebene, denn "Kommunen als direkte erste politische Ebene über den Haushalten spielen eine wichtige Rolle für die Gestaltung der Rahmenbedingungen, in denen BürgerInnen handeln«. Kommunen können »über ordnungsrechtliche Rahmenbedingungen beispielsweise durch Parkraumbewirtschaftung (Suffizienz bestärken) und den Bau von Radwegen (Suffizienz ermöglichen) direkt Handlungsoptionen der BürgerInnen beeinflussen.« Über kommunale Unternehmen können öffentliche Dienstleistungen allen Bürgerinnen und Bürgern in gleicher Weise zugänglich gemacht werden und dadurch zu einer größeren Unabhängigkeit vom Markt beitragen (Schmitt et al. 2015, S. 13). Des Weiteren wird in der aktuellen Fachliteratur beschrieben, dass Bürgerinnen und Bürger in suffizienten Praktiken bestärkt (encourage), einbezogen und motiviert (engage) werden sollten. Darüber hinaus sind Praxisbeispiele mit unmittelbar erfahrbaren Erfolgen, also mit einer deutlich beispielgebenden Wirkungsweise (exemplify) ebenfalls zu empfehlen (Schmitt et al. 2015, S. 3). Zudem wurde beispielsweise festgestellt, dass Suffizienzmaßnahmen erfolgreich konzipiert und umgesetzt werden können, wenn sie als wichtiges Handlungsfeld für den Klimaschutz erkannt werden.

Ansatzpunkt für die Anschlussfähigkeit von Suffizienzmaßnahmen sind die grundlegenden Bedürfnisse von Menschen. Im Zusammenhang mit Wohlstand sind dies drei Typen: »Güter-, Zeit- und Beziehungswohlstand« (Linz 2013, S. 95). Diese Differenzierung gilt es auch bei der Untersuchung und Einschätzung heutiger Potenziale und künftiger Entwicklungen zu berücksichtigen. Im Einzelnen heißt dies konkret: "Zum Haben gehören die objektiven Lebensbedingungen wie Nahrung, Kleidung, Wohnung, Bildung, Einkommen, Arbeitsbedingungen, Gesundheit; zum Lieben die soziale Zugehörigkeit, persönliche Beziehungen, soziale Aufgaben; und zum Sein die Selbstverwirklichung, die Möglichkeit und Fähigkeit, sich erreichbare Ziele zu setzen, die Beteiligung an den für das Leben wichtigen Entscheidungen und für viele sicher auch ein angemessenes Niveau von Alltagskultur und Kunst.« (Linz 2013, S. 76)

In Bezug auf das Erreichen einer Green Economy ist unmittelbar nachvollziehbar, dass der Typ "Haben" von größter Bedeutung ist. Dieser zieht ökologische Negativeffekte nach sich, und besitzt daher prinzipiell Potenzial zur Minimierung. Hinzu kommt, dass Suffizienz-Strategien »Vorsorge-Handeln auf Grund 
erkennbarer Anfänge von Schädigungen und spürbarer Anzeichen zukünftiger Großgefahren« darstellen (Linz 2013, S. 83).

Mehr noch als die beiden anderen Basisstrategien für eine nachhaltige Entwicklung setzt die Suffizienz-Strategie mehr oder weniger deutliche Veränderungen der Denk- und Verhaltensmuster sowohl bei Individuen als auch Organisationen in verschiedenen Bereichen und Ebenen voraus und erfordert entsprechenden Umgang mit hohen Transaktionskosten. Die Suffizienz-Strategie bedarf daher eines weitgehend partizipativen Vorgehens und ist als Bottom-up-Ansatz anzusehen, weil mit einer Veränderung der Lebensweise viele Alltagsgewohnheiten in Frage gestellt und bewusst verändert werden müssen. Im Sinne einer weiteren Ausbreitung und Transformation zur Nachhaltigkeit wären diese Aktivitäten durch angemessene politische Maßnahmen zu flankieren und zu unterstützen.

Im Einzelnen sind es beispielsweise die folgenden fünf Hemmnisse, die suffiziente Verhaltensweisen erschweren und aufwändig erscheinen lassen (nach Stengel 2011, 183 ff.):

- Barriere 1: Der Primat der individuellen Rationalität

- Barriere 2: Das materialistische Welt- und Selbstbild

- Barriere 3: Die Praxis der Majorität

- Barriere 4: Die Abgabe der Verantwortung

- Barriere 5: Verheißungen der Konsumgesellschaft

Diese und weitere Faktoren und Rahmenbedingungen können zusammengenommen als Umstände angesehen werden, die hier eine Pfadabhängigkeit erzeugen und stabilisieren bzw. reproduzieren. Aufgrund dieser Hemmnisse und Barrieren sind Verhaltensänderungen, hier die Änderung von Konsummustern, und damit letztlich auch Pfadwechsel sehr voraussetzungsvoll, eher unwahrscheinlich und selten zu beobachten.

Die Strategie der Suffizienz hängt unmittelbar mit kulturellem Wandel und Einstellungsveränderungen (»Wertewandel«) in Teilen der Gesellschaft zusammen sowie mit etwaigen Engpässen und spürbaren Kostenveränderungen - bis hin zu etwaigen Katastrophen, die die Handlungsbereitschaft meist deutlich steigen lassen. Die jeweils sich ergebende Lebensgestaltung und damit auch die Nutzung bzw. der Konsum von Produkten, Ressourcen und Energie ist auf das jeweils individuelle und milieuspezifische Normale bezogen. Das Transformationspotenzial kann punktuell hoch sein (z. B. Moden im Ernährungsverhalten), ist im gesellschaftlichen Durchschnitt jedoch eher begrenzt, wandelt sich eher langsam und lässt sich nur schwer beeinflussen (demografischer und soziokultureller Wandel, z. B. Wohnungsgröße und -ausstattung, Mobilität). Verhaltensänderungen erfolgen nur unter bestimmten Voraussetzungen und Bedingungen (z. B. Werbung), deren gezielte Steuerung bzw. Beeinflussung höchst unsicher 
bzw. unklar ist. Hierbei geht es auch um individuelle und gemeinschaftliche Lernprozesse, die weniger kognitiver als vielmehr emotionaler Natur sind. Das Problemlösungspotenzial der Suffizienz-Strategie ist in manchen Bereichen hoch (z. B. Energienutzungsverhalten, Ernährung, Mobilität, allgemein umweltbewusstes Konsumverhalten), doch hängt dessen Ausschöpfung wesentlich davon $a b$, inwieweit es gelingt, einen alltagsnahen kulturellen Wandel sowie flankierende Prozesse effektiv zu gestalten. Darüber hinaus zeigen neuere sozialwissenschaftliche Ansätze auf, dass es für transformative Aktivitäten einer akteursorientierten Fokussierung bedarf, dass die konkreten Handlungsbedingungen und Gelegenheitsstrukturen systemisch zu beachten sind und dann unter Umständen

gezielt verändert werden können. Forschung im Bereich sozialer Innovationen, Praxistheorie aber auch »nudging« könne hierfür Hinweise bieten (Howaldt und Schwarz 2017; Shove et al. 2012).

\section{Grenzen: Denkweisen und Handlungsroutinen}

Die Bereitschaft zu einem solchen suffizienten Lebensstil ist jedoch sehr voraussetzungsvoll und bislang nur in spezifischen Milieus wie beispielsweise dem der Postmateriellen anschlussfähig (Kleinhückelkotten 2005). Ein kultureller Wandel hin zu Suffizienz in der Gesamtgesellschaft wird daher unter derzeitigen Umständen als unrealistisch eingeschätzt (Linz 2012; Heyen et al. 2013). Daraus ergibt sich, dass ein verbreitetes Praktizieren von Suffizienz nicht allein dem Individuum überlassen werden kann. Die geforderten Änderungen in den Konsummustern müssen vielmehr durch politische Maßnahmen und Rahmenbedingungen bestärkt und gefördert werden (Linz 2012; Schneidewind und Zahrnt 2013; Heyen et al. 2013). Politische Maßnahmen im Bereich Suffizienz umfassen vor allem Deklarieren, Fördern, Verteuern, Regulieren und Verbieten. Suffizienz-Politiken erschöpfen sich demnach nicht allein in Ordnungspolitik, in finanziellen Belastungen, in Geboten und Verboten. $\mathrm{Zu}$ ihnen gehören ebenso das Setzen von Anreizen und die Förderung lebensfähiger Alternativen (z. B. Linz 2012, 2015; Schneidewind und Zahrnt 2013).

Ein anderer Schluss hinsichtlich der Schwierigkeit eines kulturellen Wandels hin zu Suffizienz ist ein Suffizienz-Verständnis, welches die Suffizienz-Strategie von einer individuellen Bewertungsebene abkoppeln möchte. Suffizienz soll demnach bewusst weltanschaulich neutral betrachtet werden und statt auf die Motivation oder die Bewertung von Suffizienz (also: intrinsische Motivationen) auf die erzielten Suffizienzeffekte fokussieren (»innerhalb der ökologischen Tragfähigkeit bleiben«, so Fischer und Grießhammer 2013, 9 ff.). Dies betont die Dringlichkeit von Suffizienz, welche sich aus den Grenzen und Schwächen von Effizienz (Rebound) und Konsistenz (sehr langfristige Technologieentwicklung) ergibt, die um suffiziente Nutzungsänderungen ergänzt werden müssen. Im Suffizienzdiskurs sollte jedoch die Definitionsebene von der Kommunikationsebene 
unterschieden werden. So ist es für die Anschlussfähigkeit von Suffizienz in der Gesellschaft durchaus sinnvoll, ein positives Transformationsnarrativ, welches Vorteile eines ressourcenleichten Lebens herausstellt, $\mathrm{zu}$ kommunizieren (Heyen et al. 2013, S. 20).

\subsubsection{Komplementarität der drei Basisstrategien}

Durch die oben dargestellten empirischen Befunde, Erfahrungen und Erkenntnisse wird klar, dass die drei Basisstrategien - „Effizienz«, »Konsistenz« und »Suffizienz« - jeweils unterschiedliche Beiträge zur Entwicklung einer Green Economy zu leisten vermögen.

Die Effizienz-Strategie ist mit dem Ziel der Umweltentlastung und Ressourcenschonung nur vereinbar, wenn der jährliche Effizienzgewinn größer als das Wirtschaftswachstum ist - eine erhebliche Herausforderung an Technologien. Zwar findet eine Effizienzsteigerung als immanenter Prozess so gut wie immer statt, aber die Potenzialausschöpfung könnte bzw. sollte künftig noch größer und beschleunigter ausfallen. Das Potenzial beispielsweise mit Blick auf Rohstoffund Energieproduktivität in Deutschland dürfte bei zwei bis vier Prozent pro Jahr liegen, weitergehende Effizienzgewinne sind äußerst ambitiös. Die Auswirkungen auf die Höhe der absoluten Reduktion der Stoff- und Energieströme liegt in der Größenordnung von zehn bis 20 Prozent (im Laufe von 20 Jahren). Effizienzsteigerungen schaffen »Zeitgewinne« in mittlerer Perspektive, auf längere Sicht erweist sich Effizienz alleine als "nicht-nachhaltig« (Huber 2000).

Die Konsistenz-Strategie erstreckt sich vor allem auf die Erschließung von neuen Technologien, deren Stoffströme konsistenter mit natürlichen Prozessen sind. Das Umweltentlastungspotenzial lässt sich schwer abschätzen, dürfte aber grob bei 50 bis 80 Prozent liegen. Es müssen aufgrund tief greifender, struktureller Veränderungen, in denen alte Technologien abgelöst und neue Technologien etabliert werden, lange Zeiträume (30 bis 100 Jahre) für die Erschließung angenommen werden.

Die Suffizienz-Strategie setzt auf Bewusstseinswandel und Lebensstile, die dem Prinzip der ökologischeren Bedürfnisbefriedigung folgen, aber auch Genügsamkeit und Selbstbegrenzung sowie andere Maßstäbe an Lebensqualität und Sinn des Lebens umfassen. Im Kontext einer Green Economy, die in den gängigen Vorstellungen wachstumsbasiert ist, geht es primär um einen »anderen« Konsum. Hingegen ist der mögliche Stellenwert eines "geringeren« Konsums im Konzept der Green Economy noch auszutarieren (Fragestellung: Ein anderer Konsum oder weniger Konsum?). Das Umweltentastungspotenzial hierzu lässt sich ebenfalls schwer beziffern. Schätzungen gehen von einem Umweltentlastungspotenzial von zehn bis 40 Prozent aus (Huber 2000). Auch hier müssen lange Zeiträume für die Erschließung angenommen werden. 
Tabelle 3: Effizienz, Konsistenz und Suffizienz im Vergleich

\begin{tabular}{|lllc|}
\hline Strategie & Anschlussfähigkeit & Beitrag zur Transformation & Potenzial \\
\hline Effizienz & hoch, Wirtschaftslogik & inkrementell, Rebound & $10-20 \%$ \\
\hline Konsistenz & $\begin{array}{l}\text { hohe Eingriffstiefe, große } \\
\text { Widerstände }\end{array}$ & langfristig hoch wegen naturnaher Stoffstromqualität & $50-80 \%$ \\
\hline Suffizienz & $\begin{array}{l}\text { geringes sozio-kulturelles } \\
\text { Anschlusspotential }\end{array}$ & langfristig hoch bei veränderten Konsumeinstellungen & $10-40 \%$ \\
\hline
\end{tabular}

Quelle: Eigene Darstellung, Institut für Zukunftsstudien und Technologiebewertung nach Huber (2000).

Da die drei Basisstrategien sehr unterschiedlich charakterisiert sind und funktionieren, auch Unterschiedliches zu leisten vermögen, macht es wenig Sinn, nur auf eine Strategie zu setzen (z. B. nur Effizienz-Revolution oder nur SuffizienzRevolution). Es wäre aber auch zu einfach alle Strategien gleichzusetzen, nach dem Motto: »Jeder Beitrag ist willkommen!«. Vielmehr sind die Strategien komplementär (Huber 2000, S. 12; Linz 2013; Heyen et al. 2013). Es handelt sich dabei nicht um drei getrennt nebeneinanderstehende Strategien, sondern um interdependente Strategien, die aufeinander abzustimmen sind.

Effizienz, Konsistenz und Suffizienz »haben verschiedene Auswirkungen sowohl auf die Mengen als auch auf die Arten und Weisen des industriellen Metabolismus, und sie haben dies in unterschiedlichen Zeithorizonten." (Huber 2000, S. 12) Das liegt insbesondere daran, dass ihre Anschlussfähigkeiten sehr unterschiedlich sind. Am anschlussfähigsten ist die Effizienz-Strategie, die Konsistenz-Strategie stößt auf erhebliche Pfadabhängigkeiten und Barrieren. Die Suffizienz-Strategie besetzt unter den drei Nachhaltigkeitsstrategien bis dato die schwächste Position. Im Green-Economy-Diskurs spielt sie faktisch keine Rolle, auch im Green-Tech-Atlas für Deutschland (BMUB 2014) wird sie nur am Rande behandelt. Die Suffizienz-Strategie konfligiert mit Gewohnheiten, Normalitätsidealen und Interessen von Konsumentinnen und Konsumenten, Politik und Unternehmen (Bilharz 2008, S. 64). Nachhaltiges Wirtschaften ist aber ohne nachhaltigen Konsum wiederum nicht vorstellbar. Daher stellt sich die Frage, welchen Beitrag sie tatsächlich zu einer Green Economy, weiter gefasst zu einer nachhaltigen Entwicklung leisten kann (Weniger Konsum oder ein anderer Konsum?).

Nach Einschätzung des Öko-Instituts sollten Suffizienz-Maßnahmen »eingebettet werden in einen Policy-Mix mit Effizienz- und Konsistenz-Maßnahmen.« (Heyen et al. 2013, S. 19) Dementsprechend weist auch Linz darauf hin, Bezug nehmend auf das Beispiel suffiziente Regelung der Raumwärme, dass »zwischen Effizienz und Suffizienz keine Konkurrenz entsteht. Effizienz will die falsche Nutzung der physischen Ressourcen verändern, Suffizienz die falschen Denkansätze.« (Linz 2013, S. 72) Je nach Praxisbereich, Herausforderungen, dominanten Handlungsmustern und Akteurskonstellationen kann es sowohl zu sich 
ergänzenden Entwicklungen in Richtung Green Economy kommen, aber auch zu Konflikten zwischen den drei Basisstrategien. Dies bezieht sich auf alle Handlungsebenen (Individuen, Organisationen/Unternehmen, staatlich-administrative Ebenen), insofern mit den Basisstrategien jeweils unterschiedliche Präferenzen und entsprechend unterschiedliche Entscheidungen, Handlungen und Effekte verbunden sind.

Trotz offener Fragen dürfte mit Blick auf eine Green Economy »eine Gesamtstrategie der abgestuften Optionen das Beste sein«, die erstens auf einen wirtschaftlichen Strukturwandel hinwirkt (Konsistenz), zweitens die Erschließung bestehender Effizienzpotenziale ausschöpft (Effizienz) und sich schließlich drittens in gewissen Konsumgrenzen/-qualitäten (Suffizienz) bewegt.

Was bislang fehlt ist eine systematische, aufeinander bezogene Sichtweise sowie eine abgestimmte und integrierte Umsetzung der drei komplementären Strategien. Dafür spricht auch, dass sich Maßnahmen nur selten ganz präzise der Effizienz-, Konsistenz- oder Suffizienz-Strategie zuordnen lassen. Vielmehr entwickeln sich Transformationsprozesse nicht monokausal und linear, indem einseitig das eine durch das andere bestimmt wird, sondern ko-evolutiv in wechselseitiger Beeinflussung und im Zeitverlauf mit unterschiedlicher Wirkungsmacht, gegenseitiger Verstärkung oder Schwächung (siehe Abbildung 6). Einseitige Strategiediskussionen, wie sie in einer Reihe von Publikationen zum Ausdruck kommen (z. B. Paech 2012: »Befreiung vom Überfluss« mit Blick auf SuffizienzStrategie; Braungart und McDonough 2008: "Die intelligente Verschwendung« bezüglich der Konsistenz-Strategie; Schmidt-Bleek 2014: „Grüne Lügen« mit Blick auf Dematerialisierung bzw. Effizienzstrategie), sind daher wenig fruchtbar und unterstützen »Schubladendenken«. Es wird daher wichtig sein, dass sich vielmehr Mischungen solcher Strategien ergeben bzw. geschaffen werden und manche Zielvorstellungen nur zeitversetzt als Übergangspfad (z. B. Biokraftstoffe, Hybrid-Fahrzeuge) erreichbar sind (vgl. Leinfelder 2016) So werden bestimmte Lebensstile (z. B. kollaborativer Konsum) erst durch die Nutzung technischer Möglichkeiten (z.B. Internet, Digitalisierung und soziale Netzwerke) möglich. Andererseits wird die Technikgenese (z. B. Elektrofahrzeuge) wesentlich durch die entsprechenden gesellschaftlich-kulturell-ökonomischen Adaptions- und Nachfrageentwicklungen mitbestimmt. Hier werden Überschneidungen der drei Basisstrategien $\mathrm{zu}$ den beiden anderen theoretischen Ansätzen des Vorhabens (»Pfadabhängigkeit« und »Models of Change«) erkennbar.

Zugleich aber sind die Differenzen und die damit verbundenen unterschiedlichen Funktionen im Vorhaben zu unterstreichen. Mithilfe der drei Basisstrategien kann abgeschätzt werden, welche Ansätze prinzipiell große Potenziale aufweisen, um dort einen Pfadwechsel zu konzipieren und wie dies durch eine intelligente, angemessene Kombination von Maßnahmen zur Effizienz, Konsistenz und Suffizienz gestaltet werden könnte. Der Ansatz der Pfadabhängigkeit wiede- 
rum ermöglicht ein noch genaueres Verständnis für die konkreten Prozesslogiken und -dynamiken (Phasen) sowie - damit verbunden - mögliche strategische Interventionspunkte (Bifurkationen, "Stellschrauben«, windows of opportunity). Der Models-of-Change-Ansatz wiederum ermöglicht ein genaueres Verständnis und entsprechende Schlussfolgerungen für die Gestaltung von Pfaden bis hin zu Pfadwechseln durch innovative Vorgehensweisen relevanter Akteurinnen und Akteure in Wirtschaft, Gesellschaft und Politik.

Abbildung 6: Ko-Evolution von Effizienz, Konsistenz und Suffizienz

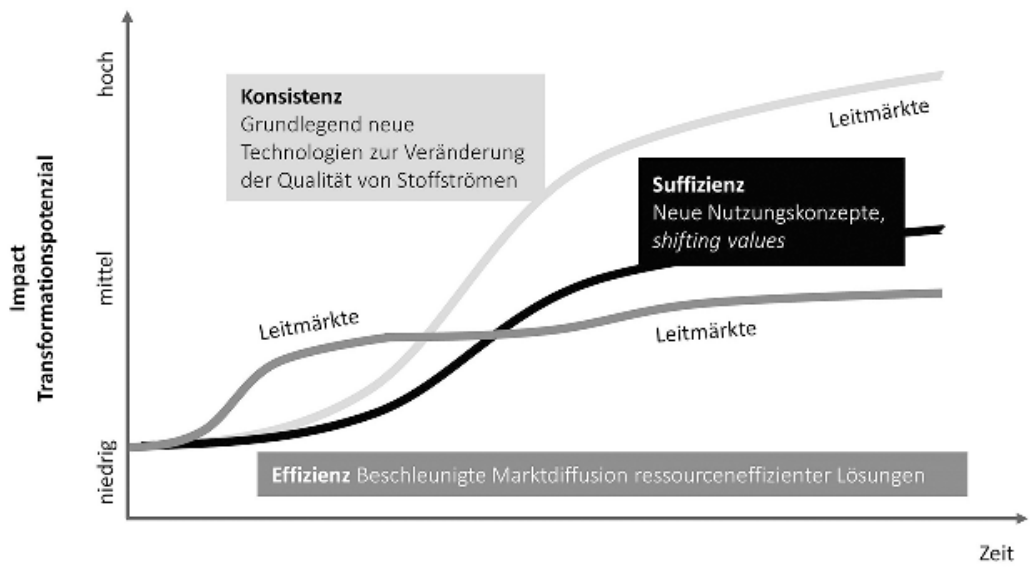

Quelle: Eigene Darstellung, Institut für Zukunftsstudien und Technologiebewertung.

In Anbetracht der möglichen Komplementarität der drei Basisstrategien stellt sich die Frage, wie ko-evolutive Transformationsprozesse zustande kommen, wie sie unterstützt und beschleunigt und die Trägheiten überwunden werden können, die Paradigmenwechseln und Pfadwechseln entgegenstehen. Die oben stehende Abbildung illustriert sowohl die vermutliche Dynamik (Zielhorizont, Zeithorizont) als auch das vermutliche Transformationspotenzial. Die gesellschaftliche Transformation muss - so die Arbeitshypothese - durch eine Kombination von Maßnahmen auf unterschiedlichen Ebenen ausgelöst, gestaltet und forciert werden. Eine Green Economy ist daher auf eine Abstimmung und Synchronisierung der Strategien angewiesen.

\section{4 Übergreifendes Systemmodell}

In den vorangegangenen Unterkapiteln wurden die drei wesentlichen Analyseansätze des Projekts ausführlich vorgestellt. Diese umfassen das Pfadabhängigkeitsmodell vor dem Hintergrund der Evolutorischen Ökonomik (Kapitel 3.1), den 
Models-of-Change-Ansatz (Kapitel 3.2) sowie die drei Basisstrategien Effizienz, Konsistenz und Suffizienz (Kapitel 3.3). An dieser Stelle folgt eine kurze Überblicksdarstellung über die wesentlichen Eckpunkte der Modelle. Daraufhin wird abschließend erläutert, wie die drei Ansätze im Rahmen eines übergreifenden Analysemodells zusammengedacht werden können und wie sie im weiteren Verlauf des Buches angewendet werden sollen.

\subsubsection{Die Analyseansätze im Überblick}

\section{Pfadabhängigkeiten und Evolutorische Ökonomik}

Pfadabhängigkeiten werden in vielen Arbeiten der Innovationsforschung als Erklärungsansatz dafür genutzt, warum sich der Wandel vielerorts so schwierig gestaltet (Nelson und Winter 1982; Nelson 1987). Auch im vorliegenden Buch dient das Konzept der Pfadabhängigkeiten in erster Linie der Analyse und Erklärung von Bindungs- und Ausriegelungskräften, welche Veränderungsprozesse hemmen oder verhindern.

In der Literatur, die sich mit Pfadabhängigkeiten beschäftigt, werden unterschiedliche Rückkopplungs- und Selbstverstärkungseffekte diskutiert (LehmannWaffenschmidt und Reichel 2000, S. 349), zumeist wird aber keine systematische Unterscheidung der verschiedenen Arten von Pfadabhängigkeiten vorgenommen. Im Rahmen des Theoriepapiers zur Evolutorischen Ökonomik für »evolution2green " (Clausen und Fichter 2016) wurde eine Systematik eingeführt, die eine grobe Abgrenzung unterschiedlicher Typen von Pfadabhängigkeiten erlaubt. Aufbauend auf der Arbeit von Fichter und Clausen (2013, S. 90) wird zwischen folgenden Haupttypen von Pfadabhängigkeiten unterschieden:

- Rechtliche Pfadabhängigkeiten, z.B. durch Gesetze, Prüfvorschriften und andere Rechtsverordnungen

- Ökonomische Pfadabhängigkeiten, z. B. durch Effekte der economies of scale und bereits getätigte Investitionen sowie die sich daraus ergebende Kapitalbindung

- Technologische Pfadabhängigkeiten, z. B. aufgrund fehlender Komplementärprodukte oder mangelnder Infrastrukturen

- Organisationale Pfadabhängigkeiten, z.B. durch Prozessroutinen und ablauforganisatorische Festlegungen oder unternehmenskulturelle Gebräuchlichkeiten

- Nutzerbezogene Pfadabhängigkeiten, z. B. durch Unsicherheiten, Verhaltensroutinen sowie kulturelle Gebräuchlichkeiten 


\section{Models of Change}

Der Models-of-Change-Ansatz stellt die zweite analytische Komponente dar (Irrek und Kristof 2000; Kristof 2010a, 2010b). Als multidimensionales, akteurszentriertes Veränderungsmodell beschreibt dieser Ansatz zentrale Rahmenbedingungen und Erfolgsfaktoren zur Gestaltung von Transformationsprozessen. Für das vorliegende Buch wurden die Dimensionen »Akteurinnen und Akteure und ihre Qualifikationen«, »Veränderungsidee/Lösungsvorschlag«, »Zeitaspekte« und »eränderungsprozesse« als Hauptkategorien für die Analyse bestimmt. Zur Operationalisierung dieser Kategorien wurden Variablen definiert, welche die Analyse von Transformationsprozessen leiten.

In der ersten Kategorie werden neben den change agents und ihren Qualifikationen explizit auch die anderen Akteurinnen und Akteure sowie Akteurskonstellationen in den Blick genommen, die einer Veränderung positiv oder skeptisch gegenüberstehen. In Bezug aufVeränderungsidee und Lösungsvorschläge werden die Passgenauigkeit der Umsetzungslösung, der (avisierte) Strategien- und Instrumentenmix sowie der Umgang mit Zielkonflikten betrachtet. Zu Zeitaspekten zählen sowohl die Betrachtung möglicher Auslöser bzw. Trigger-Ereignisse als auch Geschwindigkeit und Rhythmus von Veränderungen. Weitere, unter Veränderungsprozesse zusammengefasste Aspekte umfassen horizontale und vertikale Koordination, Institutionalisierung, Beteiligungsprozesse, Veränderungskultur, Wissensbasis und Lernprozesse, Reflexivität und Ressourcenausstattung.

\section{Basisstrategien Effizienz, Konsistenz und Suffizienz}

Als drittes Element des theoretischen Überbaus wurde die Begriffstrias aus Effizienz, Konsistenz und Suffizienz eingeführt. Als Strategiemodell bietet sie ein hilfreiches Analyseraster, um Transformationsstrategien strukturiert zu diskutieren.

Effizienz setzt einen Output - z. B. eine Dienstleistung oder ein Produkt mit den für dessen Bereitstellung eingesetzten Ressourcen ins Verhältnis. Eine Effizienzsteigerung beinhaltet einen gesunkenen Ressourceneinsatz pro erwirtschaftetem Output, beispielsweise durch technologischen Fortschritt. Das Konzept der Konsistenz zielt wiederum auf die Umweltverträglichkeit menschlicher Produktionsmuster und Stoffströme $a b$. Ein hohes Maß an Konsistenz ergibt sich dann, wenn Produktionsverfahren keine Natur- und Ökosysteme belasten, sondern vielmehr mit diesen in Einklang stehen. Suffizientes Handeln ist darauf ausgelegt, Energie- und Ressourcenverbräuche zu reduzieren. Unter den drei Leitprinzipien ist die Suffizienzstrategie daher am umstrittensten, da sie die Wachstumslogik des bestehenden Wirtschafts- und Gesellschaftssystems grundsätzlich in Frage stellt.

Wie angemerkt ist davon auszugehen, dass eine Green Economy nicht allein durch die Umsetzung effizienterer und konsistenterer Wertschöpfungs- und 
Konsumptionsmuster zu erreichen ist. Vielmehr ist auch zu fragen, wo Suffizienz einen Beitrag zur Green Economy leisten kann. Als Analyserahmen für die Untersuchung von Transformationspfaden scheint daher eine Heuristik, die alle drei Strategien und deren jeweilige Beiträge zu nachhaltigem Wirtschaften umfasst, folgerichtig.

\subsubsection{Konturen eines übergreifenden Systemmodells}

Die verschiedenen Analyseansätze unterscheiden sich sowohl in ihrer analytischen Perspektive als auch in ihrem Analysegegenstand. Dabei sind sie jedoch kombinierbar. Sie lassen sich als komplementäre, ineinandergreifende Elemente verstehen, um Transformationsprozesse hin zu einer Green Economy umfassend zu beleuchten.

- Der Ansatz der Pfadabhängigkeiten eignet sich primär zur Analyse des Status quo. Mit Blick auf die Mechanismen und Interdependenzen, die die aktuelle Situation bedingen, können sowohl Stärken als auch Schwächen des etablierten Entwicklungspfades in verschiedenen Sektoren und Problemfeldern identifiziert werden.

- Die drei Basisstrategien Effizienz, Konsistenz und Suffizienz (EKS) erlauben eine Kategorisierung von Veränderungsstrategien im Hinblick auf deren inhaltliche Ausrichtung. Sie beschreiben Zielhorizonte und Richtung der angestrebten Veränderungen.

- Der Models-of-Change-Ansatz fokussiert auf die Mechanismen und Prozesse, die eine Veränderung auslösen, befördern und zum Erfolg führen, ohne sich dabei näher mit der Frage der inhaltlichen Stoßrichtung zu befassen.

Während die Analyse von Pfadabhängigkeiten ein besseres Verständnis des zu transformierenden Wirtschafts- und Gesellschaftssystems ermöglicht, erlaubt der Models-of-Change-Ansatz die Betrachtung des Transformationsprozesses. Die EKS-Perspektive wiederum befähigt uns, notwendige Veränderungen zu benennen und einzuordnen. Beantwortet die Perspektive der Pfadabhängigkeiten also, vereinfacht ausgedrückt, die Frage des »Warum stehen wir still?«, lässt sich EKS dazu nutzen, die Frage des »Wohin wollen wir?« zu erörtern. Models of Change wiederum beschäftigt sich mit dem »Wie kommen wir dahin?«.

Die folgende Grafik (siehe Abbildung 7) verdeutlicht diesen Zusammenhang. Die Pfadabhängigkeiten im Zentrum repräsentieren den zu verändernden Ist-Zustand, gleichzeitig verdeutlichen sie in ihrer Verknüpfung die Resilienz etablierter Pfade. Effizienz-, Konsistenz- und Suffizienzstrategien im mittleren Kreis verkörpern die (vor dem Hintergrund zentraler Umweltproblematiken) intendierte Veränderung, die auf den Ist-Zustand einwirken soll. Die äußeren beiden Rin- 
ge visualisieren mit der Operationalisierung des Models-of-Change-Ansatzes die Mechanismen und Erfolgsfaktoren des Veränderungsprozesses.

Lesen lässt sich das Systemmodell sowohl von innen nach außen als auch von außen nach innen. Der Analyse- und Strategieentwicklungsprozess verläuft idealtypisch von der Betrachtung des Status quo zur Frage der gewünschten bzw. notwendigen Veränderungsrichtung hin zur Analyse der notwendigen Bedingungen und Faktoren, die die gewünschte Veränderung ermöglichen. Die Synthese und Umsetzung wiederum erfolgt in entgegengesetzter Richtung: Ausgehend von einem ineinandergreifenden Set an Ressourcen, Rahmenbedingungen und erfolgversprechenden Gestaltungsinstrumenten wird mit einer spezifischen Stoßrichtung auf das zu verändernde Wirtschafts- und Gesellschaftssystem eingewirkt.

Abbildung 7: Übergreifendes Systemmodell

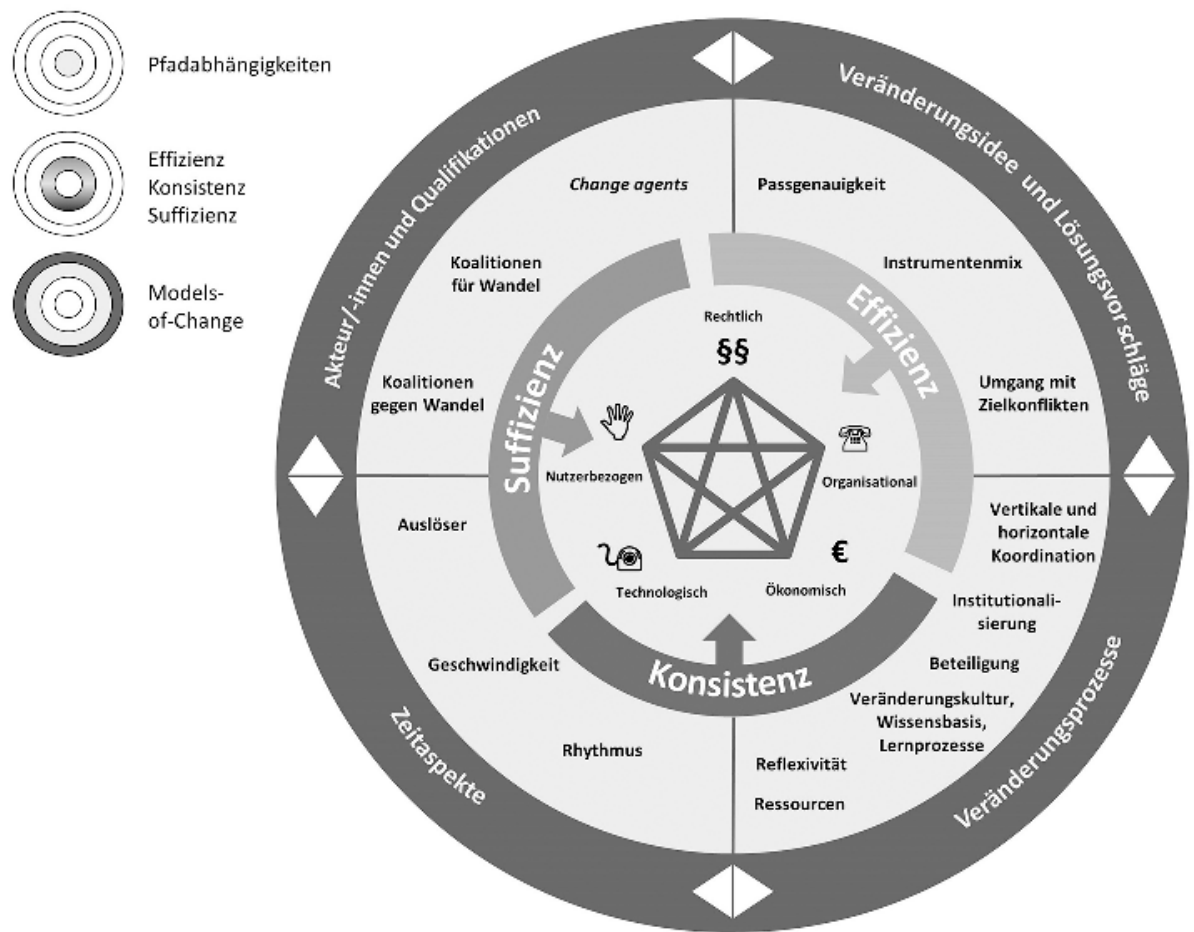

Quelle: Eigene Darstellung, adelphi.

In der Praxis ist dieser Prozess selbstverständlich nicht so eindeutig zu klassifizieren. Die Systemelemente sind eng miteinander verzahnt und bedingen sich gegenseitig. Auch einzeln betrachtet verbinden die verschiedenen Analysemodelle in unterschiedlichen Ausprägungen inhaltliche und prozessbezogene sowie normative und analytische Komponenten. Analyse, Strategieentwicklung 
und Implementierung greifen ineinander. Sowohl innerhalb als auch zwischen verschiedenen Prozessphasen bestehen Iterationsschleifen, bei denen Veränderungsgegenstand, Veränderungsrichtung und -mechanismus in schneller Folge betrachtet und aufeinander abgestimmt werden.

\subsubsection{Verwendung im Rahmen des Buches}

\section{Prozessübersicht}

Das skizzierte Systemmodell kommt in den folgenden Kapiteln als Analyserahmen sukzessive zur Anwendung. Die empirische Untersuchung bestehender Problem- bzw. Transformationsfelder (Kapitel 4.1) und erfolgreicher Transformationsbeispiele (Kapitel 4.2) sowie deren Analyse und Zusammenführung in Form von Handlungsansätzen im Rahmen von Kapitel 5 dienen dabei neben der Entwicklung konkreter Handlungsempfehlungen auch zur Validierung und Weiterentwicklung des Modells.

Im Einklang mit der skizzierten, vom Status quo ausgehenden und sich nach außen vorarbeitenden Analyserichtung steht in Kapitel 4.1 (Transformationsfeldanalysen) die Analyse von tradierten Problemfeldern unter Zuhilfenahme des Pfadabhängigkeiten-Ansatzes im Vordergrund. Gleichzeitig werden mit der Betrachtung von Akteurinnen und Akteuren sowie bestehenden Transformationsansätzen in Bezug auf die jeweiligen Problemfelder auch die äußeren Ringe des Kreises beleuchtet. Kapitel 4.2 betrachtet insbesondere den Prozess und die Mechanismen erfolgreicher Transformationen. Hier steht die Anwendung des Models-of-Change-Ansatzes im Vordergrund. Parallel wird auf die EKS-Perspektive und den Pfadabhängigkeiten-Ansatz aufgebaut und es werden Verknüpfungen hergestellt. Bei der Entwicklung von Roadmaps zu besonders relevanten Transformationsfeldern in Kapitel 5 erfolgt die Synthese der gewonnenen Erkenntnisse in Bezug auf die betrachteten Sektoren und Themenbereiche.

\section{Auswahl von Transformationsfeldern und Transformationsbeispielen}

Bei der Auswahl von Transformationsfeldern (Kapitel 4.1) und Transformationsbeispielen (Kapitel 4.2) spielen das Systemmodell und die darin enthaltenen Analyseansätze weniger für die Identifikation als für die Zusammenstellung des Sets an Transformationsfeldern und Transformationsbeispielen eine Rolle. Ausgangspunkt für die Auswahl von Transformationsfeldern waren die Indikatoren und Ziele der nationalen Nachhaltigkeitsstrategie (Bundesregierung 2008, 2012, 2014). Gleichzeitig wurde darauf geachtet, dass wesentliche Felder abgedeckt werden. Ebenso sollten in Bezug auf Pfadabhängigkeiten und Pfadwechsel auch Felder mit bisher geringeren Fortschritten bei der Erreichung der Nachhaltigkeitsziele ausgewählt werden. 
Die Auswahl der Transformationsbeispiele erfolgt auf Basis der im Kontext der Transformationsfeldanalysen identifizierten Transformationsansätze, sie beinhaltet aber auch ein breites Spektrum an effizienz-, konsistenz- und suffizienzfokussierten Beispielen. Eine Rolle spielen dabei insbesondere die beiden inneren Kreise des Systemmodells mit ihrer Fokussierung auf das Verständnis der Problemlage und der Stoßrichtung von Veränderungsprozessen.

\section{Analyse von Transformationsfeldern und Transformationsbeispielen}

Bei der Analyse von Transformationsfeldern steht der Ansatz der Pfadabhängigkeiten im Vordergrund. Unter Anwendung der entwickelten Typologie werden bestehende Pfadabhängigkeiten identifiziert und so die Stärken, aber auch Schwächen des im jeweiligen Transformationsfeld etablierten Pfades herausgearbeitet. Die ebenfalls im Rahmen der Analysen durchgeführte Betrachtung von Akteurinnen und Akteuren sowie von Transformationsansätzen wird dabei durch den Models-of-Change-Ansatz und die EKS-Perspektive strukturiert. Während die Analyse der Transformationsfelder also primär eine Betrachtung des Status quo beinhaltet, werden mit der Untersuchung von Transformationsansätzen auch die äußeren Ringe des Systemmodells angesprochen.

Die Analyse von Transformationsbeispielen erfolgt primär entlang der Operationalisierung des Models-of-Change-Ansatzes als Analyserahmen. Gleichzeitig wird eingehend betrachtet, in welcher Art und Weise bestehende Pfadabhängigkeiten mit dem Erfolg oder Misserfolg von Transformationsprozessen zusammenhängen und mit dem MoC-Ansatz interagieren. Sowohl bei der Auswahl als auch bei der anschließenden Queranalyse stellt die EKS-Perspektive ein zentrales Analysekriterium dar. Während der Fokus somit auf dem äußeren Ring des Systemmodells liegt, bauen die Untersuchungen und Ergebnisse auch auf den inneren Kreisen auf.

\section{Entwicklung von Roadmaps zu zentralen Wendethemen}

Mit der Entwicklung von Roadmaps zu zentralen Wendethemen in Kapitel 5 soll das Systemmodell von außen nach innen gelesen dabei unterstützen, konkrete Handlungsempfehlungen für Politik und andere relevante Akteurinnen und Akteure zu generieren, um von einer Grey Economy, wie sie im Systemmodell mit dem grauen Kern angedeutet wird, zu einer Green Economy zu kommen. Gleichzeitig soll dieser Schritt im Sinne einer iterativen Betrachtungsweise dazu genutzt werden, die theoretischen Erkenntnisse aus den letzten Kapiteln für eine Konsolidierung und gegebenenfalls Weiterentwicklung des Modells aufzubereiten. 


\section{Pfadabhängigkeiten, Hindernisse und Erfolgsfaktoren für Transformationsprozesse in Richtung einer Green Economy}

\subsection{Pfadabhängigkeiten und Hindernisse aus 15 Transformationsfeldern in den Bereichen Wärme, Mobilität, Ressourcen und Landwirtschaft}

\subsubsection{Die Auswahl der 15 Transformationsfelder}

Der Blick in die letzten Fortschrittsberichte zur Nachhaltigkeitsstrategie (Bundesregierung 2012, 2016) zeigt, dass sich Fortschritte keineswegs bei allen Indikatoren ergeben haben. Selbst bei der Energiewende als Vorzeigeprojekt der Bundesregierung wird zumindest im Hinblick auf die Energieproduktivität der erhoffte Effekt nicht erzielt. Auch in Bezug auf die Indikatoren »Güterverkehrsintensität«, "Flächenverbrauch", »Flächenanteil der ökologischen Landwirtschaft" und »Biodiversität« werden - wie auch in anderen Feldern - die angestrebten Verbesserungen verfehlt.

Die mitunter geringen Fortschritte sind häufig als Ergebnis des Ineinandergreifens komplexer und vielfältiger Wirkungszusammenhänge zu betrachten. Beispielhaft sei angeführt, dass z. B. im Kontext der noch reichlich vorhandenen, ungenutzten industriellen Abwärme (Pehnt et al. 2010; Grote et al. 2015) wie auch der zukünftig nötigen Nutzung von Wärme aus Solarkollektorfeldern (Nitsch et al. 2011) der Pfad der gebäudeindividuellen Wärmeversorgung teilweise verlassen und der Pfad der Versorgung über Wärmenetze verstärkt eingeschlagen werden müsste. Auch der angepeilte Ausbau des ökologischen Landbaus geht nur langsam voran. Das in der Nachhaltigkeitsstrategie von 2002 ursprünglich für das Jahr 2010 gesetzte nationale Ziel von 20 Prozent Anteil an der landwirtschaftlichen Gesamtfläche würde bei der gegenwärtigen Entwicklung erst im Jahr 2080 erreicht (Göll und Henseling 2017, S. 87).

Schon im Antrag zu »evolution2green« waren zwei Kriterien für die Auswahl der zu untersuchenden Transformationsfelder aufgeführt worden:

- Ein mangelnder Zielerreichungsgrad bei den Nachhaltigkeitsindikatoren der Bundesregierung 
- Die Abdeckung verschiedener Typen von Transformationsfeldern, z. B.

- Probleme, gegen die überhaupt noch keine Maßnahmen ergriffen wurden (z. B. Recycling von Technologiemetallen)

- Probleme, bei denen Maßnahmen bisher deutlich zu wenig Wirkung zeigen (z. B. Ökoanbaufläche)

- Ökologische Marktsegmente, die nur zu langsam aus der Nische kommen (z. B. Wärmenetze)

Von diesen Kriterien ausgehend wurde im Herbst 2015 als erster Schritt in einem dreistufigen Verfahren eine Liste von 22 möglichen Transformationsfeldern in den Bereichen Energie und Wohnen, Mobilität, Landwirtschaft und Ernährung sowie Produktion und Stoffströme aufgestellt.

Abbildung 8: Erster Auswahlschritt für die Transformationsfeldanalysen

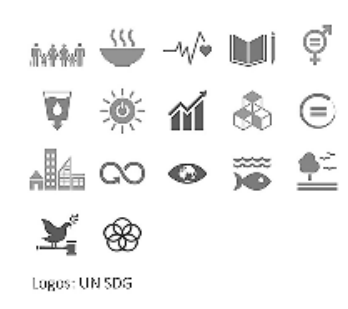

Indikatoren der Nachhaltigkeitsstrategie

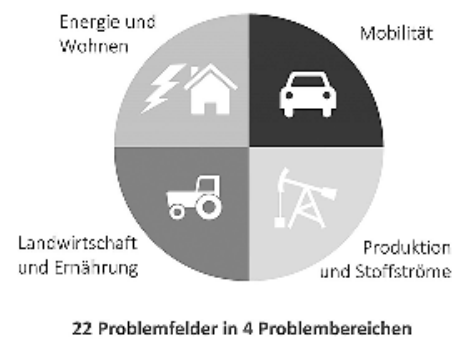

22 Problemfelder in 4 Problembereichen

Quelle: Eigene Darstellung, adelphi.

Auf der Sitzung des Expertenbeirats des Projektes wurde am 17. Dezember 2015 die Auswahl der Transformationsfelder diskutiert. Aus dem Kreis wurde eine Reihe von Aspekten benannt, die bei der Auswahl der Transformationsfelder zu berücksichtigen wären:

- Die Bedeutung der Felder für die nachhaltige Entwicklung, Mengenrelevanz

- Die Aussicht auf eine erfolgreiche Transformation, Handlungsfähigkeit und Umsetzbarkeit

- Die Berücksichtigung von Exnovationsprozessen

- Die Berücksichtigung von Globalisierung und Digitalisierung

Konkret empfohlen wurden die Transformationsfelder »Mobilität« (mit einem Fokus auf die forschungsintensive Automobilindustrie, an der Exnovationsperspektiven, Mythen und Narrative hängen), »Wärmeversorgung« (aufgrund ihrer Bedeutung für die Reduktion von Treibhausgasemissionen) sowie »Landwirtschaft und Ernährung«. 
Unter Berücksichtigung der Positionen des Expertenbeirates wurden die Untersuchungsfelder durch das Projektteam konkretisiert und auf die Wendethemen »Pkw-Verkehr«, »Wärmeversorgung von Gebäuden«, »Landwirtschaft und Ernährung« sowie »Rohstoffe/Ressourcen« zugespitzt. Außerdem wurde eine Auswahlheuristik entwickelt, anhand derer die finale Auswahl erfolgte.

Abbildung 9: Auswahlheuristik für die Transformationsfeldanalysen

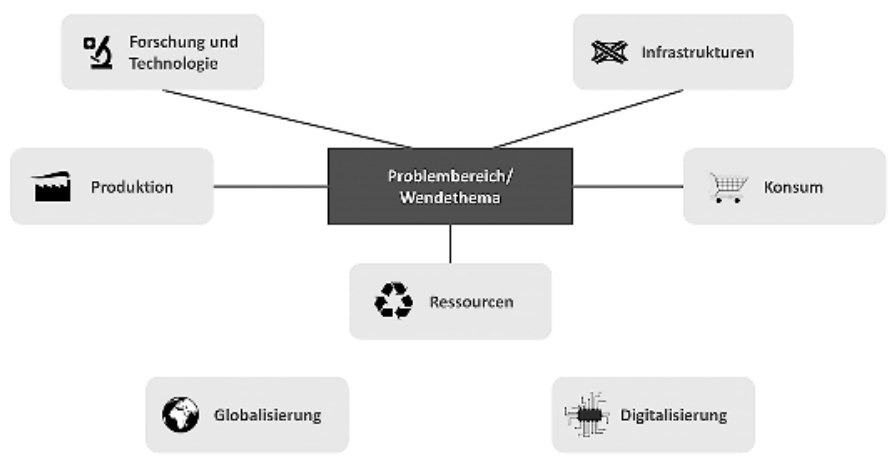

Quelle: Eigene Darstellung, adelphi.

Für jedes Wendethema wurden produktions- und konsumseitige, forschungs- und technologielastige sowie mit Infrastrukturen und Ressourcen zusammenhängende Transformationsfelder identifiziert. Globalisierung und Digitalisierung wurden dabei als Querschnittsthemen mit berücksichtigt. Die finale Auswahl der Felder ist in der folgenden Übersicht dargestellt.

\section{Abbildung 10: Finale Auswahl der Transformationsfeldanalysen}

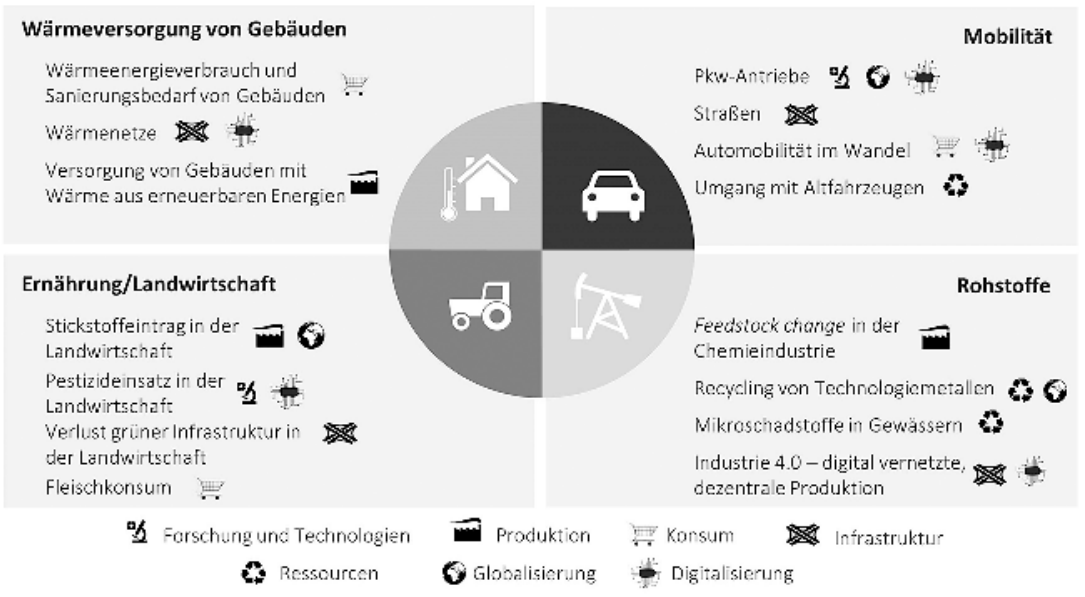

Quelle: Eigene Darstellung, adelphi. 
Eine Relevanz für die nachhaltige Entwicklung ist bei allen ausgewählten Feldern gegeben. Ebenso sind zu allen Themen mehrere Transformationsansätze bekannt. Die so getroffene Auswahl deckt mit einigen Studien auch Globalisierungsaspekte $\mathrm{ab}$ (z. B. Altautoentsorgung, Technologiemetalle), bei anderen spielen Aspekte der Digitalisierung eine Rolle (z. B. Erneuerbare-Energien-Anteil [EE-Anteil] an der Gebäudewärmeversorgung, neue Nutzungskonzepte von Pkws). Exnovation spielt bei Pkw-Antrieben, indirekt auch beim EE-Anteil an der Gebäudewärmeversorgung eine Rolle.

Zur Prüfung und weiteren Verfeinerung des Analyseansatzes wurden bis Mitte März 2015 folgende drei Pilotanalysen durchgeführt:

- Altautoentsorgung (adelphi)

- Wärmenetz-Infrastrukturen (Borderstep)

- Feedstock change (IZT)

Im Rahmen der Analyse wurden mit ausgewählten Expertinnen und Experten Interviews durchgeführt, die zum Ziel hatten, die Pfadabhängigkeit und deren Faktoren in den einzelnen Themenfeldern (Binnenlogik und -kultur) besser $\mathrm{zu}$ verstehen. Außerdem wurde eruiert, ob und welche Art von Transformationsansätzen für einen möglichen/wünschenswerten Pfadwechsel in Richtung einer nachhaltigen Entwicklung in Frage kommen und bereits diskutiert werden.

Im Wendethema "Pkw-Verkehr" wurden Pfadabhängigkeiten und Transformationsansätze im Bereich der Pkw-Antriebe (Clausen 2017d), der Straßen (Clausen 2017e), der Altautoentsorgung (Tappeser und Chichowitz 2017c) sowie neue Nutzungskonzepte von Pkw (Korte et al. 2017) untersucht. Im Wendethema "Landwirtschaft" fokussierten die Analysen auf den Fleischkonsum (Clausen und Mathes 2017), den Stickstoffeintrag in der Landwirtschaft (Tappeser und Chichowitz 2017b), den Pestizideinsatz in der Landwirtschaft (Tappeser und Chichowitz 2017a) sowie die grünen Infrastrukturen (Tappeser et al. 2017). Im Wendethema »Wärmeversorgung von Gebäuden« wurden Wärmenetze (Clausen 2017g), der Wärmeenergieverbrauch und Sanierungsbedarf von Gebäuden (Tappeser und Chichowitz 2017d) sowie der EE-Anteil an der Gebäudewärmeversorgung (Clausen und Hinterholzer 2017) untersucht. Und im Wendethema »Rohstoffversorgung" fokussierten die Transformationsfeldstudien auf feedstock change (Behrendt 2017a), Technologiemetalle (Behrendt 2017c), persistente Stoffe (novel entities) (Behrendt 2017b) sowie dezentrale Produktion (Odenbach et al. 2017). Die 15 Transformationsfeldstudien mit insgesamt 567 Seiten Umfang stehen unter www.evolution2green.de zum Download bereit. 


\subsubsection{Pfadabhängigkeiten in den vier Wendethemen}

Die Pfadabhängigkeiten in den 15 Transformationsfeldern, die den vier Wendethemen bzw. Transformationsbereichen zugeordnet sind, sind in den 15 Transformationsfeldstudien im Detail beschrieben. Die 15 Studien wurden von den jeweiligen Autorinnen und Autoren erarbeitet und von jeweils zwei weiteren Mitgliedern des Projektteams aus den anderen Instituten mit Blick auf Bedeutung, Zuordnung und Beschreibung der identifizierten Pfadabhängigkeiten geprüft. Besonders bedeutende Fragen wurden in Telefonkonferenzen diskutiert. Um Strukturen hinter den vielfältigen in der Analyse ans Licht gebrachten Pfadabhängigkeiten zu erkennen, wurde wie folgt vorgegangen:

- Alle Pfadabhängigkeiten, die in den 15 Studien erwähnt wurden, wurden tabellarisch erfasst. Die Pfadabhängigkeiten blieben dabei den fünf Typen von Pfadabhängigkeiten zugeordnet.

- Nach dem Metaplanverfahren wurden diese Pfadabhängigkeiten auf Einzelkärtchen erfasst und vom Team des Borderstep Instituts geclustert. Innerhalb der fünf Pfadtypen wurden dabei Cluster möglichst ähnlicher Pfadabhängigkeiten gebildet. Die Clusterung wurde dem gesamten Projektteam zur Kommentierung vorgelegt. Die Cluster wiederum bilden die Basis für die detaillierte Typologie von Pfadabhängigkeiten.

- In Kenntnis dieser Typologie und mit Blick auf die Spezifika der jeweiligen Transformationsfelder und Wendethemen wurden anschließend von den für die Transformationsfeldstudien verantwortlichen Autorinnen und Autoren die in den folgenden Abschnitten erstellten Grafiken erarbeitet, mit denen insbesondere die Netzwerkbeziehungen zwischen den einzelnen Pfadabhängigkeiten herausgearbeitet wurden.

- Der Abschnitt »Typische Netzwerkbeziehungen von Pfadabhängigkeiten«stellt dann den vom Projektteam erarbeiteten Versuch dar, hinter diesen Netzwerkbeziehungen typische Muster zu identifizieren und beispielhaft darzustellen.

\subsubsection{Pkw-Mobilität}

Die folgende Tabelle gibt zunächst einen Überblick über die 51 Pfadabhängigkeiten, die in den vier Transformationsfeldstudien im Feld »Pkw-Mobilität« gefunden wurden (Clausen 2017d, 2017e; Korte et al. 2017; Tappeser und Chichowitz 2017c). Eine starke Pfadabhängigkeit stabilisiert dabei aus Sicht der Autorinnen oder Autoren der jeweiligen Transformationsfeldstudie das System mit seinen Akteurinnen und Akteuren in hohem Ausmaß. Sie stellt damit ein besonders starkes Hemmnis für einen Pfadwechsel dar. 
Tabelle 4: Anzahl an Pfadabhängigkeiten im Pkw-Verkehr nach Stärke und Art

\begin{tabular}{|c|c|c|c|c|c|c|}
\hline \multirow{2}{*}{$\begin{array}{l}\text { Stärke der } \\
\text { Pfadabhängigkeit }\end{array}$} & \multicolumn{6}{|c|}{ Art der Pfadabhängigkeit } \\
\hline & Rechtlich & Ökonomisch & Technisch & Organisational & Nutzerbezogen & Summe \\
\hline stark & 10 & 5 & 7 & 5 & 3 & 30 \\
\hline $\begin{array}{l}\text { mittel und } \\
\text { schwach }\end{array}$ & 4 & 8 & 2 & 2 & 5 & 21 \\
\hline Summe & 14 & 13 & 9 & 7 & 8 & 51 \\
\hline
\end{tabular}

Quelle: Eigene Darstellung, Borderstep Institut für Innovation und Nachhaltigkeit.

Die größte Anzahl an Pfadabhängigkeiten findet sich bei den rechtlichen und ökonomischen; demgegenüber konnten nur wenige organisationale und nutzerbezogene Pfadabhängigkeiten identifiziert werden. Etwas über die Hälfte der Pfadabhängigkeiten wurde als »stark« eingestuft.

Im Bereich Pkw-Mobilität können drei Systeme von Pfadabhängigkeiten unterschieden werden. Dies ist zum einen das sich selbst stabilisierende und konsumorientierte System der Straßen in Verbindung mit der Gewöhnung an das Automobil zur Bewältigung langer Strecken. Deutlich andere Dynamiken stabilisieren das System der Verbrennungsmotoren und wiederum anders wirken die Pfadabhängigkeiten bei der Fahrzeugentsorgung zusammen.

\section{Straßenbau und Automobilität}

Das Lock-in-System in Straßenbau und Automobilität ist gekennzeichnet durch eine sehr hohe Abhängigkeit von gebauten Infrastrukturen und aus ihnen folgenden Mobilitätserfordernissen (Clausen 2017d; Korte et al. 2017).

Die Tatsachen,

- dass erhebliche Anteile der Wohn- wie auch der Gewerbegebiete durch den öffentlichen Nahverkehr nur mäßig erschlossen sind,

- dass die zur Arbeit, zum Einkaufen und zu den Schulen zurückzulegenden Strecken durch Gewöhnung an das Automobil immer länger geworden sind,

- dass wohnortnahe Versorgungsinfrastrukturen (Läden, Schulen, Ärzte usw.) in den letzten Jahrzehnten kontinuierlich ausgedünnt wurden und

- dass die Siedlungsdichte vielerorts zu niedrig ist, um Carsharing-Angebote wirtschaftlich zu betreiben und Carsharing-Angebote zum täglichen Pendeln zur Arbeit ohnehin weder ökonomisch noch ökologisch viel verbessern,

führen dazu, dass die Nutzung eines Automobils für viele Bürgerinnen und Bürger einen weiterhin hohen Nutzen im Alltag hat. Der Lock-in-Typ ist getrieben durch gebaute Infrastrukturen und vielfältige Nutzungserfordernisse. Der Komplex aus Produzenten, Lobbystrukturen und rechtlichen Regeln spielt demgegenüber eine - auch im Vergleich zu anderen Lock-in-Strukturen - eher sekundäre Rolle. 
Abbildung 11: Lock-in-System in Straßenbau und Automobilität

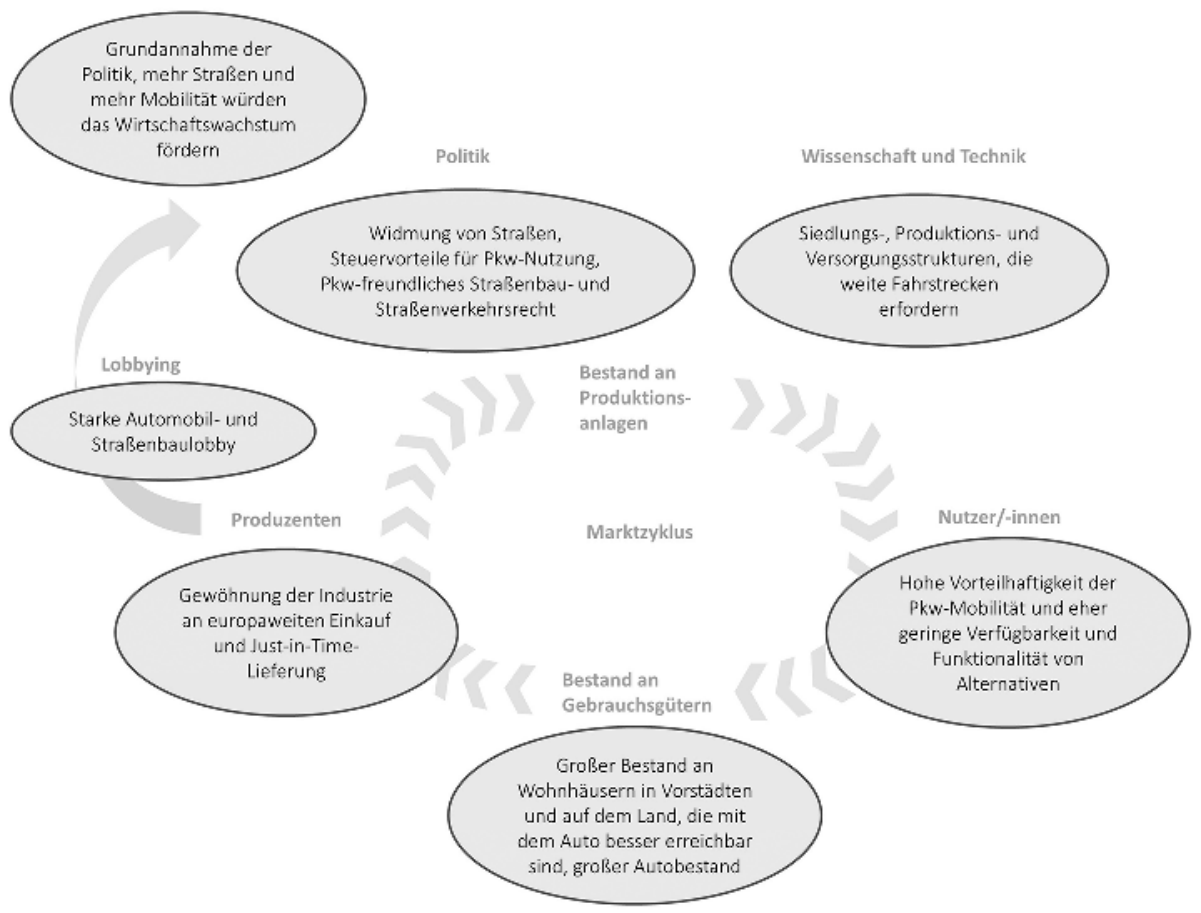

Quelle: Eigene Darstellung, Borderstep Institut für Innovation und Nachhaltigkeit.

\section{Pkw-Antriebe}

Das technologische Lock-in in die Produktion von Autos mit Benzin- und Dieselantrieb hat einen wesentlich instabileren Charakter als der Bedarf an Straßen und Autos an sich (Clausen 2017d). Zwar verfügen Millionen Nutzerinnen und Nutzer über Autos mit Verbrennungsmotor, deren mögliche Nutzungsdauer sich auf die Dauer eines möglichen Pfadwechsels auswirkt, ihre Mobilitätsbedürfnisse werden sie zukünftig aber auch mit Elektroautos ohne wesentliche Einbußen bei der Nutzung befriedigen können. Mittelfristig besteht sogar die Chance auf eine im Vergleich zum Verbrenner deutlich günstigere Automobilität.

Der Kern der Lock-in-Struktur besteht daher in den (begrenzten) technischen Fähigkeiten der Produzenten zur Produktion von Fahrzeugen mit alternativen Antrieben. Weder das Wissen in Form von Patenten (Clausen 2017d) noch die Produktionsanlagen für wesentliche Komponenten sind zur Zeit in Deutschland vorhanden. Die durch Lobbyaktivitäten geschaffenen und gegen die Erfordernisse des Klimaschutzes verteidigten Vorteile für die Verbrennertechnologie sowie das erfolgreiche Unterlaufen von Vorschriften zur Senkung des Verbrauchs und Reduktion des Schadstoffausstoßes führt die deutschen Hersteller gegenwärtig in 
Abbildung 12: Lock-in-System Pkw-Antriebe

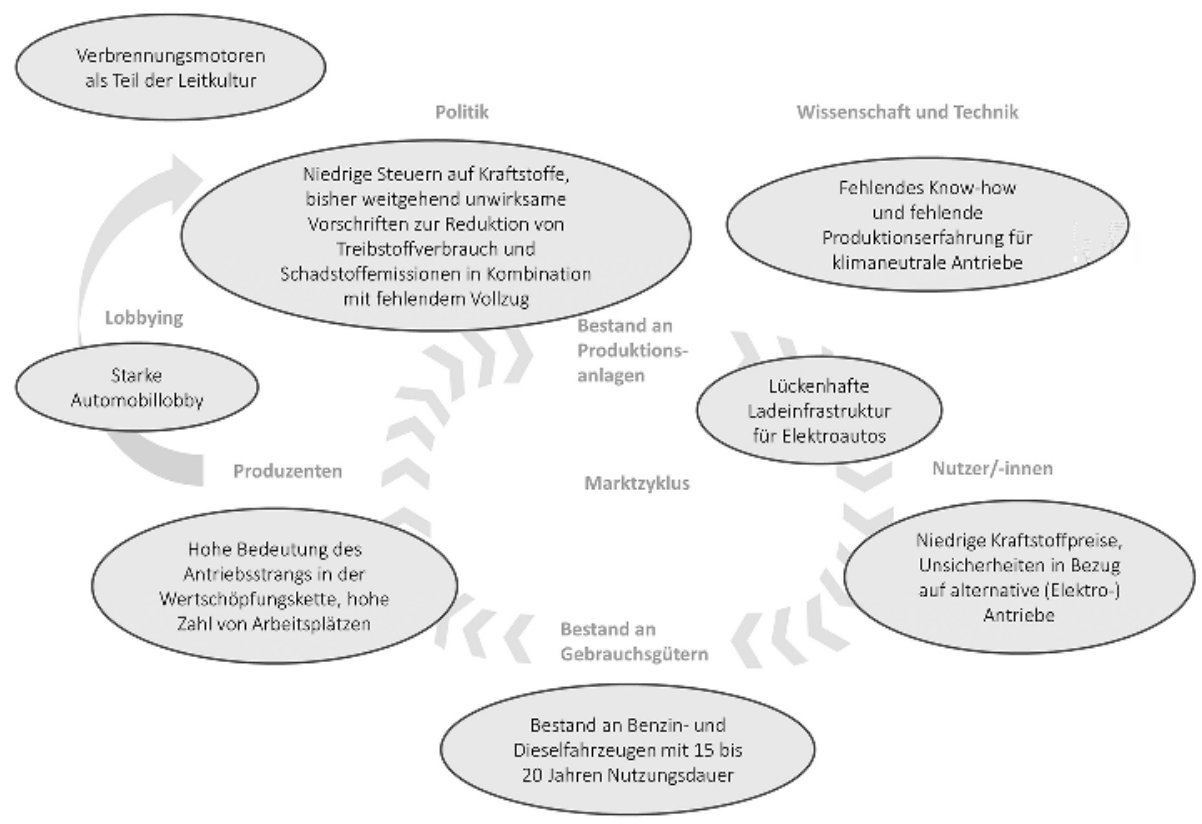

Quelle: Eigene Darstellung, Borderstep Institut für Innovation und Nachhaltigkeit.

eine Situation, die der ehemalige Umweltminister Jürgen Trittin wie folgt charakterisiert: »Wir müssen uns fragen, wie man verhindern kann, dass es den deutschen Autoherstellern so geht wie RWE und Eon: Entwicklung verpennt und plötzlich randständig« (Hannoversche Allgemeine Zeitung 2017a).

Ähnlich wie auch die landwirtschaftliche Produktion lässt sich das Lock-in als technologisch-ökonomisch-politisches System beschreiben.

\section{Pkw-Entsorgung}

Wiederum einer anderen Logik folgt die bisher aus Umweltsicht wenig erfolgreiche Entwicklung der Fahrzeugentsorgung (Tappeser und Chichowitz 2017c). Nur rund eine halbe Million der in 2013 schätzungsweise 2,86 Millionen Altfahrzeuge wurde in Deutschland als Altfahrzeug deklariert und dem offiziellen Verwertungsregime zugeführt. Mindestens 1,6 Millionen, wahrscheinlicher jedoch gut zwei Millionen Fahrzeuge verließen als Gebrauchtwagen das Land (Tappeser und Chichowitz 2017c, S. 8).

Zwar lässt sich das Lock-in auch hier als technologisch-ökonomisch-politisches System beschreiben, aber die Dynamiken sind deutlich unterschiedlich. Basierend auf dem Eigentumsrecht und dem international (noch?) gültigen Primat des Freihandels hat sich ein System entwickelt, in dem kaum noch nutzbare Altfahrzeuge 
Abbildung 13: Lock-in-System Pkw-Entsorgung

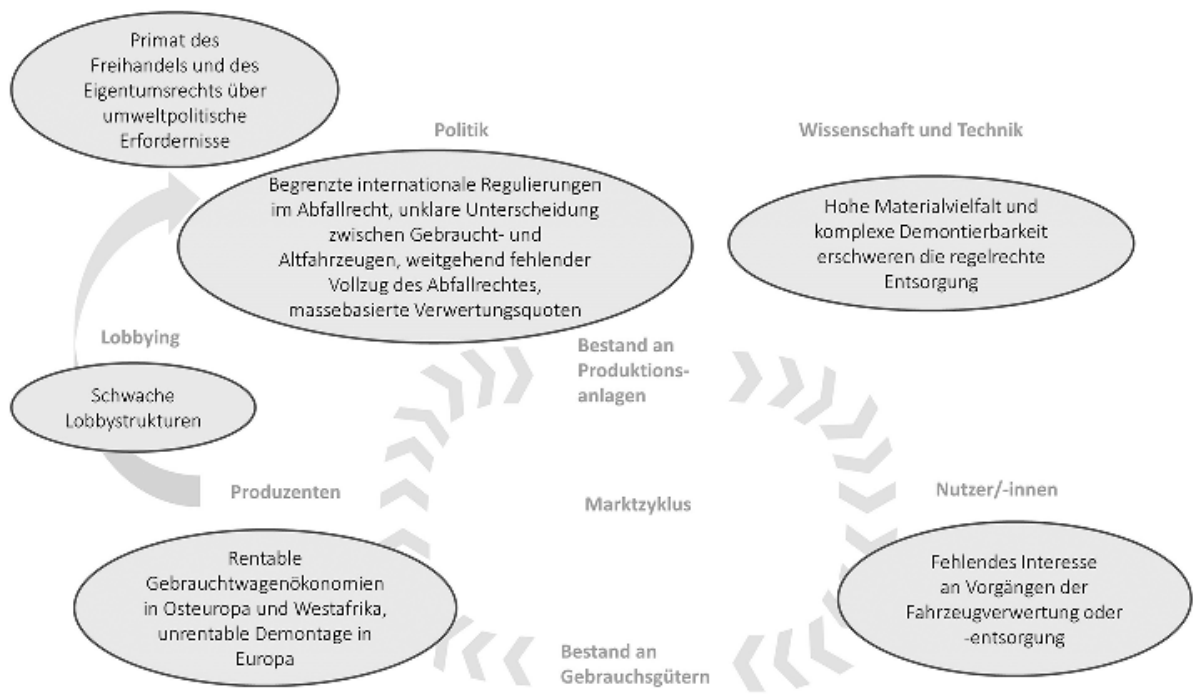

Quelle: Eigene Darstellung, Borderstep Institut für Innovation und Nachhaltigkeit.

als Gebrauchtwaren deklariert und exportiert werden. In den Zielländern werden sie zu deutlich niedrigeren Lohnkosten repariert und einer neuen Nutzungsphase zugeführt. Die Vorteile dieses Systems kommen im Wesentlichen einer Gruppe von teilweise im rechtlichen Graubereich agierenden Exporteuren sowie den Werkstätten und Nutzerinnen und Nutzern in den Zielländern zugute. Deutsche Akteurinnen und Akteure wiederum ersparen sich die aufwendige Demontage und das Recycling. Den Autobesitzerinnen und -besitzern scheint der Verbleib ihrer Fahrzeuge im Wesentlichen egal zu sein.

\subsection{Pfadabhängigkeiten und Transformationsansätze}

Als Transformationsansätze rund um die Automobilität sind aufzuführen:

- Verschiedene Konzepte der verkehrsberuhigten, fußgänger- und fahrradfreundlichen Umgestaltung von Straßen innerhalb von Siedlungen sowie das Ziel, überörtliche Straßen nicht weiter aus- oder sogar rückzubauen, als Suffizienzstrategien (Clausen 2017e)

- Die Ausrüstung des Straßennetzes mit Verkehrslenkungssystemen (vorwärtskompatibel auch als Vorbereitung auf das autonome Fahren) als Effizienzstrategie sowie mit Ladesystemen für die Elektromobilität als kombinierte Effizienz-Konsistenz-Strategie (Clausen 2017e) 
- Verschiedene Formen von Carsharing und multimodaler Mobilität sowie auch zukünftige Modelle des autonomen Fahrens (ggf. ohne eigenes Eigentum am Auto), die sämtlich Effizienzstrategien sind (Korte et al. 2017)

- Verschiedene Formen alternativer und perspektivisch klimaneutraler Antriebe, die mit Blick auf ihre Versorgung mit erneuerbarer Energie als Konsistenzansatz einzuordnen sind (Clausen $2017 \mathrm{~d}$ )

- Die recyclinggerechte Fahrzeugkonstruktion und die vermehrte Zuführung von Fahrzeugen $\mathrm{zu}$ inländischen Verwertungsprozessen und internationale Recyclingpartnerschaften, die alle Effizienz und Konsistenz (im Sinne von Materialkreisläufen) verknüpfen (Tappeser und Chichowitz 2017c)

Die Felder sind dabei stark verwoben: Infrastruktur und Antriebstechnik stehen etwa in direkten Wechselwirkungen (z. B. Aufbau ausreichender Elektro-Ladestationen), ein gleichzeitiges Vorantreiben mehrerer Aspekte ist daher unabdingbar.

Auch zur Nutzung multimodaler Mobilität oder von Carsharing stellt das Vorhandensein verschiedener, möglichst aneinander angepasster Verkehrsmittel bzw. das Vorhandensein von Carsharing-Betreibern eine notwendige Bedingung dar. Vernetzte Autos bzw. autonomes Fahren betreffen sowohl die Technikentwicklung als auch die nötige Infrastruktur.

Zur Stabilisierung tragen zunächst die mächtige Autolobby und allgemein das selbstreproduzierende System in der Autoindustrie mit der großen Zahl an von ihr abhängigen Arbeitsplätzen bei. Rechtlich wird das Auto auf mehreren Ebenen bevorzugt.

Das etablierte System der Straßen wird stabilisiert durch rechtliche Pfadabhängigkeiten im Straßenbau- und Straßenverkehrsrecht, z. B. die dauerhafte Widmung von Straßen, sowie durch vielfältige ökonomische und organisationale Pfadabhängigkeiten.

Eine Reihe spezieller Pfadabhängigkeiten wirkt sich nur auf einzelne Transformationsansätze aus:

Konstruktion und Antrieb: Bezüglich der Antriebstechnik wirken sich neben den auch durch Steuervorteile erzeugten niedrigen Preisen für fossile Kraftstoffstoffe und dem hohen Wert des Antriebsstranges am Gesamtwert auch unwirksame bzw. mangelhaft vollzogene rechtliche Instrumente (z. B. bzgl. $\mathrm{NO}_{\mathrm{x}}$ - und Feinstaubemissionen) auf die geringe Pfadwechselmotivation aus. Forschung und Entwicklung sind aufVerbrennungskraftmaschinen spezialisiert, weshalb alternative Antriebstechnologien technologisch noch nicht mit Verbrennungsmotoren gleichziehen können und es deutschen Automobilhersteller an Know-how mangelt. Bei den Herstellungskosten für Elektroautos machen die Batterien den Löwenanteil aus - in Deutschland werden jedoch keine Lithium-Ionen-Batterien hergestellt. In der Produktion spielt bisher die Möglichkeit der späteren Demontierbarkeit keine wirkliche Rolle. 
Nutzung und Infrastruktur: Die Pfadabhängigkeiten des Nutzens von Mobilität und der Infrastruktur gehen fließend ineinander über und bedingen sich wechselseitig. Unter dem Aspekt des Nutzens stellt sich der geringe relative Vorteil von Angeboten wie Carsharing oder öffentlichen Verkehrsmitteln als Pfadabhängigkeit dar. Das eigene Auto, das ja ohnehin bei vielen bereits vor der Wohnungstür steht, ist verknüpft mit Freiheits- und Unabhängigkeitsgefühlen, will als Statussymbol präsentiert werden und bietet Schutz als privater Raum. Die Zahlungsbereitschaft für Pkws ist hoch. Zudem wird das Auto als Mobilitätsmittel steuerlich bevorzugt, doch für elektrobetriebene Fahrzeuge fehlt es vielfach an (öffentlichen) Ladestationen und nutzerbezogen an Wissen hierzu.

Recycling: Relativ unabhängig von bisher genannten Pfadabhängigkeiten zeigen sich die Recycling-Ansätze. Hier macht die hohe (und im Zuge der Digitalisierung weiter zunehmende) Materialienvielfalt der Fahrzeuge eine komplexe Demontage notwendig. Unter dem Primat des Freihandels und der nicht unbedeutenden wirtschaftlichen Rentabilität zeigen sich gesetzliche Lücken, insbesondere bezogen auf die nationalen Regulierungskompetenzen sowie die Schwierigkeit der Definition von Gebraucht- und Altfahrzeugen. In den Export-Zielländern hängen viele Arbeitsplätze am Geschäft. Die erhöhte Rückführung in inländische Verwertungsstrukturen wird gehemmt durch mengenbasierte Verwertungsquoten. Nutzerinnen und Nutzer interessieren sich zudem wenig für die Restverwertung ihrer Pkws.

\subsection{Fazit}

Den geringsten Einfluss haben die existierenden Pfadabhängigkeiten auf die Suffizienzstrategien bei der Gestaltung innerörtlicher Straßen. Der Einfluss der Pfadabhängigkeiten wird im Durchschnitt als »niedrig bis mittel« eingestuft.

Die Effizienzansätze (die teilweise und abhängig von Rahmenbedingungen in einen Suffizienz- und Konsistenzcharakter übergehen) der alternativen Mobilität (Carsharing, autonomes Fahren, multimodale Mobilität), der Verkehrslenkung und des Hybridantriebs werden etwas stärker durch Pfadabhängigkeiten gebremst. Der Einfluss der Pfadabhängigkeiten wird aber auch hier durchschnittlich als »knapp mittel« eingestuft.

Die kombinierten Effizienz- und Konsistenzansätze des Fahrzeugrecyclings werden durch Pfadabhängigkeiten in »mittlerer « Stärke behindert.

Die Konsistenzansätze der Antriebe durch erneuerbaren Strom in Elektrooder Brennstoffzellenautos werden durch Pfadabhängigkeiten »knapp stark« behindert.

Die Analyse der Pfadabhängigkeiten rund um die Pkw-Mobilität führt wenig überraschend nicht zu einem völlig neuen Blick auf die Schwierigkeiten bei der Transformation der Individualmobilität. In drei Aspekten schärft sie jedoch den Blick auf Probleme bei der Veränderung: 
- Das ausgesprochen starke Lock-in aufgrund "gebauter Strukturen« in Straßenbau und Siedlungsstruktur dämpft die Erwartungen, dass mit Transformationsansätzen wie Carsharing die gesamte Pkw-Mobilität deutlich, d.h. um 20 Prozent oder mehr, reduziert werden kann.

- Die Tatsache, dass in Deutschland mehr als 40 Millionen Pkw im Einsatz sind, zeigt, dass die Transformation genügend Zeit braucht, um diese hohe Zahl an Pkws erst auszunutzen und dann auszutauschen. Der politische Planungshorizont sollte länger sein als die durchschnittliche Nutzungsdauer eines Pkws.

- Es wird deutlich, dass der mit der Autonutzung verbundene hohe Ressourcenbedarf zurzeit nur zu einem sehr kleinen Teil durch Recycling gedeckt wird. Ökonomische Pfadabhängigkeiten haben die Recyclingwirtschaft in eine Exportwirtschaft gewandelt; eine Erhöhung der Recyclingquote ist damit kein »innerdeutsches Umweltthema«, sondern ein mit der Globalisierung zusammenhängendes ökonomisch-ökologisches Thema.

\subsubsection{Landwirtschaft und Ernährung}

Im Themenfeld »Landwirtschaft« wurden insgesamt 64 Pfadabhängigkeiten dokumentiert, die in den vier Transformationsfeldstudien gefunden wurden (Clausen und Mathes 2017; Tappeser und Chichowitz 2017a, 2017b; Tappeser et al. 2017).

Tabelle 5: Anzahl an Pfadabhängigkeiten in Landwirtschaft und Ernährung nach Stärke und Art

\begin{tabular}{|c|c|c|c|c|c|c|}
\hline \multirow{2}{*}{$\begin{array}{l}\text { Stärke der } \\
\text { Pfadabhängigkeit }\end{array}$} & \multicolumn{6}{|c|}{ Art der Pfadabhängigkeit } \\
\hline & Rechtlich & Ökonomisch & Technisch & Organisational & Nutzerbezogen & Summe \\
\hline stark & 19 & 6 & 8 & 13 & 5 & 51 \\
\hline $\begin{array}{l}\text { mittel und } \\
\text { schwach }\end{array}$ & 1 & 4 & 5 & 1 & 2 & 13 \\
\hline Summe & 20 & 10 & 13 & 14 & 7 & 64 \\
\hline
\end{tabular}

Quelle: Eigene Darstellung, Borderstep Institut für Innovation und Nachhaltigkeit.

Vielen rechtlichen Pfadabhängigkeiten stehen nur wenige nutzungsbezogene Pfadabhängigkeiten gegenüber. Zahlreiche Pfadabhängigkeiten wurden als »stark« eingestuft.

Es konnten ein sich selbst stabilisierendes und konsumorientiertes System des hohen Fleischkonsums sowie ein technologisch-ökonomisch-politisches System von Pfadabhängigkeiten der landwirtschaftlichen Produktion mit seinen Transformationsfeldern »Stickstoffeinsatz«, »Pestizideinsatz« und »grüne Infrastrukturen«identifiziert werden. 


\section{Fleischkonsum}

Im Kontext des Fleischkonsums existiert ein weitgehend stabiler Markt, in dem eine hochkonzentrierte Fleischbranche in Produktion und Handel preiswerte Produkte zur Verfügung stellt, die die meisten Konsumentinnen und Konsumenten wertschätzen und kaufen (Clausen und Mathes 2017). Die Produktion baut auf der industriellen Haltung von High-Input-Rassen auf und ist durch die Sonderstellung der Landwirtschaft im Umweltrecht wie auch durch die finanzielle Förderung der konventionellen Landwirtschaft abgesichert. Die Förderlogik beruht auf der von Wirtschaft und Politik vertretenen und durch Lobbying verstärkten Annahme, dass viel billiges Fleisch wesentlicher Teil der Grundversorgung sein muss.

\section{Abbildung 14: Lock-in-System Fleischkonsum}

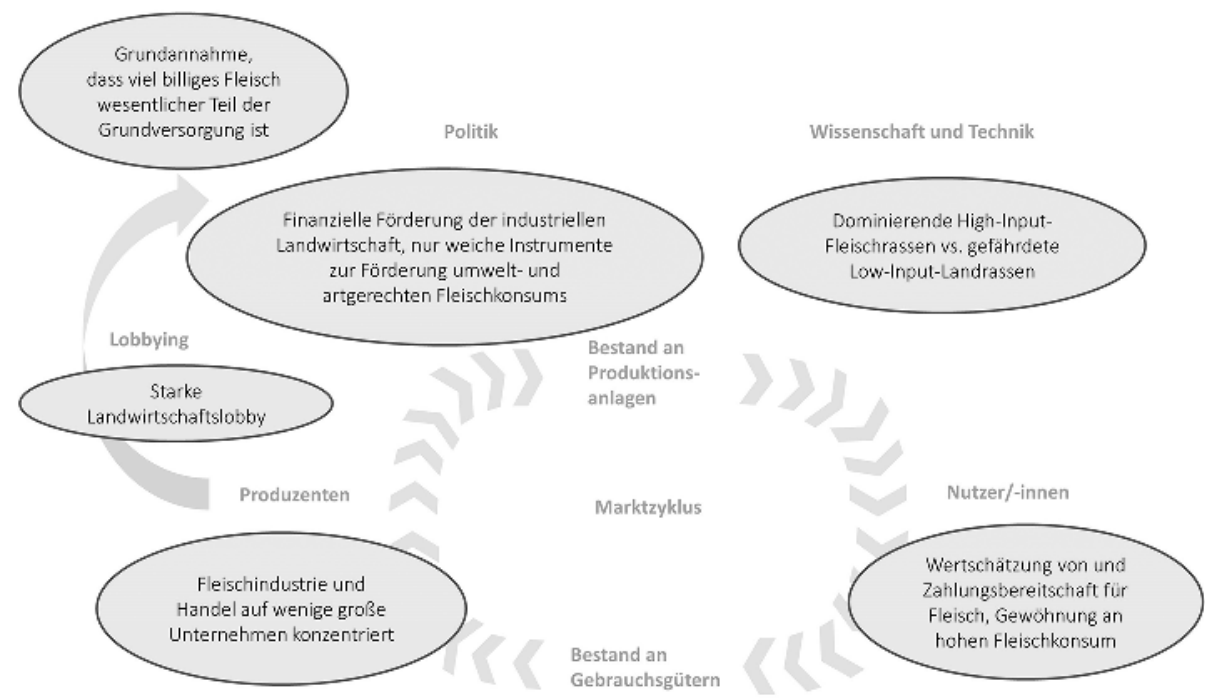

Quelle: Eigene Darstellung, Borderstep Institut für Innovation und Nachhaltigkeit.

Die Transformation dieses Regimes erfolgt einerseits dadurch, dass einzelne Konsumentinnen und Konsumenten das System »verlassen« und als Vegetarier/ -innen oder Veganer/-innen dem Fleischkonsum den Rücken kehren. Eine Reaktion des Systems auf dieses Verhalten ist kaum möglich und findet nur begrenzt und in einzelnen Fällen statt (Clausen und Fichter 2016, S. 30).

Ein zweiter Ansatz sind vielfältige und wiederkehrende Informationsbemühungen aus der Gesundheits- und Umweltpolitik, um den Fleischkonsum zu reduzieren oder den Konsum von Fleisch aus artgerechterer oder umweltschonender Produktion, z. B. Biofleisch, zu steigern. Eingesetzt werden durchweg weiche politische Instrumente der Information und Kommunikation, deren Wirkung auf 
die Transformation der Märkte bisher begrenzt ist. Der vom Umweltbundesamt im Januar 2017 ins Gespräch gebrachte Vorschlag, Fleisch durch eine Erhöhung des Mehrwertsteuersatzes zu verteuern (Köder und Burger 2016, S. 67) und so erstmals ein »härteres « Instrument einzusetzen, wurde von den Lobbys und dem Mainstream der Politik spontan und entrüstet zurückgewiesen.

\section{Einsatz von Stickstoff und Pestiziden sowie grüne Infrastrukturen}

Etwas anders sieht das Lock-in-System beim Einsatz von Stickstoff und Pestiziden sowie bei grünen Infrastrukturen aus (Tappeser et al. 2017; Tappeser und Chichowitz 2017a, 2017b). Die Rolle der Konsumentinnen und Konsumenten wie sogar der Landwirtinnen und Landwirte ist hier eher passiv. In der Produktion dominieren hochentwickelte und integrierte Technologiesysteme, typischerweise speziell gezüchtete oder gentechnisch hergestellte Pflanzensorten, deren Einsatz untrennbar mit dem Einsatz von Dünger und oft spezifischen Pestiziden verbunden ist und die kostengünstig zu produzieren und vielfach äußerst umweltschädlich sind.

Wesentliche Profiteure dieses Systems sind Agrarkonzerne sowie letztlich auch Handel und Konsumentinnen und Konsumenten, denen, so lange das System nicht zum ökologischen Kollaps führt, preiswerte Nahrungsmittel zur Verfügung stehen.

Die zentrale Stabilisierung des Systems erfolgt auf Basis der Grundannahme, dass die Landwirtschaft aufgrund ihres Beitrages zur Grundversorgung eine Sonderstellung im Umweltrecht hat. Diese Sonderstellung hat zur Folge, dass viele umweltbezogene Vorschriften in der Landwirtschaft bisher wenig wirksam sind, dass ein nur lückenhaftes Monitoring stattfindet und der Vollzug ebenso große Schwächen aufweist. Wird dennoch ein Rechtsverstoß entdeckt, fehlt manchmal eine Strafbewehrung. Hinzu kommt die finanzielle Förderung der industriellen Landwirtschaft.

Als grundsätzliche Alternative zu diesem vorherrschenden System der Agrarproduktion bietet sich der ökologische Landbau an, der jedoch seinen Marktanteil aufgrund hoher Kostennachteile nur langsam und in engen Grenzen ausweitet. Die staatlichen Labelsysteme und die finanzielle Förderung des Ökolandbaus reichen bis dato nicht aus, seine Kostennachteile am Markt auszugleichen.

Der Einsatz von Pestiziden und Dünger soll auch durch Effizienzstrategien verringert werden. Der Ausbau bzw. die Sicherung grüner Infrastrukturen soll durch Unterschutzstellung und Renaturierung erfolgen. 
Abbildung 15: Lock-in-System beim Einsatz von Stickstoff und Pestiziden sowie bei grünen Infrastrukturen

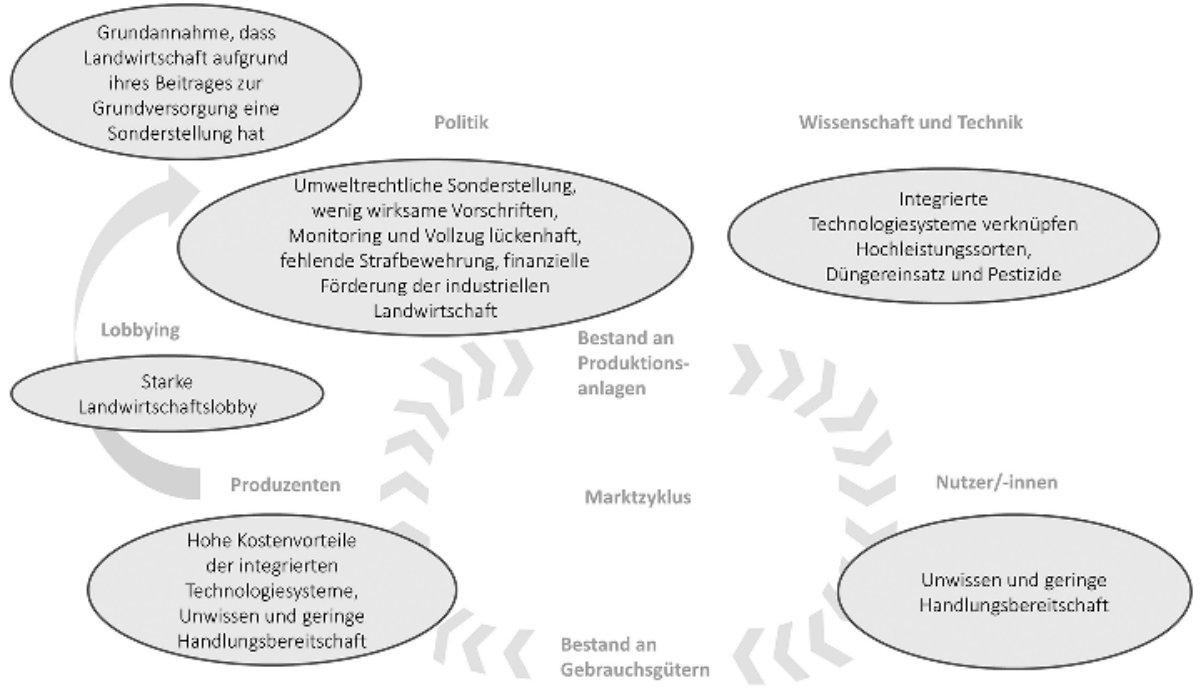

Quelle: Eigene Darstellung, Borderstep Institut für Innovation und Nachhaltigkeit.

\subsection{Pfadabhängigkeiten und Transformationsansätze}

Als Transformationsansätze sind im Bereich Landwirtschaft und Ernährung aufzuführen:

- Vegetarismus und Veganismus als individuelle und suffiziente Wege, das System des Fleischkonsums zu verlassen (Clausen und Mathes 2017)

- Der ökologische Landbau, der als konsistenter Ansatz die in vielfältiger Weise umweltgerechtere Agrarproduktion mit artgerechterer Tierhaltung verbindet (Clausen und Mathes 2017; Tappeser und Chichowitz 2017a, 2017b; Tappeser et al. 2017)

- Mengenreduktionsstrategien, mit denen als Effizienzansätze und vielfach im Rahmen einer »Digitalisierung der Landwirtschaft« die innerbetriebliche Stickstoffbilanz verbessert, der überregionale Nährstoffausgleich gefördert oder Pestizide gezielter und in kleinen Mengen ausgebracht werden sollen (Tappeser und Chichowitz 2017a, 2017b)

- Der Schutz von Landschaftselementen und die Renaturierung von Lebensräumen, die Konsistenz und Suffizienz verbinden (Tappeser et al. 2017)

Die Betrachtung der Pfadabhängigkeiten bezogen auf Transformationsansätze zeigt eine Art Schalensystem: Im Kern stehen die einzelnen Transformationsansätze, welche durch spezifische Pfadabhängigkeiten gehemmt werden. Die zweite 
Schale formt der nationale rechtliche und organisatorische Rahmen. Die internationale Konkurrenz schließlich bildet die dritte Ebene der Diffusionshemmnisse.

\section{Erste Schale: spezifisch wirkende Pfadabhängigkeiten}

Bezüglich des Ansatzes der innerbetrieblichen Stickstoffeffizienz zeigt sich:

- Das Düngerecht ist wenig wirksam, die Förderung von Bio-Energie durch das Erneuerbare-Energien-Gesetz (EEG) sorgt für erhöhte Stickstoffeinträge.

- Hochleistungs-Hybridsorten sind hochrentierlich, benötigen eine hohe Stickstoffverfügbarkeit sowie oft auch den Einsatz spezifischer Pestizide und bilden als System eine starke ökonomische Pfadabhängigkeit.

- Die schwierige Abschätzbarkeit des Nährstoffgehaltes von Wirtschaftsdünger macht seine optimale Nutzung kompliziert.

Dem Ansatz des überregionalen Nährstoffaustausches mangelt es zur Umsetzung an Technologien und Praktiken.

Die Reduktion der Pestizidverwendung in der Landwirtschaft wird u. a. gehemmt durch

- den hohen Bedarf an Pestiziden durch Monokulturen und Hochleistungssorten,

- die letztlich hohe Rentabilität der Pestizidverwendung,

- den Mangel an landwirtschaftlichem Fachwissen im Umgang mit geringen Pestizidmengen.

Im Bereich "grüne Infrastruktur« zeigen sich insbesondere ökonomische Pfadabhängigkeiten. Für Landwirtinnen und Landwirte ist es schlicht nicht wirtschaftlich, Grünstreifen zu ermöglichen. Rechtliche Rahmenbedingungen, etwa in Form des Natura-2000-Schutzgebietsnetzwerks, sind nicht effizient.

Innerhalb der Fleischwirtschaft sind es wenige große Firmen, welche vielen Menschen einen Arbeitsplatz bieten. Die Fleischproduktion wird indirekt durch Agrarsubventionen stark gefördert, während Kontrollen von Umweltauflagen und der artgerechten Tierhaltung lückenhaft sind.

Pfadabhängigkeiten, die Vegetarismus bzw. Veganismus im Wege stehen, sind auf der Nutzerebene anzusiedeln: Allgemein ist die Wertschätzung wie auch der durchschnittliche Konsum von Fleisch und tierischen Lebensmitteln hoch. Der Konsum ist tief in der alläglichen Lebenswelt verankert und gilt geradezu als Teil deutscher Leitkultur.

Dem ökologischen Landbau ist als spezifisch wirkende Pfadabhängigkeit nur der Fachkräftemangel zuzuordnen, er wird jedoch durch fast alle anderen Pfadabhängigkeiten indirekt ebenfalls behindert. 


\section{Zweite Schale: nationale rechtliche und organisatorische Konstrukte}

Auf nationaler Ebene ist die sehr starke und mächtige Landwirtschaftslobby auszumachen, die zudem Einfluss auf die rechtliche Trennung von Landwirtschaftsund Umweltverwaltung hat. Landwirtschafts- und Umweltverwaltung sind auf allen Ebenen getrennt und folgen ihrem System nach unterschiedlichen Logiken.

Rechtliche Konstrukte zum Schutz der Umwelt erweisen sich daher sowohl in der Konstruktion als auch im Vollzug an vielen Stellen als lückenhaft; die Landwirtschaft kann ihre Sonderstellung unhinterfragt behaupten. Hinzu kommen als unterkomplex bewertete Modelle der Risikobewertung.

Landwirtschaft findet in Deutschland im Spannungsfeld eines hohen Kostendrucks statt, getrieben durch die Nachfrage der Verbraucherinnen und Verbraucher nach preiswerten Lebensmitteln. Quer zu den spezifischen Pfadabhängigkeiten wirkt daher die Problematik der mangelnden Internalisierung externer Kosten im etablierten System, wodurch nachhaltige Produkte und Verfahren meist kaum wettbewerbsfähig mit den konventionellen Produkten sind.

Nicht-nachhaltige Ansätze werden zusätzlich durch die oft nicht-nachhaltigen »Grundzüge der Gemeinsamen Agrarpolitik« der EU stabilisiert.

\section{Dritte Schale: internationaler Wettbewerb}

Selbst wenn nationale Hemmnisse im hohen Maße beseitigt wären, ist noch immer mit der internationalen Konkurrenz zu rechnen, welche schon jetzt Massen an vielfältigen Agrarprodukten zu sehr günstigen Preisen auch auf den deutschen Markt bringt und so einen großen Konkurrenz- und Kostendruck erzeugt.

\subsection{Fazit}

Den geringsten Einfluss haben die existierenden Pfadabhängigkeiten auf die Suffizienzstrategien Vegetarismus und Veganismus. Dem freiwilligen Verlassen des Systems können die etablierten Strukturen des Regimes nichts entgegensetzen. Der Einfluss der Pfadabhängigkeiten wird im Mittel als »niedrig« eingestuft.

Die Effizienzansätze der Mengenreduktionsstrategien werden durch Pfadabhängigkeiten deutlich stärker behindert. Der Einfluss der Pfadabhängigkeiten wird durchschnittlich als »mittel bis stark« eingestuft.

Die Strategien zum Schutz grüner Infrastrukturen verbinden Konsistenz und Suffizienz; sie werden ebenfalls durch Pfadabhängigkeiten »mittel bis stark« behindert.

Der Ökolandbau erweist sich als die universellste Transformationsstrategie. Er würde in allen vier hier untersuchten Transformationsfeldern die Entwicklung entscheidend und positiv vorantreiben. In allen vier Studien wurde jedoch die Stärke sämtlicher gegen die Verbreitung des Ökolandbaus wirkender Pfadabhängigkeiten als »stark« beurteilt. Die Überwindung der gegen diese Transfor- 
mationsstrategie wirkenden Pfadabhängigkeiten stellt damit die größte Herausforderung dar.

Die Analyse der Pfadabhängigkeiten rund um die Landwirtschaft weist auf einen zentralen stabilisierenden Faktor hin. Gleichzeitig lässt sie einen wichtigen Treiber eines möglichen Lock-out erkennen:

- Die Basis der Stabilität des landwirtschaftlichen Produktionssystems ist die Grundannahme, dass die Landwirtschaft aufgrund ihres Beitrages zur Grundversorgung einer Sonderstellung im Umweltrecht bedarf. Die Durchsetzung von Umweltvorschriften findet zudem teilweise nicht durch die Umwelt-, sondern durch die Landwirtschaftsverwaltung statt. Der Vollzug weist vielfältige Lücken auf.

- Charakteristisch für den Fleischkonsum ist der individuelle Charakter der Entscheidung, was man isst. Dabei hat das etablierte Regime kaum eine Möglichkeit zu verhindern, dass einzelne Konsumentinnen und Konsumenten das System des Fleischkonsums »verlassen « und zu einer vegetarischen oder veganen Ernährung wechseln. Vielmehr wird das alternative Angebot weiter ausgebaut.

\subsubsection{Wärmeversorgung}

In den drei Transformationsfeldstudien des Bereichs »Wärmeversorgung« (Clausen 2017g; Clausen und Hinterholzer 2017; Tappeser und Chichowitz 2017d) wurden insgesamt 49 Pfadabhängigkeiten identifiziert.

Tabelle 6: Anzahl an Pfadabhängigkeiten in der Wärmeversorgung nach Stärke und Art

\begin{tabular}{|c|c|c|c|c|c|c|}
\hline \multirow{2}{*}{$\begin{array}{l}\text { Stärke der } \\
\text { Pfadabhängigkeit }\end{array}$} & \multicolumn{6}{|c|}{ Art der Pfadabhängigkeit } \\
\hline & Rechtlich & Ökonomisch & Technisch & Organisational & Nutzerbezogen & Summe \\
\hline stark & 12 & 7 & 5 & 6 & 4 & 34 \\
\hline $\begin{array}{l}\text { mittel und } \\
\text { schwach }\end{array}$ & 1 & 4 & 4 & 5 & 1 & 15 \\
\hline Summe & 13 & 11 & 9 & 11 & 5 & 49 \\
\hline
\end{tabular}

Quelle: Eigene Darstellung, Borderstep Institut für Innovation und Nachhaltigkeit.

Betrachtet man die Anzahl und Stärke der verschiedenen Pfadabhängigkeiten, zeigt sich eine leichte Dominanz rechtlicher Pfadabhängigkeiten; aber auch ökonomische, organisationale und technische Pfadabhängigkeiten spielen eine wichtige Rolle. Nutzerbezogene Pfadabhängigkeiten sind weniger häufig, werden jedoch größtenteils als »stark« bewertet.

Die drei untersuchten Transformationsfelder »Energieverbrauch von Gebäuden mit Fokus auf den Sanierungsbedarf im Bestand«, »Wärmebereitstellung in Einzelgebäuden« und »Wärmenetze« lassen zwei eng miteinander verknüpfte Systeme von Pfadabhängigkeiten erkennen. Einerseits besteht auf der Verbrauchsseite ein Geflecht von ökonomischen, nutzerbezogenen, rechtlichen und organisationalen 
Pfadabhängigkeiten, die eine höhere Sanierungstätigkeit verhindern. Andererseits besteht auf Bereitstellungsseite ein technologisch-ökonomisches System der fossilen Wärmeerzeugung auf Einzelhausbasis, das nur schwierig zu durchbrechen ist.

\section{Wärmeenergieverbrauch von Gebäuden}

Im Kontext der Sanierung werden langlebige Gebäude in oft sehr kleinteiligen Besitzstrukturen zuverlässig und vergleichsweise günstig durch fossile Wärme versorgt, sodass Nutzerinnen und Nutzer sowie Besitzende wenig Eigeninteresse an einer energetischen Sanierung entwickeln und entsprechend nur in geringem Maße bereit für Investitionen, Aufwand, Lärm und Unannehmlichkeiten sind. In Mietgebäuden und bei Eigentümergemeinschaften sind Finanzierung und Entscheidungsfindung besonders schwierig. Dienstleistungen zur Durchführung der Sanierung sind für Kundinnen und Kunden wenig überschaubar und mit Risiken behaftet, die durch Mythen und negative Schlagzeilen im öffentlichen Diskurs medial verstärkt werden. Gesetzliche Vorgaben sind denkbar kompliziert, werden kaum kontrolliert und beruhen auf der durch Eigentümerlobbys verstärkten Grundannahme, dass energetische Sanierungen nur im Falle einer freiwilligen Sowieso-Sanierung und unter dem Gebot der Wirtschaftlichkeit stattzufinden haben.

\section{Abbildung 16: Lock-in in einen hohen Wärmeenergieverbrauch von Gebäuden}

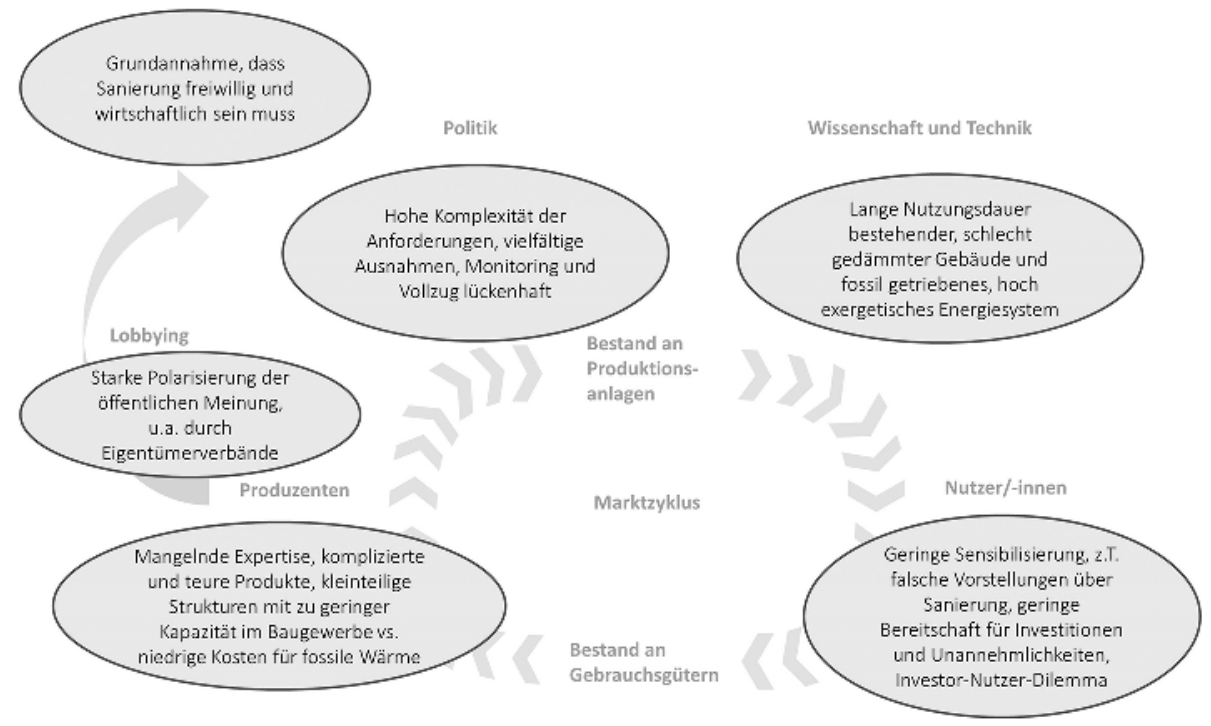

Quelle: Eigene Darstellung, Borderstep Institut für Innovation und Nachhaltigkeit.

Gleichzeitig erfolgt mit der schrittweisen Dämmung von Fenstern, Wänden, Dächern und Kellerdecken des Gebäudebestands bei gleichzeitig steigenden Wär- 
meschutzanforderungen schon ein langsamer Wandel. Strategien, um diesen zu beschleunigen, bestehen insbesondere in staatlichen Finanzzuschüssen und einer verbesserten Beratung und Planung mit Hilfe von Sanierungsfahrplänen. Weiterreichende Verpflichtungen werden nicht diskutiert.

\section{Wärmeversorgung von Gebäuden}

Im Bereich der Wärmeversorgung stellt sich die Situation zum Teil ähnlich dar bzw. ist eng mit den die Sanierungspraxis bedingenden Pfadabhängigkeiten verknüpft. Auch hier sind die Nutzungszyklen eines Versorgungssystems lang und ein Wechsel aufwendig, was das bestehende System stabilisiert. Etwaige Investitionen in eine Heizungsanlage auf Basis erneuerbarer Energien sind ebenso wie die Nutzung eines (zusätzlich zu entwickelnden) Niedrigenergiewärmenetzes im Gebäudebestand meist nur in Kombination mit umfangreichen Dämmungsmaßnahmen und dem Wechsel von Heizkörpern sinnvoll. Nutzer/-innen und Besitzer/-innen sind ähnlich wenig sensibilisiert oder investitionsbereit. Rechtliche Vorgaben sind von Ausnahmen und Vollzugsdefiziten geprägt, die Heizungsindustrie ist auf fossile Energieträger fokussiert und tritt gemeinsam mit der Brennstofflobby für einen technologieoffenen, sprich: weniger von erneuerbaren Energien geprägten Wärmemarkt ein. Wärmespeicher und -transformatoren stellen einen technologischen Engpass dar. Zusätzlich ist im Fall von Wärmenetzen ein erhöhter, externer Koordinationsaufwand notwendig.

\section{Abbildung 17: Lock-in in fossile Wärmeversorgung von Gebäuden}

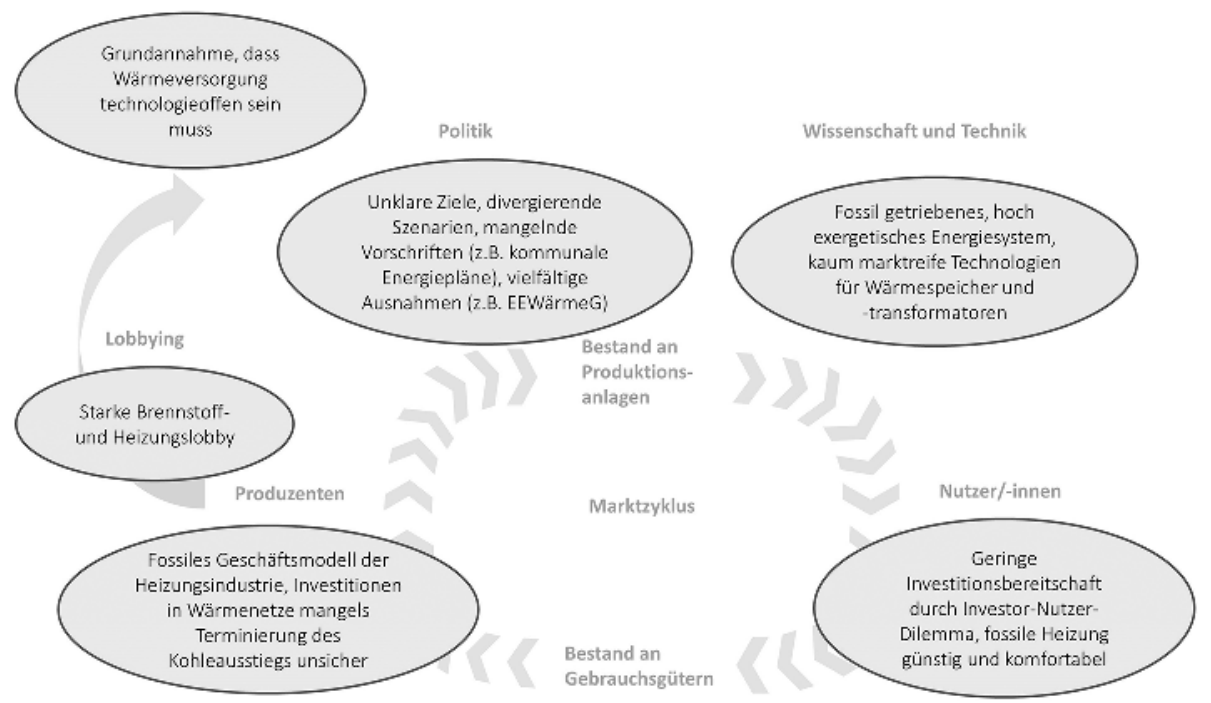

Quelle: Eigene Darstellung, Borderstep Institut für Innovation und Nachhaltigkeit. 
Alternativen zum bestehenden System der Wärmeversorgung finden sich in verschiedenen Technologien zur Wärmebereitstellung und Verteilung aus erneuerbaren Energien u.a. durch Solar- und Geothermie, kalte Netze, Wärmepumpen sowie Holz- und Pelletheizungen.

\section{Hierarchie von gebäudeübergreifender Mesoebene und Einzelgebäuden}

Beim Blick auf die Transformationsansätze im Themenbereich »Wärmeversorgung« fällt eine Hierarchie zwischen Ansätzen auf der gebäudeübergreifenden Mesoebene und Ansätzen auf der Ebene individueller Wärmenetze bzw. Gebäude auf. Weiter sind Unterschiede zwischen der Fortführung des High-Exergy-Pfades und des mit Blick auf die Begrenztheit fossiler Rohstoffe zukunftsweisenden Low-Exergy-Pfades von Bedeutung. Zudem charakterisieren sich die so zu bildenden Gruppen von Transformationsansätzen durch einige unterschiedliche, jeweils spezifische Pfadabhängigkeiten.

\section{Abbildung 18: Netzwerkbeziehungen von Pfadabhängigkeiten}

Wärmenetze
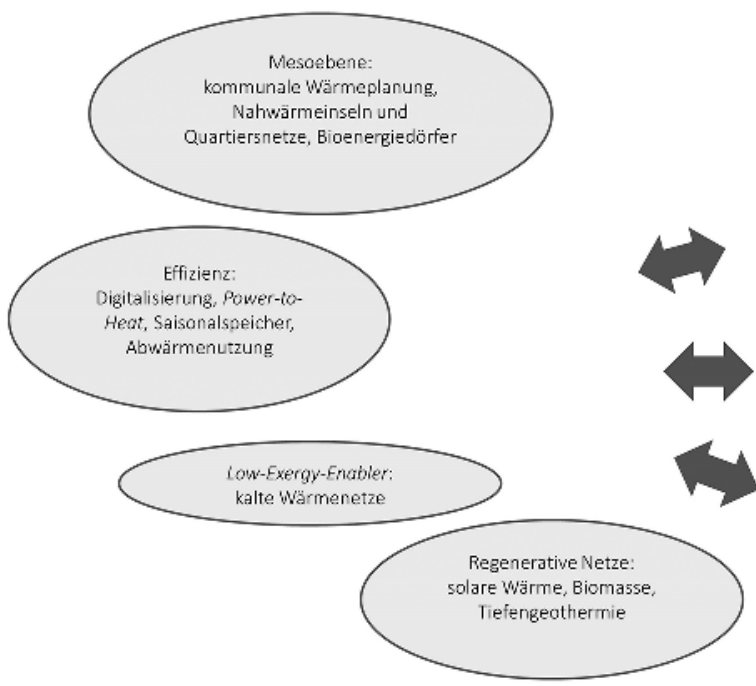

Einzelhäuser

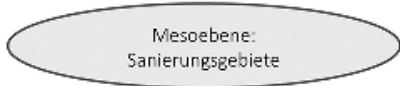

Gebăudeenergieeffizienz: Geschossdecken, Fassaden, Fenster
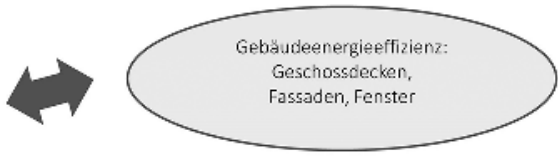

Low-Exergy-Enobler: Flächenheizungen

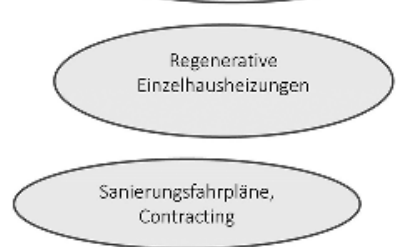

Quelle: Eigene Darstellung, Borderstep Institut für Innovation und Nachhaltigkeit.

Konsistenzbasierte Ansätze setzen zumeist eine Verknüpfung von Dämmmaßnahmen und dem Wechsel zu erneuerbaren Energien zur Wärmeproduktion voraus. Gerade solar- und geothermische Anlagen sowie kalte (low exergy) Nahund Fernwärmenetze sind auf gute Wärmedämmung und die Installation von Flächenheizungen angewiesen. Während die Installation von biomassebasierten 
Heizungen ohne grundlegende Änderungen an bestehenden Gebäuden und Wärmeverteilsystemen möglich ist, stehen deren limitierte Verfügbarkeit und Nutzungskonflikte einer flächendeckenden Ausbreitung entgegen. Effizienzbasierte Einzelansätze wie eine Erneuerung fossiler Heizungsanlagen oder die Dämmung einzelner Gebäudeelemente sind wesentlich weiter verbreitet, führen im Resultat jedoch nicht unbedingt zu einer Erreichung bestehender Ziele im Wärmesektor.

Auf einer Mesoebene lassen sich im Bereich der Wärmenetze die Transformationsansätze der kommunalen Wärmeplanung, der Nahwärmeinseln und der Quartiersnetze sowie auf dem Land auch der Bioenergiedörfer identifizieren. Diese Ansätze umfassen neben ggf. technischen Maßnahmen zusätzlich bzw. vornehmlich auch organisatorische Konzepte und stecken einen Rahmen ab, in dem dann konkrete technische Projekte umgesetzt werden können.

Mit Fokus auf Wärmenetze lassen sich darüber hinaus drei Gruppen von technischen Ansätzen identifizieren:

- Effizienzorientierte Ansätze, mit denen sowohl konventionelle (fossile) Wärmenetze effizienter gemacht werden können, aber auch die Voraussetzungen für Low-Exergy-Netze (regenerativ) verbessert werden

- Die kalten Wärmenetze, mit denen insbesondere die Möglichkeit der Nutzung von Low-Exergy- Energiequellen wie solarer Wärme mit Saisonalspeicher, aber auch Abwärme verbessert werden

- Regenerative Wärmequellen zur Versorgung von Wärmenetzen, etwa große Solarthermiefelder, geothermische Wärme und Biomassekraftwerke

In Bezug auf Einzelhäuser existiert eine Mesoebene dann, wenn z. B. die Kommune Sanierungsgebiete festlegt und in diesen bestimmte organisatorische oder finanzielle Hilfen für die wärmetechnische Sanierung durch die Gebäudeeigentümer bereitstellt. Auch mit Fokus auf Gebäude lassen sich verschiedene Maßnahmentypen identifizieren:

- Maßnahmen zur Senkung des Energieverbrauchs wie die Verbesserung der Wärmedämmung an Wänden, Fenstern und Türen sowie Geschossdecken und Dächern

- Der Einbau von Flächenheizungen, die niedrigere Vorlauftemperaturen zulassen und so die Möglichkeit der Nutzung von Low-Exergy-Energiequellen wie solarer Wärme oder Wärme aus kalten Wärmenetzen verbessern

- Der Einbau von Heizungsanlagen auf Basis erneuerbarer Energien wie Solarwärme, Wärmepumpen oder Biomasse

- Planerische Konzepte (Sanierungsfahrpläne) und ökonomische Ansätze wie Contracting, die die Realisierungschancen technischer Maßnahmen verbessern 
Zwischen der Realisierung von Maßnahmen auf der Einzelgebäudeseite und bei den Wärmenetzen bestehen Wechselwirkungen:

- Fortschreitende Wärmedämmung im Bestand senkt die Wärmeabnahme und wirkt sich so auf den Betrieb von Wärmenetzen aus. Hier können bei gleichbleibender Wärmelieferung zusätzliche Gebäude angeschlossen werden, um den Wärmeabsatz sicherzustellen. Die zusätzlichen Ausbaukosten der Netze wirken sich genau wie der sinkende Absatz negativ auf die Wirtschaftlichkeit der Netze aus.

- Die effizienzsteigernde Optimierung von Wärmenetzen durch Methoden der Digitalisierung stößt dort an Grenzen, wo das Wärmenetz über keine gebäude- und nutzerindividuellen Daten verfügt. Das smart building wird so zur Voraussetzung für ein intelligentes Netz.

- Die Existenz oder der Neubau von Netzen können planerisch durch die Kommune anhand eines Fernwärmevorranggebiets gefördert bzw. festgeschrieben werden. In solchen Fällen unterliegt die Veränderung von Heizungsanlagen in Einzelgebäuden Einschränkungen.

\subsection{Pfadabhängigkeiten und Transformationsansätze}

Eine Reihe von Pfadabhängigkeiten, welche den etablierten High-Exergy-Pfad stabilisieren, wirken sich übergreifend auf alle Transformationsänsätze aus.

- Übergreifend wirken zunächst rechtliche Pfadabhängigkeiten; sie behindern so die Umsetzung fast aller Transformationsansätze. Rechtliche Instrumente im Wärmekontext sind häufig sehr komplex (z. B. Sanierungsvorschriften) oder von Ausnahmen durchzogen (z. B. Lücken in der Förderpolitik von Wärmenetzen, Lücken im Erneuerbare-Energien-Wärmegesetz [EEWärmeG], »Oma-Regelung«). Rechtliche Vorschriften unterliegen zudem z.T. einem mangelnden Vollzug (z. B. Sanierungsvorschriften).

- Eine wichtige ökonomische Pfadabhängigkeit ist der Wert der bereits gebauten Infrastruktur (etwa die Gasnetze) und der Wert des derzeitigen Gebäudebestandes. Die hohen Pfadwechselkosten, die mit fast allen Transformationsansätzen rund um die Wärme verbunden sind, wirken sich übergreifend aus.

- Über alle Transformationsansätze hinweg wirken sich auch die niedrigen Kosten fossiler Energieträger aus, die indirekt auch Einfluss auf die Politik haben. Der politische Anspruch einer preiswerten Grundversorgung für die Bevölkerung hemmt den Willen und die Möglichkeit der Politik dafür zu sorgen, dass die Energiepreise die »ökologische Wahrheit sagen«. Viele Eigentümer/-innen bzw. Mieter/-innen schlecht sanierter Häuser und Wohnungen sind aufgrund der niedrigen Energiepreise und daher niedrigen Heizkosten mit dem gegenwärtigen Status zufrieden und spüren keinen Handlungsdruck. 
- Eine weitere generell wirksame Pfadabhängigkeit besteht in den wirkmächtigen Akteurinnen und Akteuren, welche ein Interesse an der Beibehaltung des derzeitigen Pfades haben und sich in verschiedenen Lobbyverbänden einsetzen. Dabei handelt es sich um die Betreiber fossiler Kraftwerke, die Wärmenetze speisen, und auch um die thermotechnische Industrie mit ihrem Verband, dem Bundesverband der Deutschen Heizungsindustrie (BDH).

Eine Reihe von Pfadabhängigkeiten wirkt sich aber auch fokussiert auf einzelne Transaktionsansätze aus. Am auffälligsten ist hier, dass besonders die Transformationsansätze auf der Mesoebene der Wärmenetze durch Pfadabhängigkeiten stark behindert werden.

- Als technische Pfadabhängigkeit wirkt sich hier zunächst der Zustand aus, dass es zwar umfangreiche Gasnetze (50 Prozent Anschlussgrad der Haushalte), aber nur wenig Wärmenetze (13 Prozent Anschlussgrad der Haushalte) gibt.

- Der Grundsatz der Technologieneutralität und auch die Angst, in Freiheitsrechte (Wahl der eigenen Heizung) einzugreifen, hemmen die kommunale Wärmeplanung und die Ausweisung von Fernwärmevorranggebieten.

- Die Geschäftsmodelle der etablierten Wärmeversorger lassen sich nur begrenzt auf kleine Quartiersnetze übertragen und es herrscht ein deutlicher Mangel an Akteurinnen und Akteuren, die Wärmenetze betreiben wollen und können. So findet sich z. B. selbst bei Existenz einer nutzbaren Abwärmequelle häufig kein/-e Akteur/-in, der bzw. die diese zu den Kundinnen und Kunden transportiert.

- Die Wirtschaftlichkeit fossiler Kraftwerke, der umstritten terminierte Kohleausstieg, aber auch Gewerkschaften wie die IGBCE und Verdi hemmen kommunale Planungsansätze ebenfalls, wenn diese etablierte Strukturen in Frage stellen. Zudem befinden sich viele Kommunen in einem Dilemma, da die Anteile an ihren Stadtwerken für sie eine wichtige finanzielle Stütze bilden.

$\mathrm{Zu}$ einer zögerlichen Umsetzung führt auch, dass kalte Wärmenetze als Schlüsseltechnologie einer Low-Exergy-Wärmeversorgung noch nicht ausgereift sind. Hier wirken sich vornehmlich technische Pfadabhängigkeiten aus:

- Komponenten wie der Saisonalspeicher und der Wärmetransformator (eine wärmegetriebene Wärmepumpe) sind wenig erprobt und es finden sich national kaum Hersteller mit Erfahrung.

- Die Legionellenprophylaxe erfordert hohen Aufwand.

- Es mangelt an Demonstrationsprojekten. 
Bezogen auf gebäudeindividuelle Sanierungsmaßnahmen ist zum einen die Besitzstruktur der Wohngebäude und Wohnungen problematisch. Ca. 54 Prozent der Wohnungen werden vermietet. Damit verbunden ist das Investor-Nutzer-Dilemma bei Sanierungsmaßnahmen. Und Einzeleigentümerinnen und -eigentümer von Wohnraum stellen ebenfalls ein Hemmnis dar, da sie sich z. B. als Besitzende von Wohnungen in Mehrfamilienhäusern, aber auch von einem einzelnen Reihenhaus auf Sanierungskonzepte einigen müssen, was häufig nicht gelingt. Auch Unkenntnis, Mythen und Angst vor Unbequemlichkeiten sind als Hemmnisse bei Sanierungsmaßnahmen wirksam.

Mit Fokus auf gebäudeindividuelle Maßnahmen ist auch die mangelnde Fachexpertise von Planerinnen und Planern sowie Handwerksunternehmen von Bedeutung.

Als materielles Hemmnis bei der Wahl der Heizungsanlage ist aus gesellschaftlicher Perspektive von Bedeutung, dass die Verfügbarkeit von Biomasse beschränkt ist. So bietet eine Biomasseheizung zwar für den Einzelnutzenden die Vorteile, dass das Wärmeverteilsystem mit Konvektorheizkörpern wie bei jeder anderen High-Exergy-Heizung genutzt werden kann, aus übergreifender Perspektive ist aber eben wichtig, dass der Anteil von Biomasseheizungen nicht beliebig gesteigert werden kann.

\subsection{Fazit}

Die Analyse der Pfadabhängigkeiten rund um die Wärmeversorgung lässt drei zentrale Pfadabhängigkeiten erkennen:

- Sowohl die vorhandenen, unsanierten Gebäude als auch die fehlenden Wärmenetzstrukturen erfordern hohe Pfadwechselkosten und dementsprechend eine lange Zeit für den Pfadwechsel.

- Die verteilten und manchmal komplexen Eigentumsstrukturen (Eigentümergemeinschaften) stellen im Kontext hoher Pfadwechselkosten eine besondere Herausforderung dar.

- Der rechtliche Rahmen führt zu niedrigen Preisen für fossile Energie und ist in Bezug auf die Vorschriften, die den Wandel fördern sollen, von Inkonsistenz und einem fast komplett fehlenden Vollzug geprägt. Die Anreizwirkung des Rechtsrahmens für den Pfadwechsel ist noch sehr begrenzt.

\subsubsection{Rohstoffversorgung}

Der Rohstoffverbrauch spiegelt die Entwicklung von Produktion und Konsum wider. Pfadabhängigkeiten, die einer Rohstoffwende entgegenstehen, sind damit grundsätzlich tief eingebettet in die Entwicklung der Industriegesellschaft und deren strukturellen Wandel, der mit den Begriffen Dienstleistungs- und Informationsgesellschaft gekennzeichnet werden kann. Globalisierungsprozesse im 
Sinne von grenzüberschreitender Produktion und Handel und weltweit sich vernetzenden Wertschöpfungsketten prägen die Pfadabhängigkeiten mit Blick auf die Rohstoffversorgung. Historisch betrachtet vollzog sich im Zuge der industriellen Revolution ein Wandel vom Verbrauch nachwachsender Rohstoffe hin zur Nutzung von Erdöl und Kohle (Exner 2016). Im Jahr 1859 wurde die erste Erdölraffinerie errichtet. In den Folgejahren wurden mehr Raffinerien gebaut, die Preise für Erdöl sanken. Petroleum und andere Öle fanden unter anderem als Leuchtmittel Verwendung. Während Erdöl nach der Erfindung des elektrischen Lichts zwischenzeitlich an Attraktivität verlor, gewann es bald wieder als Fahrzeugtreibstoff (Benzin) an Bedeutung. Zudem bildete Erdöl die Basis für die industrielle Kunststoffproduktion und die Herstellung anderer Produkte der Chemieindustrie. Zugleich stiegen der Bedarf und die Produktion von Stahl und Eisen (z. B. für Schiffe, Eisenbahnen, Gebäude, Maschinen). Während für vorindustrielle Zeiten um das Jahr 1700 von einer Produktion von etwa 300.000 Tonnen ausgegangen wird, lag diese im Jahr 1850 bereits bei etwa 12 Millionen Tonnen. Im Jahr 1980 betrug das Volumen ca. 1,2 Milliarden Tonnen, und bis zum Jahr 2005 kam es zu einem weiteren Anstieg auf über 1,9 Milliarden Tonnen (Exner 2016; Ökosystem Erde 2017).

Im Zuge dieser Entwicklungen haben sich seit der Industrialisierung koevolutiv in wechselseitiger Beeinflussung (teilweise inkrementell, teilweise schubartig) Pfadabhängigkeiten herausgebildet. In den vier Transformationsfeldstudien (Behrendt 2017a, 2017b, 2017c; Odenbach et al. 2017) wurden 48 Pfadabhängigkeiten gefunden.

Tabelle 7: Anzahl an Pfadabhängigkeiten in der Rohstoffversorgung nach Stärke und Art

\begin{tabular}{|c|c|c|c|c|c|c|}
\hline \multirow{2}{*}{$\begin{array}{l}\text { Stärke der } \\
\text { Pfadabhängigkeit }\end{array}$} & \multicolumn{6}{|c|}{ Art der Pfadabhängigkeit } \\
\hline & Rechtlich & Ökonomisch & Technisch & Organisational & Nutzerbezogen & Summe \\
\hline stark & 6 & 7 & 13 & 5 & 5 & 36 \\
\hline $\begin{array}{l}\text { mittel und } \\
\text { schwach }\end{array}$ & 3 & 2 & 4 & 1 & 2 & 12 \\
\hline Summe & 9 & 9 & 17 & 6 & 7 & 48 \\
\hline
\end{tabular}

Quelle: Borderstep Institut für Innovation und Nachhaltigkeit

Es dominieren deutlich technologische Pfadabhängigkeiten, gefolgt von rechtlichen und ökonomischen. Auch was die Stärke betrifft, überwiegen technologische Pfadabhängigkeiten, was darauf hinweist, dass es sich um vielstufige und vielfach verzweigte Prozessketten und Infrastrukturen handelt, die sich nur schwer transformieren lassen.

Jenseits dieser allgemeinen Betrachtung ist festzustellen, dass der Lock-in jeweils spezifisch für Rohstoffe und die damit verbundenen Rohstoffregimes ist, die sich herausgebildet haben. Öl ist nicht gleich Kupfer. Die Rohstoffregimes und 
die sich in ihnen konstituierenden Pfadabhängigkeiten sowie die damit verbundenen Möglichkeiten für einen Pfadwechsel unterscheiden sich stark.

\section{Massebasiertes Recycling}

Der Lock-in in ein Recyclingsystem, das wesentlich auf Masseströme ausgerichtet ist, ist auf ein Zusammenspiel verschiedener Faktoren zurückzuführen. Seit Mitte des letzten Jahrhunderts wurde zunächst eine geordnete, nachgeschaltete Abfallwirtschaft aufgebaut, die sich sukzessive zu einer verzweigten Entsorgungsund Verwertungswirtschaft entwickelte. Stoffliche und thermische Verwertung dienten zunächst vor allem der unmittelbaren Gefahrenabwehr und der Reduktion der Restabfallmengen angesichts befürchteter Entsorgungsnotstände. Das Abfallwirtschaftsgesetz (1980er-Jahre) markiert mit dem Konzept der Kreislaufwirtschaft den Übergang zum integrierten Umwelt- und Ressourcenschutz, blieb aber bis heute im Wesentlichen massebasiert. Unter den Technologiemetallen wurde lediglich das Recycling von Edelmetallen und einigen Sondermetallen forciert. Bei den meisten anderen Technologiemetallen fehlten die Preisanreize für ein Recycling. Hinzu kamen die geringen Materialmengen für eine Verwertung. Erst in den letzten Jahren sind viele der Technologien für ein Recycling mengenrelevant geworden oder werden dies in Zukunft sein (z. B. Photovoltaik, Li-IonenBatterien).

Nicht zuletzt spielt die fehlende Bereitschaft der Wirtschaft, in eine Sammelund Aufarbeitungslogistik und Recyclingverfahren zu investieren, eine Rolle. Dies hat sich auch durch den zeitweisen Rohstoffboom nicht wesentlich geändert. Insbesondere die seither hohen Preisvolatilitäten an den Primärrohstoffmärkten stellen ein Risiko für die Recyclingwirtschaft dar. Eine wesentliche Variable für Preisniveauprognosen sind staatliche Markteingriffe und oligopolartige Angebotssituationen (Erdmann et al. 2011). Beide Faktoren sprechen für volatile Preise, was Investitionen in Techniken und Anlagen zur Verwertung von Technologiemetallen erschwert. Hinzu kommt eine zunehmende dissipative Verwendung von Technologiemetallen in Endprodukten, die eine Erhöhung des Rückgewinnungsgrades erschwert. Weitere Pfadabhängigkeiten bestehen in den vorhandenen Sammel- und Logistik(infra)strukturen, die sich in Jahrzehnten entwickelt und etabliert haben. Die Aufarbeitungsverfahren für Altprodukte und Schrotte sind heute noch weitestgehend auf Massenströme ausgelegt. Die Demontage dient in erster Linie der Schadstoffentfrachtung, nur in geringem Maße werden verwertbare Komponenten freigelegt. Begünstigt wird die Ausrichtung auf eine Verwertung der Massenströme durch die WEEE-Direktive bzw. durch nationale Gesetze zur Rücknahme von Altgeräten und Produkten wie das Elektro-Gesetz, die massebasierte Verwertungsquoten vorgeben. 
Abbildung 19: Lock-in in ein massebasiertes Recycling

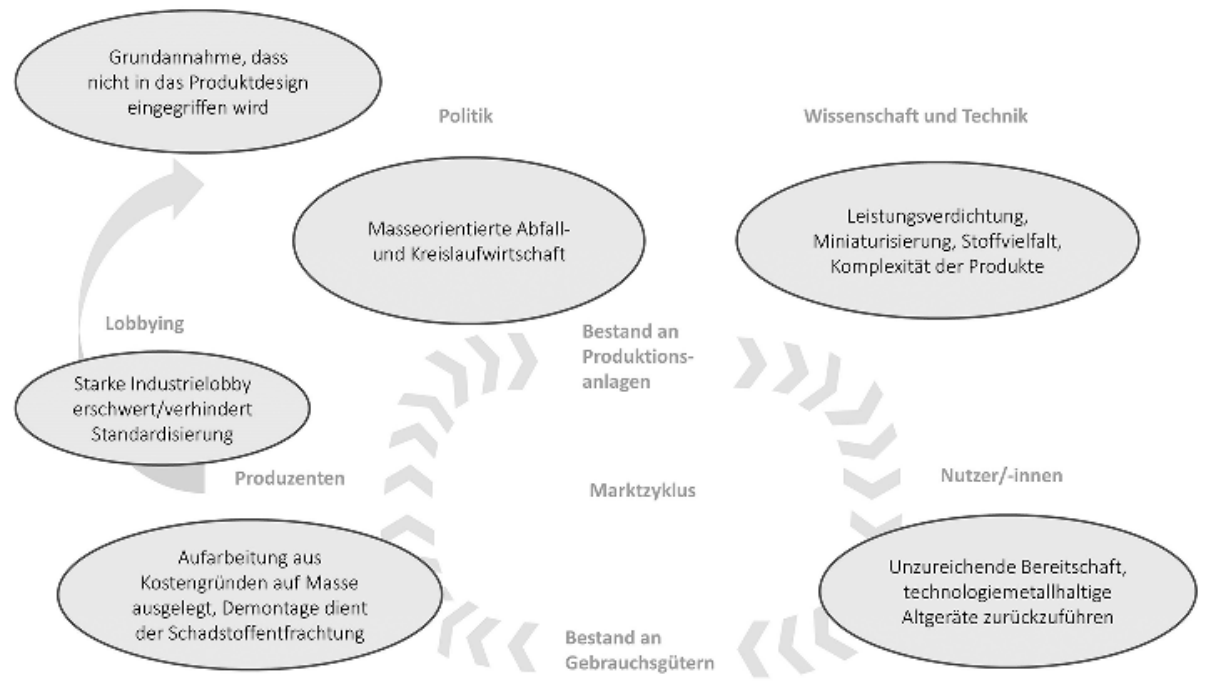

Quelle: Eigene Darstellung, Borderstep Institut für Innovation und Nachhaltigkeit.

Die Transformation erfordert das Zusammenspiel dreier Ebenen: Erstens ist ein recyclinggerechtes Produktdesign erforderlich, zweitens ist eine Erfassung der Altprodukte zu gewährleisten und drittens ist eine angepasste Aufarbeitung der Produkte erforderlich, sodass möglichst wenig Technologiemetalle verloren gehen. Da Technologie- und Marktentwicklungen die Dissipation tendenziell erhöhen, sind rechtliche Rahmenbedingungen zu entwickeln, die Anreize für das Recycling von Technologiemetallen schaffen. Produktkennzeichnungen und Informationsmaßnahmen sind nicht ausreichend, vielmehr sind Mindestanforderungen an die Recyclingfähigkeit verpflichtend vorzuschreiben. Über massebasierten Quoten hinaus sollten zusätzlich für Technologiemetalle spezifische Verwertungsraten und -anforderungen definiert werden. Zwar wird die Berücksichtigung rohstofflicher Anforderungen generell als notwendig erachtet, erste Schritte zur Erweiterung der vor allem auf die Energieeffizienz ausgerichteten EU-Ökodesign-Richtlinie sind erfolgt (Progress II), werden aber tatsächlich bis dato nur sehr zögerlich aufgegriffen.

\section{Erdölbasierte Chemieindustrie}

Bei Erdöl, das heute die Rohstoffbasis der Chemieindustrie dominiert, ist der Lock-in ungefähr Mitte des 20. Jahrhunderts zu verorten. Erdöl hat seinerzeit die Kohle abgelöst, da es kostengünstig war und zudem neue Möglichkeiten zur Herstellung von Chemikalien bot. Die Chemieindustrie nutzt heute kostengünstige Stoffströme aus der erdölverarbeitenden Industrie, um daraus Basischemikalien 
und Endprodukte herzustellen. Im Laufe von Jahrzehnten sind vielstufige und verzweigte Wertschöpfungsketten entstanden, in denen über komplexe und sehr unterschiedliche Prozesse mehr als 100.000 Produkte hergestellt werden. Aufgrund dieser Pfadabhängigkeit kann die Chemieindustrie die Herstellung von Basischemikalien nicht einfach umstellen. Sie würde einen wesentlichen Wettbewerbsvorteil aufgeben, nämlich die Verbundproduktion, das heißt aufeinander abgestimmte, hocheffiziente Produktionsverbünde, in denen der Stoff einer Produktionsanlage zum Grundstoff einer anderen wird.

Abbildung 20: Lock-in in eine erdölbasierte Chemieindustrie

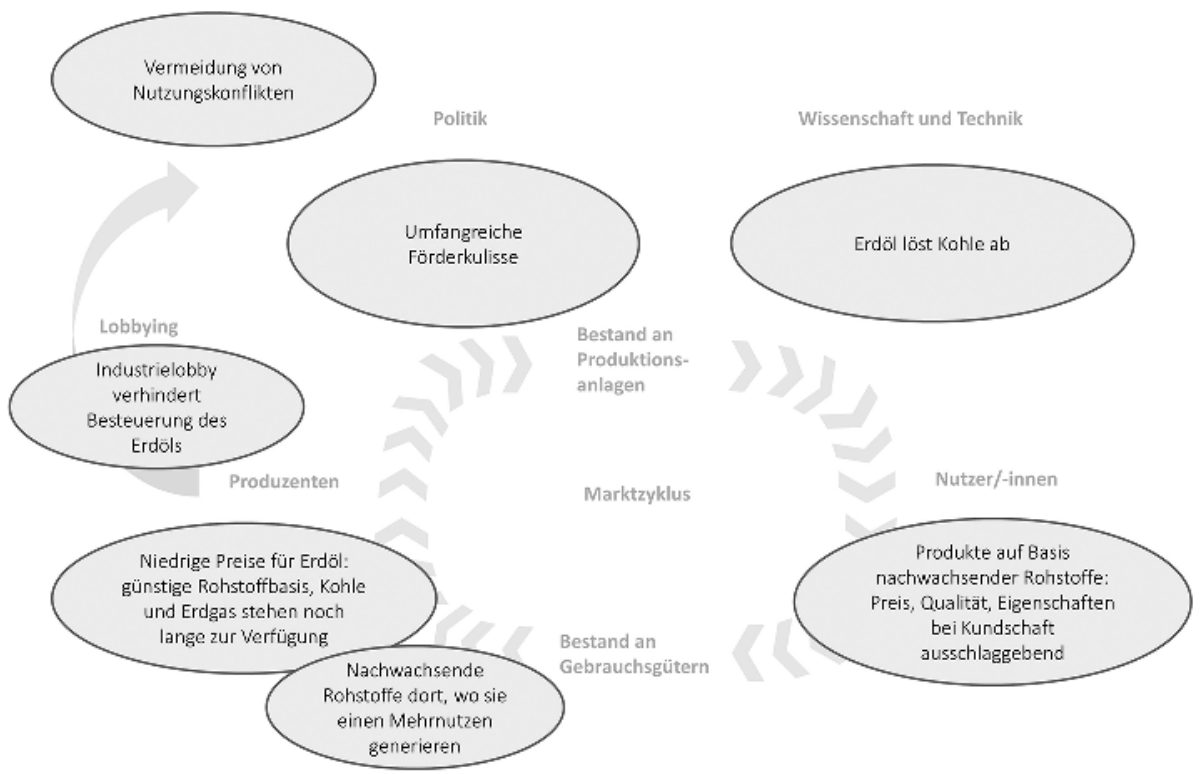

Quelle: Eigene Darstellung, Borderstep Institut für Innovation und Nachhaltigkeit.

Im Fokus zum feedstock change der Chemieindustrie stehen Effizienzsteigerungen, die verstärkte Nutzung von Non-Food-Biomasse, die Optimierung der Verarbeitungsprozesse und die Entwicklung neuer Produkte (Behrendt 2017a). Für den Übergang zu nachwachsenden Rohstoffen sind neue Synthesestrategien und Herstellungsprozesse erforderlich, die es erlauben, chemische Produkte aus heterogener Biomasse herzustellen und in bestehende Prozesse kaskadenförmig $\mathrm{zu}$ integrieren. Eine Schlüsselrolle nimmt dabei die industrielle Biotechnologie ein. In Anbetracht der enormen wirtschaftlichen Stärken und hervorragenden Strukturen ist aber auf absehbare Zeit kein feedstock change wahrscheinlich; vielmehr ist zu erwarten, dass biologische Verfahren dort zum Einsatz kommen, wo die Produkte sich durch verbesserte Eigenschaften im Markt durchsetzen oder 
wirtschaftlicher herzustellen sind als mit herkömmlichen chemischen Synthesen. Selbst langfristig ist bei steigenden Erdölpreisen nicht unbedingt mit einem Aufbrechen der Pfadabhängigkeiten zugunsten eines Wechsels hin zu nachwachsenden Rohstoffen zu rechnen. Einerseits stehen noch lange Kohle und Erdgas als alternative Rohstoffquellen für Erdöl zu Verfügung, anderseits ist davon auszugehen, dass Verfügbarkeit und Preis von nachwachsenden Rohstoffen aufgrund der Nutzungskonkurrenzen limitierende Faktoren für einen umfassenden feedstock change der chemischen Industrie sein werden. Dies betrifft insbesondere die Knappheit des Angebots an Biomasse, das nur unter Inkaufnahme von ökologischen Nebeneffekten stark ausgeweitet werden kann, wie von (Behrendt 2017a) herausgearbeitet wurde.

\section{Nachsorgende Abwasserreinigung}

Ergänzend wurden die Pfadabhängigkeiten bei synthetischen organischen Substanzen untersucht, die in Folge ihrer Freisetzung als sogenannte Mikroschadstoffe die Gewässer belasten (Behrendt 2017b). Es handelt sich um eine Vielzahl von Chemikalien und Arzneistoffen, auch Mikroplastik gehört dazu. Der Lock-in vollzog sich im Laufe des letzten Jahrhunderts als Reaktion auf zunehmende Umweltprobleme im Bereich der Gewässer. Im Lauf der Zeit wurde eine umfangreiche Infrastruktur aus Kanalisationen und Kläranlagen aufgebaut, die sich mit dem Stand der Technik entwickelt hat (mechanische, biologische und chemische Verfahren). Auf diese Weise konnte der Eintrag sauerstoffzehrender Substanzen und auch die Einträge der Nährstoffe aus dichter besiedelten Gebieten in Gewässer deutlich reduziert werden. Seit den 9oer-Jahren werden zunehmend Belastungen durch Arzneimittel, Industriechemikalien und Pestizide festgestellt. Entsprechend der Grundlogik der nachsorgenden Abwasserreinigung wurden Techniken entwickelt, mit denen Kläranlagen um eine vierte Reinigungsstufe nachgerüstet werden können, um Mikroschadstoffe zu eliminieren. Sie werden großtechnisch bereits in verschiedenen Kläranlagen erprobt. Dabei zeigt sich, dass ein breites Spektrum an Mikroschadstoffen in hohem Umfang aus dem Abwasser entfernt werden kann, allerdings nicht vollständig.

Ein Pfadwechsel bedeutet, dass neben der Aufrüstung von Kläranlagen mit einer vierten Reinigungsstufe (end of pipe) den quellenorientierten Maßnahmen ein höheres Gewicht eingeräumt wird. Dazu gehören die Substitution von Stoffen, die Veränderung der Produkte, die Reduzierung der Anwendungen in Industrie, Gewerbe und Haushalten sowie ein verantwortungsvollerer Umgang beim Gebrauch und bei der Entsorgung von Produkten. Die Potenziale hierfür sind jeweils stoffspezifisch.

Quer zu den Fallstudien wurde die Digitalisierung und Automatisierung der Produktion untersucht (Odenbach et al. 2017). Unter dem Stichwort "Industrie 4.0 « werden die Möglichkeiten einer stärkeren Verschmelzung von physischer und 
Abbildung 21: Lock-in in eine nachsorgende Abwasserreinigung

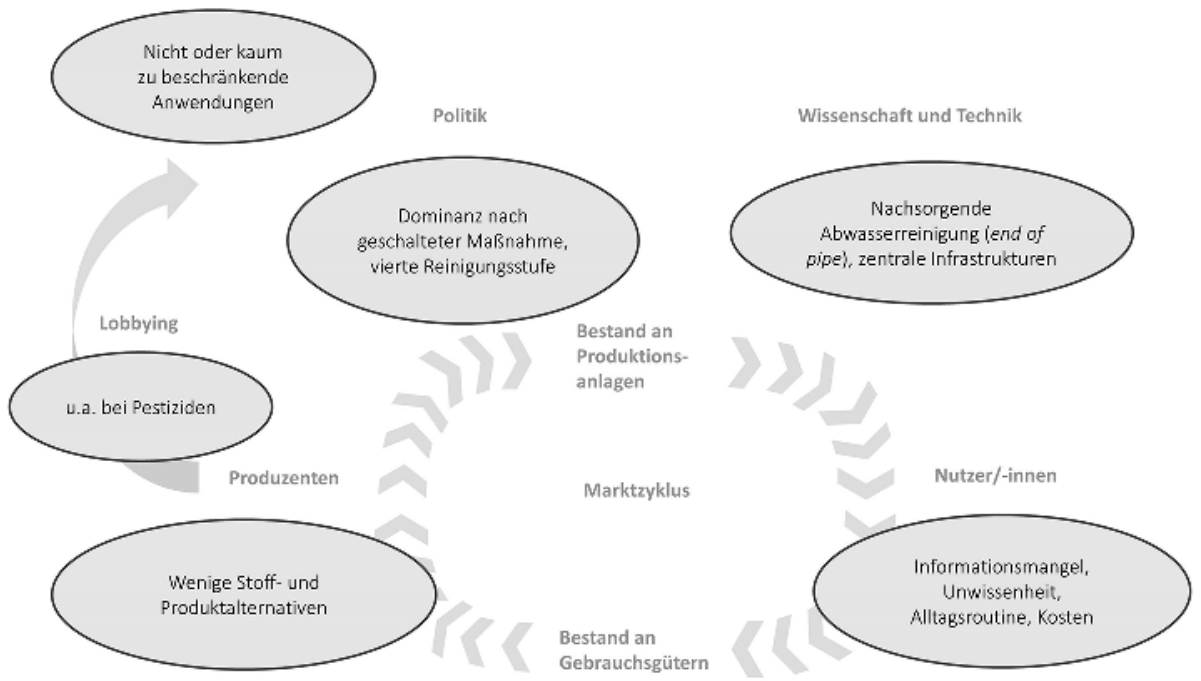

Quelle: Eigene Darstellung, Borderstep Institut für Innovation und Nachhaltigkeit.

virtueller Welt zusammengefasst. Die Maschine-zu-Maschine-Kommunikation, ad-hoc-vernetzte und umgebungssensitive Mikrosysteme können eine Effizienzsteigerung in Produktion, Logistik und Service bewirken. Am Ende der Kette sind Demontagefabriken denkbar, die automatisch Produkte zerlegen und damit händische Prozesse ablösen. Hinzu kommt, dass eine so weitgehende Vision der Durchdringung der Wertschöpfungsketten mit digitalen Komponenten, die immer und überall eingeschaltet und weitgehend drahtlos vernetzt sind, neue Produktionskonzepte möglich machen. Solche neuen Konzepte sind beispielsweise der 3D-Druck oder dezentrale Produktionsformen. Grundsätzlich sind Pfadwechsel vorstellbar. Allerdings sind die Effekte höchst ambivalent. Auffällig ist, dass der Idee einer »Industrie 4.0« ein hohes Energie- und Rohstoffeffizienzpotenzial attestiert wird, aber mit der Debatte um eine Green Economy kaum verknüpft, geschweige denn mit praktischen Projekten unterlegt ist.

\subsection{Pfadabhängigkeiten und Transformationansätze}

Die Transformationsansätze im Themenfeld Rohstoffe lassen sich anhand des Produktlebenszyklus kategorisieren. Eine Rolle spielen dabei die Auswahl der genutzten Rohstoffe und die Art und Weise der Weiterverarbeitung in der Produktion und Anwendung, aber auch die kontrollierte Entsorgung sowie ein anschließendes Recycling. 
- Rohstoffauswahl bzw. Produktgestaltung: Ansätze der Transformation bilden im Themenfeld feedstock change die nachhaltige Erzeugung und Bereitstellung nachwachsender Rohstoffe, im Bereich Metalle das Ökodesign unter dem Aspekt der leichten Demontage sowie in Bezug auf die Vermeidung von Mikroschadstoffen die Stoffsubstitutionen.

- Produktion und Anwendung: Die Transformationsansätze zielen zum einen auf die Verbesserung bestehender Produktionstechniken, zum anderen auf die Erschließung neuer ab: Sie umfassen die Steigerung der Ressourceneffizienz durch die Möglichkeiten der Industrie 4.0, die dezentrale Produktion, integrierte chemische Prozesse und neue Wertschöpfungsketten sowie die Optimierung bestehender und neuer Bioproduktionssysteme im Bereich feedstock change.

- Entsorgung und Recycling: Die gezielte Rückführung bzw. Sammlung von nicht mehr gebrauchten Produkten ist eine Vorbedingung des Recyclings. Im Bereich Mikroschadstoffe kann dies durch die geregelte Entsorgung schadstoffhaltiger Produkte geschehen, in der Industrie spricht man von Retro-Logistik. Recycling-Partnerschaften könnten der globalen Verantwortung Rechnung tragen. Am Ende des Produktionszyklus bilden Pre-Shreddering-Verfahren, z. B. automatische Demontagefabriken (smart disassembly factories), Ansätze in Richtung einer Green Economy.

Das etablierte, nicht-nachhaltige System der Rohstoffwirtschaft wird von einigen stark wirkenden und zu einem großen Teil technischen Pfadabhängigkeiten stabilisiert. Im Themenfeld Rohstoffe sind nur wenig übergreifende und vergleichsweise viele auf die einzelnen Problemfelder wirkenden Pfadabhängigkeiten auszumachen. Eine übergreifend wirksame Pfadabhängigkeit stellt die Vorstellung dar, dass billige Rohstoffe für die Wirtschaft notwendig sind.

In Bezug auf Rohstoffauswahl- und Produktgestaltung zeigen sich folgende Pfadabhängigkeiten:

Ökodesign Technologiemetalle: Die dissipative Verwendung von Technologiemetallen an vielen Stellen im Produkt ist etabliert; Käuferinnen und Käufer legen wenig Wert auf die Umweltverträglichkeit als Produkteigenschaft.

Produktgestaltung Mikroschadstoffe: Das Vorhandensein heterogener Anwendungskontexte für eine Vielzahl von Stoffen sorgt für eine große Komplexität; Stoffsubstitutionen sind nur eingeschränkt möglich, zudem können diese mit hohen Kosten verbunden sein. Die Stoffregelungskompetenzen liegen bei der EU. Vorkonfektionierte Packungsgrößen bei Medikamenten sorgen von vornherein für Überschüsse. 
Nutzung nachwachsender Rohstoffe für den feedstock change: Die Preise für Erdöl sind niedrig, während die Preisentwicklung für erneuerbare Rohstoffe volatil und schwer langfristig planbar ist, was auch auf die Konkurrenz mit anderen Nutzungsansprüchen zurückzuführen ist; auch das EEG sorgt für die Verteuerung von Biomasse. Weiter ist die Verfügbarkeit nachwachsender Rohstoffe begrenzt.

Zahlreiche Pfadabhängigkeiten hemmen auch die Veränderung der Rohstoffwirtschaft auf der Ebene der Produktion, allerdings recht selektiv.

Mit Blick auf die Idee der »Industrie 4.0« konnten z. B. keine spezifischen Pfadabhängigkeiten im Kontext der Realisierung von Industrie 4.0 in etablierten Wertschöpfungsketten ausgemacht werden.

Verknüpft sich der Gedanke von »Industrie 4.0« jedoch mit der Idee der »dezentralen Produktion« auf Basis von Open-Source-Know-how, hemmen zahlreiche Pfadabhängigkeiten die Entwicklung:

- Rechtlich sind viele Fragen der industrial security (z. B. des Datenschutzes) nicht geklärt. Auch der Anspruch auf den Schutz firmeninternen Know-hows steht Kooperationen auf Open-Source-Basis entgegen, denn Schutzrechte können in dezentralen Produktionsstrukturen auf Open-Source-Basis nicht wahrgenommen werden. Bei Kooperationen kleiner vernetzter ökonomischer Einheiten liegen für den letztendlichen Inverkehrbringer der Produkte spezifische Haftungs- und Gewährleistungspflichten vor. Auch hemmt der Mangel an offenen Standards ökonomisch nicht-integrierte Produktionsketten.

- Die Entwicklung dezentraler Produktionsstrukturen ist zudem kostenintensiv. Mit Blick auf die Unsicherheit des Produktabsatzes bleibt damit eine hohe Unsicherheit, wann Investitionen in eine dezentrale Produktion rentabel sind. Vorbedingung für eine dezentrale Produktion ist weiter ein flächendeckendes leistungsfähiges (Glasfaser-)Netz, welches in Deutschland gegenwärtig noch wesentliche Lücken hat.

- Sollten die Stückzahlen aus dezentraler Produktion steigen, dann ist aus der etablierten Produktion Widerstand zu erwarten. Denn hochentwickelte funktionale zentrale Produktionsstrukturen sind vorhanden und ein wachsender dezentraler Produktionssektor könnte zu Befürchtungen bzgl. eines Kompetenz- und Arbeitsplatzverlustes bei etablierten Anbietern führen.

Der feedstock change der chemischen Industrie erfordert integrierte chemische Prozesse und die Optimierung bestehender Bioproduktionssysteme und wird ebenfalls von Pfadabhängigkeiten deutlich behindert: 
- Technisch machen vielfach verzweigte Wertschöpfungsketten sowie der Mangel an »rohstofftoleranten« Prozessen Probleme. Die Verknüpfung von Einzeltechnologien zu komplexen Biotechnologien steht noch aus und es bestehen hohe Mengenschwellen für sehr effiziente Anlagen. Auch sind Produkte von Bioraffinerien oft inkompatibel mit herkömmlichen chemischen Wertschöpfungsketten.

- Weitere Pfadabhängigkeiten bestehen in den Risiken einer weitgehend neuen Bio-Produkt- und Rohstoffstrategie, der begrenzten Verfügbarkeit von Biomasse, den oft noch unklaren ökologischen Vorteilen biobasierter Produkte sowie in der Tatsache, dass Kundinnen und Kunden biomassebasierte Produkte nicht unbedingt als Vorteil sehen.

Im Bereich Entsorgung und Recycling zeigt sich bezüglich der Retro-Logistik von Technologiemetallen die auf nationale Grenzen beschränkte Regulierungskompetenz als hemmend. An einer klaren Definition zwischen gebrauchter Ware und Schrott mangelt es, sodass Exporte von Altprodukten oft in einem rechtlichen Graubereich stattfinden. Die getrennte Erfassung und Sammlung von Produkten mit Technologiemetallen funktioniert kaum.

Die Rückgewinnung von Technologiemetallen durch Demontage und Recycling wird gehemmt durch technische Pfadabhängigkeiten, da z. B. die Zugabe von "Gewürzmetallen« die Stoffvielfalt erhöht und die dominierende Shredder-Technologie die vielen unterschiedlichen Materialien nicht separieren kann. Metallkombinationen in der Produktion passen manchmal nicht zu etablierten Scheideverfahren. Mengenquoten setzen falsche Anreize im Recycling, und Anreize für (hohe) Investitionen in Recyclinganlagen sind durch niedrige und volatile Rohstoffpreise und aufgrund unklarer Anlagenrentabilität kaum gegeben.

Für Mikroschadstoffe sind Kläranlagen zentrale Orte der Sammlung und Eliminierung. Aufgrund dieser zentralen und kontrollierten Reinigung von Abwässern bietet sich eine zusätzliche vierte oder fünfte Reinigungsstufe an. Aber die Forschung zu Abwasserreinigungstechnologien ist weiter als die Anwendung, hier gilt es eine Lücke zu schließen. Rechtliche Vorschriften fordern zwar Gewässerqualität, sind jedoch oft maßnahmenoffen. Auch die Wasserrahmenrichtlinie begrenzt die Einleitung von lediglich 45 prioritären Stoffen.

\subsection{Fazit}

Die Analyse der Pfadabhängigkeiten rund um die Rohstoffversorgung zeigt eine starke Fragmentierung der Rohstoffwirtschaft mit ihren zahlreichen Materialien und Produkten als zentrales Problem:

- Die Zahl der in Produkten eingesetzten Elemente hat sich in den letzten Jahrzehnten stark erhöht. Das Recyclingsystem ist aber immer noch auf Mengen- 
rohstoffe wie Stahl, Papier und Glas ausgerichtet. Die vorhandene Technologie (z. B. Shredderanlagen) stabilisiert die Ausrichtung auf Massenrohstoffe.

- Das Recycling von Kleinmengenrohstoffen wie Technologiemetallen wird dadurch erschwert, dass diese verteilt in sehr vielen Produkten eingesetzt werden. Ökodesign, welches versucht, die Stoffvielfalt zu reduzieren und bestimmte Stoffe so einzusetzen, dass sie einfach demontierbar sind (z. B. durch das Clustern von Elektronikkomponenten), ist mit Blick auf die Produktvielfalt und deren weltweite Herkunft nur schwer umzusetzen.

- Der chemischen Industrie stehen die für einen feedstock change erforderlichen Mengen nachwachsender Rohstoffe nicht zur Verfügung, da diese im Lebensmittel- und Energiesektor eingesetzt werden.

\subsubsection{Pfadabhängigkeiten: Typen und Zusammenhänge}

In diesem Kapitel werden die in der Vorstudie (Clausen und Fichter 2016) systematisierten sowie die in den Transformationsfeldstudien identifizierten Pfadabhängigkeiten im Überblick betrachtet. Es werden nach einem kurzen Überblick über Zahl und Stärke der gefundenen Pfadabhängigkeiten zunächst in einer Detailanalyse die bereits aufgestellte Typologie von Pfadabhängigkeiten auf empirischer Basis weiter verfeinert und danach die Netzwerkbeziehungen von Pfadabhängigkeiten analysiert.

\section{Abbildung 22: Anzahl und Typ von Pfadabhängigkeiten in den vier Wendethemen}

25

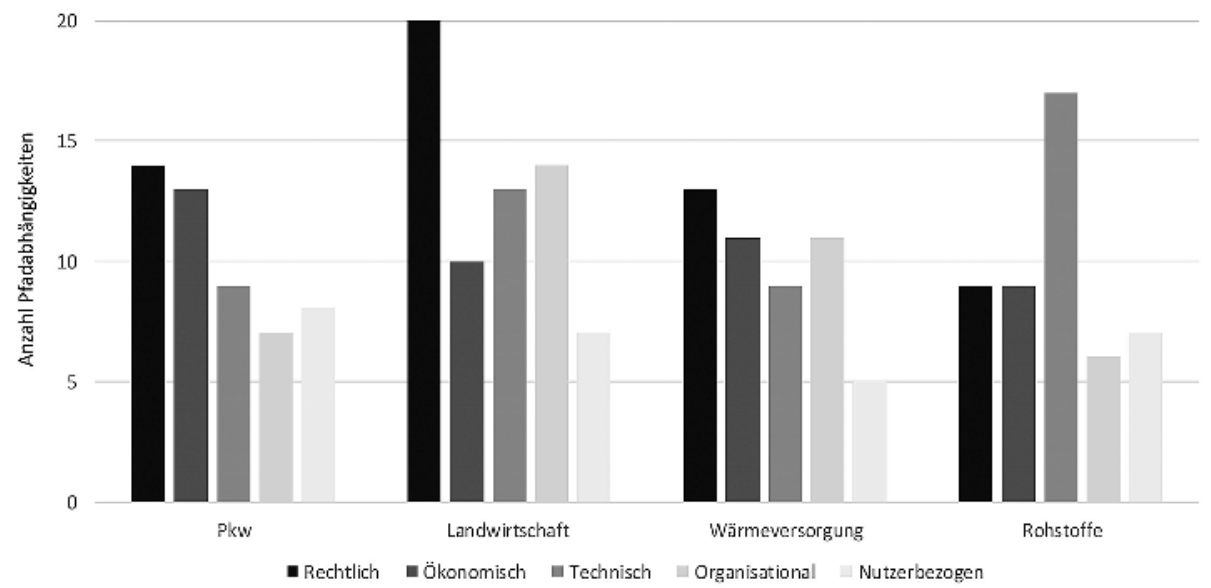

Quelle: Eigene Darstellung, Borderstep Institut für Innovation und Nachhaltigkeit. 
Der Überblick über die Typen von Pfadabhängigkeiten in den vier Wendethemen lässt erkennen, dass in der Landwirtschaft rechtliche Pfadabhängigkeiten in großer Zahl vorhanden sind und auch technische und organisationale das Regime stabilisieren. In der Rohstoffwirtschaft dagegen wurden besonders viele technische Pfadabhängigkeiten identifiziert. Die Verteilung der Pfadabhängigkeiten rund um die Pkw-Nutzung und in der Wärmeversorgung dagegen lässt auf einen technisch-ökonomischen Komplex schließen, der rechtlich gut abgesichert ist.

Von den insgesamt 212 identifizierten Pfadabhängigkeiten wurden 61 (29 Prozent) als »schwach oder mittelstark« ausgeprägt, 151 (71 Prozent) als »starke« Pfadabhängigkeiten charakterisiert. Die Verteilung starker und schwacher Pfadabhängigkeiten über die Wendethemen ist weitgehend ausgeglichen.

Abbildung 23: Anzahl an starken sowie mittleren und schwachen Pfadabhängigkeiten in den vier Wendethemen

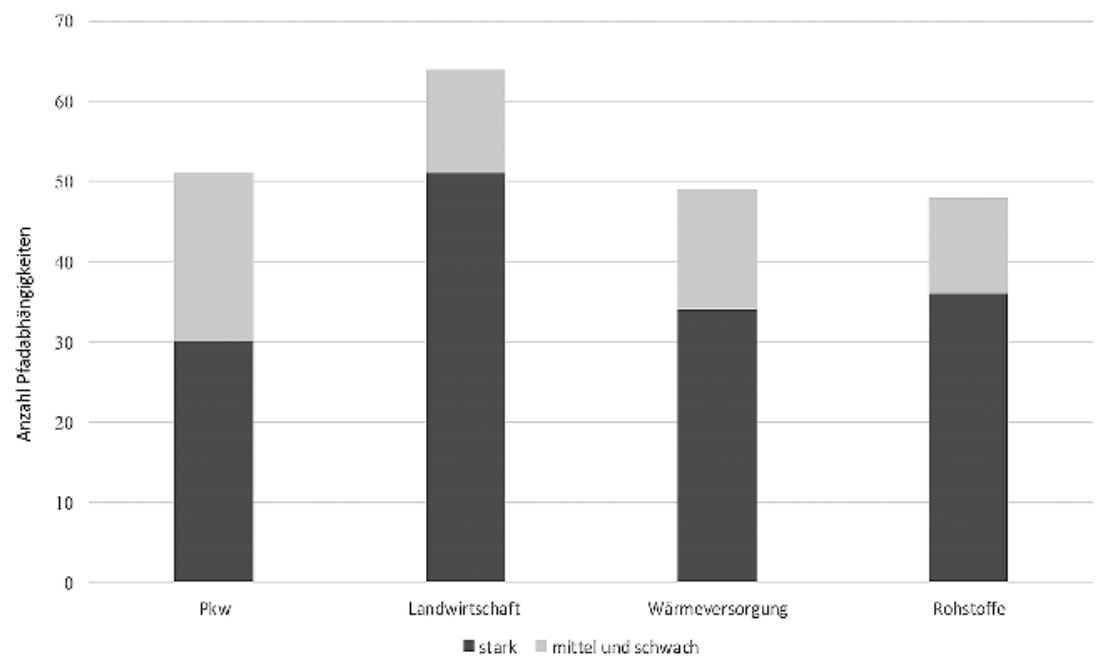

Quelle: Eigene Darstellung, Borderstep Institut für Innovation und Nachhaltigkeit.

Bei der Verteilung starker und schwacher Pfadabhängigkeiten anhand der Typologie fällt auf, dass besonders bei den ökonomischen und technischen Pfadabhängigkeiten ein vergleichsweise hoher Anteil als »nicht so stark« eingestuft wurde. 
Abbildung 24: Anzahl an starken sowie mittleren und schwachen Pfadabhängigkeiten nach Typ

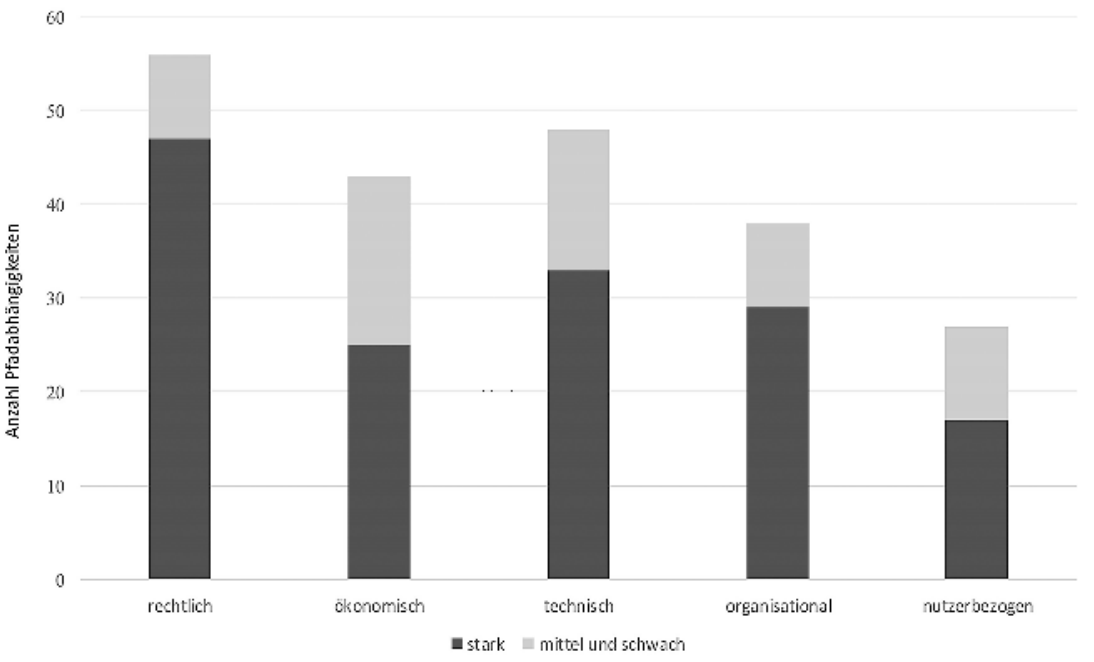

Quelle: Eigene Darstellung, Borderstep Institut für Innovation und Nachhaltigkeit.

\subsubsection{Typen von Pfadabhängigkeiten}

In diesem Abschnitt werden die Pfadabhängigkeiten vor dem Hintergrund der empirischen Erhebung typisiert und dabei das in der Vorstudie aufgestellte System von fünf Typen (Clausen und Fichter 2016) weiter detailliert.

\subsection{Rechtliche Pfadabhängigkeiten}

Die Analyse der 15 Transformationsfelder förderte Hinweise auf 56 rechtliche Pfadabhängigkeiten zutage. Der Fokus der Analyse lag dabei auf Pfadabhängigkeiten, die einerseits das vorherrschende System stabilisieren und dabei andererseits die Transformation des Systems verhindern. Im Ergebnis lassen die im Feld »rechtliche Pfadabhängigkeiten« gefundenen Strukturen auf sehr erfolgreiche Tätigkeiten von Lobbyisten im politischen Prozess schließen. 
Abbildung 25: Überblick über Typen rechtlicher Pfadabhängigkeiten und Anzahl der jeweils gefundenen Beispiele

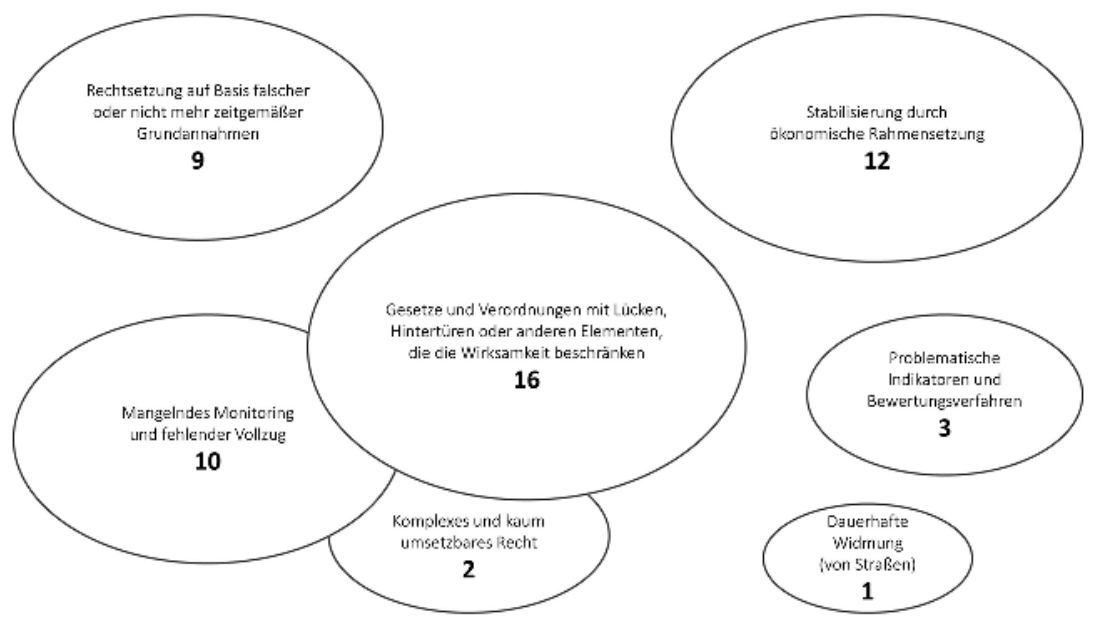

Quelle: Eigene Darstellung, Borderstep Institut für Innovation und Nachhaltigkeit.

Die Basis der rechtlichen Pfadabhängigkeiten bilden falsche oder - angesichts der ökologischen Herausforderung - nicht mehr zeitgemäße Grundannahmen, auf deren Grundlage Recht entsteht. Gefunden wurde hierfür eine Reihe von Beispielen:

- Das Primat des Freihandels über das Umweltrecht, welches gerade im internationalen Warenverkehr der Durchsetzung ökologischer Ziele Grenzen setzt

- Die grundlegende Ausnahme der Landwirtschaft aus dem Umweltrecht, die der Sicherstellung der Lebensmittelproduktion dienen soll und dabei doch langfristig zur Zerstörung der Grundlagen ebendieser Lebensmittelproduktion führt

- Der Grundsatz der Technologieneutralität, der die Förderung konkreter Technologien erschwert oder weniger wirksam macht

Im Zentrum des gegenwärtig an viel zu vielen Stellen wenig wirksamen Umweltrechts fanden wir Gesetze und Verordnungen mit Lücken, Hintertüren oder anderen Elementen, die ihre Wirksamkeit beschränken. Oft ist auch ein fehlendes Monitoring oder ein unzureichender Vollzug Hauptgrund der Unwirksamkeit. Beispielhaft sind aufzuführen:

- Maßnahmenoffene Vorschriften

- Fehlende Zielklarheit der Vorschriften

- Eingebaute Hintertüren wie beim EEWärmeG, welches im Ergebnis überwiegend zu effizienzsteigernden Maßnahmen führt 
- Vorschriften, die aufgrund ihrer Komplexität kaum umsetzbar und noch weniger vollziehbar sind, z. B. im Baurecht, aber außerhalb unserer Untersuchungen letztlich auch im Emissionshandel

- Fehlende Vorschriften zum Monitoring

- Fehlende Vorschriften oder Mittel zur Organisation eines wirksamen Vollzugs

Ein weiteres wesentliches rechtliches Instrument zur Verhinderung von Wandel besteht im Steuer- und Abgaberecht, das auf verschiedene Weise zur Stabilisierung des Systems beitragen kann:

- Niedrige Steuern auf fossile Energieträge und auf Rohstoffe,

- Ein Förderrahmen der Gemeinsamen Agrarpolitik, der den industriellen und umweltbelastenden Landbau bevorzugt und ökologische Ziele nicht wirksam verfolgt.

Abgerundet wird das Bild eines zur Transformation weitgehend ungeeigneten Rechtsrahmens durch eine Reihe von Vorschriften, mit denen zur Messung des Erfolgs ungeeignete Indikatoren vorgeschrieben werden. So setzen die Mengenquoten im Recycling keine Anreize zur Kreislaufführung von nur in kleinen Mengen enthaltenen Technologiemetallen. Andere Vorschriften fehlen ganz, z. B. eine Verpflichtung der Kommunen, kommunale Wärmeversorgungspläne zu erstellen.

\subsubsection{2 Ökonomische Pfadabhängigkeiten}

In 15 Transformationsfeldern fanden wir 43 ökonomische Pfadabhängigkeiten.

Abbildung 26: Überblick über Typen ökonomischer Pfadabhängigkeiten und Anzahl der jeweils gefundenen Beispiele

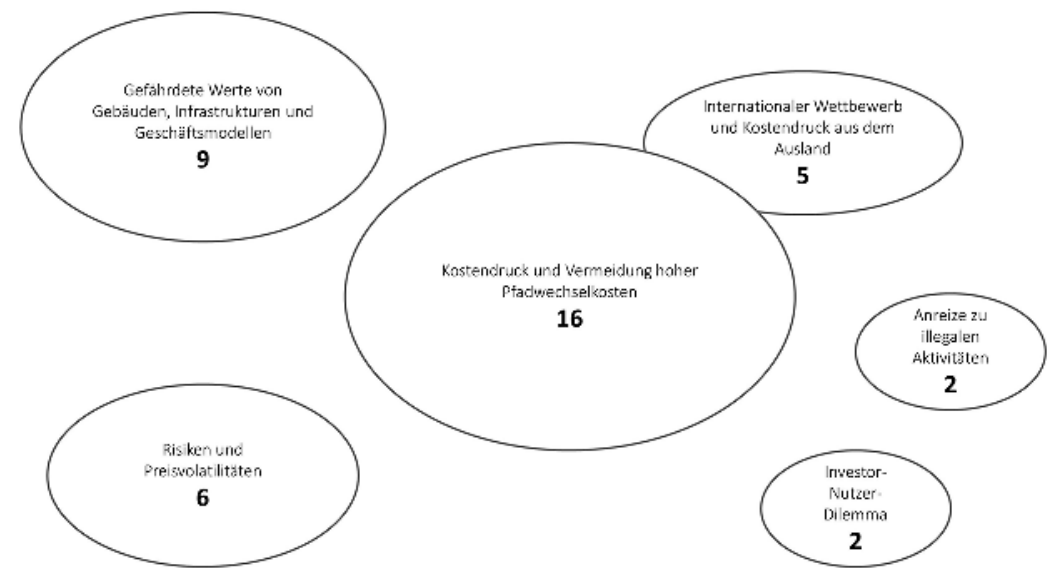

Quelle: Eigene Darstellung, Borderstep Institut für Innovation und Nachhaltigkeit. 
Im Zentrum der ökonomischen Pfadabhängigkeiten steht der Kostendruck. Gefunden wurde hierfür eine Reihe von Beispielen:

- Die (noch) bestehende faktische Unmöglichkeit, in der Anschaffung teurere regenerative Heizungstechnologien wie Wärmepumpen in einem Markt abzusetzen, der durch billige Gasthermen und billiges Gas dominiert wird

- Die gleichermaßen faktische Unmöglichkeit, die heute noch teureren Elektroautos in Ländern abzusetzen, in denen der Preisunterschied nicht über Förderungen oder steuerliche Vorteile ausgeglichen wird

- Die in der Landwirtschaft aufgrund des Kostendrucks etablierte Spirale aus preiswerter und umweltbelastender Produktion, die den Anspruch auf preiswerte Lebensmittel immer neu entstehen lässt

- Ähnliche Effekte aufgrund der internationalen Wettbewerbssituation

Wesentlich sind auch die Werte, die Industrie und Konsumenten in existierende Artefakte sowie Strukturen gesteckt haben und deren Wert sie langfristig nutzen wollen. Hierzu gehören:

- Das Eigentum der öffentlichen Hand (Straßen, Infrastrukturen) wie auch der privaten Haushalte (Häuser, Autos und Geräte) und der Industrie (Produktionsanlage und Geschäftsmodelle)

- Der Wert von etablierten Geschäftsmodellen der Unternehmen

- Der Wert von Anlageinvestitionen, z. B. kommunale Beteiligungen an fossil ausgerichteten Energieversorgern, der direkt in die politische Entscheidungsfindung zurückwirkt

Unsicherheiten und Preisvolatilitäten schaffen weitere Pfadabhängigkeiten, besonders in den Rohstoffmärkten, aber auch z. B. hinsichtlich des (wann?) kommenden Kohleausstiegs. Weiter werden bestimmte Strukturen dadurch stabilisiert, dass sie mit illegalen Wettbewerbern mithalten müssen, z. B. im Pestizidhandel und im Recycling von Elektronikkomponenten. Das Investor-Nutzer-Dilemma schließt die Aufzählung ökonomischer Pfadabhängigkeiten ab. 


\subsection{Technische Pfadabhängigkeiten}

Die Analyse ergab Hinweise auf 48 technische Pfadabhängigkeiten in den 15 Transformationsfeldern.

Abbildung 27: Überblick über Typen technischer Pfadabhängigkeiten und Anzahl der jeweils gefundenen Beispiele

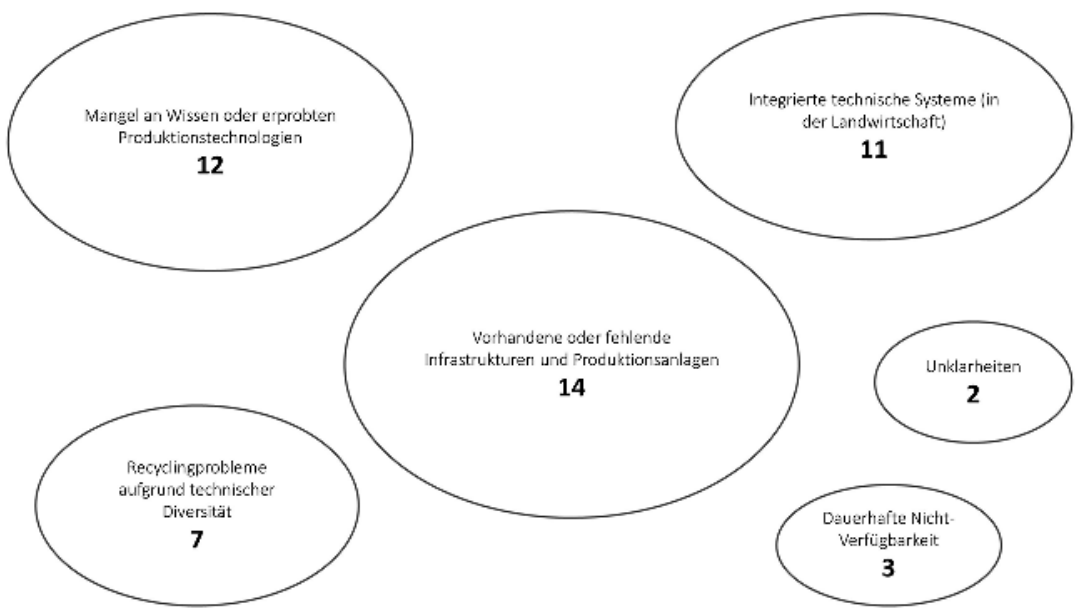

Quelle: Eigene Darstellung, Borderstep Institut für Innovation und Nachhaltigkeit.

Fundamental sind dabei die bisher wenigen technischen Unmöglichkeiten aufgrund der dauerhaften Nicht-Verfügbarkeit von Ressourcen. An zwei Stellen konnten wir entsprechende Pfadabhängigkeiten identifizieren:

- Die Unmöglichkeit, auf einer begrenzten landwirtschaftlichen Fläche Rohstoffe für die (fast unbegrenzten) Bedarfe der Lebensmittelwirtschaft, der Energiewirtschaft (nachwachsende Rohstoffe) und der chemischen Industrie (feedstock change) zu produzieren

- Die Unmöglichkeit der Nutzung von alten und standortangepassten landwirtschaftlichen Pflanzensorten und Tierrassen, wenn diese ausgestorben sind

Im Zentrum der technischen Pfadabhängigkeiten steht die normative Kraft der faktisch vorhandenen oder fehlenden Infrastrukturen und Produktionsanlagen. Beispiele sind hier:

- Die Siedlungs-, Produktions- und Versorgungsstrukturen mit ihren langen Wegen

- Die Straßen-, Schienen- und Wasserwege mit ihren Kapazitätsgrenzen 
- Die teils gut (Gasnetze), teils schlecht (Wärmenetze) ausgebauten Energieversorgungsstrukturen

- Die im internationalen Vergleich insgesamt und besonders in ländlichen Regionen schlechten digitalen Infrastrukturen

- Dominierende Industriestrukturen wie z. B. das Recyclingsystem mit seinen Großshreddern

Besonders in der Landwirtschaft sind integrierte technische Systeme von Bedeutung. Pflanzensorten sind in Kombination mit bestimmten Anforderungen an die Düngung und den Pflanzenschutz gezüchtet oder gentechnisch optimiert worden und können wirtschaftlich nur als »Gesamtsystem« genutzt werden.

Weiter wurden einige Fälle mangelnder technischer Kompetenz bzw. fehlender erprobter Produktionstechnologien identifiziert:

- In der Elektromobilität fehlt es an Wissen und Erfahrung mit diversen Technologien des elektrischen Antriebsstrangs. Auch fehlen in Deutschland wettbewerbsfähige Produktionstechnologien für Zellen und Batterien.

- In der Energiewirtschaft sind die Technologien zur Energiespeicherung nicht weit genug entwickelt, und auch Low-Exergy-Technologien wie der Wärmetransformator sind nicht serienreif. Technologien der Wärmeverteilung sind häufig nicht mit Low-Exergy-Energiequellen kompatibel.

- In der Rohstoffwirtschaft fehlen komplexe Bioraffinerien, deren Produkte kompatibel mit herkömmlichen Wertschöpfungsketten sind, sowie Nachfolgetechnologien für die zu grob sortierenden Shredderanlagen.

- In der Landwirtschaft fehlt es an Methoden zur sicheren Bestimmung des Nährstoffgehalts von Wirtschaftsdünger und einem Konzept zur überregionalen Verteilung und gezielten Substitution von Kunstdünger.

- In der Recyclingwirtschaft existiert das sich gegenwärtig kontinuierlich verschärfende Problem der technischen Diversität. Immer mehr unterschiedliche Stoffe und Materialien werden in Produkten verarbeitet und erschweren die sorten- bzw. stoffreine Trennung nach Ablauf der Nutzungsdauer, was besonders im Kontext der knappen Technologiemetalle und seltenen Erden von Bedeutung ist.

Einige technisch-naturwissenschaftliche Fragen sind zu wenig geklärt und hemmen daher die Entwicklung. Dies gilt z. B. für die Vorteile biomassebasierter Produkte, aber auch für den Datenschutz in dezentralen Produktionssystemen. 


\subsection{Organisationale Pfadabhängigkeiten}

In 15 Transformationsfeldern ergaben sich Hinweise auf 38 organisationale Pfadabhängigkeiten.

Abbildung 28: Überblick über Typen organisationaler Pfadabhängigkeiten und Anzahl der jeweils gefundenen Beispiele

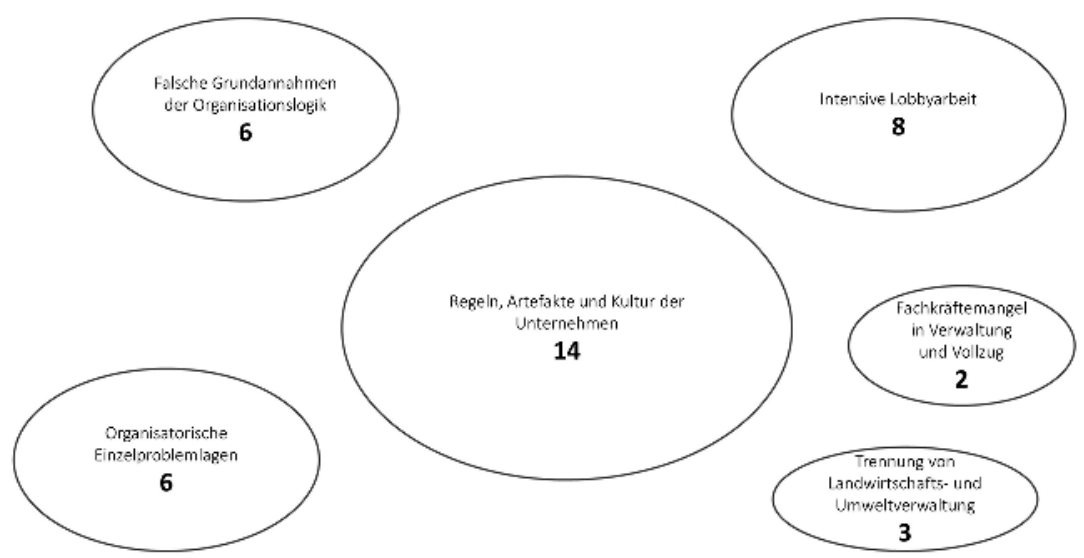

Quelle: Eigene Darstellung, Borderstep Institut für Innovation und Nachhaltigkeit.

Auch im Kontext der organisationalen Pfadabhängigkeiten sind falsche oder ökologisch unzeitgemäße Prämissen eine wichtige Ursache für die im Sinne einer Transformation wenig dynamische Führung von Organisationen. Folgende Beispiele können genannt werden:

- Niedrige Preise für Lebensmittel und Heizenergie gelten implizit als Teil der Grundversorgung der Bevölkerung und des Sozialsystems. Nach dem jüngsten Vorschlag des Umweltbundesamtes, die Mehrwertsteuer für Fleisch auf 19 Prozent zu erhöhen, kommentierte der Landwirtschaftsminister dies und sagte, er wolle »den Bürgern nicht durch Strafsteuern vorschreiben, was auf den Tisch kommt« (Zeit Online 2017). Letztlich wird nach der römischen Devise regiert und dafür gesorgt, dass das Volk panem et circensis bekommt.

- Eine weitere offensichtlich falsche Grundannahme besteht in dem immer wieder unterstellten Zusammenhang zwischen verstärktem Straßenbau und Wirtschaftswachstum.

- In der Politik wird als selbstverständlich angenommen, dass die Landwirtschaft im Umweltrecht einer Sonderstellung bedarf, weil sie mit der Lebensmittelproduktion maßgeblich zur Grundversorgung der Bevölkerung beiträgt. Dass diese Ausnahme, wie die Beispiele der Nitratverseuchung des Grundwassers und die aufgrund des hohen Pestizideinsatzes zusammenbrechende 
Biodiversität zeigen, offensichtlich dazu führt, dass die Landwirtschaft eben diese Funktion in absehbarer Zeit nicht mehr erfüllen kann, irritiert in der jeweils laufenden Legislaturperiode kaum jemanden. ${ }^{1}$

Wesentlich für organisatorische Stabilitäten sind auch eingeübte Prozesse und die Kulturen in Verwaltung und Unternehmen. So ist festzustellen,

- dass Unternehmen oft (zu?) lange an erprobten Verfahren und Prozessen festhalten, was sich z. B. in der langen Aufrechterhaltung von auf fossile Energien ausgerichteten Geschäftsmodellen von Energieversorgern trotz erklärter Energiewende und in der Produktion von Fahrzeugen mit Verbrennungsmotoren trotz klarer Erkenntnis ihrer Klimaeffekte zeigt,

- dass in einigen Fällen Zurückhaltung in der F\&E (z. B. im Kontext von LowExergy-Technologien) und in Folge dessen fehlende Qualifikationen im Kontext neuer Technologien und Lösungen zu beobachten sind und

- dass Unsicherheiten sowie die grundsätzliche Strategie der Vermeidung von Risiken Organisationen zögern lassen, sich Neuem zuzuwenden.

Die Aufrechterhaltung erprobter Geschäftsmodelle und Produktionsweisen zeigt sich oft als gemeinsames Interesse von Unternehmen einer Branche. Diese setzen ihre Verbände dann als Vertreter von Lobbyinteressen in Bewegung. Die Unternehmerseite, oft über die gesamte Wertschöpfungskette hinweg, vertritt ihre Lobbyinteressen in der Landwirtschaft, der Pkw-Produktion und auch in der Heizungstechnik aktiv und wirksam. Unterstützend werden in manchen Branchen, z. B. Energie und Fahrzeugbau, die Gewerkschaften mit dem Argument der Erhaltung von Arbeitsplätzen aktiv.

Ein "Spezialproblem « in der Landwirtschaft besteht darin, dass die Landwirtschaft (zum Teil aus gutem Grund, aber) im Gegensatz zu den meisten anderen produzierenden Gewerben und Industriezweigen durch ein eigenes politisches Ressort verwaltet wird. Die Landwirtschaftsverwaltung übernimmt dabei auch Aufgaben, die in anderen Wirtschaftszweigen den Umweltbehörden obliegen. Aufgrund der historisch gewachsenen Verflechtung von Landwirtschaftsverwaltung und landwirtschaftlicher Praxis wiegen die Interessen der Landwirtschaft in diesem Zusammenhang oftmals schwerer als umweltrechtliche Ziele, während die Umweltverwaltung im Kontext der Landwirtschaft zu wenig Einfluss und Möglichkeiten zum Vollzug hat.

1 Im Januar 2017 kündigte der BDEW stark steigende Wasserpreise an, da der Entwurf der Düngeverordnung gegenwärtig nicht ausreiche, um Boden und Crundwasser vor den Folgen einer weiter zunehmenden Überdüngung zu schützen. In vielen Wasserversorgungsgebieten würde daher eine aufwendige Denitrifizierung notwendig (Hannoversche Allgemeine Zeitung 2017b). 
In allen vier Transformationsbereichen haben wir auch einzelne organisationale Pfadabhängigkeiten identifiziert, die sich kaum gruppieren lassen. Dies gilt z. B. für folgende Sachverhalte:

- So ist z. B. die solare Wärmeversorgung in den Statistiken verkaufter Wärmeversorger nicht sichtbar, weil hier nur »Erstgeräte« erfasst werden. Elektroautos wurden in den Zulassungsstatistiken des Kraftfahrt-Bundesamtes teilweise in falsche Leistungsklassen eingestuft, da die Behörde die Einheiten $\mathrm{kW}$ für Leistung und kWh für Energie nicht zu unterscheiden in der Lage war (Kraftfahrtbundesamt 2016, S. 43).

- Die kommunale Planungspraxis ist nicht darauf ausgerichtet, Wärmeversorgung als gebäudeübergreifende Planungsaufgabe wahrzunehmen.

- Es existiert keine Lösung, das Eigentum an Korridorflächen (grüne Infrastrukturen) einfach und zentral zu verwalten, wie dies z. B. in Großbritannien für viele Flächen unter Naturschutz im National Trust erfolgt (National Trust 2017).

\subsection{Nutzungsbezogene Pfadabhängigkeiten}

In der Analyse der 15 Transformationsfelder wurden 27 nutzungsbezogene Pfadabhängigkeiten ermittelt.

Abbildung 29: Überblick über Typen nutzungsbezogener Pfadabhängigkeiten und Anzahl derjeweils gefundenen Beispiele

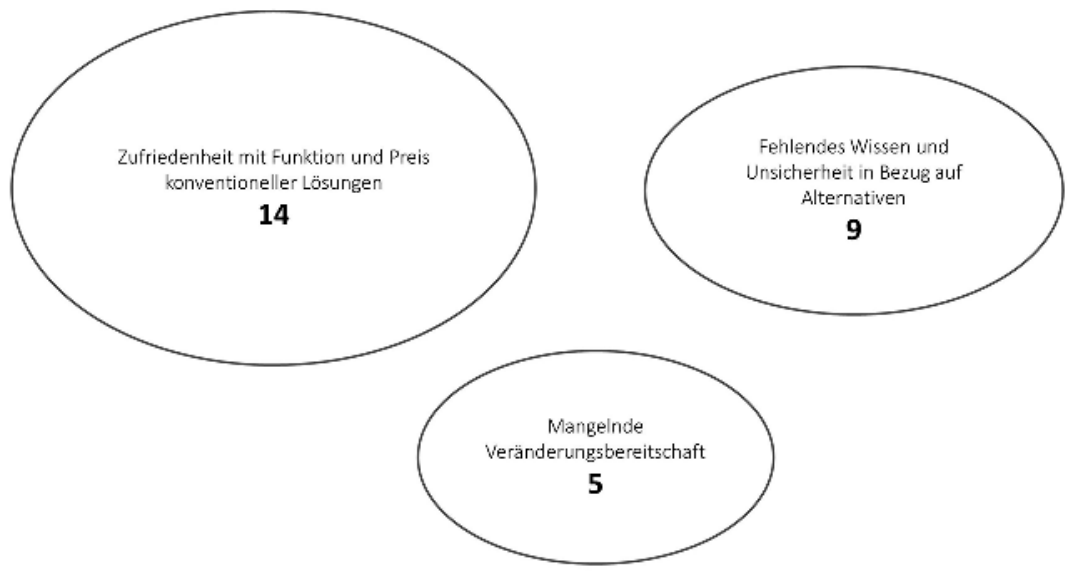

Quelle: Eigene Darstellung, Borderstep Institut für Innovation und Nachhaltigkeit.

Die nutzungsbezogenen Pfadabhängigkeiten lassen sich drei Kategorien zuordnen. Im Zentrum steht die Zufriedenheit mit Funktion und Preis konventioneller Lösungen: 
- Große Teile der Bevölkerung sind mit dem etablierten Regime und seinen Produkten und Services einfach zufrieden. Preiswertes Fleisch, große, schlecht sanierte, aber aufgrund niedriger Energiepreise billig zu heizende Wohnungen, hochpreisige Rennreiselimousinen mit viel Komfort: Viele von uns sehen bei diesen Angeboten Preis und Leistung in einem vernünftigen Verhältnis.

- Durch Gewöhnung verstärkt sich dieser Effekt der Zufriedenheit, z. B. auch durch das Erleben von »Freiheit« bei der Mobilität im eigenen Auto.

- Vorhandene Alternativen können mangels besserer Funktion bei vergleichbarem Preis den Wunsch zum Wandel oftmals nicht wecken, wie z. B. die Betrachtung der regenerativen Heizanlagen, des Carsharings oder noch in 2016 der Elektromobilität zeigt.

- Folgeprobleme, die z. B. durch die Wahl recycelbarer Produkte oder durch die ordnungsgemäße Entsorgung des eigenen Altwagens vermieden werden könnten, spielen für die Konsumentscheidung der Masse kaum eine Rolle.

Stabilisiert wird das Aufrechterhalten gewohnter Konsumformen durch fehlendes Wissen und Unsicherheiten in Bezug auf Alternativen.

- Konsumentinnen und Konsumenten sowie teilweise auch Landwirtinnen und Landwirte wissen so wenig über die umweltbelastenden Folgen des konventionellen Landbaus und die Vorteile des Ökolandbaus, dass sie ihre Konsumentscheidungen und Produktionsweisen nicht ändern.

- Unsicherheiten in Bezug auf Funktion, Reichweite und Ladesysteme führen über das Problem der zu hohen Anschaffungspreise hinaus zu einer nur sehr zögerlichen Markterschließung durch Elektromobile.

- Falsche Vorstellungen über die Kosten der Sanierung und Unkenntnis über die Vorteile hinsichtlich des Wohnkomforts drücken die Zahl der Hausbesitzerinnen und -besitzer, die eine umfassende energetische Sanierung angehen.

Abgerundet werden diese Pfadabhängigkeiten durch einen verbreiteten Mangel an Bereitschaft, Alternativen zu nutzen oder auszuprobieren.

\subsubsection{Typische Netzwerkbeziehungen von Pfadabhängigkeiten}

Mit Blick auf diese Analysen lassen sich mehrere Systeme identifizieren, in denen zusammenwirkende Pfadabhängigkeiten Regime in jeweils unterschiedlichen Konstellationen stabilisieren. Der unterschiedliche Charakter der stabilisierenden Wirkung ist dabei aus zwei Gründen von Bedeutung:

- Je nach Dauerhaftigkeit der zugrunde liegenden Abhängigkeiten hat der Charakter der das Regime stabilisierenden Lock-in-Systeme Auswirkungen auf die Zeitdauer jeder möglichen Transformation der jeweiligen Strukturen. 
- Je nach involvierten Schlüsselakteurinnen und -akteuren können sehr unterschiedliche Transformationsstrategien angemessen sein.

Folgende Systeme von zusammenwirkenden Pfadabhängigkeiten konnten identifiziert werden:

\subsection{Gebaute Infrastrukturen und Nutzungsnotwendigkeiten}

Als einziges Beispiel dieses wohl dauerhaftesten Lock-ins wurde das Lock-in-System in Straßenbau und Automobilität identifiziert.

Basis der äußerst dauerhaften Stabilität dieses Systems ist die gebaute Wohn-, Produktions- und Versorgungsinfrastruktur, die neben den Straßen letztlich auch alle über Straßen und mit Automobilen besser und schneller erreichbaren Gebäude und Destinationen umfasst.

\section{Abbildung 30: Lock-in-System in Straßenbau und Automobilität}

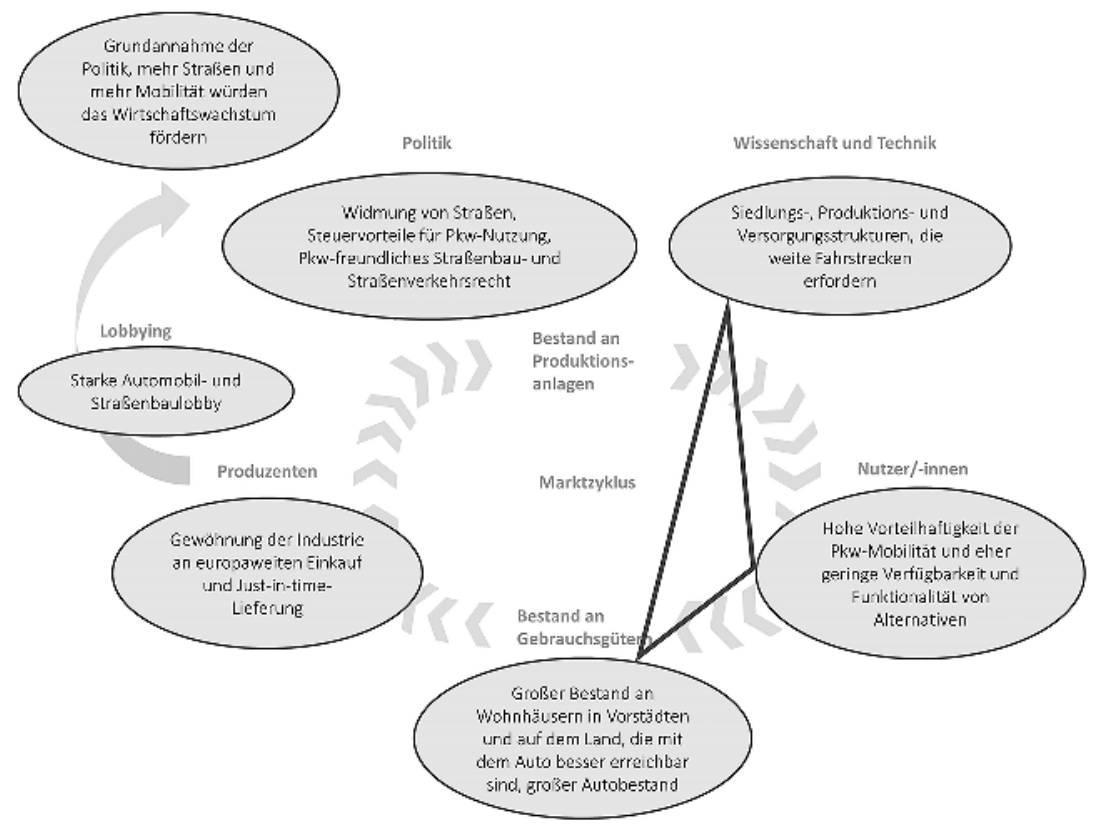

Quelle: Eigene Darstellung, Borderstep Institut für Innovation und Nachhaltigkeit.

Wohngebäude, Versorgungs- und Produktionsanlagen dürften mit zu den höchsten Werten gehören, über die unsere Gesellschaft verfügt. Ihre Nutzung ist für die gewohnte Lebensführung zentral. Die hohen hier festliegenden Werte sind untrennbar damit verbunden, dass diese Strukturen nur in äußerst langen Zeiträumen verändert werden können. 
Wichtig ist der Blick auf das Faktum, dass das hohe Maß an individueller Automobilität zum Ausbau dezentraler Siedlungsstrukturen (z. B. Neubaugebiete in Vororten und Dörfern) beigetragen und so zu weiten Wegen in der täglichen Lebensführung vieler Menschen geführt hat, sodass das Auto heute von vielen als unverzichtbar empfunden wird. Ein seit Jahrzehnten zugunsten des Autos verschobener Modal Split hat zu einem deutlichen Ausdünnen des öffentlichen Nahverkehrs in ländlichen Regionen und Vororten geführt. Alternative Mobilitätskonzepte wie stationäres und Free-Floating-Carsharing, Online-Mitfahrzentralen und der Umweltverbund haben zwar das Potenzial, jeweils fokussiert auf einzelne geografische Gebiete oder soziale Milieus Beiträge zu einem umweltverträglicheren Verkehr zu leisten, die Um- oder Durchsetzung einer autofreien oder auch nur autoarmen Gesellschaft liegt heute aber politisch in weiter Ferne.

Es folgt die politische Konsequenz, dass es zur Realisierung eines klimaneutralen Individualverkehrs nicht ausreicht, auf die zunehmende Verbreitung alternativer Mobilitätsangebote zu warten. Diese werden zwar in den kommenden Jahren den Modal Split um einige Prozentpunkte verschieben, z. B. durch ein weiteres Anwachsen des Fahrradverkehrs, aber klimaneutrale Automobilität, z. B. mit Elektro- oder Brennstoffzellenantrieb, wird dennoch eine zentrale Säule einer realistischen Umweltpolitik im Verkehr darstellen müssen.

Die wärmetechnische Sanierung von Gebäuden betrifft zwar ebenfalls die "gebauten Infrastrukturen«, erzwingt aber keine Änderung ihrer Nutzung. Die der Sanierung entgegenstehenden Pfadabhängigkeiten sind daher als deutlich weniger ausgeprägt einzustufen.

\subsection{Persistente Nutzungsgewohnheiten}

Als ebenso einziges Beispiel eines im Wesentlichen auf Gewohnheiten basierenden Lock-in-Systems wird der Fleischkonsum vorgestellt.

Im Gegensatz zur Nutzung des Automobils ist der Konsum von Fleisch keine Notwendigkeit. Er könnte grundsätzlich von jedem von uns von einem Tag auf den anderen eingestellt werden. Aufgrund der Gewöhnung an Fleisch, der Wertschätzung für Fleisch und einer Reihe gesellschaftlicher Konventionen im Kontext des gemeinsamen Essens geschieht dies nur durch eine bisher überschaubare Zahl von Menschen, die sich vegetarisch oder vegan ernährt, sowie anteilig durch eine etwas größere Zahl von Menschen, die weniger Fleisch isst. Im Zentrum des Lock-in-Systems steht hier der Marktzyklus, bei dem eine hohe Wertschätzung und eine angemessene Zahlungsbereitschaft auf Seiten der Kundschaft und eine leistungsfähige und profitable Branche auf der anderen Seite stehen. 
Abbildung 31: Lock-in-System im Fleischkonsum

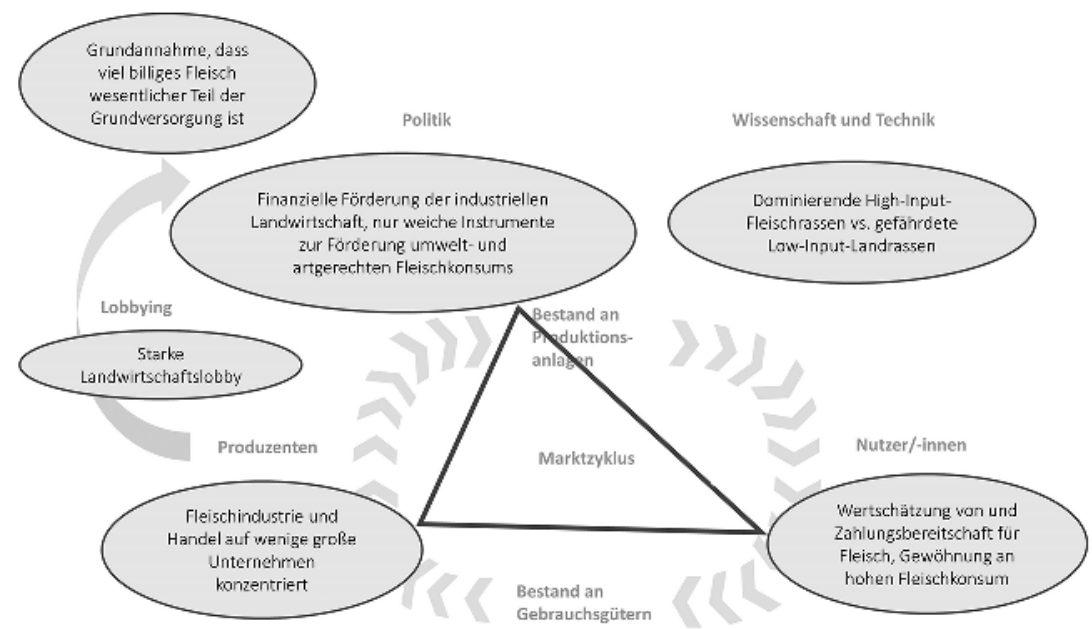

Quelle: Eigene Darstellung, Borderstep Institut für Innovation und Nachhaltigkeit.

Wesentlich für die dauerhafte Funktion des Lock-in-Systems für den Massenkonsum von Fleisch ist ein vorteilhaftes Preisniveau mit der Folge, dass es notwendig wird, die Kosten und Verpflichtungen von Landwirtschaft und Agrarindustrie aus sozialen und umweltbezogenen Ansprüchen heraus niedrig zu halten. Der Rechtsrahmen wird hierzu geeignet gestaltet. Hier wirken Wirtschaftslobbys und wechselnde Regierungen zusammen. Das Leitmotiv ist, dass preiswertes Fleisch (und in größerem Rahmen preiswerte Lebensmittel) als notwendig für die Versorgung der Bevölkerung postuliert werden. Die schon von den Römern formulierte Maxime, das Volk brauche panem et circencis, ist hier immer noch nützlich. Das zentrale Problem dieses Lock-ins ist, dass im Namen der billigen Currywurst das Grundwasser in weiten Teilen Deutschlands nicht mehr trinkbar sein und die Biodiversität bis zu einem Zusammenbruch der biologischen Systeme geschädigt werden wird. Hier ist auch auf den engen Zusammenhang der Fleisch- und Futtermittelproduktion zu den Problemfeldern »Stickstoffeintrag«, »Pestizideinsatz« und "grüne Infrastrukturen « (Tappeser und Chichowitz 2017a, 2017b; Tappeser et al. 2017) hinzuweisen.

Die politische Grundannahme, dass die Landwirtschaft aufgrund ihres Beitrages zur Grundversorgung eine Sonderstellung in der Umweltpolitik benötige, muss also letztlich durch eine verantwortliche Politik in Frage gestellt und ein wirksamer Schutz der Umweltgüter durchgesetzt werden. Nur so kann verhindert werden, dass sich die Landwirtschaft selbst die Möglichkeit nimmt, zur Grundversorgung der Bevölkerung beizutragen.

Die zeitliche Charakteristik der Pfadabhängigkeiten wird in diesem Problemfeld eher durch die Durchsetzbarkeit politischer Änderungen wie auch durch die 
Fähigkeit des landwirtschaftlichen Systems bestimmt, andere Produktionsformen überhaupt zu realisieren. Von Einfluss könnten hier mindestens sein:

- Die Notwendigkeit, die Kenntnisse in ökologischem oder zumindest ökologischerem Landbau von der Gruppe der etwa 6,5 Prozent (Flächenanteil) nach Richtlinien des Ökolandbaus arbeitenden Landwirte auf die restlichen 93,5 Prozent der Landwirte zu übertragen

- Die Tier- und Pflanzenzucht auf Rassen und Sorten umzustellen, die sich im ökologischen Landbau bewähren

- Die Produkte aus dem Dünger- und Pflanzenschutzkontext auf für Ökolandbau geeignete Produkte umzustellen

Im Vergleich zu den Zeitskalen, in denen die gebauten Infrastrukturen geändert werden können, dürfte sich dies bei vorhandenem Willen von Wirtschaft und Gesellschaft in ca. zwei Jahrzehnten bewältigen lassen. Als zeitkritisch könnte sich dabei die Tier- und Pflanzenzucht erweisen.

Ein ähnlicher Fall persistenter Nutzungsgewohnheiten scheint bei oberflächlicher Betrachtung im Kontext der preiswerten fossilen Heizung unsanierter Gebäude vorzuliegen. Da aber hier das eigentliche Bedürfnis an warmen und komfortablen Wohnräumen besteht und dieses auch mit einer regenerativen Heizanlage erfüllt werden kann, ist dieser Fall deutlich anders zu beurteilen.

\subsection{Technisch-institutionelle Komplexe mit starken technischen Defiziten} Lock-in-Systeme technisch-institutioneller Art, die durch starke technische Defizite gekennzeichnet sind, wurden in insgesamt sechs Fällen identifiziert und verteilen sich auf drei Wendethemen:

- Im Pkw-Verkehr mit den Pkw-Antrieben und der Altautoentsorgung

- In der Wärmeversorgung mit Wärmenetzen

- Im Feld der Rohstoffe und Ressourcen beim feedstock change, bei Technologiemetallen und Mikroschadstoffen

Die technisch-institutionellen Komplexe kennzeichnet zunächst, dass die Rolle der Nutzerinnen und Nutzer durchweg eher passiv oder randständig ist. Die Anbieterseite, also die Produkthersteller und die mit ihnen verknüpfte Wertschöpfungskette, kann also weitgehend machen »was sie will«, solange dabei ein funktionales, sicheres und preislich angemessenes Produkt herauskommt.

Bei denjenigen technisch-institutionellen Komplexen mit starken technischen Defiziten ist das Lock-in aber nicht nur in der Gewöhnung an ein profitables Geschäftsmodell begründet. Bei ihnen ist zusätzlich zu beachten, dass die Entwicklung technischer Alternativen entweder grundsätzlich starke Lücken aufweist 
oder aber ihre Realisierung so aufwendig ist, dass eine lange Zeitspanne dafür veranschlagt werden muss. Die Gründe hierfür sind unterschiedlich:

- Bei Pkw-Antrieben mangelt es in Deutschland an technischem Wissen und Produktionsanlagen für Elektroantriebe und ihre Komponenten.

- In der Wärmeversorgung mit Wärmenetzen mangelt es an Erfahrung mit technischen Komponenten wie Niedrigtemperatur-Wärmenetzbetrieb, Saisonalspeichern und Wärmetransformatoren; aber auch darüber hinaus ist der Netzaufbau aufwendig und langwierig.

- Im Feld des Recyclings von Technologiemetallen, z. B. in der Altautoentsorgung, ist die Stoffvielfalt und die schlechte Demontierbarkeit ein Problem, welches insoweit schwer zu lösen ist, als dass sehr viele Produkte und Produktionsketten betroffen sind. Ähnlich ist es auch bei der Stoffvielfalt bei Mikroschadstoffen, deren Vermeidung an der Quelle ebenfalls unzählige Produkte betreffen würde.

- Beim feedstock change in der chemischen Industrie liegt das technische Problem in der Nicht-Verfügbarkeit der notwendigen Mengen nachwachsender Rohstoffe und in der begrenzten Fähigkeit chemischer Prozesse, mit schwankenden Rohstoffqualitäten umzugehen.

Beispielhaft liegt ein solcher Fall im Lock-in in den Verbrennungsmotor vor.

Abbildung 32: Lock-in-System der Pkw mit Verbrennungsmotor

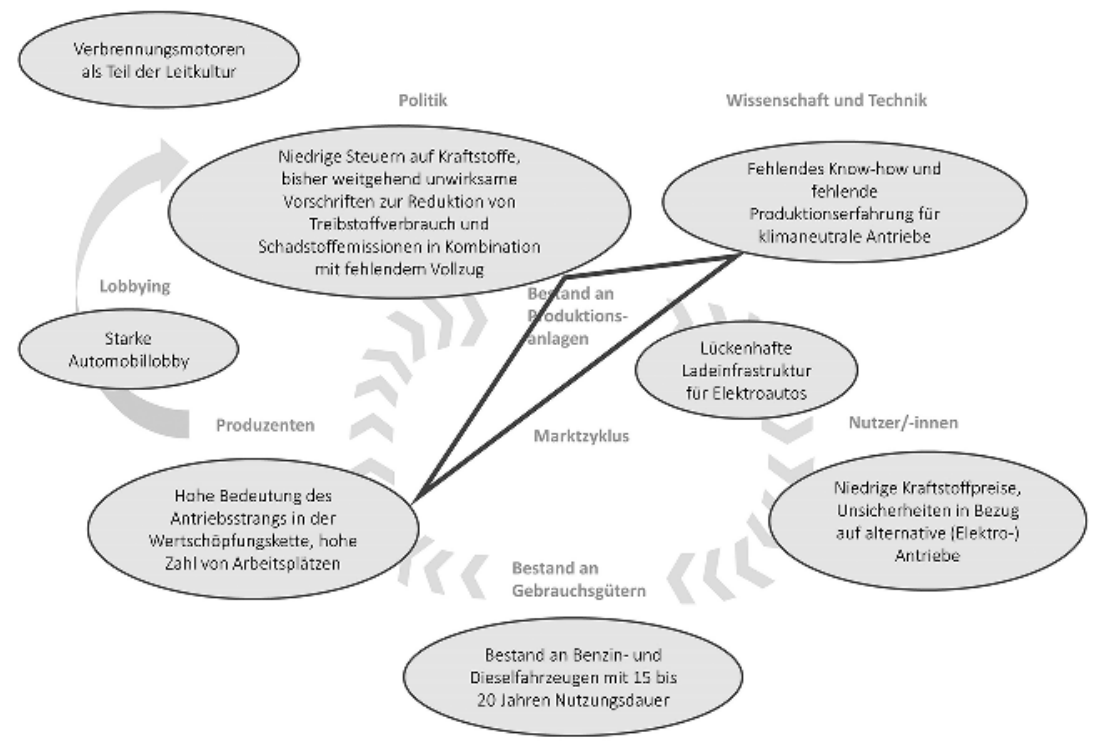

Quelle: Eigene Darstellung, Borderstep Institut für Innovation und Nachhaltigkeit. 
Die Gründe für das Lock-in lassen sich wie folgt beschreiben:

- Produkte und/oder Produktionskonzepte haben sich sowohl funktional als auch preislich als tauglich für den Massenmarkt erwiesen.

- Sowohl durch die kontinuierliche Verbesserung der zur Verfügung stehenden Technologien und Produktionsmittel als auch über economy of scale, ggf. auch durch Export, sammelt das Regime Erfahrungen, steigert die Umsätze und realisiert dauerhafte Profite.

- Durch die Argumentation mit volkswirtschaftlichen Beiträgen wie die Zahl der Arbeitsplätze oder die Exportquote lässt sich ein Wohlwollen der Politik erreichen. Durch kontinuierliches Lobbying werden Rechtsetzungen im Sinne der Branche gestaltet und so der ökonomische Rahmen langfristig verbessert.

- Letztlich kommt hinzu, dass der langfristige Erfolg mit dem dominanten Design und die scheinbar stabilen Beziehungen zur Politik die Branche vergessen ließ, sich außer um den Kontinuierlichen Verbesserungsprozess (KVP) auch um Grundlageninnovationen zu kümmern.

Letztlich ist so nicht nur ein erfolgreiches Produktionsregime stabil, sein Infragestellen scheitert überdies am Nichtvorhandensein wesentlicher Komponenten bzw. Alternativen. Die Vorbereitung solcher Alternativen in Nischen ist nur begrenzt zu beobachten, ein unvorbereiteter Pfadwechsel ist aber kaum möglich bzw. aufgrund der für die F \& E erforderlichen Fristen langwierig.

\subsection{Technisch-institutionelle Komplexe mit verfügbarer Alternative}

Technisch-institutionelle Komplexe mit verfügbarer Alternative sind zu finden

- in der Wärmeversorgung mit dem Sanierungsbedarf von Gebäuden sowie beim EE-Anteil an der Gebäudewärmeversorgung,

- in Landwirtschaft und Ernährung mit dem Stickstoffeintrag und dem Pestizideinsatz sowie im Kontext grüner Infrastrukturen.

Auch die technisch-institutionellen Komplexe mit verfügbarer Alternative kennzeichnet eine eher passive Rolle der Nutzerinnen und Nutzer. Solange die Anbieterseite funktionale, sichere und preislich angemessene Produkt erzeugt, nehmen die oft schlecht informierten und wenig zu aktivem Handeln motivierten Nutzerinnen und Nutzer kaum Einfluss auf die Wertschöpfungskette. 
Abbildung 33: Lock-in-System in der landwirtschaftlichen Produktion

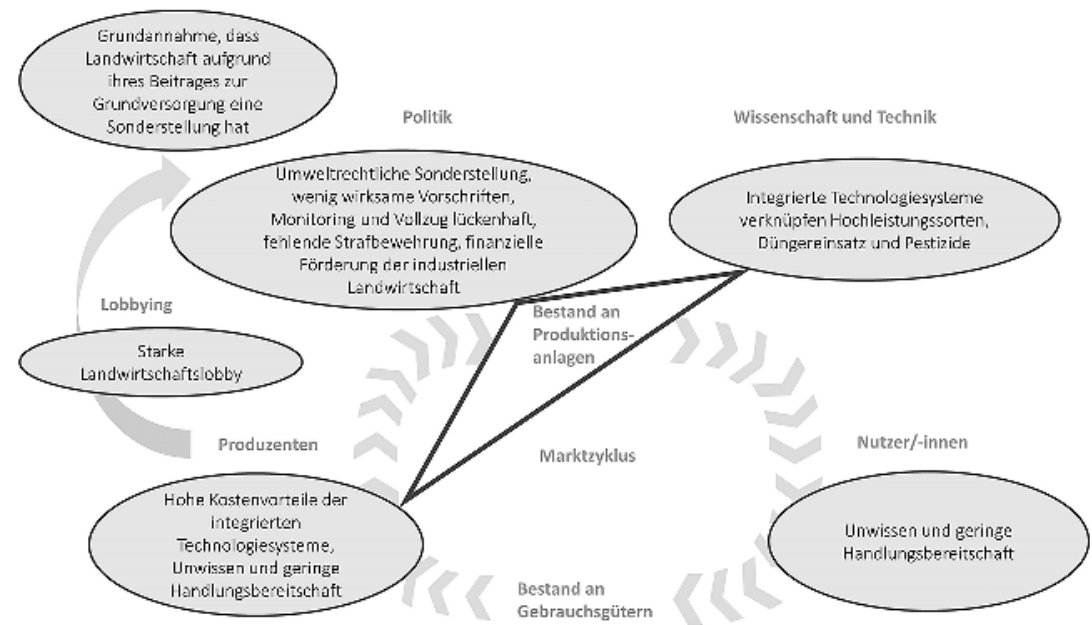

Quelle: Eigene Darstellung, Borderstep Institut für Innovation und Nachhaltigkeit.

Die Stabilität solcher Systeme liegt ganz zentral in ihrer wirtschaftlichen Vorteilhaftigkeit. Die wesentlichen Dynamiken sind ähnlich wie bei den technisch-institutionellen Komplexen mit starken technischen Defiziten. Die Lock-in-Systeme stützen sich auf massenmarkttaugliche Erzeugnisse und sichern Umsätze und Profite durch die langfristige Weiterentwicklung der Herstellungsroutinen und Produkte sowie durch die Vorteile der economy of scale. Auch volkswirtschaftlich ist das Regime aufgrund der Zahl der Arbeitsplätze und der Exportquote von hoher Bedeutung für die Politik. Lobbybemühungen bewirken häufig eine aus Branchenperspektive günstige Rechtsetzung und tragen zusätzlich zur langfristigen Stabilisierung des Regimes bei.

Der Kern der Wirksamkeit eines solchen Systems der Pfadabhängigkeiten besteht in der Entwicklung technisch-ökonomisch-rechtlicher Synergien mit den Zielen des Wachstums der Märkte und der Erzielung von Gewinn. Förderliche ökonomische Randbedingungen, z. B. niedrige Kosten für Energie und Rohstoffe sowie geringe gesetzliche Ansprüche an Sozialleistungen und Umweltschutz, sind hier wichtig.

Die Angreifbarkeit solcher Systeme beruht letztlich auf der Aktivität gesellschaftlicher Stakeholder wie Vertreterinnen und Vertreter der Interessen der Arbeitnehmerinnen und Arbeitnehmer, der Umwelt, von internationalen Zulieferern oder Verbraucherinnen und Verbrauchern.

Die Dauerhaftigkeit solcher Systeme ist bedroht durch Alternativtechnologien, die in Nischen entwickelt und erprobt wurden. Solche Alternativen sind für die oben angeführten Felder verfügbar: 
- Sanierungstechnologien für die Wärmesanierung sind in einer erheblichen Angebotsbreite verfügbar.

- Gleiches gilt für Heizungsanlagen auf Basis regenerativer Energie: Solarthermie, Biomasseheizungen und Wärmepumpen sind serienreif und können grundsätzlich am Markt beschafft werden. Gleiches gilt mit Ausnahme einiger Technologiekomponenten für den Ausbau der Wärmenetze.

- Als Alternative zu der stark umweltbelastenden industriellen Landwirtschaft bietet sich der ökologische Landbau als Gesamtkonzept oder mit seinen Einzelverfahren an.

Mit Blick auf diese Lock-in-Situationen ist der Pfadwechsel grundsätzlich abhängig von seiner politischen Durchsetzbarkeit sowie von denjenigen Fristen, die es braucht, die verfügbaren Alternativen zu skalieren.

\subsubsection{Zentrale Erkenntnisse zu Pfadabhängigkeiten}

Es gibt verschiedene Typen von Pfadabhängigkeiten, die auf unterschiedliche Weisen und unter deutlicher Verstärkung ihrer einzelnen Wirkung verknüpft sein können.

Ein zentraler externer Faktor für Veränderungen ist beispielsweise die technische Leistungsfähigkeit. Solange nur Japan und die USA über genügend Knowhow (gemessen an Patenten und der Anzahl der tatsächlich verkauften Autos) verfügten, um hochwertige Elektroautos zu bauen, hat die europäische Automobilindustrie, der das einschlägige technische Wissen noch fehlte, den Wandel systematisch verlangsamt. Die von den starken Autolobbyverbänden geprägte Politik zielte darauf, den historischen Pfad der Benzin- und Dieselautomobile zu verlängern. Die technische Pfadabhängigkeit des mangelnden technischen Wissens verknüpft sich mit ökonomischen Interessen von Unternehmen wie auch Arbeitnehmerinnen und Arbeitnehmern und der Grundüberzeugung, dass Automobile nur mit einem Brennstoff gefahren werden können, zu einer äußerst wirksamen Barriere für grundsätzliche Veränderungen.

Etablierte Unternehmen sind dabei oft wichtige Akteure der Transformation, da ihr Einfluss auf die Verbreitung neuer Lösungen hoch ist (Fichter und Clausen 2013, 2016). Unsere Analyse ergab, dass in der Unternehmenskultur etablierter Unternehmen wichtige Pfadabhängigkeiten bestehen können. Nicht jedes Management in der Wirtschaft oder in der Politik teilt z. B. die Vision von 100 Prozent erneuerbaren Energien, und einige können sich nicht einmal vorstellen, dass das Pariser Klimaabkommen jemals wirklich umgesetzt werden wird. Sie können sich eine Welt ohne Benzin- und Dieselautos, ohne Benzin- und Ölzentralheizung oder ohne die Gewohnheit, neue Straßen zu bauen, einfach nicht vorstellen. Auch wenn sie sich auf strenge Klimaschutzziele einigen, sind sie überzeugt, dass wir auch in Zukunft fossile Artefakte brauchen werden. 
Wir sind überzeugt, dass eine sorgfältige Analyse der Pfadabhängigkeiten eine realistische Sicht auf das Transformationsprojekt in den verschiedenen Sektoren erleichtert. Die Analyse von Pfadabhängigkeiten ermöglicht es oft, Gewinner/-innen und Verlierer/-innen zu identifizieren (es gibt kein Transformationsfeld nur mit Win-Win-Situationen) und erleichtert die Entwicklung einer Politik, die möglichst viele Beteiligte und Gruppen in das Transformationsprojekt einbezieht und für die negativ Betroffenen plant.

Aber politische Regelungen zur Steuerung der Transformation sind sorgfältig und mit Blick auf Wirksamkeit und Vollzug zu entwickeln. Unsere Analyse hat zahlreiche Regelungen identifiziert, die den Wandel zu unterstützen scheinen, während sie aber letztlich alte Praktiken durch eine Vielzahl von Lücken in die Zukunft übertragen. Tatsächlich unterstützen einige dieser Vorschriften überhaupt keine Änderungen. Solche »Regelungen ohne Wirkung « dienen als politisches Mittel zur Befriedung kritischer Gruppen und change agents, während sie andererseits ein Licht auf Politikerinnen und Politiker werfen, die selbst nicht an echte Veränderungen glauben oder diese anstreben.

Die Analyse der fünf Typen von Pfadabhängigkeiten führt insgesamt zu fünf zentralen Erkenntnissen:

- Zunächst fußen viele rechtliche und organisationale Pfadabhängigkeiten auf Grundannahmen, die mit Blick auf Fragen der Nachhaltigkeit überholt oder falsch sind. Auf Basis solcher Grundannahmen entsteht Recht und werden Organisationen geführt. Solche von Schein (1985, S. 14) als »unhinterfragte Grundannahme« charakterisierten Entscheidungsgrundlagen können sehr effektiv und langanhaltend Änderungen hemmen.

- Sowohl die fallweise falschen Grundannahmen und Ziele der Rechtssetzung wie auch die Aktivität von Lobbygruppen führen dann dazu, dass gegenwärtig an viel zu vielen Stellen wenig wirksames Umweltrecht entsteht. Die Gesetze und Verordnungen, die nach langen Debatten im politischen Prozess beschlossen werden, haben oft den Charakter von Kompromissen unterschiedlicher Zielsetzungen und weisen dann Lücken oder Hintertüren auf. Bei solch »lückenhaftem Umweltrecht« scheint es sich nicht nur um Einzelfälle, sondern vielmehr um ein generelles Prinzip zu handeln, das einer vertieften Untersuchung bedarf.

- Kostendruck und hohe Pfadwechselkosten stehen einem Pfadwechsel genauso entgegen wie fehlende Technologien und Infrastrukturen, deren Entwicklung bzw. Bereitstellung ihrerseits mit Pfadwechselkosten verbunden sind. Der Minimierung von Pfadwechselkosten sollte unter Umständen höhere Aufmerksamkeit zukommen.

- Die Zufriedenheit vieler Nutzerinnen und Nutzer mit den Eigenschaften und Kosten »klassischer« Produkte (bzw. mit den angenommenen und kommu- 
nizierten Zusatznutzen neuer Produkte) ist ein möglicherweise von der Umweltpolitik häufig unterschätztes Faktum. Es bietet sich hier ein vertieftes Studium des Zusammenhangs von Werthaltungen und Einkommen in verschiedenen gesellschaftlichen Milieus und der möglicherweise zwischen den Milieus sehr unterschiedlichen Vorbildfunktion von innovativen Leitkundinnen und -kunden an. Hierzu gehört auch eine allgemeine Veränderungsbereitschaft (»Macht der Gewohnheit«).

- Im Zentrum der Transformationsdynamik steht eine Vielzahl von Märkten, in denen Waren verkauft und gekauft werden. Viele der Waren oder Technologien sind Teil größerer technologischer Regimes (Kemp, 1994), und die Transformation solcher Märkte ist eng miteinander verknüpft. Diese Güter oder technologischen Systeme können nachhaltig sein oder nicht, und sowohl Lieferbetriebe als auch Nutzerinnen und Nutzer sind an Gewohnheiten, Organisationsstrukturen oder einfach an Investitionen und Infrastrukturen gebunden, die es leicht machen, den alten Weg zu gehen.

\subsection{Erfolgsfaktoren aus 20 Transformationsbeispielen in den Bereichen Wärme, Mobilität und Ressourcen}

Nachdem im Kapitel 4.1 zentrale Erkenntnisse zu Pfadabhängigkeiten und Hindernissen für eine Transformation zu einer Green Economy herausgearbeitet wurden, zielt dieses Kapitel darauf ab, wesentliche Voraussetzungen und Praktiken für erfolgreiche Pfadwechsel zu identifizieren. Dabei sollen sowohl übergreifende als auch transformationsfeldspezifische Erfolgsfaktoren für Transformationsprozesse bestimmt werden. Im Zentrum steht die Betrachtung von insgesamt 20 Transformationsbeispielen, die als Best-Practice-Fälle Anschauungsmaterial für (potenziell) erfolgreiche Transformationsansätze bieten. 17 der Transformationsbeispiele können inhaltlich den drei Wendethemen »Elektromobilität«, »Wärmeversorgung « und »Ressourceneffizienz« zugeordnet werden. Zusätzlich wurden drei übergreifende Transformationsbeispiele untersucht.

Der Analyserahmen für die Untersuchung der Transformationsbeispiele beruht auf dem in Kapitel 3.2 erläuterten und für dieses Vorhaben angepassten Models-of-Change-Ansatz. Gleichzeitig spielen auch Pfadabhängigkeiten (siehe Kapitel 3.1) und die Perspektive der drei Basisstrategien Effizienz, Konsistenz und Suffizienz (siehe Kapitel 3.3) eine wichtige Rolle in der Betrachtung der Transformationsbeispiele.

Die zentrale Forschungsfrage betrifft die Identifikation dessen, was Transformationsprozesse erfolgreich macht. In anderen Worten: 
- Was sind zentrale Variablen und Erfolgsfaktoren in Transformationsprozessen in Richtung einer nachhaltigen Wirtschaft?

- In welchem Ausmaß überlappen sie oder unterscheiden sie sich in unterschiedlichen Sektoren und Phasen von Transformation?

- Welche Steuerungsmöglichkeiten ergeben sich hieraus für die Politik und was sind weitere relevante Schlussfolgerungen für Theorie und Praxis?

Das Kapitel ist wie folgt aufgebaut: In Unterkapitel 4.2.1 werden zunächst die Auswahlkriterien für die der Analyse zugrundeliegenden Transformationsbeispiele genannt und diese in einer Vergleichsdarstellung präsentiert. Darauf folgt eine kurze Erläuterung zum Ablauf des Analysevorgangs, mit dessen Hilfe die Erfolgsfaktoren für Transformationsprozesse im Rahmen des Projekts herausgearbeitet wurden. Als Kern des Kapitels schließen sich die Abschnitte 4.2.2 bis 4.2.4 an. Jeder Abschnitt beinhaltet eine Querschnittsanalyse, welche die Transformationsfälle aus einer bestimmten Untersuchungsperspektive in den Blick nimmt - namentlich Analysen entlang zentraler Erfolgsfaktoren, unterschiedlicher Typen von Transformationsbeispielen sowie entlang des Prozessverlaufs. In 4.2.5 schließt das Kapitel mit einer kurzen Zusammenfassung und einigen Schlussfolgerungen.

\subsubsection{Transformationsbeispiele und Analysemethodik}

\subsubsection{Transformationsbeispiele: Selektionskriterien und Überblick}

\section{Auswahl der Transformationsbeispiele}

Die Auswahl der Transformationsbeispiele erfolgte anhand eines zuvor definierten Selektionsrasters, das mehrere Auswahlkriterien beinhaltete.

Ein zentrales Kriterium bestand in der thematischen Eignung der Transformationsbeispiele im Rahmen der gewählten Schwerpunktthemen. 17 der untersuchten Transformationsbeispiele sind daher in den Bereichen der Wärmeversorgung von Gebäuden, der E-Mobilität oder der Nutzung von Rohstoffen angesiedelt. Sie stellen Schwerpunktthemen des Vorhabens dar und spiegeln sich sowohl in den zuvor durchgeführten 15 Pfadabhängigkeitsanalysen als auch in den Roadmaps für einen Pfadwechsel in ausgewählten Transformationsbereichen (vgl. Kapitel 5). Um auch Beispiele von übergreifender Relevanz zu berücksichtigen, wurden zusätzlich drei Fälle ausgewählt, die sich nicht in einen der drei Themenschwerpunkte einordnen lassen, aber aufgrund anderer Merkmale von besonderem Interesse sind. Alle Fallstudien betrachten dabei Innovationen mit transformativem Potenzial oder aber bereits abgeschlossene Transformationen.

Eine Diversifizierung der Beispiele war ein weiteres wichtiges Kriterium für die Fallstudienauswahl. Dementsprechend wurden bewusst Beispiele mit unter- 
schiedlichen Eigenschaften gewählt, um übergreifende Erfolgsfaktoren sowie signifikante Unterschiede oder divergierende Ausgangsbedingungen zwischen verschiedenen Beispieltypen zu identifizieren. So decken die ausgewählten Beispiele bewusst verschiedene geografische Skalen ab (lokal bis global), betrachten Fälle im Inland und Ausland in unterschiedlichen Umsetzungs- bzw. Diffusionsstadien, Prozesse mit unterschiedlichen Geschwindigkeiten und unterschiedlichen Transformationsstrategien (Effizienz, Konsistenz, Suffizienz).

Neben der thematischen Eignung und der Diversifizierung wurden für die Auswahl noch weitere Kriterien zur Orientierung herangezogen. Diese beinhalten ein erkennbares Potenzial für einen disruptiven Prozess, das Aufzeigen oder die Entwicklung von Strategien für ein erfolgreiches Lock-out des etablierten Pfades, die Relevanz für den deutschen Kontext und eine erkennbare Intention zur (bzw. einen sichtbaren Erfolg bei) der Veränderung des dominierenden Regimes. Weiterhin sollten die beteiligten Akteurskonstellationen möglichst nachvollziehbar und erforschbar und das betrachtete Beispiel relativ komplex sein sowie eine breitenwirksame Transformation zum Ziel haben. Diese Charakteristika sind nicht bei jedem Beispiel vollständig erfüllt, haben den Auswahlprozess jedoch beeinflusst. Schlussendlich war auch die Praktikabilität der Beispiele im Hinblick auf Quellenverfügbarkeit und Analysierbarkeit im gegebenen Rahmen ein wichtiges Kriterium für die Auswahl.

In Abbildung 34 sind die 20 Transformationsbeispiele geordnet nach thematischem Überbegriff aufgeführt.

\section{Abbildung 34: Überblick über die Transformationsbeispiele}

\section{Wärmeversorgung von Gebäuden}

- Energiesprong Niederlande

- Wärmenetze in Dänemark

- InnovationCity Bottrop

- Plusenergiesiedlung Wüstenrot

- Klimasmartes Hyllie

Übergreifende Beispiele

- Nichtraucherschutz und Rauchverbote

- Stromeinspeisungsgesetz und EEG

- Vertikale Landwirtschaft

\section{E-Mobilität}

- E-Mobilität in Norwegen

- E-Mobilität in Kalifornien

- E-Mobilität in China

- E-Mobilität in den Niederlanden

- Post StreetScooter

- Tesla

Rohstoffe

- Online-Gebrauchtwarenhandel eBay \& Co.

- Reparaturwerkstätten Vangerow GmbH

- Repair Cafés

- Fairphone

- Verpackungsarmes/-freies Einkaufen

- Carsharing

Quelle: Eigene Darstellung, adelphi. 


\section{Einzelanalyse der Transformationsbeispiele}

Die einzelnen Fallbeispiele wurden auf Basis einer einheitlichen Gliederung und dem im Vorhaben entwickelten und auf dem Models-of-Change-Ansatz (Kristof 2010a; Kahlenborn et al. 2016) beruhenden Analyserasters untersucht und ausgewertet. Basis der Fallstudien waren neben Vorarbeiten der drei Institute in den jeweiligen Feldern umfangreiche Analysen der Literatur und der verfügbaren Internetquellen. In einzelnen Fällen erweiterten Experteninterviews die Datengrundlage.

Primäres Ergebnis bei jedem Transformationsbeispiel war eine Beschreibung der zentralen Erfolgsfaktoren entlang der MoC-spezifischen Analysekategorien. Vor dem Hintergrund fallspezifischer Landschaftsmerkmale und Rahmenbedingungen waren dies 1) Akteure und ihre Qualifikationen, 2) die Veränderungsidee und Lösungsvorschläge, 3) Zeitaspekte und 4) der Veränderungs- bzw. Transformationsprozess.

Zur Erfassung zentraler Merkmale der Fallbeispiele wurde zudem für jede Fallstudie ein Steckbrief erstellt, der die Fälle anhand ausgewählter Kriterien charakterisiert. Dazu gehört u.a. die Einordnung der Beispiele in Transformationsphasen. Diese Einordnung lehnt sich konzeptuell an die von Rogers (2003) im Kontext der Innovationsforschung entwickelten Diffusionsphasen sowie die darauf aufbauende Phaseneinteilung von Transformationsprozessen des Sachverständigenrats für Umweltfragen (2016) an. In der ersten Analyse wurde zwischen folgenden Transformationsphasen unterschieden:

- Eine Vorphase (o), in der der eigentliche Transformationsprozess noch nicht in Gang gesetzt wurde

- Eine Nischenphase (I), in der der Prozess zwar schon deutlich sichtbar, jedoch noch auf einzelne Nischenaktivitäten beschränkt ist

- Eine Wachstumsphase (II), in der der Transformationsprozess und damit verbundene Innovationen beginnen, in ein breiteres sozio-technisches bzw. sozio-kulturelles Regime hinein zu diffundieren

- Eine Stabilisierungsphase (III), in der die angestoßenen Veränderungen zum Teil des dominanten Regimes geworden sind und sich im Mainstream verfestigen

Neben der Phasenbestimmung wurden auch Merkmale wie die geografische Bezugsgröße (lokal/regional, national, transnational/global) sowie angewandte Transformationsstrategien (Effizienz, Konsistenz, Suffizienz; auf Basis von Behrendt et al. 2016) spezifiziert.

Die wichtigsten Ergebnisse der MoC-Analyse wurden zudem in einer auf dem Analyseraster beruhenden Auswertungstabelle zusammengefasst. Die $17 \mathrm{MoC}$ Analysekategorien sowie drei Kontextvariablen zu Landschaftsmerkmalen und 
Rahmenbedingungen wurden (soweit anwendbar) einzeln für jeden Fall mit einer Kurzbeschreibung versehen und durch die Autorinnen und Autoren im Hinblick auf ihre Relevanz für den Transformationserfolg bewertet. Alle Fallstudien inklusive der Relevanz-Bewertungen wurden einem projektinternen Peer-Review unterzogen.

\section{Die Transformationsbeispiele im Überblick}

Während des Projekts wurden insgesamt 20 Fallstudien durchgeführt. Eine Übersicht über die Transformationsbeispiele mit ausgewählten Merkmalen findet sich in Tabelle 8.

Wie bereits erläutert, lassen sich die Transformationsbeispiele nach unterschiedlichen Merkmalen klassifizieren und entsprechende Gemeinsamkeiten und Unterschiede feststellen. Hinsichtlich des thematischen Zuschnitts sind die Bereiche »Mobilität« und »Ressourcen« mit jeweils sechs und das Thema »Wärmeversorgung« mit fünf Fällen vertreten.

- Im Bereich »E-Mobilität« lag der Fokus auf dem Pfadwechsel vom Verbrennungsmotor zum Elektroantrieb im Individualverkehr. Hierfür wurden die Marktentwicklung und begleitende regulatorische Maßnahmen in Vorreiterländern (Norwegen, Kalifornien, China, Niederlande) sowie die Entwicklung erfolgreicher E-Fahrzeughersteller (Tesla, Post StreetScooter) untersucht.

- Im Bereich »Wärme« wurde sowohl auf die Verbesserung der Dämmung von Gebäuden als auch auf die Nutzung erneuerbarer Energien und den Ausbau von Wärmenetzen abgestellt. Hierfür wurden insbesondere Pilotprojekte und Initiativen auf kommunaler Ebene (Wüstenrot, Bottrop, Hyllie) analysiert. Gleichzeitig wurden auch langfristige Sektorentwicklungen (Wärmenetze in Dänemark) und eher mittelfristige, spezifische Prozesse (Energiesprong NL) auf nationaler Ebene betrachtet.

- Im Bereich »Ressourcen«stand mit der Verlängerung der Nutzung von Produkten eine Thematik im Vordergrund, die verstärkt auf die Veränderung von Konsummustern und damit einhergehende Prozesse abzielt. Untersucht wurden sowohl entstehende soziale Praktiken in der Nischen- und Beschleunigungsphase (Repair Cafés, verpackungsarmes Einkaufen, Carsharing) als auch Unternehmen mit unterschiedlicher Reichweite (Reparaturwerkstätten Vangerow GmbH, Fairphone und eBay).

- Bei den übergreifenden Fallbeispielen wurden mit dem Nichtraucherschutz und dem Rauchverbot sowie mit dem Stromeinspeisungsgesetz und dem EEG zwei Regulierungsinitiativen auf Bundesebene gewählt, die sich als erstaunlich erfolgreich erwiesen haben. Das Fallbeispiel zu vertikaler Landwirtschaft führt als explorative Technologiestudie den Projektstrang zu Landwirtschaftsfragen weiter fort. 
Tabelle 8: Übersicht über die Transformationsbeispiele mit ausgewählten Kriterien

\begin{tabular}{|c|c|c|c|c|c|}
\hline Titel der Fallstudie & Typ & $\begin{array}{l}\text { Geografische } \\
\text { Bezugsebene }\end{array}$ & $\begin{array}{l}\text { Transformations- } \\
\text { phase }\end{array}$ & $\begin{array}{l}\text { Treiber/ } \\
\text { Perspektive }\end{array}$ & Strategie \\
\hline \multicolumn{6}{|c|}{ Fallstudien im Bereich E-Mobilität } \\
\hline $\begin{array}{l}\text { Elektromobilität in } \\
\text { China }\end{array}$ & $\begin{array}{l}\text { Nationale } \\
\text { Sektorentwicklung }\end{array}$ & $\begin{array}{l}\text { Volksrepublik } \\
\text { China }\end{array}$ & Beschleunigung & Politik & EK \\
\hline $\begin{array}{l}\text { Elektromobilität in den } \\
\text { Niederlanden }\end{array}$ & $\begin{array}{l}\text { Nationale } \\
\text { Sektorentwicklung }\end{array}$ & Niederlande & Beschleunigung & Politik & EK \\
\hline $\begin{array}{l}\text { Elektromobilität in Kali- } \\
\text { fornien }\end{array}$ & $\begin{array}{l}\text { Nationale } \\
\text { Sektorentwicklung }\end{array}$ & Kalifornien & Beschleunigung & Politik & EK \\
\hline $\begin{array}{l}\text { Elektromobilität in Nor- } \\
\text { wegen }\end{array}$ & $\begin{array}{l}\text { Nationale } \\
\text { Sektorentwicklung }\end{array}$ & Norwegen & Beschleunigung & Politik & EK \\
\hline Post StreetScooter & Unternehmen & Deutschland & Beschleunigung & Wirtschaft & EK \\
\hline Tesla & Unternehmen & Welt & Beschleunigung & Wirtschaft & EK \\
\hline \multicolumn{6}{|c|}{ Fallstudien im Bereich Ressourcen/"Produkte länger nutzen" } \\
\hline $\begin{array}{l}\text { Online-Gebrauchtwa- } \\
\text { renhandel, ebay \& Co. }\end{array}$ & Unternehmen & $\begin{array}{l}\text { Deutschland, } \\
\text { Welt }\end{array}$ & Stabilisierung & Wirtschaft & ES \\
\hline Fairphone & Unternehmen & $\begin{array}{l}\text { Deutschland, } \\
\text { Österreich, } \\
\text { Schweiz, } \\
\text { Niederlande }\end{array}$ & Nische & Zivilgesellschaft & EKS \\
\hline $\begin{array}{l}\text { Reparaturwerkstätten } \\
\text { Vangerow GmbH }\end{array}$ & Unternehmen & Deutschland & Nische & Wirtschaft & ES \\
\hline Repair Cafés & $\begin{array}{l}\text { Technologie/soziale } \\
\text { Praxis }\end{array}$ & Deutschland & Nische & Zivilgesellschaft & $\mathrm{S}$ \\
\hline Carsharing & $\begin{array}{l}\text { Technologie/soziale } \\
\text { Praxis }\end{array}$ & Deutschland & Beschleunigung & $\begin{array}{l}\text { Zivilgesellschaft } \\
\text { Wirtschaft }\end{array}$ & ES \\
\hline $\begin{array}{l}\text { Verpackungsarmes/ } \\
\text {-freies Einkaufen }\end{array}$ & $\begin{array}{l}\text { Technologie/soziale } \\
\text { Praxis }\end{array}$ & Deutschland & Nische & Zivilgesellschaft & EKS \\
\hline \multicolumn{6}{|c|}{ Fallstudien im Bereich Wärme } \\
\hline Klimasmartes Hyllie & Kommunale Initiative & $\begin{array}{l}\text { Malmö, } \\
\text { Schweden }\end{array}$ & Beschleunigung & Wirtschaft & EK \\
\hline $\begin{array}{l}\text { Plusenergiegemeinde } \\
\text { Wüstenrot }\end{array}$ & Kommunale Initiative & $\begin{array}{l}\text { Wüstenrot, } \\
\text { Deutschland }\end{array}$ & Stabilisierung & Politik & EK \\
\hline $\begin{array}{l}\text { InnovationCity Ruhr- } \\
\text { Modellstadt Bottrop }\end{array}$ & Kommunale Initiative & $\begin{array}{l}\text { Bottrop, } \\
\text { Deutschland }\end{array}$ & Beschleunigung & Wirtschaft & EK \\
\hline $\begin{array}{l}\text { Wärmenetze in } \\
\text { Dänemark }\end{array}$ & $\begin{array}{l}\text { Nationale } \\
\text { Sektorentwicklung }\end{array}$ & Dänemark & Stabilisierung & Politik & EK \\
\hline $\begin{array}{l}\text { Energiesprong } \\
\text { Niederlande }\end{array}$ & $\begin{array}{l}\text { Nationale } \\
\text { Regierungsinitiative }\end{array}$ & Niederlande & Beschleunigung & Politik & EK \\
\hline \multicolumn{6}{|c|}{ Übergreifende Fallstudien } \\
\hline $\begin{array}{l}\text { Nichtraucherschutz und } \\
\text { Rauchverbot }\end{array}$ & $\begin{array}{l}\text { Nationale } \\
\text { Regierungsinitiative }\end{array}$ & Deutschland & Stabilisierung & Politik & KS \\
\hline $\begin{array}{l}\text { Stromeinsparungsge- } \\
\text { setz und EEG }\end{array}$ & $\begin{array}{l}\text { Nationale } \\
\text { Regierungsinitiative }\end{array}$ & Deutschland & Beschleunigung & Politik & K \\
\hline $\begin{array}{l}\text { Vertikale } \\
\text { Landwirtschaft }\end{array}$ & $\begin{array}{l}\text { Technologie/ } \\
\text { soziale Praxis }\end{array}$ & Welt & Nische & Wirtschaft & EK \\
\hline
\end{tabular}

Quelle: Eigene Darstellung, adelphi.

Themenübergreifend lassen sich unterschiedliche Typen von Transformationsbeispielen ausmachen. Vier Fallstudien befassen sich mit der Entwicklung bestimmter Technologien und sozialer Praktiken wie Carsharing oder vertikaler 
Landwirtschaft. Fünf Studien haben nationale Sektorentwicklungen zum Inhalt, beispielsweise im Bereich der Elektromobilität in China oder auf dem Gebiet der Wärmenetze in Dänemark. Ebenfalls fünf Fälle beschreiben Unternehmen wie Tesla oder eBay, weitere drei befassen sich mit kommunalen Initiativen wie der InnovationCity in Bottrop. Zuletzt lassen sich drei Fallstudien als Entwicklung nationaler Regierungsinitiativen kategorisieren (wie z. B. Nichtraucherschutz).

In Bezug auf die geografische Bezugsebene lassen sich neun Fallstudien identifizieren, die Beispiele innerhalb Deutschlands betreffen (zwei auf lokaler und sieben auf nationaler Ebene). Sieben Fallstudien betreffen Beispiele im Ausland (eines auflokaler, sechs auf nationaler Ebene). Insgesamt vier Fallstudien sind aus internationaler bzw. globaler Perspektive zu betrachten. Auf das gesamte Fallset gesehen beschäftigt sich also der Großteil der Fälle mit Transformationsprozessen auf nationaler Ebene (13 von 20).

Mit Blick auf die Transformationsphase, also den Entwicklungsstand der Beispiele zwischen Nische, Wachstum und Stabilisierung im dominanten Regime (siehe Kapitel 3.2 für eine Erläuterung), lassen sich vier Beispiele der Nischenphase zuordnen, während sich knapp über die Hälfte der Fallbeispiele in der Beschleunigungsphase befindet. Fünf Transformationsbeispiele sind bereits der Stabilisierungsphase zuzuordnen.

Die folgende Abbildung gibt einen Überblick über die Verteilung der Beispiele hinsichtlich der Transformationsphasen und der geographischen Bezugsebene sowie des thematischen Zuschnitts und Typs:

Abbildung 35: Überblick über die Fallbeispiele nach Transformationsphase, geografischer Bezugsebene und thematischem Zuschnitt.

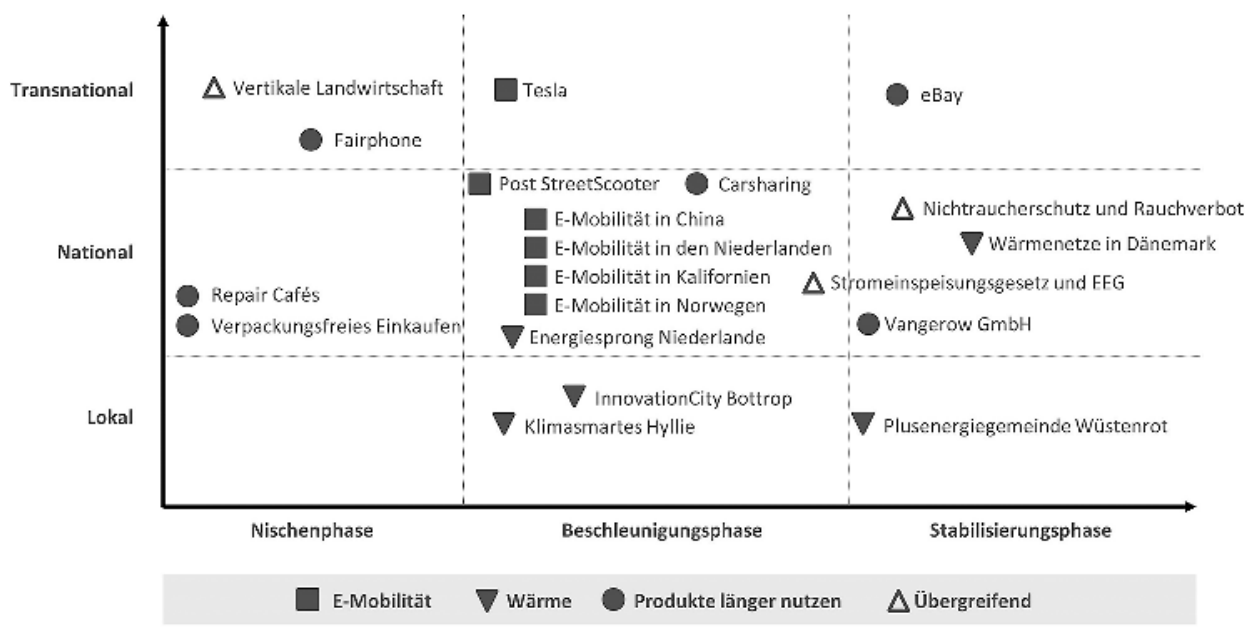

Quelle: Eigene Darstellung, adelphi. 
Auffällig sind insbesondere die Dominanz der auf nationaler Ebene angesiedelten Beispiele in der Beschleunigungsphase, die relative Homogenität der Fallbeispiele aus dem Bereich E-Mobilität sowie die größtenteils lokale Perspektive im Bereich der Wärmebeispiele.

In Bezug auf den jeweils wichtigsten Treiber dominieren politisch motivierte gegenüber wirtschaftlich und zivilgesellschaftlich getriebenen Prozessen (siehe Abbildung 36). Gleichzeitig besteht hinsichtlich der Transformationsstrategie eine deutliche Dominanz von Effizienz- und Konsistenzansätzen gegenüber Beispielen, in denen Suffizienz eine zentrale Rolle spielt. Effizienz ist bei fast allen Beispielen ein wichtiger Ansatz (Ausnahmen sind Repair Cafés, EEG und Nichtraucherschutz), Konsistenz bei über drei Viertel der Fälle (mit Ausnahme von Repair Cafés, den Reparaturwerkstätten Vangerow, Carsharing und eBay). Suffizienzansätze spielen hingegen nur bei ca. einem Drittel der Beispiele eine Rolle (Repair Cafés, Reparaturwerkstätten Vangerow, Carsharing, eBay, Nichtraucherschutz sowie bis zu einem gewissen Grad Bottrop und Wüstenrot).

Die Transformationsstrategien Effizienz, Konsistenz und Suffizienz sind je nach Treiber unterschiedlich stark ausgeprägt. Bei politisch motivierten Prozessen sind Konsistenzstrategien am dominantesten. Von wirtschaftlichen Akteurinnen und Akteuren getragene Prozesse verfolgen verstärkt Effizienzstrategien. Die Prozesse, in denen Suffizienzansätze deutlicher zum Tragen kommen, werden hingegen insbesondere von zivilgesellschaftlichen Akteurinnen und Akteuren getragen (siehe Abbildung 36).

\section{Abbildung 36: Dominante Treiber und Strategien in den Transformationsbeispielen}

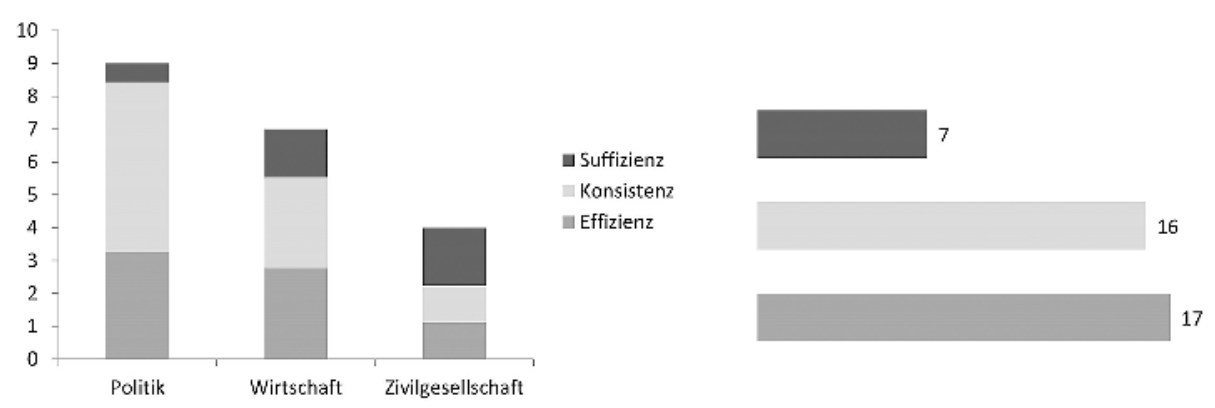

Diagramm links: Verteilung der 20 Fallstudien auf die jeweils wichtigsten Treiber (Politik, Wirtschaft, Zivilgesellschaft) sowie relatives Cewicht der einzelnen Transformationsstrategien innerhalb dieser Kategorien.

Diagramm rechts: Anzahl der Fallstudien, in denen die jeweilige Strategie eine Rolle spielt (ein Fallbeispiel kann mehrere Strategien in sich vereinen)

Quelle: Eigene Darstellung, adelphi. 


\subsubsection{Querschnittsanalyse und methodisches Vorgehen}

Wie bereits angedeutet, bestand das Ziel der Analyse darin, durch eine systematische Gesamtbetrachtung des Fallsets wesentliche Erfolgsfaktoren abzuleiten, die zu einem Pfadwechsel zu einer Green Economy beitragen können.

Für die Gesamtbetrachtung wurden qualitative und semi-quantitative Analysemethoden trianguliert, um die relevantesten Variablen und Erfolgsfaktoren sowie ihre spezifischen Ausprägungen und Wechselbeziehungen zu identifizieren. Eine explorative, statistische Auswertung verschiedener ordinalskalierter Bewertungsdimensionen (s. u.) wurde dabei als Ausgangspunkt für eine qualitative Analyse genutzt und in einem iterativen Prozess erweitert. Die Bandbreite der untersuchten Fallbeispiele erwies sich hierbei sowohl als Vorteil als auch als Herausforderung und ermöglichte ein produktives Spannungsfeld zwischen Analysetiefe und -breite.

Der Analyseprozess lässt sich in drei Schritte einteilen, die im Folgenden näher beschrieben werden. In einem ersten Schritt wurden die Bewertung der Fallbeispiele in Bezug auf die MoC-Faktoren erweitert und die Daten für die Analyse vorbereitet. Im zweiten Schritt erfolgte die eigentliche Auswertung von Erfolgsfaktoren und Entwicklungsmustern von Transformationsprozessen im Rahmen einer Drei-Wege-Analyse. In einem dritten Schritt wurden die Ergebnisse des vorherigen Schrittes abstrahiert und Schlussfolgerungen für Politik, Theorie und Praxis entwickelt.

\section{Erster Schritt: Erweiterung der Bewertung nach MoC-Faktoren und Vorbereitung der Daten}

Um eine detailliertere Auswertung zu ermöglichen, erfolgten nach der Fertigstellung der Einzelstudien eine Sichtung aller Fallstudien sowie eine zusätzliche Bewertung der MoC-Faktoren nach Ausprägung, Effekt und Potenzial durch das Projektteam von adelphi. Für die Bewertung wurden Ordinalskalen entwickelt, die eine qualitative Einschätzung, aber auch eine statistische Querauswertung zur Unterstützung der qualitativen Mustererkennung über die einzelnen Fallbeispiele hinweg ermöglichen. Die Bewertungsmethode und die statistische Auswertung sind dabei nicht als wissenschaftlich und erkenntnistheoretisch abgesicherte Verfahren zu verstehen, sondern als ein exploratives Tool, um die Mustererkennung der qualitativen Analyse zu unterstützen (siehe Tabelle 9 für eine Übersicht der Indikatoren und verwendeten Skalen). 
Tabelle 9: Indikatoren zur Bewertung der Analysekategorien nach MoC

\section{Merkmal/Indikator}

\section{Ausprägung}

gibt an, wie deutlich das jeweilige Merkmal bzw. der jeweilige Erfolgsfaktor im

Fallbeispiel vorhanden ist unklar/nicht vorhanden (0) sehr niedrig (1) niedrig (2) mittel (3) hoch (4) sehr hoch (5)

\section{Effekt}

gibt an, welcher Effekt von der Ausprägung des Merkmals (siehe oben) im Hinblick auf einen intendierten Pfadwechsel ausgeht, relativ zu einem Nichtvorhandensein (Ausprägung $=$ nicht vorhanden) des Merkmals

sehr stark negativ $(-5)$

stark negativ (-4) mittelstark negativ (-3) leicht negativ (-2) sehr leicht negativ (-1) neutral/unklar (0) sehr leicht positiv (1) leicht positiv (2) mittelstark positiv (3) stark positiv (4) sehr stark positiv (5) sehr stark negativ (-5) stark negativ (-4)

\section{Potenzial}

gibt an, welcher Effekt aus Sicht der Autorinnen und Autoren in Zukunft vom jeweiligen Merkmal erwartbar ausgehen kann mittelstark negativ (-3) leicht negativ (-2)

sehr leicht negativ (-1) neutral/unklar (0) sehr leicht positiv (1) leicht positiv (2) mittelstark positiv (3) stark positiv (4)

sehr stark positiv (5) unklar/nicht vorhanden (0) sehr niedrig (1) niedrig (2) mittel (3) hoch (4) sehr hoch (5)

Quelle: Eigene Darstellung, adelphi.

Für die Indikatoren »Ausprägung«, »Effekt « und »Potenzial« wurde zudem eine Unterscheidung nach Entwicklungsphasen vorgenommen. Da sich zahlreiche Fallbeispiele mit Transformationsprozessen beschäftigen, die sich noch in der Nischen- oder Wachstumsphase befinden, wurden Ausprägung und Effekt nur für diejenigen Phasen bestimmt, für die eine Datengrundlage bestand. Einschätzungen zum Potenzial wurden, wo sinnvoll durchführbar, jeweils für die auf den aktuellen Stand folgende Phase getroffen (siehe Darstellung in Tabelle 10). 
Tabelle 10: Beispielhafter Auszug aus der Bewertungstabelle der Fallstudie zu Elektromobilität in China

\begin{tabular}{|c|c|c|c|c|c|c|}
\hline \multirow{3}{*}{$\begin{array}{l}\text { Analysekriterien } \\
\text { Erfolgsfaktoren } \\
\text { Transformationsprozess }\end{array}$} & \multirow[t]{3}{*}{ Zusammenfassung } & \multicolumn{5}{|l|}{ Bewertung } \\
\hline & & \multirow{2}{*}{ Dimension } & \multicolumn{4}{|c|}{ Phase } \\
\hline & & & 0 & I & ॥ & III \\
\hline \multirow[t]{4}{*}{ Ressourcenausstattung } & \multirow{4}{*}{$\begin{array}{l}\text { Förderungen finanzieller Art waren } \\
\text { notwendig, um den Prozess anzustoßen. } \\
\text { Diese waren anfangs noch eher gering, } \\
\text { ab } 2009 \text { wurden große Summen auch } \\
\text { im Rahmen von direkten Kaufanreizen } \\
\text { gewährleistet. Genaue Zahlen sind nicht } \\
\text { bekannt. }\end{array}$} & Ausprägung & & 3 & 4 & \\
\hline & & Effekt & & 4 & 5 & \\
\hline & & Potenzial & & & & 4 \\
\hline & & Relevanz & \multicolumn{4}{|c|}{ hoch } \\
\hline
\end{tabular}

Quelle: Eigene Darstellung, adelphi, nach Beigang und Clausen (2017).

Um die weitere Auswertung zu ermöglichen, wurden die Bewertungen und Kurzbeschreibungen zudem in eine Tabellenkalkulation überführt, über das gesamte Fallstudienset auf Konsistenz hin überprüft, wo notwendig angepasst und so aufbereitet, dass sie für die weiteren Analyseschritte einfach nutzbar sind.

\section{Zweiter Schritt: Drei-Wege-Analyse zur Identifikation zentraler Erfolgsfaktoren und Entwicklungsmuster von Transformationsprozessen}

Für die eigentliche Analyse von Erfolgsfaktoren und Entwicklungsmustern von Transformationsprozessen wurden drei verschiedene Analysewege gewählt und miteinander trianguliert, um ein möglichst umfassendes Bild von beispielübergreifenden Entwicklungsmustern, Erfolgsfaktoren und Einwirkungsmöglichkeiten auf Transformationsprozesse zu gewinnen. Neben einer von den zuvor festgelegten MoC-Faktoren ausgehenden Queranalyse (Weg 1) wurden ausgehend von den einzelnen Fallstudien mithilfe deduktiver und induktiver Kategorienbzw. Clusterbildungsmethoden (Wege 2 und 3) typische Entwicklungsmuster von Transformationsprozessen anhand unterschiedlicher Unterscheidungsmerkmale identifiziert und ausgewertet. Die Auswertung erfolgte dabei sowohl zwischen den unterschiedlichen Analysewegen als auch zwischen statistischen und qualitativen Ansätzen iterativ, um eine gegenseitige Befruchtung der verschiedenen Perspektiven zu ermöglichen.

\section{Weg 1: Betrachtung der Fallbeispiele aus der Perspektive einzelner MoC-Faktoren} Zunächst wurden aus Perspektive einzelner MoC-Faktoren (z. B. change agents und ihre Qualifikationen) die jeweiligen Ausprägungen über das gesamte Fallstudienset hinweg betrachtet und Gemeinsamkeiten und Unterschiede identifiziert. Einen ersten Ausgangspunkt stellten hierbei Relevanzbewertungen der jeweiligen MoC-Faktoren über das Fallstudienset hinweg dar. Während der Durchschnitt der Bewertungen eine erste Einschätzung zum relativen Gewicht der MoC-Faktoren ermöglichte, wurden auf Basis der Unterschiede zwischen den einzelnen 
Bewertungen Zusammenhänge zwischen unterschiedlichen Fallbeispielen identifiziert und mit Hilfe der Beschreibungen in den Fallstudien validiert, erweitert und kontextualisiert.

Weg 2: Deduktive Kategorienbildung zur Identifikation charakteristischer Muster und Typen von Transformationsprozessen

Im Rahmen der Bearbeitung der einzelnen Fallstudien wurden schon erste Klassifizierungen der Fallbeispiele vorgenommen. Neben der Verortung in den einzelnen Wendethemen zählen hierzu die Transformationsphase, die im Kontext des Beispiels zur Anwendung kommenden Basisstrategien (Effizienz, Konsistenz, Suffizienz) und die geografische Bezugsebene. Diese Klassifizierung wurde im Rahmen der Analyse um zusätzliche Variablen erweitert. U.a. wurden unterschiedliche Fallstudientypen definiert und die zentralen Treiber nach gesellschaftlichen Anspruchsgruppen (Politik, Wirtschaft, Wissenschaft und Zivilgesellschaft) ausdifferenziert. Die so entstehenden Gruppierungsschemata und daraus resultierenden Gruppen von Fallstudien (z. B. primär von wirtschaftlichen Interessen getriebene Fallbeispiele vs. primär von zivilgesellschaftlichen Akteuren getriebene Fälle oder »kleine«, lokale vs. "große«, übergreifendere Transformationsprozesse) wurden auf Basis der MoC-Bewertungen und der Beschreibung der Entwicklung der Fallbeispiele auf verbindende und differenzierende Charakteristika hin untersucht und für die jeweiligen Gruppierungen typische Entwicklungsmuster und Einfluss- bzw. Erfolgsfaktoren identifiziert.

Weg 3: Induktives Clustering zur Identifikation charakteristischer Muster und Typen von Transformationsprozessen sowie zusätzlicher Erfolgsfaktoren

Um die im Wesentlichen deduktiv gewonnenen Erkenntnisse zu den wichtigsten Erfolgsfaktoren auf Basis der MoC-Faktoren aus Analyseweg 1 und die identifizierten Muster und Typen von Transformationsprozessen aus Analyseweg $2 \mathrm{zu}$ validieren und ggf. nicht berücksichtigte Elemente und Muster zu identifizieren, wurde ausgehend von den einzelnen Fallstudien ein induktives Clustering und eine Detailanalyse der Fallbeispiele vorgenommen. Hierbei kamen mehrere Analyseansätze zum Einsatz:

- Hierarchische Clusterbildung auf Basis statistischer Ähnlichkeiten der Relevanzbewertung von MoC-Faktoren (Single-Linkage-Verfahren auf Basis euklidischer Distanz; vgl. Schels 2008) mit anschließender qualitativer Analyse der resultierenden Cluster

- Systematischer Vergleich von Entwicklungsprozessen der Fallbeispiele mit induktiver Kategorienbildung (angelehnt an die qualitative Inhaltsanalyse nach Mayring 2010) 


\section{Dritter Schritt: Entwicklung von Schlussfolgerungen für Praxis, Politik und Theorie}

Abschließend wurden die in den einzelnen Analyseschritten gewonnenen Erkenntnisse zusammengeführt und wesentliche Schlussfolgerungen für Praxis, Politik und Theorie abgeleitet. Während zum Abschluss dieses Kapitels bereits einige ausgewählte Aspekte thematisiert werden, findet eine vertiefte Auseinandersetzung mit diesen Fragen im Fazit (Kapitel 6) dieses Buches statt.

\subsubsection{Analyse entlang zentraler Erfolgsfaktoren}

In der ersten der drei Querschnittsanalysen erfolgt eine Betrachtung des gesamten Fallstudiensets aus Perspektive der einzelnen MoC-Faktoren. Diese beinhalten die Kontextfaktoren, die Akteurinnen und Akteure, die Veränderungsidee und Lösungsvorschläge, den Transformationsprozess, Zeitaspekte sowie die diesen Kategorien jeweils zugeordneten Teildimensionen.

\subsubsection{Kontextfaktoren: Landschaftsmerkmale und Rahmenbedingungen}

\section{Problemlage und Wahrnehmung}

Die Problemlage und deren Wahrnehmung sind von hoher bis sehr hoher Relevanz in fast allen untersuchten Beispielen. Einzig für die Entwicklung des Gebrauchtwarenhandels über eBay stand kein konkretes, bis dahin als solches wahrgenommenes Problem im Vordergrund der Entwicklung, sondern eine aus neuen technischen Möglichkeiten heraus entstandene Geschäftsidee ohne zunächst offenkundigen Nachhaltigkeitsbezug (Behrendt 2018b). Hinsichtlich der anderen Fälle lässt sich trotz durchgehend hoher Relevanz eine Reihe unterschiedlicher Konstellationen erkennen.

Während ein generelles Umweltbewusstsein und eine zunehmende Aufmerksamkeit für den Klimaschutz in den meisten Fällen eine gewisse Rolle gespielt haben, waren »anfassbarere und dringendere Umweltprobleme - z. B. Luftverschmutzung und Lärmbelastung sowie die daraus resultierenden Gesundheitsprobleme in großen Städten - wesentlich wichtiger für den relativen Erfolg von Transformationsprozessen. Dies lässt sich beispielsweise bei der Entwicklung von E-Mobilität in Kalifornien, den Niederlanden, Norwegen oder China beobachten, die alle mit starker Luftverschmutzung in ihren Metropolregionen $\mathrm{zu}$ kämpfen hatten (Clausen 2017b, 2017c; Beigang und Clausen 2017; Perleberg und Clausen 2017). Auch die Entwicklung des Nichtraucherschutzes und die damit verbundenen regulatorischen Maßnahmen und Verhaltensänderungen lassen sich auf konkrete und immer besser belegbare Gesundheitsbeeinträchtigungen und Geruchsbelästigung, also konkrete körperlich-sinnliche Betroffenheit, zurückführen (Göll 2017). 
Weiterhin scheinen Fälle, in denen ein ganzer Sektor transformiert wurde oder das Potenzial hierfür erkennbar ist, vor allem durch wirtschaftliche oder sicherheitsrelevante Risiken bzw. durch die daraus erwachsenden Gewinnmöglichkeiten motiviert zu sein. Ein Beispiel hierfür findet sich in Dänemark, wo nach der Ölkrise Überlegungen zur Energiesicherheit ein wichtiger Anstoß für den Ausbau des dortigen Wärmenetzes und den verstärkten Einsatz erneuerbarer Energien waren (Tappeser und Fromm 2017a). Auch die Angst vor höheren Energiepreisen war ein vergleichbar relevanter Faktor für die Entscheidung der Gemeinde Wüstenrot, bis 2020 eine unabhängige, auf Erneuerbaren basierende Energieversorgung zu entwickeln (Tappeser 2018b). Dieses Muster lässt sich ähnlich auch für die Transformationsbeispiele der Innovation City Bottrop und den Stadtteil Hyllie in Malmö erkennen, wo langanhaltende Strukturwandelprozesse zu einer wirtschaftlichen Problemlage geführt haben, der mit innovativen Ansätzen für die Erreichung einer klimaneutralen Wärmeversorgung beigekommen werden sollte (Tappeser und Fromm 2018a, 2018b) - mit dem Unterschied, dass die o.g. ökonomischen Risiken sich in diesen beiden Fällen schon vor der Entwicklung einer Transformationsagenda in Richtung Nachhaltigkeit materialisiert hatten. Noch stärker auf wirtschaftliche Chancen ausgerichtet, aber jeweils verbunden mit Problemlagen, die das aktuell dominante Regime betreffen, ist der Fall der E-Mobilität in China. Ausgehend von der Einsicht, dass der technische Vorsprung der westlichen Automobilindustrie in Bezug auf die Verbrennertechnologie nicht aufzuholen ist, sah man in der E-Mobilität eine neue Chance für die Erreichung von Marktführerschaft im Automobilsektor (Beigang und Clausen 2017).

Für Beispiele in der Nische, die stärker zivilgesellschaftlich getragen sind z. B. die Entwicklung von Repair Cafés oder die Entstehung von Unverpackt-Läden im Ressourcenbereich (Mundt und Göll 2018; Göll und Zwiers 2018) - stellt die identifizierte Problemlage von Ressourcenverschwendung und damit verbundenen Umweltbelastungen zwar einen zentralen Beweggrund für die Initiatorinnen und Initiatoren sowie für die Nutzer/-innen solcher Einrichtungen und Praktiken dar. Gleichzeitig ist die mangelnde Wahrnehmung und die direkte Betroffenheit der breiteren Bevölkerung (z. B. hinsichtlich Gesundheit, Versorgungssicherheit oder des wirtschaftlichen Wohlstands) wohl ein wesentliches Hemmnis für die weitere Verbreitung dieser Ansätze.

\section{Pfadabhängigkeiten und Hindernisse}

Die Rolle von Pfadabhängigkeiten und Hindernissen ist über die verschiedenen Fallstudien hinweg recht unterschiedlich ausgeprägt.

Für die Fallstudien in den Bereichen E-Mobilität und Wärmeversorgung, die sich auf Transformationsbeispiele in anderen Ländern beziehen, scheint das Nicht-Vorhandensein bzw. die weniger starke Ausprägung von Pfadabhängigkeiten und Hindernissen, die eine Transformation in Deutschland behindern, einen 
Schlüsselfaktor für die dort jeweils beobachtbaren, vielversprechenden Entwicklungen darzustellen. So konnten sich Kalifornien, die Niederlande und Norwegen zu Vorreitern bei der E-Mobilität entwickeln, da es keine lokal ansässige Automobilindustrie gab, die sich gegen eine entsprechende Gesetzgebung und Förderung hätte wehren können (Clausen 2017b, 2017c; Perleberg und Clausen 2017). In Dänemark und den Niederlanden erlauben wiederum unterschiedliche Besitz- und Industriestrukturen, neue und innovative Ansätze bei der Wärmeversorgung und Gebäudeisolierung einfacher umzusetzen (Tappeser und Fromm 2017b; Tappeser 2018a).

Für andere Transformationsprozesse und -beiträge von Unternehmen scheint die Rolle von Pfadabhängigkeiten und Hindernissen vor allem deshalb vergleichsweise gering, weil sie auf unterschiedliche Art und Weise aus der bestehenden Logik ausbrechen. Dies erklärt sich zum einen durch ungewöhnliche Akteurskonstellationen (z. B. Tesla, welches sich mit Elon Musk aus der IT-Branche heraus entwickelt hat, oder der Post StreetScooter, der durch einen Logistikdienstleister finanziert und aus Perspektive der Produktionstechnik anstelle des Kraftfahrzeugbaus heraus entwickelt wurde; Clausen und Perleberg 2017; Clausen 2017a). Zum anderen relativiert sich die Wirkung von Pfadabhängigkeiten auf Unternehmen durch die Nutzung neuer technischer Möglichkeiten (neben Tesla und StreetScooter z. B. auch die Beispiele eBay und Car-Sharing; Behrendt 2018a, 2018b).

In fast allen Fallstudien in den Bereichen Mobilität und Wärme betreffen wirksame Pfadabhängigkeiten die Preisvorteile konventioneller Lösungen (Verbrennungsmotoren, fossile Wärmeversorgung) aufgrund von bestehenden Infrastrukturen und Skaleneffekten. Gleichzeitig bestehen mit alternativen Technologien, die durch Kostensenkungen bei der Herstellung, Komfortsteigerungen, Subventionierung und Regulierung beginnen, den bestehenden Entwicklungspfad aufzubrechen, Ansätze, um sowohl technologische als auch ökonomische Pfadabhängigkeiten zu überwinden.

Etwas anders gelagert sind die Beispiele im Bereich Ressourcen. Hier sind für die analysierten Fälle zur Verringerung des Ressourcenverbrauchs fast immer technologische und ökonomische Pfadabhängigkeiten wirksam (z. B. kurze Innovationszyklen sowie kostenoptimierte Produktions- und Logistikverfahren), die in enger Verbindung zu Komfortansprüchen und Konsumgewohnheiten der Nutzerinnen und Nutzer stehen. Um sich etablieren zu können, erfordern alternative Technologien oder Praktiken daher (bislang) zusätzlich zur oberflächlichen Anpassung von Konsumgewohnheiten einen tiefergreifenden kulturellen Wandel sowie eine Überwindung bestehender Umsatzinteressen und Produktionslogiken der Hersteller (z. B. in Hinblick auf Reparierbarkeit oder auf den Verzicht auf Verpackungen). 


\section{Weitere Rahmenbedingungen}

Für die Fallbeispiele aus dem Ausland sind insbesondere historisch gewachsene Strukturen und Besonderheiten des jeweiligen politischen Systems von Relevanz, was die Übertragbarkeit der dortigen Erfahrungen zumindest erschwert. Regierungsgestützte Aktivitäten in den Niederlanden, sowohl im Bereich der Wärmeversorgung als auch der E-Mobilität, bauen beispielsweise auf eine jahrhundertealte, korporative Kultur intersektoraler Zusammenarbeit und Kompromissfindung auf, die sich kaum anderswo replizieren lässt (Tappeser 2018a; Perleberg und Clausen 2017). Ähnlich schwierig gestaltet sich ein Transfer des chinesischen Ansatzes zur Förderung von Elektromobilität, der stark von der großen Gestaltungsmacht der chinesischen Regierung getrieben ist und kaum als Blaupause für den deutschen Kontext dienen kann (Beigang und Clausen 2017).

Andere Beispiele bauen wiederum auf Vorgängervorhaben und bestehenden Initiativen auf (z. B. Hyllie, Vangerow; Tappeser und Fromm 2018a; Röben und Behrendt 2018) oder sind stark durch gesellschaftliche Phänomene und Dynamiken wie den Individualismus (z. B. beim Nichtraucherschutz; Göll 2017) oder eine ausgeprägte Konsumkultur beeinflusst (z. B. verpackungsfreies Einkaufen, eBay; Göll und Zwiers 2018; Behrendt 2018b).

\subsubsection{Akteure}

\section{Change agents und ihre Qualifikationen}

Während die sehr zentrale Rolle von Landschaftsmerkmalen und Rahmenbedingungen den Eindruck erwecken mag, dass die Einflussmöglichkeiten durch individuelles Handeln äußerst begrenzt sind, stellen change agents, Promotorinnen und Promotoren sowie ihre spezifischen Qualifikationen den durchgängig am wichtigsten bewerteten Erfolgsfaktor für die untersuchten Transformationsprozesse dar.

Individuelle oder institutionelle change agents, die jeweils eine spezifische Kombination von (Zugang zu) Expertise, Einfluss, Ressourcen und Entscheidungskompetenz mitbringen, waren in allen untersuchten Fallbeispielen essenziell. Gleichzeitig gehören die einzelnen change agents zumeist nicht zum dominanten Regime, sondern sind eher Außenseiter bzw. Schnittstellenakteurinnen und -akteure, die ihre Durchsetzungsstärke und Fähigkeit zur Einflussnahme aus anderen Quellen als den zuvor bestehenden Strukturen im betrachteten sozio-technischen oder sozio-kulturellen System beziehen.

Ein wesentlicher Typus ist hierbei der oder die individuelle Entrepreneur/-in, prototypisch darstellbar an der Person von Elon Musk als Gründer von Tesla (Clausen und Perleberg 2017). Als Silicon-Valley-Unternehmer kommt er aus einer anderen Industrie als seine Konkurrenz in der etablierten Automobilwirtschaft. Gleichzeitig verfügt er über ausreichend Kapital, Kontakte und Gewicht, um dem 
bestehenden Regime von außen etwas entgegenzusetzen. Als charismatische Führungsfigur mit technischem Sachverstand und einer klaren Vision besitzt er zudem die persönlichen Eigenschaften und Qualifikationen, um den ersten wirklich erfolgreichen Hersteller von elektrisch betriebenen Pkw aufzubauen, insbesondere aber das Image der Elektromobilität im öffentlichen Diskurs umzukrempeln und so die etablierte Automobilindustrie vor sich herzutreiben. Ein ähnliches Bild lässt sich im Fall des Post StreetScooter zeichnen, bei dem der Gründer Prof. Schuh als Produktionstechniker und Universitätsprofessor wiederum aus einem ganz anderen Kontext als dem der Automobilindustrie entstammt und mit dem Post-Vorstand Jürgen Gerdes ein unkonventionelles Bündnis zur Entwicklung eines elektrischen Zustellfahrzeugs einging (Clausen 2017a). Andere Beispiele betreffen den ehemaligen Künstler Bas van Abel, der zum Gründer von Fairphone wurde (Zwiers et al. 2018), oder Pierre Omidyar, den Gründer von eBay, der zwar ein vergleichsweise typischer Tech-Entrepreneur ist, aber gleichzeitig zahlreiche abweichende Interessen pflegt (Behrendt 2018b).

Einzelne change agents spielen jedoch nicht nur in Unternehmenskontexten eine Rolle. Auch in den betrachteten kommunalen Initiativen und nationalen Sektorentwicklungen sind zahlreiche Schlüsselakteurinnen und -akteure auszumachen, die die jeweiligen Transformationsprozesse maßgeblich vorangetrieben haben. Hierzu zählen z. B. die Bürgermeister Tischler und Nägele von Bottrop und Wüstenrot (Tappeser und Fromm 2018b; Tappeser 2018b), aber auch Verwaltungsangestellte wie Thomas Löffelhardt aus der Gemeindeverwaltung von Wüstenrot oder Don Drachnand vom California Clean Air and Resources Board (CARB; Clausen 2017b). Weiterhin zu nennen sind policy entrepreneurs (Kingdon und Thurber 1984), die sich auf politischer Ebene für das Vorantreiben von Transformationsprozessen einsetzen. Im Kontext des Stromeinspeisegesetzes spielte beispielsweise Erich Maaß eine wichtige Rolle (Clausen 2017f), ebenso wie der Bundestagsabgeordnete Lothar Binding im Kontext des Nichtraucherschutzes (Göll 2017).

Gleichzeitig lassen sich auch Teams als zentrale change agents in den Fallbeispielen ausmachen, die ihre Netzwerke, Ressourcen und Expertise zusammenbringen, um gemeinsam als Triebkraft für die Beförderung von Transformationsprozessen zu wirken. Dieses Muster ist beispielsweise im Fall von Energiesprong erkennbar (Tappeser 2018a). Die drei Protagonisten ${ }^{2}$ des Marktentwicklungsprograms für Nullenergiesanierungen kamen aus unterschiedlichen Feldern (Bauwirtschaft, Energiewirtschaft und Wissenschaft). Zudem vereinten sie sehr unterschiedliche Charaktereigenschaften, die von einem mitreißenden Redner über einen begabten Netzwerker bis hin zu einem überlegten Theoretiker reichten und somit unterschiedliche Promotorenrollen erfüllten (Machtpromotor, Beziehungspromotor, Fachpromotor).

2 Jan Willem van der Groep, Jasper van den Munckhof und Ivo Opstelten. 
Institutionen wie Energiesprong oder auch das CARB in Kalifornien (Clausen 2017b) lassen sich dabei auch auf institutioneller Ebene als change agents verstehen. Gemein mit den Einzelakteurinnen und -akteuren ist ihnen insbesondere die Brückenfunktion zwischen verschiedenen Akteursgruppen, die es ihnen erlaubt, zwischen den einzelnen Interessen und Akteurinnen und Akteuren zu vermitteln sowie außerhalb der jeweiligen Partikularlogiken neue Lösungsansätze zu entwickeln.

\section{Koalitionen, die einer Transformation positiv gegenüberstehen}

Über die Fallstudien hinweg lassen sich im Wesentlichen vier Gruppen von Akteurinnen und Akteuren identifizieren, die die betrachteten Transformationsprozesse unterstützt haben:

- Unternehmen und Wirtschaftsakteure, die von einem Transformationsprozess wirtschaftlich profitieren oder darauf hoffen. Dies wird besonders deutlich im Kontext von E-Mobilität in China und Kalifornien (Beigang und Clausen 2017; Clausen 2017b), aber auch in Norwegen, wo der Versuch, eine eigene E-Mobilitätsindustrie aufzubauen, letztendlich scheiterte (Clausen 2017c).

- Umweltinstitutionen, deren Interessen bedient werden. Hier sind Institutionen wie das Umweltbundesamt (z. B. in den Fällen von Vangerow, eBay und Carsharing; Röben und Behrendt 2018; Behrendt 2018b, 2018a), Umweltverbände (z. B. Kalifornien, Carsharing, eBay und Vangerow; Clausen 2017b; Behrendt 2018a, 2018b; Röben und Behrendt 2018) oder auch das CARB (im Falle von Kalifornien, Clausen 2017b) zu nennen.

- Politikvertreter/-innen und -institutionen, die sich Profilierungsmöglichkeiten und/oder wirtschaftliche Entwicklung erhoffen. Identifizierbar sind sie z. B. bei der Entwicklung von Wärmenetzen in Dänemark (Parteien des linken Spektrums, die sich für die Etablierung dezentraler Wärmenetze als eine Alternative zur Atomkraft einsetzten; Tappeser und Fromm 2017b) oder der E-Mobilität in China (Ministerien der Zentralregierung, die die Entwicklung einer chinesischen E-Mobilitätsindustrie fördern; Beigang und Clausen 2017).

- Teile der Gesellschaft, die sich als (Umwelt-)Bewegung formieren oder schlicht durch den Status quo benachteiligt werden (z. B. Nichtraucher/-innen im Falle des Nichtraucherschutzes; Göll 2017)

Am erfolgreichsten und relevantesten scheinen diese unterstützenden Akteursgruppen allerdings in Fällen gewesen zu sein, bei denen diese gruppenübergreifende Koalitionen gebildet haben. In Norwegen beispielsweise war eine Koalition von Industriellen, Umwelt-Aktivistinnen und -Aktivisten sowie Fördergebern zentral für die Entwicklung von E-Mobilitäts-Förderprogrammen und Gesetzgebung (Clausen 2017c). Für die Entwicklung von Nullenergie-Sanierungskonzep- 
ten in den Niederlanden spielte wiederum eine Koalition aus Bauunternehmen, Wohnbaugesellschaften,Teilen der Energiewirtschaft und politischen Akteur/ -innen eine wichtige Rolle (Tappeser 2018a). Ebenfalls von Relevanz waren PublicPrivate-Partnerships in Fällen wie der InnovationCity Bottrop oder dem Modellviertel Hyllie in Malmö (Tappeser und Fromm 2018b, 2018a).

\section{Koalitionen, die einem Wandel skeptisch gegenüberstehen}

Skeptische Akteurinnen und Akteure sowie »Bremser« lassen sich in den untersuchten Fallbeispielen an zahlreichen Punkten identifizieren. Gerade bei den Auslandsbeispielen ist jedoch oftmals die Abwesenheit solcher Akteurinnen und Akteure bzw. ein geringes Level an Aufmerksamkeit und Handlungsmöglichkeiten oder ein geringes Level an Kooperation zwischen Akteurinnen und Akteuren mit konvergierenden, dem betrachteten Transformationsprozess zuwiderlaufenden Interessen auffällig und relevant für den Transformationserfolg.

Wie schon erwähnt zeichnen sich die untersuchten Vorreiterstaaten im Bereich E-Mobilität (i.e. Norwegen, Niederlande und Kalifornien; Clausen 2017c; Perleberg und Clausen 2017; Clausen 2017b) nicht zuletzt dadurch aus, dass es keine lokal ansässige Automobilindustrie gibt, während die Automobilindustrie in Deutschland und anderen Ländern alles daran setzt, den Entwicklungspfad des Verbrennungsmotors - im Sinne von veto players - so lange wie möglich fortzuführen (Clausen 2017d). In China besteht zwar eine große lokale Automobilindustrie, ihr Marktanteil innerhalb Chinas beträgt jedoch weniger als 30 Prozent. Mit der Entwicklung von Elektroantrieben besteht die Chance, den Vorsprung westlicher Hersteller zu überwinden (Beigang und Clausen 2017). Etwas anders stellt sich die Situation bei den untersuchten Unternehmen dar. Während einerseits die Relevanz von Bremsern und Kontra-Koalitionen im Kontext unternehmerischer Transformationsbeiträge nicht so hoch ist wie auf Ebene nationaler Sektorentwicklungen, lässt sich in den Fällen von Tesla und StreetScooter zusätzlich beobachten, dass die etablierte Automobilindustrie die Wettbewerber lange Zeit nicht ernst genommen und unterschätzt hat (Clausen und Perleberg 2017; Clausen 2017a).

Potenzielle Bremser einer Verbesserung der Energieeffizienz von Gebäuden und eines Umbaus der Wärmeversorgung in Richtung erneuerbarer Energien (insbesondere die fossile Brennstoff- sowie Teile der Heiztechnikindustrie) sind vor allem auf nationaler Ebene aktiv (Clausen und Hinterholzer 2017; Tappeser und Chichowitz 2017d), wurden in Dänemark jedoch schon in den 1980er-Jahren erfolgreich in den Wandel eingebunden und treten, wohl aufgrund der großen Entfernung von den eigentlichen Regimeakteurinnen und -akteuren, in den untersuchten lokalen Beispielen kaum in Erscheinung. Einzig der lokale Schornsteinfeger in Wüstenrot zeigte sich als durch das bestehende Regime getragener Akteur skeptisch gegenüber einer Umstellung der Wärmeversorgung auf dezentrale Wärmenetze und Wärmepumpen (Tappeser 2018b). 


\subsubsection{Veränderungsidee und Lösungsvorschläge}

\section{Passgenaue und dynamische Umsetzungslösung}

Die Existenz einer passgenauen und dynamischen Umsetzungslösung gehört zu den durchgängig am relevantesten bewerteten Erfolgsfaktoren der betrachteten Fallbeispiele. Was das heißt, ist je nach Fall jedoch wiederum sehr unterschiedlich.

Ein Großteil der Beispiele verknüpft Umwelt- und Nachhaltigkeitszielstellungen mit wirtschaftlichen Interessen, sei es der Aufbau einer international wettbewerbsfähigen Automobilindustrie in China (Beigang und Clausen 2017), der wirtschaftliche Aufschwung im strukturschwachen Bottrop (Tappeser und Fromm 2018b) oder die Effizienzsteigerung von Reparaturdienstleistungen zur Sicherung des wirtschaftlichen Überlebens im Falle der Reparaturwerkstätten Vangerow (Röben und Behrendt 2018). Weiterhin werden z. T. gesundheitliche Interessen bedient (vgl. Fallstudien zu Elektromobilität in Kalifornien, China, Norwegen und den Niederlanden sowie Nichtraucherschutz; Clausen 2017b, 2017c; Beigang und Clausen 2017; Perleberg und Clausen 2017; Göll 2017) oder auf bestehenden Traditionen und Strukturen aufgebaut (z. B. die Reparatur von Haushaltsgegenständen im Fall von Repair Cafés oder die genossenschaftlich und dezentral organisierte Energieversorgung in Dänemark; Mundt und Göll 2018; Tappeser und Fromm 2017b).

Die Adressierung dieser zusätzlichen Mehrwerte scheint dabei für den Transformationserfolg von hoher Bedeutung (siehe auch den Abschnitt zu Co-Benefits). Vor allem auf Nachhaltigkeitsaspekte fokussierende Umsetzungsansätze hingegen, die für die jeweiligen Nutzerinnen und Nutzer und für die Betroffenen eher voraussetzungsvoll sind, scheinen nur relativ geringe Chancen zu haben, sich über eine kleine Nische von nachhaltigkeitsaffinen Stakeholdergruppen hinaus etablieren zu können. Sie wirken somit vor allem als Diskussionsimpulse und Experimentierräume, die Entwicklungen mit zusätzlichen Mehrwerten und Skalierungspotenzial vorbereiten, aber kaum aus sich selbst heraus entwickeln können (z. B. Unverpackt-Läden; Göll und Zwiers 2018).

Die untersuchten Fälle hingegen, in denen eine umfassende Transformation des dominanten Regimes entweder schon stattgefunden hat oder bei denen zumindest ein hohes Potenzial hierfür zu erkennen ist, sind dabei fast alle von einer mutigen Vision und einer Skalierungsstrategie getrieben, die unterschiedliche Stakeholdergruppen einzeln und oftmals nacheinander adressiert und mit ihren spezifischen Interessen und Bedürfnissen abholt bzw. einbindet. Dabei lassen sich folgende Typen identifizieren:

- Im Wesentlichen markt- und technologiegetriebene Prozesse, die von einzelnen Unternehmerinnen und Unternehmern in Gang gesetzt wurden. Zum Beispiel verfolgte Tesla den Ansatz, zuerst einen Luxussportwagen für wohl- 
habende early adopter zu produzieren und daraufhin durch die Entwicklung günstigerer Modelle in den Massenmarkt vorzustoßen (Clausen und Perleberg 2017). Der Post StreetScooter hingegen verfolgt mit seinem frugalen Innovationsansatz die Strategie, zunächst sehr günstige Elektroautos für einen spezifischen Anwendungszweck zu produzieren und von dieser Basis aus weitere Kundengruppen zu erreichen (Clausen 2017a).

- Sektorübergreifende Initiativen wie die Innovation City Bottrop, in der zielgruppenspezifisch Programme entwickelt werden, um energetische Sanierungen und erneuerbare Wärmekonzepte zu realisieren (Tappeser und Fromm 2018b)

- Politisch gestützte Prozesse wie Energiesprong in den Niederlanden, in dem ein regierungsfinanziertes Marktentwicklungsteam für Nullenergiesanierungen als deal broker gezielt verschiedene Stakeholdergruppen zusammengebracht und geeignete Rahmenbedingungen und Anreizstrukturen geschaffen hat, um einen dynamischen Prozess loszutreten (Tappeser 2018a)

- Politische Regulierung und Planung wie im Falle der Entwicklung dänischer Wärmenetze oder des EEG in Deutschland (Tappeser und Fromm 2017b; Clausen 2017f)

Obwohl sehr unterschiedlich, zeichnen sich all diese Fälle durch eine klare Vision und eine Skalierungsstrategie aus, die gleichzeitig dynamisch aufVeränderungen und neue Erkenntnisse reagieren kann.

\section{Strategien- und Instrumentenmix}

Die in den betrachteten Beispielen angewandten politischen Strategien und Instrumente zählen größtenteils zum etablierten Repertoire von Finanzierung für Forschung und Entwicklung und Kaufanreizen, der Subventionierung von Infrastruktur, unterschiedlichen Regulierungsansätzen, öffentlichen (Pilot-)Aufträgen und Beteiligungsprozessen, wobei letztere vor allem bei den lokalen Beispielen im Wärmebereich (Wüstenrot, Bottrop und Hyllie) und nicht so sehr bei den nationalen Prozessen von Relevanz waren. Konventionelle Maßnahmen und Anreize zeigten sich bei entsprechend ambitionierter (und im Falle von Fördermaßnahmen gut finanzierter) Ausgestaltung als durchaus effektiv, sobald funktionierende Alternativlösungen auch wirklich verfügbar waren (z. B. E-Mobilität in den Niederlanden, Norwegen, China; Perleberg und Clausen 2017; Clausen 2017c; Beigang und Clausen 2017). Weniger kostenintensive, flankierende und gleichzeitig vergleichsweise erfolgreiche Instrumente betreffen im Bereich Elektromobilität insbesondere die Bevorzugung von E-Fahrzeugen im Verkehr durch die Freigabe von Bus- und Carpooling-Spuren, Parkplätzen und durch die Befreiung von Fahrverboten. Im Wärmebereich sind vor allem die Festschreibung des Gemeinnützigkeitsprinzips in der Wärmeversorgung und eine umfassende 
Wärmeleitplanung in Dänemark zu nennen sowie die zur Marktentwicklung von Nullenergiesanierungen in den Niederlanden umgesetzte Stimulierung von Innovation und Angebotsentwicklung durch Bündelung und Sichtbarmachung von Nachfrage einerseits und forciertem Wissenstransfer zwischen konkurrierenden Anbietern über mehrstufige, wettbewerbliche Dialogverfahren andererseits (Tappeser 2018a).

Die untersuchten Transformationsbeiträge in Form von unternehmerischen Aktivitäten und Initiativen zeichnen sich auf Strategie- und Instrumentenebene vor allem durch schnelle Geschwindigkeit und Professionalisierung sowie durch ausgeprägte Lobby-Aktivitäten und Vernetzung aus. Hierfür spielten oftmals externe Investoren eine wichtige Rolle, aber auch Zukäufe (z. B. im Fall von eBay; Behrendt 2018b) oder der Aufbau von Franchise-Systemen und Gründungshilfen zur dezentralen Verbreitung eines Konzepts (z. B. bei Repair Cafés und Unverpackt-Läden; Mundt und Göll 2018; Göll und Zwiers 2018).

\section{Umgang mit Zielkonflikten}

Häufig in den Fallbeispielen anzutreffende Zielkonflikte bestehen zwischen unterschiedlichen Organisationsebenen (z. B. zwischen Lokal- und Zentralregierung in China bei der E-Mobilitätsförderung oder im Fall von Vangerow zwischen Reparaturnetzwerk und einzelnen Betrieben; Beigang und Clausen 2017; Röben und Behrendt 2018), zwischen stärker von Ideen starker Nachhaltigkeit getriebenen und gewinnorientierten Modellen (z. B. Carsharing, verpackungsarmes Einkaufen, eBay), sozialer und ökologischer Nachhaltigkeit (Fairphone), unterschiedlichen Anspruchsgruppen (z. B. Raucher/-innen, Nichtraucher/-innen und der Tabakindustrie im Kontext des Nichtraucherschutzes), aber auch zwischen unterschiedlichen Umweltzielen wie der Reduktion von Energie- und Flächenverbrauch (vertikale Landwirtschaft).

Die hierzu in den Fallstudien identifizierbaren Lösungsansätze waren sowohl temporaler und dialogischer als auch eher planerisch-rationaler Natur. Im Falle des Nullemissionsfahrzeug-Mandats (zero emission vehicle, ZEV) in Kalifornien wurden immer wieder Verkaufsziele für Fahrzeughersteller gesetzt und dann gestreckt, um den Druck aufrecht zu erhalten und gleichzeitig mehr Zeit für die technologische Bearbeitung von Zielkonflikten zwischen Komforterwartungen der Nutzer/-innen, Umsatzerwartungen der Hersteller und Umweltzielen des CARB zu entschärfen (Clausen 2017b). Dialogische Ansätze umfassen sowohl in diesem als auch in anderen Fallbeispielen (z. B. Wüstenrot, Energiesprong, Dänemark) Beteiligungsprozesse und ein kooperatives Vorgehen, in denen Zielkonflikte früh erkannt und Lösungsansätze entwickelt werden konnten (Tappeser 2018b, 2018a; Tappeser und Fromm 2017b). Im Kontext von Wärmenetzen in Dänemark spielen zudem planerisch-rationale Ansätze eine wichtige Rolle. Im Rahmen der Wärmeleitplanung werden beispielsweise auf lokaler Ebene und in Kombination 
mit Beteiligungsverfahren umfassende Kosten-Nutzen-Analysen durchgeführt, um Zielkonflikte sichtbar zu machen und eine transparente Entscheidungsgrundlage herzustellen (Tappeser und Fromm 2017b).

Neben diesen eher auf die Auflösung von Zielkonflikten ausgerichteten Strategien und Mechanismen, an deren Ende Kompromisslösungen bzw. idealerweise Win-Win-Situationen geschaffen wurden, ließen sich in den Fallbeispielen auch Mechanismen identifizieren, die vor allem auf die Durchsetzung einer konkreten Zielstellung ausgelegt waren, ohne auf konkurrierende Zielstellungen Rücksicht zu nehmen. $\mathrm{Zu}$ nennen sind hier insbesondere starke Konzentrationen von Entscheidungskompetenzen einzelner Akteurinnen und Akteure, die ein Durchregieren von oben erlauben (z. B. im Kontext der E-Mobilität in China sowie in Unternehmenskontexten wie Tesla oder eBay) sowie die Nutzung von Gelegenheiten, in denen gegenläufige Interessen und Zielstellungen aus unterschiedlichen Gründen nur geringe Wirkung entfalten (siehe hierzu auch den Abschnitt »Gelegenheitsfenster«).

\subsubsection{Transformationsprozess}

\section{Horizontale Koordination}

Horizontale Koordination zwischen verschiedenen Akteursgruppen scheint gerade in umfassenderen Transformationsprozessen von zentraler Bedeutung zu sein. Sowohl die nationalen Sektorentwicklungen im Bereich E-Mobilität (Kalifornien, Niederlande, Norwegen) als auch der Aufbau eines Marktes für Nullenergiesanierungen in den Niederlanden (Energiesprong) zeichnen sich durch eine enge Kooperation zwischen verschiedenen Sektoren aus. Bestehen stark gegenläufige Interessen, wie bei der Auseinandersetzung zwischen Atomenergiebefürworterinnen und -befürwortern sowie -gegnerinnen und -gegnern im Kontext der Entwicklung von Wärmenetzen in Dänemark, lässt sich auch eine zunächst eher konfrontative Dynamik beobachten, die im weiteren Verlauf zu einer stärkeren Koordination zwischen unterschiedlichen Sektoren führt.

\section{Vertikale Koordination}

Vertikale Koordination findet sich in den betrachteten Fallbeispielen in unterschiedlichen Formen. Zahlreiche Beispiele zeichnen sich durch eine enge Koordination und gegenseitige Ergänzung unterschiedlicher Ebenen aus (insbesondere Kalifornien, Niederlande und Norwegen im Bereich Elektromobilität sowie Dänemark im Wärmebereich). Die Entwicklung von Elektromobilität in China sowie in kleinerem Maßstab - die Entwicklung von Unternehmen wie Tesla oder eBay sind vor allem durch hierarchische Command-and-Control-Strukturen geprägt. Im Kontext des Nichtraucherschutzes lässt sich wiederum eine Trickle-Down-Beziehung erkennen, in der Impulse auf WHO- und EU-Ebene zu nationalen Ent- 
wicklungen geführt haben, die wiederum Bundesländer und Lokalregierungen dazu bewegt haben, Rauchverbote und damit verbundene Maßnahmen umzusetzen.

\section{Institutionalisierung}

Institutionalisierungsaktivitäten waren ebenfalls von hoher Bedeutung in fast allen untersuchten Fallbeispielen. Zunächst ist hier der Aufbau von Organisationsstrukturen zu nennen, sei es in Form von Strukturen innerhalb von Unternehmen (Tesla, StreetScooter, Vangerow, Fairphone) oder in Form unterschiedlicher Organisationen und Träger innerhalb eines untersuchten Felds (z. B. Vereine, Genossenschaften und Unternehmen im Kontext von Carsharing). Weiterhin ließ sich die Institutionalisierung von Kooperationen und Netzwerken zwischen verschiedenen Akteuren im Rahmen gemeinsamer Projekte beobachten (z. B. Umsetzungsagenturen im Rahmen von public-private-partnerships wie in Bottrop, Netzwerke von Fachbetrieben für spezialisierte Reparaturdienstleistungen durch Vangerow).

In Verbindung zur politischen Ebene spielen Lobby- und Dachverbände ebenfalls eine wichtige Rolle in den untersuchten Beispielen. Ob im Kontext von EMobilität in Norwegen, Repair Cafés, Energiesprong in den Niederlanden oder im Bereich des Nichtraucherschutzes haben in Vereinen und Stiftungen institutionalisierte Lobbyverbände eine zentrale Rolle gespielt, um das jeweilige Thema auf politischer Ebene sichtbar zu machen und Änderungen anzustoßen.

Institutionalisierungsansätze auf politischer Ebene umfassen wiederum unterschiedliche Erscheinungsformen, von Gesetzen und Vorschriften (z. B. zu Elektromobilität in Norwegen) zu Beschlüssen (z. B. zur Klimaneutralität durch den Gemeinderat in Wüstenrot), Verträgen (z. B. dem Klimavertrag in Hyllie) und Plänen (z. B. den politischen 5-Jahres-Plänen in China).

\section{Nischenaktivitäten}

Nischenaktivitäten sind für fast alle Transformationsbeispiele von hoher Bedeutung, entweder weil sie selbst noch als Nischenaktivität verstanden werden können oder weil sie aus solchen hervorgegangen sind. Teilweise spielen auch parallel laufende Nischenaktivitäten eine wichtige Rolle. In China beispielsweise sind immer mehr kleine Hersteller auf dem Land aktiv, die einfache Elektroautos sowie insbesondere Elektroscooter produzieren und so recht geräuschlos die Elektromobilität für die Bevölkerung erfahrbar machen.

\section{Beteiligung}

Die in den Fallbeispielen identifizierbaren Beteiligungsformen reichen von indirekten Bemühungen wie der Herstellung von Transparenz gegenüber Anspruchsgruppen (z. B. Fairphone) hin zu umfassenden Co-Design-Prozessen mit Nutzerinnen und Nutzern (z. B. StreetScooter) und Kooperationen zwischen unterschiedlichen 
Anspruchsgruppen (z. B. im Kontext von Energiesprong). Zur Anwendung kamen dabei sowohl formelle Anhörungen und Arbeitsgruppen mit Stakeholderbeteiligung, aber auch informelle Beteiligungsprozesse, z. B. im Rahmen von Gesetzgebungsverfahren im Kontext des EEG oder des Nichtraucherschutzes.

\section{Co-Benefits}

Co-Benefits, im Sinne einer Schaffung von zusätzlichen Vorteilen, die keinen direkten Bezug zu Nachhaltigkeits- und insbesondere Umweltgesichtspunkten im engeren Sinn haben, spielen in den meisten Beispielen eine sehr zentrale Rolle. Hierbei handelt es sich zumeist um ökonomische Vorteile, wie

- die Schaffung einer günstigeren Alternative zu konventionellen Lösungen (z. B. im Fall von Energiesprong oder dem Post StreetScooter; Tappeser 2018a; Clausen 2017a),

- die Erschließung neuer Geschäftsmodelle und Gewinnmöglichkeiten für einzelne Unternehmen (z. B. im Fall von Tesla, eBay, Vangerow; Clausen und Perleberg 2017; Behrendt 2018b; Röben und Behrendt 2018)

- oder die Entwicklung eines ganzen Industriezweiges wie im Fall der E-Mobilität in China (Beigang und Clausen 2017).

Gleichzeitig spielen aber auch weniger bzw. nur indirekt monetär getriebene CoBenefits eine wichtige Rolle, z.B. die Verringerung von Gesundheitsrisiken im Kontext von E-Mobilität auf Länderebene, Komfortzuwächse bei der Entscheidung für Investitionen in energetische Sanierung oder Autonomiebestrebungen bei der Wärmeversorgung (sowohl auf kommunaler Ebene in Wüstenrot als auch auf nationaler Ebene in Dänemark).

\section{Veränderungskultur und Wissensbasis}

Betrachtet man die Entstehungskontexte der untersuchten Transformationsbeispiele, lässt sich feststellen, dass fast alle Beispiele aus einem eher als dynamisch und innovativ charakterisierbaren Hintergrund heraus erwachsen sind.

Die untersuchten Vorreiterländer im Bereich Elektromobilität zählen allesamt zu den innovativsten und wissenslastigsten Volkswirtschaften weltweit. Kalifornien ist als Technologie- und IKT-Paradies bekannt. Die Niederlande werden insbesondere für ihre Kompetenz und Innovationsfähigkeit in den Bereichen Landwirtschaft, Wasser, Medizin, Chemie, Energie und Logistik geachtet. Norwegen treibt mit Hilfe hoher Öleinnahmen schon seit Jahren den Umbau seiner Wirtschaft voran und auch China wird inzwischen als hochdynamische Technologiemacht wahrgenommen. Die betrachteten E-Mobilitätsunternehmen Tesla und StreetScooter entstammen dem Silicon Valley und dem Dunstkreis der gerade im technischen Bereich sehr renommierten RWTH Aachen. 
Im Wärmebereich kann die Entwicklung von Nullenergiesanierungen in den Niederlanden vom dortigen Innovationsklima ebenso profitieren wie die Entwicklung von Elektromobilität. Dänemark gilt als hochinnovative Volkswirtschaft mit ausgezeichneten Hochschulen. Bei den drei untersuchten kommunalen Initiativen lassen sich wiederum leichte Unterschiede feststellen. Die Gemeinde Wüstenrot, gelegen zwischen Heilbronn und Stuttgart, ließe sich eher als strukturkonservativ bezeichnen. Gleichzeitig profitiert sie von den dynamischen Industrien (insbesondere von der Automobil-, aber auch von der Solarindustrie und IKT) in der Region. Die Veränderungskultur in Bottrop und Hyllie ist hingegen stärker aus der Not heraus geboren: Der Niedergang alter Industrien hat hier zu einem hohen Veränderungsdruck geführt. Gleichzeitig konnten die jeweiligen Transformationsprozesse von Wissensinfrastrukturen und Universitäten im näheren Umland profitieren (Wuppertal und Duisburg-Essen im Fall von Bottrop, Lund im Fall von Hyllie/Malmö).

Die Fallbeispiele im Bereich Ressourcen entspringen größtenteils dynamischen und gut gebildeten urbanen Milieus (Carsharing, Repair Cafés, verpackungsfreies Einkaufen), die Unternehmen eBay und Fairphone wiederum technologie-affinen Kreisen im Silicon Valley und den Niederlanden, die auf entsprechende Wissensressourcen zurückgreifen konnten.

\section{Reflexivität und Erfolgskontrolle}

Reflexive Prozesse und unterschiedliche Formen der Erfolgskontrolle sind integraler Bestandteil fast aller untersuchten Transformationsbeispiele.

Offizielle Evaluationen von politischer Seite sind in allen breiteren und auf nationaler Ebene verankerten Prozessen durchgeführt worden. Beispielhaft genannt seien die Vorbereitung der 5-Jahres-Pläne in China, die regelmäßige Überarbeitung und Anpassung des ZEV-Mandats in Kalifornien, zahlreiche Kursanpassungen in den Niederlanden oder Evaluationsstudien und Kurskorrekturen in Norwegen. Auch in Dänemark wurden die Rahmenbedingungen und Zielsetzungen zum Ausbau von Wärmenetzen über die Jahre hinweg mehrfach angepasst und auf Basis gemachter Erfahrungen überarbeitet. Für den relativ schnell und kompakt verlaufenden Marktentwicklungsprozess des Energiesprong-Programms in den Niederlanden war insbesondere eine hohe Flexibilität hinsichtlich der Ausgestaltung des Programms von Bedeutung, die kurze Iterationsschleifen und eine schnelle Übersetzung empirischer Erkenntnisse und Erfahrungen in Kurskorrekturen ermöglichte.

Die betrachteten kommunalen Initiativen wurden allesamt von wissenschaftlichen Begleitforschungsprojekten flankiert, deren Zwischenergebnisse dazu genutzt wurden, Kursänderungen vorzunehmen. Im Kontext einzelner Unternehmen und Initiativen spielten vor allem interne Lernprozesse und Strategieanpassungen eine Rolle. 


\section{Ressourcenausstattung}

Für einen Großteil der Fallbeispiele war eine ausreichende staatliche Förderung eine ganz zentrale Bedingung, um die jeweiligen Prozesse anzustoßen und am Laufen zu halten.

Im Bereich E-Mobilität stechen insbesondere Norwegen und China mit hohen staatlichen Fördersummen hervor, wobei gerade Norwegen schon früh im Prozess sehr viel (staatliches und privates) Geld investierte. Auch in den Niederlanden spielen relativ hohe Förderungen und Steuererleichterungen eine Rolle. In Kalifornien sollte zunächst vor allem die Industrie die notwendigen Investitionen stemmen - erst ab 2012, als bereits erste Markterfolge von Tesla ersichtlich waren, wurde eine Beteiligung durch ein Rabattprogramm eingeführt. Für die Beispiele im Wärmebereich war staatliche Förderung ebenfalls essenziell - sei es für den Aufbau von Netzinfrastrukturen in Dänemark oder die kommunalen Initiativen Wüstenrot, Bottrop und Hyllie, die allesamt lokale, nationale und EU-Mittel bündeln, um ihre Ziele zu erreichen. Im Kontext von Energiesprong war insbesondere die Verfügbarkeit einer recht hohen Summe von Geldern zur freien Verfügbarkeit für unterschiedlichste Experimente von Bedeutung, während öffentliche $\mathrm{Zu}$ schüsse in der Umsetzung keine Rolle spielten.

Bei den Unternehmensfallstudien spielten zudem Gelder von Investorinnen und Investoren eine wichtige Rolle. Insbesondere Tesla und eBay hätten ohne Investorenkapital nicht ihre heutige Größe erreichen können.

\subsubsection{Zeitaspekte}

\section{Auslöser und Fensternutzung}

Auslöser und die strategische Nutzung von Gelegenheitsfenstern scheinen in fast allen Fallbeispielen eine zentrale Rolle für den Erfolg der betrachteten Transformationsprozesse zu spielen. Hierbei sind unterschiedliche Muster erkennbar:

\section{Technologiegetriebene Auslöser und Gelegenheitsfenster}

Technische Innovationen spielen in zahlreichen Fallbeispielen eine große Rolle und stellen einen wichtigen Auslöser für viele der betrachteten Transformationsprozesse dar. Aus wirtschaftlicher Perspektive besteht mit der Verfügbarkeit einer neuen Technologie immer auch ein Gelegenheitsfenster, sich durch frühe Nutzung einen Vorsprung und entsprechend große Marktanteile zu sichern.

Die technologische Entwicklung von Lithium-Ionen-Akkus und damit die erstmalige Verfügbarkeit von Batterien mit ausreichender Energiedichte für die Herstellung von Elektrofahrzeugen mit attraktiver Reichweite und Ladedauer kann beispielsweise als zentraler Auslöser für die Entwicklung von Elektromobilität in allen untersuchten Beispielen in diesem Bereich gesehen werden. 
Gerade im Fall von China, das als aufstrebende Großmacht bisher nicht in der Lage war, der etablierten europäischen, US-amerikanischen, japanischen und südkoreanischen Automobilindustrie etwas entgegenzusetzten, spielt das Gelegenheitsfenster, den technologischen Vorsprung westlicher Hersteller mit dem anstehenden Technologiewechsel aufzuholen, eine ganz wesentliche Rolle für den ambitionierten Einstieg in die Elektromobilität (Beigang und Clausen 2017). Ähnlich verhält es sich mit dem Hersteller Tesla, der mit dem First-Mover-Vorteil und der vom Dotcom-Boom ausgelösten Kapitalschwemme im Silicon Valley gleich zwei parallele Gelegenheitsfenster für sich nutzen konnte (Clausen und Perleberg 2017).

Für den Aufbau der Online-Handelsplattform eBay und der damit verbundenen Transformation des Gebrauchtwarenhandels stellte das zum Gründungszeitpunkt 1996 noch in den Anfängen steckende Internet Auslöser, disruptive Innovation und Gelegenheitsfenster zugleich dar. Während sich später zahlreiche andere Online-Handelsplattformen entwickelten, konnte eBay als erster im Markt eine entsprechend dominante Position aufbauen.

Im Fall von Energiesprong waren ebenfalls technische Innovationen von zentraler Bedeutung, um den Prozess loszutreten. Insbesondere die zunehmend einfache Nutzbarkeit digitaler Gebäudemodelle (building information modelling; BIM) und die Möglichkeit, diese für die computergestützte Vorfertigung von Fassadenmodulen zu verwenden, können als Auslöser verstanden werden. Ein wesentlicher Beweggrund für die niederländische Bauindustrie, sich an dem Programm $\mathrm{zu}$ beteiligen, war dabei wiederum das sich ergebende Gelegenheitsfenster, auch international einen Technologievorsprung zu erlangen.

\section{Krisen}

Eine weitere Kategorie von Auslösern und Gelegenheitsfenstern stellen unterschiedliche Krisen dar. Während hier latent auch die globale Umwelt- und Klimakrise eine Rolle spielt, sind vor allem konkretere und direkter erfahrbare Ereignisse wichtige Auslöser in den untersuchten Beispielen. Für die Entwicklung der Wärmenetze in Dänemark waren beispielsweise die beiden Ölkrisen der 1970erJahre wichtige Trigger-Momente, die den Prozess zum Ausbau von Wärmenetzen, dezentralen KWK-Anlagen und erneuerbaren Energien in Gang setzten. Die als problematisch angesehene Luftverschmutzung in Metropolregionen (in China, Kalifornien, den Niederlanden und Norwegen) stellte - neben der technischen Machbarkeit (s.o.) - wiederum einen zentralen Auslöser für Bestrebungen zur Förderung von Elektromobilität auf nationaler Ebene dar. Ähnlich gelagert sind auch Strukturwandelprozesse in Bottrop und Malmö als Auslöser für die dortigen Bestrebungen zur Transformation des Wärmesystems in Verbindung mit der Hebung von damit verbundenen wirtschaftlichen Potenzialen. 


\section{Ablenkung von Veto-Spielern}

Ein weiteres, in manchen untersuchten Fällen erkennbares Gelegenheitsfenster besteht in der Ablenkung von Veto-Spielern durch parallel laufende Prozesse (Krisen oder auch andere Umstände). Diese ermöglichen es change agents, Entwicklungen loszutreten, die ansonsten kaum möglich gewesen wären. So scheint der Zeitpunkt kurz nach der deutschen Wiedervereinigung für die erfolgreiche Verabschiedung des Stromeinspeisegesetzes als Vorläufer des EEG und der späteren Energiewende besonders günstig gewesen zu sein, da die großen Energiekonzerne damals damit beschäftigt waren, die Übernahme der ostdeutschen Netze und Kraftwerke zu organisieren (Clausen 2017f). Ähnlich verhält es sich im Fall von Energiesprong in den Niederlanden. Hier hatte das Projekt erheblichen Spielraum, um zu experimentieren und neue Strategien zu entwickeln, weil ein Regierungswechsel dazu geführt hatte, dass keine klare Zuständigkeit für das Vorhaben auf ministerieller Seite bestand (Tappeser 2018a).

\section{Veränderungen in regulatorischen Rahmenbedingungen}

Für zahlreiche Fallbeispiele stellten auch Veränderungen in gesetzlichen Vorgaben oder politischen Prioritäten einen wichtigen Auslöser dar, die zum Teil wieder auf andere Auslöser zurückgehen können. So lassen sich E-Mobilitätsbestrebungen in den Niederlanden nicht zuletzt auf neue Grenzwerte zur Luftverschmutzung auf EU-Ebene zurückführen (Perleberg und Clausen 2017). Vor der Entwicklung des klimasmarten Viertels Hyllie stand die Verabschiedung eines Klimaschutzplans durch die Stadt Malmö (Tappeser und Fromm 2018a). Schließlich war das Auslaufen des Konzessionsvertrags des lokalen Stromnetzes mit dem Energieversorger EnBW ein zentrales Movens für die Entscheidung der Gemeinde Wüstenrot, bis $2020 \mathrm{zu}$ einer Plusenergiegemeinde zu werden (Tappeser 2018b).

\section{Geschwindigkeit und Rhythmus}

Hinsichtlich der Geschwindigkeit und des Rhythmus lässt sich ebenfalls eine Anzahl unterschiedlicher Muster erkennen.

\section{Lange Vorlaufzeiten}

Den betrachteten Transformationsprozessen gingen oftmals lange Vorlaufzeiten voraus. Im Kontext von Elektromobilität beispielsweise musste ca. 20 Jahre auf die technologische Reifung und Verfügbarkeit ausreichend leistungsstarker Batterien gewartet werden. In Bottrop und Malmö stand im Vorfeld des betrachteten Transformationsprozesses ein längerer Strukturwandel - mit negativen Auswirkungen und auch einem in der Folge zunehmenden Veränderungsdruck. 
Rückschläge und Wartephasen

Ähnlich den Vorlaufzeiten sind auch Rückschläge oder längere Wartephasen in den Beispielen identifizierbar. Dazu gehört aus Perspektive nachhaltigkeitsorientierter Verbraucherinnen und Verbraucher beispielsweise die schwankende Verfügbarkeit von Elektroautos in Kalifornien. Während ab 1998 erste Modelle wie der GM Impact oder EV1 verkauft wurden, waren in den Jahren 2001 bis 2009 vergleichbare Autos kaum im Angebot. Erst mit dem Markteinstieg größerer Automobilhersteller ab etwa 2009 nahm der Sektor wieder Schwung auf.

\subsubsection{Gesamtbetrachtung der Fallbeispiele}

Die Auswertung der Relevanzbewertung von Erfolgsfaktoren nach dem Modelsof-Change-Ansatz über die 20 hier betrachteten Fallstudien hinweg hat gezeigt, dass die analysierten Erfolgsfaktoren und Kontextvariablen in der Tat alle relevant für die Entwicklung von Transformationsprozessen in Richtung einer Green Economy zu sein scheinen. Im Schnitt weisen alle betrachteten Erfolgs- und Kontextfaktoren eine mittlere bis hohe Relevanz auf. Gleichzeitig wird deutlich, dass einige Faktoren wichtiger sind als andere und dass signifikante Unterschiede bestehen zwischen unterschiedlichen Beispielen, Sektoren, Skalen und Phasen von Transformationsprozessen.

In Bezug auf Landschaftsmerkmale und Rahmenbedingungen wurden vor allem die Problemlage und Wahrnehmung, aber auch weitere Rahmenbedingungen für sehr relevant befunden (siehe Abbildung 37) Pfadabhängigkeiten und Hindernisse spielen ebenfalls in fast allen Beispielen eine wichtige bis sehr wichtige Rolle. Gleichzeitig zeigen sich im Detail und über die allgemeine Bedeutung der jeweiligen Kategorien hinaus deutliche Unterschiede hinsichtlich der Art und Ausprägung der Variablen sowie des damit verbundenen Einflusses auf die Entwicklung der jeweiligen Transformationsprozesse.

Hinsichtlich der Erfolgsfaktoren stechen insbesondere die zentrale Bedeutung der

- Existenz einer passgenauen und dynamischen Umsetzungslösung im Bereich der Veränderungsidee,

- Nischenaktivitäten und Co-Benefits im Bereich der Prozesskategorien (Transformationsprozess),

- change agents und ihrer Qualifikationen im Bereich der Akteurinnen und Akteure sowie der

- Nutzung von Trigger-Ereignissen (Auslöser) und Gelegenheitsfenstern im Bereich der Zeitaspekte

als durchgängig relevant für die verschiedenen Fallbeispiele heraus. Andere Kategorien wie »Institutionalisierung«, »Beteiligungsprozesse« oder der »Umgang mit 
Zielkonflikten « sind wesentlich abhängiger von der Art des betrachteten Beispiels und nicht in allen Fällen relevant.

Abbildung 37: Heatmap der für die Transformationsprozesse relevantesten Erfolgsfaktoren nach $\mathrm{MoC}$

\begin{tabular}{|c|c|}
\hline Landscape und Rahmenbedingungen & Transformationsprozess \\
\hline Problemlage und Wahrnehmung & Horizontale Koordination zwischen Sektoren \\
\hline Pfadabhängigkeiten und Hindernisse & Vertikale Koordidnation zwischen politischen Ebenen \\
\hline Weitere Rahmenbedingungen & Institutionalisierung \\
\hline Veränderungsidee & Nischenaktivitäten \\
\hline Umsetzungslösung & Beteiligungsprozesse \\
\hline Strategien und Instrumentenmix & Co-Benefits \\
\hline Umgang mit Zielkanflikten & Veränderungskultur und Wissensbasis \\
\hline Akteure & Reflexivităt, Erfolgskontrolle und Lemprozesse \\
\hline \multirow{3}{*}{$\begin{array}{l}\text { Change Agents und ihre Qualifikationen } \\
\text { unterstützende Akteure und Koalitionen } \\
\text { skeptische Akteure und Koalitionen }\end{array}$} & Ressourcenausstattung \\
\hline & Zeitaspekte \\
\hline & Auslöser und Fensternutzung \\
\hline & Prozessgeschwindigkeit und -rhythmus \\
\hline
\end{tabular}

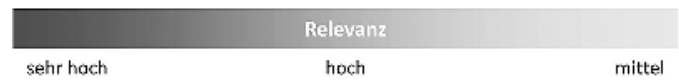

Die Schattierungen spiegeln die jeweils durchschnittliche Relevanzbewertung für die einzelnen MoC-Faktoren über alle Fallstudien hinweg wider

Quelle: Eigene Darstellung, adelphi.

\subsubsection{Analyse entlang unterschiedlicher Typen von Transformationsbeispielen}

Die vorangegangene Analyse zielte vor allem darauf ab, jene Faktoren des Modelsof-Change-Ansatzes zu identifizieren, welche sich bei Betrachtung des gesamten Fallstudienquerschnitts für den Erfolg der Transformationsbeispiele als am relevantesten herausgestellt haben. Nun folgt, als zweiter Analysestrang, eine Betrachtung ähnlicher Typen bzw. Gruppen gleichartiger Transformationsbeispiele. Ziel ist es, gruppenspezifische Erfolgsfaktoren zu bestimmen. Insgesamt fünf Typen ähnlicher Transformationsbeispiele wurden gebildet: »kommunale Initiativen«, »Unternehmen«, »nationale Regierungsinitiativen«, »nationale Sektorentwicklungen« sowie die Gruppe vom Typus »Technologie und soziale Praxis«.

Betrachtet man die einzelnen Gruppen hinsichtlich ihrer Ausrichtung auf die verschiedenen Transformationsbereiche, ergibt sich für die meisten Gruppen ein 
eher heterogenes Bild. Während die drei kommunalen Initiativen allesamt dem Bereich der Wärmeversorgung zuzurechnen sind (Bottrop, Wüstenrot, Hyllie), verteilen sich die fünf unternehmerischen Transformationsfälle einerseits auf den Bereich Elektromobilität (Tesla, StreetScooter), andererseits auf den Bereich Ressourcen (Fairphone, eBay, Vangerow). Die nationalen Regierungsinitiativen setzen sich aus zwei themenübergreifenden Beispielen (EEG, Nichtraucherschutz) und einem Wärmewendefall (Energiesprong) zusammen. Die cases der Gruppe nationale Sektorenentwicklung kommen überwiegend aus dem Feld der Elektromobilität (Niederlande, Kalifornien, China, Norwegen), lediglich die Studie zu Wärmenetzen in Dänemark bildet hier eine Ausnahme. Zuletzt finden sich beim Gruppentyp »Technologie und soziale Praxis« drei Transformationsbeispiele aus dem Bereich Ressourcen (Repair Cafés, Carsharing, verpackungsfreies Einkaufen) sowie mit der Studie zu vertikaler Landwirtschaft ein themenübergreifendes Beispiel.

Hinsichtlich der MoC-Kategorien sind die Unterschiede zwischen den einzelnen Typen insgesamt relativ gering, allerdings zeigen sich trotzdem gewisse Differenzen.

\subsubsection{Kommunale Initiativen}

Wie erwähnt betreffen die untersuchten kommunalen Initiativen ausschließlich Beispiele im Bereich Wärme. Deswegen ist eine Übertragbarkeit auf andere Bereiche unter Umständen nur bedingt gegeben. Gleichzeitig deutet sich an, dass gerade auf der lokalen Ebene Beteiligungsprozesse und der geeignete Umgang mit Zielkonflikten sowie ein umfassender Strategien- und Instrumentenmix besonders wichtig für den Transformationserfolg zu sein scheinen.

Auch lässt sich bei allen drei Beispielen in diesem Bereich eine enge Kooperation von Politik, Wirtschaft und Wissenschaft identifizieren. Bei allen stand dabei die Belebung der lokalen Wirtschaft im Vordergrund. Jedoch lassen sich keine eindeutigen Rückschlüsse auf die Initiatorinnen und Initiatoren ziehen: Während der Impuls für die Projekte in Bottrop und in Hyllie von wirtschaftlichen Akteurinnen und Akteuren ausging, lag die Initiative in Wüstenrot bei der Politik bzw. der Gemeindeverwaltung. Konkrete Umweltwirkungen einer transformierten Wärmeversorgung spielten zwar argumentativ eine Rolle, im Vordergrund stand jedoch vor allem die Sicherung von Zukunftsfähigkeit, Lebensqualität und einer langfristigen wirtschaftlichen Perspektive. Ein zentraler Beweggrund für die Einbindung wissenschaftlicher Akteurinnen und Akteure war dem Anschein nach vor allem der dadurch ermöglichte Zugang zu zusätzlichen Fördermitteln sowie die durch eine wissenschaftliche Begleitung geförderte Legitimation der Vorhaben. Gleichzeitig stellten auch das fachliche Wissen sowie das Monitoring und die Erfolgskontrolle durch wissenschaftliche Akteurinnen und Akteure einen wesentlichen Mehrwert für die Projekte dar. 
Zuletzt ist den drei Projekten gemein, dass die Transformation der Wärmeversorgung nur einen Teilaspekt einer breiteren Entwicklungsagenda darstellt. Zwar sind Wärmeversorgung und Klimaneutralität jeweils zentrale Bausteine der Initiativen, diese wurden jedoch mit anderen Zielstellungen wie Familienfreundlichkeit, Bildungsangeboten und der Schaffung von Arbeitsplätzen verknüpft.

\subsubsection{Unternehmen}

Betrachtet man die analysierten Unternehmen, ist eine recht große Bandbreite an unterschiedlichen Beispielen zu finden. Mit eBay und Tesla sind zwei der bekanntesten Unternehmen der Welt mit Umsätzen in Milliardenhöhe und Zehntausenden von Mitarbeitenden vertreten (Behrendt 2018b; Clausen und Perleberg 2017). ${ }^{3}$ Der Post StreetScooter gehört zwar seit 2014 zu einem in Bezug auf die Mitarbeiterzahl noch wesentlich größeren multinationalen Konzern und will ab Mitte 2018 20.000 Fahrzeuge pro Jahr produzieren, ist aber für sich genommen noch ein vergleichsweise kleines Unternehmen (Clausen 2017a). Fairphone ist ebenfalls noch überschaubar in der Größe. Zwar wurden laut Herstellerangaben inzwischen über 100.000 Fairphones verkauft, insgesamt beschäftigt das Unternehmen jedoch erst 80 Mitarbeiterinnen und Mitarbeiter (Zwiers et al. 2018; Fairphone 2018). Die Vangerow $\mathrm{GmbH}$ ist das zugleich älteste und kleinste Unternehmen - sie betreibt mit einer Handvoll Mitarbeiterinnen und Mitarbeiter verschiedene OnlinePortale, die Kundinnen und Kunden mit einem Netzwerk unabhängiger Reparaturwerkstätten und Gutachter/-innen verknüpft und blickt auf eine 60-jährige Firmengeschichte zurück (Röben und Behrendt 2018).

Trotz der sehr unterschiedlich gelagerten Unternehmensbeispiele lassen sich Erfolgsfaktoren erkennen, die bei allen Beispielen eine besondere Rolle gespielt haben. Geschwindigkeit und damit verbunden die Nutzung von Gelegenheitsfenstern war ebenso wie die Existenz einer passgenauen und dynamischen Umsetzungslösung für alle untersuchten Unternehmen von besonders hoher Bedeutung. Fast alle profitierten dabei von der Entwicklung und/oder Nutzung neuer technischer Möglichkeiten, die ihnen ein Alleinstellungsmerkmal auf dem Markt verschafften - sei es die Entwicklung besonders luxuriöser und reichweitenstarker (Tesla) oder besonders günstiger und praktischer (Post StreetScooter) Elektrofahrzeuge oder die frühe Nutzung der Möglichkeiten des Internets (eBay und Vangerow; beide starteten schon 1995 mit der Entwicklung von Online-Portalen; Röben und Behrendt 2018; Behrendt 2018b). Gemein ist diesen vier Beispielen auch die zentrale Rolle einzelner Unternehmerpersönlichkeiten, die ihre jeweilige Idee und Vision vorangetrieben haben und entscheidend für den Erfolg der Unternehmen sind (Elon Musk bei Tesla, Pierre Omidyar bei eBay, Prof. Günther

3 eBay Inc.: Umsatz von 8,98 Mrd. USD und ca 12.600 Mitarbeitende in 2016 (Statista 2018a); Tesla Motors Inc.: Umsatz von 7 Mrd. USD und ca. 13.000 Mitarbeitende in 2016 (Statista 2018b). 
Schuh bei StreetScooter, Detlef Vangerow bei der Vangerow GmbH, Bas van Abel bei Fairphone).

Während alle diese Unternehmen auf unterschiedliche Art und Weise einen wichtigen Beitrag zur Mobilitätswende bzw. zur Verringerung des Ressourcenverbrauchs leisten, sind wirtschaftlicher Erfolg und Wachstum in fast allen Fällen zentrale Treiber und Motivation der Prozesse (im Fall von eBay hat das Element der Ressourcenschonung zu Anfang z. B. gar keine Rolle gespielt; Behrendt 2018b). Etwas anders gelagert ist Fairphone: Aus der gemeinnützigen Waag Society für Kunst, Wissenschaft und Technologie heraus entstanden, wird es vom Gründer eher als Kunstprojekt denn als Unternehmen gesehen - Wachstum und wirtschaftlicher Erfolg stellen eher ein in Kauf zu nehmendes Übel als den originären Unternehmenszweck dar (Handelsblatt 2017).

\subsubsection{Nationale Regierungsinitiativen}

$\mathrm{Zu}$ den nationalen Regierungsinitiativen gehören sehr unterschiedliche Beispiele. Das Stromeinspeisegesetz und das EEG sowie Energiesprong befassen sich mit der Förderung von Innovationen durch die Subventionierung erneuerbarer Energien bzw. mit der Schaffung von geeigneten Rahmenbedingungen und Starthilfe für den Aufbau eines Marktes für Nullenergiesanierungen (Clausen 2017f; Tappeser 2018a). Das Beispiel Nichtraucherschutz betrifft zwar, ähnlich wie das Beispiel zu Stromeinspeisegesetz und EEG, einen legislativen Prozess, hat jedoch weniger eine technologische Innovation als die Eindämmung bzw. Exnovation einer gesundheitsschädlichen sozialen Praktik zum Ziel (Göll 2017).

Gemeinsam ist den Beispielen, dass sie jeweils auf eine recht lange Entwicklungsgeschichte zurückführbar sind. $\mathrm{Zu}$ Beginn standen dabei jeweils vor allem zivilgesellschaftliche und wissenschaftliche Nischenakteurinnen und -akteure, die z. B. auf die Gefahren des Rauchens oder die Probleme mit fossiler Energiegewinnung aufmerksam gemacht und sich für die Etablierung von Alternativen eingesetzt haben. Im Falle des Stromeinspeisegesetzes und später des EEG entwickelten sich aus dieser Bewegung heraus zahlreiche Gründer/-innen und Erfinder/-innen, die kleine Windkraftunternehmen etablierten und schließlich die Unterstützung von lokalen Bundestagsabgeordneten gewinnen konnten. Weitere Unterstützung wurde parteiübergreifend durch die Bündelung der Interessen eines Wasserkraftbetreibers aus der CSU und von Landwirtschaftsakteurinnen und -akteuren (Biomasseproduktion) gewonnen, sodass schließlich eine Mehrheit für das Gesetz zustande kam. Im Kontext des Rauchverbots war der Besuch eines Bundestagsabgeordneten beim Deutschen Krebsforschungszentrum (DKFZ) und die Auseinandersetzung mit einer Studie des DKFZ ausschlaggebend für das Ingangsetzen des legislativen Prozesses. Im Fall von Energiesprong waren es ebenfalls von der Umweltbewegung geprägte, wissenschaftliche Akteurinnen und Akteure, die im Rahmen eines regierungsfinanzierten Forschungsprojekts den 
Transition-Management-Ansatz entwickelten. Aus diesem erwuchs ein umfassender Agenda- und Beteiligungsprozess und dieser gab schließlich den Impetus für Energiesprong als Marktentwicklungsprogramm für Nullenergiesanierungen.

In Bezug auf die MoC-Faktoren sticht in diesem Kontext insbesondere die Bedeutung eines umfassenden Instrumentenmixes als Erfolgsfaktor für die beschriebenen Beispiele heraus. Während das Stromeinspeisegesetz und das EEG einzelne Instrumente beschreiben, stehen sie im Kontext einer Vielzahl von Förderinstrumenten und Regularien. Diese beförderten die Entwicklung und die Verbreitung erneuerbarer Energien in Deutschland, indem vor allem wirtschaftliche Co-Benefits für private und kommerzielle Anlagenbetreiber geschaffen wurden. Die Erfolgsgeschichte des Nichtraucherschutzes lässt sich ebenfalls auf eine Vielzahl ineinandergreifender Einzelansätze zurückführen, die neben Rauchverboten in unterschiedlichen Kontexten auch Werberestriktionen und Informationskampagnen umfassen. Das Energiesprong-Programm hat wiederum finanzielle Förderung, Prozessbegleitung, die Anpassung von Regularien und Innovationsmanagement erfolgreich in einer Initiative vereint, um einen neuen Sanierungsmarkt zu schaffen.

Im Vergleich insbesondere zu den Unternehmensbeispielen, aber auch zu den kommunalen Initiativen sticht bei den nationalen Regierungsinitiativen die Vielzahl unterschiedlicher Akteurinnen und Akteure sowie change agents heraus, die gemeinsam maßgeblich für die Einleitung und den Erfolg der jeweiligen Prozesse verantwortlich waren.

\subsubsection{Nationale Sektorenentwicklungen}

Die betrachteten nationalen Sektorentwicklungen sind die "größten« Transformationsprozesse, die im Rahmen der Fallstudien untersucht wurden. Der Fokus lag dabei jeweils auf der Verbreitung einer bestimmten Technologie (E-Mobilität bzw. Wärmenetze im Fall von Dänemark) sowie auf der Betrachtung der Entstehung und Wirkungsweise unterstützender politischer Maßnahmen in den jeweiligen Ländern. Ähnlich wie bei den Regierungsinitiativen spielen auch in diesen Beispielen die Vorgeschichte und die in den jeweiligen Ländern vorherrschenden Rahmenbedingungen sowie ein ausgewogener Instrumentenmix eine zentrale Rolle für den Transformationsprozess. In Dänemark wurden in den Städten schon früh Wärmenetze etabliert, zudem bestand eine kleinteilige, oftmals kooperativ organisierte Strom- und Wärmewirtschaft, auf deren Strukturen die Förderung und Weiterentwicklung von Wärmenetzen ab den 1980er-Jahren aufbauen konnten (Tappeser und Fromm 2017b). In den Niederlanden, Kalifornien und Norwegen gab es keine etablierte Automobilindustrie, die sich als Gegner der Förderung von Elektromobilität hätte aufstellen können. In China wiederum wurde eine Chance erkannt, sich zum Technologieführer zu entwickeln. In allen Fallbeispielen spielten dabei die mit den konventionellen Lösungen (Verbrennungsmotor 
und Ölheizungen) verbundenen Risiken und Nachteile (Luftverschmutzung und Versorgungssicherheit bzw. Abhängigkeit von Importen) eine wichtige Rolle für den Impuls, die Entwicklung bzw. Förderung nachhaltigerer Alternativen anzuschieben.

\subsubsection{Technologien und soziale Praktiken}

Die untersuchten Technologien und sozialen Praktiken sind ebenfalls recht heterogen. Einerseits stellen Repair Cafés und verpackungsfreies Einkaufen in sogenannten Unverpackt-Läden Nischenphänomene dar, die trotz wachsender Beliebtheit nur einen sehr kleinen Teil der Gesellschaft ansprechen (Mundt und Göll 2018; Göll und Zwiers 2018). Gleichzeitig erfreut sich Carsharing inzwischen immer größerer Verbreitung in urbanen Milieus, jedoch ohne dass Umweltschutz als Motivation zwingend im Vordergrund steht (Behrendt 2018a). Vertikale Landwirtschaft wiederum ist ein technologischer Ansatz, der sich noch weitestgehend im experimentellen Stadium befindet (Kahlenborn und Tappeser 2018). Insgesamt ist die Analyse von Erfolgsfaktoren für diese Beispiele schwierig, da bisher kaum mengenrelevante Erfolge aus der Nische heraus zu beobachten sind. Einzig das Beispiel Carsharing verdeutlicht bis zu einem gewissen Grad, wie sich aus zivilgesellschaftlichen und eher von einer Vision getriebenen Nischen heraus skalierbare Lösungen für die breitere Masse entwickeln können - mit der gleichzeitigen Einschränkung, dass der Umweltvorteil, der aus dieser Entwicklung erwächst, zunehmend umstritten ist (Behrendt 2018a).

\subsubsection{Analyse entlang des Prozessverlaufs}

Nach den ersten beiden Teiluntersuchungen - zunächst aus Perspektive der Models-of-Change-Kategorien, daraufhin mit Blick auf Spezifika innerhalb der fünf Fallbeispieltypen - folgt nun eine dritte Analyse entlang des Prozessverlaufs. Dieser Betrachtungsweise liegt die Annahme zugrunde, dass Transformationsprozesse verschiedene Phasen durchlaufen, in denen jeweils unterschiedliche Faktoren eine signifikante Rolle für den weiteren Prozessverlauf spielen. Vor diesem Hintergrund sollen auf Grundlage der betrachteten Fallstudien besondere Charakteristika und relevante Erfolgsfaktoren in Abhängigkeit vom Entwicklungsstadium der Transformationsbeispiele analysiert werden. Einschränkend sei jedoch angemerkt, dass nur ein kleiner Teil der Fallstudien Transformationsprozesse betrachtet, die bereits so weit fortgeschritten sind, dass eine Analyse des gesamten Prozessverlaufs möglich ist. Ein Großteil der Fälle befindet sich in der Beschleunigungsphase, einige auch noch im Nischenstadium. Eine Analyse, die sich mit der Relevanz unterschiedlicher Erfolgsfaktoren im Zeitverlauf beschäftigt, kann für spätere Phasen des Prozesses nur auf eine dementsprechend dünnere Datenbasis zurückgreifen (siehe Abbildung 38). Trotz dieser Einschränkung 
lassen sich einige interessante Erkenntnisse aus der übergreifenden Betrachtung der Studien im Zeitverlauf und im Hinblick auf die MoC-Kategorien ableiten.

Abbildung 38: Übersicht über die Fallstudien nach Transformationsphase

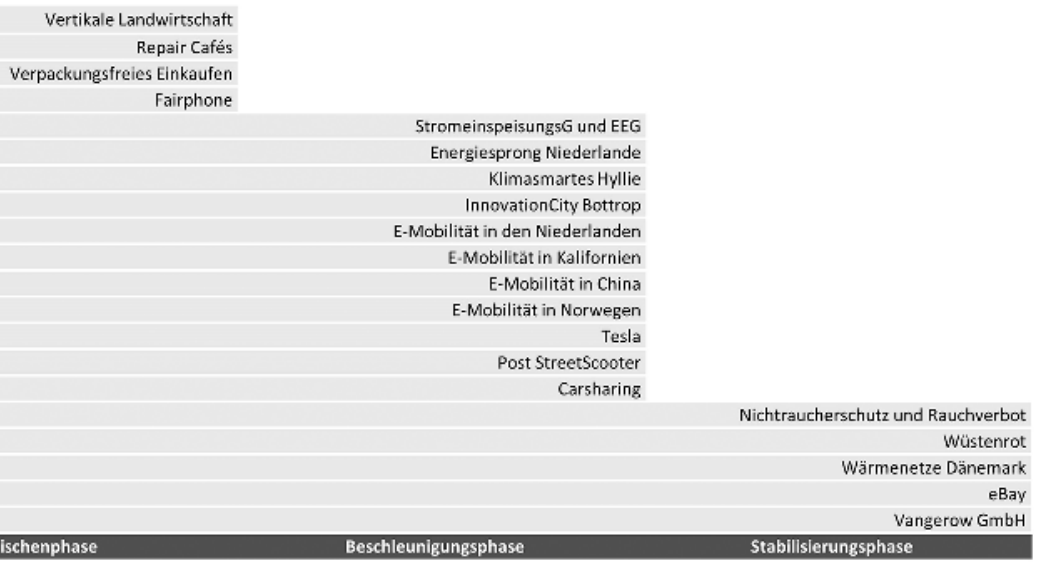

Quelle: Eigene Darstellung, adelphi.

Zunächst wurde im Rahmen der Analyse der Prozessverläufe in den Fallstudien deutlich, dass die in der Literatur gebräuchliche Unterteilung der Prozessdynamik in Diffusions- (Rogers 2003) bzw. Transformationsphasen (Sachverständigenrat für Umweltfragen 2016) nur bedingt hilfreich für das Verständnis komplexer Transformationsprozesse ist. Mit der darauf basierenden Differenzierung in Nischenphase, Beschleunigungsphase und Stabilisierungsphase ließ sich die reale Entwicklung der Transfomationsprozesse nur unzureichend erfassen. Diese Beobachtung deckt sich auch mit der für unterschiedliche Phasenmodelle bestehenden Kritik anderer Autorinnen und Autoren, die die empirische Validität der Modelle in Frage stellen (z. B. Jann und Wegrich 2009; Karnowski et al. 2006).

Ein geeigneteres Betrachtungsmodell scheint die insbesondere von Geels (2002; 2011) entwickelte Multi-Level-Perspektive (MLP) und das in diesem Zusammenhang entwickelte Verständnis von technologischen Transformationen als evolutionäre Rekonfiguration darzustellen. Gleichzeitig zeigt sich recht deutlich, dass das Modell einerseits großes Erklärungspotenzial bietet, andererseits aber auch einzelne Elemente unterbelichtet. Entwicklungen, die sich in der Nische etablieren, aber keinen destabilisierenden Effekt auf das Regime ausüben, sind im bestehenden Modell unterrepräsentiert. Ebenso werden Nischenentwicklungen, die an verschiedenen Punkten scheitern und sich nicht durchsetzen, nur unzureichend erklärt (siehe Abbildung 39). 
Abbildung 39: Multi-Level-Perspektive nach Geels

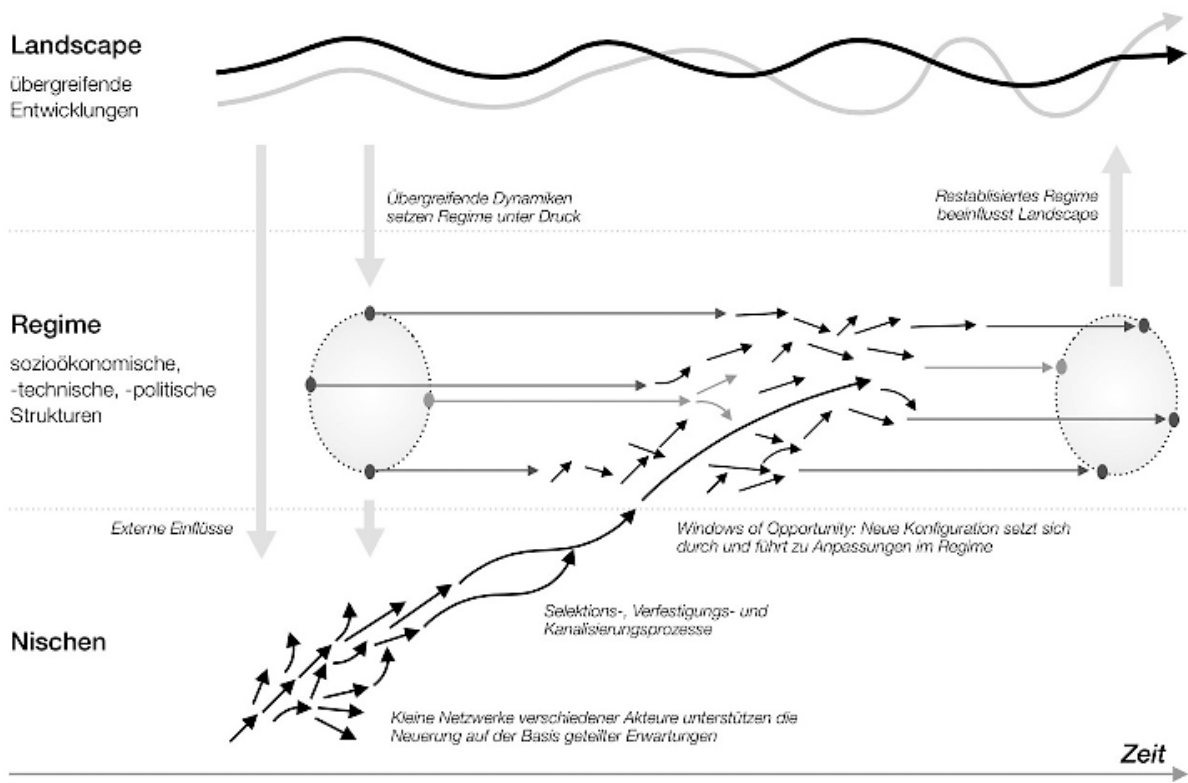

Quelle: Gedankenstrich.org (2014) auf Basis von Ceels (2002) und Geels und Schot (2007).

Der im Rahmen dieser Analyse eingenommene Fokus auf Erfolgsfaktoren wirft im Kontext der Multi-Level-Perspektive zusätzlich die Frage auf, welche charakteristischen Konstellationen und Entscheidungsmomente sich aus den betrachteten Transformationsbeispielen destillieren lassen, in denen jeweils spezifische Faktoren eine Rolle dafür spielen, wie sich der weitere Prozessverlauf gestaltet. Idealtypisch wurden sieben solcher Konstellationen identifiziert, die auch als Eingriffspunkte für unterstützende politische Aktivitäten dienen können und zum Teil auf Überlegungen von Reinhard Loske (2014) zu Diffusionsmustern sozial-ökologischer Wenden aufbauen. ${ }^{4}$ Sie beinhalten die Problemerkennung (1), die Entwicklung von Alternativen (2), den Take-off (3), die Destabilisierung des alten Regimes (4), den Rebound (5), die Etablierung eines neuen Regimes (6) und die Stabilisierung in der Nische (7). Die Konstellationen lassen sich an verschiedenen Punkten in der MLP-Evolutionsdynamik verorten (siehe Abbildung 40) wobei in unterschiedlichen Prozessen nicht alle Konstellationen in gleichem Ausmaß zu beobachten sind.

4 Reinhard Loske (2014) identifiziert im Kontext der Energiewende fünf Diffusionsmuster: »Die Negation des Falschen« (1), »Die Entwicklung von Alternativen in der Nische« (2), »Politischer Wandel braucht Pioniere, Ausbreitung braucht Reformpolitik« (3), »Soziale Bewegungen als Treibende Kraft« (4), »Erfolgreiche Diffusion bei Substanzverlust durch Mainstreaming«(5). 
Abbildung 40: Multi-Level-Perspektive auf Basis der Transformationsbeispiele

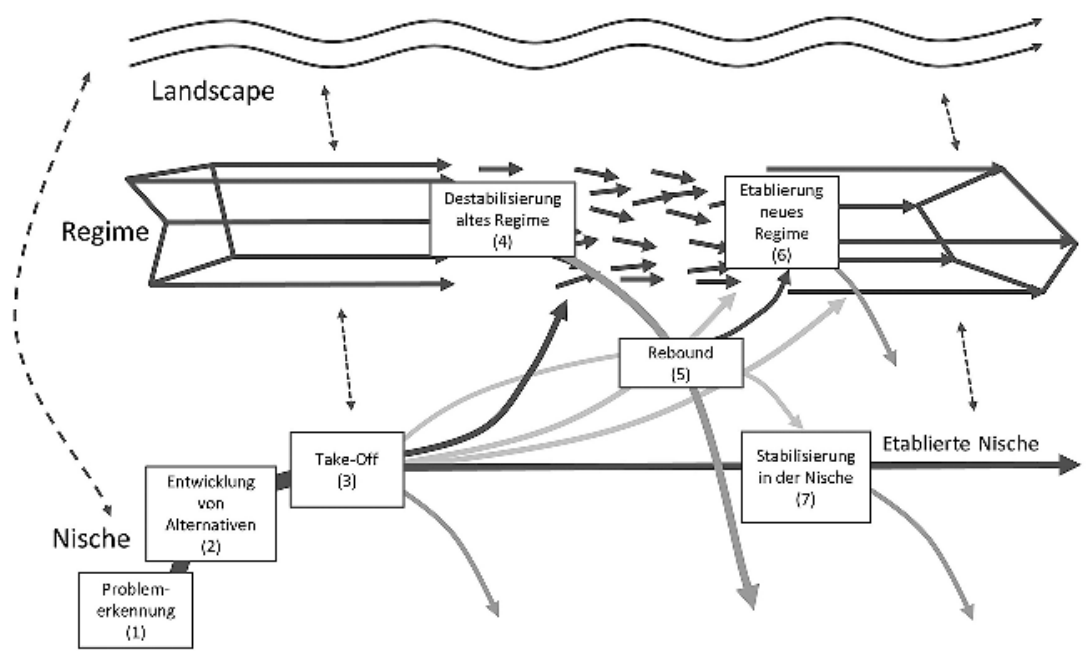

Quelle: Eigene Darstellung, adelphi.

Im Folgenden wird auf Basis der Fallbeispiele analysiert, welche Erfolgsfaktoren in Bezug auf die einzelnen Konstellationen für den weiteren Prozessverlauf von besonderer Bedeutung sind.

\section{Konstellation 1: Problemerkennung}

Noch vor der Entwicklung von Neuerungen in kleineren Nischen steht in fast allen Fällen die Erkenntnis einer relativen Dysfunktionalität des bestehenden Regimes, die sich vor allem durch Interaktionen zwischen Nischenakteuren und Landschaftsentwicklungen auszeichnet. Von sehr hoher Relevanz sind daher Faktoren, die mit der Identifikation von Missständen zu tun haben. Hierunter fallen bestimmte Typen von change agents mit der notwendigen analytischen und kommunikativen Kompetenz, um Missstände zu erkennen und diese effektiv zu kommunizieren. Im Kontext von Umweltproblematiken spielen hier oftmals einzelne Wissenschaftlerinnen und Wissenschaftler eine Rolle, die Fehlentwicklungen identifizieren und empirisch belegen können. Für die Beispiele in den Bereichen Wärmeversorgung und Elektromobilität sind hier z. B. frühe Klimaforscherinnen und -forscher zu nennen, die die Problematik einer dauerhaften Nutzung fossiler Brennstoffe und die Gefahr eines anthropogenen Klimawandels erkannt haben und als vorbereitende Akteurinnen und Akteure für die weitere wissenschaftliche, zivilgesellschaftliche und politische Bearbeitung der Klimafrage zentral waren.

Akteurskoalitionen, die für einen (nicht unbedingt näher spezifizierten) Wandel eintreten und das Problembewusstsein in der Bevölkerung fördern, spielen ebenfalls eine wichtige Rolle. Sie bereiten die Entwicklung von Lösungsansätzen 
in der Nische vor und bauen auf der Ebene der Landschaft Druck auf das Regime auf - hier sind vor allem Multiplikatorinnen und Multiplikatoren aus der Zivilgesellschaft von besonderer Bedeutung. In den einzelnen Transformationsbeispielen lassen sich diesbezüglich z. B. lokale Vertreter/-innen der Umweltbewegung identifizieren, die mit den Studentenbewegungen der 6oer- und 70er-Jahre Auftrieb erlangten (insb. relevant bei den Länderfallstudien zu E-Mobilität in Norwegen, Kalifornien und den Niederlanden sowie zu Wärmenetzen in Dänemark). Ebenfalls relevant für die Identifikation von Problemlagen sind eine ausreichende Wissensbasis und funktionierende Lernprozesse sowie u. U. Trigger-Events (Auslöser), durch die bestehende Missstände sichtbar werden. Für die Entwicklung der E-Mobilität sind hier frühe Smog-Katastrophen zu nennen, beispielsweise London 1952, wo eine besondere Wetterlage in Verbindung mit zunehmendem Verkehrsaufkommen und Schornsteinabgasen bis zu 12.000 Todesopfer mit sich brachte und die weltweite öffentliche Wahrnehmung von Luftverschmutzung nachhaltig beeinflusste (Bell et al. 2004). Die Ölkrisen der 1970er-Jahre sind sowohl in Bezug auf die Mobilitäts- und Wärme- als auch auf die Ressourcenbeispiele ein relevanter Auslöser gewesen. Insgesamt deckt sich diese Konstellation weitestgehend mit dem von Loske (2014) identifizierten Muster der »Negation des Falschen«.

\section{Konstellation 2: Entwicklung von Alternativen}

Die Entwicklung von Alternativen ist häufig gleichbedeutend mit dem Aufweichen bzw. der Überwindung technologischer Pfadabhängigkeiten, ohne die nach der Problemerkennung ein Pfadwechsel nicht stattfinden kann. Ein solcher Prozess ist in der Regel geprägt von lösungsorientierten change agents, die neue Technologien, aber auch soziale Praktiken und Prozessinnovationen entwickeln und propagieren. In den Länderfallstudien zu E-Mobilität sind hier beispielsweise frühe Prototypen und Hersteller von Elektrofahrzeugen in Norwegen und Kalifornien zu nennen. Ähnlich wichtig für den Fall von Energiesprong war die Möglichkeit, mit unterschiedlichen Sanierungskonzepten experimentieren sowie unterschiedliche Methoden der Marktaktivierung anwenden zu können (Förderungen, Wettbewerbe, Plattformen zum Erfahrungsaustausch etc.). Ein weiteres Beispiel für die Entwicklung möglicher Alternativen - wenn auch noch nicht mit dem Erfolg der obigen Beispiele - ist die vertikale Landwirtschaft, die auf die Raumknappheit in Ballungsräumen mit dem Ansatz mehrstöckiger Gewächshäuser eine flächeneffiziente Alternative zu traditionellen Anbaumethoden schafft. Doch nicht immer braucht es eine Neuentwicklung - oft genügt es, auf Lösungswege zu rekurrieren, die zwischenzeitlich vergessen oder marginalisiert worden sind: In den Fallstudien zu Vangerow und den Repair Cafés greifen die »neuen" Alternativen de facto auf traditionelle Verfahren zurück, da das Reparieren von Produkten erst durch die Wegwerfkultur seit den 1980er-Jahren verdrängt wor- 
den war. Ähnliches gilt für verpackungsarmes bzw. -freies Einkaufen. Nischenaktivitäten und Experimente, aber auch Reflexivität und Erfolgskontrolle sowie funktionierende Lernprozesse sind in dieser Phase zentral, um Lösungsansätze $\mathrm{zu}$ testen, zu verbessern und für einen breiteren Einsatz vorzubereiten.

\section{Konstellation 3: Take-off}

In dieser Konstellation findet für in der Nische entwickelte Alternativen eine Weichenstellung statt - hier entscheidet sich, ob und in welcher Geschwindigkeit eine Diffusion in den Mainstream und damit eine Veränderung des Regimes stattfindet, die Alternative wieder von der Bildfläche verschwindet oder sich zunächst in der Nische halten kann.

Wesentliche Bedingungen, die einen Take-off als eine Diffusion in den Mainstream hinein begünstigen, bestehen in der Passgenauigkeit und Anpassungsfähigkeit der entwickelten Lösungsansätze. Die Umsetzungslösung muss den Bedürfnissen und Interessen einer zunehmend größeren Gruppe von Anwenderinnen und Anwendern oder Unterstützerinnen und Unterstützern gerecht werden und mit den bestehenden Rahmenbedingungen zumindest insoweit in Einklang gebracht werden, dass der Verbreitungsprozess an Fahrt aufnehmen kann. Dies lässt sich konkret im Kontext der Entwicklung von Produkten beobachten, beispielsweise anhand der Entwicklungsfolge der Fahrzeuge von Tesla. Während mit dem Tesla Roadster zuerst ein reines Nischenprodukt auf den Markt gebracht wurde, folgten darauf eine Limousine, die auch für den täglichen Gebrauch und Familien nutzbar ist, sowie zuletzt ein preisgünstigerer Mittelklassewagen, der erkennbar auf den Massenmarkt ausgerichtet ist (Clausen und Perleberg 2017). Für das Vorantreiben der Energiewende war wiederum die anspruchsgruppengerechte Umsetzung einer Gesetzesinitiative wie des Stromeinspeisegesetzes zentral. Während des Gesetzgebungsprozesses wurden beispielsweise die Interessen der Windindustrie maßgeblich mitberücksichtigt, da diese angebotsseitig eine wesentliche Rolle für das Mainstreaming nachhaltiger Energieversorgung spielen würde (Clausen 2017f). Die Ressourcenausstattung wird wichtiger, genauso der Strategien- und Instrumentenmix, die Geschwindigkeit und die Nutzung von Gelegenheitsfenstern. In den betrachteten Beispielen sind jeweils einzelne (im Kontext der Unternehmensfallstudien, aber auch bei den kommunalen Initiativen) oder zahlreiche (im Kontext der Länderfallstudien) change agents identifizierbar, die die Skalierung der jeweiligen Transformationsansätze mit auf die jeweils relevanten Anspruchsgruppen zugeschnittenen Strategien vorantreiben.

\section{Konstellation 4: Destabilisierung des alten Regimes}

Für die Destabilisierung des alten Regimes gibt es in den betrachteten Transformationsbeispielen kaum empirisches Anschauungsmaterial. Dies begründet sich auch daraus, dass diese Konstellation in einigen Fällen, z. B. bei der Elektromobi- 
lität in den Niederlanden, schlicht entfiel. Dort war eine Regimedestabilisierung kaum notwendig, da der Wandel einen Bereich betraf, in dem das Regime durch das Fehlen einer Autoindustrie ohnehin schwach war und damit kein großes Transformationshindernis darstellte (Clausen 2017a, 2017b; Perleberg und Clausen 2017).

Als einziges nennenswertes Beispiel für eine erfolgreiche Regimedestabilisierung gilt die dänische Wärmeversorgung, in welcher das alte Regime erfolgreich destabilisiert und inzwischen ganz ersetzt worden ist (Tappeser \& Fromm, 2018). $\mathrm{Zu}$ Beginn des Wandels der dänischen Wärmeversorgung in den 1970er-Jahren hatte sich eine Koalition der SDP-geführten Minderheitenregierung sowie der größeren Energieversorgungsunternehmen gebildet, die eine alternative, auf $\mathrm{Nu}-$ klearenergie basierende Variante der Energieversorgung bevorzugte. Diese Koalition wurde aber schon 1979 erfolgreich in die neue Energiestrategie integriert, wodurch die letzten wesentlichen Widerstände gegen einen Pfadwechsel beseitigt wurden

Die Analyse von Pfadabhängigkeiten zeigt, dass der Destabilisierung der »alten" Akteurinnen und Akteure in der Phase des Take-off hohe Bedeutung zukommt. Rechtliche, ökonomische und organisatorische Pfadabhängigkeiten müssen in dieser Phase überwunden werden, wenn der Wandel gelingen soll. Der größte Widerstand des alten Regimes ist erst dann zu erwarten, wenn neue Lösungen deutlich Marktanteile gewinnen. Schritte zur Destabilisierung des alten Regimes (Beyer 2006) werden schnell als Einstieg in die Exnovation erkannt (Arnold et al. 2015; Heyen 2016) und entschlossen bekämpft.

\section{Konstellation 5: Rebound}

Die Konstellation des Rebound verknüpft verschiedene Effekte, in denen entweder schon an Bedeutung verlierende Regime-Technologien und -Institutionen erneut an Bedeutung gewinnen (z. B. durch verstärkte Lobby-Aktivitäten oder leichte Veränderungen an bestehenden Technologien) oder schon in einer verstärkten Verbreitung begriffene Alternativen wieder in die Nische zurückgedrängt werden. Faktoren, die hier eine Rolle spielen, sind insbesondere die Resilienz und Beharrungskraft von Regimestrukturen und Akteurinnen und Akteuren.

\section{Konstellation 6: Etablierung des neuen Regimes}

Die Etablierung eines neuen Regimes ließ sich im Rahmen der Fallbeispiele vor allem im Fall der Wärmenetze in Dänemark beobachten, kann aber auch im Automobilsektor in Norwegen beobachtet werden (Figenbaum 2017). Weiter ist sie auch in der chinesischen Automobilproduktion in den nächsten Jahren zu erwarten (Beigang \& Clausen, 2017). In dieser Konstellation zeigt sich, dass es häufig organisationale und rechtliche Pfadabhängigkeiten zu überwinden gilt und vor diesem Hintergrund die Institutionalisierung von Prozessen sowie vertikale und 
horizontale Koordination im Vergleich zu den anderen Konstellationen nochmals wichtiger werden, um den neuen Regimezustand zu festigen. Dies lässt sich etwa am Beispiel der Wärmenetze in Dänemark nachzeichnen. Dort wurden Fernwärmesysteme über Jahrzehnte hinweg kontinuierlich ausgebaut, dezentrale Strukturen, Kraft-Wärme-Kopplung (KWK) und die Nutzung erneuerbarer Energien gelten als etabliert. Erfolgskritisch war die weitgehend kollaborative, sektorübergreifende Zusammenarbeit von Politik und Wirtschaft, mit welcher die zu Beginn noch konfrontativere Haltung der Sektoren überwunden werden konnte. Zudem trug aktives politisch-gesetzgeberisches Handeln, wie beim Beschluss des Energieversorgungsgesetzes 1979 oder der Verabschiedung der Energiepläne, wesentlich zur Institutionalisierung der dänischen Wärmewende bei. Nicht unterschätzt werden sollte zuletzt die Einbindung der Bürgerinnen und Bürger, die sich durch Genossenschaften aktiv in die Neuausrichtung des Wärmesystems einbringen konnten. Dadurch genoss bzw. genießt das Wärmesystem breite gesellschaftliche Akzeptanz, was wiederum auch zur Beseitigung nutzerbezogener Pfadabhängigkeiten beiträgt.

\section{Konstellation 7: Stabilisierung in der Nische}

Gelingt die Destabilisierung des dominanten Regimes und die Etablierung eines Neuen nicht, droht die Stabilisierung der Alternative in der Nische. Dies ließ sich insbesondere im Kontext der Fallstudie zu den Reparaturnetzwerken Vangerow erkennen: Hier bildete sich über längere Zeit eine stabile Nische, die zwar in sich gefestigt ist, das bestehende Regime jedoch nicht ernsthaft gefährdet oder verändert. Andere Transformationsbeispiele im Ressourcenbereich wie Fairphone oder Repair Cafés könnten sich in eine ähnliche Richtung entwickeln. In beiden Fälllen besteht eine recht stabile Unterstützergemeinde, die sich aus überzeugten (Früh-) Nutzenden und engagierten Anhängerinnen und Anhängern der dahintersteckenden Grundidee rekrutiert. Gleichzeitig gelingt es aber bis dato noch nicht, eine nachhaltige Verbreitung der jeweiligen Alternativlösung zu lancieren. Auch in den Länderstudien zu Norwegen und Kalifornien ist in den Jahren 1990 bis 2010 eine über lange Jahre stabile Nische zu beobachten, in der meist kleine und wenig massenmarkttaugliche Elektroautos produziert und von kleinen Gruppen auch genutzt wurden. Hier jedoch erweckt die gegenwärtige Situation den Anschein, als würde der "Schlaf in der Nische« seit einigen Jahren beendet sein. Nach einer langen und stabilen Nischenphase beginnt in beiden Ländern seit einigen Jahren erneut der Take-off. 


\subsubsection{Fazit}

Das vorliegende Kapitel zielte darauf ab, zentrale Variablen und Erfolgsfaktoren von Transformationsprozessen in Richtung einer nachhaltigen Wirtschaft $\mathrm{zu}$ identifizieren und entlang von Sektoren, Typen und Phasen zu betrachten. Auf Grundlage dieser Analyse sollen im Kapitel 5 Steuerungsmöglichkeiten für die Theorie und Praxis herauskristallisiert werden.

Vor dem Hintergrund fallspezifischer Landschaftsmerkmale und Rahmenbedingungen wurden zur Identifikation der Erfolgsfaktoren von Transformationen gängige Indikatoren des MoC-Ansatzes - Akteure, Veränderungsidee und Lösungsvorschläge, Zeitaspekte sowie Veränderungs- und Transformationsprozess aus drei unterschiedlichen Blickwinkeln gründlich untersucht. In der Zusammenschau ergeben sich acht Beobachtungen, die im Folgenden vorgestellt werden.

\section{Beobachtung 1: Landschaftsmerkmale und Rahmenbedingungen zählen}

Landschaftsmerkmale und Rahmenbedingungen sind in fast allen analysierten Fällen erfolgsentscheidend. Erfolgreiche Transformationen außerhalb Deutschlands in den verschiedenen betrachteten Bereichen sind oftmals durch langjährig gewachsene Strukturen und akteursübergreifende Kooperation begünstigt. In den Niederlanden etwa sind regierungsgestützte Aktivitäten in den Bereichen Elektromobilität und Sanierung von Nullenergiehäusern auf eine jahrhundertealte korporatistische Kultur intersektoraler Kooperation und Kompromissfindung zurückzuführen.

Gleichzeitig ist ein freies Spielfeld, also die Abwesenheit von Pfadabhängigkeiten, ein entscheidender Faktor für erfolgreiche Transformationsprozesse. Im Falle Chinas stellte die Kombination aus bisher wenig erfolgreicher konventioneller Automobilindustrie sowie dem Anspruch Chinas, Weltmarktführer im neuen Bereich E-Mobilität zu werden, einen wegweisenden Faktor zur erfolgreichen Transformation dar. Das Fehlen von markt- und systembeherrschenden Akteuren und von ihnen geprägten Pfadabhängigkeiten als positive Rahmenbedingung lässt sich auch in den anderen Beispielen im E-Mobilitätssektor (Kalifornien, Niederlande und Norwegen) nachweisen. In Deutschland existiert eine stark verankerte Lobby der Automobilindustrie, sodass Innovationen größeren Hindernissen gegenüberstehen. Durch innovative Akteurskonstellationen konnte dennoch der Post-E-Scooter aus den bestehenden Pfadabhängigkeiten ausbrechen und sich - zunächst unbemerkt von der großen konkurrierenden Lobby - in einer Nische entwickeln. Dabei besteht jedoch permanent das Risiko, dass ein solcher Transformationsprozess in seinem Verlauf abbricht anstatt fortzuschreiten und langfristig in einem Nischenstadium verharrt.

Ein weiteres, oft vorzufindendes Hindernis als Teil der allgemeinen Rahmenbedingungen ist der große Kostenvorteil bestehender Lösungen. Er ist meistens 
ein Ausdruck ökonomischer Pfadabhängigkeiten. Die Mehrheit der untersuchten Fallbeispiele belegt jedoch, dass durch gezielte Subventionierungen und Kostensenkungen in der Herstellung, also durch Eingriffe in die bestehenden Rahmenbedingungen, technologische und ökonomische Pfadabhängigkeiten zumindest teilweise ausgeglichen bzw. überwunden werden können. Schwieriger ist es bei den nutzerbedingten Pfadabhängigkeiten, die auch Teil der allgemeinen Rahmenbedingungen sind. Nur geringe Veränderungen von Verhaltensweisen reichen für Transformationsprozesse kaum aus und größere Änderungen sind schwer zu erzielen.

\section{Beobachtung 2: Existenz und Einfluss von change agents sind von entscheidender Bedeutung}

In allen untersuchten Fallbeispielen spielen change agents, die sich oft als Außenseiter neben einem dominanten Regime herausbildeten, eine entscheidende Rolle in der erfolgreichen Implementierung einer nachhaltigen Idee. Change agents agieren dabei als Promotorinnen und Promotoren, die einen Wandlungsprozess initiieren und gestalten. Entsprechend dem Models-of-Change-Ansatz, ausführlich beschrieben in Kapitel 3.2, lässt sich eine Kategorisierung in Fach-, Prozess-, Macht- und Beziehungspromotorinnen und -promotoren vornehmen. Eine weitere mögliche Kategorie von change agents lässt sich als "Problempromotoren « bezeichnen. Hiermit sind Akteure gemeint, die vor allen anderen ein Problem erkennen und kontinuierlich auf dieses hinweisen.

Auffällig ist, dass Persönlichkeiten, die diese Transformationsprozesse einleiten, oftmals nicht dem Kern des etablierten Regimes entstammen, das sie verändern. Dennoch verfügen change agents notwendigerweise über die benötigten Ressourcen, etwa in Form von Fachwissen und Kontakten, um Rahmenbedingungen effektiv zu nutzen und Innovationen zum Durchbruch zu verhelfen. Dabei nehmen sie oftmals eine Brückenfunktion ein, die es ihnen erlaubt, sich mit anderen Akteuren zu vernetzen und zwischen den Stakeholdern zu vermitteln. Change agents können individuelle Persönlichkeiten in Unternehmen, wie Elon Musk im Falle von Tesla, oder aber auch Politikerinnen und Politiker auf kommunaler und nationaler Ebene sein (z. B. die Bürgermeister Tischler und Nägele von Bottrop und Wüstenrot). Ebenso lassen sich Teams als zentrale change agents ausmachen (Energiesprong). Zuletzt können auch Institutionen als change agents verstanden werden, indem sie konkurrierende Anspruchsgruppen zusammenbringen, Zielkonflikte lösen und geeignetere Rahmenbedingungen für technologische Durchbrüche schaffen. Das CARB als Regierungskommission des Bundesstaates Kalifornien bereitet beispielsweise strenge Gesetzesvorschläge zur Luftreinhaltung vor und schafft somit positive Kontextfaktoren. Insgesamt variieren der Einsatz, der Kooperationsgrad und die Wirkungskraft der change agents stark im Prozessverlauf. Oftmals erfahren Initiativen von Wirtschaftsakteuren, Umweltinstitu- 
tionen, Politikvertreterinnen und -vertretern sowie der Zivilgesellschaft, die sich gruppenübergreifend zusammenschließen, besondere Unterstützung im Transformationsprozess.

Die besondere Bedeutung von change agents lässt sich transformationstypübergreifend feststellen. Die wichtige Funktion einzelner Personen oder bestimmter Gruppen von Personen lässt auf den ersten Blick vermuten, dass der Erfolg von Transformationsprozessen vielfach rein zufällig sei und davon abhinge, dass die richtige Person zur richtigen Zeit am richtigen Ort ist. Die Untersuchungen belegen dies jedoch nicht. Möglich ist durchaus auch, dass es sehr viele potenzielle change agents gibt, es jedoch von den konkreten Situationen abhängt, welcher von ihnen wann seine Funktion auch tatsächlich wahrnehmen kann.

\section{Beobachtung 3: Die erfolgreiche Umsetzung einer Transformation adressiert mehr als nur eine nachhaltige Lösung}

Das allgemeine Umweltbewusstsein und die zunehmende Beachtung der Probleme des Klimawandels spielen zwar diskursiv eine große Rolle, für das tatsächliche Gelingen von Transformationsprozessen sind konkret erfahrbare und dringliche Umweltprobleme von größerer Bedeutung. Als Beispiele dienen hier Ballungsgebiete in Kalifornien, Norwegen und China, die verstärkt mit Luftverschmutzung zu kämpfen hatten, was Anstrengungen hin zu umweltfreundlicheren Technologien verstärkte und den Weg für die erfolgreiche Etablierung des E-Mobilitätssektors ebnete.

Neben einer unmittelbar erfahrbaren Lösung für Umweltprobleme sind noch weitere co-benefits entscheidende Erfolgsfaktoren für Transformationsprozesse. Diese betreffen beispielsweise ökonomische, aber auch sozialpolitische und gesundheitsrelevante Aspekte. So lässt sich feststellen, dass in von Unternehmen vorangetriebenen Transformationen der wirtschaftliche Erfolg meist der ausschlaggebende Faktor für eine erfolgreiche Transformation darstellte. Auch im Rahmen von auf kommunaler Ebene ablaufenden Transformationsprozessen standen meist wirtschaftliche Aspekte sowie eine langfristige Sicherung der Lebensqualität im Zentrum der Initiativen. Bei breitenwirksamen Transformationen wie nationalen Sektorenentwicklungen helfen co-benefits, übergreifende Narrative zu schaffen und Stakeholder zusammenzubringen. Beispielhaft kann hier der Transformationsprozess im Bereich Wärme in Dänemark genannt werden. Nach der Ölkrise wurde mit dem Ausbau des Wärmenetzes und der verstärkten Unterstützung erneuerbarer Energien eine erhöhte Energiesicherheit angestrebt.

In den betrachteten Beispielen wird auf individueller Nutzerebene deutlich, dass sich alternative Lösungen, Praktiken und Technologien nur dann über eine Nische hinaus verbreiten, wenn diese im Vergleich zu bestehenden Lösungen gleichwertig in Bezug auf Nutzen, Komfort und Kosten abschneiden. Grundsätzlich lässt sich sagen, dass individuelle und gruppenspezifische Bedürnisse stärker 
handlungsleitend sind als gesamtgesellschaftliche oder globale Überlegungen. Eine Herausforderung für die Skalierbarkeit von Transformationen ist in diesem Zusammenhang, dass sich Bedürfnisse in verschiedenen Millieus unterscheiden können.

Aus den Untersuchungen ergibt sich, dass co-benefits für alle Transformationstypen relevant sind. Im Umkehrschluss stehen Transformationsprozesse vor erheblichen Herausforderungen, wenn sie im dominanten Regime nur wenige co-benefits versprechen. So verbleiben suffizienzbasierte Transformationsstrategien oftmals im Nischenstadium.

\section{Beobachtung 4: Für eine erfolgreiche Transformation braucht es eine passgenaue Lösung und eine reflexive Anpassung von Strategien} Um co-benefits einer Transformation realisieren zu können, bedarf es einer passgenauen Umsetzungslösung, die Umwelt- und Nachhaltigkeitszielstellungen mit wirtschaftlichen Interessen verknüpft. Eine solche Lösung wurde in den untersuchten Beispielen meist mit Hilfe einer mutigen Vision und Skalierungsstrategie umgesetzt. Insbesondere wurden relevante Stakeholdergruppen nacheinander eingebunden und deren spezifische Bedürfnisse berücksichtigt.

Dabei wurden einzelne Zielgruppen individuell oder nacheinander adressiert, um Raum für Reaktionen und Anpassungen bei Fehlschlägen zu lassen. Damit sind Passgenauigkeit und Anpassungsfähigkeit von entscheidender Bedeutung für eine erfolgreiche Transformation. Dazu wurde je nach Sektor und Prozessstufe ein Instrumentenmix angewandt, der unterschiedliche Schwerpunkte auf Beteiligungsprozesse, Kaufanreize oder Kundensegmente legte. Auch ein Strategiemix aus Effizienz und Konsistenz wurde oftmals eingesetzt. Gerade zum Zeitpunkt der Marktaktivierung nutzten privatwirtschaftliche Akteure den Trialand-Error-Modus, um die Wirkungskraft diverser Maßnahmen auszuprobieren, ihre Initiativen $\mathrm{zu}$ bewerben und ihre Ressourcenausstattung zu verbessern. $\mathrm{Na}-$ tionale und kommunale Initiativen haben ihre politischen Strukturen und weitere Hebel wie Gesetze, Verbote und Förderinstrumente eingesetzt, um nachhaltige Initiativen gezielt in der Zivilgesellschaft zu platzieren und zu promoten.

Ein weiteres Merkmal erfolgreicher Transformationen ist Reflexivität: Neue Erkenntnisse werden systematisch und dynamisch in die zugrundeliegende Strategie integriert. Diese Reflexivität lässt sich in unterschiedlichen Prozessen finden. Auf der einen Seite des Spektrums stehen politische Regulierung und Planung, zum Beispiel im Falle des EEG in Deutschland oder der Entwicklung dänischer Wärmenetze. Hier wurden mehrfach Evaluationen von politischer Seite durchgeführt und Anpassungen vorgenommen. Auf der anderen Seite stehen markt- und technologiegetriebene Prozesse, die von einzelnen Unternehmen initiiert wurden. Mit dem Post StreetScooter wird der Ansatz verfolgt, zunächst günstige und funktionale Elektroautos zu produzieren, bevor weitere Kunden- 
segmente erschlossen werden. Tesla hingegen konzentrierte sich zunächst auf die Automobilproduktion für wohlhabende early adopter, um anschließend mit preisgünstigeren Modellen in den Massenmarkt vorzudringen. Mit Hilfe von internen Lernprozessen wurden anschließend Strategieanpassungen vorgenommen, um weitere Kundensegmente zu erschließen.

Beobachtung 5: Die strategische Nutzung von Gelegenheitsfenstern ist entscheidend

Zeitaspekte wie spezielle Auslöser und Zeitfenster, die oftmals strategisch genutzt werden, waren in den meisten untersuchten Beispielen ausschlaggebend. Dabei lassen sich drei wichtige Arten von Zeitfenstern unterscheiden:

1. Historische Ereignisse und Krisen, die zu größeren Umwälzungen führten: Im Zuge der Ölkrise konnten change agents den dänischen Heizungssektor umgestalten. Im deutschen Kontext band die Wiedervereinigung und die damit einhergehende Übernahme ostdeutscher Infrastruktur die Aufmerksamkeit großer Energiekonzerne. Dies wiederum erlaubte den Unterstützerinnen und Unterstützern von erneuerbaren Energien, regulatorische Prozesse anzustoßen, die letztlich im EEG mündeten. Eine aktive Gestaltung und strategische Nutzung dieser Art von Gelegenheitsfenstern lässt sich durch eine Vorbereitung auf solche Opportunitäten und schnelle, gezielte Reaktionen auf ihr Auftreten erreichen.

2. Politische und regulatorische Neuerungen: Neue Gesetze, Richtlinien und Verpflichtungen können eine disruptive Wirkung entfalten und gleichzeitig Akteurinnen und Akteuren Möglichkeiten für die Entwicklung neuer Geschäftsmodelle geben. Auch diese Art von Zeitfenster lässt sich strategisch nutzen, um einen Transformationsprozess zu initiieren oder zu fördern.

3. Disruptive technologische Innovationen: Technologische Neuerungen erlauben die Entwicklung neuer Lösungen und die Verdrängung etablierter Stakeholder. So hat beispielsweise die Entwicklung leistungsfähigerer LithiumIonen-Akkus die Entwicklung der E-Mobilität entscheidend beeinflusst. Im Allgemeinen eröffnet gerade auch die Digitalisierung Fenster für Transformationsprozesse, z. B. im Fall von Carsharing.

Insbesondere bei Transformationsprozessen durch regulatorische Neuerungen und technologische Innovationen hatten sich die handelnden Akteurinnen und Akteure gezielt auf mögliche Auslöser und Zeitfenster vorbereitet. 
Beobachtung 6: Selektive Koordination stellt einen wichtigen Erfolgsfaktor für einen erfolgreichen Transformationsprozess dar

Die Relevanz von Prozessvariablen variiert stark von Fall zu Fall. Dabei kommt horizontaler Koordination zwischen Akteurinnen und Akteuren aus Politik, Wirtschaft, Zivilgesellschaft und Wissenschaft eine vergleichsweise hohe Bedeutung zu.

Grundsätzlich lassen sich zwei Szenarien unterscheiden: Koordination wirkt sich dann positiv aus, wenn zur Initiierung des Transformationsprozesses unterschiedliche Arten von Wissen und Kompetenzen benötigt werden oder wenn politische Mehrheiten für gesetzgeberische Änderungen beschafft werden müssen. Während zu Beginn eines Transformationsprozesses oftmals die Koordination in der Nische oder zwischen verschiedenen Nischen genügt, sind mit zunehmender Größe eine Vielzahl von Akteurinnen und Akteuren von der Transformation betroffen. Insbesondere nationale Sektorenentwicklungen, also umfangreiche Transformationen, zeichnen sich durch eine enge Kooperation verschiedener Sektoren aus. Am erfolgreichsten ist Koordination, wenn es gelingt, gruppenübergreifende Koalitionen zu bilden. Das zeigt sich am Beispiel Norwegen. Hier war eine heterogene Koalition aus Industriellen, Fördergebern und Umweltaktivistinnen und -aktivisten zentral für die Entwicklung der Gesetzgebung zur Förderung der E-Mobilität.

In manchen Fällen ist der Widerstand von wirtschaftlichen oder politischen Akteurinnen und Akteuren im vorherrschenden Regime gegen Veränderungen allerdings so groß, dass keine Koalitionsbildung möglich ist. In diesen Konstellationen würde eine Koordination mit allen Akteurinnen und Akteuren dem Wandel entgegenstehen, da die Interessen zu stark divergieren. Dann beruht der Erfolg von Veränderungen darauf, dass einzelne Akteurinnen und Akteure als change agents voranschreiten, ohne ihr Handeln abzustimmen. So war die relative Unabhängigkeit des CARB entscheidend dafür, dass bereits in den 1990er-Jahren die regulatorischen Rahmenbedingungen für die E-Mobilität in Kalifornien geschaffen werden konnten. Darauf aufbauend gelang es Tesla ohne größere Koordinationsbemühungen, Elektroautos auf dem Markt durchzusetzen und eine Ladestruktur aufzubauen. Tendenziell sind unternehmens- und technologiegetriebene Transformationen, die co-benefits für Nutzerinnen und Nutzer schaffen, weniger stark auf Koordination angewiesen.

\section{Beobachtung 7: Größere Transformationen erfordern größere Ressourcenausstattungen}

Zur Initiierung von Nischenaktivitäten wie dem Aufbau von Repair-Cafés in lokalen Gemeinschaften oder verpackungsfreiem Einkaufen werden oftmals keine oder nur geringe finanzielle Ressourcen benötigt. Viel entscheidender sind hier das Engagement und der Veränderungswillen Einzelner, die oftmals das Fort- 
bestehen der kleinen sozialen Innovationen oder Nischenneuerungen durch vergleichsweise höhere Kaufpreise sichern.

Die Umgestaltung größerer sozio-technischer Systeme und ganzer Sektorentwicklungen macht hingegen umfangreiche Investitionen erforderlich. Beispiele sind die Umgestaltung des auf dem Verbrennungsmotor basierenden Individualverkehrs, die Neuausrichtung des gängigen Produktdesigns hin zu höherer Kreislaufwirtschaftsfähigkeit oder eine Erneuerung unzureichend gedämmter Gebäude und fossiler Heizungssysteme.

In Fällen, in denen Regierungen und Investoren signifikante Ressourcen zur Skalierung von alternativen Lösungen zur Verfügung gestellt haben, wurden Transformationen entscheidend beeinflusst und Innovationen sowie Marktverschiebungen ermöglicht. Mit Subventionen, Steuervergünstigungen und Infrastrukturinvestitionen wurden beispielsweise in Deutschland erneuerbare Energien gefördert oder die Entwicklung der E-Mobilität in China und anderen Ländern vorangetrieben.

Trotz weitreichender öffentlicher Finanzierungsmöglichkeiten wird aus den betrachteten Beispielen auch deutlich, dass für eine vollständige Transformation in großen Sektoren umfangreiche private Investitionen erforderlich sind. Das bedeutet im Umkehrschluss, dass privatwirtschaftliche Akteure mit signifikanten Ressourcen die Transformation vorantreiben müssen oder diese durch Koalitionsbemühungen als Unterstützer gewonnen werden müssen. Dies setzt wiederum voraus, dass in einem hohen Maße Vertrauen in den Transformationsprozess geschaffen werden kann.

Nicht zu vergessen ist schließlich, dass neben Kapital langfristige personelle Ressourcen eine wichtige Rolle spielen: Bei den betrachteten Transformationsbeispielen bedurfte es oftmals langer Vorlaufphasen technologischer Entwicklung. Im Kontext der Elektromobilität beispielsweise dauerte die technologische Reifung ausreichend leistungsstarker Batterien ca. 20 Jahre.

\section{Beobachtung 8: Die Ausprägung der Erfolgsfaktoren hängt von den Transformationstypen ab}

Im Rahmen der Analyse entlang unterschiedlicher Arten von Transformationsbeispielen (Kapitel 4.2.3) konnten auch transformationstypabhängige Aussagen zu den Erfolgsfaktoren identifiziert werden. Aufgrund der jeweils recht begrenzten Datengrundlage von drei bis fünf Beispielen sind diese Erkenntnisse jedoch als vorläufig zu betrachten. Hier wären grundsätzlich weitere Untersuchungen hilfreich.

Auf kommunaler und lokaler Ebene spielen Beteiligungsprozesse und der geeignete Umgang mit Zielkonflikten eine vergleichsweise große Rolle. Während die Initiatoren aus unterschiedlichen Sektoren stammen, waren vor allem lokale wirtschaftliche Interessen ausschlaggebend für den Anstoß von Transforma- 
tionsprozessen. Obwohl alle untersuchten kommunalen Beispiele dem Transformationsfeld Wärme zugeordnet werden können, war die Transformation der Wärmeversorgung nur ein Teilaspekt einer größeren Strategie lokaler Entwicklung. Wissenschaftliche Begleitung lieferte sowohl einen Mehrwert in Form von fachlichem Wissen als auch von zusätzlichen finanziellen Ressourcen.

Die Bandbreite analysierter Unternehmen ist groß und reicht von kleinen, langjährig bestehenden Unternehmen wie der Vangerow $\mathrm{GmbH}$ bis hin zu relativ jungen multi-nationalen Konzernen wie Tesla. Dennoch lassen sich einige Gemeinsamkeiten feststellen. Hier sind vor allem dynamische und passgenaue Lösungen von großer Bedeutung, die mit hoher Geschwindigkeit und in günstigen Gelegenheitsfenstern umgesetzt werden. Dabei kamen fast immer neue Technologien zum Einsatz, die ein Alleinstellungsmerkmal auf dem Markt verschafften oder disruptiv wirkten. Hier wird wieder die Rolle von co-benefits deutlich. Zentrale Treiber sind in den meisten Fällen wirtschaftlicher Erfolg und Wachstum. Normative Überlegungen spielen - mit Ausnahme von Fairphone - nur eine untergeordnete Rolle.

Nationale Initiativen betreffen sehr unterschiedliche Aspekte wie die Förderung von technologischen Innovationen (EEG) und die Bekämpfung von Externalitäten (Nichtraucherschutz). Sie entstehen meist über lange Zeiträume hinweg in zivilgesellschaftlichen oder wissenschaftlichen Nischen. Für ihre Umsetzung ist ein umfassender Instrumentenmix der zentrale Erfolgsfaktor. Zudem wird eine größere Zahl an change agents als in kommunalen oder unternehmerischen Beispielen deutlich. Transformationen von Sektoren auf nationaler Ebene wie E-Mobilität und Wärmenetze sind die umfangreichsten der untersuchten Transformationsbeispiele. Ziel war hier jeweils die Verbreitung einer bestimmten Technologie (E-Mobilität bzw. Wärmenetze). Analog zu den anderen nationalen Initiativen sind eine lange Vorlaufzeit und ein umfassender Instrumentenmix besonders wichtig. Aufgrund ihrer Größe sind darüber hinaus auch die weiteren Rahmenbedingungen von besonderer Bedeutung, vor allem die Abwesenheit von mächtigen Akteurinnen und Akteuren, die der Transformation entgegenstehen.

Es wird deutlich, dass Synergien zwischen den drei Transformationstypen und den jeweils dominierenden Akteurinnen und Akteuren bestehen. Während Unternehmen vergleichsweise schnell passsgenaue Lösungen entwickeln und anbieten können, die es aber oft nicht aus der Nische heraus schaffen, ist nur die nationale Regierung in der Lage, die Rahmenbedingugnen so zu verändern, dass eine rasche Diffusion umweltentlastender Innovationen auch eintritt und das dominante nicht-nachhaltige Regime sich ändert. Die nationalen Regierungen müssen diesen Beitrag leisten, da er von den innovativen Unternehmen selbst nicht erbracht werden kann. Auf regionaler Ebene besteht dann die Chance, die Innovationen im Kontext der neuen Rahmenbedingugnen aufzugreifen und in lokaler Kooperation aller Akteursgruppen »das beste daraus zu machen«. 


\section{Den Pfadwechsel gestalten - Handlungsansätze für die Wende in drei Transformationsfeldern}

\subsection{Pfadwechselkonzeption für eine Green Economy}

Voraussetzung für eine systematische, empirisch fundierte, evidenzbasierte und vor allem effektive und zielgerichtete Strategieentwicklung zur Überwindung der zahlreichen eingefahrenen, nicht-nachhaltigen Wirtschaftsaktivitäten, der althergebrachten Denkgewohnheiten und der obsoleten Alltagsroutinen und Politiken ist das angemessene Verständnis der Ausgangslage und deren Entstehung sowie der maßgeblichen Faktoren und Bedingungen für einen Pfadwechsel. Dies wurde in den vorhergehenden Kapiteln dargestellt. In diesem Abschnitt geht es darum, die empirischen und konzeptionellen Erfahrungen und Einschätzungen für ausgewählte, als prioritär erachtete Transformationsbereiche in Roadmaps für einen Pfadwechsel zu übersetzen.

Das aus der Zukunftsforschung stammende methodische Konzept des Roadmappings ist ein bewährtes Instrument zur Erarbeitung eines "Innovationsfahrplans«, der viele Einzelthemen bündelt, Handlungsoptionen identifiziert und Prioritäten benennt. Weiterhin ermöglicht es eine Sensibilisierung für Problemlagen und Chancen sowie die Erzeugung von Problemsichten und Perspektiven bei relevanten Akteurinnen und Akteuren. Mit Blick auf anstehende Pfadwechsel wird die Herausforderung zur Erstellung einer Roadmap besonders groß. Dies gilt besonders dann, wenn eine hohe Dringlichkeit vorliegt und der notwendige Umbruch zeitlich nicht mehr in weiter Ferne ist, sondern bereits in der nahen Zukunft zu erwarten bzw. erforderlich ist. Daher muss konzeptionell geklärt werden, wie das Instrument zur Überwindung von Pfadabhängigkeiten und zur Gestaltung von Transformationspfaden genutzt werden kann. Roadmaps sind ein Instrument der Forschungs- und Entwicklungsplanung und können dort den intuitiv-strukturierten Suchverfahren zugeordnet werden (Specht und Behrens 2002; Möhrle und Isenmann 2007, 2017; Behrendt 2010). Kurz gefasst ist der Vorgang des Roadmappings ein transparent gestalteter Suchprozess, der Informationen über die Art, Geschwindigkeit und Richtung möglicher Innovationsprozesse im Kontext von Rahmenbedingungen liefert, mögliche Herausforderungen bün- 
delt und in Aktivitäten, Anforderungen und Meilensteine überführbar und somit gestaltbar zu machen versucht.

Roadmaps, die Wege für einen Pfadwechsel aufzeigen sollen, stellen besondere über klassische Roadmaps hinausgehende - Anforderungen an die Komplexität von Systembetrachtungen, an die Abschätzung ökologischer und sozialer Wirkungen und den Umgang mit möglicherweise auftretenden Konflikten zwischen ökonomischen, ökologischen und gesellschaftlichen Akteurinnen und Akteuren und ihren Logiken und Zielsetzungen. Unter einem Pfadwechsel wird hier ein Vorgang verstanden, in dem ein bisher dominierendes technisch-ökonomisches System und dessen Verhaltens- und Prozessmuster durch ein neues (hier: nachhaltiges) System mit neuen Verhaltens- und Prozessmustern abgelöst wird. Ein solcher Pfadwechsel umfasst eine Reihe von idealtypischen Phasen, in denen dem Roadmapping unterschiedliche Bedeutungen zukommen:

1) In der den Pfadwechsel vorbereitenden Phase entstehen die einzelnen Innovationen und (Sub-)Systeme, mit denen Potenziale und Voraussetzungen geschaffen werden, die später den Pfadwechsel ermöglichen (notwendige Bedingungen). Durch problemorientiertes Roadmapping wie auch durch forschungspolitische Roadmaps kann die Genese des neuen Pfades unterstützt und die Entstehung von beispielsweise ökonomischen Nischen gefördert werden.

2) In der zentralen Phase des Pfadwechsels spitzen sich die Widersprüche und Konflikte zwischen dem alten und dem neuen Pfad zu. Auf Seite des neuen Pfades haben sich bereits starke Akteurinnen und Akteure in Nischen etabliert. Early mover des alten Pfades haben "Zweitstrategien" auf Seiten der neuen Lösung begonnen. Andere etablierte Akteurinnen und Akteure versuchen, den Pfadwechsel zu verhindern oder zumindest zu verzögern. Teilweise wird die Notwendigkeit des Wandels auch nur verdrängt. Roadmaps sind in dieser Phase einerseits ein Mittel zu Konsensfindung, andererseits aber auch ein wissenschaftliches Instrument zur Konkretisierung und Austragung des Konflikts zwischen alter und neuer Lösung.

3) In einer dritten Phase geht es schließlich um die Konsolidierung des neuen Systems, also den Aufbau von kommunikativen, wirtschaftlichen und finanziellen, schließlich auch rechtlichen Beziehungen und Strukturen.

Für ein auf einen Pfadwechsel abzielendes Roadmapping kann auf etablierte Vorgehensweisen des problemorientierten Roadmappings aufgebaut werden. Zum einen werden dabei etablierte Trends identifiziert und analysiert, zum anderen über Trends hinausgehende mögliche Entwicklungsverläufe aufgezeigt. Aus der Kombination von Trends und Analyse der Pfadabhängigkeiten lassen sich mögliche Handlungsoptionen für Pfadwechsel ableiten und in Maßnahmen, Anforderungen und Meilensteine (also in eine Roadmap) überführen. 
Mit Blick auf Pfadwechsel zeichnet sich das Konzept der Roadmap durch zwei Besonderheiten aus: den Fokus auf Pfadabhängigkeiten und den auf ihre Überwindung. Die empirische Basis liefern die im Rahmen des Projektes durchgeführten Analysen zu Pfadabhängigkeiten (in den 15 Transformationsfeldern) und Erfolgsfaktoren für Transformationen (in den 20 Fallstudien).

An dieser Stelle wird die Bezugnahme und Analyse der relevanten Akteurinnen und Akteure bedeutsam. Soll die Roadmap konkrete Ergebnisse liefern, setzt sie auch einen analytischen und akteursbezogenen Blick voraus, um für relevante Handlungsfelder praktisch bedeutsame Ansatzpunkte identifizieren zu können, wie Transformationen im Wechselspiel von Politik, Wirtschaft, Wissenschaft und Zivilgesellschaft angestoßen, gestaltet und synchronisiert werden können. Die Erarbeitung der Roadmap stützt sich deshalb auf einen strukturierten und vorbereiteten Dialog mit ausgewählten Akteurinnen und Akteuren. Gemeinsam mit Wissenschaft, Wirtschaft, Behörden und Politik sind Handlungsbedarfe und Anforderungen zu identifizieren, um Ansatzpunkte und Maßnahmen für einen Pfadwechsel transparent und systematisch einschätzen zu können.

In dem Dialogprozess stellten Zukunftswerkstätten ein zentrales Format dar. Die Zukunftswerkstatt ist eine in den 1970er-Jahren von Robert Jungk, Rüdiger Lutz und Norbert R. Müllert begründete Methode, eingefahrene Denkmuster kritisch zu reflektieren, die Phantasie anzuregen und out of the box zu denken, um mit neuen Ideen angemessenere Lösungen für gesellschaftliche Probleme zu entwickeln und diese umzusetzen. In Anlehnung daran wurde die Methode an unsere Aufgabenstellung angepasst. Die Zukunftswerkstätten sollten bisherige Forschungsergebnisse sowie relevante Praxiserfahrungen, Erkenntnisse und Einschätzungen insbesondere der Teilnehmenden aufgreifen und nutzen sowie Bausteine für Roadmaps liefern, die möglichst konkrete Orientierungen für Pfadwechsel bzw. Transformationen in ausgewählten, für eine Green Economy wichtigen Bereichen geben. Dabei konnten die beteiligten Fachleute aus unterschiedlichen Bereichen gemeinsam klären, wer mit wem und mit welchen Maßnahmen einen Pfadwechsel unterstützen kann.

In den folgenden drei Kapiteln zu den Themen »Wärmewende«, »E-Mobilität« und »Produkte länger nutzen« wird dieser integrative Blick für Pfadwechsel eingenommen.

\section{Roadmap: Wärmewende}

Als ein wichtiges Transformationsfeld hin zu einer Green Economy bzw. zu nachhaltigem Wirtschaften wurde das Themenfeld »Wärmewende« aufgegriffen. Im Mittelpunkt stehen hier die Steigerung der Sanierungsrate und die Deckung des Energiebedarfs von Gebäuden aus erneuerbaren Energien. 


\section{Roadmap: E-Mobilität}

Als weiteres Transformationsfeld wurde die E-Mobilität betrachtet. Hier geht es darum, Maßnahmen für eine Trendwende zur Elektromobilität zu identifizieren, die durch den Staat und die Wirtschaft ergriffen werden müssten, um die Wettbewerbsfähigkeit der deutschen Automobilhersteller und ihrer Zulieferer zu sichern.

\section{Roadmap: Produkte länger nutzen}

Als drittes Transformationsfeld wurde die Verlängerung der Nutzungsdauer von Produkten, speziell elektronischer und elektrotechnischer Produkte, thematisiert. Den schnellen Verbrauch von Konsumgütern durch eine längere Nutzung von Produkten zu reduzieren ist eine Schlüsselstrategie einer ökologischen Transformation. Dabei geht es darum, neue Wege zu eröffnen, den dominanten, nichtnachhaltigen Trend zu beenden und so einen Pfadwechsel zur längeren Nutzung von Produkten zu ermöglichen. Dafür wurden Bausteine für eine Roadmap entwickelt, die den Handlungsbedarf konkretisiert und für relevante Akteurinnen und Akteure in Maßnahmen übersetzbar macht.

\subsection{Roadmap für eine Wärmewende im Gebäudesektor}

\subsubsection{Herausforderung Wärmewende}

Die Energiewende im Stromsektor dominierte bisher den energiepolitischen Diskurs, obgleich nur etwa ein Fünftel des deutschen Endenergieverbrauchs dem Stromsektor zuzuordnen ist. Demgegenüber steht der Wärmesektor mit einem erheblich höheren Anteil. Auf ihn entfallen derzeit ca. 56 Prozent des Endenergieverbrauchs sowie ca. 40 Prozent der energiebedingten Treibhausgasemissionen. Auf Raumwärme und Warmwasser in Gebäuden entfallen ca. 30 Prozent des gesamten Endenergieverbrauchs (Ministerium für Umwelt, Energie, Ernährung und Forsten Rheinland-Pfalz 2017, S. 2).

Die Energiewende erfordert damit zu einem wesentlichen Teil eine Wärmewende. Dafür ist es notwendig, zwei strategische Pfade gleichzeitig einzuschlagen: Zum einen muss der Wärmebedarf nachfrageseitig deutlich gesenkt und damit das - in der Energieeffizienzstrategie Gebäude (ESG) festgeschriebene Prinzip »Efficiency First« operationalisiert werden; zum anderen muss die Wärmeversorgung angebotsseitig schnellstmöglich auf erneuerbare Energien umgestellt werden (Schneller et al. 2018).

Die Bundesregierung hat sich zum Ziel gesetzt, den Primärenergiebedarf von Gebäuden bis 2050 um 80 Prozent gegenüber 2008 zu senken. Die Energieeffizienzstrategie Gebäude und der Klimaschutzplan verankern außerdem das Ziel 
eines nahezu klimaneutralen Gebäudebestands bis 2050. Bisher wurden jedoch lediglich Primärenergiebedarf-Einsparungen von 15,9 Prozent im Vergleich zu 2008 erreicht (Abbildung 41) und damit nur ein Bruchteil der angestrebten Minderung bis zum Jahr 2050. Ein ähnliches Bild ergibt sich für den Wärmebedarf von Gebäuden (-11 Prozent) und den Anteil erneuerbarer Energien am Wärmeverbrauch (13,2 Prozent im Jahr 2015).

Abbildung 41: Zielerreichung im Gebäudesektor nach Klimaschutzplan 2050

\begin{tabular}{|c|c|c|c|c|c|}
\hline & 2015 & 2020 & 2030 & 2040 & 2050 \\
\hline \multicolumn{6}{|l|}{ Effizienz und Verbrauch } \\
\hline $\begin{array}{l}\text { Primärenergiebedarf Gebäude } \\
\text { (gegenüber 2008) }\end{array}$ & $-15,9 \%$ & & & & $\rightarrow-80 \%$ \\
\hline $\begin{array}{l}\text { Wärmebedarf Gebäude } \\
\text { (gegenüber 2008) }\end{array}$ & $-11,1 \%$ & $-20 \%$ & & & \\
\hline \multicolumn{6}{|l|}{ Erneuerbare Energien } \\
\hline Anteil am Wärmeverbrauch & $13,2 \%$ & $14 \%$ & & & \\
\hline
\end{tabular}

Quelle: BMWi (2016, S. 7).

Zur Zielerreichung ist neben steigenden energetischen Standards im Neubau eine deutliche Steigerung der aktuellen Sanierungstätigkeit (Sanierungsrate) im Gebäudebestand erforderlich. Gleichzeitig müssen eine Erhöhung der Sanierungstiefe sowie ein konsequentes Umschwenken auf erneuerbare Energien zur Wärmeversorgung ermöglicht werden.

Für Neubauten bestehen bereits hohe Anforderungen an deren energetische Qualität, die erheblich zur Senkung der $\mathrm{CO}_{2}$-Emissionen von Gebäuden beitragen. Seit dem 1. Januar 2016 gelten für den Neubau zusätzlich strengere Anforderungen. Im Vergleich zu 2009 sinkt der zulässige Jahres-Primärenergiebedarf um 25 Prozent. Der Wärmeschutz der Gebäudehülle muss parallel dazu um 20 Prozent steigen (Tuschinski 2016, S. 36).

Weitaus mehr Transformationsbedarf als für den Neubau besteht in Bezug auf energetische Sanierungsmaßnahmen am Gebäude-Altbestand. Damit sind bauliche Maßnahmen gemeint, die zur Verbesserung der thermischen Hülle von Gebäuden und somit zu deren Energieeffizienz beitragen, beispielsweise Dach-, Fassaden-, Obergeschossdecken- oder Kellerdecken-Dämmung sowie die Modernisierung der Fenster.

Dass eine Steigerung der Sanierungsrate im Bestand zwingend notwendig ist, um die Ziele der Bundesregierung zu erreichen und den Wärmeenergiebedarf von Gebäuden langfristig zu senken, ergibt sich aus den Daten zum Bestand und dem entsprechenden Energieverbrauch. Knapp zwei Drittel (64 Prozent) von etwa 41 Millionen Wohnungen in Deutschland befinden sich in Gebäuden, die vor 1979 
gebaut wurden, also bevor nennenswerte Wärmeschutzanforderungen existierten (BMWi 2014b, S. 5). 68 Prozent des Endenergiebedarfs werden demnach in Wohngebäuden verursacht, die vor 1979 errichtet wurden (Abbildung 42). Im Jahr 2009 hatten nur rund 36 Prozent dieser Gebäude eine gedämmte Außenwand (Diefenbach et al. 2010). Die alten Bestandsgebäude sind damit das größte Problem oder positiv formuliert: Sie bieten die größten Effizienzpotenziale. Obwohl das große Potenzial des Gebäudebestands für die Senkung des Energieverbrauchs und den Klimaschutz seit langem bekannt ist und spätestens mit der Vorlage des Energiekonzepts 2010 der Bundesregierung eine erhöhte politische Aufmerksamkeit genießt (Bundesregierung 2010), wird in Deutschland zu wenig saniert. Umfangreiche Beratungs- und Förderprogramme konnten die Sanierungsrate bislang nicht steigern.

Abbildung 42: Anteil am Endenergieverbrauch für Raumwärme und Warmwasser in Wohngebäuden nach Baujahr

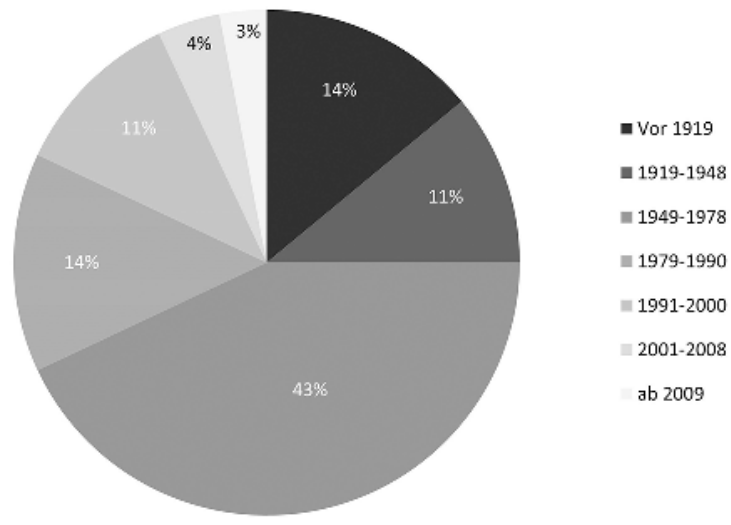

Quelle: dena (2016), Daten: Statistisches Bundesamt.

Zur Erreichung der Zielsetzung der Bundesregierung wäre je nach Berechnung eine jährliche Sanierungsrate von zwei (BMWi 2015a) bis drei Prozent (Purper et al. 2015) erforderlich. Aktuell stagniert sie bei ca. einem Prozent. Die angestrebte Verdoppelung der Sanierungsrate zeichnet sich derzeit nicht ab (Abbildung 43). Gleichzeitig gibt es trotz der großen politischen Bedeutung derzeit keine verlässliche Quelle, die die jährliche energetische Sanierungsrate präzise erfasst.

Gleichzeitig zeichnet sich die bestehende, vordergründig auf Effizienzsteigerungen ausgerichtete Sanierungspraxis durch die Verwendung von ökologisch zum Teil problematischen Materialien (insbesondere Polystyrol) aus. Die Verwendung von Naturdämmstoffen im Sinne einer stärkeren Berücksichtigung von Konsistenz (Behrendt et al. 2016) ist bisher nur vereinzelt zu beobachten. 
Abbildung 43: Sanierungsrate 2005 bis 2008 für Bauteile nach Gebäudeart und Region

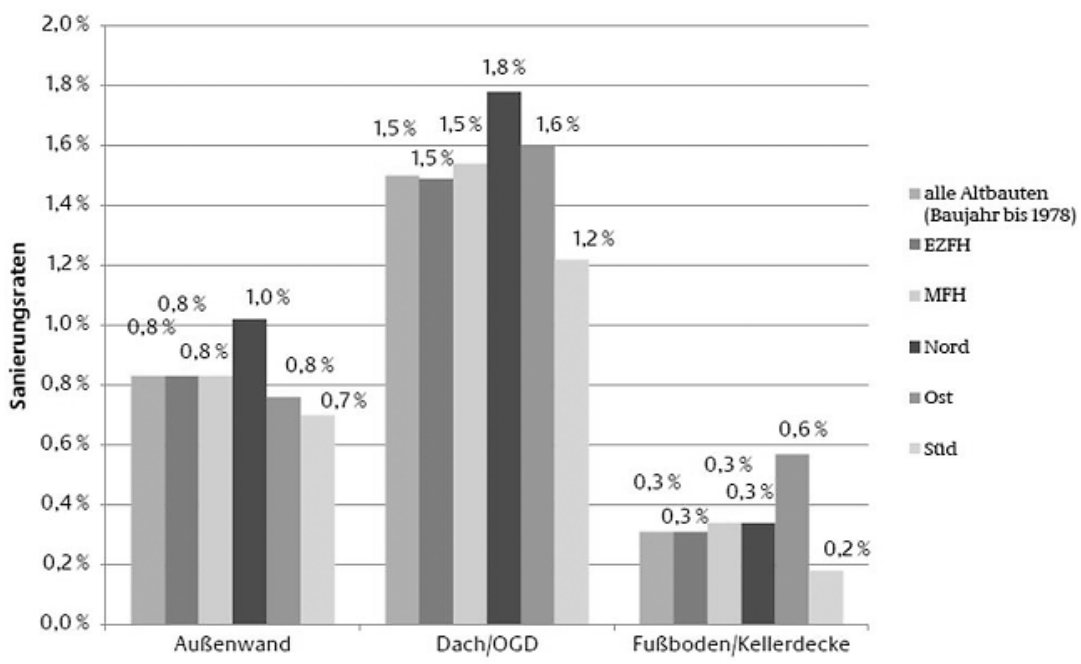

Quelle: dena (2016), Daten: IWU.

Abbildung 44: Anteil erneuerbarer Energien am Endenergieverbrauch für Wärme und Kälte

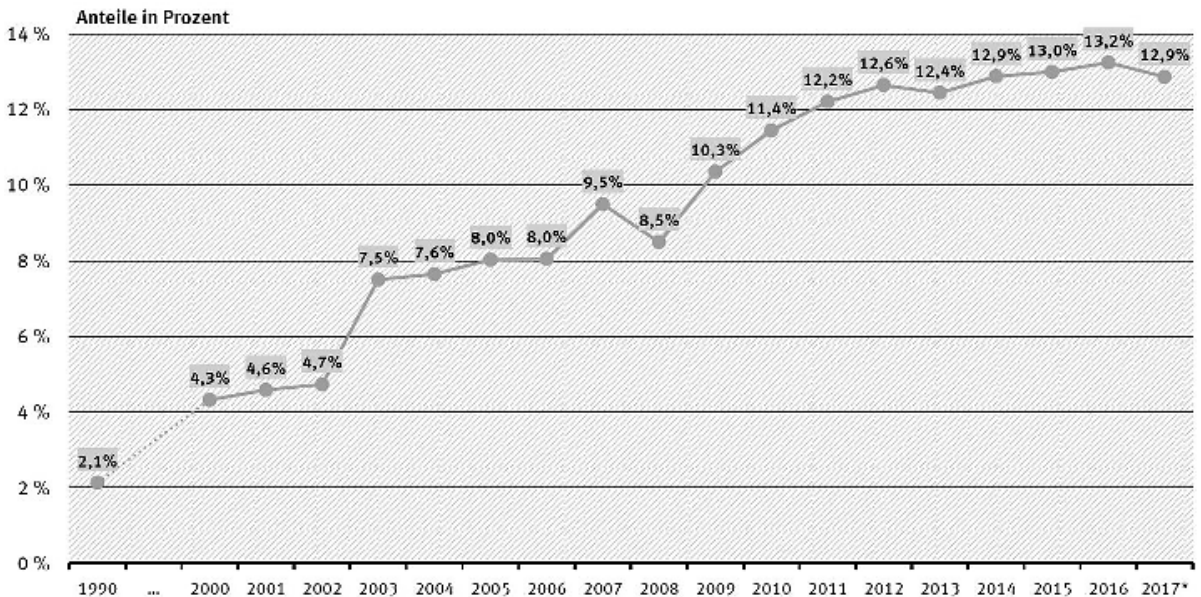

*vorläufige Daten

Quelle: UBA (2018) auf Basis AGEE-Stat, Stand 02/2018.

Weiterhin geht die Dekarbonisierung der Wärmeversorgung nur schleppend voran (Abbildung 44). In Einzelgebäuden werden bisher primär Gas- und Ölheizungen verbaut. Der Anteil von Wohngebäuden, die auf Basis regenerativer Energien beheizt werden, ist derzeit sehr gering. Im Jahr 2014 betrug der Anteil des Wohnungsbestands in Deutschland, der mit Gas beheizt wurde, 49,3 Prozent, mit 
Heizöl 26,8 Prozent, mit Strom 2,9 Prozent, mit Wärmepumpen 1,5 Prozent und mit »Kohle, Holz und weiteren festen Brennstoffen« 6,0 Prozent. 13,5 Prozent des Wohnungsbestandes werden über Fernheizungen versorgt (Arbeitsgemeinschaft Energiebilanzen e. V. 2015, S. 14).

Wie Clausen und Hinterholzer (2017) ausführlich darstellen, verläuft die Marktentwicklung der Heizungstechnologien auf Basis erneuerbarer Energien außerdem derzeit nur langsam (Baulinks 2016):

- Biomassekessel mussten in 2017 Markteinbußen von 8 Prozent bei den Absatzahlen hinnehmen (26.500 abgesetzte Wärmeerzeuger) und hatten nur einen sehr geringen Marktanteil. Als Ursache hierfür gelten der niedrige Ölpreis und die vergleichsweise hohen Investitionskosten.

- Wärmepumpen wiederum hatten hinsichtlich der Wettbewerbsfähigkeit wie in den Vorjahren unter den hohen Strompreisen zu leiden. Mit $78.000 \mathrm{ab}$ gesetzten Geräten und einem deutlichen Plus von 17 Prozent konnte sich die Technologie jedoch am Markt behaupten und nimmt mittlerweile den zweiten Platz bei den verkauften Heizungstechnologien ein.

- Als Folge der verbesserten Förderung im Rahmen des Marktanreizprogramms konnte sich die Solarthermie in den vergangenen Jahren ebenfalls sukzessive am Markt etablieren, schloss allerdings auf das Jahr 2017 gesehen mit einem Minus von 16 Prozent der neu installierten Anlagen (ebenfalls 78.000 Stück) $\mathrm{ab}$.

Zum Vergleich: In Deutschland wurden im Jahr 2017 insgesamt 712.000 Wärmeerzeuger abgesetzt, die Gas-Brennwerttechnik dominierte mit 472.500 abgesetzten Anlagen den Markt deutlich, ölbasierte Systeme landeten hinter den Wärmepumpen mit insgesamt 63.500 abgesetzten Geräten immer noch auf Platz drei in der Gunst der Nachfrage. Damit verlaufen die Modernisierung mit Anlagen auf Basis erneuerbarer Energien und die Marktdurchdringung bei Neubauten weiterhin nur sehr langsam.

Es lässt sich somit konstatieren, dass sich in Deutschland zum einen ein Lockin-Effekt bei Heiztechnologien auf Basis fossiler Brennstoffe eingestellt hat und zum anderen im Gebäudebestand ein erhebliches Sanierungsdefizit vorhanden ist. Die jeweiligen Pfadabhängigkeiten, die zu dieser Situation geführt haben, gilt es im Folgenden näher zu untersuchen.

\subsubsection{Pfadabhängigkeiten}

Aktuell sind die Kosten und der Aufwand von Sanierungen für Hausbesitzerinnen und Hausbesitzer oftmals zu hoch, um eine Marktdynamik zu entfalten. Komplexe Regulierungen, Vollzugsdefizite, kleinteilige Besitzstrukturen bei Gebäuden, 
die fehlende Anreizwirkung durch das Investor-Nutzer-Dilemma in Mietverhältnissen wie auch personelle Engpässe im Baugewerbe stellen weitere Herausforderungen dar. Diese Verschränkung unterschiedlicher Sanierungshemmnisse gilt es zu überwinden. Im Rahmen einer detaillierteren Problemanalyse muss dabei zunächst zwischen den ökonomischen, strukturellen, rechtlichen, organisationalen und nutzerbezogenen Pfadabhängigkeiten unterschieden werden, die nachfolgend näher erläutert werden.

\subsubsection{1 Ökonomische Pfadabhängigkeiten}

Eine Hauptursache dafür, dass es derzeit wenig ökonomische Anreize für eine klimaschonende Wärmeversorgung mit hohem Anteil erneuerbarer Energien gibt, ist die aktuelle Preisstruktur des Wärmemarktes mit relativ niedrigen Preisen für fossile Energieträger (Dunkelberg et al. 2017). Der politische Anspruch einer preiswerten Grundversorgung für die Bevölkerung hemmt den Willen und die Möglichkeit der Politik, dafür zu sorgen, dass die Energiepreise die »ökologische Wahrheit sagen«. Viele der Eigentums- bzw. Mietparteien schlecht sanierter Häuser und Wohnungen sind aufgrund der niedrigen Energiepreise und daher niedrigen Heizkosten mit dem gegenwärtigen Status zufrieden und spüren keinen Handlungsdruck.

Abbildung 45: Entwicklung der Brennstoffpreise im Zeitraum 2006 bis 2017

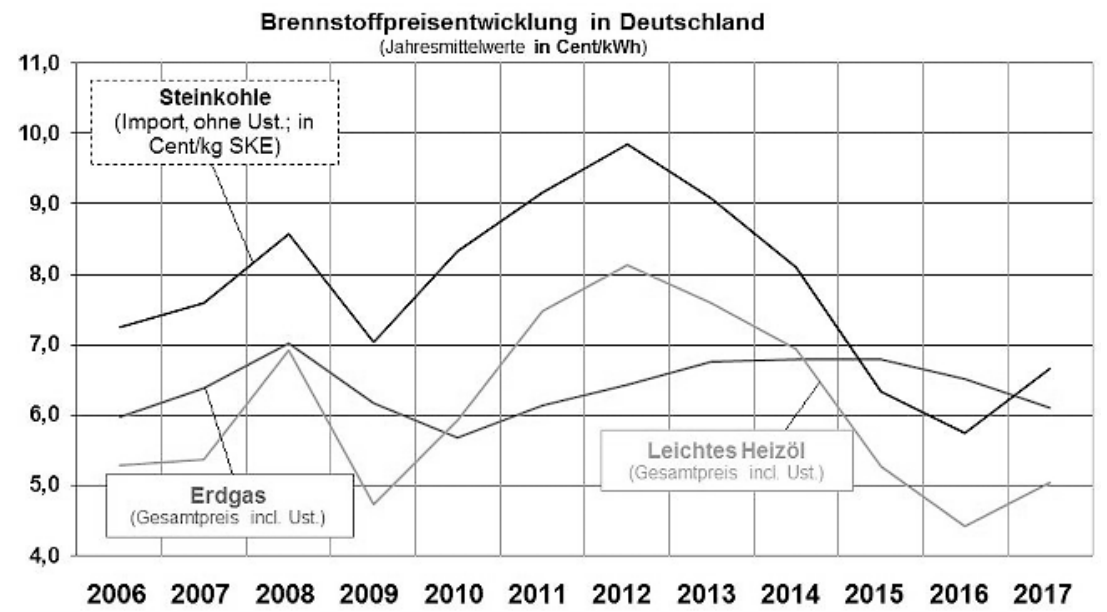

Quelle: Bund der Energieverbraucher (2017), Daten: Statistisches Bundesamt.

In den Jahren 2016 und 2017 lagen beispielsweise die Preise für leichtes Heizöl deutlich unter dem früheren Preisniveau. Zudem haben sich die Gas- und Steinkohlepreise ebenfalls auf einem niedrigen Niveau eingependelt (Abbildung 45). Die mit den niedrigen fossilen Brennstoffpreisen verbundene fehlende Interna- 
lisierung von Klimafolgekosten stellt somit ein großes Hemmnis für die Transformation hin zu einer klimafreundlichen Wärmeversorgung dar. Die niedrigen Preise führen dazu, dass objektbezogene Öl- und Gaskessel bei einigen Verbrauchergruppen die günstigste Wärmeversorgungsoption darstellen. Auch die gasund kohlebasierte Wärmeerzeugung in zentralen Heizkraftwerken bleibt weiterhin konkurrenzfähig (Dunkelberg et al. 2017).

Die relativ günstigen Preise für fossile Energieträger sorgen dafür, dass sich eine angemessene Wohnraumtemperatur trotz niedriger energetischer Qualität der Gebäude vergleichsweise günstig herstellen lässt. Ausgenommen von dieser Betrachtungsweise sind einkommensschwache Haushalte, für die die Heizkosten bereits unter den derzeitigen Rahmenbedingungen eine erhebliche Belastung darstellen können (Schneller und Kahlenborn 2018).

Im vermieteten Wohngebäudebestand kommt erschwerend hinzu, dass das Wirtschaftlichkeitsgebot bei den Nebenkosten greift. Dies bedeutet, dass Vermietende hinsichtlich ihrer Handlungsmöglichkeiten beim Wechsel zu einer teureren Wärmeversorgung stark eingeschränkt sind. Ältere, un- und teilsanierte Mehrfamilienhäuser stellen somit eine Verbrauchergruppe dar, die unter den aktuellen Marktbedingungen voraussichtlich nur schwer für Investitionen in technische Lösungen mit einem hohen Anteil erneuerbarer Energien zu motivieren sind (Dunkelberg et al. 2017).

Betrachtet man die Problematik der Energieeffizienz von Gebäuden als Ganzes, besteht zudem eine wesentliche ökonomische Pfadabhängigkeit in den schon getätigten Investitionen zur Errichtung des derzeitigen Gebäudebestandes mit seinen geringen Effizienzwerten sowie dessen Instandhaltung im Zuge von Sanierungs- und Modernisierungsarbeiten, die keine oder nur geringfügige energetische Verbesserungen zum Ziel hatten.

Bei umfassenden Sanierungsarbeiten stellen die Mehrkosten einer gleichzeitigen energetischen Sanierung zumeist nur ungefähr ein Drittel der Gesamtkosten dar. Die übrigen Kostenbestandteile für die Bauinfrastruktur, Baumaterialien usw. fallen bei jeder Sanierungsmaßnahme an. Wirtschaftlich rentable Verbesserungen der Wärmedämmung sind somit in hohem Grade vom Zyklus der Instandsetzungsmaßnahmen von Gebäuden abhängig, was sich auch im Kopplungsprinzip der Energieeinsparverordnung (EnEV) widerspiegelt, welche energetische Maßnahmen nur im Falle ohnehin anstehender Sanierungsarbeiten verpflichtend macht. Wurden Decken, die Fassade oder das Dach eines Hauses "angefasst", ohne dass in diesem Zuge ambitioniert gedämmt wurde, ist die Gelegenheit zumeist für die nächsten 20 bis 30 Jahre vertan. Gleichzeitig spielt die Rentabilität energetischer Sanierungsmaßnahmen vielfach eine wichtige Rolle bei der Entscheidung, ob in nächster Zeit überhaupt saniert wird oder nicht. 


\subsubsection{Strukturelle Pfadabhängigkeit: Das Investor-Nutzer-Dilemma}

Eine weitere Pfadabhängigkeit ergibt sich aus der Besitzstruktur des deutschen Wohnungsbestandes. Von rund 40 Millionen Wohnungen in Deutschland werden ca. 54 Prozent vermietet (BMWi 2014b). Dieser im internationalen Vergleich recht hohe Mietanteil führt zu einem Dilemma bezüglich der Finanzierung von Investitionen in Sanierungsmaßnahmen. Die Kosten für die Sanierung sind zunächst von den Vermietenden zu tragen. Von den daraus resultierenden Einsparungen an Heizenergie und dem zusätzlichen Wohnkomfort profitieren jedoch unmittelbar nur die Mieterinnen und Mieter.

Für letztere sind die mit (energetischen) Sanierungen einhergehenden Mietzinserhöhungen jedoch oftmals ein enormer Kostenfaktor. Geringere Wärmekosten gleichen die von den Vermieterinnen und Vermietern angesetzten Kaltmieterhöhungen häufig nicht aus (Sachverständigenrat für Umweltfragen 2016, S. 10). Einer Steigerung der Kaltmiete von zwei Euro pro Quadratmeter steht vielfach lediglich eine Einsparung der Heizkosten von etwa 0,50 Euro pro Quadratmeter gegenüber (Sachverständigenrat für Umweltfragen 2016, S. 213). Insbesondere dann, wenn die energetische Sanierung mit weiteren Instandsetzungsmaßnahmen einhergeht und die Umlage der Investitionen entsprechend hoch ist, entsteht eine überproportional hohe Belastung für einkommensschwache Haushalte (Sachverständigenrat für Umweltfragen 2016, S. 213). Allerdings sind nicht nur armutsgefährdete Mietparteien nicht willens, Mietpreiserhöhungen für eine energetische Sanierung in Kauf zu nehmen. Laut einer Umfrage der Immowelt AG sind nur 50 Prozent der Mietenden bereit, für eine besser gedämmte Wohnung mehr Miete zu bezahlen (Immowelt 2014).

Während das Investor-Nutzer-Dilemma die Dynamiken und Hemmnisse für eine energetische Gebäudesanierung im Mietmarkt also trotz Umlageverfahren erheblich prägt, ist die Situation für Personen mit Eigenheimen aus ökonomischer Perspektive überschaubarer. Hier spielen die Kosten von energetischen Sanierungsmaßnahmen und ihre rechnerische Wirtschaftlichkeit zwar auch eine zentrale Rolle. Die individuelle Liquidität der Besitzenden stellt jedoch ein wesentlich wichtigeres Entscheidungskriterium dar (Albrecht und Zundel 2010). Mit dem Sanierungskalkül eng verbunden sind allerdings nicht nur wirtschaftliche Faktoren, sondern auch individuelle Prioritäten und Unsicherheiten der Haushalte sowie die zeitlichen und emotionalen Auswirkungen von Sanierungsmaßnahmen (Bellmann et al. 2015).

\subsubsection{Rechtliche Pfadabhängigkeiten}

Ein weiteres wesentliches Hemmnis hinsichtlich der Sanierungstätigkeiten begründet sich mit der Komplexität ordnungsrechtlicher Vorgaben und Regularien, die sich aus der historischen Entwicklung verschiedener Verordnungen, Gesetze und Normen ergibt. Zwar wurden mit der ersten EnEV von 2002 die Verordnun- 
gen zu Wärmeschutz und Heizungsanlagen zusammengelegt, das ErneuerbareEnergien-Wärmegesetz hat die Lage bezüglich der energetischen Vorgaben jedoch nochmals verkompliziert. Zusätzlich zu beachten sind bestehende Vorgaben aus dem Baurecht sowie in den jeweiligen Verordnungen referenzierte DIN-Normen, die zum Teil mehrere Tausend Seiten Text umfassen. Daraus ergibt sich selbst für Fachpersonal aus Energieberatung, Planung, Architektur und Handwerk, aber auch für die Kontrollbehörden eine fast unüberschaubare Fülle an Anforderungen. Unterschiedliche Regelungen auf Bundes- und Länderebene tragen zusätzlich zur Komplexität bei.

Auch bezüglich des Monitorings sind die Vorgaben denkbar komplex. Mit der Einführung des Energieausweises für Gebäude wurde im Zuge der EnEV 2007 das Ziel verfolgt, Transparenz herzustellen und einen Marktanreiz für die energetische Gebäudesanierung zu schaffen. Mit der Etablierung zweier unterschiedlicher Ausweise - dem Bedarfs- und dem Verbrauchsausweis - wurde jedoch viel Verwirrung gestiftet. Der aufwendigere und teurere Bedarfsausweis erlaubt eine objektive Bewertung des Gebäudes auf Basis der Bausubstanz. Anhand der jeweiligen Ausstattung (Dämmung, Fenster, Heizungsanlage) wird ein theoretischer Bedarf ermittelt, der sich mit anderen Gebäuden vergleichen lässt. Für Gebäude mit mindestens fünf Wohnungen, die entweder nach der ersten Wärmeschutzverordnung (WSVO) 1977 errichtet oder nachgerüstet wurden, ist jedoch auch der günstigere Verbrauchsausweis zulässig. Er bezieht sich auf den reellen Verbrauch, wobei das individuelle Heizverhalten der Bewohnenden zu deutlichen Unterschieden bei gleicher Bauweise führen kann. Ein Großteil der intendierten Wirkung des Energieausweises wurde so, zumindest in Deutschland, durch die geringe Vergleichbarkeit verfehlt.

Eng mit der Komplexität der gesetzlichen Vorgaben verknüpft sind die zahlreichen Ausnahmen, die es ermöglichen, energetische Sanierungsanforderungen zu umgehen. Bezüglich der seit der EnEV 2009 verpflichtenden Dämmung freiliegender Warmwasser- und Heizungsrohre, der Keller- und obersten Geschossdecken sowie des Austauschs von über 30 Jahre alten Heizkesseln auch ohne bestehendes Sanierungsvorhaben gilt eine Ausnahme für Gebäude mit maximal zwei Wohnungen, die schon 2002 von den aktuellen Besitzerinnen und Besitzern bewohnt wurden (sog. »Oma-Regelung«).

Andere Maßnahmen wie die Dämmung der Außenwände sind nur bei ohnehin anstehenden Sanierungsvorhaben verpflichtend, die über zehn Prozent der jeweiligen Bauteilfläche des Gebäudes betreffen. Zudem stehen sie unter dem Gebot der Wirtschaftlichkeit, sprich: Die zusätzlichen Kosten von Dämmungsmaßnahmen müssen sich langfristig über die Energieeinsparungen amortisieren (Sachverständigenrat für Umweltfragen 2016). Während die Wirtschaftlichkeit verschiedener Maßnahmen in vielen Studien nachgewiesen wird (IWU 2013; Enseling und Hinz 2015; Holm et al. 2015), kann sich diese aufgrund unterschied- 
licher Bewertungsmaßstäbe aus Perspektive der Immobilienbesitzenden durchaus anders darstellen (Holm et al. 2015; Albrecht und Zundel 2010). Auch können letztere ihre Verpflichtung im Falle einer anstehenden Sanierung mit der Vorlage überteuerter Kostenvoranschläge leicht umgehen.

In der Gesamtschau beruhen die bestehenden Ausnahmen in den Gesetzen und Verordnungen zum Teil auf legitimen Abwägungen bezüglich der Wirtschaftlichkeit und Finanzierbarkeit energetischer Sanierungsmaßnahmen und sollen mögliche soziale Härten verhindern. Sie sind jedoch so weit gefasst, dass die Wirksamkeit der Regularien geschwächt wird, was angesichts des klimapolitischen Ambitionsniveaus baldige Änderungen erfordert.

\subsubsection{Organisationale Pfadabhängigkeit}

Eine wesentliche organisationale Pfadabhängigkeit besteht in der mangelnden flächendeckenden Verfügbarkeit von Fachexpertise. Der Pfad unzureichender Wärmedämmung wird hierbei sowohl über die langsame Entwicklung des Berufszweigs der Energieberaterinnen und Energieberater als auch über die fehlende Erfahrung im Architektur- und Handwerksgewerbe mit innovativen Sanierungskonzepten verlängert, was sich wiederum aus der Ausbildungsorganisation und Beschäftigungsstruktur der jeweiligen Branchen ergibt.

Im Hinblick auf Energieberaterinnen und Energieberater hat sich zwar seit den 1990er-Jahren ein Markt entwickelt, die Berufsbezeichnung ist jedoch weiterhin nicht geschützt (Feser et al. 2015). Entsprechend hoch sind die Qualitätsdefizite. Die Stiftung Warentest (2012) bewertete in einer Erhebung durchschnittlich sieben von neun Fachleuten aus der Energieberatung mit der Note mangelhaft. Die unterschiedlichen Ausbildungshintergründe (Handwerker/-innen, Ingenieurinnen und Ingenieure, Architektinnen und Architekten) erschweren zusätzlich eine Vereinheitlichung von Ausbildungsmaßnahmen und Standards. Die staatliche Förderung der Energieberatung sowie deren Schlüsselrolle im Hinblick auf die Beantragung von KfW-Fördergeldern führen laut einer Expertenbefragung der Universität Göttingen vielfach dazu, dass Energieberatungen vor allem wegen ihres Legitimierungseffekts für Banken und Förderinstitutionen und weniger wegen ihrer Beratungsleistungen in Anspruch genommen werden (Feser et al. 2015). Fachkräfte aus Architektur und Handwerk sind insbesondere ohne Zusatzqualifikation in der Energieberatung oftmals nur geringfügig mit den Möglichkeiten energetischer Sanierung vertraut.

Mit Angeboten wie dem BUILD-UP-Skills-Programm der EU wurden zwar verstärkt Angebote zur Weiterbildung geschaffen, die Personalentwicklungspraxis in den Baubetrieben ist jedoch weiterhin problembehaftet. Eine strategische Mitarbeiterförderung ist kaum vorhanden. In 40 Prozent der Betriebe fanden in den letzten fünf Jahren keine Weiterbildungen für Gesellinnen und Gesellen statt (Buschfeld et al. 2016). Zusätzlich ist aufgrund des demografischen Wandels ein 
erheblicher Fachkräftemangel in sanierungsrelevanten Gewerken zu erwarten, der einer langfristigen Steigerung der Sanierungsrate im Wege stehen könnte (Runst und Ohlendorf 2015) .

\subsubsection{Nutzerbezogene Pfadabhängigkeit}

Nutzerbezogene Pfadabhängigkeiten im Hinblick auf die energetische Gebäudesanierung bestehen in der Vielzahl von Mythen, die sowohl auf Seiten der Immobilienbesitzenden und Mietenden, vielfach aber auch bei Fachkräften aus Handwerk und Architektur verbreitet sind. Beispielsweise gehört die Annahme, dass Wände atmen müssten und eine Dämmung diese Funktion verhindere, zu den weit verbreiteten Irrtümern. Andere Thesen beziehen sich auf die im Vergleich zur Dämmung überragende Relevanz schwerer Mauern als Energiespeicher, einen die Energieeinspareffekte übertreffenden Einsatz von Energie bei der Herstellung von Dämmmaterialien oder die Dämmung als Ursache für Schimmel oder überhöhte Brandgefährdung (Deutsche Umwelthilfe 2014). Auch wenn eine Fülle von Publikationen und Informationsangeboten diese Thesen inzwischen regelmäßig widerlegt, haben sie sich doch in vielen Köpfen festgesetzt.

Die Verwendung des giftigen Brandhemmers HBCD in Polystyrol-Dämmungen, gepaart mit einer sichtbaren Interessenkonvergenz zwischen Dämmstoffherstellern und umweltpolitischen Akteurinnen und Akteuren in der Ausgestaltung der EnEV, hat in den letzten Jahren zudem einen Diskurs über den »Dämmwahn« (Frankfurter Allgemeine Zeitung 2014; 3sat 2015) und die »Dämm-Lobby« (Die Welt Online 2014) losgetreten. Die Skepsis gegenüber energetischen Sanierungsmaßnahmen auf Seite der Verbraucher/-innen, ob Eigentums- oder Mietpartei, stellt somit ein durchaus wichtiges Hemmnis dar, das Sanierungsentscheidungen beeinflusst.

Im Umkehrschluss sind auch die Vorteile einer energetischen Sanierung nur wenig bekannt. Dies betrifft sowohl die Potenziale zur Energieeinsparung mit den entsprechenden positiven wirtschaftlichen und ökologischen Effekten, die für den Laien zumeist nicht abschätzbar sind, als auch die Erhöhung des Wohnkomforts (BMWi 2014b). Während die Sanierung des Bades oder der Bau eines Wintergartens ein erhebliches emotionales Resonanzpotenzial bietet und durchaus etwas kosten darf, besteht über die Verbesserung der Wohnqualität durch energetische Sanierung kaum ein Bewusstsein. Zwar bestätigen Bewohner/-innen sanierter Gebäude, dass sich der Wohnkomfort mit der Sanierung erheblich gesteigert habe. Dieses subjektive Empfinden lässt sich jedoch schwer weitervermitteln und wird nicht in demselben Maße mit einem sozialen Statusgewinn honoriert.

Der finanzielle Nutzen hingegen stellt sich erst nach Amortisation der Maßnahmen ein und ist zudem von Unsicherheit hinsichtlich der Energiepreisentwicklung geprägt. In Bezug auf den Umweltnutzen sind die Effekte ebenso wenig spürbar, sodass hieraus nur wenige Verbraucherinnen und Verbraucher eine kon- 
krete Handlungsmotivation beziehen, zumal die subtilen Vorteile zunächst sehr offensichtlichen Unannehmlichkeiten gegenüber stehen. Hierzu zählen sowohl die Komplexität einer umfassenden Sanierung und die teils mangelnde Verlässlichkeit bei der Beratung, Planung und Bauausführung (BMWi 2014b), aber auch Dreck und Lärm sowie zusätzlicher organisatorischer Aufwand und Stress.

\subsubsection{Den Pfadwechsel gestalten: Politische Maßnahmen und Initiativen}

Für eine erfolgreiche Wärmewende ist es zum einen notwendig, den Wärmebedarf deutlich zu senken und damit das in der ESG festgeschriebene Prinzip »Efficiency First« zu operationalisieren; zum anderen muss die Wärmeversorgung schnellstmöglich auf erneuerbare Energien umgestellt werden (Schneller et al. 2018). Für beide Ziele wurden im Rahmen von Fallstudien vielversprechende Technologien und Konzepte mit Innovationscharakter analysiert, wovon zwei ausgewählte Fallbeispiele nachfolgend überblickshaft dargestellt werden (Tappeser und Fromm 2017a; Tappeser 2018a). Ziel ist es, anhand der Beispiele exemplarisch Wege aufzuzeigen, um in Bezug auf das energie- und klimapolitische Ziel der Wärmewende langfristig "vom Potenzial zur Umsetzung« zu gelangen und die bestehenden Pfadabhängigkeiten zu durchbrechen. Bei der Auswahl der Fallbeispiele wurde insbesondere auf die Umsetzbarkeit und Übertragbarkeit auf den deutschen Kontext geachtet.

\subsubsection{Zwei Pfadwechselbeispiele mit Innovationscharakter}

Ein unter dem Gesichtspunkt der Senkung des Energiebedarfs Erfolg versprechender Ansatz zur Steigerung der Sanierungsrate bei gleichzeitig höherer Sanierungstiefe kommt aus den Niederlanden. Im Rahmen von "Energiesprong«, einem regierungsfinanzierten Marktentwicklungsprogramm, wurden dort Wohnungsbaugesellschaften und Bauunternehmen zusammengebracht, um große Volumina gleichartiger Gebäude mit vorgefertigten Fassaden- und Dachelementen innerhalb kurzer Zeit zu Nullenergiehäusern umzurüsten. Dieses Konzept kann besonders geeignet sein, um mit einem technisch-wirtschaftlichen Ansatz auch in Deutschland strukturelle und organisationale Pfadabhängigkeiten zu überwinden (siehe unten).

Pfadwechselbeispiel: Energetische Sanierungskonzepte durch industrielle Vorfertigung - Energiesprong Niederlande

Im Rahmen von Energiesprong, einem regierungsfinanzierten Marktentwicklungsprogramm, wurden in den Niederlanden Wohnungsbaugesellschaften und Bauunternehmen zusammengebracht, um große Volumina gleichartiger Gebäude mit vorgefertigten Fassaden- und Dachelementen innerhalb kurzer 
Zeit zu Nullenergiehäusern umzurüsten. Das Konzept wurde im Rahmen einer e2g-Fallstudie ausführlich analysiert (Tappeser 2018a). Seit kurzem wird auch in Deutschland an einer Umsetzung dieses Programms gearbeitet (dena o. J.). Kosteneinsparungen durch digital unterstützte Vorfertigung und Standardisierung sowie ein ausgeklügeltes Finanzierungsmodell sollen Warmmietenneutralität für die Bewohnenden erlauben.

Ein wesentlicher Baustein des Projektes war die Entwicklung eines Standards für Null-auf-dem-Zähler-Energieverbrauch (nul-op-de-meter; NOM), was in etwa einem Nullenergiehaus entspricht. Für die Realisierung des Projektes wurde als Voraussetzung eine deutliche Senkung der Sanierungskosten bei gleichzeitiger Erreichung des NOM-Standards gesehen. Durch das größere potenzielle Auftragsvolumen konnten sowohl die Baufirmen als auch Zuliefererbetriebe $\mathrm{zu}$ weiteren Innovationsschritten angeregt werden, sodass schon Ende 2013 mit ersten Prototypen (u.a. in Arnhem) die Sanierungskosten auf 60.000 Euro je Wohneinheit gesenkt werden konnten. Ein weiteres wichtiges Element bestand in der Entwicklung eines Instruments zur Umleitung der eingesparten Energiekosten von den Mietenden hin zu den Vermietenden (in diesem Fall den Wohnbaugesellschaften). Hierfür wurde das Konzept einer Energieeinsparvergütung entwickelt, bei der auf Basis des bestehenden Energieverbrauchs ein Vertrag geschlossen wird, der den Mieterinnen und Mietern eine Mindestmenge an Strom, Warmwasser sowie Raumtemperatur garantiert.

Die Einsparungen speisen sich aus den erzielten Einspareffekten durch die energetische Sanierung des Gebäudes und der Stromeigenproduktion durch Photovoltaikmodule. Im Gegenzug zahlen die Mieter/-innen statt der vorherigen Stromrechnung eine vergleichbare Gebühr an die Vermieter/-innen. Da eine solche Gebühr zuvor rechtlich nicht vorgesehen war, setzten sich Energiesprong sowie die Vertragsparteien der Stroomversnelling für die Verabschiedung eines Gesetzes ein, das seit September 2016 die Energieeinsparvergütung ermöglicht (Overheid.nl 2016).

Ebenfalls von zentraler Bedeutung für eine erfolgreiche Umsetzung ist die Verknüpfung der Sanierung mit einer Performancegarantie durch das ausführende Bauunternehmen. Entgegen der üblichen Standards im Baubereich wurde im Rahmen der Stroomversnelling-Vereinbarung festgelegt, dass die Bauunternehmen ihre Sanierungsprodukte grundsätzlich mit einer Performancegarantie für Energieverbrauch und -produktion sowie einer Anzahl von Komfortkriterien zu unterlegen haben, die sich über einen Zeitraum von mindestens 15 Jahren (typischerweise 30 Jahre) erstrecken und eventuelle Wartungsarbeiten beinhalten (Energiesprong 2013).

Zur Absicherung dieser Performancegarantie etablierte sich ein Modell, in dem externe Versicherungsdienstleister (z. B. Bouwgarant) mögliche Män- 
gel versichern (Borsboom et al. 2015). Die Performancegarantien stellen die Grundlage für die Energieeinsparvergütungen der Vermieter/-innen dar und ermöglichen zusammen eine Erhöhung der Kreditlinien für die Wohnbaugesellschaften. ${ }^{1}$

Eine Replikation des Ansatzes in Deutschland könnte zu einer Überwindung der genannten Pfadabhängigkeiten beitragen und eine Transformation des Gebäudesektors anstoßen, müsste jedoch auf die divergierenden Ausgangsbedingungen eingehen. Für die Marktentwicklung kann das insgesamt größere Volumen des deutschen Marktes von Vorteil sein. Gleichzeitig stellt die höhere Fragmentierung sowohl der Bawwirtschaft als auch der Besitzstrukturen und des regulatorischen Rahmens eine Herausforderung dar. In der Pilotphase könnte eine Ausschreibung für Liegenschaften im Bundesbesitz einen Initialimpuls für die Produkt- und Geschäftsmodellentwicklung liefern. Mittelfristig scheinen jedoch vor allem eine erfolgreiche Einbindung des Bauhandwerks und eine Anpassung des Angebots für Privatkunden von Relevanz zu sein, um den Massenmarkt zu erreichen.

Für die Realisierung der langfristigen Zielstellung einer Wärmeversorgung auf Basis erneuerbarer Energien können zentrale Versorgungslösungen, etwa innovative Niedrigtemperatur-Wärmenetze, eine wichtige Rolle spielen. Die besondere Bedeutung der Transformation der Nah- und Fernwärmeversorgung für die Umsetzung der Klimaschutzziele wird u. a. in der Strategy on Heating and Cooling der EU-Kommission betont (Europäische Kommission 2016, S. 2). Wärmenetze können ein wichtiges Bindeglied zur Einbindung von erneuerbaren Energiequellen in die Wärmeversorgung sein und erleichtern die Nutzung von energieeffizienter Kraft-Wärme-Kopplung und Abwärme. So lassen sich gewerbliche und industrielle Abwärmequellen nur durch die Infrastruktur leitungsgebundener Wärmeversorgung sinnvoll erschließen. Die leitungsgebundene Wärmeversorgung begünstigt zudem die Nutzung solarthermischer Großanlagen sowie (tiefen-)geothermischer Potenziale (Maaß et al. 2015, S. 7). Sie ist daher insbesondere in Ballungsgebieten geeignet, die Energiewende auch im Wärmesektor umzusetzen (Schneller et al. 2018) (siehe unten).

1 Wohnbaugesellschaften des sozialen Wohnungsbaus wickeln ihre Kredite größtenteils über die Bank der niederländischen Cemeinden ab und werden durch den Garantiefonds für den sozialen Wohnungsbau (Waarborgfonds Sociale Woningbouw; WSW) abgesichert (Stroomversnelling 2015). Der WSW kann die garantierten Kreditlinien auf Basis der neuen Einkommensströme durch abgesichterte Energieeinsparvergütungen erhöhen. 


\section{Pfadwechselbeispiel: Innovative Wärmenetze der vierten Generation}

Der Anteil erneuerbarer Energien an der Erzeugung von Fernwärme liegt in Deutschland derzeit nur bei etwa zehn Prozent und beruht überwiegend auf der Verbrennung von Biomasse aus Abfallprodukten. Es dominieren fossile Brennstoffe: 83 Prozent der Wärme aus KWK-Anlagen und Heizwerken, die in Wärmenetze eingespeist wird, wird durch Verbrennung von Steinkohle, Braunkohle und Erdgas erzeugt.

In Anbetracht der städtisch geprägten Siedlungsstruktur in Deutschland sind emissionsarme Versorgungslösungen vor allem im urbanen und suburbanen Raum erforderlich. In urbanen Gebieten besteht zudem das Problem des Flächenmangels für die notwendigen zentralen Wärmeerzeugungskapazitäten: Größere solarthermische Anlagen, Biogasanlagen etc. können nur bedingt zum Einsatz kommen. Hinzu kommen hohe Infrastrukturkosten durch die notwendige Trassenverlegung und Nutzungskonkurrenzen im Planungsrecht (Schneller et al. 2018).

In der historischen Rückschau hat die Fernwärme einen Entwicklungsprozess über mehr als ein Jahrhundert durchlaufen. Abbildung 46 veranschaulicht den Übergang zwischen den Generationen im Zeitverlauf und den idealtypischen Verlauf der Effizienzsteigerungen verbunden mit niedrigeren Temperaturniveaus und der Erschließung neuer Energiequellen. Die zukünftige vierte Generation mit Betriebstemperaturen deutlich unterhalb vergangener Werte soll die Flexibilisierungsmöglichkeiten von Wärmenetzen voll ausschöpfen und die Effizienz des Gesamtsystems deutlich steigern (Lund et al. 2014). Alle lokal verfügbaren erneuerbaren Wärmequellen sollen bestmöglich eingebunden werden können.

Abbildung 46: Vier Generationen leitungsgebundener Wärme

$$
\text { 1G Dampf } 2 \text { G Heißwasser } \quad \text { GG LowEx }
$$
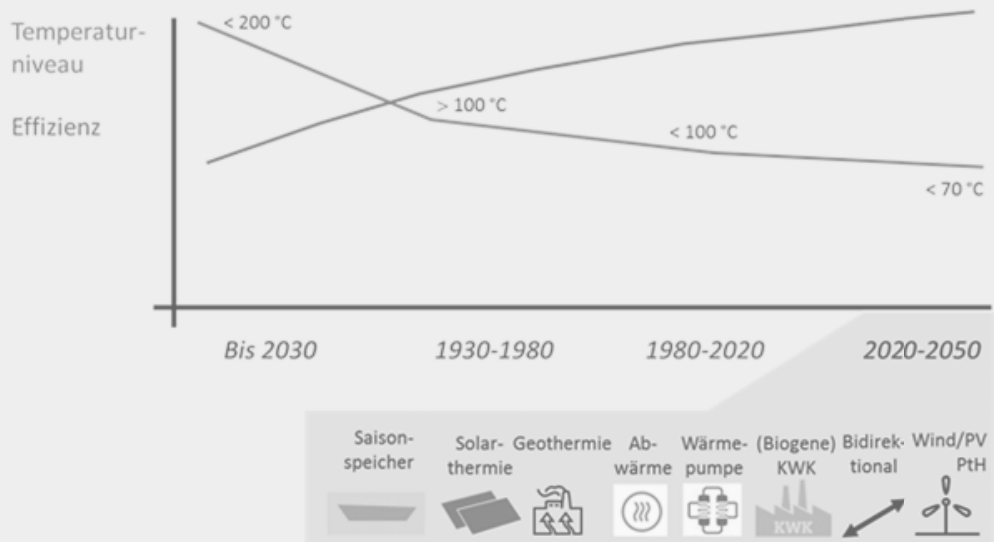

Quelle: Darstellung Pehnt et al. (2017, S. 20), nach Lund et al. (2014) 
Die niedrigeren Netztemperaturen von Niedrig-Exergie-Netzen (LowEx-Netzen) der vierten Generationen sollen dabei unter anderem folgende Vorteile bieten:

- Verbesserte und kostengünstigere Einbindung erneuerbarer Energiequellen

- Erschließung neuer Wärmequellen in Randgebieten, z. B. durch die Nutzung von Abwärme und die nötige Flächenverfügbarkeit für Solarthermie

- Systemische Effizienzsteigerungen durch Senkung der Netzverluste, insbesondere in Gebieten mit geringer Wärmedichte

- Vermeidung von Degradation durch verringerte thermische Spannungen in den Rohrmaterialien

- Steigerung der Akzeptanz durch partizipative Betreibermodelle und partizipative Organisationsstrukturen

Eine teilweise Übertragung der genannten Elemente auf den deutschen Kontext ist möglich und unter dem Gesichtspunkt der Zukunftsfähigkeit der Fernwärmeversorgung auch geboten. So ließe sich durch eine dezidiertere Förderung von Energiegenossenschaften die Ausbildung von Strukturen und Netzwerken fördern, mit der auch außerhalb der großen Ballungszentren integrierte Energiekonzepte mit Wärmenetzen erarbeitet und betrieben werden können. Ein solches Vorgehen würde jedoch implizit die »Lufthoheit« der regionalen und überregionalen Energieversorger einschränken, die diese aus den Zeiten der Gebietsmonopole heraus immer noch empfinden könnten. Gleichzeitig wäre eine schrittweise Einführung von Reinvestitionszielen in Anlehnung an das dänische Gemeinnützigkeitsprinzip denkbar, um langfristige Investitionen zu ermöglichen.

Ähnlich förderlich war die Entscheidung der dänischen Regierungen, fossile Brennstoffe kontinuierlich hoch zu besteuern und gleichzeitig Biomasse als Brennstoff von der Besteuerung auszunehmen. Auch in Deutschland existieren steuerliche Förderungen und Subventionen für Biomasse und KWKs, der Effekt ist jedoch augenscheinlich begrenzt (BMJV 2006). Insbesondere eine Verteuerung fossiler Brennstoffe könnte hier zu einer nachhaltigen Veränderung der Anreizstrukturen führen. Das Ziel sollte hierbei jedoch in Deutschland eher in einer Verschiebung hin zu solarer Wärmegewinnung und Abwärmenutzung liegen, da die Biomassenutzung in Deutschland zwar nicht erschöpft, aber nur noch sehr begrenzt ausbaufähig ist.

Ebenso wären eine umfassende Wärmeleitplanung sowie technologiespezifische Ziele und Sektorvereinbarungen denkbar, die in Dänemark durchaus erfolgreich darin waren, den Anteil an Fernwärme und die Nutzung erneuerbarer Energieträger im Wärmesystem zu erhöhen. Während in Deutschland bisher verstärkt auf energetische Gebäudesanierungen gesetzt wird (Maaß 
et al. 2015; Tappeser und Chichowitz 2017d) und Vorgaben für erneuerbare Wärmebereitstellung im EEWärmeG durch Effizienzmaßnahmen umgangen werden können (Clausen 2017g), sich so auch das Potenzial von Wärmenetzen und Wärmespeichern für die Integration von Solar- und Windenergie in das Energiesystem besser nutzen.

Von Seiten der Politik wird bisher nur sehr zaghaft versucht, die rechtlichen und ökonomischen Rahmenbedingungen zu schaffen, um eine Steigerung der Sanierungstätigkeit zu erreichen. Gleichzeitig bestehen in diesem Bereich zahlreiche Pfadabhängigkeiten und daher entsprechender Handlungsbedarf, um einem umfassenden Wandel anzustoßen.

Es gibt jedoch kein Allheilmittel auf dem Weg zum klimaneutralen Gebäudebestand. Wie in anderen politischen Handlungsfeldern auch ist ein Instrumentenmix erforderlich. Einige instrumentelle Ansätze, die zukünftig Bestandteile eines energiepolitischen Maßnahmenpakets sein sollten, werden nachfolgend kurz dargestellt. Dabei handelt es sich um Einzelmaßnahmen, die durch weitere flankierende Instrumente, wie etwa in der Energieeffizienzstrategie Gebäude (BMWi 2015a) beschrieben, ergänzt werden müssen.

\subsubsection{2 Übergreifendes Instrument $\mathrm{CO}_{2}$-Bepreisung}

Auf staatlicher Ebene sind niedrige Preise für fossile Energieträger durch die nur mäßigen Impulse des EU-Emissionshandels und die im Vergleich zu Vorreiterländern der Nachhaltigkeitstransformation wie Dänemark oder Norwegen niedrigen Energiesteuern auf lange Zeit festgeschrieben. Aufgrund des niedrigen Preisniveaus besteht aus Sicht der Marktteilnehmer/-innen nur ein geringer wirtschaftlicher Anreiz, erneuerbare Energieträger zu erschließen. Höhere Preise für fossile Energieträger, die als level playing field für alle in der Wärmeversorgung aktiven Akteurinnen und Akteure gelten müssten, sind deshalb eine zentrale Voraussetzung für die Wärmewende (Clausen 2017g).

Es ist daher sinnvoll und notwendig, dass die Folgekosten von $\mathrm{CO}_{2}$-Emissionen stärker als bisher internalisiert werden und sich der ökologische Nutzen erneuerbarer Energieträger in einem Preisvorteil ausdrückt. Höhere Kosten von gas- oder ölbetriebenen Wärmeversorgungsanlagen, beispielsweise durch eine $\mathrm{CO}_{2}$-Bepreisung, würden die Wettbewerbsfähigkeit verschiedener klimaschonender Wärmeversorgungsoptionen unmittelbar verbessern.

Die Einführung einer $\mathrm{CO}_{2}$-Bepreisung wird beispielsweise als wesentliche Bedingung für die Transformation der dänischen Wärmeversorgung angesehen. In Dänemark ist Biomasse als Wärmequelle von der Besteuerung ausgenommen, während auf fossile Brennstoffe eine gesonderte Steuer erhoben wird, sodass die Strom- und Wärmeerzeugung auf Grundlage von Biomasse eine gute Wettbewerbsposition innehat. 
Eine $\mathrm{CO}_{2}$-Bepreisung auf fossile Brennstoffe wäre außerdem gut geeignet, um angemessen auf veränderte Marktbedingungen durch Preisschwankungen bei fossilen Energieträgern zu reagieren. Die Steuer könnte bei sinkenden Öl-, Gasoder Kohlepreisen kurzfristig erhöht werden, um die externen Kosten der fossilen Energieträger weiterhin in ausreichendem Maße abzubilden und den gewünschten Ausbauanreiz für Wärmeerzeugungsanlagen auf EE-Basis zu erhalten.

\subsubsection{Weiterentwicklung ordnungspolitischer Ansätze}

Ein im Hinblick auf die unübersichtliche Rechtslage und damit verbundene Pfadabhängigkeiten zentraler Transformationsansatz bezieht sich auf die Vereinfachung und Harmonisierung ordnungsrechtlicher Vorgaben bei gleichzeitiger Erhöhung des Ambitionsniveaus im Hinblick auf die energetische Sanierung.

Hinsichtlich einer Vereinfachung der Gesetzeslage besteht zwischen den verschiedenen Akteurinnen und Akteuren weitestgehend Einigkeit, dass EnEV, Energieeinspargesetz und EEWärmeG zusammengeführt werden sollten. Dieser Prozess ist jedoch trotz langwieriger Diskussion bisher immer noch nicht abgeschlossen (Stand: November 2018). Anforderungen an die Energieeinsparung und die Nutzung erneuerbarer Energien würden dann direkt in diesem Gesetz geregelt. Die EnEV solle sich stärker auf technische Details fokussieren (Naturschutzbund Deutschland 2016).

Unklar ist, inwieweit eine Zusammenführung mit einer gleichzeitigen Erhöhung des Ambitionsniveaus in Bezug auf die energetische Gebäudesanierung verbunden werden kann. Für das Bundesministerium für Wirtschaft und Energie (BMWi) stellen der Wirtschaftlichkeitsgrundsatz und die Technologieoffenheit zentrale Grundbedingungen für das neue Gesetzesvorhaben dar. Vieles hängt jedoch an deren spezifischer Ausgestaltung. Fordert beispielsweise der Bundesverband der Deutschen Industrie (BDI), dass das Wirtschaftlichkeitsgebot sowie bestehende Ausnahmen für Sanierungspflichten in ihrer jetzigen Form zu erhalten seien (BDI 2016), so setzt sich die Gebäude-Allianz für eine Lebenszyklusbetrachtung ein. Diese soll sowohl bei der Berechnung der Wirtschaftlichkeit in Bezug auf die Kosten als auch bei der Berechnung energetischer Kennwerte Anwendung finden und die Ökobilanzen verschiedener Dämmstofftypen von der Produktion bis hin zur Entsorgung einschließen. Bestehende Ausnahmen und Auslegungsspielräume sollen hingegen weitestgehend abgebaut werden. Stattdessen wird eine Betrachtung der wirtschaftlichen Situation der jeweiligen Eigentums- und Nutzungsparteien zur Vermeidung sozialer Härten befürwortet (Gebäude-Allianz 2016). 


\subsubsection{Ausbau der staatlichen Förderung und Schaffung steuerlicher Anreize}

Seit 2006 fördert die Bundesregierung über das von der $\mathrm{KfW}$ betriebene $\mathrm{CO}_{2}$-Gebäudesanierungsprogramm die energetische Sanierung, aber auch den Neubau energieeffizienter Gebäude. Das Fördervolumen wurde schrittweise erhöht und liegt nach einer Aufstockung um 200 Millionen Euro im Rahmen des 2014 verabschiedeten Nationalen Aktionsplans Energieeffizienz (NAPE) bei zwei Milliarden Euro jährlich. Während die Bundesregierung mit den Erfolgen des Programms wirbt (Bezuschussung von Baumaßnahmen an vier Millionen Wohnungen, Auslösung privater Investitionen von 214 Milliarden Euro, Sicherung von 300.000 Arbeitsplätzen sowie Einsparungen von sechs Millionen Tonnen $\mathrm{CO}_{2}$; BMUB 2015), wurden im Rahmen des NAPE geplante steuerliche Anreize in Höhe von einer Milliarde Euro (BMWi 2014a) bisher nicht verwirklicht. Eine eigens eingerichtete Bund-Länder-Arbeitsgruppe wurde im Februar 2015 aufgrund des Widerstands der bayrischen Landesregierung ergebnislos aufgelöst (Neumann 2015). Stattdessen wurde ein Programm über 165 Millionen Euro zur Förderung von Qualifizierungsmaßnahmen sowie der Modernisierung von Heizungsanlagen und Lüftungssystemen beschlossen (BMWi 2015b).

Aus Sicht zahlreicher Akteurinnen und Akteure ist das aktuelle Fördervolumen jedoch nicht ausreichend. Ein von Prognos, dem Institut für Energie und Umwelt (ifeu) und dem Institut für Wohnen und Umwelt (IWU) erstelltes Hintergrundpapier zur Energieeffizienzstrategie Gebäude (Thamling et al. 2015) sieht eine mittelfristige Steigerung des Fördervolumens im Rahmen des $\mathrm{CO}_{2}$-Gebäudesanierungsprogramms auf vier bis fünf Milliarden Euro jährlich vor. Weitere empfohlene Maßnahmen beziehen sich auf eine übergreifende Anhebung der Energiesteuer sowie eine Rückerstattung der Grunderwerbssteuer bei ambitionierter Gebäudesanierung (Thamling et al. 2015).

\subsubsection{Gebäudeindividuelle Sanierungsfahrpläne}

In der Energieeffizienzstrategie Gebäude (BMWi 2015a) wird die Absicht geäußert, gebäudeindividuelle Sanierungsfahrpläne auf Bundesebene einzuführen. Die Umsetzung dieses Vorhabens steht allerdings noch aus. In Baden-Württemberg hingegen ist am 1. Juli 2015 die Verordnung zum Sanierungsfahrplan Baden-Württemberg (SFP-VO) in Kraft getreten, die das Beratungsinstrument für Eigentümerinnen und Eigentümer sowohl von Wohngebäuden als auch von Nichtwohngebäuden einführt. In der Praxis werden im Rahmen einer Energieberatung der Ist-Zustand eines Gebäudes analysiert und entweder eine umfassende Gesamtmaßnahme oder Teilschritte vorgeschlagen. Zusätzlich zu den Maßnahmen enthält die Dokumentation der Energieberatung Informationen zum Klimaschutz, Berechnungen zum Energiebedarf, die Ermittlung des Energieverbrauchs und Angaben zu Energiekosten und Einsparpotenzialen. Verpflichtend ist die Umsetzung der vorgeschlagenen Maßnahmen allerdings nicht. Der Plan 
soll darlegen, welche Sanierungsschritte in welcher Reihenfolge für die Immobilie sinnvoll sind. Er liefert Informationen und Entscheidungsgrundlagen, die helfen können, die energetische Verbesserung des Gebäudes optimal zu planen und umzusetzen.

\subsubsection{Auflösung des Investor-Nutzer-Dilemmas}

Ein weiterer strategischer Hebel ist die Steigerung der energetischen Sanierungsrate im Mietwohnungsbestand. In Deutschland lebt die Mehrheit der Haushalte zur Miete ${ }^{2}$, somit kommt der Modernisierung des vermieteten Gebäudebestands eine wichtige Rolle bei der angestrebten Emissionsreduktion zu. Dafür sind Rahmenbedingungen notwendig, die Vermieter/-innen, Mieter/-innen und den Staat angemessen an den Kosten und Erträgen energetischer Sanierungen beteiligen (Drittelmodell).

Die Durchführung von energetischen und damit langfristig klimafreundlichen Modernisierungen von vermieteten Bestandsgebäuden setzt jedoch zunächst einmal voraus, dass Sanierungsmaßnahmen auf Mieter- und Vermieterseite auf grundsätzliche Zustimmung stoßen. Die mietrechtlichen Bestimmungen beeinflussen die Investitionsbereitschaft auf Vermieterseite und die Akzeptanz auf Mieterseite für energieeffizienzgetriebene Sanierungen dabei in wesentlichem Maße (BMWi 2015a, S. 78).

Zur Verbesserung der Rahmenbedingungen ist deshalb ein Abbau existierender Sanierungshemmnisse erforderlich: Neben finanziellen Investitionshürden und der Komplexität der vielschichtigen Entscheidungsprozesse aufgrund großer Akteursvielfalt spielen insbesondere "geteilte Anreize« in Gestalt des Mieter-Vermieter-Dilemmas (allgemein: Investor-Nutzer-Dilemma) eine zentrale Rolle (Klinski et al. 2009, S. 76).

In Fällen, bei denen sich energetische Modernisierungsmaßnahmen nicht über die eingesparten Energiekosten amortisieren lassen, liegt die Vermutung nahe, dass Vermieter/-innen (etwa bei Selbstnutzung) die Maßnahme ohne Gegenfinanzierung durch die Mieter/-innen gemäß dem Wirtschaftlichkeitsgrundsatz nicht durchgeführt hätten (Wild 2017, S. 3). Wird die mögliche Energieeinsparung einer energetischen Modernisierungsmaßnahme überschätzt bzw. die Amortisationszeit unterschätzt, liegt für die Sanierungsmaßnahme aus Mietersicht ein Legitimationsdefizit vor (Techem 2016, S. 72).

Gleichzeitig wird mieterseitig angemahnt, dass die jetzigen Rahmenbedingungen den Vermietenden umfangreiche, kostenintensive und nur in geringem Maße energiesparende Maßnahmen ermöglichen, die nur zu einer begrenzten Einsparung von »warmen Betriebskosten« für die Mietparteien führen und bei

2 Laut den Ergebnissen der Einkommens- und Verbrauchsstichprobe 2013 des Statistischen Bundesamts leben 57 Prozent der Haushalte in Deutschland zur Miete (Statista 2019). 
hohen umlagefähigen Kosten finanzielle Härten mit sich bringen können (Wild 2017, S. 3). Anders als bei reinen Wohnwertsteigerungen, etwa durch eine Aufwertung der sanitären Anlagen oder einen Balkonanbau, sollte bei energetischen Modernisierungsmaßnahmen aus Wirtschaftlichkeitsgesichtspunkten eine Warmmietenneutralität die Zielstellung sein. Im Ergebnis sollten die Mieterinnen und Mieter im Anschluss an die energetische Modernisierung zwar eine höhere Kaltmiete zahlen; die warmen Betriebskosten sollten aber in gleichem Maße sinken.

\subsubsection{Roadmap Wärmewende im Überblick}

Die in Kapitel 3.1 genannten Pfadabhängigkeiten bedingen sich in vielerlei Hinsicht gegenseitig. Die geringe Rentabilität von Maßnahmen unter den bestehenden Marktbedingungen niedriger Preise für fossile Energieträger verringert die Wirksamkeit ordnungsrechtlicher Vorgaben, die unter das Wirtschaftlichkeitsgebot fallen. Es bestehen zu wenige wirtschaftliche Anreize, um den ambitionierten Vorgaben nachzukommen. Dies gilt insbesondere für den vermieteten Gebäudebestand aufgrund des Investor-Nutzer-Dilemmas. Gleichzeitig verhindern Informationsdefizite auf allen Seiten, allgemeine Imageprobleme, die mangelnde Expertise und Verfügbarkeit von Fachkräften und die Komplexität rechtlicher Vorgaben Nachfrageimpulse nach energetischen Sanierungsmaßnahmen.

Verschiedene Maßnahmen werden diskutiert, um den Problemlagen beizukommen - eine glaubhafte und von den wesentlichen Beteiligten mitgetragene Gesamtstrategie ist bisher jedoch kaum zu erkennen. Wie zuvor ausführlich beschrieben, ist es für eine erfolgreiche Wärmewende notwendig zwei strategische Pfade gleichzeitig einzuschlagen: Zum einen muss der Wärmebedarf deutlich gesenkt und damit das - in der ESG festgeschriebene - Prinzip »Efficiency First« operationalisiert werden. Zum anderen muss die Wärmeversorgung schnellstmöglich auf erneuerbare Energien umgestellt werden. Durch eine angemessene Kombination der beiden strategischen Ansätze lassen sich Lösungen hin zu einem nahezu klimaneutralen Gebäudebereich realisieren. Wichtig ist dabei sicherzustellen, dass entsprechende Maßnahmen praktikabel, sozialverträglich, wirtschaftlich und nutzerfreundlich sind.

Für beide strategischen Ansatzpunkte wurden zuvor sowohl vielversprechende Konzepte mit Innovationscharakter analysiert sowie geeignete politische Instrumente zur Überwindung der in Kapitel 5.2.2 aufgeführten Pfadabhängigkeiten vorgestellt. Für die erfolgreiche Transformation des Gebäudesektors sind als Ergebnis sowohl kurz- wie auch mittel- und langfristige Maßnahmen erforderlich, die in der Roadmap (Abbildung 47) zusammenfassend dargestellt werden.

Die Aufgabe der kurzfristigen Maßnahmen muss darin bestehen, bereits existierende ordnungsrechtliche Vorgaben an die klimapolitischen Erfordernisse anzupassen und zu vereinfachen sowie einen Ausbau der staatlichen Förderung 
für emissionsarme Wärmeversorgungslösungen und die Schaffung steuerlicher Anreize für energetische Sanierungsmaßnahmen auszuweiten. Die bestehende Subventionierung fossiler Energieträger, z. B. von Öl-Brennwertkesseln, sollte zeitnah beendet werden.

Mittelfristig müssen außerdem gebäudeindividuelle Sanierungsfahrpläne, Monitoring-Instrumente wie Gebäudeenergieausweise sowie die Möglichkeiten des Sanierungs-Contractings gezielt gestärkt und ausgeweitet werden. Die Information und Beratung durch qualifizierte Energieberater/-innen ist in diesem Zusammenhang sowohl aus wirtschaftlichen als aus Akzeptanzgesichtspunkten zentral. Weiterhin gilt es, die genannten Instrumente zur Minderung des Investor-Nutzer-Dilemmas als eines der zentralen Sanierungshemmnisse zu implementieren.

Auf lange Sicht müssen erneuerbare Energien zur Versorgung von Gebäuden fossile Energieträger vollständig ablösen. Dazu müssen die Wettbewerbsbedingungen erneuerbarer Heizungstechnologien deutlich verbessert und eine für Investorinnen und Investoren langfristig kalkulierbare $\mathrm{CO}_{2}$-Bepreisung als übergeordnetes Instrument eingeführt werden. Die $\mathrm{CO}_{2}$-Bepreisung sollte auf einem verlässlich definierten Pfad schrittweise angehoben werden, um die nötigen Modernisierungsimpulse auszulösen und den Marktakteurinnen und Marktakteuren sicherere Randbedingungen für Investitionen $\mathrm{zu}$ bieten.

Um die Verfügbarkeit und Effizienz der erforderlichen Heizungstechnologien auf Basis erneuerbarer Energien sicherzustellen und signifikante Kostensenkungen $\mathrm{zu}$ erreichen, ist eine umfangreiche Forschungsförderung essentiell. Dabei müssen zukünftig die Wechselwirkungen zwischen den Sektoren und die Potenziale der Sektorkopplung besser erforscht werden.

Aus- und Weiterbildungsmaßnahmen für Fachkräfte aus Handwerk und Bausektor gilt es ebenfalls langfristig zu fördern, um dem drohenden Fachkräftemangel entgegenzutreten, die Kapazitäten auf dem Bau zu erhöhen und die Qualität von energetischen Sanierungsmaßnahmen zu steigern. Zur angestrebten Verdoppelung der energetischen Sanierungsraten ist zudem ein kultureller Wandel erforderlich, der bestehende Sanierungsmythen verdrängt und die Partizipation der Bürgerinnen und Bürger für das Großprojekt Wärmewende stärkt.

Das Transformationsfeld der energetischen Gebäudesanierungen zeichnet sich insgesamt durch komplexe Akteurskonstellationen aus, die bei der Überwindung struktureller und organisationaler Pfadabhängigkeiten zentral sind. Die Immobilienbranche, der Wohnungs- und Mietmarkt, das Baugewerbe, Beteiligte aus Handwerk und Architektur, Politik, Wissenschaft und zivilgesellschaftliche Akteurinnen und Akteure beeinflussen die Geschwindigkeit der Transformation erheblich. Die Gebäudeeigentümerinnen und -eigentümer als wichtigste Akteursgruppe im Hinblick auf eine Verbesserung der Energieeffizienz von Gebäuden sind dabei von sehr heterogenen Interessen und Möglichkeiten geprägt. Eine spezifischere Betrachtung ihrer Bedürfnisse und Überzeugungen sowie 
damit zusammenhängend der jeweiligen Mietergruppen scheint zentral, um die verschiedenen Interessen im Kontext der Gebäudesanierung besser bedienen $\mathrm{zu}$ können. Mit Umweltverbänden und ökologisch motivierten Immobilieneigentümerinnen und -eigentümern bestehen intrinsisch motivierte Akteursgruppen, die es für Kooperationen zu berücksichtigen gilt. Ebenso sind Baugewerbe und -industrie, Fachkräfte aus dem Handwerk und der Energieberatung mit ihrem ökonomischen Eigeninteresse an einer erhöhten Sanierungstätigkeit wichtige Verbündete bei der Erhöhung der Sanierungsrate.

Die Klimaschutzziele der Bundesregierung werden außerdem nur erreicht werden, wenn energetische Quartiers- und Stadtentwicklung, Fragen des umweltund klimafreundlichen Wohnens und Bauens sowie der Energieeffizienz und der Einsatz erneuerbarer Energien im Gebäudebereich zukünftig Hand in Hand gehen.

Abbildung 47: Roadmap für eine Wärmewende im Gebäudesektor im Überblick
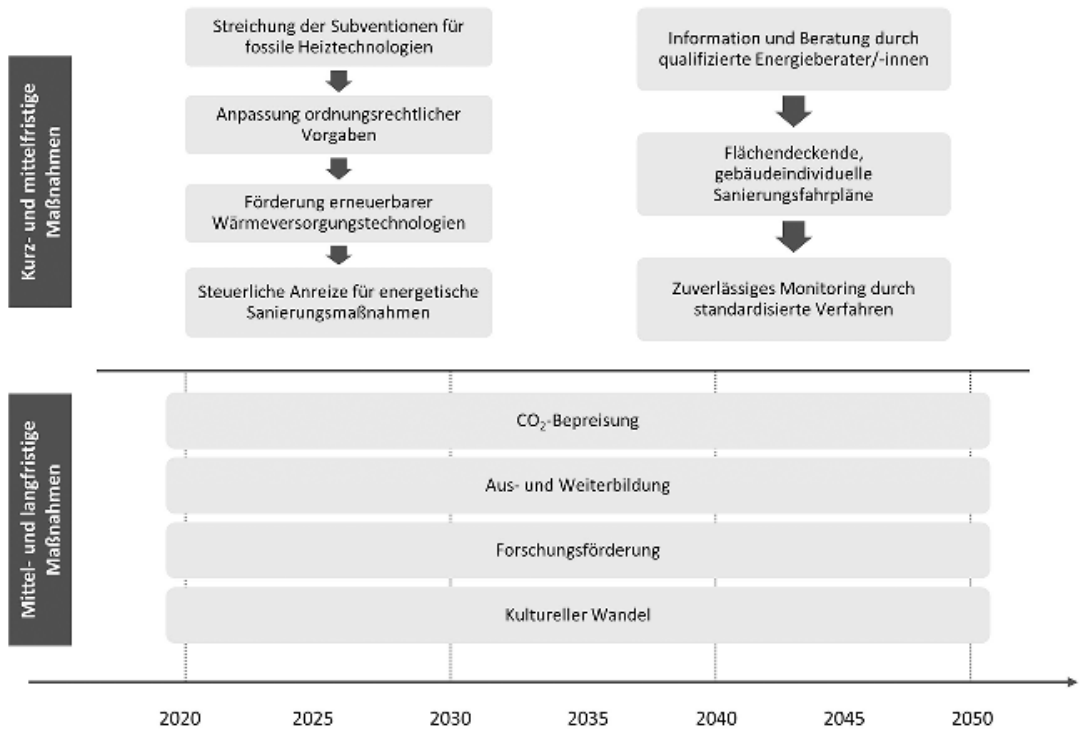

Quelle: Eigene Darstellung, adelphi.

\subsection{Roadmap E-Mobilität}

Um die deutschen Klimaziele $\mathrm{zu}$ erreichen ist ein $\mathrm{zu} 100$ Prozent klimaneutraler Pkw-Verkehr bis 2050 notwendig. Die einfachen Hybrid-Pkw wie auch verbesserte Benziner und Diesel oder Erdgasfahrzeuge stellen dabei keinen Beitrag zu wirklich klimaneutralen Pkw-Antrieben dar. Die Gruppe der Plug-in-Hybride ist dagegen schon eher geeignet, die Durchschnittsverbräuche an Energie sowie die 
Emissionen in einer Übergangsphase zu reduzieren. Brennstoffzellenfahrzeuge sind zwar potenziell klimaneutral zu betreiben und man kann sie schon zu hohen Preisen kaufen. Ihre Produktion in großen, den Markt verändernden Stückzahlen scheint jedoch vor Ende der 2020er-Jahre eher unwahrscheinlich (Clausen 2017d). Die vorliegende Roadmap fokussiert daher auf batterieelektrische Fahrzeuge (BEV), die zum einen in Kombination mit regenerativer Stromerzeugung zumindest für die Zukunft klimaneutrale Automobilität ermöglichen werden und die zum anderen mit heute schon weltweit in die Millionen gehenden Produktionszahlen auch von der Verfügbarkeit am Markt her am ehesten eine marktverändernde Alternative sind.

\subsubsection{Batterieelektrische Fahrzeuge}

Das Umweltbundesamt (Helms et al. 2016, S. 19) sieht beim Pkw ein Kontinuum vom konventionellen Antrieb durch Verbrennungsmotor über verschiedene Hybridvarianten (Hybrid, Plug-In, Range Extender) bis zum reinen Elektrofahrzeug. Für die Beurteilung der Klimawirkung von BEV ist dabei neben der Batteriegröße die Frage, ob der Strom aus dem Deutschlandmix oder aus erneuerbaren Quellen, z. B. Windstrom, kommt, entscheidend. Weitere Verbesserungen können durch die Versorgung der Fahrzeug- wie der Batteriezellproduktion mit erneuerbarem Strom erzielt werden.

Abbildung 48: Treibhausgasemissionen verschiedener Fahrzeugkonzepte unter heute durchschnittlichen Bedingungen in Deutschland in $\mathrm{CO}_{2} / \mathrm{km}$

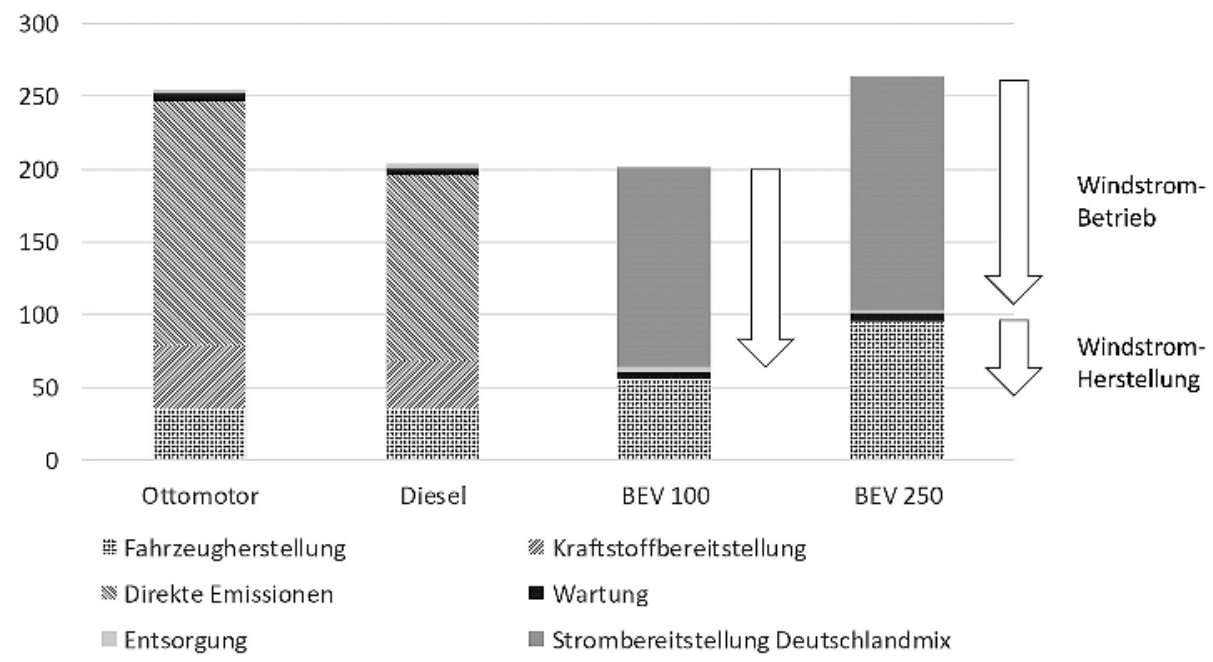

Quelle: In Anlehnung an Helms et al. (2016, S. 19). 
Für den reinen Elektroantrieb eines Pkw wird der Verbrennungsmotor mit seinen Komponenten fortgelassen und stattdessen ein oder zwei Elektromotoren an den Achsen oder in den Radnaben sowie eine Leistungselektronik zur Motorsteuerung und eine Batterie eingebaut. Obwohl Leistungselektronik und Elektromotoren erheblich leichter sind als ein Verbrennungsmotor, ergibt sich ein Gewichtsnachteil, da die Batterien noch sehr schwer sind. Die Herstellung einer Batterie ist zudem teuer und mit erheblichem Energie- und Ressourcenaufwand verbunden. Vielfältige Studien untersuchen die Herstellung von Li-Ionen Batterien ökobilanziell, zuletzt die von Romare und Dahllöf (2017), die bei der Batterieherstellung aus Umweltsicht einen erheblichen Bedarf zur Steigerung der Öko-Effizienz sehen. Mit Blick auf die Vielfalt der gegenwärtig in Entwicklung befindlichen Batteriesysteme, beispielhaft das Konzept einer Natrium-Glas-Batterie mit deutlich gesteigerter Kapazität pro Kilogramm (Braga et al. 2017) sowie eine Batterie mit ultrakurzer Ladezeit von sechs Minuten für $32 \mathrm{kWh}$ (Toshiba Corp Japan 2017), ist es nicht unwahrscheinlich, dass bei dieser Technologie noch erhebliche Effizienzpotenziale erschlossen werden können.

Helms et al. (2016) kommen aktuell zu dem Schluss, dass Elektrofahrzeuge, wenn sie mit erneuerbarem Strom betrieben werden, sowohl in der Nutzungsphase als auch in der Betrachtung über den gesamten Lebenszyklus klare Klimavorteile haben und der heute noch erforderliche Aufwand der Fahrzeugherstellung durch Verbesserung spezifischer Batterieeigenschaften, Folgenutzungen (second life) und verstärktes Recycling auch aus ökonomischen Gründen deutlich effizienter werden wird. Durch die Herstellung unter Nutzung regenerativer Energie kann dieser Vorteil weiter ausgebaut werden.

Elektrofahrzeuge stoßen in der Nutzung keine Emissionen wie $\mathrm{NO}_{\mathrm{x}}$ oder Feinstaub aus. Schädliche Emissionen sind eher mit der Herstellung verbunden, die Innenstädte würden also deutlich entlastet. Da die Geräuschentwicklung des Pkw-Antriebs neben den Windgeräuschen und den Reifen nur eine von drei wichtigen Ursachen ist, wirkt sich der leise Elektroantrieb auf die Lärmemissionen nur bei niedrigen Geschwindigkeiten bis $\mathrm{zu} 30 \mathrm{~km} / \mathrm{h}$ aus.

Nachdem erste im Vergleich zu Verbrennern wirtschaftlichere Elektrofahrzeuge bereits verfügbar sind (ADAC Fahrzeugtechnik 2018), ist mit ihrer Verbreitung relativ bald zu rechnen. Kostenvorteile entwickeln diese Fahrzeuge zunächst auf der Kurzstrecke. Ihre kleinen und damit preiswerten Batterien ermöglichen Reichweiten von 100 bis 150 Kilometern.

Parallel wird das Netz an Ladesäulen intensiv ausgebaut; die Reichweite der verfügbaren Fahrzeuge wird bis 2020 deutlich ansteigen, womit sich die Vorteilhaftigkeit der Elektrofahrzeuge auf weitere Marktsegmente ausdehnen wird. Auch die Fahrdynamik der Elektrofahrzeuge dürfte bei ihrer weiteren Diffusion eine wesentliche Rolle spielen (Clausen 2017d, S. 27; Valentine-Urbschat und Valentine-Urbschat 2014, S. 22). 


\subsubsection{Beurteilung der alternativen Antriebe mit Blick auf das Jahr 2050}

Im Zentrum des folgenden Szenarios stehen batterieelektrische Fahrzeuge. Brennstoffzellenfahrzeuge haben neben den noch sehr niedrigen Produktionszahlen den Nachteil einer im Vergleich zum batterieelektrischen Fahrzeug etwa um den Faktor 2,5 schlechteren Systemeffizienz. Eine solche niedrige Systemeffizienz weisen ebenso alle Technologien für die Herstellung flüssiger Kraftstoffe auf Basis von regenerativer Elektrizität, Wasser und Luft auf. So ist es zwar theoretisch möglich, klimaneutrale Kraftstoffe herzustellen, der kumulierte Energieaufwand ist aber sehr groß (Forschungsvereinigung Verbrennungskraftmaschinen 2013). Dies zeigt sich bei der Errechnung der Stromerzeugung, die notwendig ist, um genügend Energie für die gegenwärtig 626 Milliarden Kilometer Fahrleistung von Pkw in Deutschland (Kraftfahrtbundesamt 2017) zu haben. Für die verschiedenen klimaneutralen Antriebsarten wäre für den Betrieb der gesamten Pkw-Flotte in 2050 - gleiche Fahrleistung wie heute vorausgesetzt - die Erhöhung der Stromerzeugung um ca. 23 Prozent für den Batterieantrieb, um ca. 60 Prozent für eine Brennstoffzellenflotte und um bis zu 230 Prozent für den Antrieb durch »regeneratives Benzin« notwendig. Diese Steigerung der Stromerzeugung muss grundsätzlich auf Basis regenerativer Technologien erfolgen.

Abbildung 49: Notwendige Stromerzeugung für 626 Milliarden Kilometer Fahrleistung von Pkw (2016) für verschiedene klimaneutrale Antriebsarten

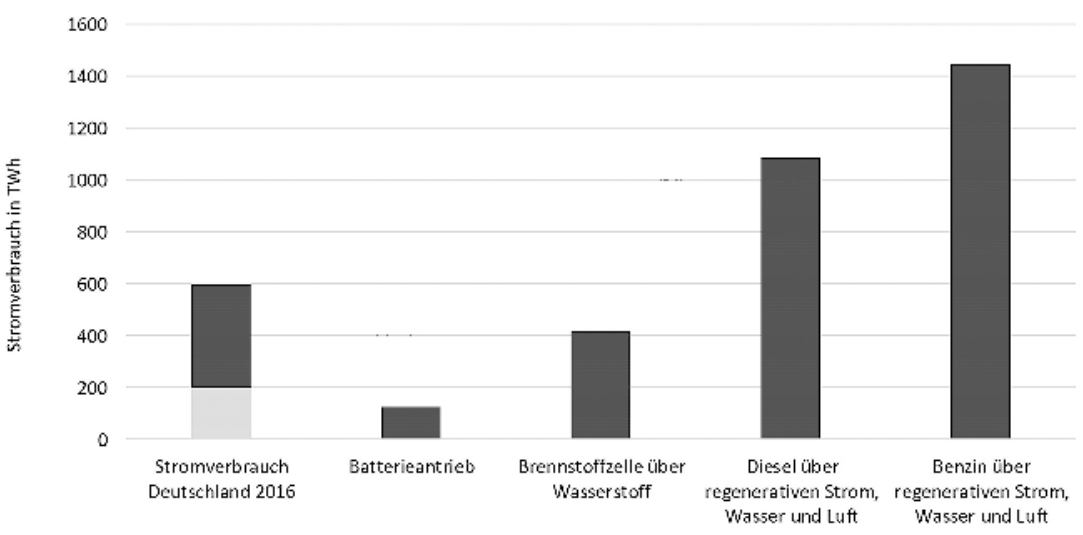

Quelle: Eigene Berechnung, Borderstep Institut für Innovation und Nachhaltigkeit.

Die Transformation der Pkw-Antriebe hin zu verbrauchseffizienten und schadstoffarmen Antrieben muss daher aus energiepolitischer Sicht im Wesentlichen durch batterieelektrische Fahrzeuge erfolgen.

Dabei dürfte die Batterie mit der durch sie bestimmten Reichweite und Ladezeit, ihren Kosten und dem notwendigen Aufwand zur Herstellung das zentrale 
Bauteil sein. Die Frage der Rohstoffsicherheit für die Batterieproduktion wird dabei gegenwärtig intensiv diskutiert. Aber parallel werden sehr unterschiedliche, leistungsfähige Batteriekonzepte entwickelt, die jeweils unterschiedliche Rohstoffe erfordern. Es wird nötig sein, bei der Auswahl der Batteriekonzepte für die Großserie nicht nur deren Leistung und Kosten, sondern auch deren Rohstoffbasis und Umweltverträglichkeit sowie die sozialen Aspekte der Rohstoffproduktion ins Kalkül zu ziehen.

\subsubsection{Ziele und Trends in der Elektromobilität}

Das zentrale Ziel der Transformation der Pkw-Antriebe in Richtung Nachhaltigkeit besteht darin, drei wesentliche Umweltauswirkungen zu reduzieren:

- Geringere $\mathrm{CO}_{2}$-Emissionen durch höhere Systemeffizienz sowie das Potenzial zur effizienten Nutzung regenerativer Energie

- Schadstofffreiheit im Fahrzeugbetrieb

- Reduktion der innerörtlichen Lärmemission

Das Ziel der Klimaneutralität in 2050 ist aus Sicht der Umweltpolitik imperativ und aus heutiger Sicht nur mit BEV erreichbar.

Die radikalste Lösung ist dabei die Umstellung auf einen reinen Elektroantrieb, langfristig immer im Zusammenhang mit einer Versorgung mit 100 Prozent Regenerativstrom gedacht. Im auf die Einhaltung des $2^{\circ} \mathrm{C}$-Ziels hin entwickelten Szenario 450 der Internationalen Energieagentur errechnete die IEA schon 2009 die Notwendigkeit, in 2030 im globalen Mittel im Neuwagenmarkt einen Anteil von 7 Prozent BEV, 21 Prozent Plug-in electric vehicles (PEV) und 29 Prozent hybrid electric vehicles (HEV) abzusetzen (Valentine-Urbschat und Valentine-Urbschat 2014, S. 145). Schon 2030 sollten in diesem Szenario nur noch 43 Prozent des globalen Marktes bei Benzin- und Dieselfahrzeugen verbleiben.

Valentine-Urbschat und Valentine-Urbschat (2014, S. 145) leiten aus den Zahlen zur Reduktion des Energieeinsatzes für die Mobilität im Szenario 450 die plausible Notwendigkeit ab, bis 2030 einen Anteil von ca. 25 Prozent BEV im Fahrzeugbestand der OECD-Länder zu etablieren. Das deutsche Ziel lautet seit 2009, in 2020 eine Million Elektrofahrzeuge auf die Straße zu bringen (Bundesregierung 2009). Das Szenario 450 würde es erforderlich machen, dass es schon 2030 ungefähr 10 Millionen Elektrofahrzeuge sind.

Auch die zivilgesellschaftlichen Kräfte in der Klima-Allianz Deutschland (2016, S. 20) fordern den Markthochlauf alternativer Antriebe. Aus der Sicht der Umweltverbände verspricht die Elektromobilität als Element einer Transformation zur Nachhaltigkeit nur dann eine wirklich umweltentlastende Wirkung, wenn sie Teil einer Verkehrswende ist und die Energiewende parallel und erfolgreich fortgesetzt wird (Lottsiepen 2014). Nur durch den Erfolg der Energie- 
wende lässt sich eine erneuerbare Stromversorgung der Elektromobile sichern und nur durch eine erfolgreiche Verkehrswende lässt sich der Modal Split soweit vom Pkw weg verlagern, dass wir in der Lage sein könnten, die Materialkreisläufe rund um die große Zahl schwerer Wagen zu bewältigen. Canzler und Knie (2014) gehen in ihrem Zukunftsbild sogar noch weiter und sehen sowohl Strom als auch Wärme und Verkehr als Teile einer gesellschaftlichen Versorgungsstruktur, die zu einem synergetischen »Gesamtkunstwerk« weiter entwickelt werden muss.

Die Bundesregierung hat lange Zeit ihr Ziel von einer Million Elektrofahrzeugen in 2020 betont (Nationale Plattform Elektromobilität 2016), welches aber von Jahr zu Jahr unrealistischer und zunehmend in Frage gestellt wird. Andere Länder treiben die Elektromobilität deutlich entschlossener voran, z. B. Norwegen (Clausen 2017c), Kalifornien (Clausen 2017b) und die Niederlande (Perleberg und Clausen 2017). Aber in diesen drei Ländern gibt es kaum eine Automobilbranche, die Fahrzeuge mit Verbrennungsmotor herstellt und gegen eine Politik der Elektromobilität Widerstand leisten würde; es existieren dort keine veto player.

Anders ist es in China. Nach dem aktuellen chinesischen "Development Plan for Fuel-efficient and New Energy Vehicles « im Zeitraum 2016 bis 2020 soll die Entwicklung von Elektro- und Hybridfahrzeugen in China entschlossen vorangetrieben werden (Beigang und Clausen 2017). Bis 2020 sollen chinesische Hersteller die weltweite Technologieführerschaft erlangen und an die Spitze der Verkaufszahlen von new energy vehicles aufsteigen (Tagscherer 2012, S. 4). In China sollen in 2020 schon 2,1 Millionen, fünf Jahre später 5,25 Millionen und 2030 dann 15,2 Millionen Elektrofahrzeuge abgesetzt werden, was 2030 einem Marktanteil von 40 Prozent entsprechen würde (Mizuho Bank 2017).

Chinesische Hersteller wie BYD führen schon heute die Liste der Unternehmen mit der höchsten Produktionszahl an BEV an. Zusammen mit Tesla, Streetscooter, Sono Motors und der e.Go Mobile AG wächst damit die neue Start-upSzene in der Automobilbranche zusehends.

Die deutschen Hersteller Volkswagen, BMW, Audi und Mercedes haben im Herbst 2018 alle schon vollelektrische Autos im Angebot. BMW will 2018 schon 140.000 Elektroautos verkaufen, in 2025 plant Volkswagen 2 bis 2,5 Millionen, BMW 500.000 und Daimler 400.000 Elektroautos zu produzieren. Zusammen wären das 2,9 bis 3,4 Millionen Wagen. Bei einer Batteriekapazität von 35 bis $50 \mathrm{kWh}$ pro Auto errechnet sich ein Batteriebedarf von 100 bis $170 \mathrm{GWh}$, während die Nationale Plattform Elektromobilität (NPE) für 2025 eine nationale Produktion von 13 GWh anstrebt (Nationale Plattform Elektromobilität 2016, S. 13). Ziel der NPE wäre damit ein Importanteil bei Batterien von 85 bis 93 Prozent. 


\subsubsection{Pfadabhängigkeiten und Hemmnisse}

Durch die zunehmende Zahl von Automobilen in der Gesellschaft verschob sich seit den 1950er-Jahren der Modal Split immer mehr zum motorisierten Individualverkehr. Parallel wuchsen die Größe der Automobilhersteller und Zulieferer sowie die Zahl der Beschäftigten in Produktion und Werkstätten. Dabei ist die (Pfad-)Abhängigkeit vom Automobil selbst größer als die vom »Bauteil« Verbrennungsmotor. Als bedeutendste Pfadabhängigkeiten des Verbrennungsmotors als Pkw-Antrieb sehen wir die folgenden (Clausen 2017d, S. 40):

\section{Technologische Pfadabhängigkeiten}

Deutschland hat (auch aus chinesischer Sicht) einen kaum einholbaren Technologievorsprung bei der Konstruktion und dem Bau von Verbrennungsmotoren. Nicht zuletzt durch anspruchsvolle Kundinnen und Kunden hierzulande haben sich die deutschen Automobile der Mittel- und Oberklasse zu internationalen Exportschlagern entwickelt. Der Pfadwechsel zum Elektrofahrzeug gefährdet diese Position. Nicht nur, dass es hierzulande noch kaum Kundschaft für Elektrofahrzeuge gibt, deren Reaktion und Feedback für die Hersteller zur Realisierung internationaler Top-Qualität unverzichtbar sind, auch die Zahl der Patente für viele der Schlüsseltechnologien der Elektromobilität war in Deutschland im internationalen Vergleich noch vor wenigen Jahren eher klein (e-mobil bw 2015); der Verband der Automobilindustrie (VDA) berichtete 2017 allerdings von einer deutlichen Trendwende (Wilkens 2017). Die Lieferkette weist zumindest im Batteriebau deutliche Lücken auf. Das durch die Nationale Plattform Elektromobilität aufgestellte Ziel, bis 2020 in Deutschland einen internationalen Leitmarkt für Elektromobilität aufzubauen, scheint mit dieser Ausgangsposition kaum erreichbar (Nationale Plattform Elektromobilität 2016, S. 14).

Denn bis in die 1990er-Jahre hinein wurden keine ernsthaften Anstrengungen zur Entwicklung alternativer Antriebe gemacht. Elektroantriebe wurden zwar für stationäre Anwendungen in der Industrie gebraucht und daher kontinuierlich verbessert; da sie aber nicht mobil eingesetzt wurden, verschwand die Batterietechnik langsam aus der universitären wie privaten deutschen Forschungslandschaft. Heute wird ein Großteil der Patente für den elektrischen Antriebsstrang von Unternehmen in Ostasien gehalten (Clausen 2017d, S. 19). Auch das Start-up Tesla in Kalifornien ist aktiv. Die deutsche Automobilbranche hat zwar ein wenig in Wasserstoffantriebe und Brennstoffzellen investiert, bietet aber im Gegensatz zu Toyota und Honda noch keine verkaufsfähigen Produkte an. Auch beim Verkauf von Fahrzeugen mit Elektroantrieben lag sie in Europa 2017 keineswegs an der Spitze.

Weitere Hemmnisse für batterieelektrische Fahrzeuge liegen in der begrenzten Reichweite in Verbindung mit einer erst im Aufbau befindlichen Ladeinfra- 
struktur. Batterien, die heute einigermaßen bezahlbar sind, bieten bis zu 100 oder 150 Kilometer Reichweite. Höhere Reichweiten sind mit höheren Kosten und höheren Umweltbelastungen in der Herstellung verbunden. Je kleiner aber die Reichweite, desto bedeutender das Problem der Ladeinfrastruktur, wobei nicht nur die Zahl der Säulen noch zu gering ist, auch die Bezahlsysteme sind noch nicht so weit entwickelt, dass wirklich jedes Elektrofahrzeug an jeder Säule laden kann.

\section{Ökonomische Pfadabhängigkeiten}

Niedrige Preise für Benzin und Diesel ermöglichen das Autofahren - mit Verbrenner - zu einem Preis, den die Autofahrerinnen und Autofahrer offensichtlich zu zahlen bereit sind. Auch die Zahlungsbereitschaft für den Kauf des Automobils selbst ist im breiten Publikum vorhanden. Flossen 1970 noch 12,5 Prozent der Konsumausgaben privater Haushalte in den Verkehr, so waren es 1990 schon 20,3 Prozent und 2010 24,9 Prozent (Statistisches Bundesamt 2019).

Etwa ein Viertel der Arbeitsplätze in der Zulieferbranche sind konkret an den Antriebsstrang für Verbrennungsmaschinen gebunden. Mit den hochmotorisierten Premiummodellen verdienen Hersteller und Zulieferer viel Geld. Auch volkswirtschaftlich besteht eine hohe Bedeutung und damit eine Abhängigkeit von der Automobilbranche mit ihren 471.300 Mitarbeiterinnen und Mitarbeitern zzgl. 302.700 Mitarbeitenden in deutschen Zulieferbetrieben. Die Automobilindustrie hat im Jahr 2016 ihren Umsatz auf 404,6 Mrd. Euro gesteigert und damit einen neuen Rekord erreicht. Mit 256,3 Mrd. Euro kamen fast zwei Drittel des Umsatzes aus dem Export (Verband der Automobilindustrie 2018). Die Exporte von Autos machen über 20 Prozent aller deutschen Exporte aus. Erfolg und Wettbewerbsfähigkeit der Automobilbranche sind damit wesentliche Faktoren für den nationalen Wohlstand.

\section{Organisationale Pfadabhängigkeiten}

Die Bindung der Hersteller wie auch der Zulieferer an den Verbrennungsmotor ist stark. Der große Hersteller Volkswagen konnte noch vor wenigen Jahren nicht glauben, dass die Post wirklich ein elektrisch angetriebenes Lieferfahrzeug wollte, worauf die Post es dann eigenständig mit einem Start-up der RWTH Aachen auf die Beine stellte (Clausen 2017a). Noch Ende 2017 erwarteten 54 Prozent der europäischen Automanager/-innen ein Scheitern des Elektroautos und 69 Prozent stimmten der Erwartung zu, dass erst die Brennstoffzelle mit dem »Brennstoff" $\mathrm{H}_{2}$ den Durchbruch der Elektromobilität bringen werde (KPMG 2018).

\section{Nutzerspezifische Pfadabhängigkeiten}

Das Automobil - mit Verbrennungsmotor - entwickelte sich zum selbstverständlichen Element des Alltags, und schrittweise wurden Infrastrukturen in eine bestimmte Richtung verändert: Wohnungen wurden fern von Arbeitsplätzen und 
Schulen gebaut oder gemietet, Geschäfte entstanden auf der grünen Wiese statt in den Innenstädten. Der Pfad des motorisierten Individualverkehrs stabilisierte sich kontinuierlich (Clausen 2017e).

Spezifische Hemmnisse für batterieelektrische Fahrzeuge liegen in der Einschränkung der Nutzung aufgrund der begrenzten Reichweite in Verbindung mit einer erst im Aufbau befindlichen Ladeinfrastruktur. Unsicherheiten bezüglich der Reichweitenplanung, der praktischen Durchführung des Ladevorgangs und der Bezahlung sowie der durch das Fahrzeug real möglichen Reichweite wurden durch die Presse phasenweise so stark geschürt, dass sie schon fast wie eine Scheindebatte wirkten. Der Mythos, dass nur mit einem »Brennstoff« gefahren werden kann, scheint sich nicht nur in der Autobranche, sondern auch in der Presse hartnäckig zu halten.

\section{Rechtliche Pfadabhängigkeiten}

Dem Wechsel vom Verbrenner zum Elektroantrieb stehen keine wesentlichen Vorschriften entgegen. Wenig wirksame umweltrechtliche Vorschriften zur Reduktion des Ausstoßes von Treibhausgasen, Stickoxiden und Feinstaub und die über Jahre fehlende Kontrolle machten es aber für die Hersteller attraktiv, kleinschrittige Verbesserungen durchzuführen anstatt auf grundsätzliche Änderungen des Antriebskonzeptes zu setzen (Clausen $2017 \mathrm{~d}$ ).

\section{Zusammenhänge}

Zwischen den verschiedenen Pfadabhängigkeiten bestehen insoweit Zusammenhänge, als sie nur zusammen die hohe Stabilität des sozio-technischen Systems »Automobilität mit Verbrennungsmotor« erklären können. Niedrige Treibstoffpreise zusammen mit der durch immense Werbeausgaben geförderten hohen Zahlungsbereitschaft der Kundschaft und einem komplett fehlenden bzw. wenig wirksamen umweltpolitischen Druck zur Veränderung ließen Hersteller und Zulieferer immer mehr immer leistungsstärkere Fahrzeuge mit Verbrennungsmotor entwickeln und erfolgreich vermarkten.

Auf die Verbreitung alternativer Antriebe wirken sich die Pfadabhängigkeiten mit unterschiedlicher Intensität aus. Herstellung und Nutzung von Hybrid-Pkw, die den bisher üblichen Verbrennern recht ähnlich - also »anschlussfähig« - sind, werden weniger stark behindert als die Vermarktung von batterieelektrischen Fahrzeugen und solchen mit Brennstoffzelle. 


\subsubsection{Ansatzpunkte eines Pfadwechsels}

\subsubsection{Chancen}

Mit einem grundlegenden Pfadwechsel vom Antrieb durch Verbrennungsmotoren zur Elektromobilität sind Chancen in der Umwelt- wie in der Wirtschaftspolitik verbunden.

Wirtschaftspolitisch besteht eine Chance darin, Deutschland in den nächsten Jahren zu einem Leitmarkt der Elektromobilität zu entwickeln. Ein solcher Leitmarkt müsste die normative Idee eines über den gesamten Pkw-Lebenszyklus wirksamen klimaneutralen Pkw-Verkehrs mit dem großen Vorsprung zusammenführen, den die deutsche Automobilbranche in der Produktion qualitativ hochwertiger Pkw hat. In Anlehnung an das Verständnis von Clustern bei Porter (1998, S. 6) ist hier darauf hinzuweisen, dass Cluster und Leitmärkte leistungsstarker Forschung, Hersteller und Zulieferer bedürfen, aber ebenso ohne eine Gruppe anspruchsvoller Kundinnen und Kunden nicht denkbar sind. Die Realisierung eines deutschen Leitmarktes für Elektromobilität und damit die angestrebte Technologieführerschaft deutscher Hersteller bedarf daher eines schnellen Wachstums des nationalen Absatzmarktes.

Umweltpolitisch kann die Elektromobilität aus heutiger Sicht einen großen und vergleichsweise rasch wirkenden Beitrag zur Bekämpfung des Treibhauseffektes, zur Reduktion der Schadstoffbelastung in Innenstädten sowie auch zur Reduktion der innerörtlichen Lärmemissionen leisten.

Bezogen auf den Anstoß eines Pfadwechsels besteht eine, wenn auch letztlich ambivalente, Chance aus Dieselgate ${ }^{3}$ heraus. Zwar hat Dieselgate die Befürworterinnen und Befürworter des Antriebs durch Verbrennungsmotoren auf den Plan gerufen, aber gerade bei in Innenstädten aktiven Flottenbetreibern wie der Post (Clausen 2017a), der Deutschen See (2017) oder der Caritas (e.GO Mobile AG 2017) fallen gegenwärtig erste Entscheidungen, größere Fahrzeugflotten auf Elektroantrieb umzustellen. Dabei ist durch Dieselgate neben Kosten und Klimafreundlichkeit auch die Schadstofffreiheit des Fahrzeugbetriebs ein wesentliches Argument geworden.

Diese Entscheidungen der Flottenbetreiber weisen auch auf eine weitere Chance der Elektromobilität hin. Die zweite Generation von Elektrofahrzeugen bietet deutlich günstigere total cost of ownership (TCO) als die bisher gefertigten hochpreisigen Wagen und ist auch gegenüber Fahrzeugen mit Antrieb durch Verbrennungsmotoren wettbewerbsfähig. Ein gutes Kosten-Nutzen-Verhältnis ist für die Verbreitung von Innovationen zentral; damit ist die weitere Kostensen-

3 Gemeint ist die im September 2015 durch eine Klage der US-amerikanischen Umweltschutzbehörde gegenüber VW ausgelöste Kontroverse um Abschaltvorrichtungen für Abgasreinigungssysteme von Dieselmotoren. 
kung, die sich auch bei den großen Herstellern andeutet, eine weitere wesentliche Chance für den Pfadwechsel.

\subsubsection{Risiken}

Die wirtschaftspolitischen Risiken sind gravierend. Gelingt der Aufbau eines Leitmarktes nicht rasch und bevor die Unternehmen anderer Nationen einen wesentlichen Vorsprung erreicht haben, dann droht der Verlust der Technologieführerschaft und ggf. auch ein drastisches Absinken des Exports. Zwar gibt es eine Gruppe innovativer Start-ups in der Elektromobilität nicht nur international (Geely, BYD, Tesla, Polestar), sondern auch national (Streetscooter, Sono Motors, e-Go Mobile AG), aber die Präsenz gerade der großen Unternehmen der Automobilbranche im Markt für Elektroautos ist noch nicht da, wo sie sein sollte. Genau wie in Politik und Gesellschaft dürften auch innerhalb der deutschen Autobranche Befürworter/-innen und Gegner/-innen des Pfadwechsels noch um die strategische Führung ringen. Eine wirksame nationale Koordination und ein gutes Narrativ der "starken deutschen Hersteller mit den weltbesten PremiumElektro-Angeboten « könnten auch in der Gruppe der unternehmerischen Akteure Entwicklungen beschleunigen und mehr Einigkeit schaffen.

Umweltpolitisch bestehen die Risiken in einem Misserfolg bei der Begrenzung des Klimawandels und entsprechend katastrophalen und globalen Langfristfolgen, wobei sich der Klimaschutzbeitrag durch die Elektromobilität in Deutschland durch internationale Effekte über die Vorbildwirkung von Deutschland potenzieren könnte. Ein weiteres umweltpolitisches Risiko besteht darin, dass es nicht gelingt, die Herstellung der Batterien durch Fortschritte in der Effizienz deutlich material- und energieeffizienter zu machen. Auch die ressourcenpolitisch notwendige Realisierung eines besseren Recyclings und Materialkreislaufs in der Automobilwirtschaft steht noch aus (Tappeser und Chichowitz 2017c).

Mit Blick auf die Koordination des Pfadwechsels zu einer Elektromobilität mit starken deutschen Herstellern und Marktführerschaft bei Premium-Elektro-Angeboten besteht ein erhebliches Risiko in der bisher wenig ausgeprägten Fähigkeit der "Deutschland AG« mit ihren politischen und industriellen Akteurinnen und Akteuren, einen wirksamen wirtschaftspolitischen Rahmen zu setzen und Förderaktivitäten optimal zu koordinieren. Dazu bedarf es eines kohärenten und koordinierten Vorgehens politischer Akteurinnen und Akteure aus verschiedenen Ministerien, die sich nicht permanent durch Lobbyinteressen ablenken oder durcheinander bringen lassen. Ein zu starkes Bremsen durch die bisher am Verbrennungsmotor hängenden Anspruchsgruppen von der Industrie über die Gewerkschaften bis hin zu Regionen und Bundesländern mit bisher starken Produktionsstandorten könnte die Realisierung der oben aufgeführten Chancen so verzögern, dass mit Blick auf das Zieljahr 2030 alle national beteiligten Akteurinnen und Akteure auf der Verliererseite stehen könnten. 
Einiges deutet sogar darauf hin, dass wesentliche Akteurinnen und Akteure immer noch kein wirkliches Interesse am Aufbau eines Leitmarktes für Elektromobilität haben: zum einen die oft immer noch schlechte Kaufberatung in den Autohäusern der großen Hersteller (Helmer und Gnannt 2017), zum anderen die immer wieder kritisch statt konstruktiv geführte Debatte um die öffentliche Ladeinfrastruktur, die teilweise den Charakter einer Scheindebatte annimmt. Während sich Elektrofahrzeuge zunächst offenbar eher als Zweitwagen und Lieferfahrzeuge etablieren, die - wie auch die norwegischen Erfahrungen zeigen (Clausen 2017c) - für Millionen von Haushalten daheim geladen werden können, wird in Deutschland das Elektrofahrzeug immer wieder an der Nutzung des Pkws mit dem One-fits-all-Ansatz gemessen. Die »Reichweitenangst « zu schüren ist sogar mit dem Risiko verbunden, zu viel Geld in eine Ladeinfrastruktur zu stecken, die z. B. mit Blick auf die Innenstädte angesichts einer weiterentwickelten Batterietechnik schon in zehn Jahren wieder überholt sein könnte.

\subsubsection{Maßnahmen und politische Initiativen}

Für die erfolgreiche Einführung von Elektrofahrzeugen in großer Zahl in den deutschen Automobilbestand sind sowohl kurz- (bis 2020) als auch mittelfristige (bis 2025) Maßnahmen erforderlich. Langfristige Entwicklungen, z. B. das ab 2025 oder $2030 \mathrm{zu}$ erwartende autonome Fahren, werden nicht berücksichtigt.

Die Aufgabe der kurzfristigen Maßnahmen besteht primär darin, die Ladeinfrastrukturen systematisch aufzubauen und den Absatzmarkt für die um das Jahr 2020 herum geplante Markteinführung zahlreicher elektrischer Modelle der großen deutschen Hersteller vorzubereiten.

Die mittel- und langfristigen Maßnahmen bauen auf dem Erfolg der kurzfristigen Maßnahmen auf und zielen auf die dauerhafte Sicherung und den Ausbau der Wettbewerbsfähigkeit der deutschen Automobilhersteller mit Premium-Elektrofahrzeugen im Weltmarkt der zweiten Hälfte der zwanziger Jahre.

\subsubsection{Einfache und praxisgerechte Ladeinfrastruktur}

Prioritär sollten in den Jahren 2019 und 2020 die Probleme der Ladeinfrastruktur gelöst werden. Ladeangebote müssen für daheim, für den Arbeitsplatz und für unterwegs aufgebaut werden. Die richtigen Lösungen variieren dabei mit den Fortschritten der Batterietechnik. Gelingt z. B. Toshiba die Serienproduktion des 32 kWh-Akkus, der sich in sechs Minuten laden lässt (Toshiba Corp Japan 2017), könnten Innenstadtbewohner auch zukünftig zu einer »Tankstelle« fahren und dort auf das Laden warten. Ein flächendeckendes Ladenetz in Stadtteilen mit mehrstöckiger Bebauung wäre dann verzichtbar. Aus heutiger Sicht sind neben der Anzahl und Leistung der Ladepunkte auch Probleme der Bezahlsysteme von hoher Bedeutung: 
- Wichtig ist eine hinreichende Zahl öffentlicher Ladepunkte in Städten und an Autobahnraststätten.

- Die Ausstattung öffentlicher Parkplätze mit Lademöglichkeiten ist zu verbessern. Dabei ist die Sichtbarkeit zu optimieren und an Ladeplätzen für Elektroautos muss ein Halteverbot für Fahrzeuge mit Verbrennungsmotoren ermöglicht und wirksam durchgesetzt werden.

- Es sollte ein »Recht auf das Laden zu Hause« auch in Mietwohnungen und Eigentumswohnungen mit Stellplatz oder Garage gesetzlich verankert werden.

- Wichtig sind weiter Roaming-Vereinbarungen für Ladesysteme, sodass man mit der eigenen Tankkarte überall zahlen kann, sowie transparente Kosten, sodass man von den Kosten der Ladeaktivität nicht überrascht wird. Bei beidem dürfte analog zu den Aktivitäten der Bundesnetzagentur eine ordnungsrechtliche Regulierung erforderlich sein.

- Hilfreich könnte die integrierte Entwicklung von Elektromobilität und Digitalisierung sein, z. B. der Einsatz von Apps bei der Suche nach freien Ladeplätzen, bei der Abrechnung sowie hinsichtlich der Schnittstellen zur multimodalen Mobilität (ÖPNV, E-Bikes, Fahrradmiete, Carsharing).

\subsubsection{Preiswerte und nutzergerechte Elektroautos}

Von zentraler Bedeutung ist, dass Elektroautos im Vergleich zu Autos mit Antrieb durch einen Verbrennungsmotor wettbewerbsfähiger werden, sowohl was den Verkaufspreis als auch die Reichweite und eine nutzergerechte Variantenvielfalt angeht. Dies lässt sich auf verschiedenen Wegen erreichen:

Wichtig ist die Entwicklung von Automobilen, die von vornherein »elektrisch « angelegt sind und so preiswerter produziert werden können als Wagen, die quasi umgebaute Verbrenner sind, z. B. heute der E-Golf oder der E-Up. Dass dieser Weg aussichtsreich ist, zeigen die Entwicklung des Streetscooter, des e.GO Life, des Tesla Model 3 und auch der Modulare Elektrobaukasten (MEB) von Volkswagen mit seinen geplanten Modellen. Das jetzt in Entwicklung befindliche Grundmodell des Volkswagen ID soll eine mit dem Golf vergleichbare Preisgestaltung haben. Über günstigere Betriebskosten würden sich Vorteile in den total cost of ownership ergeben. Als mögliche Aktivität bietet sich an:

\section{- Entwicklung preiswerter Modelle durch die Hersteller}

Wichtig ist auch ein breiteres Angebot von Modellen mit höherer Reichweite. Während zur Zeit Elektrofahrzeuge primär in der Luxusklasse (Tesla S, Tesla X), der Kleinwagenklasse (Renault ZOE, Nissan Leaf, VW E-Up, BMW i3) und bei Transportern (Streetscooter, E-Crafter, Mercedes-Benz e-Vito) angeboten werden, fehlt es noch an Mittelklassewagen, Vans, Kombis, SUVs oder Cabriolets. Da sich die von den Kundinnen und Kunden gewünschte Fahrzeugklasse nicht am Antrieb 
orientiert, ist zu erwarten, dass mit einem breiteren Angebot an Modellen auch die Zahl der Käuferinnen und Käufer eines Elektroautos zunimmt. Weiter ist es erforderlich, die Reichweite der angebotenen Modelle auf das Mindestniveau von nominell ca. 400 Kilometern zu steigern. Handlungsoptionen sind hier:

- Entwicklung breiter Modellfamilien mit Mindestreichweite von 400 Kilometern durch die Hersteller

- Aufklärungskampagnen, dass eine nominelle Reichweite von 400 Kilometern völlig ausreichend ist

\subsubsection{Nutzergruppen für die Elektromobilität erschließen}

Die Änderung von Wunschvorstellungen, Erwartungen und Routinen bei breiten Kundenkreisen in Bezug auf Automobile ist erforderlich. Heute noch dürfte sich die unhinterfragte Grundvorstellung in vielen Köpfen befinden, dass ein Auto brummt, bei Vollgas aufheult und sich in wenigen Minuten volltanken lässt. Es wird erwartet, dass man auch spontan zu langen Fahrten aufbrechen kann. Einige dieser Vorstellungen müssen sich, zumindest mit Blick auf die heutige Batterietechnik und Ladeinfrastruktur, ändern. Zwar ist möglich, dass mit radikal kürzeren Ladezeiten in Zukunft diese Unterschiede zwischen Verbrenner und Batteriefahrzeug fast verschwinden, heute aber sind sie ohne Zweifel vorhanden. Die Änderung dieser unhinterfragten Vorstellungen und Wünsche ist letztlich ein Vorgang der Änderung der Mobilitätskultur und es ist zu erwarten, dass dieser Vorgang vergleichsweise viel Zeit - in jedem Falle mehrere Jahre - benötigt. Hier könnte der »kritische Pfad« des Pfadwechsels zur Elektromobilität liegen. Als Maßnahmen bieten sich folgende Aktivitäten an:

- Erhöhung der Kaufprämie für einen begrenzten Zeitraum von ca. 2 bis 3 Jahren, um möglichst schnell eine zunehmende Zahl von privaten und professionellen Nutzerinnen und Nutzern »umzugewöhnen« sowie die Zahl der Elektromobilistinnen und -mobilisten zu vergrößern, die ihre positiven Erfahrungen in ihrem jeweiligen sozialen Umfeld bzw. ihrer jeweiligen Region verbreiten.

- Einfache Möglichkeiten, ein Elektrofahrzeug einen oder mehrere Tage probezufahren, und niederschwellige Testangebote wie der Nordseeflitzer ${ }^{4}$ dürften sich als zentrales Instrument erweisen, die Erfahrung des elektrischen Fahrens zu verbreiten und Hemmschwellen abzubauen.

4 Der Nordseeflitzer kann in einigen Touristengemeinden Ostfrieslands mit der Kurkarte einmal gratis ausgeliehen und darüber hinaus gemietet werden. Mit dem elektrischen Nordseeflitzer, vom dem es sechs Exemplare des Renault ZOE in sechs Kommunen gibt, machen viele Touristen ihre ersten Erfahrungen mit der Elektromobilität. Die kostenlose Möglichkeit einer »Schnupperleihe« ist attraktiv. 
- Dadurch, dass E-Fahrzeuge als Dienstfahrzeuge zur Verfügung stehen, gewöhnen sich die betroffenen Mitarbeiterinnen und Mitarbeiter an diese Fahrzeuge und meist entsteht eine erfreuliche Akzeptanz. So kann eine »ökonomische« Fahrweise erlernt werden, um die geplanten Reichweiten zu erreichen und Unsicherheiten abzubauen. Die Etablierung von Elektrofahrzeugen in Fahrzeugpools mit vielen verschiedenen Nutzerinnen und Nutzern ist damit von Bedeutung.

- Vorteile der Nutzung von Elektroautos sollten für die Nutzerinnen und Nutzer deutlich spürbar und erlebbar werden. Eine Umschichtung der EEG-Umlage auf alle Energieformen (Strom, Heizöl, Gas, Benzin und Diesel) würde den Strompreis relativ senken und so die relativen Kosten der Elektromobilität verringern. Bei der Kfz-Steuer und den Regelungen zur Dienstwagenbesteuerung sollten ebenfalls Vorteile für Elektroautos deutlich werden. Exklusive und kostengünstige Parkmöglichkeiten sollten geschaffen werden.

\subsubsection{Regionale Aktivitäten und Kommunikationsmaßnahmen}

Durch regionale Bündnisse kann der Pfadwechsel zur Elektromobilität unterstützt werden. Diese könnten sowohl die Entwicklung in Produktion, Herstellung und Standortmarketing rund um mögliche Cluster »Elektromobilität« in Bewegung bringen. Aber auch die Verbreitung und Nutzung von Elektromobilen im Tourismus, durch Privatleute und durch Flottenkundinnen und -kunden kann mithilfe solcher Bündnisse gefördert werden. Infoveranstaltung für Autohäuser, Flotten- und Privatkundinnen und -kunden wären durch solche Bündnisse durchzuführen. Die Kooperation mit den Gemeinden und Stadtwerken bietet sich hier genauso an wie die Zusammenarbeit mit Stromversorgern, um auch die Ladeinfrastruktur koordiniert, sinnvoll und zukunftsfähig weiter auszubauen. Die Koordination der Einzelaktivitäten könnte zu regelmäßigen Rückmeldungen an die Bundesregierung führen, deren Rahmenbedingungen für die gesamte Entwicklung sehr bedeutend sind, deren Wirkung in der regionalen Praxis aber der regelmäßigen Rückmeldung bedarf. Als Maßnahmen bieten sich an:

- Bildung regionaler Bündnisse für Elektromobilität

- Aktivitäten zum Ausbau der Ladeinfrastruktur sollten durch kommunikative Maßnahmen begleitet werden, um potenzielle Kundinnen und Kunden davon zu überzeugen, dass eine vollständige und leistungsfähige Ladeinfrastruktur in 2020 zur Verfügung steht.

- Kommunikationsaktivitäten, die die (positiven) Erfahrungen von privaten wie auch professionellen Elektromobilistinnen und -mobilisten verbreiten, sollten von Herstellern und der Nationalen Plattform Elektromobilität unterstützt werden. Möglich wären einfache Formate wie »Die Sendung mit der Maus«, aber auch grenzüberschreitende Öffentlichkeitsarbeit, z. B. Stammtische für 
E-Auto-Nutzerinnen und -Nutzer in Autohäusern, die öffentlich auffallen, durch die aber auch zahlreiche Autoverkäuferinnen und -verkäufer in Kontakt zu positiven E-Auto-Erfahrungen kämen. Über große Infoveranstaltung für Autohäuser und Flottenkundinnen und -kunden könnten Hersteller und Kommunen professionelle Nutzerinnen und Nutzer sowie Intermediäre ansprechen, informieren und für die Elektromobilität gewinnen.

\subsubsection{Politische Priorisierung und Zuordnung der Elektromobilität}

Seit 2010 existiert die Nationale Plattform Elektromobilität. Noch 2016 veröffentlichte sie im Wegweiser Elektromobilität (Nationale Plattform Elektromobilität 2016) die Prognose, dass in 2017 ca. 200.000 Elektromobile im deutschen Fahrzeugbestand sein würden. Real waren es zum 31. Dezember 2016 allerdings nur 34.000 Wagen. Die Arbeit der NPE insgesamt ist bisher alles andere als erfolgreich. Die Zahl der Publikationen, mit der die NPE das für die wirtschaftliche Zukunft des Landes zentrale Thema begleitet, liegt seit ihrer Gründung bis 2017 bei etwa zwei Publikationen pro Jahr. Mit dem Förderprogramm zum Aufbau der Ladeinfrastruktur hat das Bundesministerium für Verkehr und digitale Infrastruktur ausgerechnet die Nationale Organisation Wasserstoff- und Brennstoffzellentechnologie (NOW) beauftragt, also eine Organisation, die im Kern zur Förderung einer deutlich weniger effizienten Alternative gegründet wurde. Die Etablierung eines nationalen Leitmarktes ist nach wie vor in weiter Ferne. Das langjährige Ziel, in 2020 eine Million Elektrofahrzeuge auf deutschen Straßen zu haben, hält die Bundeskanzlerin aktuell für unrealistisch (Der Tagesspiegel 2017). Referate für Elektromobilität existieren in verschiedenen Bundesministerien.

- Es sollte erwogen werden, das Thema aufgrund seiner Bedeutung für Wirtschaft, Energie und Umweltschutz primär dem BMWi gemeinsam mit dem BMUB zuzuordnen und hier eine hinreichend ausgestattete und wirksame Koordinationsstelle einzurichten, die die Aufgaben der NPE übernimmt und insbesondere die Themen Energiewende und Elektromobilität aufeinander abstimmt. Alternativ ist eine deutliche Aufwertung der NPE denkbar. Eine zukünftige Koordinierungsstelle Elektromobilität sollte auf einem politischen Langfristziel und einem starken Narrativ aufbauen, von allen beteiligten Bundesministerien unterstützt werden und über ausreichende Mittel verfügen bzw. diese mobilisieren können, um einen erfolgreichen Pfadwechsel zu begleiten. Wesentlich ist auch die Koordinierung der Arbeiten zur Anpassung des rechtlichen Rahmens.

- Für die Verkehrspolitik ist das Thema der Pkw-Antriebe dagegen von eher geringem Belang, da die Art des Antriebs sich nur marginal auf die Möglichkeiten der Mobilität auswirkt. Während die Ladeinfrastrukturen relativ eindeutig in die Zuständigkeit der Verkehrspolitik fallen, ist dies für die Antriebstechnik 
kritisch zu sehen. Dies gilt besonders deshalb, weil nur etwa 40 Prozent der in Deutschland gebauten Autos hierzulande zum Einsatz kommen. Die wirtschaftspolitische Bedeutung für Arbeitsplätze und Export sowie die umweltpolitische Bedeutung für den Klimaschutz erfordern hier ein Umdenken.

\subsubsection{Langfristige Wettbewerbsfähigkeit der deutschen Automobilindustrie im Weltmarkt}

In der deutschen Automobilindustrie arbeiten über 800.000 Menschen. Sie exportiert ca. 60 Prozent ihrer Produkte im Wert von ungefähr 200 Milliarden Euro p.a. Dass diese Branche auch nach dem Pfadwechsel zur Elektromobilität wettbewerbsfähig ist und hochpreisige Premium-Fahrzeuge erfolgreich exportiert, ist für den Wirtschaftsstandort Deutschland zentral.

- Von kaum zu überschätzender Bedeutung ist die Batterietechnik, von der Reichweite, Ladezeiten, Kosten und Ökoeffizienz der Herstellung von Elektrofahrzeugen entscheidend abhängen. Koordiniert durch die Bundesregierung und unterstützt durch Forschungsförderung sollte sowohl an der Forschung und Entwicklung wie an der Skalierung der Produktion von Batteriezellen und Batterien gearbeitet werden. Die F\&E zu neuen, leistungsfähigeren, preisgünstigeren und ressourcenschonenderen Batteriekonzepten sollte, ähnlich wie in Japan (The Japan Times 2018), deutlich gefördert werden. Die Skalierung der Produktion sollte durch die Hersteller erfolgen und das Ziel des Volumens der inländischen Batterieproduktion sollte von $13 \mathrm{GWh}$ in 2025 (Nationale Plattform Elektromobilität 2016, S. 13) auf mindestens $100 \mathrm{GWh}$ hochgesetzt werden. Im neuen Fortschrittsbericht 2018 der NPE wird der Batterietechnologie eine hohe Bedeutung zugesprochen, neue Zahlen werden aber nicht genannt (Nationale Plattform Elektromobilität 2018, S. 36).

- Eine zentrale Stärke der deutschen Hersteller ist die Fähigkeit, hochqualitative Premiummodelle zu produzieren. Dies eröffnet ihnen sogar objektiv die Chance, als me too Elektroautos zwar nicht als erste einzuführen, sie dafür aber »besser« zu machen. Aber auch hierzu benötigen die deutschen Hersteller einen starken Leitmarkt mit anspruchsvollen Kundinnen und Kunden in Deutschland, für die noch für einige Jahre erhebliche Kaufanreize erforderlich sein werden. Nur aus einem starken Heimatmarkt können Rückmeldungen und Impulse in die F\&E der Hersteller kommen, die die Premium-Produktion deutscher Hersteller sichern helfen.

\subsubsection{Klimaneutrale Autoproduktion}

Während bei Fahrzeugen mit Verbrennungsmotor die Fahrzeugherstellung nur 15 bis 20 Prozent der gesamten Treibhausgasemissionen ausmacht, erhöht sich dieser Wert für Elektrofahrzeuge, die mit erneuerbarem Strom angetrieben werden, 
auf über 90 Prozent (Helms et al. 2016, S. 19). Eine klimaneutrale Produktion ist also zur langfristigen Optimierung dieser Fahrzeuge mit dem Ziel der Klimaneutralität von besonderer Bedeutung.

Der ab 2021 geltende Grenzwert für Flottenemissionen von $95 \mathrm{C} \mathrm{CO}_{2} / \mathrm{km}$ für alle neu zugelassenen $\mathrm{Pkw}$ kann durch eine $\mathrm{CO}_{2}$-freie Autoproduktion bislang nicht reduziert werden, da hierzu die politischen Instrumente fehlen. Eine klimaneutrale (Fahrzeug-)Produktion in Deutschland sollte aber politisch unterstützt werden, da durch sie ein im Laufe der Zeit immer wichtigerer relativer Beitrag zur Erreichung der Klimaziele geleistet werden muss. Regenerativer Strom steht vielerorts zu wettbewerbsfähigen Gestehungskosten zur Verfügung, wird aber durch institutionell bedingte Kosten des Energiesystems so verteuert, dass der Bezug bei den Herstellern intern wirtschaftlich kaum durchgesetzt werden kann. Die gleichen Problematiken, die im Energierecht die dezentrale Erzeugung immer wieder benachteiligen, werden zukünftig auch die "physische« regenerative und ortsnahe Versorgung energieintensiver Produktionsstandorte behindern. Für eine klimaneutrale Autoproduktion sollten folgende Maßnahmen in Betracht gezogen werden:

- Die Versorgung von Produktionsstandorten mit "physisch« regionalem und erneuerbarem Strom sollte im Energierecht als Ziel verankert und so institutionalisiert werden, dass die Vorteile geringer Durchleitungsentfernungen sich in Kostenvorteilen niederschlagen. Eine solche Netzentgeltstruktur dürfte auch dazu führen, dass Netze effizienter und kostengünstiger betrieben werden.

- Fahrzeuge, produziert mit erneuerbaren Energien, sollten mit einem Faktor und in Abhängigkeit von der Antriebsart für die Erreichung des $959-\mathrm{CO}_{2} / \mathrm{km}$ Ziels berücksichtigt werden.

- Eine klimaneutrale Produktion sollte als Argument der Absatzförderung gegenüber Flotten- und Großkundinnen und -kunden sowie zur Imagepflege mit geeigneten Marketingmaßnahmen durch den Vertrieb der Hersteller genutzt werden.

- Zur Erzielung einer möglichst klimaneutralen Produktion ist die energieintensive Batteriezellfabrikation zentral. Die entstehenden Produktionsstätten der Batteriezellproduktion sollten daher prioritär an Standorten angesiedelt werden, an denen eine möglichst hohe anteilige Versorgung durch erneuerbaren Strom unproblematisch möglich ist.

\subsubsection{Koordination des Ausstiegs aus der Verbrenner-Technologie}

Die immer wieder emotional geführte Debatte um den Ausstieg aus dem Verbrennungsmotor verstellt den Blick dafür, dass ein solcher Ausstieg in den nächsten 30 Jahren, ob wir ihn wollen oder nicht, aller Voraussicht nach kommen wird. Damit 
dies keine vermeidbaren Verwerfungen bei Herstellern und Zulieferern zur Folge hat und dadurch unnötig private wie auch volkswirtschaftliche Werte vernichtet werden, bedarf es einer weniger emotional geführten, sachlichen Debatte darüber, wie ein solcher Ausstieg gestaltet und wie seine Folgen abgemildert werden können. Schon heute ist ja sichtbar, dass sich große Gruppen der weitsichtigeren Hersteller und Zulieferer auf den Ausstieg vorbereiten.

- Innovative Hersteller und Zulieferer beginnen, in der F \& E einen Plan B vorzubereiten. Neben den Entwicklungslinien für Produkte für Verbrennungsmotoren wird analysiert, welche Produktlinien und Fertigungskompetenzen in der Zeit der Elektromobilität Potenzial haben werden (Stieber 2017). Die weniger innovativen Unternehmen sollten in diesem Prozess öffentlich, z. B. durch Landesinitiativen der Wirtschaftsförderung, unterstützt werden.

- Neue Produkte bedeuten oft, dass neue Qualifikationen benötigt werden. Angebote für die Aus- und Weiterbildung sind erforderlich.

- Ein politisches Ausstiegsszenario könnte hilfreich sein, um auch die weniger beweglichen und innovativen Akteurinnen und Akteure dazu zu motivieren, sich auf eine veränderte Zukunft vorzubereiten. Da der Pfadwechsel zur Elektromobilität eher markt- als politikgetrieben sein dürfte, kommt politischen Ausstiegsplänen u. U. gar keine besondere Bedeutung zu. Es ist aber denkbar, dass sie als Signal an die Nachzügler helfen können, den Schaden des Pfadwechsels zu begrenzen.

- Für Unternehmen, deren Ausgangsposition zu schlecht oder deren Bemühungen im Pfadwechsel letztlich nicht erfolgreich sind, sollte ein Notfonds für Sozialpläne bereitgehalten werden.

\subsubsection{Roadmap Elektromobilität im Überblick}

Für einen erfolgreichen Transformationsprozess hin zu Elektrofahrzeugen sind kurz-, mittel- und langfristige Maßnahmen notwendig. Kurzfristig muss die Ladeinfrastruktur erweitert werden, um die zeitnah absehbare Markteinführung zahlreicher elektrischer Modelle der großen deutschen Hersteller zu begleiten. In den dann folgenden Jahren soll sich ein nationaler Leitmarkt entwickeln, dessen anspruchsvolle Kundschaft die Automobilhersteller herausfordert und unterstützt.

Die mittel- und langfristigen Maßnahmen nutzen die Kraft des Leitmarktes und haben die Absicherung und weitere Steigerung der Wettbewerbsfähigkeit der deutschen Autobranche im Bereich hochwertiger Elektroautos in der zweiten Hälfte der kommenden Dekade zum Ziel. 
Abbildung 50: Roadmap für den Ausbau der Elektromobilität in Deutschland im Überblick
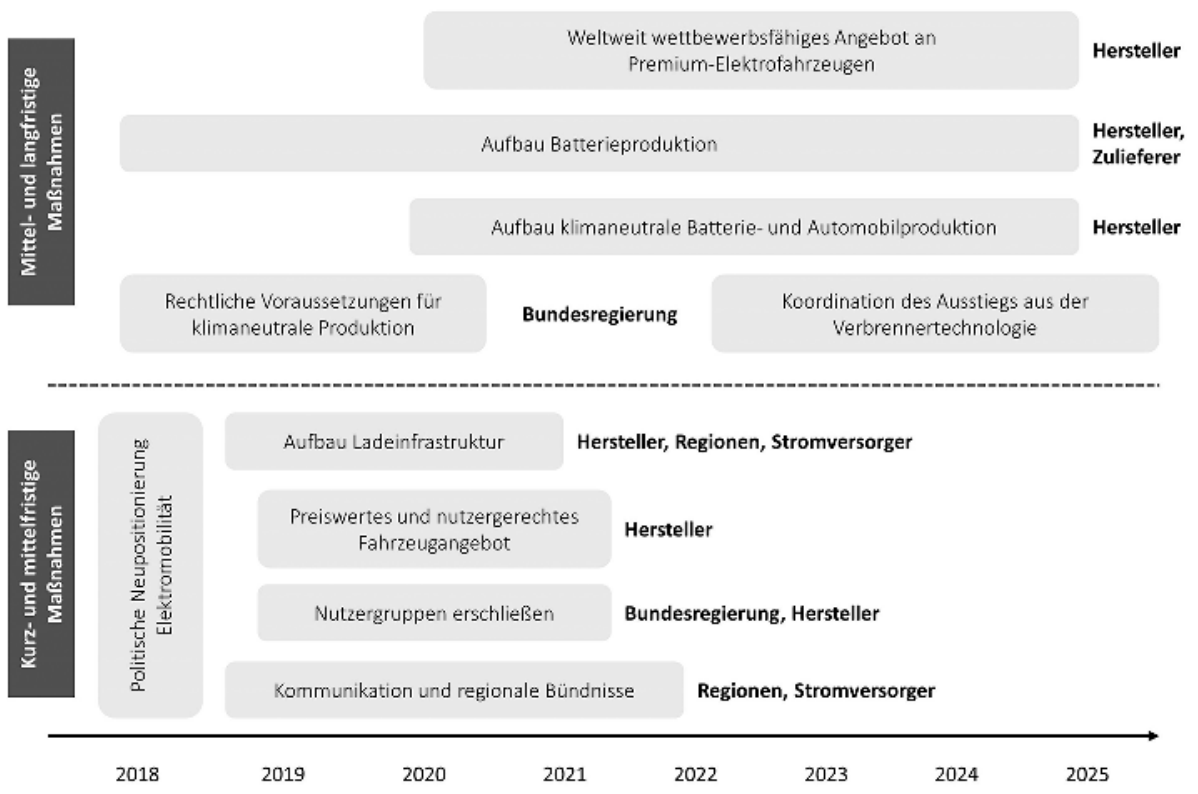

Quelle: Eigene Darstellung, Borderstep Institut für Innovation und Nachhaltigkeit.

Die Darstellung von Maßnahmen in einer Roadmap erweckt grundsätzlich den Eindruck von politischer Steuerbarkeit. Es ist daher wichtig zu betonen, dass der Pfadwechsel zur Elektromobilität einige Aspekte aufweist, die eher auf einen marktgetriebenen Pfadwechsel als auf ein politisches Transformationsprojekt hinweisen. Solche Aspekte sind:

- Die deutlich niedrigere Teilezahl in einem Elektroauto ermöglicht mittelfristig niedrigere Verkaufspreise, wie es sich bei Streetscooter und e.GO Life schon andeutet. Zusammen mit den ohnehin niedrigeren Betriebskosten von Elektroautomobilen böte dies einen hohen Kaufanreiz.

- Die Innovationen in der Batterietechnik könnten mittelfristig leistungsfähigere Batterien mit radikal kürzeren Ladezeiten, niedrigeren Kosten und weniger Rohstoffproblemen verfügbar machen. Auch dies wäre ein erheblicher zusätzlicher Kaufanreiz.

- Die Digitalisierung des Fahrens rückt das Automobil dichter an die IKT heran. Über zusätzliche Nutzenversprechen sind gerade in der IKT marktgetriebene Prozesse vorherrschend.

Für marktgetriebene Pfadwechsel, als Beispiel sei der Flachbildmonitor genannt, sind aber sehr hohe Geschwindigkeiten des Wandels typisch. Das Ziel der mittel- 
fristigen Wettbewerbsfähigkeit der deutschen Automobilbranche in dem Umfeld eines schnellen Pfadwechsels sollte daher von all den Akteurinnen und Akteuren mitbedacht werden, die gegenwärtig noch auf eine Verzögerung des Wandels setzen.

Als Akteure des Pfadwechsels stehen die Automobilhersteller und ihre $\mathrm{Zu}$ lieferer im Mittelpunkt, deren Kernaufgabe in der Entwicklung und Produktion von preiswerten und nutzergerechten Elektrofahrzeugen besteht, die aber auch an vielen anderen Aktivitäten schon aus dem eigenen Interesse heraus mitwirken sollten. Am Aufbau der Ladeinfrastruktur können und sollten auch Energieversorger und regionale Planungsakteurinnen und -akteure mitarbeiten. Regionale Akteurinnen und Akteure können auch dafür sorgen, dass die Aktivitäten regional anschlussfähig und in Kooperation mit regionalen Netzwerken durchgeführt werden. Für eine Reihe von ordnungsrechtlichen und förderpolitischen Aufgaben ist die Bundesregierung in der Pflicht.

In vielen großen und entscheidenden Akteursgruppen, besonders innerhalb der Automobilunternehmen, der Politik und der Gewerkschaften, bestehen Gegensätze zwischen denjenigen, die die Elektromobilität vorantreiben wollen und Gruppen, die den Pfadwechsel eher verzögern wollen. Deren Argumente fokussieren auf einen befürchteten, mit dem Pfadwechsel einhergehenden Verlust von Arbeitsplätzen, auf Befürchtungen von Rohstoffknappheit für die Batterieproduktion und vielem mehr. Eine bessere und parteiübergreifende Vernetzung und Koordination der Befürworterinnen und Befürworter scheint erforderlich, um die Kräfte der innovation community hinter der Elektromobilität zur Entfaltung zu bringen (Fichter und Beucker 2012a, 2012b). Dabei dürfte auch die Unterstützung von Wissenschaft und Zivilgesellschaft von Bedeutung sein.

\subsection{Roadmap Produkte länger nutzen}

\subsubsection{Das Problem der Obsoleszenz}

Den schnellen Verbrauch von Konsumgütern durch eine längere Nutzung von Produkten zu reduzieren ist eine Schlüsselstrategie für eine ökologische Transformation zu einer Green Economy (Behrendt und Göll 2019). Sie beruht im Wesentlichen darauf, dass eine längere Nutzungsdauer von Produkten geringere Neukaufraten erfordert und so Umweltbelastungen durch Neuanschaffung vermieden werden können.

Obgleich die ökologischen Vorteile einer längeren Produktnutzung offensichtlich sind, lässt sich faktisch ein gegenteiliger Trend beobachten. Die Dynamisierung von Innovationsprozessen, der harte Preiswettbewerb, schnelle Produktzyklen und Modeerscheinungen tragen erheblich dazu bei, dass insbesondere 
Elektro- und Elektronikprodukte immer kürzer genutzt werden. Ihre zunehmende Vernetzung, das »Internet der Dinge«, wird diese Entwicklung wohl noch beschleunigen. In der Folge der verkürzten Nutzungsdauer entstehen kurzlebige Produkte, die unsere Umwelt grundsätzlich deutlich drastischer belasten als Geräte mit langer Nutzungsdauer. Dieser Trend zur kurzlebigen Produktnutzung läuft einer Green Economy entgegen. Aufgrund des zunehmenden Wachstums von Mittelschichten in vielen Ländern, insbesondere den Schwellenländern, und entsprechend »westlich-kapitalistisch« ausgerichteter Konsumweisen gewinnt diese Thematik zusätzlich eine besonders hohe Bedeutung und Dringlichkeit.

Obwohl eine möglichst effiziente Lebens- und Nutzungsdauer von Produkten ein lang verfolgtes Kernanliegen der Umweltpolitik und Thema weitreichender politischer Debatten ist, die sich in Foren (Wider die Verschwendung I, II, III) und politischen Programmen niederschlagen, von denen insbesondere das Abfallvermeidungsprogramm, ProgRess I und II sowie auf EU-Ebene das Kreislaufwirtschaftspaket herausragen, fehlt es bis dato an einer umfassenden und kohärenten nationalen und europäischen Strategie für einen Pfadwechsel.

Im Folgenden werden nach der Analyse der Pfadabhängigkeiten auf Basis von Fallstudien (u.a. zu Fairphone, Ebay, Repair-Cafés, Reparaturnetzwerken) und einer Zukunftswerkstatt innovative Ansätze und Lösungswege für einen Pfadwechsel aufgezeigt.

\subsubsection{Pfadabhängigkeiten}

Die Gründe für die Zunahme der Konsumgegenstände und den Rückgang ihrer Nutzungsdauer sind vielfältig, unterschiedlich und produktgruppenspezifisch. Der Lock-in, also die Tatsache, dass vorherrschende Standards und Trends sowie bestimmte Marktbedingungen und Nutzungsweisen nicht zulassen, dass insbesondere Elektro- und Elektronikgeräte länger genutzt werden, dass sie stattdessen sogar immer schneller ausgetauscht werden und quantitativ zunehmen, ist auf ein Zusammenspiel von unterschiedlichen Faktoren zurückzuführen. Es bestehen grundlegende Pfadabhängigkeiten mit erheblichen Bindungskräften, die den Pfad für lange Zeit stabilisieren und gegen Änderungen jeder Art sehr widerstandsfähig machen können. Eine gezielte positive Abweichung vom Pfad $\mathrm{zu}$ erreichen wird dann sehr schwierig. Bisherige Ansätze zur Produktdauerverlängerung liefen weitgehend ins Leere, blieben auf Nischen beschränkt oder konnten zumindest den allgemeinen Trend zu kürzerer Produktnutzung nicht aufhalten.

Eine Erhöhung der Produktnutzungsdauer stößt vor allem auf folgende Pfadabhängigkeiten: 


\section{Ökonomische Pfadabhängigkeiten}

Ökonomische Pfadabhängigkeiten bestehen darin, dass Unternehmen in einem marktwirtschaftlich-expansiven System auf eine Steigerung ihrer Umsätze und dadurch ihrer Verkaufsraten hinarbeiten. Unternehmen sind zudem einem Preiswettbewerb untereinander ausgesetzt, Geräte immer günstiger anzubieten, was zulasten der Qualität und der Lebensdauer gehen kann. Dieser Mechanismus tritt vor allem in gesättigten Märkten auf, wo sich im Zuge der Marktsegmentierung Billigmärkte entwickeln, wie dies beispielsweise bei Waschmaschinen $\mathrm{zu}$ beobachten ist. Auch wenn sich keine geplante Obsoleszenz im Sinne bewusst eingebauter Sollbruchstellen nachweisen lässt, werden Schwachstellen aber in Kauf genommen. Solche Schwachstellen sind beispielsweise hitzeempfindliche Bauteile an Stellen, an denen es zur Wärmentwicklung kommt, minderwertige Verarbeitung an empfindlichen Stellen des Gerätes oder billige und wenig stabile Kunststoffbestandteile (Prakash et al. 2016). Auch Akkus (z. B. von elektrischen Zahnbürsten), die nicht ausgetauscht werden können, verringern die Nutzungsdauer unnötig. In gesättigten Märkten können Unternehmen ihre Umsätze in erster Linie dadurch steigern, dass sie auf dem Markt erhältliche Produkte »obsolet« machen. Das heißt, man lässt Produkte bewusst veralten - nicht durch Schwachstellen, sondern indem man immer wieder neue Produkte mit neuen Leistungsmerkmalen in den Markt bringt und den Konsumentinnen und Konsumenten das Gefühl gibt, nur mit neuen Produkten auf der Höhe der Zeit zu sein (Wallbrecher 2008). Durch Marketing zielen Unternehmen und ihre Werbedienstleister auf eine Bedarfsweckung für neue Produkte, während alte Produkte kontinuierlich entwertet werden. Des Weiteren bestehen ökonomische Pfadabhängigkeiten, die Reparaturen erschweren und verhindern. Sie müssen mit Skalenvorteilen in der industriellen Massenfertigung, welche den Erwerb von Neugeräten begünstigen, konkurrieren. Kosten für die Lagerhaltung der Ersatzteile und hohe Reparaturkosten führen in vielen Fällen dazu, dass eine Neuanschaffung kostengünstiger ausfällt als eine Reparatur oder der Kauf von Gebrauchtprodukten. Eine Reparatur lohnt oft nicht, sodass Verbraucherinnen und Verbraucher neue Elektround Elektronikgeräte anschaffen. Eine Umfrage der Verbraucherzentrale Bundesverband zeigt, dass ökonomische Überlegungen mit Abstand der häufigste Grund sind (74 Prozent), keine Reparatur vorzunehmen (VZBV 2017). In der Folge schrumpft der Markt für Reparaturdienstleistungen.

\section{Technologische Pfadabhängigkeiten}

Infolge technologischer Fortschritte werden Innovations- und Produktzyklen kürzer und die Produktkomplexität nimmt zu. Durch zunehmende Miniaturisierung und Leistungsverdichtung werden Komponenten fest verbaut oder geklebt, was dazu führt, dass defekte Einzelteile nicht mehr ausgetauscht oder einfach repariert werden können. Besonders ausgeprägt ist dieses Wirkungsgefüge bei 
Informations- und Kommunikationsgeräten. Die Fortschritte der Mikroelektronik führen zu permanenten Leistungszuwächsen und gleichzeitigem Preisverfall der Geräte. Anspruchsvolle Medientechnik wird dadurch immer erschwinglicher. Die Miniaturisierung und Verbilligung elektronischer Schaltkreise führt auch dazu, dass Informations- und Kommunikationstechniken zunehmend in andere Hausgeräte eingebettet werden, wodurch sich die Innovationsdynamik auf diese Produkte überträgt (Behrendt 2012). Der Übergang von stationären zu mobilen Endgeräten führt zu einer Verkürzung der durchschnittlichen Nutzungsdauer der Hardware, da mobile Geräte weniger lange im Gebrauch sind u. a. weil es schwerer ist, Einzelkomponenten auszutauschen (Hilty et al. 2015). Die Verkürzung der Produktzyklen, neue Funktionalitäten und Technologiesprünge lassen vorhandene Produkte »alt« aussehen. Sie sind häufig der Auslöser für den Kauf neuer Geräte.

\section{Organisationale Pfadabhängigkeiten}

Organisationale Pfadabhängigkeiten ergeben sich aus Prozessroutinen, Verfahren oder der Unternehmenskultur. Unter Prozessroutinen werden Artefakte, Werte und Grundannahmen verstanden, wobei den Artefakten Verhaltensweisen, Gebräuchlichkeiten und die Architektur der Unternehmensabläufe zugeordnet werden (Schein 1995). Auch Lobbying wird zu den organisationalen Pfadabhängigkeiten gezählt. Ökonomische und technologische Voraussetzungen bestimmen die Prozessroutinen von Unternehmen, aber auch von anderen Arten von Gesellschaften (Organisationen, Verbände etc.) mit. Zu den organisationalen Pfadabhängigkeiten von Unternehmen kann auch die fehlende Antizipation neuer Technologieentwicklungen gezählt werden. Es fehlt ein Innovationsmanagement, das zukünftige Technologieentwicklungen frühzeitig bei laufenden Produktlinien berücksichtigt, etwa über eine vorausschauende Planung von Schnittstellen. Weitere für eine Transformation hinderliche, aber mit einigem Aufwand mittelfristig potenziell überwindbare organisationale Pfadabhängigkeiten bestehen auf der Ebene der Vertragsbeziehungen. Bei Mobiltelefonen werden die Kundinnen und Kunden durch die Preisgestaltung dazu animiert, bei Vertragsverlängerung ihre Geräte zu ersetzen. Oft werden auch Reparaturen für herstellerunabhängige Reparaturbetriebe organisatorisch durch fehlende Bauteileunterlagen, einen komplizierten Zugang zu Ersatzteilen oder die Notwendigkeit, Spezialwerkzeuge einsetzen zu müssen, erschwert oder unmöglich gemacht. Schwieriger gestaltet sich eine Transformation auf der Ebene der Werte und Grundannahmen. Hierzu zählen - zusätzlich zu system-ökonomisch bedingten Notwendigkeiten - unternehmerische Fixierungen auf monetären Gewinn und betriebliche Expansion vor Werten des Gemeinwohls. Letztere sind erwartungsgemäß mit einem größeren Interesse an einer längeren Nutzungsdauer von Geräten korreliert. Es ist anzunehmen, dass dies von Unternehmen zu Unternehmen verschieden ist, wobei 
Indikatoren hierfür die Unternehmensgröße, das Vorhandensein und die Ausprägung internationaler Wettbewerbsfähigkeit und die Gesellschaftsform sein dürften.

\section{Nutzerbezogene Pfadabhängigkeiten}

Nutzerbezogene Pfadabhängigkeiten bestehen auf der Grundlage von Wahrnehmungen, Verhaltensroutinen, Lebensstilen und kulturellen Normen. Kulturelle liberale Normen bilden anspruchsvoll zu adressierende, jedoch handfeste benutzerspezifische Pfadabhängigkeiten, die einen gesellschaftlichen Wandel hin zu längeren Nutzungsdauern von insbesondere elektronischen Geräten verhindern. Hierunter fallen Individualismus und das Streben nach extrinsischen Zielen wie Reichtum und Macht (Rosenmann et al. 2016), da länger genutzte Geräte mit Einbußen beim Erreichen dieser Normen einhergehen können.

Soziale Settings und die jeweiligen sozialen Bedeutungen von Handlungsweisen prägen das alltägliche Verhalten von Menschen (John et al. 2016; Shove et al. 2012). Der Besitz und Gebrauch moderner Konsumgüter ist an vielen gesellschaftlichen Orten (etwa auf Arbeit, in der Schule, an öffentlichen Orten) Grundlage für Teilhabe und soziale Anerkennung. Dies gilt insbesondere, jedoch nicht nur, für Informations- und Kommunikationstechnik (IKT) sowie Unterhaltungselektronik (v. a. Smartphones). Diese Konsumbereiche haben entsprechend einen hohen Stellenwert. Geschickte (teure) Werbung generiert und verstärkt den Wunsch nach einem neuen, besseren Gerät und prägt das Konsumverhalten, was dazu führt, dass häufig Produkte neu gekauft werden, obwohl die vorhandenen noch gut funktionieren. Eine repräsentative Studie von TNS Infratest im Auftrag von eBay ergab, dass durchschnittlich 217 Gegenstände in deutschen Haushalten ungenutzt lagern; geschätzter Gesamtwert pro Haushalt: 3.223 Euro. Insbesondere Haushaltsgeräte und elektronische Gadgets, darunter 43 Millionen Smartphones und Handys, bleiben ungenutzt (ebay 2015). Benutzerspezifische Pfadabhängigkeiten kommen auch durch eine Abwärtsspirale der Erwartungen hinsichtlich der Lebens- und Nutzungsdauer von elektronischen Geräten zustande. Die schlechten Erfahrungen mit der Lebensdauer von Produkten senken das Vertrauen und infolgedessen auch die Bereitschaft, mehr für ein langlebigeres Produkt auszugeben. Umgekehrt werden die Hersteller darin bestärkt, anzunehmen, dass Konsumentinnen und Konsumenten immer das Neueste verlangen, was dazu führt, dass Produkte mit noch kürzerer Lebensdauer hergestellt werden. In dieser Interaktion ergibt sich eine bedenkliche Abwärtsspirale der Erwartungen, die zu einer Beschleunigung der Ersatzkaufraten führt (Wieser und Tröger 2015). 


\section{Rechtliche Pfadabhängigkeiten}

Fehlende, unzureichende und falsche Anreize kennzeichnen rechtliche Pfadabhängigkeiten. Die derzeit geltenden Gewährleistungsregelungen geben keine Anreize, die Lebensdauer zu erhöhen. Zum einen endet die Gewährleistung nach 24 Monaten, viele Geräte verschleißen erst danach. Zum anderen besteht für Hersteller lediglich innerhalb der ersten sechs Monate die Nachweispflicht, dass der Schaden nicht bereits beim Kauf bestand. Danach liegt die Beweislast bei den Verbraucherinnen und Verbrauchern, was dazu führt, dass berechtigte Ansprüche kaum geltend gemacht werden können. Rechtliche Handlungsmöglichkeiten im Rahmen der Ökodesign-Richtlinie sind bislang in der Durchführungsverordnung auf Energieeffizienz fokussiert. In Bezug auf Normen existiert bereits eine Reihe von Standards zur Prüfung der Sicherheit und Gebrauchstauglichkeit von Elektro- und Elektronikgeräten. Was vielfach fehlt sind jedoch auf die Lebensdauer der Produkte bezogene Prüfungen.

Zwischen den Pfadabhängigkeiten bestehen insoweit Zusammenhänge, als sie nur in ihrer Wechselwirkung die hohe Stabilität des Trends erklären können. So erzeugen Preiswettbewerb und Innovationsdynamik permanent neue Produkte, die den Wunsch nach Neuem anregen und die Erwartungen bezüglich der Lebens- und Nutzungsdauer der Geräte senken. Technologische Fortschritte führen $\mathrm{zu}$ permanenten Leistungszuwächsen bei gleichzeitigem Preisverfall von elektronischen Geräten. Geräte mit höheren Leistungen werden dadurch immer erschwinglicher. Diese Dynamiken stehen einem Richtungswechsel entgegen, der es ermöglichen würde, die Lebens- und Nutzungsdauer von Elektro- und Elektronikgeräten deutlich zu steigern.

\subsubsection{Herausforderungen und Maßnahmen für einen Pfadwechsel}

Das Thema der Obsoleszenz von Konsumprodukten wird seit mehreren Jahren breit in der Öffentlichkeit diskutiert. Dazu beigetragen haben mehrere Dokumentationen im Fernsehen, die die Obsoleszenz als "geplant" skandalisiert hatten, darunter insbesondere die vielbeachtete arte-Dokumentation »Kaufen für die Müllhalde«. In der Nachfolge haben verschiedene andere Medien das Thema immer wieder aufgegriffen. Obsoleszenz ist auch politisch in den Parteien zum Thema geworden. NGOs setzen sich mit dem Thema auseinander. Obsoleszenz ist seit Jahren auch Gegenstand wissenschaftlicher Untersuchungen, sodass mittlerweile eine Informationsgrundlage über den Einfluss der Nutzungsdauer von Produkten auf ihre Umweltwirkung vorliegt.

Anknüpfungspunkte für die Umsetzung von Maßnahmen zur Verlängerung der Nutzungsdauer liefern verschiedene politische Initiativen und Aktivitäten. Dazu zählen insbesondere das Nationale Programm Nachhaltiger Konsum, der Beschluss des Staatssekretärsausschusses für nachhaltige Entwicklung vom 1. Juni 
2015 und der Beschluss der Verbraucherschutzministerkonferenz vom 8. Mai 2015. Außerdem thematisiert das Ressourceneffizienzprogramm der Bundesregierung (ProgRess II) die Verlängerung der Produktlebens- und -nutzungsdauer mit dem Ziel, das Angebot von und die Nachfrage nach ressourcenschonenden Produkten mit einer längeren Produktnutzungs- und/oder -lebensdauer zu fördern. Mit Blick darauf wurden zahlreiche Prüfaufträge vergeben. ${ }^{5}$ Das Umweltbundesamt hat im Mai 2017 ein Positionspapier zu »Strategien gegen Obsoleszenz« veröffentlicht, das Handlungsempfehlungen zur Verlängerung der Produktnutzungsdauer gibt. Auf EU-Ebene bietet das »Circular Economy Package« der EU politische Anknüpfungspunkte. Mit der Ökodesign-Richtlinie ist ein passender rechtlicher Rahmen bereits vorhanden. Die Richtlinie ist Teil der Umsetzung einer integrierten europäischen Produktpolitik. Der Arbeitsplan 2016 bis 2019 sieht vor, Anforderungen bezüglich der Produktlebensdauer zu prüfen. Das Europäische Parlament hat im Juni 2017 den Bericht »Längere Lebensdauer für Produkte: Vorteile für Verbraucher und Unternehmen« verabschiedet (Europäisches Parlament 2016). Darin wird die Kommission aufgefordert, darauf hinzuwirken, dass für alle Produktkategorien ab der Konstruktionsphase - soweit dies möglich ist geltende Mindestkriterien für die Beständigkeit festgelegt werden, die sich auf die Bereiche Robustheit, Reparierbarkeit und Nachrüstbarkeit erstrecken und auf Normen beruhen, die von allen Mitgliedern der Europäischen Normungsorganisation, d.h. dem CEN, dem CENELEC und dem ETSI, ausgearbeitet werden. Das EU-Parlament verlangt Maßnahmen für eine längere Produktnutzung u.a. die Angabe eines Mindestzeitraums, in dem für Betriebssysteme Sicherheitsupdates bereitgestellt werden, Informationen über die Verfügbarkeit von Ersatzteilen, die Förderung »zirkulärer Wirtschaftsmodelle« etwa über Steueranreize. EU-Kommission und Rat haben 2015 einen Vorschlag für eine Richtlinie des Europäischen Parlaments und des Rates über bestimmte vertragsrechtliche Aspekte des OnlineWarenhandels und anderer Formen des Fernabsatzes von Waren angenommen. Die gesetzliche Gewährleistungsdauer ist Gegenstand des Vorschlags. Der Richt-

5 Untersuchung und gegebenenfalls Einführung von Mindest- und Informationsanforderungen an Produzenten zu Materialeffizienz, Lebensdauer und Recyclingfähigkeit von Produkten im Rahmen der Umsetzung der EU-Ökodesign-Richtlinie und der EU-Energieverbrauchskennzeichnungsrichtlinie; Prüfung einer Einführung und Stärkung rechtlicher Instrumente, die eine Nutzungsverlängerung von Produkten und nachhaltiges Konsumverhalten unterstützen; Verstärkte Unterstützung der Ressourcenschonung durch Normung; Verstetigung des Bundespreises Ecodesign als Plattform für ökologische und ästhetisch besonders hochwertige Produktangebote; Prüfung nationaler rechtlicher Maßnahmen, um das Angebot von und die Nachfrage nach ressourcenschonenden Produkten mit einer längeren Produktnutzungs- und/oder-lebensdauer zu fördern; Förderung von Modellen, Projekten und Akteuren für eine Sharing Economy (Nutzen statt Besitzen); Verlängerung der Nutzungsdauer von Produkten durch eine Stärkung des Cebrauchtwarenhandels. 
linienvorschlag der EU-Kommission über »bestimmte vertragsrechtliche Aspekte der Bereitstellung digitaler Inhalte« enthält in der Fassung, wie sie von den Mitgliedsstaaten auf dem Rat für Justiz und Inneres am 8. Juni 2017 beschlossen wurde, eine Frist für die Beweislastumkehr von einem Jahr. Die Bundesregierung hat dem Richtlinien-Text zugestimmt.

Impulse kommen auch aus anderen Mitgliedstaaten der EU, wie der Bericht des EU-Parlamentes dokumentiert (Europäisches Parlament 2016):

»Belgien war in diesem Bereich mit der Annahme einer Entschließung des Senats zur Bekämpfung der geplanten Obsoleszenz energieverbrauchsrelevanter Produkte im Februar 2012 Vorreiter. In dieser Entschließung wird unter anderem die Empfehlung ausgesprochen, auf europäischer Ebene eine Kennzeichnung zur Lebensdauer energieverbrauchsrelevanter Produkte (Glühbirnen, Computer, Mobiltelefone usw.) sowie zur Reparierbarkeit dieser Produkte einzuführen.«

Frankreich hat - parallel zu den Initiativen französischer Unternehmen - seinen Rechtsrahmen geändert und im August 2015 ein Energiewende-Gesetz, in dessen Rahmen die geplante Obsoleszenz strafbar ist, sowie im März 2014 ein Gesetz über den Energieverbrauch, in dem die Rechte der Verbraucherinnen und Verbraucher in Bezug auf die Gewährleistung und die Verfügbarkeit von Ersatzteilen dargelegt werden, angenommen.

Die Rechtsvorschriften der Niederlande sehen vor, dass die gesetzliche Gewährleistungsfrist von zwei Jahren lediglich als Mindestanforderung zu werten ist. Für bestimmte Produkte, darunter insbesondere Kraftfahrzeuge, Waschmaschinen und andere Gebrauchsgüter, kann eine längere Gewährleistungsfrist gelten, die sich an der durchschnittlichen Lebensdauer bemisst, von der die Verbraucherinnen und Verbraucher nach vernünftigem Ermessen ausgehen können.

Auch Finnland plant, die gesetzliche Gewährleistungsfrist $\mathrm{zu}$ ändern, und zwar im Rahmen eines Verbraucherschutzgesetzes. Gemäß der Präambel des Gesetzes haftet der Verkäufer - etwa bei Kraftfahrzeugen, Baumaterial und Elektrogeräten - auch für Herstellungsmängel, die erst mehr als zwei Jahre nach der Lieferung des jeweiligen Produkts auftreten. Dieser Ansatz ähnelt dem Konzept, das in den Niederlanden verfolgt wird. Für die Festlegung der Lebensdauer einzelner Produkte ist eine Mediatorin oder ein Mediator zuständig, die ihre Bewertung anhand verschiedener Kriterien erstellt - etwa auf der Grundlage des Produktpreises, des Preises der Bestandteile oder auch der Nutzung, also darauf, wie oft ein Gerät verwendet wird. Der Gesetzgeber selbst hat keine Liste zu der zzu erwartenden Lebensdauer konkreter Produkte verfasst. Allerdings können Einzelfälle im Rahmen der Empfehlungen des »Consumer Dispute Board« geprüft werden.

In Spanien wurde im Rahmen einer Konferenz über neue Konsummodelle, zu der der Europäische Wirtschafts- und Sozialrat geladen hatte, am 24. Juni 2014 
die `Entschließung von Madrid zu bewährten Verfahren in den Bereichen gemeinschaftlicher Konsum und geplante Obsoleszenz angenommen.

In Österreich wurde ein Gütezeichen für langlebige, reparaturfreundlich konstruierte elektrische und elektronische Geräte eingeführt.

In Schweden wurden verschiedene steuerliche Maßnahmen getroffen, die im Januar 2017 in Kraft traten. Ziel ist die Stärkung der Reparaturbranche sowie der Recycling- und der Kreislaufwirtschaft. Vorgesehen ist Folgendes: Reparaturen sollen günstiger werden, indem der Mehrwertsteuersatz für bestimmte Produkte von 25 auf zwölf Prozent gesenkt wird (z. B. für Fahrräder, Schuhe und Kleidungsstücke). Verbraucherinnen und Verbrauchern, die Elektrogeräte reparieren lassen, soll die Möglichkeit offenstehen, 50 Prozent der Lohnkosten von der Steuer abzusetzen. Produkte, die Stoffe enthalten, die nicht oder nur schwer recycelt und repariert werden können, sollen besteuert werden (Europäisches Parlament 2016).

Die Vielzahl politischer Aktivitäten und Initiativen, die in jüngster Zeit ergriffen worden sind, zeigen, dass das Thema politisch mittlerweile hoch auf der Agenda steht. Das Zeitfenster (window of opportunity) ist relativ günstig, in den nächsten Jahren ein effektives Regelungsregime zu entwickeln und mit Maßnahmen $\mathrm{zu}$ flankieren, die tendenziell eine Langlebigkeitskultur für Konsumprodukte in Verbindung mit einer Sharing-, Reparatur- und Wiederverkaufskultur fördern und damit einen Pfadwechsel herbeiführen könnten.

Mit Blick darauf wurden die verschiedenen Ansatz- und Anknüpfungspunkte für eine Verlängerung der Produktnutzungsdauer reflektiert, wobei vor allem die Frage interessiert, mit welchen Maßnahmen ein Pfadwechsel erreicht werden kann, wie anschlussfähig diese sind und wie sie von wem erfolgreich umgesetzt werden können. Im Folgenden wurde eine Reihe von Ansätzen identifiziert und der Handlungsbedarf für Politik, Wirtschaft und Zivilgesellschaft herausgearbeitet.

\subsubsection{Produktverantwortung der Hersteller: Gewährleistung einer Mindestlebensdauer und Reparierbarkeit von Produkten}

Eine starke Maßnahme für eine Trendwende ist es, sicherzustellen, dass Produkte eine verlässliche Mindestlebensdauer haben, ohne dass in dieser Zeit die Geräte ausfallen und Reparaturen notwendig werden. Dies wäre vor allem für Billigprodukte relevant, die häufiger schon nach kurzer Zeit ausfallen. Wer einen Staubsauger für weniger als 80 Euro kauft, wird ein Gerät bekommen, das schneller kaputt geht. Bei Waschmaschinen liegt die Grenze etwa bei 500 Euro (nach Die Mittelbayerische [2015]). Da die Produktlebensdauer eine planbare Größe ist, müssten Hersteller verpflichtet werden, ihre Produkte so zu gestalten, dass eine bestimmte Anzahl an Betriebsstunden oder Nutzungszyklen erreicht wird. Um tatsächlich eine Trendwende einzuleiten, müssten sich diesbezügliche Standards an der Qualität langlebiger Referenzprodukte orientieren. So müssen beispielsweise 
Haartrockner nach den Anforderungen des Blauen Engels einen Dauertest von 400 Stunden bestehen. Mobiltelefone mit Blauem Engel müssen einen WechselAkkumulator und eine Garantiedauer von mindestens zwei Jahren haben. Frühund Zufallsausfälle könnten ausgeschlossen oder verringert werden. Dies setzt eine "geplante Obsoleszenz in der Form voraus, dass die Ausfallraten als planbare Größe verringert werden. Von Verbraucherinnen und Verbrauchern wird erwartet, dass Gebrauchsgüter deutlich länger halten, als sie genutzt werden. Anhaltspunkte darüber, in welcher Größenordnung eine längere Lebensdauer gewünscht wird, liefert die österreichische Untersuchung von Wieser und Tröger (2015). Ihr zufolge ist die gewünschte Lebensdauer je nach Produkt unterschiedlich, für Laptops ist sie um das 2,19-fache, für Handys um das 2,5-fache höher als die derzeitige tatsächliche Nutzungsdauer.

Forderungen nach einer Mindestlebensdauer für Elektro- und Elektronikgeräte werden von verschiedenen Akteurinnen und Akteuren zunehmend gestellt. So fordert u.a. der Europäische Wirtschafts- und Sozialausschuss die Einführung einer Mindestlebensdauer für Produkte (EWSA 2013). Verbraucherexpertinnen und -experten von der Linken, der SPD und den Grünen fordern Mindestnutzungszeiten für Elektro- und Elektronikgeräte. Einen rechtlichen Rahmen solche Forderungen umzusetzen bietet die Ökodesign-Richtlinie. Zur Umsetzung gibt es noch Forschungsbedarf. Dieser betrifft insbesondere die Frage, wie bestehende Sicherheitsnormen und Standards auf Komponentenebene mit Blick auf Lebensdauer- und Haltbarkeitsprüfungen angepasst werden sollten. Im Bereich der Reparatur ist eine offene Frage, wie ein effektiver Zugang zu Ersatzteilen für Elektro- und Elektronikgeräte mit Blick auf Importe in den EU-Binnenmarkt sichergestellt werden kann. Bezüglich der Herstellergarantiepflicht und der Erweiterung der Verbandsklagebefugnisse ist die rechtliche Umsetzbarkeit zu prüfen. Unklar sind auch noch mögliche Lenkungseffekte der Veränderung verbraucherrechtlicher Regelungen mit Blick auf die Verlängerung der Produktlebens- und Nutzungsdauer. Auch bezüglich der Frage, welche Lebens- und Nutzungsdauern Verbraucherinnen und Verbraucher für die verschiedenen Produkte erwarten und wie sie auf verbraucherrechtliche Änderungen reagieren werden, gibt es Forschungsbedarf.

\section{Maßnahmen}

1. Normung und Ökodesign-Richtlinie: Es ist zu erwarten, das aus den Normungsaktivitäten wesentliche Impulse und Vorschläge für Produktanforderungen, Informationspflichten, Prüfmethoden und Normen resultieren. Auf dieser Basis sind rechtliche Maßnahmen im Rahmen der Ökodesign-Richtlinie auf EU-Ebene und national abzuleiten und umzusetzen. Die Produktanforderungen müssten wissenschaftlich valide, technisch machbar und wirtschaftlich vertretbar sein. Hinzu kommt, dass sie im Zuge der Marktüberwachung 
mit vertretbarem Aufwand überprüft werden können. Die Methoden zur Bestimmung der Lebensdauer sind noch nicht ausreichend entwickelt. Vielfach ist der Aufwand für eine Lebensdauerprüfung so hoch, dass sie mit hohen Kosten verbunden sind. Dies gilt insbesondere für Dauertests von langlebigen und intensiv genutzten Geräten wie Fernseher, Kühlschränke oder Waschmaschinen. Prüfungen und Anforderungen an Komponenten und Bauteile elektrischer Hausgeräte sind in einer Reihe von Normen enthalten. Hintergrund ist meist die Sicherheit des Produktes. Probleme sind die Reproduzierbarkeit der Ergebnisse sowie aufwändige, teure und zeitintensive Prüfverfahren. ${ }^{6}$

2. Entwicklung von Standards für eine upgrade- und reparaturfreundliche Produktentwicklung: Notwendig sind eine leichtere Reparatur von sensibler Elektronik (physics of failures) und die Möglichkeit zum Upgrade. Dies setzt auch die Schaffung von Schnittstellen (z. B. Android) für neue (mit Kopplung an alte) Technologien gerade in Hinblick auf die Digitalisierungswelle voraus. Allerdings ist nicht zu erwarten, dass dies umfassend marktgetrieben erfolgt, sodass gesetzgeberische Maßnahmen notwendig sind, um förderliche Rahmenbedingungen zu schaffen. So lassen sich Schnittstellen vorsehen, die eine spätere Erweiterung ermöglichen. Das Spektrum der Ansatzpunkte reicht von Schnittstellen für neue Technologien, der Entkopplung von Hardware und Software, der Entwicklung von innovativen und modularen SoftwareLösungen und einer ausreichend langen Vorhaltung von Software-Treibern bis hin zu open source, verpflichtenden Hardware- und Software-Updates und verpflichtenden Fehlerdiagnosefunktionen (Prakash et al. 2016). Voraussetzung für eine bedarfsgerechte Steuerung der Geräte wäre eine konsequent modulare Softwarearchitektur (und damit eine Rückbesinnung auf klassische Prinzipien der Informatik). Die Auswahl der Module kann bei der Installation und Konfiguration oder auch im laufenden Betrieb erfolgen (bei webbasierter Software entfällt die Installation ohnehin).

3. Marktüberwachung und Monitoring der Lebensdauer der Geräte: Um festzustellen, ob Maßnahmen greifen und inwieweit der Trend kürzer werdender Produktnutzung gestoppt wird und in die andere Richtung gedreht werden kann, bedarf es darüber hinaus eines kontinuierlichen Monitorings der Entwicklung der Lebensdauer der Geräte. Bislang fehlt ein differenziertes, methodisch einheitliches und kontinuierliches nationales Monitoring in Deutschland. Nur auf Basis eines verlässlichen Monitorings lassen sich langfristige Orientierungen und Entscheidungsgrundlagen sowohl für die Politik als auch für die Hersteller und Verbraucher/-innen schaffen. Vor diesem Hin-

6 So wurde der Vorschlag für ein Prüfverfahren zur Lebensdauerprüfung von Waschmaschinen bei der europäischen Normungsorganisation CENELEC in den 9oer-Jahren mit der Begründung abgelehnt, dass das Prüfverfahren zu zeit- und kostenintensiv sei (Oehme und Jacob 2017). 
tergrund ist es erforderlich, ein kontinuierliches nationales Monitoring aufzubauen. Dieses sollte mit der Marktüberwachung verbunden werden.

\subsubsection{Empowerment der Nutzer/-innen: \\ Transparenz und Ausweitung der Verbraucherrechte}

Die derzeit geltenden verbraucherrechtlichen Gewährleistungsregelungen sind nicht geeignet, die Verbraucherinnen und Verbraucher vor Frühausfällen zu schützen. In der jetzigen Form bieten die Gewährleistungsregelungen (24 Monate, Beweislast liegt bis sechs Monate bei den Verkäuferinnen und Verkäufern, danach bei den Käuferinnen und Käufern) keine Anreize für eine längere Produktlebensdauer. Auch die Garantie, die von den Herstellern freiwillig gegeben wird, erzeugt keine marktdifferenzierenden Lenkungseffekte für eine längere Produktlebensdauer. Die Garantie ist nicht gesetzlich geregelt. Sie ist eine freiwillige Leistung des Herstellers. Deshalb kann der Hersteller selbst entscheiden, was die Garantie abdeckt und wie lange sie gilt. Häufig wird garantiert, dass das Produkt ein bis zwei Jahre funktioniert, wenn der Verbraucher oder die Verbraucherin es normal nutzt. Dabei ist es egal, ob ein Mangel von Anfang an bestand oder erst später entstanden ist. Verschleißteile sind aber meist von der Garantie ausgenommen (Pöhler 2017).

Um bessere Voraussetzungen für langlebige Produkte herzustellen, müssten die Verbraucherrechte deutlich gestärkt werden. Hierzu sind drei Optionen zu verfolgen: 1) die Ausdehnung der Beweislastumkehr bei Vorliegen eines Mangels von derzeit sechs Monate auf 24 Monate, 2) die Verlängerung der Gewährleistungsfristen und 3) die Einführung einer Herstellergarantieaussagepflicht. Handlungsbedarf besteht außerdem bei der Schaffung von geeigneten Kennzeichnungs- und Informationsregelungen für Verbraucherinnen und Verbraucher (Angaben zur Haltbarkeit), um bewusste Kaufentscheidungen mit Blick auf die Lebensdauer zu unterstützen. Man sieht dem Produkt nicht an, für welche Lebensdauer es konzipiert wurde. Auch der Preis ist nicht immer ein zuverlässiger Indikator. Kennzeichnungs- und Informationsregelungen sollen daher helfen, die mangelnde Transparenz für die Verbraucherinnen und Verbraucher abzubauen. Mit dem Blauen Engel und dem Bundespreis Ecodesign existieren zwei Instrumente, die Anforderungen an die Langlebigkeit und Reparaturfreundlichkeit stellen. Die Ökodesign-Richtlinie fordert vereinzelt Informationen, beispielsweise zu den Ladezyklen von Akkumulatoren bei Notebooks. Über unabhängige Warentests werden regelmäßig elektronische und elektrotechnische Produkte auch auf Mängel bei der Haltbarkeit getestet, die in Qualitätsurteile einfließen.

All diese Aktivitäten, die zur Verbesserung der Markttransparenz beitragen, reichen aber nicht aus. So ist der Blaue Engel Deutschlands bekanntestes Umweltzeichen, bezüglich der elektronischen und elektrotechnischen Geräte spielt er aber kaum eine Rolle. Hinzu kommt, dass die Wirkung von Kennzeichnungs- 
und Informationsregelungen darunter leidet, dass es bereits unzählige Label und Informationsquellen gibt, was den Verbraucherinnen und Verbrauchern oft die Orientierung erschwert. Zur Verbesserung der Verbraucherinformation ist die generelle Angabe der Produktlebensdauer, eine Deklaration spezifischer Nutzungs- und Wartungsbedingungen sowie Informationen zu Ersatzteilen und Reparaturservice notwendig.

\section{Maßnahmen}

1. Anforderungen zur Angabe der Produktlebensdauer: Dabei müssen Informations- und Kennzeichnungsanforderungen für die Marktaufsichtsbehörden mit vertretbarem Aufwand überprüfbar sein. Das Hauptproblem von Informations- und Kennzeichnungsregelungen zur Lebensdauer von Produkten liegt in der Bestimmung der erwarteten Lebensdauer. Die Methoden dafür sind größtenteils noch nicht verlässlich genug und damit noch nicht umsetzungsreif. Hier besteht erheblicher Forschungsbedarf. Die Bestimmung der erwartbaren Lebensdauer erfordert ausgiebige Tests, auf deren Resultate sich die betroffenen Akteure (Hersteller, Prüfinstitute, Marktüberwachungsbehörden etc.) dann einigen müssten. »In der Regel würde die Geschwindigkeit eines solchen Prozesses bei vielen Produkten wahrscheinlich der technologischen Entwicklung hinterher hinken.« (Wieser und Tröger 2015) Angaben zur Produktlebensdauer werden unter der Ökodesign-Richtlinie nur »fallspezifisch für einzelne Produktgruppen« sinnvoll möglich sein, eher leistbar sind Informationsanforderungen zu Komponenten (Ladezyklen von Akkumulatoren, Laufzeit des Motors etc.).

2. Stärkung der unabhängigen Verbrauchereinrichtungen: Um angesichts der Produktvielfalt und Marktdynamik sowie der Mächtigkeit der Werbung die Transparenz beim Kauf von Produkten zu verbessern, sind die Verbrauchereinrichtungen und unabhängigen Prüfinstitute, wie die Stiftung Warentest, zu stärken. Neben den klassischen Instrumenten der Verbraucherinformation und Verbraucherbildung können insbesondere Kampagnen im Rahmen der von der UN aufgestellten Sustainable Development Goals (SDGs) einen Beitrag dazu leisten, den vorherrschenden Konsumstil zu hinterfragen und zukunftsfähige Leitbilder, zu denen der Kauf und die Nutzung langlebiger Produkte zählen, zu vermitteln. Um diese Aufgabe ernsthaft wahrnehmen zu können, sind die Verbrauchereinrichtungen deutlich stärker mit Ressourcen auszustatten und zu fördern.

3. Änderung des Gewährleistungsrechts (Verbraucherrechte-Richtlinie): Im kaufrechtlichen Gewährleistungsrecht ist die Beweislastumkehr bei Existenz eines Defektes von derzeit sechs Monaten auf 24 Monate zu erhöhen. Außerdem ist zu prüfen, um welche Zeitspanne die Gewährleistungsfristen verlängert werden sollten, sodass sie Lenkungseffekte bezüglich der Produktlebensdauer 
erzielen. Im Rahmen der Erarbeitung der EU-Verbraucherrechte-Richtlinie wurde von einigen EU-Staaten eine Verlängerung der Gewährleistungsfrist auf vier Jahre angeregt.

4. Einführung einer Herstellergarantieaussagepflicht und Deklarationspflicht: Der Hersteller muss explizit darstellen, für welche Dauer er die Garantie eines Produkts und die Reparaturkosten übernimmt. Dadurch könnte ein Wettbewerb zwischen Herstellern um längere Garantiezeiten gefördert werden.

\subsubsection{Soziale Innovationen für eine suffiziente Konsumkultur}

Wenn der Wandel hin zu einer längeren Nutzung von Produkten gelingen soll, reichen Mindestlebensdauer und Empowerment der Verbraucherinnen und Verbraucher durch Stärkung der Verbraucherrechte nicht aus. Vielmehr setzt ein Pfadwechsel auch soziale Innovationen und innovative Geschäftsmodelle voraus, die weiterreichende Alternativen zur »Wegwerfmentalität« liefern. Dazu gehört das Teilen von Produkten (Produkt-Sharing etc.), die Reparatur von Produkten (Repair-Cafés etc.) und die Wiedernutzung gebrauchter Produkte (re-commerce etc.). In den vergangenen Jahren haben sich zunehmend innovative Formen des Konsums entwickelt, die sich durch eine längere bzw. intensivere Nutzung von Konsumgütern auszeichnen. Dadurch steigt der funktionale Nutzen, den die Produkte liefern, und solange der dafür nötige Ressourcenverbrauch nicht überproportional steigt, nimmt insgesamt die Ressourcenproduktivität zu. Der Handel mit Gebrauchtwaren hat sich bereits in den letzten Jahren dynamisch entwickelt.

Durch eBay, dem weltweit größten Online-Portal für gebrauchte Produkte, hat es einen regelrechten »Quantensprung« im Gebrauchtwarenhandel gegeben, der sich vor allem in dem enormen Anwachsen der Anzahl der Marktteilnehmer/ -innen, Käufer/-innen und Verkäufer/-innen von gebrauchten Produkten zeigt. Mittlerweile existieren in Deutschland 36 Wiederverkaufsportale für gebrauchte Produkte. Der Handel von Gebrauchtprodukten über digitale Plattformen hat ein Niveau erreicht, das ökologisch mengenrelevant ist. Für Deutschland wird geschätzt, das pro Jahr 119.616 Tonnen Elektro- und Elektronikgeräte allein über eBay einer weiteren Nutzung zugeführt werden. Vergleicht man diese Menge mit dem Aufkommen an Elektroaltgeräten in Höhe von 603.052 Tonnen (in 2014), so ergibt sich ein Anteil von 19,8 Prozent (Gries et al. 2017). Der Wiederverkauf von Produkten trägt also bereits spürbar zur Ressourcenschonung bei und könnte in Zukunft sogar noch erheblich ausgeweitet werden. Heute befinden sich in einem deutschen Haushalt schätzungsweise durchschnittlich 10.000 Gegenstände (Destatis, zit. n. RNZ 2014). Viele werden selten oder nicht genutzt. Unter den hochwertigen Gegenständen befinden sich vor allem Haushaltsgeräte und elektronische Geräte, die meist voll funktionsfähig sind und sich somit sehr gut für einen Wiederverkauf bzw. eine Wiedernutzung eignen würden. Allein 43 Millionen Mobiltelefone werden nicht genutzt, also pro Haushalt mehr als ein Handy oder 
Smartphone. In jedem zweiten Haushalt findet sich im Schnitt eine ungenutzte Kaffeemaschine, eine Digitalkamera und eine Spielekonsole. Der geschätzte Gesamtwert der ungenutzten Gegenstände beträgt (gemessen an dem durchschnittlichen Erlös, den diese Gegenstände bei Verkauf auf Ebay erbringen würden) 3223 Euro pro Haushalt. ${ }^{7}$ Hochgerechnet auf alle Haushalte in Deutschland beträgt der Wert rund 130 Milliarden Euro. Die Herausforderung besteht darin, über soziale Innovationen dieses Potenzial zu erschließen.

In der aktuellen Fortschreibung des Deutschen Ressourceneffizienzprogramms, ProgRess II (BMUB 2016), haben sich die Bundesregierung bzw. die entsprechenden Ressorts und Bundesbehörden folgende Ziele gesetzt: die Stärkung des öffentlichen Dialogs über die Potenziale sozialer Innovationen für Ressourcenschonung (z.B. durch die Etablierung eines Zukunftsforums), den Aufbau eines nationalen Netzwerks als Ort gesellschaftlichen Experimentierens und Lernens, die Initiierung eines runden Tisches unter Beteiligung von Banken, Stiftungen und gesellschaftlichen Netzwerken, um neue Finanzierungsinstrumente sozialer Innovationen zu etablieren (z. B. Crowdfunding-Modell, Joint Venture Capital) sowie der Aufbau einer nationalen Kontaktstelle für soziale Innovationen und kollaborativen Konsum. (Behrendt und Henseling 2019)

\section{Maßnahmen}

1. Repair-Cafés, Leihstationen, Wiederverwendungs- und Reparaturzentren fördern und strukturell stärken: Während kommerzielle Plattformen sich durch marktlich-wettbewerbliche Mechanismen dynamisch entwickeln, fällt die Dynamik bei gemeinwohlorientierten Plattformen gering aus. Trotzdem bilden sie einen Resonanzboden, auf dem sich neue soziale Praktiken entfalten, die für die Lösung von Nachhaltigkeitsherausforderungen wichtige Impulse geben. Ihnen kommt als Orte des gemeinschaftlichen Teilens und Reparierens und des Austauschs über einen reflektierten Umgang mit Konsum in besonderer Weise eine transformative Rolle für eine auf nachhaltige Entwicklung ausgerichtete Konsumkultur zu. Daher ist es notwendig soziale Experimentierfelder in Form von Reallaboren $\mathrm{zu}$ fördern und Initiativen strukturell $\mathrm{zu}$ stärken (z. B. durch Bereitstellung von kommunalen Infrastrukturen für gemeinwohlorientierte Angebote).

2. Frugale Innovationen haben das Potenzial, suffiziente Konsumweisen $\mathrm{zu}$ unterstützen. Frugale Innovationen stehen für robuste, benutzerfreundliche und wartungsarme Produkte (Tiwari und Herstatt 2014; Jänicke 2014). Falls nachgewiesen werden kann, dass bei einer zunehmenden Anzahl von Produkten deren Kernfunktionalität sowie deren Gesamtkosten für Anschaffung und

7 Bezogen auf die in deutschen Haushalten durchschnittlich 217 nicht genutzten Gegenstände, gemäß repräsentativer Befragung von TNS im Auftrag von Ebay (2015). 
Nutzung kaufentscheidend sind statt immer höherwertige Produktmerkmale und gesteigerte Premiumqualität, wäre ein Umdenken im gesamten Innovationsprozess notwendig. Die häufig technikgetriebene Produktentwicklung müsste ihren Fokus weg von immer ausgefeilteren und besser ausgestatteten Produkten hin zu kostengünstigen und dennoch qualitativ hochwertigen Marktlösungen richten.

3. Der Zugang zu Reparaturanleitungen, Ersatzteilen und Diagnosesoftware für herstellerunabhängige Reparaturbetriebe und -initiativen ist zu verbessern. Hier sind rechtliche Fragen u. a. zur Nutzung von Datensätzen der Hersteller klärungsbedürftig. Hersteller müssen Diagnose-Software kostenfrei zugänglich machen. Ohne Verbesserung der rechtlichen Rahmenbedingungen wird sich die schwierige Situation im Bereich der Reparaturen nicht verbessern lassen.

4. Auszeichnung von Produkten nach ihrem Wiederverkaufswert: Ein Ansatzpunkt zur Förderung einer Wiederverkaufskultur ist die Auszeichnung von Produkten nach ihrem Wiederverkaufswert (analog der "Schwacke-Liste« im Fahrzeugmarkt). Auf diese Weise könnten hochwertige und langlebige Produkte von Billigprodukten mit geringer Chance für eine Wiederverkaufsmöglichkeit unterschieden werden.

5. Flankierend können Kampagnen für langlebige Produkte das Bewusstsein für die Wertigkeit von Produkten erhöhen. Einen Rahmen liefert dafür das 2016 von der Bundesregierung verabschiedete »Nationale Programm für nachhaltigen Konsum«. Auch das Programm »Bildung für nachhaltige Entwicklung" bietet dafür einen Rahmen. Bis dato wird die Thematik in Einzelprojekten aufgegriffen, müsste aber, um Breitenwirkung zu entfalten, systematisch im Nationalen Aktionsplan verankert werden.

6. Förderung von fablabs und makers spaces als Reallabore, in denen abseits von Fabriken kreative, neuartige Produkte selbst produziert und dabei digitale Werkzeuge (3-D-Drucker, Vinyl Cutter, Apps \& Co.) genutzt werden. Speziell der 3-D-Druck bietet hier neue Möglichkeiten zur Bereitstellung von Ersatzteilen, die vom Hersteller aufgekündigt wurden ${ }^{8}$. Open-Source-HardwareLösungen tendieren oft zur Modularität und können dazu beitragen, Produkte einfacher zu warten, zu reparieren, aufzubereiten und wieder zu nutzen?.

8 In dem vom BMUB geförderten Projekt »Wiederverwendung durch Reparatur stärken - Potentiale des 3D-Drucks zur Ersatzteilbeschaffung nutzen« werden die derzeitigen Möglichkeiten und Grenzen des 3-Drucks zur Bereitstellung von Ersatzteilen untersucht. Das Projekt wird Datensätze auf einer Microwebsite bereitstellen. Außerdem zielt das Projekt darauf, fablabs, maker spaces, Repair-Cafés, Reparaturwerkstätten und Reparaturinitiativen zu vernetzen. Damit soll die Verfügbarkeit von Ersatzteilen für die Reparatur verbessert werden.

9 Eine Inspiration hierfür bietet das Open-Structures-Projekt (Zimmermann 2018). 
7. Entwicklung neuer Geschäftsmodelle für re-commerce: Potenziale zur Umsetzung einer ressourceneffizienten Produktnutzung liegen in innovativen Geschäftsmodellen, die daraufabzielen, nicht nur gebrauchte Produkte zu kaufen und wieder zu verkaufen, sondern auch zu reparieren und aufzurüsten sowie eine Garantiezeit zu gewähren, die über den üblichen Garantiezeiten bei Neuprodukten liegt. Damit könnte ein wesentliches Hemmnis für eine Ausweitung des Gebrauchtwarenhandels bei elektrotechnischen Geräten abgebaut werden.

\subsubsection{Preisliche Anreize für langlebige Produktgestaltung und -nutzung}

Bei Billigprodukten werden Umweltkosten infolge einer kürzeren Produktlebens- und -nutzungsdauer externalisiert. Vergleichbare Kosten für die Konsumentinnen und Konsumenten zwischen der Nutzung langlebiger und kurzlebiger Produkte können (zeitbezogen) als ein Indiz für die Externalisierung von Kosten interpretiert werden. Laut Umweltbundesamt belasten die kurzlebigen Elektround Elektronikprodukte die Umwelt grundsätzlich deutlich stärker als Geräte mit langer Lebensdauer (Oehme und Jacob 2017). Beim Billigpreissegment ergibt sich außerdem durch häufige Neugründungen das Problem, dass sich Hersteller ihrer Produktverantwortung entziehen. Zur Umkehr des Trends immer kürzer werdender Produktlebensdauer und Nutzungszeiten ist eine Internalisierung der externen Kosten notwendig. Folgende Maßnahmen sind zu verfolgen:

\section{Maßnahmen}

1. Steuerliche Förderung von Reparaturen: $\mathrm{Zu}$ prüfen ist, inwieweit eine steuerliche Förderung von Reparaturen entsprechende Lenkungseffekte erzeugt und wie sie umgesetzt werden könnte. Bei Reparaturen von Haushaltsgeräten vor Ort können in Deutschland bereits Arbeitskosten steuerlich geltend gemacht werden. Darüber hinaus könnte ein reduzierter Mehrwertsteuersatz für Reparaturleistungen die Reparatur verbilligen. Die EU-Mehrwertsteuerrichtlinie lässt bisher einen verringerten Mehrwertsteuersatz nur für kleinere Reparaturdienstleistungen (nämlich bei Fahrrädern, Schuhen etc.) zu. Der existierende europarechtliche Spielraum sollte auf nationaler Ebene aber intensiv genutzt werden. Dazu sollte die Ausweitung auf weitere Reparaturdienstleistungen geprüft und in den europäischen Diskussionsprozess eingebracht werden (Oehme und Jacob 2017).

2. Zweites Preisschild: Einführung eines »Zweiten Preisschildes«, wie vom Bundesumweltministerium angedacht. Dieses soll die Umweltwirkungen, also unter anderem Umweltschäden und Ressourcenverbrauch, von besonders umweltrelevanten Produkten und Dienstleistungen darstellen. Elektro- und Elektronikgeräte könnten so mit Angaben zu den verbrauchten Ressourcen gekennzeichnet werden. Langfristiges Ziel des BMUB ist, dass bei möglichst vielen Produkten und Dienstleistungen der Preis die tatsächlichen gesellschaftlichen 
Kosten widerspiegelt (BMUB 2016). Die Methoden zur Bestimmung des Ressourcenverbrauchs sind mit Blick auf Langlebigkeit noch zu entwickeln. Hier besteht Forschungsbedarf.

3. Rohstoffabgabe bzw. Rohstoffsteuer: Zur Internalisierung externer Kosten wird außerdem von verschiedenen Seiten eine Rohstoffabgabe oder Rohstoffsteuer diskutiert. Eine Ressourcenabgabe könnte für Hersteller zum Anreiz werden, langlebige, reparaturfreundliche und qualitativ hochwertige Produkte zu produzieren. Die Besteuerung von Rohstoffen kann aber nicht singulär für eine bestimmte Produktgruppe erfolgen, sondern muss in ein Gesamtkonzept, welches ökonomische und ökologische Aspekte der Langlebigkeit genauso berücksichtigt wie Substituierbarkeit und Knappheit von Ressourcen im Kontext nationaler und internationaler Ressourcenströme, eingebettet sein.

\subsubsection{Akteure und Zeithorizont}

\subsubsection{Roadmap im Überblick}

Wie die verschiedenen Maßnahmen für einen Pfadwechsel zeitlich eingeordnet werden, zeigt die folgende Abbildung.

Abbildung 51: Roadmap für einen Pfadwechsel im Bereich »Produkte länger nutzen«im Überblick

\begin{tabular}{|c|c|c|}
\hline \multirow{5}{*}{$\begin{array}{l}\text { Politik } \\
\text { auf europäischer } \\
\text { und Bundesebene }\end{array}$} & $\begin{array}{l}\text { Okodesign-Richtlinie Arbeitsplan 2016- } \\
\text { 2019, Umsetzung ab } 2019\end{array}$ & Steuerliche Anreize für Reparatur \\
\hline & Marktuberwachung & Änderung des Gewährleistungsrechts \\
\hline & \multirow{2}{*}{ Kampagnen fur langlebige Produkte } & Herstellergarantieaussagepflicht \\
\hline & & Zweites Preisschild \\
\hline & $\begin{array}{l}\text { Forderung von fablabs und } \\
\text { makerspaces }\end{array}$ & Rohstoffabgabe \\
\hline \multirow{3}{*}{$\begin{array}{l}\text { Kooperationen } \\
\text { Normungsorganisationen } \\
\text { Warentester } \\
\text { Netzwerke }\end{array}$} & $\begin{array}{l}\text { Standards für upgrade- und } \\
\text { reparaturfreundliche Produktentwicklung }\end{array}$ & \multirow[t]{3}{*}{$\begin{array}{l}\text { Anforderung zur Angabe der } \\
\text { Produktlebensdauer }\end{array}$} \\
\hline & $\begin{array}{l}\text { Stärkung der unabhängigen } \\
\text { Verbrauchereinrichtungen }\end{array}$ & \\
\hline & Soziale Experimentierfelder & \\
\hline \multirow{3}{*}{$\begin{array}{l}\text { Wirtschaft } \\
\text { Hersteller } \\
\text { Dienstleister } \\
\text { Start-ups }\end{array}$} & $\begin{array}{l}\text { Zugang zu Reparaturanleitungen und } \\
\text { Ersatzteilen }\end{array}$ & \multirow[t]{3}{*}{ Kennzeichnung Wiederverkaufswert } \\
\hline & Frugale Innovationen & \\
\hline & $\begin{array}{l}\text { Neve Geschäftsmodelle für } \\
\text { Re-Commerce }\end{array}$ & \\
\hline
\end{tabular}

Quelle: Eigene Darstellung, Institut für Zukunftsstudien und Technologiebewertung, auf Grundlage der Zukunftswerkstatt»Produkte länger nutzen«am 8.6.2017, Berlin. 
Kurz- bis mittelfristig ist die Produktverantwortung der Hersteller und Vertreiber von Konsumprodukten auszuweiten. Dazu bedarf es rechtlicher Rahmenbedingungen für die Sicherung einer Mindestlebensdauer für Produkte und deren Reparierbarkeit. Den Rahmen liefert die Umsetzung des EU-Kreislaufwirtschaftspaketes und des Arbeitsplans 2016 bis 2019 der Ökodesign-Richtlinie. Hier geht es darum, Produktstandards, Anforderungen und Informationspflichten zur Produktlebensdauer und Reparatur zu prüfen und festzulegen. Dies geschieht in Verbindung mit Normungsmandat $\mathrm{M} / 543$.

Gleichzeitig bedarf es eines Empowerments der Nutzerinnen und Nutzer durch die Stärkung der Verbraucherrechte. Im Gewährleistungsrecht ist die Beweislastumkehr bei Vorliegen eines Defektes zeitlich auszuweiten. Es ist zu prüfen, um welche Zeitspanne die Gewährleistungsfristen verlängert werden sollten. Außerdem sollten die Hersteller verpflichtet werden, die Garantiezeit anzugeben. Verbraucherinnen und Verbraucher sollten darüber hinaus darin unterstützt werden, langlebige und nachhaltige Produkte zu identifizieren, um eine bewusste Kaufentscheidung treffen zu können. Hierfür müssen verständliche und zuverlässige Kennzeichnungs- und Informationsgrundlagen geschaffen werden.

Die Setzung von Mindeststandards für Langlebigkeit und Reparierbarkeit sowie die Stärkung der Verbraucherrechte reichen aber nicht aus, um einen Pfadwechsel herbeizuführen. Notwendig ist ein grundlegender Wandel des Konsums, der die Wertschätzung von Produkten in den Mittelpunkt rückt. Außerdem ist ein Pfadwechsel ohne Abbau der ökonomischen Pfadabhängigkeiten kaum zu erwarten. Ein Ansatz ist die Einführung eines reduzierten Mehrwertsteuersatzes für Reparaturdienstleistungen. Tiefgreifender wären Rohstoffabgaben bzw. -steuern, die sich auch nach der Haltbarkeit der Produkte richten. Abgaben oder eine Besteuerung nach Haltbarkeit und Reparaturfähigkeit könnten langlebigen Produkten einen Wettbewerbsvorteil verschaffen. Für eine umfassende Besteuerung des Ressourceneinsatzes (unter Langlebigkeitsgesichtspunkten, Reparaturfähigkeit etc.) ist erst noch eine geeignete Konzeption zu entwickeln.

Da sich die Verlängerung der Produktnutzung nicht marktgetrieben vollzieht, sondern die dominanten Marktlogiken eine Trendwende ausbremsen, wenn nicht gar verhindern, kommt der Politik für einen Pfadwechsel eine besondere Rolle zu. Die Verlängerung der Nutzungsdauer setzt nicht nur neue Marktangebote, sondern auch Veränderungen des Verbraucherverhaltens voraus. Märkte für langlebige Produkte lassen sich nur mit einem entsprechenden Wandel von Konsumstilen und Wertschätzungen entwickeln. Die Umsetzung von Strategien einer längeren Produktnutzungsdauer ist daher auf eine Synchronisierung des angebots- und nachfrageseitigen Wandels angewiesen (vgl. Fichter 2005). Um Prozesse im Konsumbereich anzustoßen und zu verstärken, bedürfen Initiativen flankierender Maßnahmen auf anderen Entscheidungsebenen und der kooperativen Mobilisierung finanzieller und personeller Ressourcen. Ein Pfadwechsel von der kürzeren 
hin zur längeren Produktnutzung bedarf das Zusammenspiel von Herstellern, Politik, Handel, Wissenschaft, NGOs, Marktüberwachungsbehörden, Warentestern, Normungsorganisationen und Konsumentinnen und Konsumenten. Neben Normungsaktivitäten und politischen Regulierungen, staatlichen Finanzierungsund Fördermaßnahmen spielt die Zusammenarbeit von Unternehmen, Start-ups, Verbraucherinnen und Verbrauchern und gesellschaftlichen Gruppen in sozialen Experimentierfeldern für eine suffiziente Konsumkultur eine wichtige Rolle.

Während weiche (informatorische und auf Eigenverantwortung setzende) Instrumente breite Akzeptanz bei wirtschaftlichen Akteuren finden, erweisen sich ordnungsrechtliche Instrumente (z. B. Ökodesignvorschriften zur Mindestlebensdauer, Verlängerung der Gewährleistungsfrist und Verlängerung der Beweislastumkehr über die derzeit geltende Frist von sechs Monaten hinaus) oder Ansätze der Kosteninternalisierung (Ressourcenbesteuerung, erweiterte Produzentenverantwortung) als durchweg kontrovers. Dabei stellt sich die grundlegende Frage, inwieweit die politischen Institutionen, insbesondere auf EU-Ebene die Kommission, das EU- Parlament sowie der Europäische Rat und auf nationaler Ebene die Bundesregierung mit den entsprechenden Ressorts und zugeordneten Behörden bereit sind, in die bestehenden Produktions- und Konsumstrukturen einzugreifen. Mindeststandards sind aus regulatorischer Sicht eine Maßnahme mit hoher Eingriffstiefe in die Produktgestaltung der Unternehmen und Märkte. Eine solche Maßnahme stößt daher auf großen Widerstand seitens der Hersteller. Ein Konfliktfeld ist die geringe Kooperationsbereitschaft betroffener Hersteller (vor allem in niedrigpreisigen Marktsegmenten) und Verbände, die in Mindestanforderungen für Langlebigkeit und Reparierbarkeit nachteilige Auswirkungen auf ihre Produkt- und Vermarktungsstrategien sehen (Bertling et al. 2014). Hier prallen Interessen aufeinander. Ein Pfadwechsel hängt davon $a b$, ob und inwieweit an den Schnittstellen zwischen Umwelt-, Wirtschafts- und Verbraucherpolitik europäisch und national solche Lösungen durchgesetzt werden können, die bessere Rahmenbedingungen für die Herstellung langlebiger Produkte, für ihre Reparatur und ihre Wiedernutzbarkeit sicherstellen. Dabei spielen Pioniere (Repair Cafés, Vangerow, Fairphone etc.), NGOs (NABU, BUND etc.) und Netzwerke (Runder Tisch Reparatur, Open Source Circular Economy Days etc.) eine wichtige Rolle im Transformationsprozess. Die besondere Leistung von Pionieren besteht in der Schaffung eines Resonanzbodens, auf dem sich neue soziale Praktiken, die wichtige Impulse für einen Pfadwechsel geben, entfalten. 



\section{Fazit}

Ökologische Systeme weltweit geraten zunehmend an ihre Grenzen und die Folgen des Klimawandels werden immer stärker sichtbar (Steffen et al. 2015; IPCC 2014). Dennoch haben sich bis jetzt weder auf internationaler noch nationaler Ebene politische, wirtschaftliche und gesellschaftliche Transformationen vollzogen, die in der Summe auf eine signifikante Änderung dieser Entwicklungen hindeuten. Beim Klimawandel etwa, als einem besonders markanten internationalen Umweltproblem, ist bisher stark zu bezweifeln, dass das politisch angestrebte 2-Grad-Ziel auch erreicht wird, und für die Einhaltung des vom IPCC angeratenen 1,5-Grad-Zieles fehlt es definitiv an einer entsprechenden internationalen Regelungsgrundlage. In vielen anderen umweltpolitischen Bereichen sehen die Voraussetzungen noch deutlich ungünstiger aus.

Ausmaß und Charakter von Umweltproblemen haben sich in den letzten Jahrzehnten deutlich geändert. Zum einen wird immer offenkundiger, dass viele Umweltfragen nicht mehr lokaler, regionaler oder nationaler Natur sind, sondern einen globalen Maßstab angenommen haben. Gleichzeitig sind, nachdem teilweise deutliche Erfolge in bestimmten Umweltbereichen erzielt werden konnten, die komplexeren Aspekte übrig geblieben, wie etwa die zahlreichen Stoffeinträge künstlicher Substanzen oder eben die Zerstörung von Ökosystemen. Gleichzeitig sind die Erfolge der bisherigen Umweltpolitik, so wichtig sie auch waren, in vielen Fällen nicht ausreichend und weitere entschiedene Maßnahmen müssen ergriffen werden. Wie schon in der Einleitung des Buches geschrieben, kann es angesichts der verschiedenen Problemlagen keinen Zweifel mehr geben, dass die klassische Umweltpolitik an ihre Grenzen gestoßen ist.

Um auf die aktuellen und absehbaren Herausforderungen zu reagieren, bedarf es eines ganz neuen Ansatzes, einer transformativen Umweltpolitik, also einer Umweltpolitik, die gezielt die Transformation ganzer gesellschaftlicher und wirtschaftlicher Systeme anstrebt. Eine solche Umweltpolitik zu implementieren ist gerade für Deutschland nicht einfach. Die oft schon sehr ausdifferenzierten vorhandenen wirtschaftlichen Systeme und die vielfältige Zersplitterung der politischen und rechtlichen Zuständigkeiten erschweren es erheblich, sich auf stark einschneidende, systemändernde Maßnahmen zu verständigen. Ohne eine Umweltpolitik, die bereit ist, die Führung bei der Lösung der bevorstehenden He- 
rausforderungen zu übernehmen, wird es aber nicht möglich sein, die benötigten Transformationen anzustoßen.

Im Rahmen des Forschungsprojektes sind vielfältige Faktoren identifiziert worden, die für die Frage von Erfolg oder Misserfolg eines Transformationsprozesses von Bedeutung sind. Aus den verschiedenen Fallbeispielen sind zahlreiche Erkenntnisse erwachsen. Auf der Basis dieser Erkenntnisse ist es möglich, diverse Anregungen für die Gestaltung einer transformativen Umweltpolitik zu formulieren.

Grundsätzlich hat sich gezeigt, dass viele der Erfolgs- wie auch der Hemmnisfaktoren von nachhaltigen Transformationen durchaus politischen Maßnahmen zugänglich sind. Damit ergibt sich auch, dass Transformationsprozesse zwar nicht beliebig politisch steuerbar, aber durchaus gestaltbar sind. Wenn die Umweltpolitik hier eine entscheidende Rolle übernehmen will, muss sie allerdings bereit sein, sich nicht als Gegenspieler der Gesellschaft, sondern als Akteur im gesellschaftlichen System zu verstehen. Gleichzeitig muss die Gesellschaft bereit sein, dieses neue Rollenverständnis der Umweltpolitik zu akzeptieren. Umweltpolitik als Gesellschaftspolitik funktioniert nur, wenn die Umweltpolitik auch innerhalb der Gesellschaft wirkt und nicht lediglich von außen an sie herantritt.

Mit Blick auf die Ansatzpunkte einer transformativen Umweltpolitik ist zu beachten, dass Transformationsprozesse komplex sind sowie parallel und nicht-linear ablaufen. Für die politische Praxis hat das zur Folge, dass kein chronologisch ablaufender Maßnahmenkatalog definiert werden kann. Politische Akteurinnen und Akteure sind gut beraten, Maßnahmen flexibel und dynamisch an veränderte Gegebenheiten anzupassen und Fehlschläge als natürlichen Teil des Prozesses zu antizipieren. Gleichzeitig gibt es diverse Ansatzpunkte, als Teil eines größeren Governance-Systems Veränderungen anzuregen und zu beeinflussen. Deshalb können und sollten Transformationen so gut wie möglich strategisch geplant und einzelne Aspekte wo möglich bewusst beeinflusst werden.

Als Schlussfolgerungen der Untersuchungen werden nachfolgend verschiedene Vorschläge vorgestellt, die sich aus den untersuchten Transformationsbeispielen ergeben. Die Vorschläge können als empirisch informierte und priorisierende Ergänzung zur allgemeinen Transformationsliteratur und zu bereits existierenden transformationsorientierten Strategien betrachtet werden.

\section{Stakeholder-Management}

Schon im Rahmen der Entwicklung des MoC-Ansatzes hat sich die Bedeutung von change agents erwiesen und verschiedene Untersuchungen in der Nachfolge haben dies bestätigt (vgl. etwa Eucker et al. 2017). Die im Rahmen des evolutionzgreen-Vorhabens durchgeführten Fallstudien weisen ebenfalls auf die große Bedeutung günstiger Akteurskonstellationen und des Vorhandenseins von change agents für nachhaltige Transformationen hin. Change agents müssen dabei nicht 
aus unternehmerischen Kontexten stammen. Auch Akteurinnen und Akteure aus Wissenschaft (Beispiel Post StreetScooter) oder Zivilgesellschaft, wie Verbände, Gewerkschaften und Kirchen, können eine Rolle spielen. Am Beispiel von Fairphone zeigt sich, dass Transformationsprozesse sogar aus dem künstlerischen Umfeld heraus angestoßen werden können.

Change agents sind oft außerhalb des »Umweltthemas« und bestehenden Regimes zu finden. Da etablierte Marktführer ihre Ressourcen oft nur für Verbesserungen bestehender Produkte nutzen anstatt technologische Innovationen einzuführen (Christensen 1997), sind gestaltende Pioniere umso wichtiger. Dies wurde auch vom Wissenschaftlichen Beirat der Bundesregierung für Globale Umweltveränderungen anerkannt:

»[Die gesellschaftliche Dynamik für die Transformation in Richtung Klimaschutz] stützt sich stark auf Pioniere des Wandels, welche die Optionen für die Überwindung einer auf der Nutzung fossiler Ressourcen beruhenden Ökonomie testen und vorantreiben und so neue Leitbilder bzw. Visionen entwickeln helfen, an denen sich der gesellschaftliche Wandel orientieren kann. Die Pioniere agieren zunächst als Nischenakteure, können dann aber zunehmend Wirkungskraft entfalten und die Transformation entscheidend befördern« (Wissenschaftlicher Beirat der Bundesregierung Globale Umweltveränderungen 2011).

Nicht nur einzelne Personen können als change agents agieren, sondern auch Teams, Organisationen und Institutionen. Wichtig ist, dass Akteure und Akteurskonstellationen letztlich über eine ausreichende Mischung an transformationsnotwendigem Wissen, Kompetenzen und Ressourcen verfügen. In den untersuchten Beispielen waren oftmals gruppenübergreifende Konstellationen am erfolgreichsten.

Aus umweltpolitischer Sicht entscheidend ist, dass sowohl das Vorhandensein von change agents als auch das Zusammenspiel von Akteurskonstellationen im Rahmen eines Stakeholder Managements gezielt beeinflusst werden können: change agents können aufgebaut werden und ihre Vernetzung untereinander kann entscheidend gefördert werden, wie die Einrichtung des CARB zeigt (vgl. Kapitel 4.2.5, Beobachtung 2 und 6). Ein Beispiel dafür, dass Stakeholder Management noch weiter gehen kann und dabei auch in den gesellschaftlichen Bereich hinausgegriffen werden kann, ist die Entstehung von Eurosif, des Europäischen Dachverbandes der Sustainable Investment Foren. Der Verband wurde um die Jahrtausendwende konkret auf Anregung der Europäischen Kommission gegründet und hat in seinen ersten Jahren einige finanzielle und politische Unterstützung bei seinem Aufbau durch die Generaldirektion für Beschäftigung, Soziales und Integration erhalten. Auf diese Weise hat die Kommission einen Gegenspieler zu den rein an finanziellen Interessen ausgerichteten klassischen Verbänden der Finanzindustrie geschaffen. Gleichzeitig wurde damit auch ein Anstoß gegeben, 
auf nationaler Ebene weitere solcher Verbände zu gründen, die inzwischen vielerorts eine wichtige Rolle bei der Transformation des Finanzwesens in Richtung von mehr Nachhaltigkeit spielen. Gleichzeitig wirken die Verbände wie der europäische Dachverband als wichtige Vernetzungsmöglichkeit der verschiedenen Akteure, die an einer solchen Transformation interessiert sind.

Stakeholder Management im Rahmen einer transformativen Umweltpolitik bedeutet mithin nicht nur change agents zu identifizieren und sich mit ihnen auszutauschen. Ein aktives Stakeholder Management kann darüber deutlich hinausgehen. Es identifiziert Lücken und Schwachstellen im Akteursgefüge und ergreift dann Maßnahmen diese Lücken zu füllen bzw. die Schwachstellen zu stärken, indem gemeinsam mit anderen gesellschaftlichen Akteuren geeignete Initiativen ergriffen werden.

\section{Management von windows of opportunity}

Gelegenheitsfenster ergeben sich aus Umbrüchen gesellschaftlicher, rechtlicher oder technologischer Rahmenbedingungen (vgl. Kapitel 4.2.5, Beobachtung 5). Grundsätzlich sind Gelegenheitsfenster Situationen, in denen ein etabliertes System durch externe Faktoren oder interne Probleme destabilisiert wird. Dabei ist das zeitliche Zusammenspiel von drei Strömen wichtig, damit ein Thema nicht nur allgemein diskutiert wird, sondern auch wichtige Entscheidungen fallen (Larkin 2012): Diese drei Ströme sind der Problem-Strom, der Policy-Strom und der Politik-Strom. Dies bedeutet, dass Gelegenheitsfenster im Rahmen der Politik-Agenda nur weiterverfolgt werden, wenn ein relevantes Problem auftritt oder aufgezeigt wird (z. B. ein plötzliches Desaster oder wiederholtes Aufgreifen eines Themas durch die Medien), zeitgleich Elemente aus dem Policy-Strom einfließen (z. B. Lösungsvorschläge aus der akademischen Forschung) sowie der PolitikStrom mit einbezogen wird (mit z. B. Veränderungen des Regierungsapparates). Ein Gelegenheitsfenster bietet damit die Basis für einen zeitlichen Spielraum, in denen ein ansonsten stabiles System gegenüber Innovationen offen ist (Tongur und Engwall 2017).

In der Vergangenheit wurden Gelegenheitsfenster oftmals nicht oder nur unzureichend genutzt. So hat beispielsweise die Finanz- und Wirtschaftskrise 2007 nicht zu nachhaltigen Finanzmarktreformen geführt. Manche historische Ereignisse sind dem Zufall geschuldet und können nicht gezielt vorbereitet werden. Dazu zählt beispielsweise die Ölkrise in den 1970er Jahren, die die Volkswirtschaften unvermittelt traf. Die Fallstudien (so etwa die Transformation des Wärmesektors in Dänemark, vgl. Kapitel 4.2) haben aber gezeigt, dass auch in solchen Fällen ein rasches Aufgreifen von Gelegenheitsfenstern durchaus noch möglich ist.

Für eine transformative Umweltpolitik ist es wichtig, Gelegenheitsfenster frühzeitig, also möglichst im Vorfeld zu identifizieren. Auf diese Weise kann man 
sich bereits vorbereiten, Lösungsvorschläge entwickeln, andere Akteure frühzeitig einbinden und Ansatzmöglichkeiten schaffen, um das Thema in den Politikstrom aufzunehmen (vgl. Wolff et al. 2018). Um günstige Zeitpunkte in Zukunft besser zu identifizieren, ist die Nutzung von Strategic foresight von Bedeutung. Durch diese Art der Vorausschau lässt sich eine gezielte Vorbereitung des Politikstroms verwirklichen und Gelegenheitsfenster können effektiv genutzt werden.

Neben Gelegenheitsfenstern, die sich durch die Anwendung von Strategic foresight im Vorhinein identifizieren lassen, gibt es auch Fälle, in denen es keine solche Methodik braucht, um Gelegenheiten vorherzusehen. So ist bereits absehbar, dass es in Zukunft vermehrt zu Extremwetterereignissen kommen wird, die politisch genutzt werden können, um die Klimaschutz- und -anpassungspolitik zu fördern, Gesetzesinitiativen anzustoßen und umzusetzen. Der Hitzesommer 2018, welcher als der wärmste seit Beginn der regelmäßigen Wetteraufzeichnungen gilt und vom Großteil der deutschen Bevölkerung sehr unmittelbar wahrgenommen wurde (Mühr et al. 2018), stellt etwa eine gute situative Ausgangslage für Gesetzesänderungen dar. Auch ergeben sich mit einer gewissen Regelmäßigkeit medienwirksame Skandale in der Landwirtschaft, die als Anlass für politische Initiativen genutzt werden können. Auf solche und ähnliche Gelegenheiten kann sich eine transformative Umweltpolitik bereits einstellen und entsprechende Reaktionen vorbereiten.

Jenseits dieser Prozesse sollte das Management von Gelegenheitsfenstern nicht nur in der Vorbereitung auf unabhängig von der Politik eintretenden Ereignissen bestehen. Eine transformative Umweltpolitik muss dynamisch vorgehen und Teil einer solchen Umweltpolitik ist die aktive Schaffung neuer Gelegenheitsfenster.

Dies ist zunächst in einfachem Maße möglich, indem wiederkehrende Gelegenheitsfenster in den Politikprozess integriert werden. Denn solche Fenster haben nicht nur als Auslöser einer Transformation eine entscheidende Bedeutung, sondern werden im weiteren Verlauf des Transformationsprozesses gebraucht, um dessen Fortschritt zu gewährleisten und den Prozess in die gewünschte Richtung zu lenken. Diese Art von wiederauftretenden Gelegenheitsfenstern lässt sich unter anderem durch fest verankerte regelmäßige Review- und Monitoringprozesse umsetzen. So kann in neuen rechtlichen Vorgaben von Beginn an ein strukturierter Review-Prozess festgelegt werden, durch den sich wiederauftretende Gelegenheitsfenster ergeben. Teils werden festgelegte Daten für Gesetzesnovellierungen bereits heute umgesetzt. Ein klares Monitoring, das deutlich über die bisherige Praxis hinausgeht, spielt für die dynamische Steuerung von Transformationen ebenfalls eine wichtige Rolle.

Transformative Umweltpolitik kann aber noch einen Schritt weitergehen und selbst Auslöser von Gelegenheitsfenstern sein. So sind zum Beispiel die umweltbezogenen Probleme im Verkehrs-, Agrar- und Chemiesektor vielfältig und gravie- 
rende Problemlagen einschließlich deutlicher Gesetzverstöße hier zahlreich. Eine in die Gesellschaft wirkende Umweltpolitik muss sich nicht darauf beschränken, dass andere Akteure eigeninitiativ diesen Problemen nachgehen und sie in die gesellschaftliche Diskussion bringen. Sie kann durchaus auch selber im Verbund mit solchen Akteuren, diese Probleme offen legen, breit thematisieren und damit den Ansatzpunkt schaffen, für Änderungen im bestehenden System. So könnte eine transformierende Umweltpolitik etwa gezielt Mittel zur Verfügung stellen, um systematische Gesetzesverstöße im Agrarbereich aufzudecken, die von den bestehenden Aufsichtsbehörden nicht beachtet werden und diese dann auch unmittelbar in eine politische Diskussion zu überführen.

Ein letzter Aspekt, der im Zusammenhang mit Gelegenheitsfenstern und einer transformativen Umweltpolitik von Bedeutung ist, bezieht sich auf zunächst themenfremde gesellschaftliche Diskurse. Ein Gelegenheitsfenster muss nicht darin bestehen, dass systemrelevante Änderungen abrupt auftreten. Es kann auch dadurch entstehen, dass gesellschaftliche und politische Diskurse aufgegriffen und im Sinne einer sozialökologischen Transformation weitergedacht werden. Hierdurch können neue Dynamiken für eine umweltpolitische Transformation entstehen. Ein Beispiel hierfür bietet die Debatte um das Einhalten der fiskalpolitischen »schwarzen Null«. Basierend auf dem Anspruch, zukünftigen Generationen keine belastenden Schulden zu hinterlassen, wird hier für eine verantwortungsvolle Haushaltspolitik argumentiert. Dies wird von weiten Teilen der Politik und Bevölkerung als richtig akzeptiert. Eine parallele Argumentationslinie für Generationengerechtigkeit ist das Hinterlassen eines intakten Planeten ohne "materielle Schulden« für nachfolgende Generationen. Eine Verlinkung dieser Debatten hat das Potential, Umweltproblematiken sowie der Frage der Pfadwechselkosten einen neuen Stellenwert zu geben und dringenden Handlungsbedarf $\mathrm{zu}$ verdeutlichen. Umweltpolitische Akteure sollten daher versuchen, wichtige Ziele angrenzender Politikbereiche oder sogar einzelner politischer Akteure, die sich mit bestimmten Thematiken profilieren möchten, zu verstehen, mit ihren Anliegen zu verknüpfen und daraus neue Gelegenheitsfenster erwachsen zu lassen.

\section{Co-Benefits}

Wie im Rahmen der Projektfallstudien vielfach herausgearbeitet, stellen über Nachhaltigkeits- und Umweltaspekte hinausgehende Co-Benefits eine entscheidende Bedingung für die Akzeptanz und das Gelingen von Transformationen dar. Wirtschaftlichen, gesundheitlichen und sozialen Nutzenaspekten wird bei realen Entscheidungsprozessen von den meisten Menschen tendenziell eine höhere Bedeutung zugemessen als ökologischen Fragen (Capstick und Whitmarsh 2018). Transformationsbestrebungen, die diese Gesichtspunkte nicht bedienen oder ihre Bedeutung dafür nicht klar genug vermitteln, laufen Gefahr in Nischen zu 
verharren. Transformationsprozesse müssen sich an solchen gesellschaftlichen und individuellen Bedürfnissen orientieren. In den untersuchten Fällen auf kommunaler Ebene war beispielsweise die Belebung der lokalen Wirtschaft und die langfristige Sicherung von Lebensqualität oftmals das Hauptziel der angestrebten Transformation. Bei nationalen Transformationsprozessen spielten neben konkreten Umweltproblemen wirtschaftliche Überlegungen sowie geopolitische Ziele eine Rolle.

Transformationspolitik kann bestehende technologische und gesellschaftliche Entwicklungen nutzen, indem sie diese in politischen Maßnahmen mit weiteren Zielen, die zu einer Green Economy führen, verbindet. Beispiele sind Trends hin zu gesunder Ernährung und einem sportlichen Lebensstil. Diese können mit Maßnahmen im Tierschutz, der ökologischen Landwirtschaft und dem Ausbau der Fahrradinfrastruktur aufgegriffen werden (Wolff et al. 2018). Ein solches Aufgreifen gesellschaftlicher Trends wurde bereits im Absatz "Gelegenheitsfenster « beschrieben und spielt ebenfalls für die Kommunikation von Co-Benefits eine entscheidende Rolle. Die mit den nachhaltigen Transformationen zusammenhängenden Zusatznutzen müssen der Zivilgesellschaft und der Wirtschaft explizit und transparent mitgeteilt werden (Jänicke et al. 2015).

Bei der Schaffung und Kommunikation der Nutzen von Transformationsprozessen gilt es gestalterisch tätig zu werden. Politische Initiativen können auch ergriffen werden, um selbst Trends zu setzen, die gewünschten Zielen zuträglich sind. Co-Benefits können von staatlicher Seite zwar oftmals nicht direkt kreiiert werden. Aber der Staat hat einigen Einfluss auf die gesellschaftliche Wahrnehmung, was einen Zusatznutzen darstellt. Diese Wahrnehmung ist der politischen Gestaltbarkeit durchaus zugänglich. Was als Co-Benefit gilt, ist nicht zuletzt eine Frage der individuellen Perzeption und an dieser Stelle verfügt der Staat über zahlreiche Möglichkeiten auf eine bestimmte Wahrnehmung hinzuwirken. Dabei spielt die Erkenntnis eine wichtige Rolle, dass Werte und Bedürfnisse, an die Transformationen Anschluss suchen und die die Wahrnehmung von Co-Benefits maßgeblich mitbestimmen, nicht statisch im derzeitigen Zustand verbleiben. Stattdessen sind diese stark durch Einstellungen und Verhalten anderer sozialer Akteure geprägt, was wiederum Bedeutung für eine erfolgreiche Kommunikation von Co-Benefits hat (Grimm et al. 2014).

Ein praktisches Beispiel ist die Kommunikation von Nichtraucherschutz als wesentlicher Vorteil einer Transformation zu einer raucherfreien Gesellschaft. Anstatt nur auf die Gesundheitsschäden für Raucher selbst hinzuweisen, wurde der Aspekt der Schädigung Unbeteiligter neu eingeführt und durch Informationskampagnen in den Vordergrund der Debatte gerückt. Damit bekam der Übergang zu einer rauchfreien Gesellschaft einen essentiellen neuen Nutzen und wurde von weiten Teilen der Bevölkerung mitgetragen (vgl. Fallstudie zum Nichtraucherschutz in Kapitel 4.2). 
Ein ähnlicher Prozess lässt sich hinsichtlich der Regulierung und der staatlichen Kommunikation zur PKW-Nutzung in Singapur feststellen. Hier existieren bereits seit längerem rigide Auflagen für die Anschaffung und Nutzung privater PKWs. Zusätzlich verfolgt die Regierung nun das Ziel, Autofahren mit einem schlechten Ruf zu verknüpfen und die schädigenden Aspekte des Autofrahrens für weite Teile der Gesellschaft aufzuzeigen. Es ist diese Art der Mitgestaltung von gesellschaftlichen Wertvorstellungen, die eine transformative Umweltpolitik benötigt.

\section{Soziale, technische und regulatorische Innovationen}

Die verschiedenen Fallstudien des Projektes haben die zentrale Bedeutung von Innovationen für Transformationsprozesse bestätigt. Das betrifft technische, soziale und regulative Innovationen. Die Förderung umweltfreundlicher Technologien ist seit langem ein wichtiger Aspekt der Umweltpolitik, sowohl was die eigentliche Erfindung anbelangt (Invention) als auch was das upscaling betrifft, die eigentliche Innovationsphase (Kahlenborn et al. 2013). Vergleichsweise wenig betrachtet wird im Kontext der technischen Innovationen die Frage, wie diese eigentlich starten. Voraussetzung für die meisten Innovationen ist, dass einzelne Personen oder Gruppen von Personen mit der bisherigen Situation nicht zufrieden sind. Es bedarf also eines grundsätzlichen Problembewusstseins. Eine wichtige Voraussetzung für die Entwicklung technischer Lösungen im Bereich von Windkraft und Solar war etwa ein deutliches Unbehagen an der Entwicklung der Atomkraft. Dabei handelt es sich anfangs um reine Nischendiskussionen.

Transformationsprozesse werden oftmals in Nischen initiiert. Nischen stellen wichtige Orte da, in denen mit neuen Technologien oder sozialen Praktiken experimentiert wird und wo Pfadabhängigkeiten aufgebrochen werden können. In günstigen Gelegenheitsfenstern kann dann aus der Nische heraus skaliert werden. In den untersuchten Beispielen entstanden breitenwirksame Transformationen wie nationale Regierungsinitiativen oftmals in zivilgesellschaftlichen Nischen. Dort wurden sie für einige Zeit entwickelt, bevor sie über legislative Prozesse ins Zentrum dominanter Regime vordrangen und diese veränderten. Dabei spielen oftmals Akteurinnen und Akteure eine Rolle, die an den Rändern oder außerhalb dominanter Regime stehen. Deshalb muss mehr Aufmerksamkeit auf die Ränder bestehender Systeme gelegt werden, um Transformationen sowie Paradigmenwechsel zu ermöglichen. Insbesondere ist es eine wichtige Aufgabe einer langfristig angelegten, transformativen Umweltpolitik, solche Nischenprozesse zu fördern. Dabei gilt es zunächst auch, einfach ein Problembewusstsein zu schaffen und den Austausch kleiner gesellschaftlicher Gruppen zu Problemfragen zu ermöglichen, auch wenn technische Lösungen noch nicht in Sicht sind. Dies gilt insbesondere für jene Transformationsprozesse, die noch ganz am Anfang stehen. So gibt es etwa nur wenige gesellschaftliche Foren, die sich mit den 
verschiedenen Risiken der Chemieindustrie auseinandersetzen und zu wichtigen Fragen, wie etwa der Freisetzung von endokrinen Substanzen, findet kaum eine Problemdiskussion statt. Diese wird aber wichtig sein für die Entwicklung von Lösungen.

Neben technischen Innovationen spielen für eine sozial-ökologische Transformation auch soziale Innovationen eine große Rolle. Gelungene Transformationen auf individueller, organisationaler wie gesamtgesellschaftlicher Ebene hängen maßgeblich von konkreten Handlungen ab. Innovationsförderung sollte sich deshalb nicht nur auf technologische Innovationen, sondern verstärkt auch auf soziale Innovationen konzentrieren. Soziale Innovationen sind die beabsichtigte Neukonfiguration sozialer Praktiken des Wirtschaftens und Zusammenlebens. Dabei muss Innovation nicht immer etwas radikal Neues bedeuten, sondern kann sich auch im Rückgriff auf bereits lange vorhandene Praktiken zeigen (Howaldt und Schwarz 2018).

Soziale Innovationen müssen in stärkerem Maße als bisher gefördert werden. Sie können zwar nicht politisch verordnet, aber durch politische Maßnahmen gefördert werden. "Experimente« mit von dominanten Regimen abweichenden Lebens-, Arbeits- und Wirtschaftsformen sind notwendig, um deren Auswirkungen und notwendige Rahmenbedingungen zu testen. Daneben müssen auch Netzwerkaktivitäten zwischen Akteurinnen und Akteuren und Sektoren gefördert werden, um soziale Innovationen zu skalieren.

Neben technologischen und sozialen Innovationen sind zudem regulatorische Innovationen vonnöten, die gezielt mit regulatorischen Rahmenbedingungen experimentieren und bei Erfolg skaliert werden. Hier bietet sich besonders viel Raum für politische Maßnahmen.

Das Beispiel CARB zeigt, dass auch Innovationen in einem regulatorischen Umfeld förderlich für Transformationen sein können. Zudem ist für eine Etablierung von Transformationen oft eine Institutionalisierung nötig, beispielsweise über geänderte Regulierung. In räumlich und zeitlich begrenzten regulatorischen Innovationszonen kann mit regulatorischen Vorgaben experimentiert und deren Auswirkungen auf technologische wie soziale Innovationen getestet werden. Dabei muss die konkrete regulatorische Fragestellung ebenso wie der Evaluationsprozess klar definiert sein (Bauknecht et al. 2015b). Aus den hier gewonnenen Erkenntnissen lassen sich wiederum Rückschlüsse auf geeignete allgemeingültige Rahmenbedingungen ziehen.

Die Voraussetzungen für regulatorische Innovationen sind in Deutschland im Allgemeinen recht gut. Jedes föderale System bietet die Möglichkeit eines regulativen Wettbewerbs und damit auch der regulativen Innovation auf begrenzter räumlicher Ebene. Leider wird dieser Vorteil bislang kaum genutzt, um eine sozial-ökologische Transformation voranzutragen. 
Angesichts sehr dynamischer Transformationsprozesse, die sich parallel zur sozial-ökologischen Transformation vollziehen - insbesondere die verschiedenen Digitalisierungsprozesse - gehört zur Frage der regulatorischen Innovation auch, den Blick darauf zu richten, inwieweit der Staat weiterhin letztendlich Haftungsgarant in allen Fragen sein kann. Aktuell geht das Rechtssystem im Wesentlichen davon aus, dass bei Einhaltung aller Vorschriften der Staat die (finanzielle) Verantwortung dafür trägt, wenn wirtschaftliche/technische Entwicklungen zu nicht tragbaren Umweltrisiken führen (vgl. etwa die Atomkraft oder aktuell auch den Rückbau der Kohlekraft oder Ausgleichszahlungen für die Landwirtschaft). Für die bevorstehenden Transformationsprozesse wird es wichtig sein, zu klären, ob hier nicht ein Perspektivwechsel dergestalt stattfinden muss, dass die jeweils einschlägigen Unternehmen bzw. der entsprechende Wirtschaftssektor ebenfalls deutlich stärker die Verantwortung tragen muss für die gesellschaftlichen Kosten, die durch ihre Aktivitäten entstehen - selbst dann, wenn sie (ursprünglich) vom Staat genehmigt wurden. So steht etwa die Frage im Raum, inwiefern sich Großunternehmen, die auch aktuell mit der Förderung von Öl und Gas sehr viel Geld verdienen, auf Dauer an den entstehenden Folgekosten beteiligen müssen. Gleiches kann beispielsweise auch eine Frage für die Chemieindustrie werden.

Diese Fragestellung ist besonders virulent im Kontext der Digitalisierung, wo der Staat mit seinem Regelungsprozess angesichts der Dynamik nicht mehr mitkommt und ständig neue Risiken entstehen bzw. unmittelbar gesellschaftliche Kosten anfallen, die Nebenprodukt einer sehr gewinnträchtigen technologischen Entwicklung sind, von den entsprechenden Konzernen aber nicht getragen werden. Hier gilt es auch, jenseits der rein finanziellen Verantwortung verstärkt die Frage zu thematisieren, inwieweit die Unternehmen dafür Sorge tragen müssen, dass die Gesellschaft insgesamt nicht unter dem Digitalisierungsprozess leidet (Kahlenborn et al. 2018).

\section{Investitionen in Transformation}

Es ist evident, dass der Wandel hin zu einer grünen Wirtschaft nicht ohne zusätzliche finanzielle Ressourcen auskommt. Ein Bericht des Entwicklungsprogramms der Vereinten Nationen schätzte 2011, dass »der Übergang zu einem kohlenstoffarmen, ressourceneffizienten Weg in zehn zentralen Wirtschaftssektoren jährlich $2 \%$ des globalen Bruttoinlandsprodukts erfordern würde«(UNEP 2011). Auch eine Studie aus dem Privatwirtschaftssektor kam zu einem ähnlichen Schluss: Die Gesamtkosten einer Transformation könnten bis 2030 jährlich 200-350 Milliarden Euro betragen (McKinsey \& Company 2009).

Auch wenn dem Staat hier eine wichtige Rolle zufällt, kann er nicht Träger all dieser Investitionen sein. Um zusätzlich privates Kapital zu kanalisieren, können politische Akteurinnen und Akteure verstärkt Alternativen zu Top-Down-Finanzierungsmodellen fördern. Beispiele sind Public-Private-Partnerships (z. B. In- 
novationCity Bottrop, Hyllie in Malmö), Crowdfunding und Genossenschaften (Jacob et al. 2015a). Ein guter Teil der benötigten Investitionen wird jedoch direkt und ausschließlich durch die Privatwirtschaft getragen werden müssen.

Eine Transformation hin zu einer Green Economy schafft allerdings grundsätzlich Unsicherheit; dies schreckt mögliche Investoren ab. Mit Blick auf die Exnovation, die Dekonstruktion des alten Systems, handelt es sich um einen durchaus gewünschten Effekt. Im Bereich der Energiewende beispielsweise sind die intensiven Diskussionen über die Beendigung von Kohleverstromung sehr hilfreich, damit sich Finanzmarktakteure weltweit aus der Finanzierung neuer Kohlekraftwerke zurückziehen.

Problematisch ist aber, dass die Transformation erheblichen neuen Investitionsbedarf auslöst. Damit sich Investoren auf die Bereitstellung dieser Mittel einlassen, brauchen sie eine gewisse Planungssicherheit. Ab dem Zeitpunkt der Hochskalierung einer Transformation besteht Bedarf nach "Verlässlichkeit im Wandel«. Transformative Umweltpolitik muss zum richtigen Zeitpunkt den Ansatzpunkt ändern. Während es zu Beginn darauf ankommt, alternative Lösungen zu suchen und auszuprobieren, ist ab einem gewissen Zeitpunkt eine Festlegung erforderlich. So erforderte die Energiewende die Festlegung auf bestimmte Formen von Energieträgern (Windkraft und solare Energieerzeugung) als zukünftige Schwergewichte; auch die Verkehrswende kann nur gelingen, wenn die Festlegung auf ein neues Antriebssystem erfolgt (Elektro- versus Wasserstoffantrieb) ${ }^{1}$.

Grundlegend für die Planungssicherheit sind zum einen entsprechende strategische Entscheidungen und Strategiefestlegungen. Bei der Formulierung von Zielen und Maßnahmen, gerade in nicht unmittelbar umweltpolitischen Bereichen, muss auf eine Kompatibilität mit Transformationsstrategien geachtet werden. Es braucht eine Analyse der fördernden und hemmenden politischen Maßnahmen, die Transformationsprozesse beeinflussen. Ein erfolgreiches Beispiel für eine langfristig und ganzheitlich durchdachte Transformationsstrategie ist die Initiative InnovationCity Bottrop (Kapitel 4.2). Mit Blick auf den durch den Kohleausstieg induzierten Strukturwandel zielte das Projekt des industriell geprägten Standorts auf einen klimafreundlichen Umbau und die gleichzeitige Sicherung des Industriestandorts ab.

1 Der Ruf nach zusätzlichen Innovationen zur Lösung von Umweltproblemen erweist sich so oft als Verzögerungstaktik. Im Rahmen von Evolution2Green wurde dies z. B. in der Frage der PKW-Antriebstechnologie deutlich. Trotz der Markt- und Produktionsreife des Elektroantriebs wird regelmäßig nach Forschungsmitteln für die mit Blick auf die Effizienz deutlich unterlegene Brennstoffzelle gerufen und betont, dass man sich alle Wege offen halten müsse. Diese von Geels 2014a kritisch als »prognostisches framing « bezeichnete Strategie erreicht, dass die Umsetzung vorhandener Lösungen mit Blick auf zukünftige - teils völlig unrealistische - Optionen über Jahrzehnte verzögert wird Geels 2014b. 
Zum anderen und eng verbunden mit der Strategieentwicklung geht es um die Gestaltung von neuen Pfadabhängigkeiten, etwa durch die Schaffung neuer Netzwerke, rechtlicher Festlegungen etc. Systemdenken muss in der Politikgestaltung stärker durchgesetzt werden, um das Entstehen von Pfadabhängigkeiten möglichst bewusst zu gestalten. Pfadabhängigkeiten können somit eine positive Wirkung haben, wenn sie in der entsprechenden Weise genutzt werden. Die vorangegangene Analyse zeigt, dass das Vorhandensein von günstigen Pfadabhängigkeiten in fast allen analysierten Fällen erfolgsentscheidend ist. Bestehenden Rahmenbedingungen und Pfadabhängigkeiten - und dem Einfluss politischer Akteurinnen und Akteure auf diese Variablen - sollte daher mehr Aufmerksamkeit gewidmet werden. Ein in weiten Teilen gutes Beispiel für die Schaffung neuer Pfadabhängigkeiten war die Einführung des EEG. Zwar wurde versäumt, frühzeitig die Transformationsdynamik vorherzusehen, sodass unnötig hohe Mittel für den Aufbau regenerativer Energiequellen bereitgestellt worden sind, aber die Schaffung konsistenter planbarer Rahmenbedingungen zum rechten Zeitpunkt der Hochskalierung war ein zentraler Faktor für den bisherigen Erfolg der Energiewende.

Zur »Verlässlichkeit im Wandel« gehört auch Transparenz über die Kosten der Transformation. Ehrlichkeit mit Blick auf die finanziellen und gesellschaftlichen Kosten der Transformation ist essenziell, um den nötigen Rückhalt in der Gesellschaft zu haben, diesen bisweilen auch schmerzhaften Prozess zu durchlaufen.

\section{Transformationsforschung und -bildung}

Transformationen werden nicht nur politisch initiiert, sondern von Menschen in allen gesellschaftlichen Sektoren angestoßen. In den untersuchten Beispielen weisen Länder und Regionen mit erfolgreichen Transformationsprozessen hohes Humankapital auf, insbesondere in Form von nachhaltigkeits- und transformationsspezifischem Wissen. Beispielhaft können hier die Niederlande und Kalifornien genannt werden. Die Vermittlung von transformationsrelevantem Wissen sollte daher ein Aspekt einer transformativen Umweltpolitik sein und als zentraler Bestandteil der gesamten formalen und informellen Aus- und Weiterbildung etabliert werden. Dies bezieht sich nicht nur auf Schule, Ausbildung und Studium, sondern auch auf Fort- und Weiterbildungen im Arbeitsleben.

Die wichtige Rolle von Transformationsforschung und -bildung wird auch vom Wissenschaftlichen Beirat der Bundesregierung zu Globalen Umweltveränderungen (WBGU) hervorgehoben. Das Hauptgutachten des WBGU aus dem Jahr 2011 »Welt im Wandel - Gesellschaftsvertrag für eine Große Transformation« spricht hierbei explizit von einer Stärkung des transformativen Quartetts der Wissensgesellschaft, welches Transformationsforschung, Transformationsbildung, transformative Forschung und transformative Bildung miteinander verbindet (Wissenschaftlicher Beirat der Bundesregierung Globale Umweltveränderungen 2011). 
Die Transformationsforschung selbst ist ein noch relativ junges wissenschaftliches Feld. Zwei Formen wissenschaftlicher Erkenntnisgewinnung können unterschieden werden, die beide in der Transformationsforschung zentral sind. Konzeptionelles Wissen (1) befähigt dazu, Systeme und Prozesse interdisziplinär beschreiben, erklären und verstehen zu können. Handlungswissen (2) hingegen erlaubt es Akteurinnen und Akteuren, Transformationsoptionen miteinander $\mathrm{zu}$ vergleichen sowie letztlich Entscheidungen $\mathrm{zu}$ fällen und $\mathrm{zu}$ handeln. Die Transformationsforschung zählt damit zu den Forschungsgebieten, die ihren Forschungsgegenstand aktiv mitgestalten. Das bedeutet, dass sich Transformationsbildung nicht nur auf die Vermittlung von bloßem konzeptionellem Wissen konzentrieren sollte. Oftmals ist bereits umfangreiches Wissen über ökologische Notwendigkeiten von Transformationen vorhanden, das sich allerdings nicht in tatsächliches Handeln übersetzt. Zu den erweiterten Kompetenzen gehört daher auch die Fähigkeit, das eigene Wissen in Handeln umzusetzen (Jacob et al. 2015b; Hölscher et al. 2017). Zentral ist in diesem Zusammenhang auch eine neue Lernkultur, die ein Scheitern von Transformationen nicht normativ bewertet, sondern als Lernmöglichkeit für andere Transformationsprozesse.

Während Transformationsforschung und -bildung eine wichtige Voraussetzung dafür darstellen, den notwendigen gesellschaftlichen Rückhalt für eine Transformationspolitik aufzubauen und die Fähigkeiten relevanter gesellschaftlicher und wirtschaftlicher Akteurinnen und Akteure zu sichern, Transformationsprozesse erfolgreich zu durchlaufen, ist Transformationsforschung für eine transformative Umweltpolitik auch noch unter einem anderen Gesichtspunkt bedeutsam: Eine umfassende Transformationsforschung ermöglicht das notwenige Systemverständnis, das entscheidend ist als Grundlage für die Entwicklung von Transformationsstrategien. Die Entwicklung und Ausgestaltung von Transformationsstrategien, die insbesondere in der Phase des Hochskalierens von Transformationen von großer Bedeutung sind (vgl. auch den Abschnitt zu Investieren in Transformation), bedarf eines genauen Verständnisses der bestehenden Pfadabhängigkeiten, der Interessenlagen der wichtigen Akteurinnen und Akteure im Transformationsprozess und der Wirkzusammenhänge zwischen verschiedenen parallel ablaufenden Transformationsprozessen. Hier ist eine intensive Transformationsforschung unentbehrlich.

\section{Strategic foresight}

Eine wichtige Voraussetzung für eine erfolgreiche transformative Umweltpolitik ist der Ausbau von strategic foresight. Strategic foresight kann wichtige Hilfestellungen bei der Identifizierung von Gelegenheitsfenstern leisten wie auch bei der Verknüpfung mit parallelen gesellschaftlichen Diskursprozessen. Es unterstützt das allgemeine Transformationsverständnis. Strategic foresight ist auch deshalb von besonderer Bedeutung, weil die sozial-ökologische Transformation parallel 
$\mathrm{zu}$ weiteren gesellschaftlichen und wirtschaftlichen Translationsprozessen abläuft.

Strategic foresight bezeichnet das Untersuchen alternativer Zukunftsvisionen sowie das Abschätzen der Konsequenzen von relevanten Entscheidungen und Handlungen, die zu diesen alternativen Zukunftsvisionen führen können (Cook et al. 2014). Es unterstützt die Politikgestaltung, indem es einerseits den themenspezifischen Wissensstand systematisch erarbeitet und zur Verfügung stellt und andererseits als Treiber für reflexive soziale Lernprozesse für politische Entscheidungsträgerinnen und -träger dient (Habegger 2010). Die wichtigsten Instrumente des strategic foresights sind horizon scanning und Szenarioanalysen (auch Szenarioplanung). Horizon scanning bezeichnet das Sammeln und Organisieren von sehr breitgefassten Informationen mit dem Ziel, neu auftretende Probleme $\mathrm{zu}$ erkennen und $\mathrm{zu}$ identifizieren sowie das Antizipieren von zukünftigen Entwicklungen (Behrendt et al. 2015b). Szenarioanalysen hingegen umfassen unterschiedliche Ansätze, um eine alternative Zukunftsvision zu simulieren (Cook et al. 2014) und sind ein geeignetes Werkzeug, um angesichts zunehmender Komplexität und Unsicherheit heutige Entscheidungen zukunftsgerichtet zu reflektieren und auszurichten.

Die mit diesen Methodiken identifizierten Entwicklungen können dann mit nachhaltigkeitsspezifischen Zielsetzungen verbunden werden. In den untersuchten Transformationsbeispielen auf kommunaler Ebene zeigt sich, dass bei erfolgreichen Transformationen oftmals mehrere Zielsetzungen gleichzeitig miteinander verknüpft werden. Ein wirkungsvoller Mechanismus kann durch ein Einklinken transformativer Umweltpolitik an gesellschaftliche Entwicklungen in anderen Bereichen erzeugt werden.

Strategic foresight spielt für alle entscheidenden »Wende«-Themen eine wichtige Rolle. Im Rahmen der Energiewende sind zwar viele Entwicklungen inzwischen sehr klar absehbar. Dies ändert aber nichts daran, dass gerade auch bei einem weiteren Blick in die Zukunft diverse, auch bedeutende Fragen nach wie vor ungeklärt und zum Teil auch ungestellt bleiben. So wird für die weitere Entwicklung in vielen, insbesondere auch aus Umweltsicht bedeutsamen Bereichen die Frage der weiteren Entwicklung der Kosten von erneuerbaren Energien eine sehr hohe Bedeutung haben. Bislang gehen die allermeisten Analysen davon aus, dass die Preise von erneuerbaren Energien zwar weiter sinken, aber in etwa auf dem Niveau der derzeitig günstigsten Energieträger Halt machen werden. Die doch sehr essenzielle Frage, inwieweit auch jenseits dessen noch deutliche Optimierungspotenziale bestehen, inwieweit neue Technologien zur Gewinnung erneuerbarer Energien noch weitere massive Kostenreduktionen mit sich bringen werden und welche Auswirkungen dies dann wiederum auf einzelne Wirtschaftssektoren und eine Entwicklung hin zu mehr Nachhaltigkeit hat, würde eigentlich weitere umfassende Untersuchungen erfordern. 
Noch offenkundiger ist die Bedeutung von strategic foresight für die Verkehrswende. Viele systemrelevante Entwicklungen treffen hier in den nächsten Jahren und Jahrzehnten aufeinander: Elektromobilität, autonomes Fahren, neue Möglichkeiten der Mikromobilität, Sharing und Digitalisierung der Verkehrsträger, veränderte Mobilitätsanforderungen durch inklusive Stadtkonzepte etc. Strategic foresight ist hier essenziell, um diese Entwicklungen besser fassen und ihr Zusammenspiel verstehen zu können. Dies wiederum ist die Grundlage dafür, eine sozial-ökologische Verkehrswende zu gestalten.

Nicht weniger wichtig ist strategic foresight im Bereich der Agrarwende und der Chemiewende. In diesen beiden Bereichen deutet sich die erforderliche Wende bisher nur schwach an. Mit Blick auf die vorherrschenden Leitbilder und Strategien scheint hier die Zeit teils noch stehen geblieben zu sein. Auch hier wird es absehbar erhebliche Änderungen geben, die die aktuellen Systeme in den Grundfesten erschüttern werden. Künstliches Fleisch etwa kann einem guten Teil der aktuellen Landwirtschaft in Deutschland die Grundlage entziehen. Urban farming hat ebenso das Potenzial, wichtige Bereiche der Landwirtschaft auf eine ganz andere Grundlage zu stellen. Die Reaktion auf solche Prozesse besteht derzeit eher in einer Abwehrhaltung, anstatt die Trends besser zu verstehen und im Sinne einer sozial-ökologische Transformation aufzugreifen und mitzugestalten.

In solchen Fällen hat strategic foresight auch die Aufgabe, Problembewusstsein erst zu schaffen und aufzuzeigen, dass der Status quo oder leichte Weiterentwicklungen der aktuellen Systeme diese von selber zum Einsturz bringen.

Strategic foresight ist darüber hinaus auch wichtig, um parallel laufende Transformationen $\mathrm{zu}$ erfassen und mit sozial-ökologischen Transformationen $\mathrm{zu}$ verknüpfen. Insbesondere die Digitalisierung stellt eine solche Transformation dar, die in vielfacher Hinsicht sowohl Chancen als auch Risiken für eine sozial-ökologische Transformation beinhaltet. Um einige Beispiele $\mathrm{zu}$ nennen: Digitale Medien machen das analoge Produzieren von Büchern überflüssig, und ein vernetzter Verkehr bewegt sich effizienter als viele nicht miteinander verbundene individuelle Verkehrsteilnehmer. Industrielle Herstellungsprozesse können ressourceneffizienter geplant werden und Second-Hand-Portale, wie im untersuchten Beispiel eBay, ermöglichen die Verlängerung von Produktlebensdauern (Lange und Santarius 2018). Zudem zeigen die untersuchten Beispiele, dass die Nutzung neuer technischer Möglichkeiten ein hohes disruptives Potenzial bietet, das beim Aufbrechen bestehender Pfadabhängigkeiten helfen kann. Neben der Digitalisierung gibt es noch diverse weitere soziale und technische Entwicklungen (wie etwa Urbanisierung und Globalisierung), die im Blick behalten werden müssen, weil sie entscheidende Auswirkungen auf eine sozial-ökologische Transformation haben.

Die Voraussetzungen für eine verstärkte Berücksichtigung von strategic foresight sind durchaus schon gegeben. In vielen Fachpolitiken wird strategic foresight 
zunehmend angewandt. Auf Bundesebene existiert hierzu schon ein reger Austausch in Fachnetzwerken. Sichtbare Ergebnisse von strategic foresight sind etwa Studien zu Konsum 4.0 oder zu Potenzialen und Chancen des 3-D-Drucks. Wichtig ist die Bereitschaft, diese Methode auch im Sinne einer transformativen Umweltpolitik systematisch zu nutzen.

\section{Schlussbetrachtung}

Die sozial-ökologische Transformation ist gestaltbar. Für die nationale Umweltpolitik wie auch für die vielen anderen relevanten Akteurinnen und Akteure gibt es dazu diverse Möglichkeiten. Damit die deutsche Umweltpolitik in diesem Transformationsprozess aber die notwendige Führungsrolle übernimmt, muss sie neu konzipiert werden. Transformative Umweltpolitik muss als Gesellschaftspolitik verstanden und implementiert werden. Eine transformative Umweltpolitik muss viel stärker als bisher in andere Politikfelder und in die Gesellschaft hineinwirken. Dabei reicht es nicht aus, anderen Akteurinnen und Akteuren zuzuhören und ihre Interessenlagen und Erwartungshaltungen $\mathrm{zu}$ berücksichtigen. Eine transformative Umweltpolitik muss darauf abzielen, auf Augenhöhe mit anderen Akteurinnen und Akteuren in Wirtschaft und Gesellschaft einen Dialog zu führen und Ziele dann gemeinsam umzusetzen. Dazu wiederum ist es wichtig, soziale, technische und ökonomische Trends frühzeitig zu erkennen, sie aufzugreifen und in Richtung einer nachhaltigen Entwicklung weiterzuführen. Die geeigneten Akteurinnen und Akteure müssen identifiziert oder gar erst aufgebaut werden; ebenso müssen strategische Gelegenheitsfenster rechtzeitig erkannt oder auch gezielt geschaffen werden. Eine transformative Umweltpolitik muss auch in gesellschaftliche Wertediskussionen eingreifen und verschiedenste gesellschaftliche Diskurse mit Blick auf ihre Zielsetzungen beeinflussen. Für die notwendige Gestaltung einer sozial-ökologischen Transformation bedarf es eines solchen neuen Ansatzes. Klassische Umweltpolitik, die sich darauf beschränkt, nur regelnd an jenen Stellen einzugreifen, wo gerade Umweltprobleme besonders sichtbar werden, reicht nicht mehr aus. In Zukunft werden ihre Instrumente zwar weiter von Bedeutung sein, ihre Begründungsbasis und ihre konzeptionelle Grundlage ist aber überholt.

Für die sehr weit ausgreifende Rolle von transformativer Umweltpolitik ist die gesellschaftliche Legitimation entscheidend. Eine transformative Umweltpolitik wird sich noch mehr rechtfertigen müssen für ihre Aktivitäten, als dies in der Vergangenheit für die Umweltpolitik insgesamt schon der Fall war. Entscheidend wird sein, Gerechtigkeit in den Transformationsprozessen explizit zu machen, neu auszuhandeln und dann herzustellen. Diese Gerechtigkeitsfrage wird in den nächsten Jahren vermutlich eine zentrale Frage der sozial-ökologischen Transformation werden. 
Ein wichtiger Aspekt ist dabei unzweifelhaft die soziale Gerechtigkeit. Die Gefahr besteht permanent, dass jene Bevölkerungsschichten, die über die geringsten Ressourcen verfügen, unter den Transformationen besonders leiden. Dies ist aber, wie die fortschreitende Energiewende zeigt, zwar eine ganz wesentliche, aber keineswegs die einzige Dimension von Gerechtigkeit im sozial-ökologischen Transformationsprozess. Eine zweite, ebenso wichtige, jedoch schwieriger zu lösende Dimension ist die der Verlierer/-innen und Gewinner/-innen im Transformationsprozess. Gerade hier wird die Behandlung von Gerechtigkeitsaspekten entscheidend sein. Eine sozial-ökologische Transformation wird nicht gelingen, wenn alle Verlierer/-innen des Transformationsprozesses sich als Benachteiligte wahrnehmen, dies gesellschaftlich auch akzeptiert wird und die Kosten der Transformationen dann durch Kompensationszahlungen erheblich erhöht werden. Eine gerechte sozial-ökologische Transformation setzt voraus, ein gesellschaftliches Verständnis darüber zu erzielen, dass viele der Benachteiligten der Transformation, sei es etwa im Energie-, sei es im Verkehrs-, sei es im Agrar- oder im Chemiesektor, die Privilegierten der Vergangenheit waren. Die Beschneidung ihrer Möglichkeiten ist nicht ein unbilliger Eingriff in ihre Rechte, sondern das Herstellen von Gerechtigkeit in einer Welt, in der wachsende Umweltrisiken von wenigen verursacht und Umweltschäden von vielen getragen werden. 



\section{Literatur}

3sat (2015): Im Dämm-Wahn. Gebäudesanierung verändert das Stadtbild (nano).

Online verfügbar unter www.3sat.de/page/?source=/nano/technik/157468/in dex.html, zuletzt geprüft am 25.04.2019.

ADAC Fahrzeugtechnik (2018): Was kosten die neuen Antriebsformen? Kostenvergleich E-Fahrzeuge + Plug-In Hybride gegen Benziner und Diesel. München.

Adler, Frank (2014): Postwachstumsvisionen. $\mathrm{Zu}$ einer neuen Tendenz in der wachstumskritischen Debatte. In: Berliner Debatte Initial 25 (2), S. 118-132.

Albrecht, Tanja; Zundel, Stefan (2010): Gefühlte Wirtschaftlichkeit - Wie Eigenheimbesitzer energetische Sanierungsmaßnahmen ökonomisch beurteilen. Hochschule Lausitz; Institut für ökologische Wirtschaftsforschung; Institut für sozial-ökologische Forschung. Senftenberg.

Aldrich, Howard; Zimmer, Catherine (1986): Entrepreneurship through social networks. In: Donald Lee Sexton und Raymond W. Smilor (Hg.): The art and science of entrepreneurship. Cambridge: Ballinger, S. 3-23.

Allen, Cameron; Clouth, Stuart (2012): A Guidebook to the Green Economy - Issue 1: Green Economy, Green Growth, and Low-Carbon Development - history, definitions and a guide to recent publications. United Nations Department of Economic and Social Affairs. New York.

Arbeitsgemeinschaft Energiebilanzen e. V. (2015): Auswertungstabellen zur Energiebilanz Deutschland. 1990 bis 2014. Berlin.

Arnold, Annika; David, Martin; Hanke, Gerolf; Sonnberger, Marco (Hg.) (2015): Innovation - Exnovation. Über Prozesse des Abschaffens und Erneuerns in der Nachhaltigkeitstransformation. Marburg: Metropolis (Ökologie und Wirtschaftsforschung, 99).

Arthur, W. Brian (1989): Competing Technologies, Increasing Returns, and Lock-In by Historical Events. In: The Economic Journal 99 (394), S. 116-131. DOI: 10.23 07/2234208.

Asara, Viviana; Otero, Iago; Demaria, Federico; Corbera, Esteve (2015): Socially sustainable degrowth as a social-ecological transformation: repoliticizing sustainability. In: Sustainability Science 10 (3), S. 375-384. DOI: 10.1007/s11625015-0321-9. 
Avelino, Flor; Wittmayer, Julia M. (2016): Shifting Power Relations in Sustainability Transitions: A Multi-actor Perspective. In: Journal of Environmental Policy \& Planning 18 (5), S. 628-649. DOI: 10.1080/1523908X.2015.1112259.

Ayres, Robert U.; Simonis, Udo E. (Hg.) (1994): Industrial metabolism. Restructuring for sustainable development. Tokyo: United Nations University Press.

Bauknecht, Dierk; Brohmann, Bettina; Grießhammer, Rainer; Bach, Matthew; Funke, Simon (2015a): Gesellschaftlicher Wandel als Mehrebenenansatz. Transformationsstrategien und Models of Change für nachhaltigen gesellschaftlichen Wandel. Bericht des AP2. Umweltbundesamt. Dessau-Roßlau (UBA Texte, 66/2015).

Bauknecht, Dierk; Heinemann, Christoph; Stronzik, Marcus; Schmitt, Stephan (2015b): Austesten von regulatorischen Innovationen - das Instrument der Regulatorischen Innovationszone. In: Energiewirtschaftliche Tagesfragen 65 (7), S. 61-64.

Baulinks (2016): Heizungsindustrie 2015: Brennwert gewinnt, Erneuerbare schwächeln. Online verfügbar unter www.baulinks.de/webplugin/2016/0259.php4, zuletzt geprüft am 25.04.2019.

Baumgartner, Frank R.; Berry, Jeffrey M.; Hojnacki, Marie; Leech, Beth L.; Kimball, David C. (2009): Lobbying and policy change: Who wins, who loses, and why. Chicago: University of Chicago Press.

Baumgartner, Frank R.; Jones, Bryan (1993): Agendas and instability in American politics. Chicago: University of Chicago Press.

Behrendt, Siegfried (2010): Integriertes Roadmapping. Nachhaltigkeitsorientierung in Innovationsprozessen des Pervasive Computing. Heidelberg, Berlin: Springer.

Behrendt, Siegfried (2012): Entlastend und belastend zugleich. Der ökologische Fußabdruck unserer digitalen Medienwelt. In: Lars Gräßer und Friedrich Hagedorn (Hg.): Medien nachhaltig nutzen. Beiträge zur Medienökologie und Medienbildung. Düsseldorf, München: kopaed, S. 19-30.

Behrendt, Siegfried (2017a): Feedstock change in der Chemieindustrie. Transformationsfeldanalyse im Rahmen des Projekts Evolution2Green - Transformationspfade zu einer Green Economy. Institut für Zukunftsstudien und Technologiebewertung. Berlin.

Behrendt, Siegfried (2017b): Mikroschadstoffe in Gewässern. Transformationsfeldanalyse im Rahmen des Projekts Evolution2Green - Transformationspfade zu einer Green Economy. Institut für Zukunftsstudien und Technologiebewertung. Berlin.

Behrendt, Siegfried (2017c): Recycling von Technologiemetallen. Transformationsfeldanalyse im Rahmen des Projekts Evolution2Green - Transformationspfade zu einer Green Economy. Institut für Zukunftsstudien und Technologiebewertung. Berlin. 
Behrendt, Siegfried (2018a): Car-Sharing. Fallstudie im Rahmen des Projekts Evolution2Green - Transformationspfade zu einer Green Economy. Institut für Zukunftsstudien und Technologiebewertung. Berlin.

Behrendt, Siegfried (2018b): Online-Gebrauchtwarenhandel eBay \& Co. Fallstudie im Rahmen des Projekts Evolution2Green - Transformationspfade zu einer Green Economy. Institut für Zukunftsstudien und Technologiebewertung. Berlin.

Behrendt, Siegfried; Göll, Edgar (2019): Pfadwechsel für eine Transformation zum nachhaltigen Wirtschaften. Perspektiven einer Rohstoffwende am Beispiel Produkte länger nutzen. Metropolis-Verlag. Marburg.

Behrendt, Siegfried; Göll, Edgar; Korte, Friederike (2016): Effizienz, Konsistenz, Suffizienz. Strategieanalytische Betrachtung für eine Green Economy. Inputpapier im Rahmen des Projekts Evolution2Green - Transformationspfade zu einer Green Economy. Institut für Zukunftsstudien und Technologiebewertung. Berlin.

Behrendt, Siegfried; Henseling, Christine (2019): Zukunftsszenarien des Peer-toPeer Sharing. In: Siegfried Behrendt, Christine Henseling und Gerd Scholl (Hg.): Digitale Kultur des Teilens. Mit Sharing nachhaltiger Wirtschaften. Wiesbaden: Springer Fachmedien Wiesbaden, S. 149-175.

Behrendt, Siegfried; Nolte, Roland; Röben, Adrian (2015a): Recycling als Rohstoffquelle. Integrierte Roadmap III Automation 2025+. Zentralverband Elektrotechnik- und Elektronikindustrie; Institut für Zukunftsstudien und Technologiebewertung. Frankfurt a. M..

Behrendt, Siegfried; Scharp, Michael; Zieschank, Roland; van Nouhuys, Jo (2015b): »Horizon Scanning u und Trendmonitoring als ein Instrument in der Umweltpolitik zur strategischen Früherkennung und effizienten Politikberatung. Konzeptstudie. Umweltbundesamt. Dessau-Roßlau (UBA Texte, 106/2015).

Beigang, Alina; Clausen, Jens (2017): Elektromobilität in China. Fallstudie im Rahmen des Projekts Evolution2Green - Transformationspfade zu einer Green Economy. Borderstep Institut für Innovation und Nachhaltigkeit. Berlin.

Bell, Michelle L.; Davis, Devra L.; Fletcher, Tony (2004): A retrospective assessment of mortality from the London smog episode of 1952. The role of influenza and pollution. In: Environmental Health Perspectives 112 (1), S. 6-8. DOI: 10.1289/ ehp.6539.

Bellmann, Erika; Bollmann, Susann; Bornholdt, Martin (2015): Eigenheim. Von der Idee zum innovativen Finanzierungsansatz und Geschäftsmodell für energetische Gebäudemodernisierung. Ein Leitfaden. Deutsche Unternehmensinitiative Energieeffizienz; WWF Deutschland. Berlin.

Benz, Arthur; Dose, Nicolai (Hg.) (2010): Governance - Regieren in komplexen Regelsystemen. Eine Einführung. 2. Aufl. Wiesbaden: VS Verlag für Sozialwissenschaften. 
Berkhout, Frans; Hertin, Julia; Gann, David M. (2006): Learning to Adapt: Organisational Adaptation to Climate Change Impacts. In: Climatic Change 78 (1), S. 135-156. DOI: 10.1007/s10584-006-9089-3.

Bertling, Jürgen; Hiebel, Markus; Pflaum, Hartmut; Nühlen, Jochen (2014): Arten und Entstehungstypen frühzeitiger Produktalterung - Entwicklung eines Obsoleszenz-Portfolios. In: Umweltmagazin 3, S. 60-61.

Beyer, Jürgen (2006): Pfadabhängigkeit. Über institutionelle Kontinuität, anfällige Stabilität und fundamentalen Wandel. Frankfurt a. M.: Campus (Schriften des Max-Planck-Instituts für Gesellschaftsforschung Köln, 56).

Biermann, Frank; Siebenhüner, Bernd; Schreyögg, Anna (Hg.) (2009): International organizations in global environmental governance. London, New York: Routledge.

Bilharz, Michael (2008): »Key Points« nachhaltigen Konsums. Ein strukturpolitisch fundierter Strategieansatz für die Nachhaltigkeitskommunikation im Kontext aktivierender Verbraucherpolitk. Marburg: Metropolis-Verlag (Wirtschaftswissenschaftliche Nachhaltigkeitsforschung, 4).

Binswanger, Mathias (2001): Technological progress and sustainable development: what about the rebound effect? In: Ecological Economics 36 (1), S. 119132. DOI: 10.1016/S0921-8009(00)00214-7.

Birkland, Thomas A. (2014): An introduction to the policy process: Theories, concepts and models of public policy making. London, New York: Routledge.

Blunden, Jessica; Arndt, Derek S.; Hartfield, Gail (2018): State of the Climate in 2017. In: Bulletin of the American Meteorological Society 99 (8), i-310. DOI: 10.1175/2018BAMSStateoftheClimate.1.

BMBF (2014): Bioökonomie - neue Konzepte zur Nutzung natürlicher Ressourcen. Berlin. Online verfügbar unter www.bmbf.de/de/biooekonomie.php, zuletzt geprüft am 25.04.2019.

BMBF (2018a): Bekanntmachung des Bundesministeriums für Bildung und Forschung von Richtlinien zur Förderung von Forschungs- und Entwicklungsvorhaben im Rahmen der Sozial-ökologischen Forschung zum Themenschwerpunkt »Nachhaltiges Wirtschaften«. Berlin. Online verfügbar unter https:// www.bmbf.de/foerderungen/bekanntmachung-883.html, zuletzt geprüft am 25.04.2019.

BMBF (2018b): Nachhaltiges Wirtschaften. Sozial-ökologische Forschung. Online verfügbar unter https://www.fona.de/de/nachhaltiges-wirtschaften-sozialoekologische-forschung-17399.html, zuletzt geprüft am 25.04.2019.

BMUB (2014): GreenTech made in Germany 4.0. Umwelttechnologie-Atlas für Deutschland. Berlin.

BMUB (2015): Umweltbericht der Bundesregierung 2015. Auf dem Weg zu einer modernen Umweltpolitik. Berlin. 
BMUB (2016): Den ökologischen Wandel gestalten. Integriertes Umweltprogramm 2030. Berlin.

BMWi (2011): Regierungsprogramm Elektromobilität. Berlin.

BMWi (2014a): Ein gutes Stück Arbeit. Mehr aus Energie machen. Nationaler Aktionsplan für Energieeffizienz. Berlin.

BMWi (2014b): Sanierungsbedarf im Gebäudebestand. Ein Beitrag zur Energieeffizienzstrategie Gebäude. Berlin.

BMWi (2015): Energieeffizienzstrategie Gebäude. Wege zu einem nahezu klimaneutralen Gebäudebestand. Berlin.

BMWi (15.05.2015): Gabriel: Neues »Anreizprogramm Energieeffizienz« als sinnvolle Alternative zur steuerlichen Förderung der energetischen Gebäudesanierung. Berlin. Online verfügbar unter www.bmwi.de/DE/Presse/pressemit teilungen,did=703790.html, zuletzt geprüft am 25.04.2019.

BMWi (2016): Energiewende Plattform Gebäude. Berlin. Online verfügbar unter www.bmwi.de/DE/Themen/Energie/Energiewende-im-Gebaeudebereich/ plattform-gebaeude,did=725442.html, zuletzt geprüft am 25.04.2019.

Born, Manfred; Kreuzer, Klaus (2002): Nachhaltigkeit Lokal. Lokale Agenda 21 in Deutschland. Eine Zwischenbilanz 10 Jahre nach Rio. Forum Umwelt und Entwicklung. Bonn.

Borsboom, Wouter; Leidelmeijer, Kees; Jacobs, Piet; van Vliet, Marcel; Jong, Peter de (2015): Resultaten uit monitoring: Eerste Ervaringen met Prestatieenergiecontracten voor Nul op de Meter Woningen. Energiesprong; TNO; van Beek; RIGO. Den Haag.

Bower, Joseph; Christensen, Clayton (1995): Disruptive Technologies: Catching the Wave. In: Harvard Business Review 73 (1), S. 43-53.

Braga, Maria H.; Grundish, Nicholas S.; Murchison, Andrew J.; Goodenough, John B.; Braga, M. H.; Grundish, N. S. et al. (2017): Alternative strategy for a safe rechargeable battery. In: Energy \& Environmental Science 10 (1), S. 331-336. DOI: 10.1039/C6EE02888H.

Brand, Ulrich (2013): Green Economy - A new paradigm? Ambiguities of Green Growth Strategies. In: Climate Change and Green Growth. Legislative achievements and Prospects, S. 61-88.

Brand, Ulrich; Brunnengräber, Achim (2013): Socio-ecological transformations in times of a multiple crises. Common ground of the debate, divergences and open questions. In: World Social Science Report 2013.

Braungart, Michael; McDonough, William (2008): Einfach intelligent produzieren. Cradle to cradle: Die Natur zeigt, wie wir die Dinge besser machen können. 3. Aufl. Berlin: BvT Berliner Taschenbuch-Verlag (Gebrauchsanweisungen für das 21. Jahrhundert). 
Brischke, Lars-Arvid (2014): Energiesuffizienz - Strategie zur absoluten Senkung des Energieverbrauchs. In: Energiewirtschaftliche Tagesfragen 64, S. 13-15. DOI: 10.1007/s15027-013-0233-5.

Brohmann, Bettina; David, Martin (2015): Tipping Point Konzeptionen im Kontext eines nachhaltigen gesellschaftlichen Wandels. Transformationsstrategien und Models of Change für nachhaltigen gesellschaftlichen Wandel. Bericht des AP3. Umweltbundesamt. Dessau-Roßlau (UBA Texte, 67/2015).

Brown, Tim (2008): Design thinking. In: Harvard Business Review 86 (6), S. 84-92. Bulkeley, Harriet (2000): Discourse coalitions and the Australian climate change policy network. In: Environment and Planning C: Government and Policy 18 (6), S. 727-748. DOI: 10.1068/c9905j.

Bund der Energieverbraucher (2017): Daten und Statistiken. Online verfügbar unter https://www.energieverbraucher.de/de/daten-und-statistiken__988/, zuletzt geprüft am 25.04.2019.

Bundesregierung (2008): Fortschrittsbericht 2008 zur nationalen Nachhaltigkeitsstrategie. Für ein nachhaltiges Deutschland. Berlin.

Bundesregierung (2009): Nationaler Entwicklungsplan Elektromobilität der Bundesregierung. Berlin.

Bundesregierung (2010): Energiekonzept für eine umweltschonende, zuverlässige und bezahlbare Energieversorgung. Berlin.

Bundesregierung (2012): Nationale Nachhaltigkeitsstrategie. Fortschrittsbericht 2012. Berlin.

Bundesregierung (2014): Meilensteine der Nachhaltigkeitspolitik. Weiterentwicklung der nationalen Nachhaltigkeitsstrategie. Berlin.

Bundesregierung (2016): Entwurf der Deutschen Nachhaltigkeitsstrategie 2016. Neuauflage 2016. Berlin.

Bundesverband der Deutschen Industrie (2016): Positionierung zur Zusammenführung von Energieeinspargesetz (EnEG)/Energieeinsparverordnung (EnEV) und Erneuerbare-Energien-Wärmegesetz (EEWärmeG). Positionspapier. Bundesverband der Deutschen Industrie e. V. Berlin.

Burns, Tom R. (2011): The Sustainability Revolution: A Societal Paradigm Shift Ethos, Innovation, Governance Transformation. In: Sociologisk Forskning 48 (3), S. 93-108.

Buschfeld, Detlef; Rasch, Katrin; Rehbold, Rolf (2016): Qualifizierung von Mitarbeitern im Kontext der Energiewende. Forschungsinstitut für Berufsbildung im Handwerk (FBH) an der Universität zu Köln. Köln.

Caldwell, Raymond (2003): Models of Change Agency. A Fourfold Classification. In: British Journal of Management 14 (2), S. 131-142. DOI: 10.1111/1467-8551.00270.

Cameron, Esther; Green, Mike (2012): Making sense of change management. A complete guide to the models, tools, and techniques of organizational change. London: Kogan Page. 
Canzler, Weert; Knie, Andreas (2014): Mobil mit selbst gemachtem Strom. In: Politische Ökologie (137), S. 46-52.

Capstick, Stuart; Whitmarsh, Lorraine (2018): Public Perceptions of Climate Change. In: Benedetta Brevini und Justin Lewis (Hg.): Climate change and the media. Second edition. New York: Peter Lang Publishing (Global crises and the media, Vol. 27).

Casado-Asensio, Juan; Steurer, Reinhard (2014): Integrated strategies on sustainable development, climate change mitigation and adaptation in Western Europe: communication rather than coordination. In: Journal of Public Policy 34 (3), S. 437-473. DOI: 10.1017/So143814X13000287.

Casson, Mark (1982): The entrepreneur. An economic theory. Oxford: Robertson. Chappelow, Craig (2004): 360-degree feedback. In: Cynthia McCauley und Ellen van Velsor (Hg.): The Center for Creative Leadership Handbook of Leadership Development. San Francisco: Jossey-Bass, S. 58-84.

Chong, Dennis; Druckman, James N. (2007): Framing theory. In: Annual Review of Political Science 10, S. 103-126. DOI: 10.1146/annurev.polisci.10.072805.103054. Clausen, Jens (2017a): Der Post StreetScooter. Fallstudie im Rahmen des Projekts Evolution2Green - Transformationspfade zu einer Green Economy. Borderstep Institut für Innovation und Nachhaltigkeit. Berlin.

Clausen, Jens (2017b): Elektromobilität in Kalifornien. Fallstudie im Rahmen des Projekts Evolution2Green - Transformationspfade zu einer Green Economy. Borderstep Institut für Innovation und Nachhaltigkeit. Berlin.

Clausen, Jens (2017c): Elektromobilität in Norwegen. Fallstudie im Rahmen des Projekts Evolution2Green - Transformationspfade zu einer Green Economy. Borderstep Institut für Innovation und Nachhaltigkeit. Berlin.

Clausen, Jens (2017d): PKW-Antriebe. Transformationsfeldanalyse im Rahmen des Projekts Evolution2Green - Transformationspfade zu einer Green Economy. Borderstep Institut für Innovation und Nachhaltigkeit. Berlin.

Clausen, Jens (2017e): Straßen. Transformationsfeldanalyse im Rahmen des Projekts Evolution2Green - Transformationspfade zu einer Green Economy. Borderstep Institut für Innovation und Nachhaltigkeit. Berlin.

Clausen, Jens (2017f): Stromeinspeisungsgesetz und EEG. Fallstudie im Rahmen des Projekts Evolution2Green - Transformationspfade zu einer Green Economy. Borderstep Institut für Innovation und Nachhaltigkeit. Berlin.

Clausen, Jens (2017g): Wärmenetze. Transformationsfeldanalyse im Rahmen des Projekts Evolution2Green - Transformationspfade zu einer Green Economy. Borderstep Institut für Innovation und Nachhaltigkeit. Berlin.

Clausen, Jens; Fichter, Klaus (2016): Pfadabhängigkeiten und evolutorische Ökonomik. Inputpapier im Rahmen des Projekts Evolution2Green - Transformationspfade zu einer Green Economy. Borderstep Institut für Innovation und Nachhaltigkeit. Berlin. 
Clausen, Jens; Fichter, Klaus; Winter, Wiebke (2011): Theoretische Grundlagen für die Erklärung von Diffusionsverläufen von Nachhaltigkeitsinnovationen. Verbundvorhaben im Rahmen der BMBF-Bekanntmachung "Innovationspolitische Handlungsfelder für die nachhaltige Entwicklung«im Rahmen der Innovations- und Technikanalyse. Borderstep Institut für Innovation und Nachhaltigkeit. Berlin.

Clausen, Jens; Hinterholzer, Simon (2017): Versorgung von Gebäuden mit Wärme aus erneuerbaren Energien. Transformationsfeldanalyse im Rahmen des Projekts Evolution2Green - Transformationspfade zu einer Green Economy. Borderstep Institut für Innovation und Nachhaltigkeit. Berlin.

Clausen, Jens; Mathes, Maite (2017): Fleischkonsum. Transformationsfeldanalyse im Rahmen des Projekts Evolution2Green - Transformationspfade zu einer Green Economy. Borderstep Institut für Innovation und Nachhaltigkeit. Berlin.

Clausen, Jens; Perleberg, Steffi (2017): Tesla Motors. Fallstudie im Rahmen des Projekts Evolution2Green - Transformationspfade zu einer Green Economy. Borderstep Institut für Innovation und Nachhaltigkeit. Berlin.

Colander, David; Kupers, Roland (2014): Complexity and the Art of Public Policy. Solving Society's Problems from the Bottom Up. Princeton: Princeton University Press.

Cook, Carly N.; Inayatullah, Sohail; Burgman, Mark A.; Sutherland, William J.; Wintle, Brendan A. (2014): Strategic foresight: how planning for the unpredictable can improve environmental decision-making. In: Trends in ecology \& evolution 29 (9), S. 531-541. DOI: 10.1016/j.tree.2014.07.005.

Cooke, Bill; Kothari, Uma (2001): Participation: The new tyranny? London, New York: Zed Books.

D'Este, Pablo; Iammarino, Simona; Savona, Maria; Tunzelmann, Nick von (2012): What hampers innovation? Revealed barriers versus deterring barriers. In: Research Policy 41 (2), S. 482-488. DOI: 10.1016/j.respol.2011.09.008.

David, Paul A. (1985): Clio and the Economics of QWERTY. In: The American Economic Review 75 (2), S. 332-337.

DeFries, Ruth S.; Ellis, Erle C.; Chapin, F. Stuart; Matson, Pamela A.; Turner, B. L.; Agrawal, Arun et al. (2012): Planetary Opportunities: A Social Contract for Global Change Science to Contribute to a Sustainable Future. In: BioScience 62 (6), S. 603-606. DOI: 10.1525/bio.2012.62.6.11.

dena (o. J.): Serielles Sanieren von Mehrfamilienhäusern. Energiesprong Deutschland. Deutsche Energie-Agentur. Online verfügbar unter https://www.dena. de/themen-projekte/projekte/gebaeude/serielles-sanieren-von-mehrfami lienhaeusern/, zuletzt geprüft am 25.04.2019.

dena (2016): dena-Gebäudereport 2016. Statistiken und Analysen zur Energieeffizienz im Gebäudebestand. Deutsche Energie-Agentur. Berlin. 
Der Tagesspiegel (2017): Merkel kassiert das Ziel von einer Million E-Autos bis 2020. Online verfügbar unter https://www.tagesspiegel.de/wirtschaft/elektromobi litaet-merkel-kassiert-das-ziel-von-einer-million-e-autos-bis-2020/19809744. html, zuletzt geprüft am 25.04.2019.

Deutsche See (2017): Wir fahren Streetscooter. Online verfügbar unter https:// www.deutschesee.de/ueber-uns/nachhaltigkeit/umwelt-energie/wir-fahrenstreetscooter/, zuletzt geprüft am 25.04.2019.

Deutsche Umwelthilfe (2014): Energetische Gebäudesanierung - Wider die falschen Mythen. Radolfzell.

Deutscher Bundestag (2011): Dreizehntes Gesetz zur Änderung des Atomgesetzes vom 31. Juli 2011. Bonn (Bundesgesetzblatt Teil I, Nr. 43).

Dickmann, Philipp (2008): Schlanker Materialfluss mit Lean Production, Kanban und Innovationen. Wiesbaden: Springer.

Die Mittelbayerische (2015): Haltbarkeitsdatum für Elektrogeräte. Online verfügbar unter www.mittelbayerische.de/wirtschaft-nachrichten/haltbarkeitsdatumfuer-elektrogeraete-21840-art1250014.html, zuletzt geprüft am 25.04.2019.

Die Welt Online (2014): Das Milliardengeschäft der Dämmstoffindustrie. Online verfügbar unter www.welt.de/finanzen/immobilien/article132802827/Das-Milli ardengeschaeft-der-Daemmstoffindustrie.html, zuletzt geprüft am 25.04.2019.

Diefenbach, Nikolaus; Cischinsky, Holger; Rodenfels, Markus; Clausnitzer, KlausDieter (2010): Datenbasis Gebäudebestand. Datenerhebung zur energetischen Qualität und zu den Modernisierungstrends im deutschen Wohngebäudebestand. Institut Wohnen und Umwelt; Bremer Energie-Institut. Darmstadt, Bremen.

Diyar, Sabit; Akparova, Aigul; Toktabayev, Azamat; Tyutunnikova, Marina (2014): Green Economy - Innovation-based Development of Kazakhstan. In: Procedia - Social and Behavioral Sciences 140, S. 695-699. DOI: 10.1016/j.sbspro. 2014.04.497.

Dovers, Stephen R.; Hezri, Adnan A. (2010): Institutions and policy processes: the means to the ends of adaptation. In: Wiley Interdisciplinary Reviews: Climate Change 1 (2), S. 212-231. DOI: 10.1007/978-3-540-34338-7.

Dunkelberg, Elisa; Bachmann, Max; Schneller, Andreas; Schröder, Sabine (2017): Rahmenbedingungen für die Umsetzbarkeit von LowEx-Mehrleiter-Wärmenetzen. Kurzzusammenfassung von Projektergebnissen. IÖW; TU Berlin; adelphi; nexus. Berlin.

e.GO Mobile AG (2017): Caritas-Verbände steigen auf Elektroautos von e.GO Mobile um. Online verfügbar unter http://e-go-mobile.com/de/newspool/caritasverbaende-steigen-auf-elektroautos-von-e.go-mobile-um/, zuletzt geprüft am 25.04.2019.

ebay (2015): TNS-Studie im Auftrag von eBay: Knapp 103 Milliarden Euro schlummern in deutschen Haushalten. Dreilinden, Berlin. Online verfügbar unter 
https://www.ebayinc.com/stories/press-room/de/tns-studie-im-auftrag-vonebay-knapp-103-milliarden-euro-schlummern-in-deutschen-haushalten/, zuletzt geprüft am 25.04.2019.

Elzen, Boelie; Wieczorek, Anna (2005): Transitions towards sustainability through system innovation. In: Technological Forecasting and Social Change 72 (6), S. 651-661. DOI: 10.1016/j.techfore.2005.04.002.

Energiesprong (2013): Stroomversnelling. Bijlagen bij de Samenwerkingsovereenkomst. Versie 10.3. Platform 31. Den Haag.

Energiesteuergesetz (2006). Online verfügbar unter https://www.gesetze-im-in ternet.de/energiestg/index.html\#BJNR153410006BJNE008100140, zuletzt geprüft am 25.04.2019.

Enseling, Andreas; Hinz, Eberhard (2015): Kurzgutachten zur wirtschaftlichen Vertretbarkeit ausgewählter Maßnahmen nach EnEV 2014, Anlage 3. Institut Wohnen und Umwelt. Darmstadt.

Erdmann, Lorenz; Behrendt, Siegfried; Feil, Moira (2011): Kritische Rohstoffe für Deutschland. "Identifikation aus Sicht deutscher Unternehmen wirtschaftlich bedeutsamer mineralischer Rohstoffe, deren Versorgungslage sich mittel- bis langfristig als kritisch erweisen könnte«. Im Auftrag der KfW Bankengruppe. Institut für Zukunftsstudien und Technologiebewertung; adelphi. Berlin.

Eucker, Dennis; Bubeck, Philipp; Kahlenborn, Walter; Boehm, Nora; Klockemann, Lena (2017): Erfolgsfaktoren im Politikprozess Klimaanpassung. Arbeitspaket 2: Gestaltungs- und Steuerungsschwerpunkte in Deutschland. Umweltbundesamt. Dessau-Roßlau.

Europäische Kommission (2016): An EU Strategy on Heating and Cooling. Communication from the Commission to the European Parliament, the Council, the European Economic and Social Committee and the Committee of the Regions. SWD (2016) 24 final. Brüssel.

Europäische Kommission (2019): Moving towards a green economy - policy. Online verfügbar unter https://ec.europa.eu/europeaid/sectors/environment/envi ronment-and-green-economy/green-economy_en, zuletzt geprüft am 25.04.2019.

Europäische Union (2014): Eine grüne Infrastruktur für Europa. Luxemburg.

Europäisches Parlament (2016): Entwurf eines Berichts über das Thema »Längere Lebensdauer für Produkte: Vorteile für Verbraucher und Unternehmen«. Europäisches Parlament. Brüssel (2016/2272[INI]).

EWSA (2013): Für einen nachhaltigeren Konsum: die Lebensdauer von Industrieprodukten und die Verbraucherinformation zugunsten eines neuen Vertrauens. Stellungnahme des Europäischen Wirtschafts- und Sozialausschusses, CCMI/112. Brüssel.

Exner, Andreas (2016): Einführung: Kritische Metalle in der Großen Transformation. In: Andreas Exner, Martin Held und Klaus Kümmerer (Hg.): Kritische 
Metalle in der Großen Transformation. Berlin, Heidelberg: Springer Spektrum, S. 1-16.

Fairphone (2018): About us. Online verfügbar unter https://www.fairphone.com/ en/about/about-us/?ref=footer, zuletzt geprüft am 25.04.2019.

Feola, Giuseppe (2015): Societal transformation in response to global environmental change: A review of emerging concepts. In: AMBIO 44 (5), S. 376-390. DOI: 10.1007/s13280-014-0582-z.

Feser, Daniel; Proeger, Till; Bizer, Kilian (2015): Die Energieberatung als der zentrale Akteur bei der energetischen Gebäudesanierung? In: Zeitschrift für Energiewirtschaft 39 (2), S. 133-145. DOI: 10.1007/s12398-015-0149-0.

Fichter, Klaus (2005): Interaktives Innovationsmanagement: Neue Potenziale durch Öffnung des Innovationsprozesses. In: Klaus Fichter, Niko Paech und Reinhard Pfriem (Hg.): Nachhaltige Zukunftsmärkte. Orientierungen für unternehmerische Innovationsprozesse im 21. Jahrhundert. Marburg: Metropolis-Verlag (Theorie der Unternehmung, 29), S. 239-269.

Fichter, Klaus; Beucker, Severin (2012a): Innovation communities. Teamworking of key persons - a success factor in radical innovation. Berlin, Heidelberg: Springer.

Fichter, Klaus; Beucker, Severin (2012b): Innovation Communities: Kooperation zahlt sich aus. Ein Leitfaden für die Praxis. Borderstep Institut für Innovation und Nachhaltigkeit. Berlin.

Fichter, Klaus; Clausen, Jens (2013): Erfolg und Scheitern "grüner« Innovationen. Warum einige Nachhaltigkeitsinnovationen am Markt erfolgreich sind und andere nicht. Marburg: Metropolis.

Fichter, Klaus; Clausen, Jens (2016): Diffusion Dynamics of Sustainable Innovation - Insights on Diffusion Patterns Based on the Analysis of 100 Sustainable Product and Service Innovations. In: Journal of Innovation Management 4 (2), S. 30-67. DOI: 10.24840/2183-0606_004.002_0004.

Figenbaum, Erik (2017): Perspectives on Norway's supercharged electric vehicle policy. In: Environmental Innovation and Societal Transitions 25, S. 14-34. DOI: 10.1016/j.eist.2016.11.002.

Fischer, Corinna; Grießhammer, Rainer (2013): Mehr als nur weniger. Suffizienz: Begriff, Begründung, Potenziale. Öko-Institut e. V. Freiburg.

Flanagan, Kieron; Uyarra, Elvira; Laranja, Manuel (2011): Reconceptualising the spolicy mix for innovation. In: Research Policy 40 (5), S. 702-713. DOI: 10.1016/j.respol.2011.02.005.

Fligstein, Neil; McAdam, Doug (2011): Toward a General Theory of Strategic Action Fields. In: Sociological Theory 29 (1), S. 1-26. DOI: 10.1111/j.1467-9558.2010. 01385.x. 
Forschungsvereinigung Verbrennungskraftmaschinen (2013): Kraftstoffstudie. Zukünftige Kraftstoffe für Verbrennungsmotoren und Gasturbinen. Forschungsvereinigung Verbrennungskraftmaschinen e. V. Frankfurt a. M..

Forum Fairer Handel (2016): Forum Fairer Handel - Das Netzwerk des fairen Handels in Deutschland. Berlin. Online verfügbar unter www.forum-fairer-han del.de/startseite/, zuletzt geprüft am 25.04.2019.

Frankfurter Allgemeine Zeitung (2014): Stoppt den Dämmwahn! Energetische Sanierung. Online verfügbar unter www.faz.net/aktuell/finanzen/meine-finan zen/mieten-und-wohnen/daemmung-ist-oekologisch-zweifelhaft-und-teu er-12933587.html?printPagedArticle=true\#pageIndex_2, zuletzt geprüft am 25.04.2019.

Fücks, Ralf (2013): Intelligent wachsen. Die grüne Revolution. München: Carl Hanser Fachbuchverlag.

Gardini, Laura; Hommes, Cars; Tramontana, Fabio; Vilder, Robin de (2009): Forward and backward dynamics in implicitly defined overlapping generations models. In: Journal of Economic Behavior \& Organization 71 (2), S. 110-129. DOI: 10.1016/j.jebo.2008.09.010.

Garud, Raghu; Kumaraswamy, Arun; Karnøe, Peter (2010): Path dependence or path creation? In: Journal of Management Studies 47 (4), S. 760-774. DOI: 10.1111/j.1467-6486.2009.00914.x.

Gavetti, Giovanni; Levinthal, Daniel (2000): Looking Forward and Looking Backward: Cognitive and Experiential Search. In: Administrative Science Quarterly 45 (1), S. 113-137. DOI: 10.2307/2666981.

Gebäude-Allianz (2016): Anforderungen an die Zusammenführung, Vereinfachung und Weiterentwicklung von Energieeinspargesetz, -verordnung und Erneuerbare-Energien-Wärmegesetz aus Sicht der Gebäude-Allianz. Naturschutzbund Deutschland. Berlin.

Gedankenstrich.org (2014): Kurze Einführung in die Multi-Level Perspective. Online verfügbar unter https://gedankenstrich.org/2014/11/kurze-einfuhrungin-die-multi-level-perspective/, zuletzt geprüft am 25.04.2019.

Geels, Frank W. (2002): Technological transitions as evolutionary reconfiguration processes: a multi-level perspective and a case-study. In: Research Policy 31 (8-9), S. 1257-1274. DOI: 10.1016/S0048-7333(02)00062-8.

Geels, Frank W. (2011): The multi-level perspective on sustainability transitions: Responses to seven criticisms. In: Environmental Innovation and Societal Transitions I (1), S. 24-40. DOI: 10.1016/j.eist.2011.02.002.

Geels, Frank W. (2014a): Reconceptualising the co-evolution of firms-in-industries and their environments: Developing an inter-disciplinary Triple Embeddedness Framework. In: Research Policy 43 (2), S. 261-277. DOI: 10.1016/j. respol.2013.10.006. 
Geels, Frank W. (2014b): Regime Resistance against Low-Carbon Transitions: Introducing Politics and Power into the Multi-Level Perspective. In: Theory, Culture \& Society 31 (5), S. 21-40. DOI: 10.1177/0263276414531627.

Geels, Frank W.; Kemp, René (2006): Transitions, Transformations, and Reproduction: Dynamics in Socio-Technical Systems. In: Maureen d. McKelvey und Magnus Holmén (Hg.): Flexibility and stability in the innovating economy. Oxford: Oxford Univ. Press, S. 227-256.

Geels, Frank W.; Schot, Johan (2007): Typology of sociotechnical transition pathways. In: Research Policy 36 (3), S. 399-417. DOI: 10.1016/j.respol.2007.01.003.

Gleich, Arnim von (1998): Bionik. Ökologische Technik nach dem Vorbild der Natur? Wiesbaden: Vieweg+Teubner Verlag.

Gleich, Arnim von; Gößling-Reisemann, Stefan (2008): Industrial Ecology. Erfolgreiche Wege zu nachhaltigen industriellen Systemen. Wiesbaden: Vieweg+Teubner Verlag/GWV Fachverlage GmbH Wiesbaden.

Göll, Edgar (2017): Nichtraucherschutz und Rauchverbote. Fallstudie im Rahmen des Projekts Evolution2Green - Transformationspfade zu einer Green Economy. Institut für Zukunftsstudien und Technologiebewertung. Berlin.

Göll, Edgar; Henseling, Christine (2017): Gesellschaftliche Trägheiten und Optionen zu ihrer Überwindung in Richtung Nachhaltigkeit. In: Jana Rückert-John und Martina Schäfer (Hg.): Governance für eine Gesellschaftstransformation. Herausforderungen des Wandels in Richtung nachhaltige Entwicklung. Wiesbaden: Springer VS, S. 87-108.

Göll, Edgar; Nolting, Katrin (2012): »Rio+20 vor Ort« - Kommunen auf dem Weg zur Nachhaltigkeit: Beispiele aus Deutschland. Institut für Zukunftsstudien und Technologiebewertung. Berlin.

Göll, Edgar; Zwiers, Jakob (2018): Verpackungsarmes/-freies Einkaufen. Fallstudie im Rahmen des Projekts Evolution2Green - Transformationspfade zu einer Green Economy. Institut für Zukunftsstudien und Technologiebewertung. Berlin.

Graedel, Thomas (1994): Industrial Ecology. Definition and Implementation. In: Robert H. Socolow (Hg.): Industrial ecology and global change. Cambridge: Cambridge University Press.

Gries, Nadja von; Wilts, Henning; Meissner, Markus (2017): Schaffung einer Datenbasis zur Erfassung der Mengen von in Deutschland wiederverwendeten Produkten. Zwischenbericht. Umweltbundesamt. Dessau-Roßlau (UBA Texte, 04/2017).

Grießhammer, Rainer; Brohmann, Bettina (2015): Wie Transformationen und gesellschaftliche Innovationen gelingen können. Transformationsstrategien und Models of Change für nachhaltigen gesellschaftlichen Wandel. BadenBaden: Nomos. 
Grießhammer, Rainer; Brommer, Eva; Gattermann, Marah; Grether, Stefanie; Krüger, Malte; Teufel, Jenny; Zimmer, Wiebke (2010): Co2-Einsparpotenziale für Verbraucher. Öko-Institut e. V. Freiburg.

Grimm, Franc; Heinrichs, Harald; Neumann, Kai (2014): Entwicklung eines Integrated Assessment Modells: Nachhaltige Entwicklung in Deutschland. Band 1: Hauptband. Umweltbundesamt. Dessau-Roßlau (UBA Texte, 74/2014).

Grote, Lars; Hoffmann, Patrick; Tänzer, Guillem (2015): Abwärmenutzung Potentiale, Hemmnisse und Umsetzungsvorschläge. Institut für Zukunftsenergiesysteme. Saarbrücken.

Groth, Uwe; Kammel, Andreas (1994): Lean management. Konzept, kritische Analyse, praktische Lösungsansätze. Wiesbaden: Gabler.

Habegger, Beat (2010): Strategic foresight in public policy: reviewing the experiences of the UK, Singapore, and The Netherlands. In: Strategic Direction 26 (9). DOI: $10.1108 / \mathrm{sd} .2010 .05626 \mathrm{iad} .004$.

Hamdouch, Abdelillah; Depret, Marc-Hubert (2010): Policy integration strategy and the development of the sgreen economy<: foundations and implementation patterns. In: Journal of Environmental Planning and Management 53 (4), S. 473-490. DOI: 10.1080/09640561003703889.

Handelsblatt (2017): Fairphone-Gründer Bas van Abel: Immer mehr, trotzdem fair. Online verfügbar unter https://www.handelsblatt.com/unternehmen/ it-medien/fairphone-gruender-bas-van-abel-immer-mehr-trotzdem-fair-/ 20302312.html?ticket=ST-1413801-UTpzIXOjipHxr417QXv4-ap3, zuletzt geprüft am 25.04.2019.

Hannoversche Allgemeine Zeitung (2017a): Trittin gibt Land Mitschuld an VWKrise. In: Hannoversche Allgemeine Zeitung, 17.01.2017.

Hannoversche Allgemeine Zeitung (2017b): Steigende Wasserpreise befürchtet. In: Hannoversche Allgemeine Zeitung, 20.01.2017.

Head, Brian W. (2008): Wicked problems in public policy. In: Public Policy 3 (2), S. 101-118.

Hege, Elisabeth (2019): Towards a sustainable Europe in 2030? An analysis of the European Commission's Reflection Paper. IDDRI. Online verfügbar unter https://www.iddri.org/en/publications-and-events/blog-post/towards-sus tainable-europe-2030-analysis-european-commissions, zuletzt geprüft am 25.04.2019.

Heitkötter, Martina; Schneider, Manuel (2004): Zeitpolitisches Glossar. Grundbegriffe - Felder - Instrumente - Strategien. Gesellschaft für Zeitpolitik. München.

Helmer, Maja; Gnannt, Nora (2017): Kaufberatung fürs Elektro-Auto (WISO). ZDF, 2017.

Helms, Hinrich; Jöhrens, Julius; Kämper, Claudia; Giegrich, Jürgen; Liebich, Axel; Vogt, Regine; Lambrecht, Udo (2016): Weiterentwicklung und vertiefte Ana- 
lyse der Umweltbilanz von Elektrofahrzeugen. Umweltbundesamt. DessauRoßlau (UBA Texte, 27/2016).

Hendricks, Barbara (2016): Rede zur 30-Jahr-Feier des Forschungszentrums für Umweltpolitik (FFU). Bundesministerium für Umwelt, Naturschutz, Bau und Reaktorsicherheit (BMUB). Berlin, 06.04.2016. Online verfügbar unter https:// www.bmu.de/rede/rede-von-dr-barbara-hendricks-zur-30-jahr-feier-desforschungszentrums-fuer-umweltpolitik-ffu/, zuletzt geprüft am 25.04.2019.

Henseleit, Meike (2011): Die Nachfrage nach Fair-Trade-Produkten in Deutschland. Eine empirische Untersuchung unter Berücksichtigung von Präferenzen für Bio-Produkte. Vortrag anlässlich der 51. Jahrestagung der GEWISOLA »Unternehmerische Landwirtschaft zwischen Marktanforderungen und gesellschaftlichen Erwartungen«. GEWISOLA. Halle, 28.09.2011. Online verfügbar unter http://ageconsearch.umn.edu/bitstream/114495/2/Henseleit.pdf, zuletzt geprüft am 25.04.2019.

Heyen, Dirk Arne (2016): Exnovation: Herausforderungen und politische Gestaltungsansätze für den Ausstieg aus nicht-nachhaltigen Strukturen. Öko-Institut e. V. Freiburg (Öko-Institut Working Paper, 3/2016).

Heyen, Dirk Arne; Fischer, Corinna; Barth, Regine; Brunn, Christoph; Grießhammer, Rainer; Keimeyer, Friedhelm; Wolff, Franziska (2013): Mehr als nur weniger. Suffizienz: Notwendigkeitund Optionen politischer Gestaltung. ÖkoInstitut e. V. Freiburg.

Hilty, Lorenz; Behrendt, Siegfried; Hintemann, Ralph; Evers-Wölk, Michaela; Fichter, Klaus; Lohmann, Wolfgang (2015): Grüne Software: Ermittlung und Erschließung von Umweltschutzpotenzialen der Informations- und Kommunikationstechnik (Green IT). Umweltbundesamt. Dessau-Roßlau (UBA Texte, 22/2015).

Hilty, Lorenz M. (Hg.) (2003): Das Vorsorgeprinzip in der Informationsgesellschaft. Auswirkungen des Pervasive Computing auf Gesundheit und Umwelt. Bern: Zentrum für Technologiefolgen-Abschätzung (Technology assessment, 46).

Hitchens, David; Trainor, Mary; Clausen, Jens; Thankappan, Samarthia; Marchi, Bruna (2003): Small and Medium Sized Companies in Europe. Environmental Performance, Competitiveness and Management: International EU Case Studies. Berlin, Heidelberg: Springer.

Holm, Andreas; Mayer, Christine; Sprengard, Christoph (2015): Wirtschaftlichkeit von wärmedämmenden Maßnahmen. Forschungsinstitut für Wärmeschutz. Gräfelfing (FIW Bericht, FO-2015/O2).

Hölscher, Katharina; Wittmayer, Julia M.; Maschmeyer, Steffen; Frantzeskaki, Niki (2017): Transition Management als Meta-Governance-Rahmenwerk zur Gestaltung von Nachhaltigkeitstransitionen. Analyse von Governance-Kapazitäten durch Transition-Management-Interventionen in Gent und Montreuil. In: Ralf Kleinfeld, Joachim Hafkesbrink und Jens Stuhldreier (Hg.): Innova- 
tives Regionalmanagement im demografischen Wandel, Bd. 39. Wiesbaden: Springer Fachmedien Wiesbaden, S. 27-57.

Howaldt, Jürgen; Schwarz, Michael (2017): Die Mechanismen transformativen Wandels erfassen: Plädoyer für ein praxistheoretisches Konzept sozialer Innovationen. In: GAIA - Ecological Perspectives for Science and Society 26 (3), S. 239-244. DOI: 10.14512/gaia.26.3.6.

Howaldt, Jürgen; Schwarz, Michael (2018): Soziale Innovation. In: Birgit BlättelMink, Ingo Schulz-Schaeffer und Arnold Windeler (Hg.): Handbuch Innovationsforschung. Heidelberg: Springer (Springer Reference), S. 1-17.

Huber, Joseph (1995): Nachhaltige Entwicklung: Strategien für eine ökologische und soziale Erdpolitik. Berlin: Edition Sigma.

Huber, Joseph (2000): Industrielle Ökologie. Konsistenz, Effizienz und Suffizienz in zyklusanalytischer Betrachtung. »Global Change« VDW Jahrestagung, Berlin, 28.-29.10.1999. In: Udo Ernst Simonis (Hg.): Global Change. Baden-Baden: Nomos.

Huber, Joseph (2013): Konsistenz - schlüssig für Nachhaltigkeit. In: Udo Ernst Simonis, Heike Leitschuh, Gerd Michelsen, Jörg Sommer und Ernst Ulrich von Weizsäcker (Hg.): Mut zu Visionen. Brücken in die Zukunft - Jahrbuch Ökologie 2014. 1. Auflage. Stuttgart: S. Hirzel Verlag (Jahrbuch Ökologie, 2014), S. 64-70.

Hunecke, Marcel (2013): Psychologie der Nachhaltigkeit. Psychische Ressourcen für Postwachstumsgesellschaften. München: Oekom.

ICC (2012): Green Economy Roadmap. Paris (213-18/8).

IEA (2015): World Energy Outlook 2015. International Energy Agency. Online verfügbar unter www.worldenergyoutlook.org/, zuletzt geprüft am 25.04.2019.

Immowelt (2014): Modernisierungsumlage: Wann die Miete nach einer energetischen Sanierung erhöht werden darf. Online verfügbar unter http://news. immowelt.de/n/2449-modernisierungsumlage-wann-die-miete-nach-einerenergetischen-sanierung-erhoeht-werden-darf.html, zuletzt geprüft am 25.04.2019.

IPCC (2012): Managing the Risks of Extreme Events and Disasters to Advance Climate Change Adaptation. Intergovernmental Panel on Climate Change. Genf. IPCC (2014): Climate Change 2014: Synthesis Report. Contribution of Working Groups I, II and III to the Fifth Assessment Report of the Intergovernmental Panel on Climate Change. Intergovernmental Panel on Climate Change. Genf. IPCC (2018): Global Warming of $1.5^{\circ} \mathrm{C}$. An IPCC Special Report on the impacts of global warming of $1.5^{\circ} \mathrm{C}$ above pre-industrial levels and related global greenhouse gas emission pathways, in the context of strengthening the global response to the threat of climate change, sustainable development, and efforts to eradicate poverty. Intergovernmental Panel on Climate Change. Genf.

Irrek, Wolfgang; Kristof, Kora (2000): Was ist neu und was ist hilfreich? Ein nutzerorientierter Blick auf die Ergebnisse der empirischen Analyse von Klima- 
schutzprozessen auf kommunaler Ebene. In: Ulla Böde und Edelgard Gruber (Hg.): Klimaschutz als sozialer Prozess. Heidelberg: Physica Verlag (Technik, Wirtschaft und Politik - Schriftenreihe des Fraunhofer-Instituts für Systemtechnik und Innovationsforschung, 44), S. 63-85.

IWU (2013): Wirtschaftlichkeit energetischer Gebäudesanierung. Anmerkungen zur aktuellen Diskussion. Institut für Wohnen und Umwelt. Darmstadt.

Jackson, Tim (2009): Prosperity without growth. Economics for a finite planet. London: Earthscan.

Jacob, Klaus; Bär, Holger; Graaf, Lisa (2015a): Metaanalyse von Visionen einer nachhaltigen Gesellschaft. Teilbericht 2 des Projektes »Nachhaltiges Deutschland 2030 bis 2050 - Wie wollen wir in Zukunft leben?«. Umweltbundesamt. Dessau-Roßlau (UBA Texte, 59/2015).

Jacob, Klaus; Bär, Holger; Graaf, Lisa (2015b): Transformative Umweltpolitik - Der

Beitrag der Umweltpolitik zu Prozessen gesellschaftlichen Wandels. Teilbericht 3 des Projektes "Nachhaltiges Deutschland 2030 bis 2050 - Wie wollen wir in Zukunft leben?«. Umweltbundesamt. Dessau-Roßlau (UBA Texte, 60/2015).

Jacob, Klaus; Jänicke, Martin (2006): Environmental Governance in Global Perspective: New Approaches to Ecological and Political Modernisation. Berlin.

Jänicke, Martin (2014): Entwicklungsländer als Vorreiter der Nachhaltigkeit? Frugale Technik. In: Ökologisches Wirtschaften, S. 30-36. DOI: 10.14512/ OEW290130.

Jänicke, Martin; Schreurs, Miranda; Töpfer, Klaus (2015): The Potential of MultiLevel Global Climate Governance. Institute for Advanced Sustainability Studies. Potsdam.

Jann, Werner; Wegrich, Kai (2009): Phasenmodelle und Politikprozesse. Der Policy Cycle. In: Klaus Schubert und Nils C. Bandelow (Hg.): Lehrbuch der Politikfeldanalyse 2.0. München: Oldenbourg, S. 71-106.

Jarillo, J. Carlos (1988): On Strategic Networks. In: Strategic Management Journal 9 (1), S. 31-41. DOI: 10.1002/smj.4250090104.

John, René; Jaeger-Erben, Melanie; Rückert-John, Jana (2016): Elusive Practices: Considerations on limits and possibilities of environmental policy for sustainable consumption. In: Environmental Policy and Governance 26 (2), S. 129-140. DOI: 10.1002/eet.1706.

Kahlenborn, Walter; Keppner, Benno; Uhle, C.; Richter, S.; Jetzke; T. (2018): Die Zukunft im Blick: Konsum 4.0: Wie Digitalisierung den Konsum verändert. Trendbericht zur Abschätzung der Umweltwirkungen. Umweltbundesamt. Dessau-Roßlau.

Kahlenborn, Walter; Mewes, Heike; Knopf, Jutta; Hauffe, Petra; Kampffmeyer, Nele; Fichter, Klaus et al. (2013): Treiber und Hemmnisse für die Transforma- 
tion der deutschen Wirtschaft zu einer "Green Economy«. adelphi; Borderstep Institut für Innovation und Nachhaltigkeit. Berlin.

Kahlenborn, Walter; Tappeser, Valentin (2018): Vertikale Landwirtschaft. Fallstudie im Rahmen des Projekts Evolution2Green - Transformationspfade zu einer Green Economy. adelphi. Berlin.

Kahlenborn, Walter; Tappeser, Valentin; Chichowitz, Lisa (2016): »Models of Change« (MoC) als Analyseansatz. Der MoC-Ansatz als Analyserahmen für Evolution2Green. Inputpapier im Rahmen des Projekts Evolution2Green Transformationspfade zu einer Green Economy. adelphi. Berlin.

Kallis, Giorgos; Kerschner, Christian; Martinez-Alier, Joan (2012): The economics of degrowth. In: Ecological Economics 84, S. 172-180. DOI: 10.1016/j.ecolecon.2012.08.017.

Kärcher-Vital, Thomas (2002): Clusterorientierte Förderstrategien in der Regionalpolitik und -entwicklung. Inputpapier für die Expertenkommission Überprüfung und Neukonzeption der Schweizer Regionalpolitik. Winterthur.

Karnøe, Peter; Garud, Raghu (2012): Path Creation: Co-creation of Heterogeneous Resources in the Emergence of the Danish Wind Turbine Cluster. In: European Planning Studies 20 (5), S. 733-752. DOI: 10.1080/09654313.2012.667923.

Karnowski, Veronika; Pape, Thilo von; Wirth, Werner (2006): Zur Diffusion neuer Medien. Kritische Bestandsaufnahme aktueller Ansätze und Überlegungen zu einer integrativen Diffusions- und Aneignungstheorie neuer Medien. In: M\&K Medien \& Kommunikationswissenschaft 54 (1), S. 56-74. DOI: 10.5771/1615-634X-2006-1-56.

Kemp, René (1994): Technology and the transition to environmental sustainability. In: Futures 26 (10), S. 1023-1046. DOI: 10.1016/0016-3287(94)90071-X.

Kemp, René; Parto, Saeed; Gibson, Robert B. (2005): Governance for sustainable development: moving from theory to practice. In: International Journal of Sustainable Development 8 (1-2), S. 12-30. DOI: 10.1504/IJSD.2005.007372.

Kemp, René; Schot, Johan; Hoogma, Remco (1998): Regime shifts to sustainability through processes of niche formation: The approach of strategic niche management. In: Technology Analysis \& Strategic Management 10 (2), S. 175-198. DOI: $10.1080 / 09537329808524310$.

Kingdon, John W. (2002): The reality of public policy making. In: Marion Danis, Carolyn Clancy und Larry Churchill (Hg.): Ethical Dimensions of Health Policy. Oxford: Oxford University Press, S. 97-116.

Kingdon, John W.; Thurber, James A. (1984): Agendas, alternatives, and public policies. Boston: Little, Brown.

Kirchner, Stefan (2008): Pfadabhängigkeit als Mehrebenenphänomen: Grundlagen und Erweiterungen des Pfadansatzes. In: Hamburg Review of Social Sciences 3 (3), S. 317-343. 
Kleinhückelkotten, Silke (2005): Suffizienz und Lebensstile. Ansätze für eine milieuorientierte Nachhaltigkeitskommunikation. Berlin: BWV Berliner Wissenschafts-Verlag (Umweltkommunikation, 2).

Klima-Allianz Deutschland (2016): Klimaschutzplan 2050 der deutschen Zivilgesellschaft. Berlin.

Klinski, Stefan; Bürger, Veit; Nast, Michael (2009): Rechtskonzepte zur Beseitigung des Staus energetischer Sanierungen im Gebäudebestand. Umweltbundesamt. Dessau-Roßlau (UBA Texte, 36/2009).

Köder, Lea; Burger, Andreas (2016): Umweltschädliche Subventionen in Deutschland. Aktualisierte Ausgabe 2016. Umweltbundesamt. Dessau-Roßlau.

Korte, Friederieke; Göll, Edgar; Behrendt, Siegfried (2017): Automobilität im Wandel. Transformationsfeldanalyse im Rahmen des Projekts EvolutionzGreen Transformationspfade zu einer Green Economy. Institut für Zukunftsstudien und Technologiebewertung. Berlin.

KPMG (2018): Electric readiness: A successful infrastructure is defined by two components: Charge point coverage and a positive charging experience. Online verfügbar unter https://gaes.kpmg.de/brain.html\#electric-readiness, zuletzt geprüft am 16.01.2018.

Kraftfahrtbundesamt (2016): Fahrzeugzulassungen (FZ). Neuzulassungen von Kraftfahrzeugen nach Umwelt-Merkmalen. Jahr 2015. FZ 14. Flensburg.

Kraftfahrtbundesamt (2017): Verkehr in Kilometern der deutschen Kraftfahrzeuge im Jahr 2016. Flensburg. Online verfügbar unter https://www.kba.de/DE/ Statistik/Kraftverkehr/VerkehrKilometer/verkehr_in_kilometern_node.html, zuletzt geprüft am 25.04.2019.

Kristof, Kora (2010a): Models of Change: Einführung und Verbreitung sozialer Innovationen und gesellschaftlicher Veränderungen in transdisziplinärer Perspektive. Zürich: vdf Hochschulverlag.

Kristof, Kora (2010b): Wege zum Wandel. Wie wir gesellschaftliche Veränderungen erfolgreicher gestalten können. München: Oekom.

Lacey, Miriam Y. (1995): Internal consulting: perspectives on the process of planned change. In: Journal of Organizational Change Management 8 (3), S. 75-84. DOI: $10.1108 / 09534819510090178$.

Lahl, Uwe; Zeschmar-Lahl, Barbara (2012): Weg vom Öl durch sfeedstock change in der Chemieindustrie. Leibniz-Institut für interdisziplinäre Studien e.V. Berlin.

Landesagentur für Elektromobilität und Brennstoffzellentechnologie BadenWürttemberg $\mathrm{GmbH}$ (2015): Elektromobilität weltweit. Baden-Württemberg im internationalen Vergleich. Stuttgart.

Lange, Steffen; Santarius, Tilman (2018): Smarte grüne Welt? Digitalisierung zwischen Überwachung, Konsum und Nachhaltigkeit. München: Oekom. 
Larkin, Paul J. (2012): John Kingdon's »Three Streams« Theory and the Antiterrorism and Effective Death Penalty Act of 1996. In: Journal of Law \& Politics 28 (25), S. 25-50.

Latouche, Serge (2010): Farewell to growth. Cambridge: Polity Press.

Leach, Melissa; Bloom, Gerald; Ely, Adrian; Nightingale, Paul; Scoones, Ian; Shah, Esha; Smith, Adrian (2007): Understanding governance: pathways to sustainability. STEPS Centre. Brighton (STEPS Working Paper, 2).

Lehmann-Waffenschmidt, Marco; Reichel, Markus (2000): Kontingenz, Pfadabhängigkeit und Lock-In als handlungsbeeinflussende Faktoren der Unternehmenspolitik. In: Thomas Beschorner und Reinhard Pfriem (Hg.): Evolutorische Ökonomik und Theorie der Unternehmung. Marburg: Metropolis, S. 337-367.

Leinfelder, Reinhold (2016): Das Haus der Zukunft (Berlin) als Ort der Partizipation. Berlin.

Lepsinger, Richard; Lucia, Anntoinette D. (2009): The art and science of 360-degree feedback. San Francisco: Jossey-Bass.

Leybourn, Evan (2013): Directing the Agile Organisation. A lean approach to business management. Ely: IT Governance Publishing.

Liebowitz, Stan J.; Margolis, Stephen E. (1995): Path Dependence, Lock-in, and History. In: Journal of Law, Economics, \& Organization 11 (1), S. 205-226. DOI: 10.2139/ssrn.1706450.

Linz, Manfred (2012): Weder Mangel noch Übermaß. Warum Suffizienz unentbehrlich ist. München: Oekom.

Linz, Manfred (2013): Suffizienz - unentbehrlich für Nachhaltigkeit. In: Udo Ernst Simonis, Heike Leitschuh, Gerd Michelsen, Jörg Sommer und Ernst Ulrich von Weizsäcker (Hg.): Mut zu Visionen. Brücken in die Zukunft - Jahrbuch Ökologie 2014. 1. Auflage. Stuttgart: S. Hirzel Verlag (Jahrbuch Ökologie, 2014).

Linz, Manfred (2015): Suffizienz als politische Praxis. Ein Katalog. Wuppertal: Wuppertal Institut für Klima, Umwelt, Energie (Wuppertal spezial, 49).

Loorbach, Derk (2010): Transition Management for Sustainable Development: A Prescriptive, Complexity-Based Governance Framework. In: Governance 23 (1), S. 161-183. DOI: 10.1111/j.1468-0491.2009.01471.x.

Loske, Reinhard (2014): Von der »Energiewende« zur »Geldwende«. Transformationsstrategien für eine nachhaltige Geldordnung. Witten-Herdecke.

Lottsiepen, Gerd (2014): Raus auf die Straße. Chancen von E-Mobilität und Elektroautos. In: Politische Ökologie (137), S. 74-80.

Lund, Henrik; Werner, Sven; Wiltshire, Robin; Svendsen, Svend; Thorsen, Jan Eric; Hvelplund, Frede; Mathiesen, Brian Vad (2014): 4th Generation District Heating (4GDH): Integrating smart thermal grids into future sustainable energy systems. In: Energy (68), S. 1-11. DOI: 10.1016/j.energy.2014.02.089.

Maaß, Christian; Sandrock, Matthias; Schaeffer, Roland (2015): Fernwärme 3.0 Strategien für eine zukunftsorientierte Fernwärmepolitik. Studie im Auftrag 
der Bundestagsfraktion Bündnis 90/Die Grünen. Hamburg Institut Research. Hamburg.

Mayntz, Renate (2008): Von der Steuerungstheorie zu Global Governance. In: Gunnar Folke und Michael Zürn (Hg.): Governance in einer sich wandelnden Welt. Berlin, Heidelberg: Springer, S. 43-61.

Mayring, Philipp (2010): Qualitative Inhaltsanalyse. In: Günter Mey und Katja Mruck (Hg.): Handbuch qualitative Forschung in der Psychologie. Wiesbaden: VS Verlag für Sozialwissenschaften, S. 601-613.

McKinsey \& Company (2009): Pathways to a low-carbon economy. Version 2 of the global greenhouse gas abatement cost curve. Online verfügbar unter https:// www.mckinsey.com/business-functions/sustainability/our-insights/path ways-to-a-low-carbon-economy, zuletzt geprüft am 25.04.2019.

Messner, Dirk; Scholz, Imme (2015): Agenda 2030 und die Sustainable Development Goals: gemeinsam für das Wohlergehen aller. In: Politische Ökologie 143, S. 18-26.

Ministerium für Umwelt, Energie, Ernährung und Forsten Rheinland-Pfalz (2017): Wärmekonzept für Rheinland-Pfalz. Mainz.

Mizuho Bank (2017): The »New Normal« in China's Auto Industry. Hongkong.

Möhrle, Martin G.; Isenmann, Ralf (Hg.) (2007): Technologie-Roadmapping. Zukunftsstrategien für Technologieunternehmen. 3. Aufl. Berlin, Heidelberg.

Möhrle, Martin G.; Isenmann, Ralf (Hg.) (2017): Technologie-Roadmapping. Zukunftsstrategien für Technologieunternehmen. 4. Aufl. Berlin, Heidelberg.

Mühr, Bernhard; Kubisch, Susanne; Marx, Andreas; Stötzer, Johanna; Wisotzky, Christina; Latt, Christian et al. (2018): Dürre \& Hitzewelle Sommer 2018 (Deutschland). Report No. 1. CEDIM Forensic Disaster Analysis. Center for Disaster Management and Risk Reduction Technology. Karlsruhe.

Müller, Michael (2007): Eine Effizienzrevolution ist machbar. In: Magazin Mitbestimmung 6.

Mundt, Ingmar; Göll, Edgar (2018): Repair-Cafés. Fallstudie im Rahmen des Projekts Evolution2Green - Transformationspfade zu einer Green Economy. Institut für Zukunftsstudien und Technologiebewertung. Berlin.

National Trust (2017): Our cause. Online verfügbar unter https://www.nationaltrust.org.uk/our-cause, zuletzt geprüft am 25.04.2019.

Nationale Plattform Elektromobilität (2014): Fortschrittsbericht 2014. Bilanz der Marktvorbereitung. Berlin.

Nationale Plattform Elektromobilität (2016): Wegweiser Elektromobilität. Handlungsempfehlungen der Nationalen Plattform Elektromobilität. Berlin.

Nationale Plattform Elektromobilität (2018): Fortschrittsbericht 2018 - Markthochlaufphase. Berlin.

Naturschutzbund Deutschland (2016): Gebäude-Allianz diskutiert die Zusammenführung von EnEV und EEWärmeG. Praxisbericht zeigt zwingenden 
Handlungsbedarf in der neuen Gesetzgebung. Berlin. Online verfügbar unter https://www.nabu.de/umwelt-und-ressourcen/energie/energieeffizienzund-gebaeudesanierung/gebaeudeallianz/20657.html, zuletzt geprüft am 25.04.2019.

Nelson, Richard R. (1987): Understanding technical change as an evolutionary process. Amsterdam: North-Holland (Professor Dr. F. de Vries lectures in economics, 8).

Nelson, Richard R.; Winter, Sidney G. (1982): An Evolutionary Theory of Economic Change. Cambridge: Harvard University Press.

Neumann, Hinrich (2015): Keine Steuererleichterung für Sanierungsmaßnahmen. Topagrar online. Online verfügbar unter www.topagrar.com/news/EnergieEnergienews-1717280.html, zuletzt geprüft am 25.04.2019.

Nill, Jan (2009): Ökologische Innovationspolitik. Eine evolutorisch-ökonomische Perspektive. Marburg: Metropolis (Wirtschaftswissenschaftliche Nachhaltigkeitsforschung, 6).

Nill, Jan; Sartorius, Christian; Zundel, Stefan (2005): Zeitfenster vorbereiten, öffnen und nutzen. Strategien für eine ökologische Innovationspolitik. In: Frank Beckenbach, Ulrich Hampicke, Christian Leipert, Georg Meran, Jürg Minsch, Hans G. Nutzinger et al. (Hg.): Innovationen und Nachhaltigkeit. Marburg: Metropolis (Jahrbuch ökologische Ökonomik, 4), S. 95-122.

Nitsch, Joachim; Pregger, Thomas; Scholz, Yvonne; Naegler, Tobias; Sterner, Michael; Gerhardt, Norman et al. (2011): Langfristszenarien und Strategien für den Ausbau der erneuerbaren Energien in Deutschland bei Berücksichtigung der Entwicklung in Europa und global. Leitstudie 2010. Deutsches Zentrum für Luft- und Raumfahrt; Fraunhofer Institut für Windenergie und Energiesystemtechnik; Ingenieurbüro für neue Energien. Stuttgart, Kassel, Teltow.

Odenbach, Jan; Göll; Edgar; Behrendt; Siegfried (2017): Industrie 4.0 - digital-vernetzte dezentrale Produktion. Transformationsfeldanalyse im Rahmen des Projekts Evolution2Green - Transformationspfade zu einer Green Economy. Institut für Zukunftsstudien und Technologiebewertung. Berlin.

OECD (2011): Towards green growth. Organisation for Economic Co-operation and Development. Paris.

Oehme, Ines; Jacob, Anett (2017): Strategien gegen Obsoleszenz. Sicherung einer Produktmindestlebensdauer sowie Verbesserung der Produktnutzungsdauer und der Verbraucherinformation. Umweltbundesamt. Dessau-Roßlau (Position, November 2017).

Oekom (Hg.) (2013): Vom rechten Maß. Suffizienz als Schlüssel zu mehr Lebensglück und Umweltschutz. München: Oekom-Verlag (Politische Ökologie, 135).

Ökosystem Erde (2017): Das Zeitalter der Industrie. Der Mensch bewegt die Erde: unsere Rohstoffe. Online verfügbar unter www.oekosystem-erde.de/html/ rohstoffe.html, zuletzt geprüft am 25.04.2019. 
Overheid.nl (2016): Besluit energieprestatievergoeding huur. Online verfügbar unter http://wetten.overheid.nl/BWBRo038456/2016-09-01, zuletzt geprüft am 25.04.2019.

Paech, Niko (2012): Befreiung vom Überfluss. Auf dem Weg in die Postwachstumsökonomie. München: Oekom-Verlag.

Pal, Nirmal; Pantaleo, Daniel (Hg.) (2005): The agile enterprise: Reinventing your organization for success in an on-demand world. New York: Springer Science \& Business Media.

Pehnt, Martin; Bödecker, Jan; Arens, Marlene; Jochem, Eberhard; Idrissova, Farikha (2010): Die Nutzung industrieller Abwärme - technisch-wirtschaftliche Potenziale und energiepolitische Umsetzung. Bericht im Rahmen des Vorhabens »Wissenschaftliche Begleitforschung zu übergreifenden technischen, ökologischen, ökonomischen und strategischen Aspekten des nationalen Teils der Klimaschutzinitiative«. Institut für Energie- und Umweltforschung; Fraunhofer Institut für System- und Innovationsforschung; Institut für Ressourceneffizienz und Energiestrategien. Heidelberg, Karlsruhe.

Pehnt, Martin; Nast, Michael; Götz, Christian; Blömer, Sebastian; Barckhausen, Anton; Schröder, David et al. (2017): Wärmenetzsysteme 4.o. Endbericht. Kurzstudie zur Umsetzung der Maßnahme »Modellvorhaben erneuerbare Energien in hocheffizienten Niedertemperaturwärmenetzen«. Institut für Energie- und Umweltforschung; adelphi; PricewaterhouseCoopers; Ecofys; Agentur für Erneuerbare Energien. Heidelberg, Berlin, Düsseldorf, Köln.

Pelling, Mark (2011): Adaptation to climate change. From resilience to transformation. London: Routledge.

Perleberg, Steff; Clausen, Jens (2017): Elektromobilität in den Niederlanden. Fallstudie im Rahmen des Projekts Evolution2Green - Transformationspfade zu einer Green Economy. Borderstep Institut für Innovation und Nachhaltigkeit. Berlin.

Persson, Asa (2004): Environmental Policy Integration: An Introduction. Stockholm Environment Institute. Stockholm (PINTS - Policy Integration for Sustainability Background Paper).

Peters, B. Guy (1998): Managing Horizontal Government: The Politics of Co-Ordination. In: Public Administration 76 (2), S. 295-311. DOI: 10.1111/1467-9299.00102.

Pettigrew, Andrew (1997): What is a processual analysis? In: Scandinavian Journal of Management 13 (4), S. 337-348. DOI: 10.1016/S0956-5221(97)00020-1.

Pöhler, Daniel (2017): So unterscheiden sich Garantie und Gewährleistung. Finanztip. Online verfügbar unter https://www.finanztip.de/garantie-gewaehrleistung/, zuletzt geprüft am 25.04.2019.

Polanyi, Karl (2001): The great transformation: The political and economic origins of our time. 3. Aufl. Boston: Beacon Press. 
Pollack, Mark A.; Hafner-Burton, Emilie M. (2010): Mainstreaming international governance: The environment, gender, and IO performance in the European Union. In: The Review of International Organizations 5 (3), S. 285-313. DOI: 10.1007/s11558-010-9091-4.

Porter, Michael E. (1998): Clusters and the new economics of competition. In: Harvard Business Review 76 (6), S. 77-90.

Prakash, Siddharth; Dehoust, Günter; Gsell, Martin; Schleicher, Tobias (2016): Einfluss der Nutzungsdauer von Produkten auf ihre Umweltwirkung: Schaffung einer Informationsgrundlage und Entwicklung von Strategien gegen »Obsoleszenz«. Umweltbundesamt. Dessau-Roßlau (UBA Texte, 11/2016).

Pregernig, Michael; Böcher, Michael (2012): Normative and analytical perspectives on the role of science and expertise in environmental governance. In: Karl Hogl, Eva Kvarda, Ralf Nordbeck und Michael Pregernig (Hg.): Environmental governance: the challenge of legitimacy and effectiveness. Cheltenham: Edward Elgar Publishing, S. 199-220.

Purper, Gabriele; Neumann, Werner; Bollmann, Marcus (2015): Energieeffizienz im Wärme- und Strombereich. Bund für Umwelt und Naturschutz Deutschland. Berlin (positionen, 63).

Rechenberg, Ingo (1973): Evolutionsstrategie. Optimierung technischer Systeme nach Prinzipien der biologischen Evolution. Stuttgart: Frommann Holzboog.

Rein, Martin; Schön, Donald (1996): Frame-critical policy analysis and frame-reflective policy practice. In: Knowledge and Policy 9 (1), S. 85-104. DOI: 10.1007/ BFo2832235.

Reisch, Lucia (2002): Ist das Thema Zeitwohlstand theoriefähig? Plädoyer für einen theoriegeleiteten Zeitwohlstandsdiskurs. In: Jürgen P. Rinderspacher (Hg.): Zeitwohlstand. Ein Konzept für einen anderen Wohlstand der Nation. Berlin: Edition Sigma (Forschung aus der Hans-Böckler-Stiftung, 39), S. 37-57. Reisch, Lucia; Bietz, Sabine (2014): Zeit für Nachhaltigkeit. Zeiten der Transformation: Elemente einer Zeitpolitik für die gesellschaftliche Transformation $\mathrm{zu}$ nachhaltigeren Lebensstilen. Transformationsstrategien und Models of Change für nachhaltigen gesellschaftlichen Wandel. Umweltbundesamt. Dessau-Roßlau (UBA Texte, 68/2014).

Renault, Jean-Francois; Schwietring (2016): Übergang in eine Green Economy: Notwendige strukturelle Veränderungen und Erfolgsbedingungen für deren tragfähige Umsetzung in Deutschland. Umweltbundesamt. Dessau-Roßlau (Umwelt, Innovation, Beschäftigung, 03/2016).

Röben, Adrian; Behrendt, Siegfried (2018): Reparaturwerkstätten - das Beispiel der Vangerow GmbH. Fallstudie im Rahmen des Projekts Evolution2Green Transformationspfade zu einer Green Economy. Institut für Zukunftsstudien und Technologiebewertung. Berlin. 
Rockström, Johan; Steffen, Will; Noone, Kevin; Persson, Asa; Chapin, F. Stuart, III; Lambin, Eric et al. (2009): Planetary Boundaries: Exploring the Safe Operating Space for Humanity. In: Ecology and Society 14 (2), S. 32. DOI: 10.5751/ ES-03180-140232.

Rogers, Everett M. (2003): Diffusion of innovations. New York, London, Toronto, Sydney: Free Press.

Romare, Mia; Dahllöf, Lisbeth (2017): The Life Cycle Energy Consumption and Greenhouse Gas Emissions from Lithium-Ion Batteries. A Study with Focus on Current Technology and Batteries for light-duty vehicles. Swedish Environmental Research Institute. Stockholm.

Rosa, Hartmut (2012): Weltbeziehungen im Zeitalter der Beschleunigung. Umrisse einer neuen Gesellschaftskritik. Berlin: Suhrkamp (Suhrkamp-Taschenbuch Wissenschaft).

Rosenmann, Amir; Reese, Gerhard; Cameron, James E. (2016): Social Identities in a Globalized World: Challenges and Opportunities for Collective Action. In: Perspectives on psychological science : a journal of the Association for Psychological Science 11 (2), S. 202-221. DOI: 10.1177/1745691615621272.

Runst, Petrik; Ohlendorf, Jana (2015): Die Rolle des Handwerks auf dem Weg zu einem klimaneutralen Gebäudebestand. Deutsches Handwerksinstitut. Göttingen (Göttinger Beiträge zur Handwerksforschung, 1).

Ruschkowski, Eick von (2002): Lokale Agenda 21 in Deutschland - eine Bilanz. In: Aus Politik und Zeitgeschichte 52 (31-32), S. 17-24.

Rydin, Yvonne; Pennington, Mark (2000): Public participation and local environmental planning: the collective action problem and the potential of social capital. In: Local environment 5 (2), S. 153-169. DOI: 10.1080/13549830050009328.

Sabatier, Paul A. (1993): Advocacy-Koalitionen, Policy-Wandel und Policy-Lernen: Eine Alternative zur Phasenheuristik. In: Adrienne Héritier (Hg.): Policy-Analyse: Kritik und Neuorientierung. Wiesbaden: VS Verlag für Sozialwissenschaften, S. 116-148.

Sabatier, Paul A. (1998): The advocacy coalition framework: revisions and relevance for Europe. In: Journal of European Public Policy 5 (1), S. 98-130. DOI: 10.1080/13501768880000051.

Sachverständigenrat für Umweltfragen (2016): Umweltgutachten 2016. Impulse für eine integrative Umweltpolitik. Berlin.

Santarius, Tilman (2012): Der Rebound-Effekt - Über die unerwünschten Folgen der erwünschten Energieeffizienz. In: Impulse zur Wachstumswende (5).

Schein, Edgar H. (1985): Organizational culture and leadership. San Francisco: Jossey-Bass.

Schein, Edgar H. (1995): Unternehmenskultur. Ein Handbuch für Führungskräfte. Frankfurt a. M.: Campus-Verlag. 
Schels, Helmut (2008): Vereinfachte Clusteranalyse mit Excel. Clusteranalyse mit Excel nach einer der hierarchischen Methoden (Single-Linkage) - Kurzbeschreibung. Verband deutscher Städtestatistiker - AG Methodik.

Schmelzer, Matthias; Passadakis, Alexis (2011): Postwachstum. Krise, ökologische Grenzen und soziale Rechte. Hamburg: VSA-Verl. (AttacBasisTexte, 36).

Schmidt, Mario (2008): Die Bedeutung der Effizienz für Nachhaltigkeit - Chancen und Grenzen. In: Susanne Hartard, Axel Schaffer und Jürgen Giegrich (Hg.): Ressourceneffizienz im Kontext der Nachhaltigkeitsdebatte. 1. Aufl. 2008. Baden-Baden: Nomos.

Schmidt-Bleek, Friedrich (2014): Grüne Lügen. Nichts für die Umwelt, alles fürs Geschäft - wie Politik und Wirtschaft die Welt zugrunde richten. München: Ludwig Verlag.

Schmitt, Corinna; Leuser, Leon; Brischke, Lars-Arvid; Duscha, Markus; Jacobsen, Sirkka (2015): Suffizienz-Maßnahmen und -Politiken in kommunalen Klimaschutzkonzepten und Masterplänen. Ein Überblick. Institut für Energie- und Umweltforschung Heidelberg. Heidelberg, Berlin.

Schneider, Cédric; Veugelers, Reinhilde (2010): On young highly innovative companies: why they matter and how (not) to policy support them. In: Industrial and Corporate Change 19 (4), S. 969-1007. DOI: 10.1093/icc/dtp052.

Schneidewind, Uwe; Zahrnt, Angelika (2013): Jenseits der Steigerungslogik. In: Politische Ökologie (135), S. 115-121.

Schneller, Andreas; Frank, Leonard; Kahlenborn, Walter (2018): Wärmenetze 4.0 im Kontext der Wärmewende. Politische Handlungsempfehlungen für eine klimafreundliche Fernwärmeversorgung. adelphi. Berlin.

Schneller, Andreas; Kahlenborn, Walter (2018): Klimaschutz und soziale Belange lokal denken. Vier Handlungsfelder für die erfolgreiche Ausgestaltung sozialverträglichen Klimaschutzes auf kommunaler Ebene. adelphi. Berlin (Policy Paper, 02/2018).

Schuh, Günther (2013): Lean Innovation. Berlin, Heidelberg: Springer.

Schulz, Christian; Affolderbach, Julia (2015): Grünes Wachstum und alternative Wirtschaftsformen. In: Geographische Rundschau 67 (5), S. 4-9.

Schumpeter, Joseph A. (1983): The theory of economic development: An inquiry into profits, capital, credit, interest, and the business cycle. New Brunswick: Transaction Books.

Sharman, Amelia; Holmes, John (2010): Evidence-based policy or policy-based evidence gathering? Biofuels, the EU and the $10 \%$ target. In: Environmental Policy and Governance 20 (5), S. 309-321. DOI: 10.1002/eet.543.

Shove, Elizabeth; Pantzar, Mika; Watson, Matt (2012): The dynamics of social practice. Everyday life and how it changes. Los Angeles: SAGE. 
Simonis, Udo Ernst (2004): Ökologische Modernisierung der Industriegesellschaft. In: Zukunftsforschung im Spannungsfeld von Visionen und Alltagshandeln. In: IZT Werkstattbericht 64, S. 29-46.

Smith, Adrian; Raven, Rob (2012): What is protective space? Reconsidering niches in transitions to sustainability. In: Research Policy 41 (6), S. 1025-1036. DOI: 10.1016/j.respol.2011.12.012.

Sommer, Bernd; Welzer, Harald (2014): Transformationsdesign. Wege in eine zukunftsfähige Moderne. München: oekom verlag (Transformationen, Band 1).

Spangenberg, Joachim (2013): Ökonomie und Nachhaltigkeit. Konferenz »Nachhaltigkeit - Verantwortung für eine begrenzte Welt«. Universität Göttingen, 23.11.2013. Online verfügbar unter www.academia.edu/5176531/\%C3\%96kono mie_und_Nachhaltigkeit, zuletzt geprüft am 25.04.2019.

Spash, Clive L. (2001): Broadening democracy in environmental policy processes. In: Environment and Planning C: Government and Policy 19 (4), S. 475-481.

Späth, Philipp; Rohracher, Harald (2010): `Energy regions‘. The transformative power of regional discourses on socio-technical futures. In: Research Policy 39 (4), S. 449-458.

Specht, Dieter; Behrens, Stefan (2002): Strategische Planung mit Roadmaps Möglichkeiten für das Innovationsmanagement und die Personalbedarfsplanung. In: Martin G. Möhrle und Ralf Isenmann (Hg.): Technologie-Roadmapping. Berlin, Heidelberg: Springer.

Statista (2018a): Statistiken zu eBay. Online verfügbar unter https://de.statista. com/themen/1278/ebay/, zuletzt geprüft am 25.04.2019.

Statista (2018b): Statistiken zu Tesla. Online verfügbar unter https://de.statista. com/themen/2418/tesla-motors/, zuletzt geprüft am 25.04.2019.

Statista (2019): Verteilung der Haushalte in Deutschland nach Miete und Eigentum von 1998 bis 2013. Online verfügbar unter https://de.statista.com/statis tik/daten/studie/237719/umfrage/verteilung-der-haushalte-in-deutschlandnach-miete-und-eigentum/, zuletzt geprüft am 25.04.2019.

Statistisches Bundesamt (2019): Konsumausgaben privater Haushalte. Online verfügbar unter https://www.destatis.de/DE/ZahlenFakten/Indikatoren/Lange Reihen/LebensunterhaltKonsum/lrlebo3.html, zuletzt geprüft am 18.01.2019.

Steffen, Will; Persson, Asa; Deutsch, Lisa; Zalasiewicz, Jan; Williams, Mark; Richardson, Katherine et al. (2011): The Anthropocene: From Global Change to Planetary Stewardship. In: AMBIO (40), S. 739-751. DOI: 10.1007/s13280-0110185-X.

Steffen, Will; Richardson, Katherine; Rockström, Johan; Cornell, Sarah E.; Fetzer, Ingo; Bennett, Elena M. et al. (2015): Planetary boundaries: Guiding human development on a changing planet. In: Science 347 (6223), S. 1259855. DOI: 10.1126/science.1259855. 
Stengel, Oliver (2011): Suffizienz. Dissertation (Wuppertaler Schriften zur Forschung für eine nachhaltige Entwicklung, 1).

Stieber, Benno (2017): Grüß Gott, Sie sind unsere Zukunft. In: Die Zeit, 31.08.2017 (36).

Stiftung Warentest (2012): Guter Rat ist rar. In: test (5), S. 60-63.

Stroomversnelling (2015): 4,5 miljoen woningen naar Nul op de Meter. Utrecht.

Tagscherer, Ulrike (2012): Electric mobility in China - A policy review. Fraunhofer Institut für System- und Innovationsforschung. Karlsruhe (Fraunhofer ISI Discussion Papers Innovation Systems and Policy Analysis, 30).

Tappeser, Valentin (2018a): Energiesprong Niederlande. Standardisierung und Skalierung energetischer Sanierungmaßnahmen im industriellen Maßstab. Fallstudie im Rahmen des Projekts Evolution2Green - Transformationspfade zu einer Green Economy. adelphi. Berlin.

Tappeser, Valentin (2018b): Plusenergiegemeinde Wüstenrot. Fallstudie im Rahmen des Projekts Evolution2Green - Transformationspfade zu einer Green Economy. adelphi. Berlin.

Tappeser, Valentin; Chichowitz, Lisa (2017a): Pestizideinsatz in der Landwirtschaft.

Transformationsfeldanalyse im Rahmen des Projekts Evolution2Green Transformationspfade zu einer Green Economy. adelphi. Berlin.

Tappeser, Valentin; Chichowitz, Lisa (2017b): Stickstoffeintrag der Landwirtschaft.

Transformationsfeldanalyse im Rahmen des Projekts Evolution2Green Transformationspfade zu einer Green Economy. adelphi. Berlin.

Tappeser, Valentin; Chichowitz, Lisa (2017c): Umgang mit Altfahrzeugen. Transformationsfeldanalyse im Rahmen des Projekts Evolution2Green - Transformationspfade zu einer Green Economy. adelphi. Berlin.

Tappeser, Valentin; Chichowitz, Lisa (2017d): Wärmeenergieverbrauch und Sanierungsbedarf von Gebäuden. Transformationsfeldanalyse im Rahmend des Projekts Evolution2Green - Transformationspfade zu einer Green Economy. adelphi. Berlin.

Tappeser, Valentin; Fromm, Carina (2017a): Wärmenetze in Dänemark. Fallstudie im Rahmen des Projekts Evolution2Green - Transformationspfade zu einer Green Economy. adelphi. Berlin.

Tappeser, Valentin; Fromm, Carina (2017b): Wärmenetze in Dänemark. Fallstudie im Rahmen des Projekts Evolution2Green - Transformationspfade zu einer Green Economy. adelphi. Berlin.

Tappeser, Valentin; Fromm, Carina (2018a): Climate Smart Hyllie. Fallstudie im Rahmen des Projekts Evolution2Green - Transformationspfade zu einer Green Economy. adelphi. Berlin.

Tappeser, Valentin; Fromm, Carina (2018b): InnovationCity Ruhr - Modellstadt Bottrop. Fallstudie im Rahmen des Projekts Evolution2Green - Transformationspfade zu einer Green Economy. adelphi. Berlin. 
Tappeser, Valentin; Kohlmorgen, Nina; Marsden, Katrina (2017): Verlust Grüner Infrastruktur in der Landwirtschaft. Transformationsfeldanalyse im Rahmen des Projekts Evolution2Green - Transformationspfade zu einer Green Economy. adelphi. Berlin.

Techem: Energiekennwerte 2016. Online verfügbar unter https://www.techem.de/ ueber_techem/klimaschutz_und_digitalisierung/klimaschutz/energiekenn werte.html, zuletzt geprüft am 28.01.2019.

Thamling, Nils; Pehnt, Martin; Kirchner, Joachim (2015): Hintergrundpapier zur Energieeffizienzstrategie Gebäude. Erstellt im Rahmen der Wissenschaftlichen Begleitforschung zur Erarbeitung einer Energieeffizienzstrategie Gebäude. Prognos; Institut für Energie- und Umweltforschung; Institut für Wohnen und Umwelt. Berlin, Heidelberg, Darmstadt.

The Japan Times (2018): Japan government-backed project aims to bolster development of next-generation battery. Online verfügbar unter https://www.japan times.co.jp/news/2018/06/15/business/japan-government-backed-projectaims-bolster-development-next-generation-battery/\#.XDMQZ3RKiUk, zuletzt geprüft am 25.04.2019.

Tiwari, Rajnish; Herstatt, Cornelius (2014): Frugale Innovationen: Analogieeinsatz als Erfolgsfaktor in Schwellenländern. In: Cornelius Herstatt, Katharina Kalogerakis und Marc Schulthess (Hg.): Innovationen durch Wissenstransfer. Mit Analogien schneller und kreativer Lösungen entwickeln. Wiesbaden: Springer Gabler, S. 83-107.

Tongur, Stefan; Engwall, Mats (2017): Exploring window of opportunity dynamics in infrastructure transformation. In: Environmental Innovation and Societal Transitions 25, S. 82-93. DOI: 10.1016/j.eist.2016.12.003.

Toshiba Corp Japan (03.10.2017): Toshiba Develops Next-Generation Lithium-ion Battery with New Anode Material. Online verfügbar unter https://www.toshi ba.co.jp/about/press/2017_10/pro301.htm, zuletzt geprüft am 25.04.2019.

Tripp, Steven D.; Bichelmeyer, Barbara (1990): Rapid prototyping: An alternative instructional design strategy. In: Educational Technology Research and Development 38 (1), S. 31-44. DOI: 10.1007/BFo2298246.

Tsebelis, George (2002): Veto players. How political institutions work. Princeton: Princeton University Press.

Tuschinski, Melita (2016): EnEV 2017 - Was kommt wann? Schritt für Schritt zur Novelle der Energieeinsparverordnung. Institut für Energie-Effiziente Architektur mit Internet-Medien. Stuttgart.

Umweltbundesamt (2018): Energieverbrauch für fossile und erneuerbare Wärme. Dessau-Roßlau. Online verfügbar unter https://www.umweltbundesamt.de/ daten/energie/energieverbrauch-fuer-fossile-erneuerbare-waerme\#textpart-1, zuletzt geprüft am 25.04.2019. 
UN (1992): Agenda 21. Konferenz der Vereinten Nationen für Umwelt und Entwicklung. Vereinte Nationen. Rio de Janeiro. Online verfügbar unter www.un.org/ Depts/german/conf/agenda21/agenda_21.pdf, zuletzt geprüft am 25.01.2019.

UNEP: Decoupling 2. Technologies, opportunities and policy options. United Nations Environment Programme. Paris.

UNEP (2011): Towards a Green Economy: Pathways to Sustainable Development and Poverty Eradication. United Nations Environment Programme. Paris.

Unmüßig, Barbara; Sachs, Wolfgang; Fatheuer, Thomas (2012): Kritik der grünen Ökonomie. Impulse für eine sozial und ökologisch gerechte Zukunft. Berlin: Heinrich-Böll-Stiftung (Schriften zur Ökologie, 22).

Unruh, Gregory C. (2000): Understanding carbon lock-in. In: Energy Policy 28 (12), S. 817-830. DOI: 10.1016/S0301-4215(00)00070-7.

Valentine-Urbschat, Michael; Valentine-Urbschat, Nancy (2014): Elektrisiert. München: Valentine-Urbschat.

Verband der Automobilindustrie (2018): Angaben zu Forschungausgaben, Umsätzen und Beschäftigten in der Automobilwirtschaft. Online verfügbar unter https://www.vda.de/de/services/zahlen-und-daten/jahreszahlen/allgemeines. html, zuletzt geprüft am 25.04.2019.

Verbraucherzentrale Bundesverband (06.06.2017): Elektroschrott verhindern- Reparaturen fördern. Online verfügbar unter https://www.vzbv.de/pressemit teilung/elektroschrott-verhindern-reparaturen-foerdern, zuletzt geprüft am 25.04.2019.

Vergne, Jean-Philippe; Durand, Rodolphe (2010): The missing link between the theory and empirics of path dependence. Conceptual clarification, testability issue, and methodological implications. In: Journal of Management Studies 47 (4), S. 736-759. DOI: 10.1111/j.1467-6486.2009.00913.x.

Walk, Heike (2008): Partizipative Governance. Beteiligungsformen und Beteiligungsrechte im Mehrebenensystem der Klimapolitik. Wiesbaden: VS Verlag für Sozialwissenschaften.

Wallbrecher, Hartmut (2008): Grundlagen der Betriebswirtschaftslehre Marketing. 6. Aufl. Berlin.

Weible, Christopher M.; Sabatier, Paul A.; McQueen, Kelly (2009): Themes and variations: Taking stock of the advocacy coalition framework. In: Policy Studies Journal 37 (1), S. 121-140. DOI: 10.1111/j.1541-0072.2008.00299.x.

Weiß, Ralf (2015): Green Economy Gründungsmonitor 2014. Grüne Wirtschaft als Gründungs- und Beschäftigungsmotor. Borderstep Institut für Innovation und Nachhaltigkeit. Berlin.

Weizsäcker, Ernst Ulrich von; Hargroves, Karlson; Smith, Michael H.; Desha, Cheryl; Stasinopoulos, Peter (2010): Faktor Fünf. Die Formel für nachhaltiges Wachstum. München: Droemer. 
Weizsäcker, Ernst Ulrich von; Lovins, Amory B.; Lovins, L. Hunter (1995): Faktor Vier. Doppelter Wohlstand - halbierter Naturverbrauch. Der neue Bericht an den Club of Rome. München: Droemer Knaur.

Welzer, Harald (2011): Mentale Infrastrukturen. Wie das Wachstum in die Welt und in die Seelen kam. Berlin: Heinrich-Böll-Stiftung (Schriften zur Ökologie, 14).

Welzer, Harald (2019): Alles könnte anders sein. Eine Gesellschaftsutopie für freie Menschen. Frankfurt a. M.: S. Fischer.

Wieser, Harald; Tröger, Nina (2015): Die Nutzungsdauer und Obsoleszenz von Gebrauchsgütern im Zeitalter der Beschleunigung. Kammer für Arbeiter und Angestellte für Wien. Wien.

Wild, Reiner (2017): Mieterhöhungen nach Modernisierungen und Energieeinsparung. Empirische Kurzstudie über 200 Maßnahmen im Berliner Mietwohngebäudebestand. Berliner Mieterverein e. V. Berlin.

Wilkens, Andreas (2017): Elektromobilität ist für den Automobil-Präsidenten sehr wichtig für den Standort Deutschland. Heise Online. Online verfügbar unter https:/www.heise.de/newsticker/meldung/Elektromobilitaet-ist-fuer-denAutomobil-Praesidenten-sehr-wichtig-fuer-den-Standort-Deutschland-3709315. html, zuletzt geprüft am 25.04.2019.

Wissenschaftlicher Beirat der Bundesregierung Globale Umweltveränderungen (2011): Welt im Wandel. Gesellschaftsvertrag für eine große Transformation. Hauptgutachten. Berlin.

Wolff, Franziska; Heyen, Dirk Arne; Brohmann, Bettina; Grießhammer, Rainer; Jakob, Klaus; Graaf, Lisa (2018): Transformative Umweltpolitik: Nachhaltige Entwicklung konsequent fördern und gestalten. Ein Wegweiser für den Geschäftsbereich des BMU. Umweltbundesamt. Dessau-Roßlau.

Wolling, Jens; Arlt, Dorothee (2014): Fukushima und die Folgen - Medienberichterstattung, Öffentliche Meinung, Politische Konsequenzen. Ilmenau: Universitätsverlag Ilmenau (NEU - Nachhaltigkeits-, Energie- und Umweltkommunikation, 2).

Wuppertal Institut (Hg.) (2005): Fair future. Begrenzte Ressourcen und globale Gerechtigkeit. Ein Report. Wuppertal Institut für Klima, Umwelt, Energie. München: Beck.

Young, Oran R.; Berkhout, Frans; Gallopin, Gilberto C.; Janssen, Marco A.; Ostrom, Elinor; van der Leeuw, Sander (2006): The globalization of socio-ecological systems: An agenda for scientific research. In: Global Environmental Change 16 (3), S. 304-316. DOI: 10.1016/j.gloenvcha.2006.03.004.

Zeit Online: Regierung gegen höhere Steuer auf Fleisch. Online verfügbar unter https:/www.zeit.de/wirtschaft/2017-o1/christian-schmidt-klimaschutzfleischsteuer-milchsteuer, zuletzt geprüft am 25.04.2019. 
Zimmermann, Lars (2018): Open Source und Empowerment der Nutzer und Verbraucher. Zukunfts-werkstatt Nachhaltige Wirtschafts-und Lebensweise Lösungsansätze, Runder Tisch Reparatur. Berlin, 18.01.2018.

Zwiers, Jakob; Behrendt, Siegfried; Göll, Edgar (2018): Fairphone. Fallstudie im Rahmen des Projekts Evolution2Green - Transformationspfade zu einer Green Economy. Institut für Zukunftsstudien und Technologiebewertung. Berlin. 


\section{Autorinnen und Autoren der Kapitel}

\section{Kapitel}

2. Einleitung

3.1 Pfadabhängigkeiten

3.2 Models of Change

3.3 Effizienz, Konistenz, Suffizienz

3.4 Übergreifendes Systemmodell

4.1 Pfadabhängigkeiten

4.2 Erfolgsfaktoren

5.1 Pfadwechselkonzeption

5.2 Roadmap Wärme

5.3 Roadmap E-Mobilität

5.4 Roadmap Produkte länger nutzen

6. Fazit

\section{Autorinnen und Autoren}

Walter Kahlenborn, Daniel Weiss

Jens Clausen, Klaus Fichter

Walter Kahlenborn, Valentin Tappeser

Siegfried Behrendt, Edgar Göll

Valentin Tappeser, Walter Kahlenborn

Jens Clausen, Klaus Fichter

Valentin Tappeser, Walter Kahlenborn, Daniel Weiss

Edgar Göll

Andreas Schneller

Jens Clausen

Siegfried Behrendt, Edgar Göll

Walter Kahlenborn, Daniel Weiss, Linda Hölscher 


\section{Soziologie}

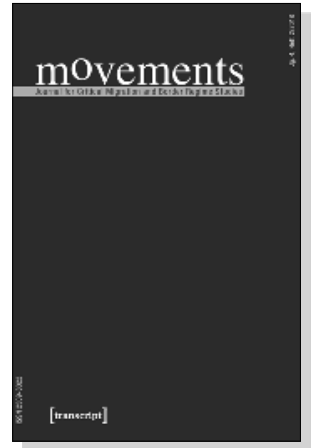

Juliane Karakayali, Bernd Kasparek (Hg.)

movements.

Journal for Critical Migration and Border Regime Studies

Jg. 4, Heft 2/2018

Februar 2019, 246 S., kart.

24,99 €(DE), 978-3-8376-4474-6

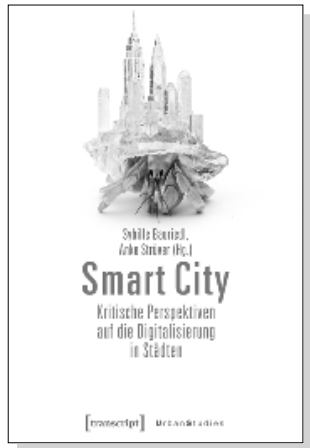

Sybille Bauriedl, Anke Strüver (Hg.)

\section{Smart City -}

Kritische Perspektiven auf die Digitalisierung in Städten

2018, 364 S., kart.

29,99€ (DE), 978-3-8376-4336-7

E-Book: 26,99 € (DE), ISBN 978-3-8394-4336-1

EPUB: $26,99 €(D E)$, ISBN 978-3-7328-4336-7

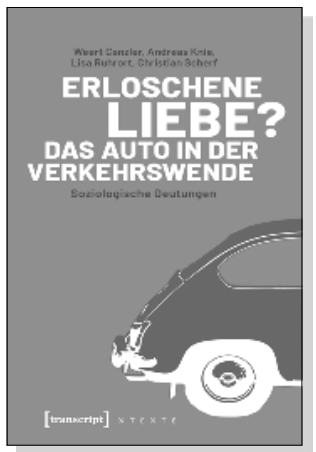

Weert Canzler, Andreas Knie, Lisa Ruhrort, Christian Scherf Erloschene Liebe?

Das Auto in der Verkehrswende

Soziologische Deutungen

2018, 174 S., kart., zahlr. Abb.

$19,99 €(D E), 978-3-8376-4568-2$

E-Book: $17,99 €(D E)$, ISBN 978-3-8394-4568-6

EPUB: $17,99 €(D E)$, ISBN 978-3-7328-4568-2 


\section{Soziologie}

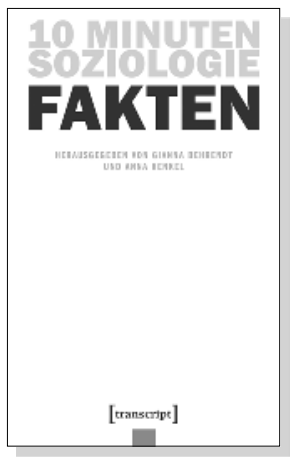

Gianna Behrendt, Anna Henkel (Hg.)

10 Minuten Soziologie: Fakten

2018, 166 S., kart.

$16,99 €(D E), 978-3-8376-4362-6$

E-Book: 14,99 € (DE), ISBN 978-3-8394-4362-0

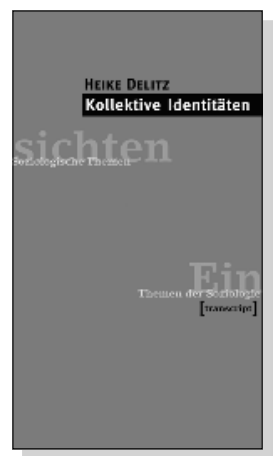

Heike Delitz

Kollektive Identitäten

2018, 160 S., kart.

$14,99 €(D E), 978-3-8376-3724-3$

E-Book: 12,99 € (DE), ISBN 978-3-8394-3724-7

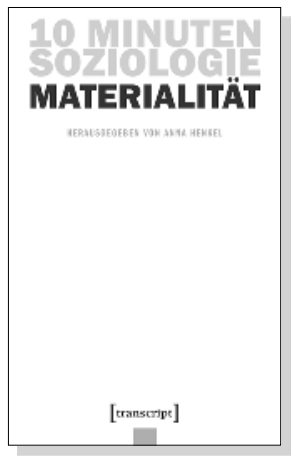

Anna Henkel (Hg.)

10 Minuten Soziologie: Materialität

2018, 122 S., kart.

$15,99 €(D E), 978-3-8376-4073-1$

E-Book: 13,99 €(DE), ISBN 978-3-8394-4073-5 
